2042

SÉMITISMES ET LATINISMES

DANS LE

PASTEUR D'HERMAS

A. HILHORST 

SEMITISMES ET LATINISMES

DANS LE PASTEUR D'HERMAS 
PROMOTOR:

PROF. DR. CHR. A. E. M. MOHRMANN 


\section{SÉMITISMES ET LATINISMES \\ DANS LE PASTEUR D'HERMAS}

Proefschrift

ter verkrijging van de graad van

doctor in de letteren

aan de Katholieke Universiteit

te Nijmegen,

op gezag van de rector magnificus

Prof. Dr. A. J. H. Vendrik

volgens besluit van het college van decanen

in het openbaar te verdedigen op

donderdag 24 juni 1976

des namiddags te 2 uur precies door

ANTONIUS HILHORST

geboren te Soestdijk 
La préparation de cet ouvrage a été rendue possible par l'appui financier de l'Organisation Néerlandaise pour le développement de la recherche scientifique (Z.W.O.). 
A MES PARENTS 

TABLE DES MATIERES

PREFACE

I. HISTOIRE DU PROBLÈME

I. L'élément latin . . . . . . . . . . . . . . . I

2. L'élément sémitique .. . . . . . . . . . 8

3. Remarques générales . . . . . . . . . . . II

II. QUESTIONS PRELIMINAIRES . . . . . . . . . . . . . . I5

I. Le texte . . . . . . . . . . . . . . . . I5

2. Un ou plusieurs auteurs? . . . . . . . . . . . I9

3. Lieu et date d'origine . . . . . . . . . . . . 3 I

III. SÉMITISMES ET LATINISMES . . . . . . . . . . . . 36

r. Phénomènes d'interférence . . . . . . . . . 36

2. Sémitismes . . . . . . . . . . . . 38

3. Latinismes . . . . . . . . . . . . . . . 45

4. Hermas . . . . . . . . . . . . . . . 47

5. Méthode à suivre . . . . . . . . . . . 5 I

IV. LE VERBE . . . . . . . . . . . . . . . . . 57

I. Le parfait . . . . . . . . . . . . . . . . 57

2. Le plus-que-parfait . . . . . . . . . . . 60

3. L'absence d'optatifs . . . . . . . . . . . . 6r 


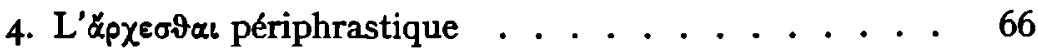

5. Périphrase avec elval + participe présent . . . . . 70

6. Elvaisis . . . . . . . . . . . . . . . . 74

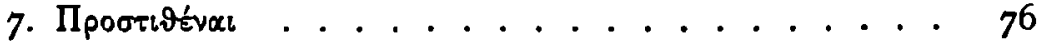

8. Formules avec des verbes signifiant dire . . . . . . 77

v. PREPOSITIONS . . . . . . . . . . . . . . 82

I. Le $\varepsilon v$ instrumental . . . . . . . . . . . 82

2. Le sjép comparatif . . . . . . . . . . . . . . 89

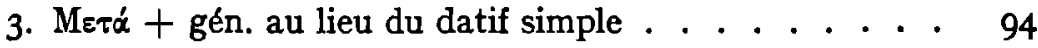

A. Merá + gén. au lieu du datif sociatif . . . . . . . 94

B. Merá + gén. «à l'égard de . . . . . . . . . . Ioo

4. Prépositions impropres . . . . . . . . . . . 104

A. 'O $\pi l \sigma \omega+$ gén . . . . . . . . . . . . 104

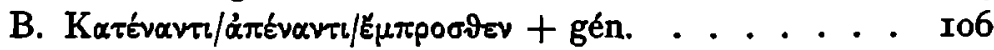

VI. AUTRES PHENOMÈNES SYNTAXIQUES . . . . . . . . . . IIO

I. Le "génitif hébraique* . . . . . . . . . . . IIo

2. Le redoublement distributif . . . . . . . . . II3

3. La parataxe dans les subordonnées . . . . . . . II6

4. Quelques latinismes supposés . . . . . . . . . I22

VII. MOTS ET EXPRESSIONS . . . . . . . . . . . . I26

I. Le pluriel oủpavol . . . . . . . . . . . . 126

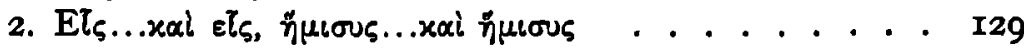

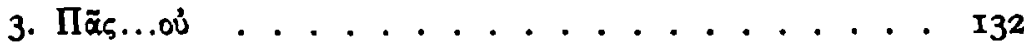

4. Le $\dot{\omega}$ comparatif . . . . . . . . . . . . . . . . I34

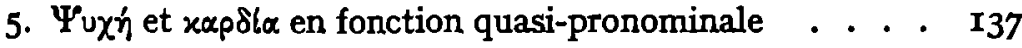

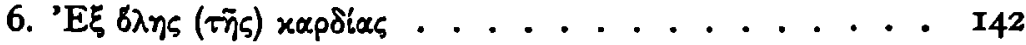

7. Ol viol $\tau \tilde{\eta} s$ ávoulas . . . . . . . . . . . . . . . I44

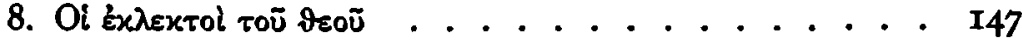

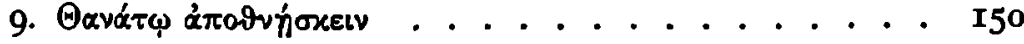

Io. Kai isoú . . . . . . . . . . . . . . . . I53

II. L'apport sémitique dans le vocabulaire . . . . . . I55

I2. Quelques latinismes supposés . . . . . . . . I60 
VIII. EMPRUNTS . . . . . . . . . . . . . . . . I63

I. Introduction . . . . . . . . . . . . I63

2. Hermas . . . . . . . . . . . . . . . I65

3. $\Sigma_{\tau \alpha \tau i \omega \nu}$. . . . . . . . . . . . . . . . I68

4. Oủat . . . . . . . . . . . . . . . . I79

CONCLUSIONS . . . . . . . . . . . . . . . . I83

INDICES . . . . . . . . . . . . . . . . . . I87

Index des mots . . . . . . . . . . . . . . . . I87

Index des citations . . . . . . . . . . . . . Igo

Index analytique . . . . . . . . . . . . . 207 

La langue du Pasteur d'Hermas comporte une série de particularités qui remontent soit à l'hébreu et à l'araméen soit au latin. Certains de ces éléments étrangers ont déjà fait l'objet de recherches, mais il manquait jusqu'à présent une étude portant sur l'ensemble du phénomène. Nous voudrions ici essayer de combler cette lacune en répondant aux deux questions qui se posent: quels phénomènes faut-il considérer comme des sémitismes ou des latinismes? comment expliquer leur présence dans le Pasteur?

Nous citons d'après le texte de Whittaker, paru dans le Corpus de Berlin $\left(\mathbf{I} 967^{2}\right)$, sans négliger pour autant l'édition de Joly, dans les Sources Chrétiennes (1968²). Les sigles des textes-témoins sont aussi ceux employés par Whittaker, excepté pour le Codex Sinaiticus, que nous indiquons par S. Sauf indication contraire, nous citons l'Ancien Testament d'après la Septante, dans l'édition de Rahlfs; pour le Nouveau Testament, nous avons utilisé l'édition de Nestle-Aland $\left(\mathrm{I} 963^{25}\right)$. Pour les papyrus et les inscriptions, nous nous servons des abréviations de Liddell-Scott-Jones, Lexicon et Supplement; IGUR signifie L. Moretti, Inscriptiones graecae urbis Romae, Romae I968sq., CMRDM, E. Lane, Corpus monumentorum religionis dei Menis, Leiden I97rsq. Pour les références bibliographiques, nous nous sommes permis d'utiliser trois abréviations: BD pour la Grammatik des neutestamentlichen Griechisch de Blass-Debrunner, SD pour la Syntax de SchwyzerDebrunner et ThWNT pour le Theologisches Wörterbuch zum Neuen Testament de Kittel. A partir du chapitre III, nous employons constamment le terme "apocryphes»: nous visons par là un certain nombre de textes énumérés dans le paragraphe 5 de ce chapitre.

Enfin, qu'il me soit permis d'exprimer ici ma reconnaissance à tous ceux, maîtres, spécialistes, amis, qui, chacun à leur manière, ont contribué à la réalisation de cet ouvrage. Il m'est impossible de les nommer tous. Je voudrais pourtant faire une exception pour ma femme, Caroline, qui, par son jugement, par la part qu'elle a prise à mes travaux et par sa patience, m'a permis de mener à bience travail. 



\section{EDITIONS, COMMENTAIRES}

AbBadie, A. D', Hermae Pastor, asthiopice primum edidit et aethiopica latine vertit, Lipsiae 1860.

ANGer, R. -Dindorf, Guil., Hermae Pastor graece, Lipsiae 1856.

Bonner, C., A papyrus codex of the Shepherd of Hermas (Similitudes 2-9) with a fragment of the Mandates, Ann Arbor 1934.

Dibelius, M., Der Hirt des Hermas erklärt, Tübingen 1923.

Dressel, A. R. M., Patrum A postolicorum Opera, Lipsiae $1857^{1}, 1863^{2}$.

Funk, F. X., Opera Patrum Apostolicorum, I, Tubingae 1878 .

-, Patres Apostolici, I, Tubingae $190 \mathrm{r}$.

GebhardT, O. DE-HARNACK, A., Hermae Pastor graece, addita versione latina recentiore e codice Palatino, Lipsiae 1877.

Hilgenfeld, A., Hermae Pastor graece, Lipsiae $1866^{1}, \mathrm{I}_{88 \mathrm{I}^{2}}$.

- Hermae Pastor, veterem latinam interpretationem e codicibus edidit, Lipsiae 1873 .

-, Hermae Pastor graece integrum ambitu, Lipsiae 1887.

JoLy, R., Hermas, Le Pasteur, Paris 19581, 1968².

LAKE, K., Facsimiles of the Athos fragments of the Shepherd of Hermas, Oxford 1907.

-, Codex Sinaiticus Petropolitanus, Oxford I9II.

-, The Apostolic Fathers, II, London Igr3.

Lefort, L.-Th., Les Peres apostoliques en copte, Louvain 1952.

Lelong, A., Le Pasteur d'Hermas, Paris 1912.

Lightroot, J. B.-Harmer, J. R., The Apostolic Fathers, London 1891'1, 1893". SNyder, G. F., The Shepherd of Hermas, Camden, N.J. 1968.

Tischendorf, AE. Fr. C., Hermae Pastor graece, Lipsiae 1856.

Whittaker, M., Der Hirt des Hermas, Berlin 19561, $1967^{2}$.

\section{GRAMM A LRES, DICTIONNAIRES, INDICES}

AgeL, F.-M., Grammaire du grec biblique suivie d'un choix de papyrus, Paris 1927. BaUer, W., Griechisch-Deutsches Wörterbuch zu den Schriften des Neuen Testaments und der übrigen urchristlichen Literatur, Berlin $195^{5}$.

Blass, Fr., Grammatik des Neutestamentlichen Griechisch, Göttingen 1896.1 Blass, Fr.-Debrunner, A., Grammatik des neutestamentlichen Griechisch, Göttingen $1913^{4}, 193^{\circ}, 1954^{\circ}$. [BD]

GeSENIUS, W.-KAUTzSCH, E., Hebräische Grammatik, Leipzig 19098.

Goodspeed, E. J., Index patristicus sive clavis Patrum Apostolicorum operum, Lipsiae $1907^{1}$, Naperville, Ill. $1960^{2}$. 
Hatch, E.-Redpath, H. A., A concordance to the Septuagint and the other Greek versions of the Old Testament, Oxford 1897-1906.

JANNARIS, A. N., An historical Greek grammar, London 1897.

Joüon, P., Grammaire de l'hébreu biblique, Rome $1947^{2}$.

Kittel, G.-Friedrich, G., Theologisches Wöterbuch zum Neuen Tetament, Stuttgart I933sq. [ThWNT]

KoeHLer, L.-BaUmgartner, W., Hebräisches und aramäisches Lexikon zum Alten Testament, Leiden 1967sq.

Kraft, H., Clavis Patrum Apostolicorum, Darmstadt 1963.

KÜHNER, R.-GerTh, B., Ausfihrliche Grammatik der griechischen Sprache, II, Satzlehre, Hannover-Leipzig 1898-19043.

Lampe, G. W. H., A patristic Greek lexicon, Oxford I96I-I 968.

Lasso de LA VeGA, J. S., Sintaxis griega, I, Madrid 1968.

Liddeli, H. G.-Scott, R.-Jones, H. S., A Greek-English lexicon, Oxford 1925-1940. A supplement, Oxford 1968.

Mayser, E., Grammatik der Papyri aus der Ptolemäerzeit, Berlin-Leipzig 1906I934.

Moulton, J. H., Einleitung in die Sprache des Neuen Testaments, Heidelberg 19 II.

Moulton, J. H.-Howard, W. F., A grammar of New Testament Greek, II, Accidence and word-formation, Edinburgh 1919-1929.

Moulton, W. F.-Geden, A. S., A concordance to the Greek Testament, Edinburgh $1963^{4}$.

Preuschen, E., Vollständiges Griechisch-Deutsches Handwörterbuch zu den Schriften des Neuen Testaments und der übrigen urchristlichen Literatur, Giessen I9IO'.

Radermacher, L., Neutestamentliche Grammatik, Tübingen $1925^{2}$.

Schwyzer, E.-Debrunner, A., Griechische Grammatik, II, Syntax und syntaktische Stilistik, München I950. [SD]

Sмyth, H. W.-Messing, G. M., Greek grammar, Cambridge, Massachusetts 1956.

Szantyr, A., Lateinische Syntax und Stilistik, München 1965.

Tавасноvitz, D., Grammatik des neutestamentlichen Griechisch, Ergänzungshett zur I2. A uflage, Göttingen 1965.

Thackeray, H. St. J., A grammar of the Old Testament in Greek according to the Septuagint, I, Cambridge Igog.

Thesaurus Linguae Latinae, Lipsiae I9oosq.

TURner, N., A grammar of New Testament Greek, III, Syntax, Edinburgh I963. Winer, G. B.-SchmiEdel, P. W., Grammatik des neutestamentlichen Sprachidioms, Göttingen I894-I898.

\section{TRAVAUX DIVERS}

Aerts, W. J., Periphrastica, an investigation into the use of elvac and Exelv as auxiliaries or pseudo-auxiliaries in Greek from Homer up to the present day, Amsterdan 1965 .

Alfonst, L., La vile e l'olmo, Vigiliae Christianae 2x, I967, pp. 8r-86.

Anlauf, G., Standard late Greek oder Attizismus? Eine Studie zum Optativgebrauch im nachklassischen Griechisch, Köln 1960.

anonyme, compte-rendu de Ae. Fr. C. Tischendorf, Hermae Pastor graece, Leipziger Repertorium der deutschen und ausländischen Literatur I5 ${ }^{\mathrm{I}}$, ${ }^{857}$, pp. I-8. 
Audet, J -P, Affinutés littéraıres et doctrinales du Manuel de Discipline (sunte), Le "Pasteur d'Hermas, Revue Biblique 60, I953, Pp 4I-82

-, compte-rendu de G F Snyder, The Shepherd of Hermas, Journal of Biblical Literature 87, 1968, PP 475-477

Bakel, H A van, De compositie van den Pastor Hermae, Amsterdam I900 BARDY, G, La question des langues dans l'Église ancienne, I, Pans 1948

BARnARD, L W, Studies in the Apostolvc Fathers and their background, Oxford I966

-, The Shepherd of Hermas in recent study, The Heythrop Journal 9, I968, pp. 29-36

BARR, J , The semantics of biblical language, Oxford $196 \mathrm{r}$

BARTELINK, G J M, Lexicologisch-semantische studie over de taal van de Apostolische Vaders, Utrecht [1952]

-, Ellipse und Bedeutungsverduchtung in der christluchen grnechuschen Literatur, Vigliae Christianae Io, I956, pp I-I3

-, Observations stylistıques et lingurstiquos chez Isidore de Peluse, Vigliae Chnstianae 18, 1964, PP I63-180

Bauernfeind, O, art dpetń. ThWNT I, 1933, pp 457-46I

Baumgartner, P, Die Einhent des Hermas-Buchs, Freiburg 1 B I889

BECKER, J H, Het begrip nefesj in het Oude Testament, Amsterdam I942

BeckmanN, G A, Die Nachfolgekonstruktionen des instrumentalen Ablativs ım Spatlatein und $2 m$ Franzosischen, Tubingen 1963

BEHM, J, art vйбtls, ThWNT IV, 1942, PP 925-935

Benort, P, L'inspiration des Septante d'apres les Peres, L'homme devant Dieu, Mélanges offerts au Père Henr de Lubac, I, Parıs 1963. pp I69-I87

BetтINi, L, Tendenze e problemi nello studio della lingua der Settanta, Atene e Roma N S I6, 197I, pp I-I2

BeYER, K, Semutische Syntax im Neuen Testament, I, Gottıngen I9682

Bickerman, E J, The Septuagint as a translation, Proceedings of the American Academy for Jewish Research 28, 1959, pp I-39

BietenHARD, $\mathrm{H}$, Die himmlische Welt im Urchristentum und Spatjudentum, Tubingen 1951

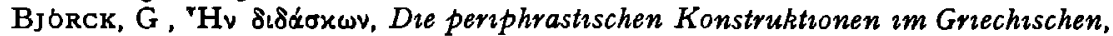
Uppsala I940

BLACK, M, An Aramaic approach to the Gospels and Acts, Oxford $1967^{3}$

Bonsirven, J , Notre statio liturgıque est-elle omprunté au culte junf ', Recherches de Science Relıgieuse I5, I925, pp 258-266

Boscherini, S, 'Osúvns úós (Menandrt, Dysc, v 88), Studi Italianı di Filologra Classica N S 3I, 1959, PP 247-253

Boyanct, P, La connarssance du grec d Rome, Revue des Etudes Latines 34, I956, PP I I I-I3I

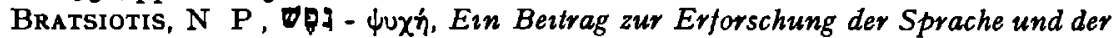
Theologie der Septuaginta, Supplements to Vetus Testamentum I5, I966, PP $58-89$

Brock, S P. The phenomenon of the Septuagent, Oudtestamentische Studien I7, I972, PP I I-36

BuRNEY, C F, The Aramaic arigen of the fourth gospel, Oxford 1922

CADBURY, H J, Luke - translator or author?. Amencan Journal of Theology 24, I 920 , PP 436-455

- Lexical notes on Luke - Acts I. Journal of Biblical Literature 44, I925, Pp. $214-227$

Cameron, A, Latin words in the Greek inscriptions of Asia Minor, The American Journal of Phulology 52, I931, pp 232-262. 
CAsPaRI, C. P., Ungedruckte, unbeachtete und wenig beachtete Quellen zur Geschichte des Tautsymbols und der Glaubensregel, III, Christiania 1875.

Champagny, Le Che De, Les Antonins (ans de J.C. 69-180), I, Paris 18631.

Chantraine, P, Histoure du parfant grec, Paris 1927.

Charles, R. H., A critical and exegetical commentary on the Revelation of St. John, Edinburgh I920

COLEBORne, W., A linguzstic approach to the problem of structure and composition of the Shepherd of Hermas, dissertation non publeee. Newcastle, N S.W 1965.

- The Shepherd of Hermas, $A$ case for a multiple authorship and some implicatons, Studia Patristica 1o, Berlin 1969, Pp. 65-70.

-. A linguistic approach to the problem of structure and composition of the Shepherd of Hermas, Colloquium 3², 1969, pp. 133-142.

ColorNi, V., L'uso del greco nella hiturgia del giudaismo ellenistico e la Novella I46 di Giustiniano, Annali di Stona del Diritto 8, 1964, pp. 19-8o

Coseriu, E., Das Problem des griechischen Einflusses auf das Vulgärlatein, Sprache und Geschichte, Festschnft fur Harn Meier zum 65. Geburtstag, Munchen I97I, pp. 135-147.

Dalman, G, Die Worte Jesu mit Berucksichtigung des nachkanonischen judischen Schrifttums und der aramäischen Sprache erörtert, I, Leipzig 18981, $193^{2}$. Danielou, J., Théologie du Judéo-christzanisme, Tournal 1958.

- Les symboles chretiens primitzts, Pans I961.

-. La littérature latine avant Tertulinen, Revue des Etudes Latines 48, 1970, PP 357-375.

DARIS, S., Il lessico latino nel greco d'Egitto, Barcelona I97I.

Dautzenberg, G, Sein Leben bewahren. $\Psi$ ux'́n in den Herrenworten der Evangelien, Munchen 1966.

DAwson, W. R., Who was who in egyptology, London $195 \mathrm{I}$.

Debrunner, A.-Scherer, A., Geschichte der griechischen Sprache, II, Berlin $1969^{2}$.

Deemter, R. van, Der Hert des Hermas, Apokalypse oder Allegorie ', Delft 1929.

Deissmann, (G.) A., Bibelstudien, Marburg I895.

-, Licht vom Osten, Tubingen 19234.

Dekkers, E., Tertullianus en de geschiedenis der liturgue, Brussel-Amsterdam 1947 .

Delekat, L., Ein Septuagintatargum, Vetus Testamentum 8, 1958, pp 225-252.

Delling, G., Partızipiale Gottesprädıkationen in den Briefen des Neuen Testaments, Studia Theologica 17, 1963. Pp. I-59

Demaray, C. E, Studies in the language of Hermas, dissertation non publiee, Ann Arbor 1940

Demetz, P., The Elm and the vine, Notes toward the history of a marriage topos, Publications of the Modern Language Association of America 73, 1958, Pp. 52I-532.

Denniston, J D., The Greek particles, Oxford $1954^{2}$.

Dirle, A et aln, art $\psi u \times \eta^{\prime}$. ThWNT IX, 1973, pp. 604-66I.

Dindorf, W.-ANGer, R., Nachträgliche Bemerkungen zu Hermas, Leipziger Repertonum der deutschen und auslandischen Literatur I4 III, I856, PP I29-I 47

DindoRF, W., Nachträgluche Bemerkungen zu Hermas. II, Leipziger Repertonum der deutschen und auslandischen Literatur 151, 1857, pp 65-79.

Donaldson, J, A critical history of Christian literature and doctrine from the death of the A postles to the Nicene Councul, I, The Apostolical Fathers, London 1864 . 
- The Apostolucal Fathers, $A$ critucal account of therr genurne writings and of their doctrines, London 1874

- The Shepherd of Hermas, The Theological Review I4, I877, Pp 504-5I9. Doudna, J C , The Greek of the Gospel of Mark, Philadelphia, Pennsylvania r96I.

ElBOGEN, I, Der judische Gottesdienst in seiner geschrchtlichen Entwucklung, Frankfurt am Main 19 I $^{3}$

Fieвig, P, Der Erzahlungsstıl der Evangelıen im Luchte des rabbınıschen Erzählungsstils untersucht, Leipzig I925

Fischer, J A, compte-rendu de S Giet, Hermas et les Pasteurs, Theologische Revue 6r, I965, col 304-308

Fitzmyer, J A, The languages of Palestine in the first century $A D$, The Catholic Biblical Quarterly 32, I970, pp 50I-53 I

Foerster, R-Munscher, K, Libanzos, Paulys Real-Encyclopadie der classischen Altertumswissenschaft, Neue Bearbeitung, XII, Stuttgart I925, col $2485-255 I$

Fohrer, G-LoHse, E et alı, art vibs, ThWNT VIII, I969, pp 334-400

Foucault, J -A DE, Recherches sur la langue et le style de Polybe, Pans I972.

FrEND, W H C, Martyrdom and persecution in the early Church, Oxford 1965.

Fritsch, C T, The anti-anthropomorphisms of the Greek Pentateuch, Pnnceton I 943

Giet, S., Hermas et les Pasteurs, Les trous auteurs du Pasteur d'Hermas, Paris I963. GoldenberG, G, Tautological Infinitive, Israel Oriental Studies I, I971, pP $36-85$

GoNDA, J, The functions of word duplication in Indonesian languages, Lingua 2, I949, PP I70-I97

Grant, R M, compte-rendu de S Giet, Hermas et les Pasteurs, Gnomon 36. I964, pp 357-359

Grobel, K, Shepherd of Hermas, Parable II, Vanderbilt Studies in the Humanities I, I95I, pp 50-55

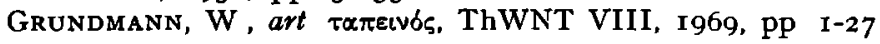

Gúnther, R, Die Präpositıonen in den griechischen Dialehtınschriften, Indogermanische Forschungen 20, 1906-1907, pp I-I63

Gú́RANGER, DoM, Sainte Cécule et la société romanne aux deux premiers siècles, l'ars $1874^{1}$

HAHN, L, Rom und Romanismus im griechrsch-römıschen Osten, Lelpzig 1906.

Hanson, A T, Hodayoth vi and vur and Hermas Sim VIII, Studia Patristica 10, Berlin I969, pp I05-108

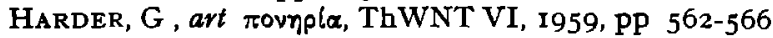

HARL, M, Y a-t-ıl une influence du "grec biblique" sur la langue spirituelle des chretiens' Exemples tirés du psaume II et de ses commentateurs d'Orngene a Théodoret, La Bible et les Pères, Pans 1971, pp 243-262

Harnack, A, Die Wiederauffindung der Athoshandschrift des Hirten des Hermas, Theologische Literaturzeitung I3, I 888, col 303-305

-, Geschichte der altchristluchen Literatur bis Eusebius, II I, Leipzig 1897

Harsing, C, De optative in chartis Aegyptris usu, Bonnae rgro

HAUGEN, E, Bilingualism in the Americas, $A$ bibliography and research guide, Alabama 1956

Hauschild, G R, Die Verbindung finiter und infiniter Verbalformen desselben Stammes in einigen Bibelsprachen, Benchte des Freien Deutschen Hochstiftes zu Frankfurt am Main N F 9, 1893, pp 99-I 27

Haussleiter, I, De versionibus pastorts Hermae latınis, Acta Seminari Philologici Erlangensis 3, 1884, pp 399-477 
Havers, W., Enumerative Redeweise, Indogermanische Forschungen 45, I927, pp. 229-25I.

-, Handbuch der evklärenden Syntax, Heidelberg 193 I.

Helning, R., Die Kasussyntax der Verba bei den Septuaginta, Göttingen 1928. HeLFMEYER, F.-J., "Gott nachfolgen in den Qumrantexten, Revue de Qumran, $7^{1}, 25,1969$, Pp. 81-104.

Hengel, M., Die Synagogeninschrift von Stobi, Zeitschrift für die neutestamentliche Wissenschaft 57, 1966, pp. 145-183.

- Judentum und Hellenismus, Tübingen $1973^{2}$.

Hering, J., Lateinisches bei Appian, Weida i. Thür. 1935.

Hilgenfeld, A., compte-rendu de A. Link, Die Einheit des Pastor Hermae et de P. Baumgärtner, Die Einheit des Hermas-Buchs, Zeitschrift für wissenschaftliche Theologie 32, I889, pp. 363-373.

HoFmanN, J. B., Beiträge zur Kenntnis des Vulgärlateins, Indogermanische Forschungen 43, I926, pp. 8o-122.

-, Lateinische Umgangssprache, Heidelberg $195^{3}$.

Humbert, J., La disparition du datif en grec (du Ier au $X^{e}$ siecle), Paris 1930.

Hunkin, J. W., "Pleonastic" kpopar in the New Testament, The Journal of Theological Studies 25, 1924, Pp. 390-402.

JallaberT, P. J., Hermas et Simonides, Etude sur la controverse récemment soulevé en Allemagne par la decouverte d'un manuscrit grec. Paris-Lyon 1858 .

Jeremias, J., Die Muttersprache des Evangelisten Matthäus, Zeitschrift für die neutestamentliche Wissenschaft 50, I959, pp. 270-274.

Jörns, K.-P., Das hymnische Evangelium, Untersuchungen zu Aufbau, Funktion und Herkunft der hymnischen Stücke in der Johannesoffenbarung, Gütersloh I97I.

Johannessohn, M., Der Gebrauch der Kasus und der Präpositionen in der Septuaginta, I, Berlin rgro.

- Der Gebrauch der Präposilionen in der Septuaginta, Berlin 1925.

$\rightarrow$ Das biblische xal lóoú in der Erzählung samt seiner hebräischen Vorlage, Zeitschrift für vergleichende Sprachforschung 66, 1939, PP. I45-195;67, 1942, Pp. 30-84.

-, Die biblische Einführungsformel xal Eđra!, Zeitschrift für die alttestamentliche Wissenschaft N.F. I8, I942-1943, pp. 129-184.

-, Et ecce und sein Ersatz in der Vulgata, Glotta 33, 1954, pp. 125-156.

Joly, R., Judaisme, christianisme et hellénisme dans le Pasteur d'Hermas, La Nouvelle Clio 5. I953, pp. 396-406.

-, compte-rendu de M. Whittaker, Der Hirt des Hermas, L'Antiquité Classique 27, 1958, pp. I88-I9o.

- Le Tableau de Cébès et la philosophie religieuse, Bruxelles I963.

- Hermas et le Pasteur, Vigiliae Christianae 21, 1967, pp. 20 I-2 8.

Jonge, M. DE, The Testaments of the Twelve Patriarchs, Assen 1953.

Joúon, P., Respondit et dixit, Biblica 13, 1932, pp. 309-314.

Katz, P., Philo's Bible, Cambridge 1950.

KAUPEL, H., Beobachtungen zur Ubersetzung des Infinitivus absolutus in der Septuaginta $(G)$, Zeitschrift für die alttestamentliche Wissenschaft N.F.20, I945-I948, Pp. I9I-192.

KIECKERS, E., Zur oratio recta in den indogermanischen Sprachen $I$, Indogermanische Forschungen 35, I9I 5, PP. I-93.

Köster, H., Synoptische Überlieferung bei den Apostolischen Vätern, Berlin 1957. KRAPP, F., Der substantivierte Infinitiv abhängig von Präpositionen und Präpositionsadverbien in der historischen Gräcität (Herodot bis Zosimus), Heidelberg I 892. 
KRAUSS, S, Grnechısche und lateinısche Lehnwōrter $\imath m$ Talmud, Mıdrasch und Targum, Berlin I 898-I899

-, Talmudische Archdologie Leipzig 1910-1912

LA PIANA, G, Foreign groups in Rome during the first centuries of the Empire, The Harvard Theological Review 20, I927, pp I83-403

LEE, J A L, A neglected sense of pepos, Antichthon 6, 1972, pp 39-42

LEON, H J, The Jews of ancient Rome, Philadelphia 1960

LiNK, A, Die Einhent des Pastor Hermae, Marburg 1888

Lipsius, R A, compte-rendu de Ae Fr C Tischendorf, Hermae Pastor graece, Literarisches Centralblatt fur Deutschland 1856, col 745-748

-, Der Hirte des Hermas und der Montanısmus in Rom, Zeitschnft fur wissenschaftliche Theologie 8, 1865, pp 266-308

- Duplik gegen Herrn von Tischendorf, Zeitschrift fur wissenschaftliche Theologe 9, I 866, pP I 22-I 30

LjUNGVIK, H, Beiträge zur Syntax der spätgriechıschen Volkssprache, Uppsala 1932

-, Zum Gebrauch einiger Adverbien im Neuen Testament, Eranos 62, 1964, pp 26-39

-, Aus der Sprache des Neuen Testaments, Eranos 66, I968, pp 24-5I

LoBeck, ChR A, Paralıpomena grammatıcae graecae, Lipsiae 1837

Lofstedt, E, Late Lain, Oslo 1959

Lowe, A D, The origin of oúd, Hermathena 105, 1967, pp 34-39

LuCKE, FR, Versuch einer vollständigen Einleitung in die Offenbarung Johannis und in die gesammte apokalyptische Litteratur, Bonn 1832

Lykurgos, A , Enthullungen uber den Simonides Dindorfschen Uranios, Leipzig I $856^{2}$

Lys, D, The Israelite soul according to the $L X X$, Vetus Testamentum 16,1966 , PP I8I-228

Mandilaras, B G, The verb in the Greek non-literary papyrz, Athens 1973

Marrou, H -I, Historve de l'éducation dans l'antiquité, Pans I965

Martin, $\mathrm{R}$ A, Syntactical evidence of Semitic sources in Greek documents, Cambridge, Massachusetts 1974

Massingberd Ford, J , A possible luturgical background to the Shepherd of Hermas, Revue de Qumran 64, 24, I969, pP 53I-55I

Masson, O, A propos de deux formules redoublés au locatıf mycennen weterweter "chaque annè, chyprote amatramatz "chaque joun, Ziva Antıka I5, I966, pP 257-266

McKAY, K L, The use of the ancient Greek perfect down to the second century $A D$, Bulletin of the Institute of Classical Studies of the University of London I2, I965, PP I-2 I

Meershoeк, G Q A, Le latın biblique d'après saint Jérome, Aspects lingutstıques de la rencontre entre la Bible et le monde classique, N1]megen-Utrecht 1966

Meillet, A , Apercu d'une histozre de la langue grecque. Pans I965?

Merkx, P A H J, Zur Syntax der Kasus und Tempora in den Traktaten des hl Cyprian, Nijmegen I939

Meuwese, A P M, De rerum gestarum divi Augusti versione graeca, Buscoducl I920

-, De versione graeca Monumentr Ancyrani quaestiones, Mnemosyne N S 54, I 926, pp 224-233

MOHRMANN, CHRIsTINe, Quelques tratts caracteristiques du latin des chretiens, Miscellanea Giovanni Mercatı, I, Citta del Vaticano 1946, pp 437-466= Études I, pp 21-50 
-, Les origines de la latinite chrêtenne a Rome, Vigiliae Chnstianae 3, 1949, pp 67-I06, I63-I $83=$ Études III, PP 67-126

-, Quelques observations sur l'onginaluté de la littérature latine chretienne, Rivista di Stona della Chiesa in Italıa 4, 1950, pp 153-163 = Études I, Pp I39-1 50

-, Le dualısme de la latınté médıévale, Revue des Études Latınes 29, 1952, pp. 330-348

-, Statio, Vigiliae Christianae 7, 1953, pp 221-245 = Études III, pp 307-330

- Problemes philologiques et litteraires, Augustınus Magister, III, Pans I954, pp 27-4I

-, Problemes stylistiques dans la litterature latıne chrétrenne, Vigiliae Christianae 9, I955, pp 222-246 = Études III, pp I 47-I70

-, The Latin of Saint Patrick, Four lectures, Dublin 1961

- Etudes sur le latin des chrétrens, $I$, Le latın des chrétiens, Roma $196 \mathrm{I}^{2}$

-. Etudes sur le latin des chrêtrens, II, Latın chrétien et médiéval, Roma 196I

-, Etudes sur le latin des chrétiens, III, Latın chrétien et liturgique, Roma I965

Mommsen, T, Bettrage zu der Lehre von den griechischen Prapositionen, Berlin 1895

MonteIL, P , La phrase relatzve en grec ancren, Paris 1963

MoretTI, L, Inscriptiones graecae urbis Romae, Romae I968sq

Morton, A Q-Mcleman, J , Christranty and the computer, London 1964

Moulton, J H , Grammatical notes from the papyn, The Classical Review 15. I9OI, Pp 31-38, 434-442

Muraoka, T, The use of is in the Greek Bible, Novum Testamentum 7, 19641965, PP 5I-72

Mussies, G, The morphology of koine Greek as used in the Apocalypse of St John, $A$ study in bilingualism, Leiden r97I

Musurillo, $\mathrm{H} \mathrm{A}$, The need of a new edition of Hermas, Theological Studies I2, I95I, PP 382-387

Nachmanson, E, Syntaktische Inschrittenstudien, Eranos 9, 1909, PP 30-8I

Norden, E, Die antrke Kunstprosa vom VI Jahrhundert v Chr bis in die Zent der Renaissance, Leipzıg-Berlın $1909^{2}$

ORBÁN, $\AA \mathrm{P}$, Les dénominations du monde chez les premiers auteurs chrétzens, Nijmegen 1970

Papanikolaou, A D, Charnton-Siudien, Untersuchungen zur Sprache und Chronologie der griechischen Romane, Gottingen 1973

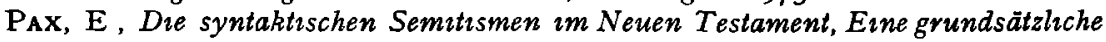
Erwagung. Studi Biblicl Franciscanı Liber Annuus, 13, I962-1963, Pp I 36-162

Percy, E, Die Probleme der Kolosser- und Epheserbriefe, Lund 1946.

Pernveden, L, The concept of the Church in the Shepherd of Hermas, Lund 1966

Peterson, E, Fruhkirche, Judentum und Gnosis, Studien und Untersuchungen, Freiburg 1959

- Guudarsmo e Cristianesimo culto giudarco e culto cristiano, Rivista di Storia e Letteratura Religiosa I, 1965, PP 367-39I

Petit, F , compte-rendu de M WhittakfR, Der Hirt des Hermas, Recherches de Théologie Ancienne et Médıévale 24, 1957, pp 380-382

Pfitzner, V C, Paul and the agon motrf, Traditional athletzc imagery in the Pauline literature, Leiden 1967

Philonfnko, M, Joseph et Aséneth, Introduction, texte critıque, traduction et notes, Leiden 1968

- La httérature intertestamentaire et le Nouveau Testament, Revue des Sciences Religieuses 47, 1973, pp 270-279 
Pinkstrer, H., On Latin adverbs, Amsterdam-London 1972.

Psichari, J., Essai sur le grec de la Septante, Revue des Études Juives 55, I908, pp. $16 \mathrm{I}-208$.

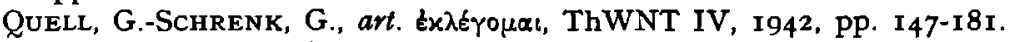

RABIN, $\mathrm{CH}_{\mathrm{H}}$., The translation process and the character of the Septuagint, Textus 6, 1968, Pp. I-26.

Radermacher, L., Griechischer Sprachbrauch, Philologus 68, I909, pp. 449-455.

-, compte-rendu de J. H. Moulton, Einleitung in die Sprache des Neuen Testaments, Indogermanische Forschungen 31, 1913, Anzeiger pp. 6-10. Ramsay, W. M., The cities and bishoprics of Phrygia, I, Oxford I895-1897.

Rapallo, U., Per una definizione diacronica e tipologica dei calchi ebraici nelle antiche versioni del Levitico (Studio sui LXX, la Vulgata e la Vetus), Rendiconti dell' Istituto Lombardo, Classe di Lettere, Scienze morali e storiche 103, 1969, pp. 369-437.

-. Calchi ebraici nelle antiche versioni del «Levitico», Roma I971.

Reckendorf, H., Uber Paronomasie in den semitisehen Sprachen, Ein Beitrag zur allgemeinen Sprachwissenschaft, Giessen I909.

ReichmanN, V., Römische Literatur in griechischer Ubersetzung, Leipzig 1943. ReILIng, J., Hermas and Christian prophecy, A study of the eleventh Mandate, Leiden 1973 .

ReInd, J., Das Angesicht Gottes im Sprachgebrauch des Allen Testaments, Leipzig 1970.

Reinhold, H., De graecitate Patrum Apostolicorum librorumque apocryphorum Novi Testamenti quaestiones grammaticae, Halis Saxonum 1898.

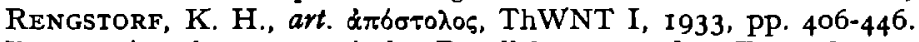

Resch, A., Aussercanonische Paralleltexte zu den Evangehen gesammelt und untersucht, Leipzig 1893-1897.

-, Agrapha, Leipzig 1906².

Rodenbusch, E., Präsentia in perfektischer Bedeutung, Indogermanische Forschungen 28, I911, pp. 252-285.

Rosên, H. B., Palestinian xouv' in vabbinic illustration, Journal of Semitic Studies 8, 1963, pp. 56-72.

-, 'Hv $\delta\llcorner\delta \alpha \sigma o \omega v$ et questions apparentes, Mises au point sur les contacts linguistiques netestamentaires, Bulletin de la Société de Linguistique de Paris 62, 1967, pp. XXI-XXVI.

RYDBEск, L., Fachprosa, vermeintliche Volkssprache und Neues Testament, Zur Beurteilung der sprachlichen Niveauunterschiede im nachklassischen Griechisch, Uppsala 1967.

- Bemerkungen zu Periphrasen mit elvar + Präsens Partizip bei Herodot und in der Koine, Glotta 47, 1969, pp. 186-200.

Salonius, A. H., Zur Sprache der griechischen Papyrusbriefe, Helsinki 1927. SCHLÄGER, G., Der Hirt des Hermas eine ursprünglich jüdische Schrift, Nieuw Theologisch Tijdschrift 16, I927, pp. 327-342.

Schlier, H., art. Yorvo, ThWNT I, 1933, pp. 738-740.

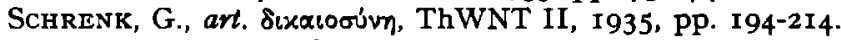

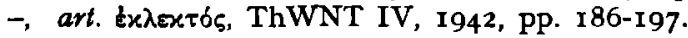

Schrijnen, J.-Mohrmann, Christine, Studien zur Syntax der Briefe des hl. Cyprian, Nijmegen 1936-1937.

ScHÜMMER, J., Die altchristliche Fastenpraxis mit besonderer Berücksichtigung der Schriften Tertullians, Münster in Westf. I933.

Schulze, GuIL., Graeca Latina, Göttingen Igor.

SchwaB, M., Transcription de mots grecs et latins en hébreu, aux premiers siècles de J.-C., Journal Asiatique IX Io, I897, pp. 4' 4-444. 
Schwartz, J., Survivances littéraires paiennes dans le "Pasteur" d'Hermas, Revue Biblique 72, I965, pp. 240-247.

Sevenster, J. N., Do you know Greek? How much Greek could the first Jewish Christians have knoun?, Leiden I968.

SHERK, R. K., Roman documents from the Greek East, Senatus Consulta and Epistulae to the age of Augustus, Baltimore, Maryland I969.

SoIsalon-SoIninen, I., Die Infinitive in der Septuaginta, Helsinki 1965.

SolÁ-SolE, J. M., L'infinitif sémitique, Paris I96r.

SolIN, H., Beiträge zur Kenninis der griechischen Personennamen in Rom, I, Helsinki I97I.

SPEYER, W., Die literarische Fälschung im heidnischen und christlichen Altertum, München r97I.

SPITtA, Fr., Zur Geschichte und Litteratur des Urchristentums, II, Der Brief des Jakobus; Studien zum Hirten des Hermas, Göttingen 1896.

Steen, H. A., Les clichés épistolaires dans les lettres sur papyrus grecques, Classica. et Mediaevalia I, I938, pp. I I9-I 76.

Strack, H. L.-Billerbeck, P., Kommentar zum Netuen Testament aus Talmud und Midrasch, München I922-1928.

Sttss, W., Studien zur lateinischen Bibel, I, Augustins Locutiones und das Problem der lateinischen Bibelsprache, Tartu 1932.

Suggs, M. J., The Christian two ways tradition: its antiquity, form and function, Studies in New Testament and Early Christian Literature, Essays in Honor of Allen P. Wikgren, Leiden 1972, Pp. 60-74.

TABachovitz, D., Die Septuaginta und das Neue Testament, Stilstudien, Lund I956.

Tanner, R. G., Latinisms in the text of Hermas, Colloquium 44, 1972, pp. 12-23. TCHERIKOVER, V. A., Jewish apologetic literature reconsidered, Eos $4^{3}$, I956, pp. I69-193.

- Corpus Papyrorum Judaicarum, I, Cambridge, Massachusetts 1957.

Teeuwen, St. W. J., Sprachlicher Bedeutungswandel bei Tertullian, Ein Beitrag zum Studium der christlichen Sondersprache, Paderborn 1926.

Thackeray, H. St. J., Renderings of the infinitive absolute in the LXX. The Journal of Theological Studies 9, I908, Pp. 597-60I.

- An unrecorded varamaism* in Josephus ("He began to say unto them"), The Journal of Theological Studies 30, 1929, pp. 361-370.

Thiersch, H. W. J., Die Kirche im apostolischen Zeitaller und die Entstehung der neutestamentlichen Schriften, Frankfurt am Main-Erlangen $185^{1}$, $185^{82}$.

Thörnell, G., compte-rendu de St. W. J. TeEuwen, Sprachlicher Bedeutungswandel bei Tertullian, Gnomon 3, 1927, Pp. 48-50.

Tнuмв, A., Die griechische Sprache im Zeitalter des Hellenismus, Beitrage zur Geschichte und Beurteilung der xoเvń, Strassburg I90I.

Torm, F., Der Pluralis oúpavol, Zeitschrift für die neutestamentliche Wissenschaft 33, I934, pp. 48-50.

TorreY, C. C., The composition and date of Acts, Cambridge r9i6.

TRAPP, E., Der Dativ und der Ersatz seiner Funktionen in der byzantinischen Vulgärdichtung bis zur Mitte des I5. Jahrhunderts, Jahrbuch der Österreichischen Byzantinischen Gesellschaft I 4, I965, pp. 2 I-34.

Traub, H., art. oúpavós, ThWNT V, 1954, pp. 496-50I, 509-536.

TrEnKNer, S., Le style xal dans le vécit attique oral, Assen I $960^{2}$.

TREU, K., Ein neuer Hermas-Papyrus, Vigiliae Christianae 24, 1970, pp. 34-39.

- Die Bedeutung des Griechischen für die Juden im römischen Reich, Kairos N.F. I5, I973, pp. I 23-I44. 
TuRner, C. H., The Shepherd of Hermas and the problem of its text, The Journal of Theological Studies 21, 1920, pp. 193-209.

Turner, N., The "Testament of Abraham": problems in Biblical Greek, New Testament Studies I, 1954-1955, Pp. 219-223.

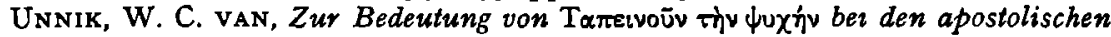
Vätern, Zeitschrift für die neutestamentliche Wissenschaft 44, 1952-I953, pp. 250-255.

- Dominus vobiscum: the background of a liturgical formula, New Testament Essays, Studies in Memory of Thomas Walter Manson 1893-1958, Manchester I959, pp. $270-305$.

Vergote, J., Grec biblique, Dictionnaire de la Bible, Supplément III, Paris I938, col. I320-I369.

VILDOMEC, V., Multilingualism, Leyden 1963.

Völter, D., Die Visionen des Hermas, die Sibylle und Clemens von Rom, Berlin I 900 .

-, Die Apostolischen Väter neu untersucht, Leiden 1904-1910.

Wackernagel, J., Hellenistica, Gottingae 1907.

-, Vorlesungen über Syntax mit besonderer Berücksichtigung von Griechisch, Lateinisch und Deutsch, Basel I926-19282.

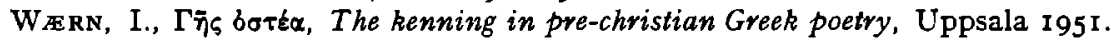

Waldis, J., Die Präpositions-Adverbien mit der Bedeutung «vor" in der Septuaginta, Luzern I922.

WeINREICH, U., Languages in contact, Findings and problems, New York 1953.

WeINRICH, H., Tempus, Besprochene und erzählte Welt, Stuttgart $197 \mathrm{I}^{2}$.

WENSINCK, A. J., Un groupe d'aramaismes dans le texte grec des évangiles, Amsterdam 1936.

Whittaker, M., Hermas, Shepherd of, New Catholic Encyclopedia 6, New York I 967, P. I074.

WIEMKeN, H., Der griechische Mimus, Bremen 1972.

WILcox, M., The semitisms of Acts, Oxford r965.

Willis, W. H., A census of the literary papyri from Egypt, Greek, Roman and Byzantine Studies 9, I968, pp. 205-24I.

Wilson, W. J., The unity of the Aramaic $A$ cts, The Harvard Theological Review I I, I 9I8, pp. 322-335.

- The career of the prophet Hermas, The Harvard Theological Review 20, I927, pp. $2 \mathrm{I}-62$.

Wolf, K., Studien zur Sprache des Malalas, II, München ror2.

Young, D., Theognis, Lipsiae I96r.

ZaHN, TH., Der Hirt des Hermas untersucht, Gotha I 868.

ZоBEL, J., Der bildliche Gebrauch der Verwandtschaftsnamen im Hebräischen mit Berücksichtigung der übrigen semitischen Sprachen, Halle (Saale) I932. 



\section{HISTOIRE DU PROBLEME}

\section{L'ELEMENT LATIN}

Bien que le Pasteur présente beaucoup moins de latinismes que de sémitismes, l'élément latin a été plus souvent mis en question que l'élément sémitique. C'est lui aussi qui, le premier, attira l'attention. Aussitôt après la découverte du texte grec on se demanda en effet si l'on avait bien affaire au texte original ou à une rétroversion tardive de la version (ou d'une version) latine du texte original. On comprend mal aujourd'hui que des spécialistes réputés comme Constantin Tischendorf n'aient pas hésité à défendre la deuxième thèse et l'on peut en effet se demander si les motifs qui déterminèrent leur prise de position étaient bien seulement d'ordre scientifique. Un bref exposé des circonstances dans lesquelles le texte fut découvert nous aidera à mieux comprendre leur attitude ${ }^{1}$.

Jusqu'en I85I, on ne possédait du Pasteur qu'une version latine complète, dite Vulgate, ainsi qu'une série de citations grecques chez les Pères de l'Église ${ }^{2}$. Cette même année, le Grec Konstantinos Simonidès découvrit dans le couvent de Saint Grégoire, sur le mont Athos, un manuscrit du XVe siècle comprenant neuf feuillets qui, sur les II4 chapitres du Pasteur, comportaient le texte intégral de I,I à I07,2. Il emporta le cinquième, le sixième et le neuvième feuillet et une copie des six autres et, en juillet 1855 , il montra les 3 feuillets au professeur Anger de Leipzig. Or, il faut savoir que Simonidès s'adonnait depuis

I. Les données suivantes sont empruntées à R. Anger, Hermae Pastor graece, Lipsiae 1856, pp.V-XXV; A. Lykurgos, Enthüllungen über den SimonidesDindorfschen Uranios, Leipzig $185^{2}$, pass.; Ae.Fr.C. Tischendorf, Hermae Pastor graece, Lipsiae I856, pp.V-XVI (repris presque sans changement dans A. R. M. Dressel, Patrum A postolicorum Opera, Lipsiae $1857^{1}, 1863^{2}$; dans la suite nous désignons cette édition par 4857 \#). Bibliographie sur Simonidès chez W. R. Dawson, Who was who in egyptology, London 195I, p.I48; W. Speyer, Die literarische Fälschung im heidnischen und christlichen Altertum, München I97I, P.323 n.4.

2. Pour la tradition manuscrite du Pasteur voir infra, pp.r5-17. 
des années à la recherche de manuscrits en vue de les vendre et qu'il ne reculait pas devant la fabrication de manuscrits quasi-anciens (de préférence des palimpsestes) d'après des textes existants ou même des textes de pure invention. Ses pratiques n'avaient pas manqué de porter des fruits; mais, à plusieurs reprises on avait démasqué l'escroquerie et sa réputation n'était plus à faire en Allemagne. Anger savait à qui il avait affaire et, bien que le manuscrit qu'on lui présentait le passionat, il eut des soupçons et pensa aussitôt qu'il pourıait s'agir d'un faux. Simonidès n'avait pas pu fabriquer lui-même le texte à partir de la version latine du Pasteur, car les trois feuillets du manuscrit étaient indubitablement du XVe siècle et le caractère du texte de la copie était en parfaite concordance avec celui des feuillets. Mais il s'agissait peut-être d'une rétroversion du texte du moyen âge, et c'est sur ce point qu'Anger examina le texte qu'on lui présentait. Ce faisant, il tomba dans le piège tendu par Simonidès. Ce dernier, en effet, outre les trois feuillets, n'avait pas donné la copie qu'il avait faite du reste au mont Athos, mais une copie de cette copie où, à dessein, il avait fait un certain nombre de modifications afin d'éviter, entre autres, qu'on ne pensât précisément que c'était une rétroversion du latin. C'est ainsi que partout il avait remplacé par des mots grecs authentiques les mots d'emprunt latins qui, à une exception près, se présentaient tous dans la partie qu'il avait recopiée. Anger, qui, on peut le supposer, espérait voir s'évanouir ses craintes, ne trouva rien qui prouvât de façon convaincante qu'il s'agissait d'une rétroversion du latin ${ }^{1}$. Il accepta le texte et, assisté par son collègue Wilhelm Dindorf, il prépara aussitôt l'édition princeps. Dès décembre le livre était prêt à être envoyé aux libraires (la page de titre portait déjà 1856 ). C'est peu après que la fraude fut découverte. Au début de février, la police retrouva dans les papiers de Simonidès la copie originale. Anger et Dindorf s'en servirent pour publier les nombreux écarts du texte imprimé par rapport à elle. Mais leur foi dans l'originalité du texte grec du Pasteur n'en fut pas ébranlée 2 .

Entre-temps, un autre collègue d'Anger, Tischendorf, se proposait d'éditer l'Hermas grec. Bien qu'il ne disposât d'aucune autre source manuscrite que le manuscrit d'Anger, l'idée lui semblait justifiée: d'une part, son édition était destinée à compléter une nouvelle édition des Pères Apostoliques que publiait Dressel; d'autre part, il estimait

I. Voir Anger, Hermae Pastor, pp.XI-XXIII.

2. W. Dindorf-R. Anger, Nachträgliche Bemerkungen zu Hermas, Leipziger Repertorium der deutschen und ausländischen Literatur I4 III, I856, pp. I 29-I 47. 
que personne ne pouvait s'acquitter mieux que lui de cette tâche. C'était en outre un coup de chance que la falsification de Simonidès ait été découverte au bon moment: pour la partie copiée il pouvait aussi utiliser la première copie. Son édition parut séparément, dès 1856 , dans un petit tirage, puis, en 1857 , dans l'édition de Dressel1.

Au cours de l'automne de 1855 , Simonidès, encouragé par son succès auprès d'Anger, se risqua à produire plusieurs autres faux. Dans la mesure où il avait pu examiner personnellement ces fabrications, Tischendorf était convaincu de leur inauthenticité et il se crut obligé de le dire. Il mit en garde Dindorf contre une pièce pour laquelle celui-ci montrait un intérêt particulier: un palimpseste qui aurait contenu une histoire complète des rois d'Égypte par Uranios, auteur à peu près inconnu jusqu'alors. Ce fut en vain: au contraire, dans cette mise en garde, Dindorf trouva une raison de plus de précipiter l'édition aussi bien d'Hermas que d' "Uranios». Mais Tischendorf put bientôt confirmer son point de vue, et, non sans satisfaction, il publia un texte d'Hermas débarrassé des fautes qui avaient échappé à Anger et Dindorf. Il ne s'en contenta pas: il crut devoir s'opposer aux premiers éditeurs trop crédules jusque sur la question de savoir si le texte d'Hermas était original. Dans l'introduction de son édition, il affirmait que le texte retrouvé par Simonidès n'était autre chose qu'une rétroversion médiévale du latin.

Mais ses arguments étaient peu solides². Il joua de malheur notamment avec un symptôme de rétroversion qu'il croyait avoir trouvé dans II,5, où le manuscrit du mont Athos comportait $\pi \alpha$ voũpyos el

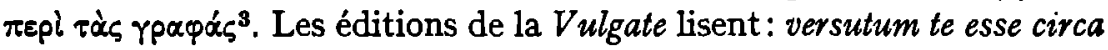
structuras, ce qui donne un texte facile. Il semblait évident qu'au cours de la tradition de la Vulgate un copiste avait par distraction remplacé structuras par scripturas, beaucoup plus fréquent dans les écrits bibliques et patristiques et que le texte grec de l'Athos avait été traduit d'après un manuscrit comportant scripturas. Malheureusement, Tischendorf n'avait pas examiné de près les bases de la leçon structuras dans les manuscrits. Bientôt R. A. Lipsius, dans son compte-rendu de l'édition de Tischendorf, fit savoir que tous les manuscrits com-

r. Cf. supra p.I n.I.

2. P. J. Jallabert en donne une ample réfutation, Hermas et Simonidès, ParisLyon I 858, surtout pp.92-I24.

3. Ce passage avait déjà suscité la méfiance d'Anger. On ne voit pas très bien, ni dans la préface d'Anger, ce qu'on comprend, ni dans Lykurgos, Enthüllungen, pp.4-5, comment malgré tout Simonidès est parvenu, dans l'édition princeps, a

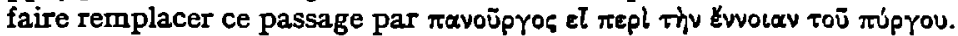


portaient scripturas et que structuras n'était probablement qu'une émendation du premier éditeur reprise par tous les éditeurs suivants ${ }^{1}$.

L'affaire prit un tour décisif en 1859, lorsque Tischendorf lui-même découvrit le Codex Sinaitıcus du IVe siècle, qui comporte le premier quart du Pasteur dans un texte qui est essentiellement le même que celui du Codex Athous. Il retira alors sa théorie d'une rétroversion médiévale, mais il refusa de reconnaitre avoir commis une faute, en tant qu'il prétendait qu'un certain nombre de passages avaient été revisés d'après une version latine ${ }^{2}$.

Les deux interprétations de Tischendorf n’ont, en général, suscité aucune réaction si ce n'est des réactions négatives. Le seul à se ranger à son avis fut l'Écossais Donaldson. Il commença par reprendre la théorie de la rétroversion et refusa même de l'abandonner lorsque Tischendorf y renonça. Il se débarrassa du Codex Sınaitıcus en le reléguant à une époque plus tardive que le IVe siècle ${ }^{\mathrm{a}}$. Plus tard, tout comme Tischendorf, il mitigea la théorie, mais en la compliquant considérablement à d'autres égards. Tout bien considéré, il estima alors que l'hypothèse la plus vraisemblable était: "that we have, as the basis of our present Greek manuscripts, a recension and modernized version belonging to the sixth or seventh century, and that the editor used all the materials at his command, having probably in his possession large portions of the original text, but filling up gaps from some Latin translation, introducing parts from some modifications of the text, such as those of pseudo-Athanasius, and clothing the whole in the language current among the Christian populace of his day ${ }^{4}$. Dans la formation de son point de vue, Donaldson a-t-il été influencé, lui aussi, de façon négative par le personnage de Simonidès? Simonıdès, en tout cas, lui fit des avances avec ce qu'il disait être la fin grecque

r. R A. Lipsius, dans son compte-rendu de Tischendorf, Hermae Pastor, Literansches Centralblatt fur Deutschland 1856, col (745-748) 747 La discussion sur ce point traîna encore pendant de longues années, vour, par exemple, R A Lipsius, Duplik gegen Herrn von Tischendorf. Zeitschnft fur wissenschaftliche Theologie 9, I866, PP I 22-130

2 Vorr, entre autres, Dressel, Patrum Apostolicorum Opera ${ }^{2}$, pp III-IV Dès I856, ll avait soumıs cette idée à ceux qui ne pourraient admettre sa théone d'une rétroversion (Hermae Pastor, p XV $=1857, \mathrm{p} \mathrm{L} \Gamma \mathrm{V}$ )

3. J Donaldson $A$ critical history of Christian literature and doctrine from the death of the Apostles to the Nicene Counctl, I, London I864, Pp 308-3II. De même, mais çà et là en termes plus prudents, dans la deuxième édition, sous le titre The A postolical Fathers, $A$ critical account of their genume writngs and of of their doctrines, London $1874, \mathrm{PP}$ 383-384, 387-39I

4 J Donaldson, The Shepherd of Hermas, The Theological Revew 14, 1877 , p (504-5I9) 5 I 4 . 
du Pasteur. Donaldson pourtant flaira aussitôt le faux et lorsque, dans les années 80 , cette fin donna lieu, une fois de plus, à une polémique en Allemagne, il fit savoir dans une lettre à Harnack ce qui lui était arrivé'.

Dans les discussions que nous venons de résumer on avait utilisé trois groupes d'arguments: mots et formes de mots tardifs, passages qui, en latin, semblaient plus originaux qu'en grec (comme la formule

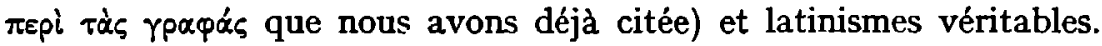
Nous ne nous arrêterons pas longtemps aux deux premiers groupes: les mots et les formes tardives venaient de fautes dans les copies de Simonidès ou s'avérèrent en fin de compte des formes plus anciennes qu'on ne l'avait cru; quant aux (passages traduits", ils s'expliquaient aussi bien ou mieux d'une autre manière. En ce qui concerne les latinismes, la plupart de ceux qui s'occupaient de la question ne signalaient comme tels dans le texte d'Hermas que les emprunts de vocabulaire. Tischendorf mentionna en outre des phénomènes comme l'asyndète, $p \eta \sigma i v$ dans la fonction d'inquit, le génitif absolu là où on s'attendrait à un participium coniunctum et une ou deux expressions dont nous ne parlerons pas $\mathrm{ici}^{2}$.

Entre-temps, on avait retrouvé non seulement la plus grande partie du texte grec, mais aussi une deuxième version latine, dite Palatine, et une version éthiopienne qui furent publiées l'une en I857 par Dressel, l'autre en 1860 par Abbadie ${ }^{3}$. Mettant à profit ces trouvailles, Hilgenfeld prépara la première édition vraiment critique du Pasteur, qui parut en 18664. On disposait maintenant d'un instrument de travail pratique. Du même coup se trouvait dépassé le stade de la vraisemblance d'une rétroversion: les latinismes pouvaient être étudiés sous un jour meilleur. Deux questions se posaient: quels latinismes trouve-t-on dans le Pasteur? Comment faut-il les expliquer si leur présence n'est pas due à une traduction trop littérale d'un texte latin qui, à son tour, serait une traduction du texte grec original?

I. A. Harnack, Die Wiederauffindung der Athoshandschrift des Hirten des Hermas, Theologische Literaturzeitung I3, I888, col (303-305) 305.

2. Voir Anger, Hermae Pastor, p.XXIII; Dindorf-Anger, Nachträgliche Bemerkungen, p.I3I; Tischendorf, Hermae Pastor, pp.XIII-XIV (= 1857, pp. LII-LIII; ici, en outre, l'addition p.I.V, n.r2, $3^{e}$ alınéa); Lipsius, compte-rendu de Ae. Fr. C. Tischendorf, Hermae Pastor graece, col.746.

3. Palatine: Dressel, Patrum A postolicorum Opera ${ }^{1}$, pp 409-57I (pages impaires). Version éthiopienne: A. d'Abbadie, Hermae Pastor, aethiopice primum edidit et aethiopica latine vertit, Lipsiae 1860 .

4. A. Hilgenfeld, Hermae Pastor, Lipsiae 1866. 
Pour la première question, si l'inventaire que Tischendorf avait commencé fut contesté sur certains points, il prit néanmoins petit à petit plus d'ampleur ${ }^{1}$. Quant à la deuxième question, elle ne reçut qu'un petit nombre de réponses, et encore le plus souvent d'une façon rudimentaire. Zahn, Caspari, Baumgärtner et Debrunner semblent considérer que l'auteur avait comme langue le grec, mais qu'il a été influencé par son entourage latin de Rome ${ }^{2}$. D'autres le virent comme un auteur dont la première langue est le latin et qui ne peut s'en départir complètement lorsqu'il utilise le grec. Dès 1856, Lipsius notait incidemment: "Wir wollen hierbei nicht die Frage aufwerfen, ob denn bei einem Griechisch schreibenden Römer einzelne Latinismen wirklich etwas ganz unerhörtes wären" ${ }^{3}$. Après lui, cette opinion fut exprimée, mais avec plus de prudence, en 1877 , par Donaldson et, en I920, par C. H. Turner.4. Mais ce n'est qu'en I949 que Christine Mohrmann lui donna un fondement circonstancié5.

Les latinismes qu'elle-même et d'autres signalent lui permettent d'abord de conclure qu'Hermas connaissait le latin et même probablement qu'il le connaissait mieux que le grec. L'emprunt $\sigma \tau \alpha \tau i \omega \nu$ en particulier lui révèle le fait que le latin était familier non seulement à Hermas mais aussi à la communauté chrétienne de Rome en général. Ce mot, en effet, s'emploie dans le sens spécifiquement chrétien de ijeûne». Il semble donc que les Chrétiens de Rome étaient déjà

I. Voir Th. Zahn, Der Hirt des Hermas untersucht, Gotha 1868, p.487, n.2; C. P. Caspari, Ungedruckte, unbeachtete und wenig beachtete Quellen zur Geschichte des Taufsymbols und der Glaubensregel, III, Christiania 1875, note p.298; Fr. Blass, Grammatik des Neutestamentlichen Griechisch, Göttingen 1896, pp.33 (n.I), 76. 225 (n.2); E. Preuschen, Vollständiges Griechisch-Deutsches Handwörterbuch zu den Schriften des Neuen Testaments und der übrigen urchristlichen Literatur, Giessen Igro, s.vv. Evớw IIb et $\mu$ vela I; A. Debrunner dans Fr. Blass-A. Debrunner, Grammatik des neutestamentlichen Griechisch, Göttingen 19134, § 197 et $193 \mathrm{I}^{\circ}, \S 347^{\mathrm{a}}$ et $3^{60^{4}}$; C. H. Turner, The Shepherd of Hermas and the problem of its text, The Journal of Theological Studies 21, r920, p.(193-209) I98; M. Dibelius, Der Hirt des Hermas, Tübingen 1923, p.435; Chr. Mohrmann, Les origines de la latinité chrétienne a Rome, Études III, Pp.(67-126) 75-76; W. Coleborne, $A$ linguistic approach to the problem of structure and composition of the Shepherd of Hermas, dissertation non publiée, Newcastle, N.S.W. 1965, Pp. 49, 227, 372, $377,382,420,439,444,592-593$.

2. Zahn, Hirt, P.487; Caspari, Quellen, note p.298; P.Baumgärtner, Die Einheit des Hermas-Buchs, Freiburg i.B. 1889, P.50; Blass, Grammatik, p.33 n.I.

3. Lipsius, compte-rendu de Ae. Fr. C. Tischendorf, Hermae Pastor graece, col. 746.

4. Donaldson, Shepherd, p.5I2. Mais p.5I4 cette opinion a fait place à celle dont nous avons parlé plus haut p.4. Tumer, Shepherd, p.198.

5. Mohrmann, Origines, PP.74-78. 
latinisés au point de s'être formé des termes techniques latins ${ }^{1}$. Mais dès lors, la question se pose de savoir pourquoi un auteur qui possédait le latin et qui écrivait dans une communauté pour une bonne part latine, s'adresse à son public en grec.

Nous abordons ici un autre problème auquel le Pasteur nous confronte: celui de l'époque à laquelle il a été écrit. Les a vis à ce sujet sont partagés et nous y reviendrons plus loin en détail. Contentonsnous ici de signaler que Christine Mohrmann opte pour l'hypothèse qu'Hermas écrivait vers le milieu du IIe siècle, mais se faisait passer pour un contemporain de Clément de Rome, qui vivait un bon demisiècle plus tôt. Cette fiction impliquait qu'il utilisât la langue employée chez les Chrétiens de Rome à l'époque de Clément, c'est-à-dire le grec. D'autre part, il ne parvint pas à effacer entièrement les traces du latin, qui lui était plus familier, d'où les latinismes.

A l'exception de Tischendorf et Donaldson, tous les auteurs cités plus haut ont supposé que le texte grec qui nous est parvenu est l'œuvre du "Père Apostolique» Hermas lui-même. La théorie de la rétroversion était, surtout après la découverte du Codex Sinaiticus, condamnée à mourir de sa belle mort; aussi, après 1877 , personne ne l'a plus défendue. Seul Resch, en I893, estima, sans d'ailleurs fournir l'ombre d'une preuve, qu'il s'agissait d'une forme très spéciale de rétroversion. Il ne doutait pas, il est vrai, de l'originalité du texte d'Hermas que nous connaissons, mais il supposait que les nombreuses allusions aux évangiles qui se rencontrent dans le Pasteur étaient dues à la rétroversion d'un très ancien texte latin des évangiles; cela expliquerait aussi pourquoi on eut tant de peine à les retrouver ${ }^{2}$. Resch ne trouva personne pour le suivre.

D'ailleurs, l'idée que notre texte d'Hermas est une traduction du latin n'a pas sombré définitivement. Au Congrès Patristique d'Oxford, en 1967 , l'Australien Tanner soutint l'hypothèse d'un original latin du Pasteur ${ }^{3}$. Il s'en tint aux Préceptes, dont il estimait que Coleborne avait prouvé que c'était la plus ancienne partie du Pasteur. Trois passages des Préceptes, à savoir 34,6, 35,2-3 et tout le chapitre 27 , contiendraient. à son avis, des obscurités qui disparaîtraient si l'on reconstitue le texte

I. Pour plus de détails à ce sujet voir son article Statio, Études III, pp. (307-330) 308-310.

2. A. Resch, Aussercanonische Paralleltexte zu den Evangelien, I I, Leipzig I893, P.4.

3. R. G. Tanner, Latinisms in the text of Hermas, Colloquium 44, 1972, Pp. I2-23. Nous tenons à remercier $\mathrm{M}$. Tanner qui a eu la bonté de nous procurer une copie de son article. 
original. Voici à titre d'exemple deux points de son commentaire de

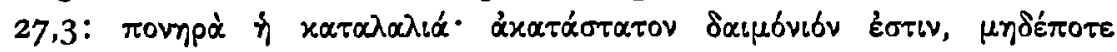

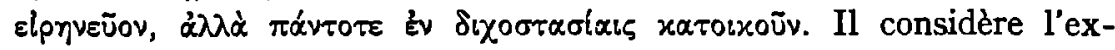

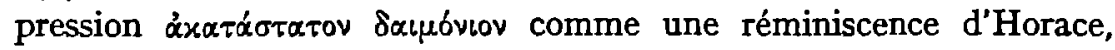
Ep. 2,2,187-189:

scit genius, natale comes qui temperat astrum,

naturae deus humanae, mortalis in unum

quodque caput, vultu mutabilis, albus et ater.

Vu sous le jour de ce passage d'Horace, il est clair, à son avis,

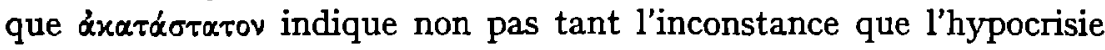
(vultu mutabilis, albus et ater). L'expression xo. fade traduction de colere aliquid, que le traducteur comprend à tort dans le sens de thabiter dans» au lieu du sens beaucoup plus fondamental de acultiver». Il construit l'original latin de ce passage comme ceci: Prava est maledictio; "genıus mutabilis" est; numquam conciliat, semper seditionem colit.

En introduisant son raisonnement, Tanner invoque les latinismes dans le Pasteur; il se réfère à ce sujet à Turner et Mohrmann. Mais, dans sa propre argumentation, ces latinismes ne jouent aucun rôle: il se fonde alors sur des fragments de phrases qu'il appelle bien des latinismes, mais qui, en réalité, ne contiennent rien qui ne soit pas grec. Ce sont, en effet, des "erroneous renderings of a Latin original" en un grec qui est correct en soi. C'est pourquoi son analyse ne peut avoir d'utilité pour l'étude des latinismes que si la thèse principale - à savoir que le Pasteur a d'abord été écrit en latin - a été démontrée d'une façon probante. Or, ce n'est pas plus le cas que ce ne le fut pour la thèse de Tischendorf avant lui.

\section{L'ELEMENT SEMITIQUE}

Pendant les premières années qui suivirent la publication du texte grec du Pasteur, la fraus Simonidis et la théorie de Tischendorf occupèrent tellement les esprits que l'attention ne se porta guère sur la question des sémitismes. Tischendorf lui-même parle incidemment d'influence sémitique lorsque'il dit considérer comme un latinisme la manière

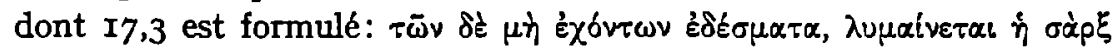
$\alpha \dot{\tau} \tau \bar{\omega}$, étant donné qu' «ad hebraismos Graecae dictionis Hermae vix concludere licet»; mais il laisse ignorer au lecteur la raison pour laquelle "ce n'est guère permis"'. L'auteur anonyme qui commente l'œuvre de r. Tischendorf, Hermae Pastor, p.XIV (= I857, p.LIII). 
Tischendorf dans le Leipziger Repertorium de 1857 , s'il est disposé à admettre la chose, signale pourtant deux autres cas d'influence

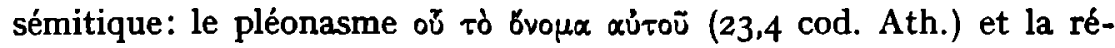

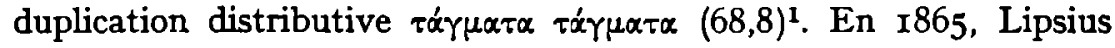
four nit des données nouvelles, surtout des expressions bibliques ou des mots à sens biblique ${ }^{2}$.

L'étude de loin la plus importante est celle de Zahn, qui parut trois ans plus tard. Dans ce livre, Zahn ajoute un appendice où il énumère la plus grande partie de la documentation de Lipsius, quelques dizaines d'autres mots et expressions "judéo-grecques» et un certain nombre de sémitismes syntaxiques comme le datif au lieu de l'accusatif de relation,

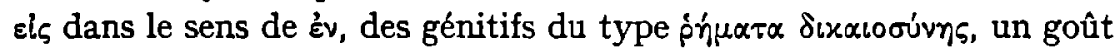
de la parataxe avec xal, év et $\mu \varepsilon \tau \dot{\alpha}$ construits avec une proposition infinitive, des constructions participiales umanquées" et l'absence de l'optatif ${ }^{3}$. Etant donné que, juste avant, il avait examiné les rapports entre Hermas et le Nouveau Testament, il ne s'occupait pas ici des réminiscences néotestamentaires qu'il avait rencontrées dans le Pasteur. Ce qui restait donc en fait d'expressions sémitisantes, il le considérait comme du ujudéo-grec», c'est-à-dire comme appartenant à l'idiome des milieux juifs où la langue usuelle était le grec, mais où souvent aussi c'était l'araméen et où l'on connaissait l'hébreu, où, en tout cas, le grec était marqué par l'influence de ces deux langues. La langue du Pasteur montrait donc que l'auteur avait grandi dans un milieu juif.

Voici ce qu'en dit Zahn: "...so wird die Behauptung zu Recht bestehn, dass Hermas nicht nur kein gebildeter, sondern überhaupt kein aus dem Geist der griechischen Sprache heraus seine Rede gestaltender Schriftsteller ist. Er hat sie vielleicht von Jugend auf gesprochen; aber seine Muttersprache im vollen Sinn des Worts ist sie nicht. Entweder er hat sie in einer Gestalt überkommen, welche auf der Grundlage hebräischer Gedanken- und Begriffsbildung ruht, oder er hat gar neben ihr die $\pi \dot{\alpha} \tau p$ tos $\gamma \lambda \omega \bar{\omega} \sigma \sigma \alpha$ der damaligen Juden gehört und gesprochen und deren ältere Grundlage gekannt. Seine Sprache böte der Annahme kein Hindernis, dass das Haus, in welchem er gross geworden ist, ein echt jüdisches gewesen sei und selbst auf palästinen-

1. Leipziger Repertorium der deutschen und ausländischen Literatur I5 $^{\mathbf{I}}$, I857, $\mathrm{p}(\mathrm{I}-8) 4$.

2. R. A. Lipsius, Der Hirte des Hermas und der Montanismus in Rom, Zeitschnft für wissenschaftliche Theologie 8, 1865, p.(266-308) 270 n.2.

3. Zahn, Hivt, pp.485-497. 
sischem Boden gestanden habe»1. Nous avons tenu à citer le passage en entier pour que ne se perdent pas les nuances. Nous y distinguons deux idées essentielles: (I) l'idiome ujudéo-grec» s'écarte à tel point du grec courant qu'on peut à peine dire de ceux dont c'était la langue véhiculaire que leur langue maternelle est le grec; (2) cet idiome "judéo-grec" sert aussi bien de langue parlée que de langue écrite.

L'étude de Zahn a très longtemps fait autorité. Mais sur certains points la controverse ne se fit pas attendre. Tout d'abord, le caractère sémitisant d'une partie des matériaux qui servaient de preuve s'avéra douteux. A cet égard, Donaldson commença par éliminer eic au sens de év; plus tard, Dibelius rejeta en outre la parataxe, le datif de relation, l'absence de l'optatif 2 . Ensuite, le bien-fondé de la notion de «judéo-grec», telle que la concevait Zahn, fut de plus en plus mis en doute $^{3}$. Et enfin, on se demanda, même indépendamment des deux considérations précédentes, s'il était indispensable de supposer l'existence d'un milieu juif pour expliquer la langue d'Hermas, étant donné qu'on pouvait déjà relever dans la Septante et chez les plus anciens Chrétiens tout ce que signalait $\mathrm{Zahn}^{4}$.

Après Zahn, on n'a guère apporté de données nouvelles ${ }^{5}$. La dernière étude originale est de Grobel et est datée de I95 ${ }^{\circ}$. Il signale au c.5I deux phénomènes qui, selon lui, sont courants dans la littérature juive de langue grecque, mais qui, dans le Pasteur et, en général, dans les écrits des premières générations chrétiennes, font complètement défaut, si ce n'est dans des passages d'origine juive: ce sont le stichochiasme (qui consiste à disposer en chiasme des membres de phrase) et l'emploi

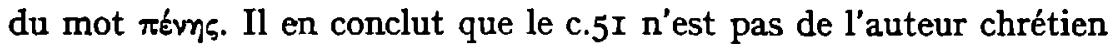
du Pasteur, mais est emprunté par celui-ci à un auteur juif?.

r. Zahn, Hirt, pp.495-496.

2. Donaldson, Shepherd, pp.509-510; Dibelius, Hirt, p.424.

3. Cf. H. Reinhold, De graecitate Patrum Apostolicorum librorumque apocryphorum Novi Testamenti quaestiones grammaticae, Halis Saxonum I898, pp.20-2I.

4. Comme le fit remarquer déjà Donaldson, Shepherd, p. 509.

5. Quelques remarques seulement chez Baumgärtner, Einheit, p.5o; D. Völter, Die Apostolischen Väter neu untersucht, I, Leiden 1904, Pp.208 (n.2), 228-229, 293-294.

6. K. Grobel, Shepherd of Hermas, Parable II, Vanderbilt Studies in the Humanities I, I95I, Pp.(50-55) 51-52.

7. Le raisonnement est très discutable. En ce qui concerne les faits: le ustichochiasme" se présente aussi chez Hermas, par exemple dans 66,7 et ro8,5, et on trouve aussi $\pi t$ th $_{\text {, }}$ en plus des passages qui sont peut-être d'origine juive comme Did. 5,2 et Ps. Barn. 20,2; chez Polycarpe 6, I. 


\section{REMARQUES GENERALES}

Les études dont nous avons parlé ci-dessus sont en général de peu d'étendue. Elles font en général partie d'études d'une plus grande ampleur et elles n'étaient pas l'essentiel pour leurs auteurs. Voilà sans doute la raison pour laquelle les sémitismes et les latinismes n'ont jamais été traités ensemble. C'est ainsi qu'il arrivait qu'un «latiniste» relevât comme un échantillon d'influence latine ce qu'un "sémitiste" attribuait à une influence sémitique et vice versa. Tischendorf, par

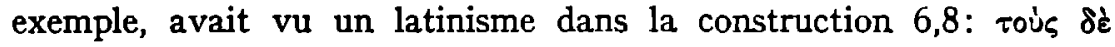

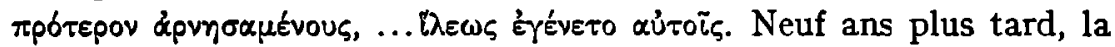
même construction trahissait pour Lipsius une influence sémitique ${ }^{1}$. C'est ainsi que le datif de relation qui, chez Hermas, prend régulièrement la place de l'accusatif de relation du grec classique, est relevé par Zahn comme un sémitisme, mais est ramené par Debrunner à l'ablatif latin correspondant2. Malheureusement, nous ne voyons jamais fournir les raisons qui déterminent un auteur à prendre le contre-pied d'un prédécesseur.

Les exemples que nous avons choisis invitent à une autre remarque: nombre de phénomènes linguistiques ont été attribués à une influence sémitique ou latine en un temps où ce qu'on appelait «le grec" était censé être encore identique au grec classique du Ve ou IVe siècle avant J.-C. Mais cette erreur n'allait plus durer longtemps: le développement de la linguistique, ainsi qu'une connaissance plus approfondie du grec tardif, allaient bientôt ouvrir la voie à des conceptions plus justes. Du coup tombèrent bon nombre de prétendus sémitismes et latinismes. C'est ainsi qu'après Tischendorf et Lipsius, personne ne s'avisa de voir dans 6,8 une construction non grecque; on retrouva des constructions parallèles non seulement dans d'autres langues, mais aussi dans les papyrus grecs et même chez les grands classiques. Le datif de relation continua à choquer, comme il le fait encore chez Giet en I963; mais, dans une grammaire comme celle de Mayser, il est mentionné depuis longtemps comme un élément authentique du grec tardif ${ }^{3}$.

I Tischendorf, Hermae Pastor, p XIV (= 1857, p LIII); Lipsius, Montanısmus,

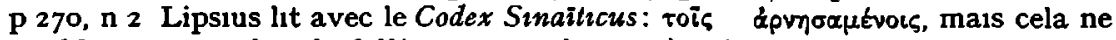
semble pas avoir fait de différence pour la manière de juger

2 Dans Fr Blass-A Debrunner, Grammatık, $\$ 197$ Voir aussı infra p 123

3 S Giet, Hermas et les Pasteurs, Paris 1963, P 284, E Mayser, Grammattk der Papyrz aus der Ptolemäerzent, II 2, I, Berln-Leipzig 1933, pp I 49-I 50 (note $\imath b$. $\mathrm{P}$ I50 Vom Einfluss des lateinischen Ablat limitationis kann in agyptischen Texten nicht die Rede sein()), voir aussi SD II, p I68 
Schématiquement, la présence de sémitismes et de latinismes peut $s^{\prime}$ expliquer de trois manières: $I^{\circ}$ notre texte grec est la version d'un original sémitique (ou bien latin); $2^{\circ}$ l'auteur s'exprime en grec, mais il a plus l'habitude d'une langue sémitique (ou du latin); $3^{\circ}$ l'auteur s'exprime dans la langue qui lui est la plus familière, le grec, mais utilise des sémitismes et des latinismes parce que le grec de son époque ou du moins de son milieu les avait déjà adoptés.

La première explication se trouve, pour les latinismes, chez Tischendorf et Donaldson. Ils tenaient notre texte pour la traduction d'une traduction latine du texte original; mais ils abandonnèrent presque entièrement cette hypothèse lorsqu'il apparut que le texte du Codex Athoiis, du XVe siècle, concordait essentiellement avec celui du Codex Sinaiticus, du IVe siècle; plus tard, on devait d'ailleurs trouver encore des fragments de papyrus du IIIe et du IIe siècles. Récemment, Tanner essaya de faire admettre un original latin pour au moins les Préceptes; il ne tirait pas ses arguments du caractère non idiomatique du grec, mais de traductions erronées en un grec correct en soi.

Personne ne s'est jamais avisé de prendre à son compte l'hypothèse d'un original sémitique. Au contraire, les rares auteurs dont l'esprit en fut effleuré les rejetèrent aussitôt ${ }^{1}$, et $\mathrm{W}$. J. Wilson, celui-là même qui admettait un original araméen derrière Actes I-I5 rangeait résolument Hermas parmi les Chrétiens d'origine paiennea.

Jusqu'à ce jour, la deuxième et la troisième explication restent en présence. C'est d'après les faits qu'il faudra décider quelle est la plus vraisemblable.

Enfin, signalons les études qui, sans parler expressément de la langue d'Hermas, arrivent à des conclusions telles qu'il faut supposer qu'Hermas connaissait parfaitement l'hébreu et l'araméen, ou le latin. Nous ne parlerons que des plus récentes. Dans un article de I953, Audet se dit frappé par les analogies dans la pensée théologique entre Hermas et le Manuel de discipline de Qumran, connu depuis peu à cette date. Il estime que la meilleure explication est de considérer Hermas comme le fils d'un membre de la communauté de Qumran, qui, après la guerre de 70 après J.-C., a été emmené en esclavage à

I. A. Hilgenfeld, Hermae Pastor, Lipsiae r8812, p.XXXI; H. J. Cadbury, Luke - translator or author?, American Journal of Theology 24, 1920 , p. $(436-455) 447$.

2. W. J. Wilson, The career of the prophet Hermas, The Harvard Theological Review 20, 1927, pp.(21-62)4I, 6I, 62. Cf. le même, The unity of the Aramaic $A c t s$, The Harvard Theological Review I1, 1918, Pp.322-335. Cf. aussi M.Black, An Aramaic approach to the Gospels and Acts, Oxford 1967', Pp. 56-57, 61. 
Rome ${ }^{1}$. Au c.5I, Hermas utilise l'image de la vigne qui pousse contre l'orme. Cette manıère de cultiver la vigne était coutumière en Italie, et c'est pourquoi Grobel voyait dans cette image la preuve qu'il fallait chercher l'auteur en Italie ${ }^{2}$. Alfonsi, de son côté, fait un pas de plus: étant donné que, dans la littérature latine, il est souvent question de la vigne qui grimpe contre l'orme, soit pour rapporter le fait, soit pour en tirer un sens symbolique, il en conclut qu'Hermas a $d \hat{u}$ trouver son inspiration chez les auteurs latıns, de sorte qu'il est évident «che Erma anche da questo indizio dimostra di essere un latino" ${ }^{9}$. Peu de temps avant, Schwartz avait déjà acquis la certitude qu'Hermas, du moins l'auteur de 24-77 et III-II4, uest un lettré qui connaît les Bucolıques de Virgile"', comme de nombreuses rémıniscences en témoigneraient.

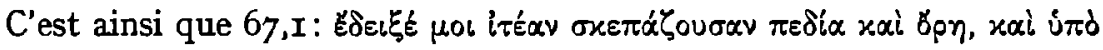

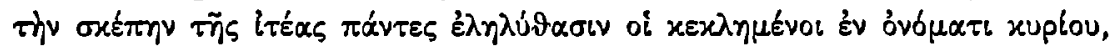
par exemple, lui faisait penser irrésistiblement à des passages comme Ecl. I0,40:

mecum inter salices lenta sub vite iaceret,

vers à propos duquel, pour être juste, il faut remarquer que la chose est d'autant plus frappante qu'il y est question de la vigne dont Hermas parle lui-même ailleurs. Nous avons déjà dit que Tanner signale que notre auteur cite Horaces.

Toutes ces conclusions nous laissent une impression d'insatisfaction. Ou bien on utilise un petit nombre de données, parfois même une seule, qui plaident en faveur de la thèse à établir, ou bien on se réclame d'une documentation qui ne suffit pas à prouver ce qu'on prétend. Lorsqu'on

I J -P Audet, Affinites litteraires et doctrinales du Manuel de Discipline (surte), Le "Pasteur" d'Hermas, Revue Biblique 60, 1953. P (4I-82)82 Cf J Reiling, Hermas and Christzan prophecy, Leiden 1973, pp 25-26, I34 (n I), I 73

2 Grobel, Parable II, pp 52-55 D'arlleurs, sa conclusion va déjà trop lon, cf, par exemple, les citations dans H G Liddell-R Scott-H S Jones, $A$ Greek-

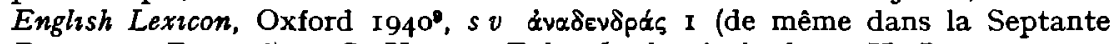
Ps 79,II, Ez 17,6) et S Krauss, Talmudische Archäologie, II, Leıpzig I9II, Pp 229-230, 605

3 L Alfonsi, La vite e l'olmo, Vigiliae Christianae 2I, 1967, pp (8I-86)85-86.

$4 \mathrm{~J}$ Schwartz, Survivances littéraires paīennes dans le "Pasteur" d'Hermas, Revue Biblique 72 , 1965, p (240-247)246 Mars sa conclusion ne concerne que l'auteur de 25-77 et I I I-I I 4, 1 l suit en cela Gret, Les Pasteurs, pass, qu1, à son avis, a démontré "d'une manière définitıve" que le Pasteur est do à trois auteurs Nous parlons de la théone de Giet plus loin, pp 20-2 I

5 Nous laissons évidemment au compte de Schwartz et de Tanner la supposition des vastes lectures d'Hermas Nous n'en avons en tout cas aucune preuve, cf les remarques sur les sources littéraures chez Chnstıne Mohrmann, Problemes philologiques et littéraires, Augustnus Magister, III, Pans I954, PP (27-4I)34-38. 
examine séparément la valeur probante de chaque fait et qu'ensuite on les rassemble, on ne tarde pas à se convaincre, qu'à s'en tenir à des données non linguistiques, il est impossible de découvrir le pays d'origine d'Hermas ou les langues qu'il connaissait, du moins d'après le Pasteur. 


\section{QUESTIONS PRÉLIMINAIRES}

\section{LE TEXTE}

La plus grande partie du texte du Pasteur nous est connue sous la forme grecque; nous disposons, en outre, de plusieurs anciennes versions, qui nous sont parvenues plus ou moins complètes'.

La tradition grecque comprend trois manuscrits incomplets: le Codex Athoüs (A), du XVe siècle, contient $95 \%$ du texte (I,I-IO7,2), le Codex Sinaiticus (S), du IVe siècle, le premier quart $(I, I-3 I, 6)$ et le Michigan Codex (M), du IIIe siècle, le troisième quart (5I,8-82,I). Dans de nombreux cas, $A$ diffère de $S$ et $M$ de telle sorte qu'il est difficile de dire quelle est la meilleure leçon et, dans beaucoup d'autres cas, A s'avère plus classique et plus littéraire que $S$ et $M$; dans un nombre beaucoup plus restreint de cas, ce sont $S$ et $M$ qui sont plus classiques. Ensuite, nous connaissons le texte grec par de nombreux fragments sur papyrus et sur parchemin ${ }^{2}$ et par des citations chez des auteurs ultérieurs.

En latin, nous possédons deux versions complètes, la Vulgate $\left(\mathrm{L}_{1}\right)$ et la Palatine $\left(\mathrm{L}_{2}\right)$. D'habitude, - mais les preuves sont faibles -, on les date l'une de la fin du II' siècle, l'autre du IVe ou du Ve siècle.

I. Pour plus de détails sur la tradition manuscrite, voir $\mathrm{C}$. Bonner, $A$ papyrus codex of the Shepherd of Hermas (Similitudines 2-9) with a fragment of the Mandates, Ann Arbor 1934, Pp.I-32; C. E. Demaray, Studies in the language of Hermas, dissertation non publiée, Ann Arbor 1940, PP.I 7-2I, 134; Giet, Les Pasteurs, pp.47-63 et les introductions des éditions critiques. De notre cóté, nous n'avons consulté la tradition grecque et latine que sous sa forme publiée et la tradition copte et éthiopienne dans ses traductions modernes. Pour ce qui est de l'étude de M. Demaray, l'auteur a eu l'extrême amabilité de nous prêter une copie de sa thèse: qu'il en soit vivement remercié.

2. D'aucun autre texte patristique on n'a retrouvé autant de fragments, cf. W. H. Willis, $A$ census of the literary papyri from Egypt, Greek, Roman and Byzantine Studies 9, I968, Pp.(205-24I)212, 227. A ses fragments s'ajoute un quinzième, un papyrus contenant $32,4-33,4$ publié par $K$. Treu, Ein neuer Hermas-Papyrus, Vigiliae Christianae 24, I970, pp.34-39. 
Lorsque l'édition critique des deux versions, qui est en préparation', sera prête, il faudra soumettre la question à un nouvel examen. Leur qualité, en tout cas, est telle qu'elles peuvent fort bien être utilisées pour reconstituer le texte original. Il existe aussi deux versions en copte, l'une akhmîmique $\left(C_{1}\right)$ et l'autre sahidique $\left(C_{2}\right)$, mais qui ne nous sont parvenues que par fragments d'ailleurs importants. Elles datent de l'Antiquité: $C_{1}$ se trouve dans un codex du IVe siècle, $C_{2}$ en partie dans un codex du $\mathrm{V}^{\mathrm{e}}$ et en partie dans un codex du VI ${ }^{\circ}-\mathrm{VII}^{\circ}$ siècle. Ces versions, elles aussi, sont considérées comme des témoins précieux du texte. Ensuite, nous avons une version éthiopienne complète qui, sans doute, date du IVe siècle, mais dont la valeur est limitée par des modifications, d'inspiration dogmatique, dans le texte et par une tendance à traduire d'une manière souvent libre et abrégée. Pour être complet, citons encore les restes peu importants et peu utilisables d'une traduction en moyen-perse.

En comparaison de l'Epitre à Diognète, par exemple, ou des œuvres de Justin, qu'on ne connaît que par un seul manuscrit de la fin du moyen âge, nous avons là une tradition enviable. Cependant, pour reconstituer le texte original, on se heurte à deux difficultés: en beaucoup de passages, les témoins du texte divergent et il est impossible de classer la tradition en familles, ce qui permettrait d'éliminer une partie des témoins. Pour certains ces difficultés paraissent insurmontables. Musurillo pense que, dans la mesure où les textes diffèrent, il n'y a rien d'autre à faire qu'à les imprimer en colonnes parallèles². Audet est d'avis qu'étant donné la diversité de la tradition, le nombre des témoins du texte est trop petit pour qu'on puisse faire un choix ${ }^{2}$. Mais ne voir que cette diversité serait injuste. Il est bien vrai que les témoins présentent de nombreuses différences; mais ces différences sont pour la plupart insignifiantes: variantes morphologiques, petits changements dans l'ordre des mots, additions ou suppressions de particules, articles, prépositions, pronoms personnels. En outre, on peut établir des critères qui permettent de choisir les meilleures leçons. Parmi ceux-ci, les deux les plus importants nous semblent être les suivants:

(a) $\mathrm{S}$ et $\mathrm{M}$ sont à préférer à $\mathbf{A}$. D'abord, ils sont plus "vulgaires»; or, la normalisation ultérieure d'un texte vulgaire est plus vraisemblable

I. Par R. A. B. Mynors d'Oxford.

2. H. A. Musurillo, The need of a new edition of Hermas, Theological Studies 12, I95 I, pp. (382-387) 386-387.

3. Audet, Pasteur, P.50 n.I. 
que le contraire'. En outre, dans le temps, ils sont considérablement plus près du texte original que $A$.

(b) Lorsque sont en face l'une de l'autre deux leçons de valeur égale, les autres témoins tranchent souvent dans un sens ou dans l'autre, précisément parce qu'ils sont indépendants les uns des autres.

Quelques années après les remarques faites par Musurillo et Audet, parurent deux éditions critiques du Pasteur, celle de Whittaker et celle de Joly², qui foumissaient la preuve qu'il était parfaitement possible d'arriver à une reconstruction satisfaisante du texte. Il est vrai que, pour I75 passages, elles présentent des divergences, mais il s'agit presque toujours de points peu importants, qui n'altèrent pas le sens et n'affectent que superficiellement le style ${ }^{3}$. Dans l'ensemble on a l'impression d'une concordance fondamentale. Et le nombre des divergences peut, à n'en pas douter, être encore réduit. Nous croyons pouvoir conclure en disant que, pour le Pasteur, nous disposons d'un texte qui présente suftisamment de garanties pour qu'on puisse y étudier la langue de l'auteur.

Voyons maintenant comment les deux éditeurs modernes, Whittaker et Joly, ont procédé et comment nous devons juger les résultats qu'ils ont obtenus. Dans l'ensemble, ils ont bien utilisé la tradition manuscrite. Pourtant, ils n'ont pas tiré tout le parti qu'il fallait des matériaux disponibles, étant donné qu'ils ont seulement pris connaissance de la tradition manuscrite sous sa forme éditée. Cela signifie deux choses: d'abord, étant donné qu'ils n'ont pas vu eux-mêmes les manuscrits, les différentes leçons qu'ils donnent ne sont pas à mettre à leur compte; ensuite, les textes témoins qui n'ont pas encore été publiés, à savoir un certain nombre de manuscrits latins et un manuscrit éthiopien, n'ont pas été utilisés. En outre, il semble bien que les éditeurs n'aient consulté les textes éthiopiens et coptes déjà publiés que dans des traductions moderness.

I. Cf. Bonner, Papyrus codex, pp.22-31.

2. M. Whittaker, Der Hirt des Hermas, Berlin 19561, r967²; R. Joly, Hermas, Le Pasteur, Paris 19581, 1968². H. Stegemann, de Marbourg, prépare une nouvelle édition.

3. Comme on le voit aisément dans la liste des variantes de l'édition Whittaker que Joly a insérée dans sa propre édition. On s'étonne de voir qu'il affirme ailleurs (compte-rendu de M. Whittaker, Der Hirt des Hermas, L'Antiquité Classique 27, 1958, p.[188-190]r89) qu'il n'y a qu'environ 60 passages où il s'écarte de Whittaker.

4. Comme on peut le conclure de leurs éditions; cf. également Whittaker, Hirt, pp.IX (n.3), X, XVI; Joly, Pasteur, p.67.

5. Cf. F. Petit, compte-rendu de M. Whittaker, Der Hirt des Hermas, Recherches de Théologie Ancienne et Médiévale 24, 1957, p.(380-382)381. 
Voilà ce que les éditions ont en commun. Passons aux différences. L'apparat critique de Whittaker est beaucoup plus détaillé que celui de Joly, comme ce dernier le souligne lui-même à plusieurs reprises. Les éditeurs ne donnent pas de détails sur la façon dont ils ont établi le texte, mais les résultats permettent d'en juger. Alors que, dans beaucoup de cas, Whittaker chosit, là où la choise est raisonnablement possible, la lectio difficilior, la tendance de Joly est de préférer une variante tardive, une variante qui n'a été transmise qu'en traduction, une conjecture sans fondement aucun dans la tradition. Il en résulte que le texte de Joly, là où il s'écarte de celui de Whittaker, est souvent plus coulant, plus littéraire, ce qui se manifeste par les formes plus classiques et les particules de liaison qu'il y intègre. Il ne donne pas les raisons de cette manière de procéder. On le soupçonne de vouloir montrer qu'Hermas a plus de talent littéraire qu'on ne lui en accorde en général ${ }^{1}$. S'il en est ainsi, il donne dans un cercle vicieux: Hermas a du talent, donc il faut donner au texte qu'on reconstruit à partir des manuscrits un tour littéraire; le texte est relativement littéraire, donc Hermas avait du talent. Jusqu'à présent, l'édition de Whittaker nous semble plus près du texte original que celle de Joly.

Whittaker l'emporte donc sur Joly tant pour l'apparat critique que pour le texte. Il n'empêche que son ouvrage contient, lui aussi, quelques erreurs ${ }^{2}$. C'est pourquoi, à l'aide des éditions des différents textes-témoins, nous avons vérifié nous-même tous les passages d'Hermas que nous étudions. Dans la mesure du possible, nous citons d'après Whittaker. Là où sa leçon est inacceptable, nous donnons le texte tel que nous avons cru devoir le reconstituer nous-même, en indiquant chaque fois que nous nous écartons de Whittaker. Remarquons enfin qu'en général nous avons traité comme des textes authentiques d'Hermas, sauf preuve du contraire, les fragments de provenances diverses qui font suite à I07,2, là où A s'arrête.

I. Cf. Joly, Pasteur, pp.55-57.

2. Son appréciation des témoins du texte n'est pas tout à fait conséquente (par exemple dans 4,3 et $1_{3}$, I elle choisit avec $S-\alpha v$ au lieu du -ov de $A$, dans $I_{0,7}$ avec $\mathrm{A}$-ov au lieu du $-\alpha v$ de $\mathrm{S}$ ), elle a trop (le clémence pour des émendations (cf. par exemple pour les optatifs infra pp.62-63) et des lectiones faciliores (par

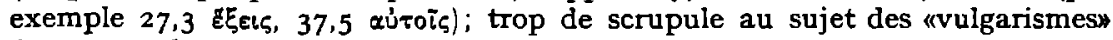
(par exemple l'accusatif -Es $2 \mathrm{I}, 3 ; 72,5.6)$. L'apparat critique n'est pas complet en ce qui concerne les variantes morphologiques (quelques omissions:

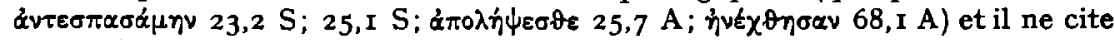
pas toujours les témoins de façon correcte (par exemple dans 57,$5 ; 63,6 ; 67,18$, les prétendues leçons du manuscrit sont en réalité des reconstructions de Bonner). 
2. UN OU PLUSIEURS AUTEURS?

Le Pasteur contient, dans le fond et dans la forme, tant de différences d'accent et tant d'inégalités que Snyder ne craignit pas de parler d'un (masterpiece of inconsistencies»' Voici quelques exemples, qu'on pourrait multiplier: dans 26,I Dieu est dit á $\chi \dot{\rho} p \eta \tau o s$ à titre exclusif, mais dans $9 I, 5$ c'est le Fils de Dieu qui reçoit ce prédicat. Dans $I, 6$, c'est à cause de l'Église, dans 47,2, à cause de l'homme, que le monde a été créé. La vision de la construction de la tour des Visions revient sur le tapis dans Similitude 9. Sim. 5 présente des traits d'adoptianisme qui s'accordent mal avec ce qui est dit dans Sim. 9 au sujet du Fils de Dieu. La morphologie et le vocabulaire sont remarquablement capricieux.

On pourrait penser que cette diversité est le propre d'un écrivain qui a peu de métier. Mais, depuis la deuxième moitié du siècle dernier, plusieurs spécialistes ont pensé qu'il faut l'expliquer en attribuant le Pasteur à deux ou à plusieurs auteurs. Du reste, pareille hypothèse ne s'appuyait pas seulement sur les inégalités de l'ouvrage lui-même, elle pouvait aussi invoquer les contradictions dans les traditions qui, de l'Antiquité, sont parvenues jusqu'à nous au sujet de l'auteur ${ }^{2}$. Le premier argument a pris de plus en plus le pas sur l'autre. Lorsqu'en I852, Thiersch situa le Pasteur original au ler siècle après J. -C. et la rédaction finale au milieu du IIe siècle, il n'avait d'autre souci que de réconcilier les traditions anciennes ${ }^{3}$. Mais de Champagny et Guéranger, qui donnent les mêmes dates d'abord aux Visions puis aux Préceptes et aux Similitudes, utilisèrent aussi des critères internes ${ }^{4}$. Et la tentative la plus complète pour attribuer le $P$ asteur à plusieurs auteurs, celle de Hilgenfeld en I88I, vise déjà avant tout à expliquer les divergences dans le livre même ${ }^{6}$. Hilgenfeld propose une solution tripartite: "Hermas pastoralis» écrivit, vers roo après J.-C., Vis. 5-Sim. 7 , "Hermas apocalypticus" y ajouta, à l'époque d'Hadrien, Vis. I-4, et

I. G. F. Snyder, The Shepherd of Hermas, Camden, N.J. I968, p.6. Voir aussi infra p.I42 n. 3.

2. Voir infra pp.32-33. Pour un aperçu plus circonstancié des différentes hypothèses voir Giet, Les Pasteurs, pp.63-7o.

3. H. W. J. Thiersch, Die Kirche im apostolischen Zeitalter und die Entstehung der neutestamentlichen Schriften, Frankfurt am Main-Erlangen 18521, P.352; $185^{2}$, p. 352 .

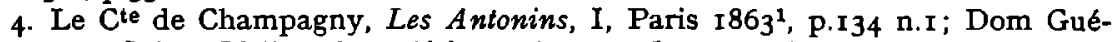
ranger, Sainte Cécile et la sociéte romaine aux deux premiers siecles, Paris $1874^{1}$, Pp. I28-1 29, 193.

5. Hilgenfeld, Hermae Pastor², Pp.XXI-XXIX, $13^{8 .}$ 
"Hermas secundarius» enfin apporta Sim. 8-ro, à l'époque de Pie Ier (I40-I55). Peu de temps après, Haussleiter date le Pasteur proprement dit ( $V$ is. 5-Sim. Io) de I50 environ et les quatre premières Visions, qui, à son avis, sont l'œuvre d'un autre auteur, d'environ $200^{1}$. Aucune de ces théories ne s'est imposée et, lorsque deux élèves de Harnack, Adolf Link et P. Baumgärtner, réfutèrent les idées de Hilgenfeld dans deux publications différentes, il sembla bien que les recherches dans ce sens étaient définitivement abandonnées ${ }^{2}$.

Vers la fin du XIXe siècle, certains commentateurs, tout en tenant compte des critiques faites à la thèse de Hilgenfeld et autres, se mirent à la défendre sous une nouvelle forme: Spitta, van Bakel, Völter et Schläger pensèrent que le Pasteur était un écrit juif dans lequel des Chrétiens avaient fait des interpolations plus ou moins importantes. Mais ils ne purent s'accorder sur les détails et, en moins de huit ans, Völter modifia jusqu'à deux fois sa théorie ${ }^{3}$. On ne s'étonne donc pas que celle-ci rencontrât un refus presque général. Au cours du $\mathrm{XX}^{\mathbf{e}}$ siècle, l'idée que le Pasteur était l'œuvre d'un seul auteur finit par s'imposer comme allant de soi. En I958, Joly exprimait l'opinion générale quand il écrivait: «l'œuvre est une et les thèses - différentes, contradictoires - d'une pluralité d'auteurs méritent à peine d'être mentionnées» ${ }^{4}$. Nous ne nous arrêterions pas plus longtemps à ce problème si, récemment, on n'avait essayé de démontrer avec plus d'arguments que jamais que le Pasteur est une œuvre composite. Il s'agit des études du Français Giet et de l'Australien Coleborne. Examinons d'abord ces études. Nous développerons ensuite notre propre point de vue sur les disparates que présente le Pasteur.

En I963, Stanislas Giet lança l'idée que nous trouvons dans le Pasteur un assemblage des cuvres de trois auteurs ${ }^{5}$. Il imaginait la genèse de notre texte de la façon suivante. Dans le premier quart du

I. I. Haussleiter, De versionibus pastoris Hermae latinis, Acta Seminarii Philologici Erlangensis 3, I884, pp.(399-477) 47 I-476.

2. A. Link, Die Einheit des Pastor Hermae, Marburg 1888; Baumgärtner, Einheit. Voir également le compte-rendu de ces livres par Hilgenfeld dans Zeitschrift für wissenschaftliche Theologie 32, I889, Pp.363-373.

3. Fr. Spitta, Zur Geschichte und Litteratur des Urchristentums, II, Göttingen 1896, Pp.24I-437; H. A. van Bakel, De compositie van den Pastor Hermae, Amsterdam 1900, pass.; D. Völter, Die Visionen des Hermas, die Sibylle und Clemens von Rom, Berlin I90o, pass.; le même, Die apostolischen Vater, I, pp. 173-327; II I, pp.61-69; G. Schläger, Der Hirt des Hermas eine ursprünglich jüdische Schrift, Nieuw Theologisch Tijdschrift 16, 1927, pp.327-342.

4. Joly, Pasteur, p.I6. Cf. Musurillo, New edition, p.383 n.5.

5. Giet, Les Pasteurs, pass. 
IIe siècle, Hermas écrivit son appel à la conversion, ce qui représente $V$ isions I-4. Vers le milieu du siècle, le succès de ce petit ouvrage incita un autre auteur à ajouter à cet appel sa doctrine sur le Fils de Dieu. C'est ainsi qu'apparut le Pasteur, qui correspond à notre Similitude 9. Un troisième auteur enfin, qui voulut remplacer les vues de son prédécesseur par ses idées adoptianistes, inséra, quelques années plus tard, sa contribution, le Pseudo-Pasteur comme l'appelle Giet, en partie entre l'ouvrage du premier et du deuxième auteur ( $\mathrm{Vis.}$-5im. 8) et en partie à la suite du deuxième ( $\operatorname{Sim}$. ro). Notre texte se divise donc en trois parties: Hermas (Vis. I-4 $=\mathrm{I}-24$ ), Pasteur (Sim. $9=78-\mathrm{IIO}$ ) et Pseudo-Pasteur ( $V$ is. 5-Sim. 8 et Sim. Io = 25-77.III-II4).

Giet n'a pu convaincre les spécialistes', comme on pouvait d'ailleurs s'y attendre. Comment, en effet, se représenter sa construction dans la réalité historique? Avec un peu de bonne volonté on pourrait encore comprendre la façon d'agir du deuxième auteur qui voulait exploiter au profit de ses idées le succès des Visions. Mais qu'est-ce qui a pu inciter l'adoptianiste à insérer son œuvre dans ce produit composite? S'il voulait une polémique, il ne fallait pas qu'elle passât inaperçue. Mais si on la remarquait, sa supercherie littéraire était inévitablement découverte et c'en était fait de sa crédibilitéa.

Ces objections valent à fortiori pour la théorie de William Coleborne qui, dans une étude volumineuse ${ }^{3}$, donne au Pasteur non moins de six auteurs. Ceux-ci auraient écrit successivement, au cours de la période qui va de 60 à environ roo après J.-C., les parties $26, I-46,3,50-66$, 25.46,4-49,5, I-24, 67-77 et 78-I07 (les derniers chapitres du Pasteur, qui ne nous sont pas parvenus en grec, ne sont pas pris en considération). Coleborne ne semble pas se rendre compte qu'il nous présente là une sorte de miracle de l'histoire littéraire. Ses arguments, qu'il tire

r. Cf. par exemple le compte-rendu de R. M. Grant, Gnomon 36, 1964, pp.357359; le compte-rendu de J. A. Fischer, Theologische Revue 6r, 1965, col.304-308; L. Pernveden, The concept of the Church in the Shepherd of Hermas, Lund I966, p.13. cf. p.70; M. Whittaker, Hermas, Shepherd of, New Catholic Encyclopedia 6, New York 1967, p.1074; L. W. Barnard, The Shepherd of Hermas in recent study, The Heythrop Journal 9. I968, pp.(29-36)31-32; Snyder, Shepherd, pp.6, 23; J.-P. Audet, compte-rendu de G. F. Snyder, The Shepherd of Hermas, Journal of Biblical Literature 87, 1968, p.(475-477) 475; Reiling, Hermas, p.23; mais surtout R.Joly, Hermas et le Pasteur, Vigiliae Christianae 21, 1967, pp.20I-218. 2. Giet ne semble pas voir de difficulté ici, cf. Les Pasteurs, p. 306.

3. Coleborne, Linguistic approach, pass. Sommaire chez le même, The Shepherd of Hermas, $A$ case for a multiple authorship and some implications, Studia Pa. tristica Io, Berlin 1969, pp.65-70, et $A$ linguistic approach to the problem of structure and composition of the Shepherd of Hermas, Colloquium $3^{2}, 1969$, PP. 133-142. 
en partie d'inconséquences offertes par le contenu, en partie des indices fournis par la tradition manuscrite, ne suffisent pas, et de loin, à nous convaincre. L'auteur cependant soumet sa théorie à une vérification linguistique.

Il établit notamment ce qu'il appelle un apparatus discernendi, liste de 162 formes d'expression linguistiques que les six auteurs étaient susceptibles d'utiliser plus ou moins souvent: genres, temps et modes des verbes, place des mots, pronoms, prépositions etc., et compte dans chacun des morceaux dans lesquels il partage le Pasteur la fréquence de ces 162 éléments. Si les différences sont grandes, c'est que les auteurs sont différents. Ce critère, juste en soi, n'est cependant pas précisé. L'auteur ne nous dit pas quand les différences deviennent significatives, combien d'éléments doivent présenter des différences significatives, de quel ordre de grandeur les textes à comparer doivent être au minimum. C'est ainsi que Coleborne trouve déjà "révélateur" que l'auteur de 26,I-46,3 (2I pages chez Whittaker) a une préférence

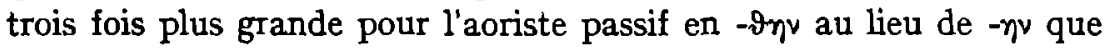
l'auteur de 46,3-49,5 (3 pages chez Whittaker) ${ }^{1}$. En général, il semble que seule une très grande égalité de fréquence dans les 162 éléments puisse le dissuader de conclure à une différence d'auteur. Mais les concordances elles-mêmes ne l'embarrassent pas. Lorsque, par exemple, l'auteur de 67-77 et celui de 78-107 se distinguent du reste par un

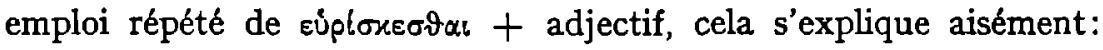
c'est que l'un imite l'autre ${ }^{2}$. Peu importe au critique, dirait-on, qu'en disant des choses pareilles, il affaiblisse sa méthode. Il va méme encore beaucoup plus loin. Car, après avoir contrôlé à l'aide de son apparatus discernendi son hypothèse des six auteurs et l'avoir jugée confirmée, il examine la valeur de l'apparatus lui-même. À cette fin, il partage en deux la partie écrite par le premier auteur, 26, I-43,6, et compare la fréquence des différents éléments dans les deux tronçons. Comme résultat, il obtient des chiffres très divergents, et son commentaire c'est que travailler avec l'apparatus discernendi n'a de sens que lorsqu'a priori il s'y ajoute d'autı es indices permettant d'attribuer l'ouvrage à plusieurs auteurs ${ }^{3}$. Nous nous trompons fort ou, en disant cela, l'auteur déclare sa méthode sans utilité. Dans ces conditions, nous ne nous occuperons plus désormais de la théorie de Coleborne.

Ailleurs, les recherches statistiques sur les questions d'authenticité

1. Coleborne, Linguistic approach, p. 86.

2. Coleborne, Linguistic approach, pp.388-389.

3. Coleborne, Linguistic approach, pp.472-476. 
ont permis d'enregistrer des résultats impressionnants. L'étude quantitative du style d'un texte pour en dépister l'auteur ou les auteurs s'est développée en une branche autonome de la linguistique et on peut supposer qu'un jour ou l'autre, ses méthodes permettront de trouver une solution satisfaisante aux problèmes qui nous occupent. Dans son étude sur l'authenticité des épitres de saint Paul, Morton a déjà examiné le Pasteur dans ce sens ${ }^{1}$. Il semble arriver à la conclusion que l'ouvrage est d'un seul auteur, mais ce qu'il en dit est malheureusement trop sommaire et ne peut être contrôlé. Nous choisirons une manière plus traditionnelle pour déterminer notre position.

Pour commencer, il ne faut pas attacher trop d'importance à l'inconséquence dans le choix des mots. Le style ancien avait à cet égard d'autres normes que le style moderne. La fantaisie qu'Hermas montre dans son vocabulaire est, en tout cas, aussi fréquente dans les textes d'auteurs indiscutés. D'autre part, un auteur n'a pas besoin d'être d'une harmonie exemplaire en son œuvre au point que Giet et Coleborne semblent exiger. Il est possible que son style laisse à désirer, qu'il oublie ce qu'il a dit précédemment, qu'il parle de choses qu'il ne connaît pas suffisamment. Une grande partie, sinon la totalité de ce qui a choqué chez Hermas s'explique de la sorte. Par exemple, le peu de logique dans sa langue est caractéristique à cet égard. C'est ce qui donne à cette langue les traits typiques du style oral: parataxe omniprésente, génitifs absolus nombreux au lieu de participia coniuncta, anacoluthes, disposition des mots dans l'ordre affectif. On ne peut pas être sûr que l'auteur tienne toujours compte de ce que le lecteur est censé savoir ou non, et nous pouvons nous estimer heureux lorsqu'il rattrape par une correction épexégétique ce qu'il avait écrit sans

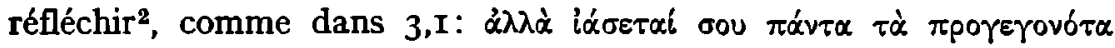

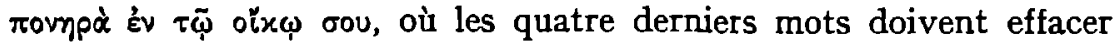
l'impression erronée faite par le premier oov: il ne s'agit pas de péchés commis par Hermas, mais par ses enfants; ou comme dans

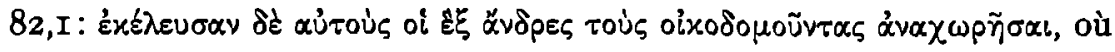

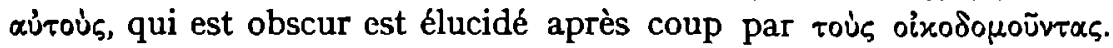

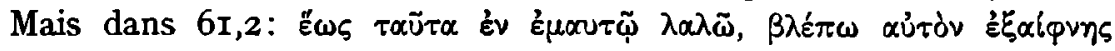

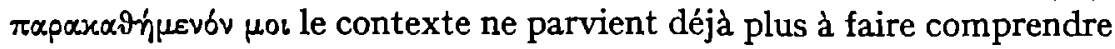
qui aủróv veut désigner, et c'est d'une négligence encore bien plus frappante qu'il se rend coupable dès le premier paragraphe du livre

I. A. Q. Morton-J. McLeman, Christianity and the computer, London I964, PP.3I, 84-85.

2. Cf. W. Havers, Handbuch der erklärenden Syntax, Heidelberg I93I, PP.48-5I. 
où il raconte sans transition qu'il fut vendu à une certaine Rhodè et qu'il la reconnut bien des années plus tard; c'est à nous de deviner qu'entre les deux événements il y eut une séparation. Dès lors nous ne nous étonnons pas qu'un auteur qui, dans des courts passages, a déjà tant de peine à se mettre à la place du lecteur, soit souvent en défaut quand il s'agit d'ensembles plus vastes.

Mais sa maladresse se manifeste de bien d'autres manières. Dans 6,2-7,4, on trouve le texte littéral de la lettre dont Hermas doit faire la lecture aux fidèles. Mais cette lettre s'adresse presque continuellement à Hermas lui-même, et ceux à qui elle est réellement destinée sont le plus souvent désignés par la troisième personne du pluriel. En outre, il n'y a pas la moindre recherche pour faire trancher le style de la lettre sur le reste du livre. Et la seule citation littérale qui se présente

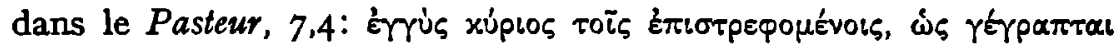

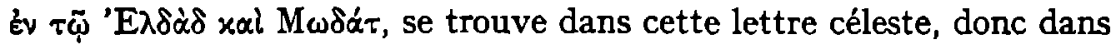
une révélation divine directe, là où on s'attendrait le moins à trouver un recours à des écrits. Dans 23,I, où l'Église est présentée comme une

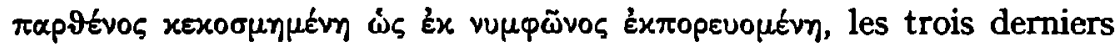

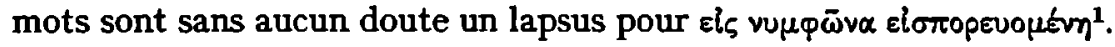
Dans Similitude 5, l'auteur a la malencontreuse idée de représenter, dans une parabole, le Fils de Dieu comme un esclave du maitre et le Saint Esprit comme le fils du maitre, ce qui a créé d'énormes difficultés pour l'exégèse. Dans 56,7, le Pasteur désigne les instructions qu'il a

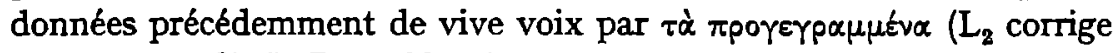
par: quae audisti). Dans 88,2, le Voyant, au lieu de passer la nuit chez les vierges, veut rentrer chez lui, oubliant que depuis 78,4 il se trouve non pas à Rome tout près de chez lui, mais en Arcadie. On peut signaler aussi les détails qu'on trouve dans les paraboles, mais qui sont omis dans l'explication et inversement, et les déclarations sur la

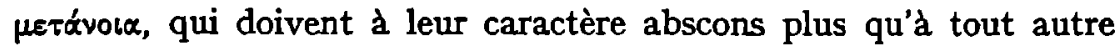
chose d'avoir été l'objet de tant de discussions. Ces exemples montrent de combien de négligences, de disparates et de contradictions est capable notre auteur ou, si l'on veut, chacun des auteurs de Coleborne et de Giet.

D'un autre côté, son style montre nombre de constantes remarquables. En voici une sélection. Les exemples que nous citons pourraient servir à contester toutes sortes de théories soutenant la pluralité des auteurs, mais il nous a paru plus utile de les confronter plus 
spécialement avec la théorie de Giet. Nous les présentons en deux parties. Dans la première, nous montrons que, dans l'ensemble, les exemples que nous avons choisis sont singulièrement fréquents dans le Pasteur par rapport au Nouveau Testament, et, dans la deuxième partie, qu'ils se présentent tout de même chez les trois auteurs de Giet. Si, pour la comparaison qui fait l'objet de la première partie, nous avons choisi le Nouveau Testament, c'est qu'il comporte une multiplicité de textes traitant de sujets apparentés: il donne une image d'autant plus représentative du grec des premières générations chrétiennes qu'il offre un grand nombre d'auteurs et de genres; en outre, on peut se fier au texte. Pour le calcul de la fréquence relative, nous avons tenu compte du fait que le Nouveau Testament est 5 fois plus volumineux que le Pasteur. Dans la deuxième partie, nous nous sommes contenté des chiffres absolus. Si, ici aussi, on voulait calculer la fréquence relative, il faudrait tenir compte du fait que 25-77. I II-I I4 constituent plus de la moitié du Pasteur et que les morceaux des deux autres auteurs sont à peu près équivalents. Après les tables que voici, nous commenterons quelques-uns des exemples cités.

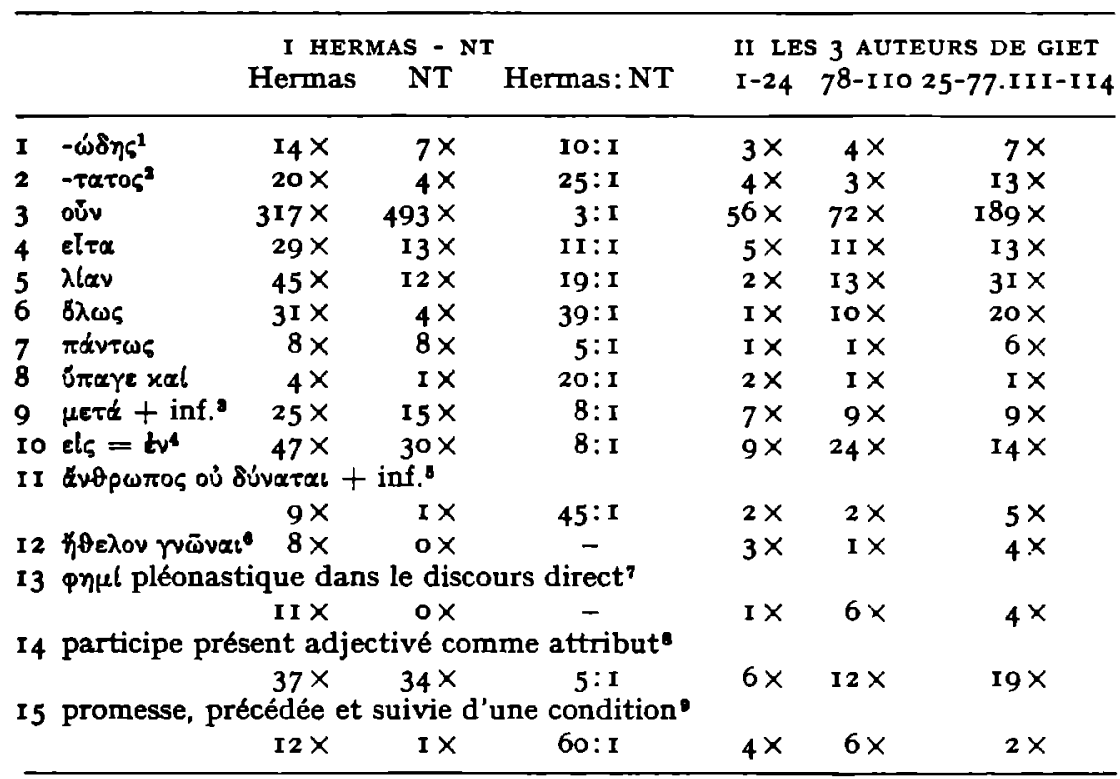

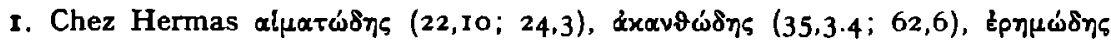

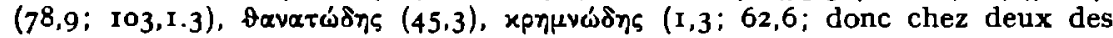

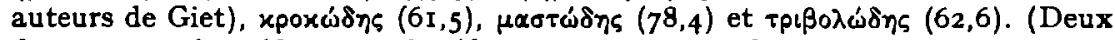

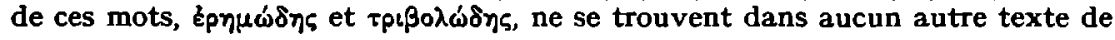


Ad 3. Ici, la fréquence s'écarte relativement moins de celle du Nouveau Testament que dans le cas des autres exemples; en revanche, la fréquence absolue est très élevée; il ne peut s'agir ici d'une coīncidence. Les données du Nouveau Testament sont encore plus frappantes lorsqu'on sait que, sur 493 cas, il y en a I94 qui se présentent dans l'évangile de saint Jean. Si l'on compare le Pasteur au Nouveau Testament sans l'évangile de saint Jean, le rapport devient de 5 à I.

Ad 7. Dans les trois parties, sans distinction, ce mot a en outre le sens exceptionnel de "peut-être"".

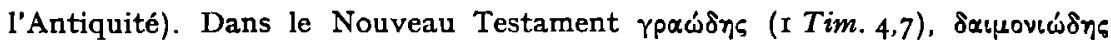

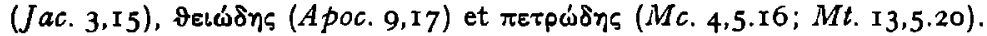

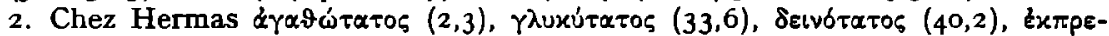

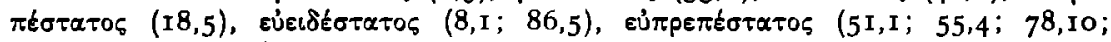

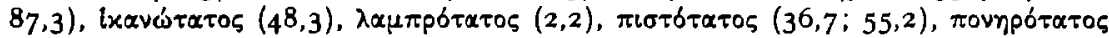

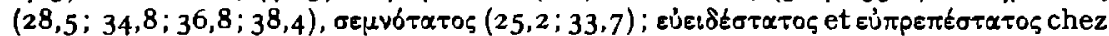
deux des auteurs de Giet; le dernier peut-être chez les trois, car $\mathrm{A}$ lit aussi dans

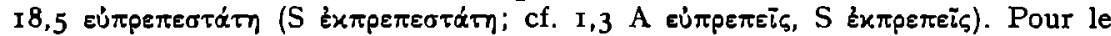
Nouveau Testament cf. BD $\S 60^{1}$.

3. Chez Hermas 2,$1 ; 3,3 ; 5,3 ; 6,4 ; 9,8 ; 17,5 ; 23,1 ; 29,7 ; 61,5 ; 67,3 ; 68,5.6 .8 .9$; 72,$1 ; 77,1 ; 78,1 ; 79,5 ; 82,2 ; 86,6 ; 94,5 ; 95,3$ bis.4; 106,4 . Pour le Nouveau Testament voir W. F. Moulton-A. S. Geden, A concordance to the Greek Testament, Edinburgh $1963^{4}$, s.v. $\mu \in \tau \dot{\alpha}$ c.acc. (2).

4. Chez Hermas I, 2; 2,2; 8,3; 9,9; 15,5; I 7,7bis; 19,$4 ; 24,1 ; 25,1 ; 32,3 ; 42,3$; $50,2.3 .7 ; 51,1 ; 53.2 ; 54,1 ; 55,2 ; 65,3 ; 66,1 ; 70,6 ; 72,6 ; 73,3$ bis. $5 ; 74,2.3$ bis; $75,2.4 ; 76,1.4 ; 78,3.8 ; 79,1.3 b i s ; 83,1.2 b i s .4 ; 90,2.4 .5 ; 92,1 ; 103,7$. Dans le Nouveau Testament Mc. I,9; 5.34; I0,10; 13.3.9.16; Lc. 4,23.44; 7.50; 8,48; 9,6I; II,7; 21,37; Joh. I,I8;Act. 2,5.27.39;4,5;7.4.12;8,23.40;9,21; I4,25; 19,22; 21,$13 ; 23,1$ Ibis; 25,4; I Petr. 5,12.

5. Chez Hermas 1,$3 ; 3,3 ; 46,4$ bis.5.6; 47,$1 ; 89.5 ; 90,2$. Dans le Nouveau Testament Joh. 3,27.

6. 12,3; 16,$6 ; 19,4 ; 33.7 ; 46,1 ; 51,2 ; 63,4 ; 82,3$. Une formule analogue,

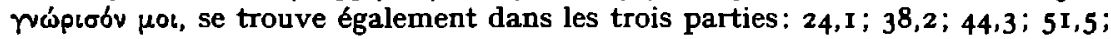
69,$1 ; 82,4$.

7. I,8;30,2;53,I; $57.3 ; 62,1 ; 63,2 ; 77,3 ; 81,8 ; 82,2 ; 84,4 ; 87.4$. Dans $57,3 \mathrm{M}$ a un deuxième $\phi \eta \sigma l v$ et lit aussi $\lambda \epsilon \gamma \omega \ldots \phi \eta \mu l$ dans 64,1 . A a un deuzième $\phi \eta \sigma l v$ dans 66,7 . Dans $69,5 \mathrm{~L}_{1}$ traduit $\lambda \varepsilon_{\gamma} \omega$ par dico...inquam; $\mathrm{L}_{12}$ ont en outre le pléonasme dans III,I-2.

8. Chez Hermas 2,1.4; 10,8; 12,2; 13,5; 19,2; 34,3sexies; 36,$4 ; 43,6.8$.I2bis; 46,4 ; 50,Iobis; 61,1.6bis; 70,6; 73,4;78,5.6.8;83,4ter; 98, Ibis.2; 99,I; 101,I ; I05,3. Dans le Nouveau Testament $M t$. 3,$15 ; 5,25 ; 12,4 ; 19,22 ; 27,33 ; M c .9,3$; 10,22; Lc. I,20bis; 9,29; I3, I Ibis; I9, I 7; Act. 2,29; 9,9; I3, I I; 18,7; 19,36; Rom. 15,14; I Cor. 11,13; 2 Cor. 6,14; 9,12bis; 12,1.4;Gal. 1,22; 4,24;Col. 2,23; Hebr. 5,1 2; Jac. 2,15; 3,15;A Poc. I,8bis; 3,2.

9. Chez Hermas 7,2 ; 13,$5 ; 21,4 ; 23,5 ; 26,2 ; 39,4 ; 50,7 ; 56,3 ; 60,4 ; 77,3 ; 100,5$; 105.3. Dans le Nouveau Testament Apoc. 2,5; cf. Joh. I3, I7.

I. Dans 25-77. III-II4, en tout cas 39,7. Pour cette signification voir $\mathbf{H}$. J. Cadbury, Lexical notes on Luke - Acts, I, Journal of Biblical Literature 44, 1925. Pp. $(214-227) 226-227$. 
Ad 8. Cette formule s'emploie pour introduire un ordre, comme par

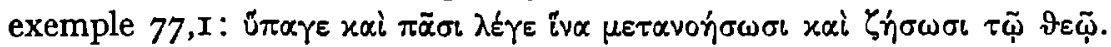
D'autre part, dans le Nouveau Testament, ü $\pi \alpha \gamma \varepsilon$ sans $x \alpha i$ ne s'emploie pas moins de ${ }^{4} 4$ fois dans cette fonction ${ }^{1}$, ce qu'on ne rencontre pas dans le Pasteur.

Ad Io. On ne peut toujours établir si $\varepsilon i \zeta$ a bien la fonction visée ici.

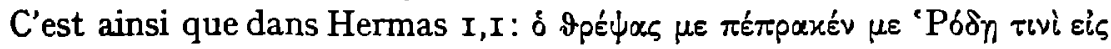

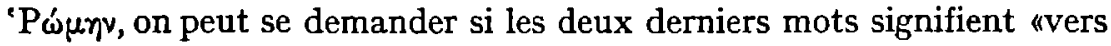

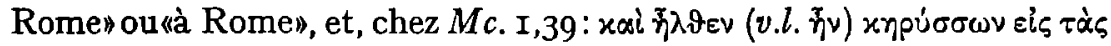

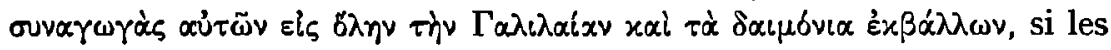
groupes de prépositions se rattachent à $\hbar \lambda \vartheta \varepsilon v$, si telle est la leçon

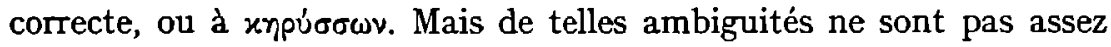
nombreuses pour compromettre les statistiques.

Ad I2. On rencontre cette formule lorsque Hermas demande des explications sur le détail d'une révélation, par exemple, 19,4: $\delta\llcorner\alpha \tau$

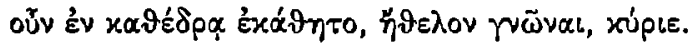

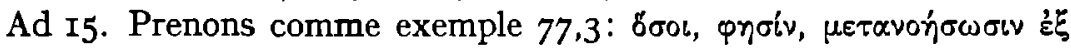

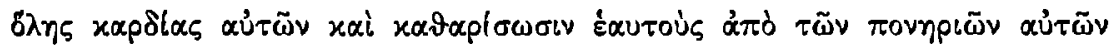

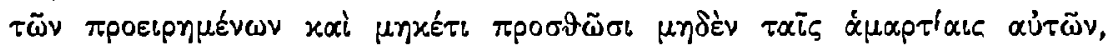

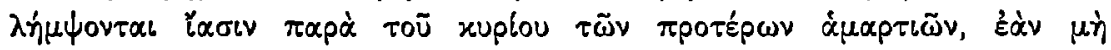

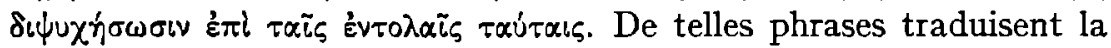
crainte que le lecteur ne tire pas la conclusion souhaitée, scrupule qui, dans chacune des trois parties, conduit celui qui pose des questions rhétoriques à y répondre 2 .

On pourrait encore étendre le champ d'investigation aux constantes lexicologiques (qui invitent pourtant plus facilement à l'imitation et, de ce fait, sont sans doute moins probantes) et à des particularités qu'on trouve chez deux des trois auteurs de Giet ${ }^{3}$. Mais les matériaux que nous avons retenus justifient, semble-t-il, la conclusion que nous avons bien affaire à un seul auteur.

Mais nous nous voyons obligé de revenir encore une fois sur les inconséquences dans son ouvrage. Avec les défenseurs de l'auteur

I. Mt. 5,$24 ; 8,4.13 ; 19,21 ; 21,28 ; 27,65 ; 28,10 ; M c .1,44 ; 6,38 ; 10,21 ; 16,7$; Joh. 4, I6;9.7; Apoc. го,8.

2. 1,$8 ; 43,12.19 ; 109,4-5$.

3. C'est ainsi qu'en ce qui concerne l'usage fréquent de formules de générali-

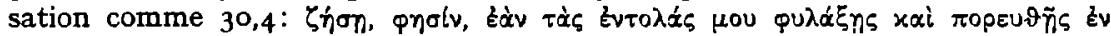

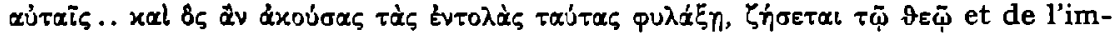

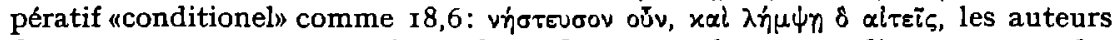
de I-24 et 25-77-III-II4 s'accordent. On trouve beaucoup d'autres exemples chez Link, Einheit, Pp.40-43. 
unique nous croyons devoir les attribuer en partie à des sources hétérogènes que l'auteur a incorporées à son œuvre, mais sans les harmoniser suffisamment. La question se pose dès lors de savoir si les passages que l'auteur a pris ailleurs peuvent servir pour étudier sa langue: à quoi nous pourrions répondre que le recours à des sources permet tout un éventail de possibilités, dont la citation littérale ou presque littérale n'est qu'un cas extrême. Or, le Pasteur contient un seul passage dont l'auteur dit lui-même que c'est une citation (voir supra, p. 24), mais presque partout ailleurs, il est à peu près impossible de départager ce qui est à lui et ce qu'il emprunter. Si, à certains endroits, on a l'impression d'être sur la piste d'un corps étranger, on y décèle aussi cependant une conformité avec le reste du livre. En voici quelques exemples.

D'abord 3,4. Dans ce qui précède, Hermas raconte que la vieille femme qui lui est apparue lui lit un livıe. La dernière partie de cette

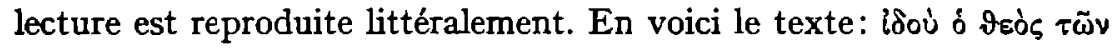

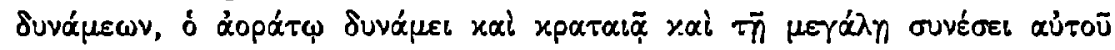

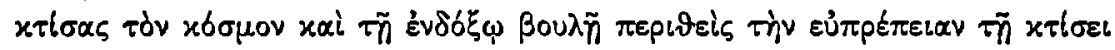

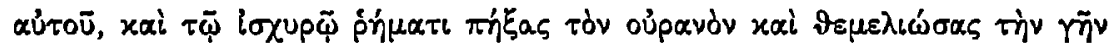

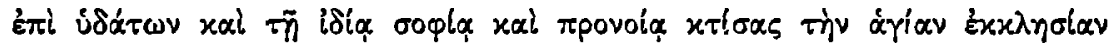

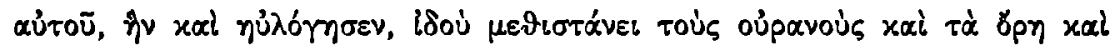

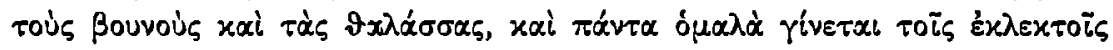

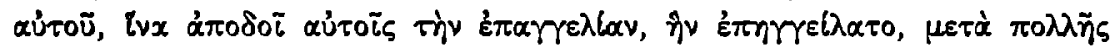

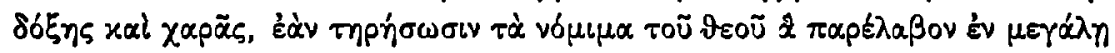

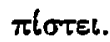

Dans son commentaire, Dibelius consacre un petit excursus à ce passage. Il est d'avis que le texte est un tout, que ce tout est un hymne et que cet hymne, tout comme les morceaux hymniques de l'Apocalypse de Jean, vient certainement de la tradition liturgique pour l'essentiel. A notre avis, les trois points sont contestables. Le texte est évidemment un dans la mesure où il se donne pour une citation littérale. Mais sa cohérence interne n'est pas très grande: Dieu est d'abord loué pour avoir bien façonné sa création et ensuite pour avoir débarrassé d'obstacles cette même création en faveur de ses élus. Et la subordonnée conditionnelle de la fin nous transporte dans une tout autre atmosphère encore. Mais s'agit-il d'un hymne? Il y a, en effet, des indices qui laissent supposer qu'Hermas entendait le texte comme tel:

I. Cf. Zahn, Hirt, P.486; M. J. Suggs, The Christran two ways tradition: its antiquity, form and function. Studies in New Testament and early Christian literature, essays in honor of Allen P. Wikgren, Leiden 1972, p. (6o-74) $7^{2}$ n.2. 


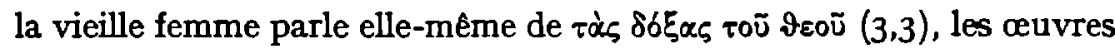
de la toute-puissance divine sont citées en manière de louange et on trouve l'élément hymnique de la série de participes qui définissent l'action de Dieu. Mais c'est tout autant une révélation prophétique: les paroles s'adressent de la part de Dieu à un être humain, dans la

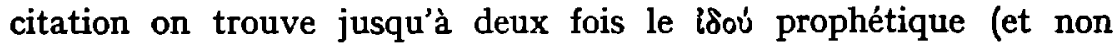
hymnique), au fur et à mesure le texte prend davantage le caractère d'une promesse, et, après la lecture, la vieille femme confirme en

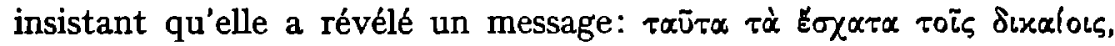

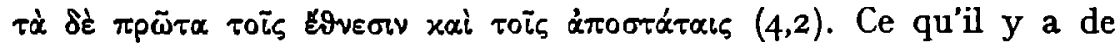
caractéristique dans ce texte nous paraît être précisément cet enchevêtrement d'éléments hymniques et prophétiques, qui font qu'on ne peut pas non plus le considérer comme un texte liturgique autonome. Et les nombreuses phrases et expressions traditionnelles qu'on a remarquées dans notre texte ${ }^{1}$ prouvent une seule chose: c'est qu'Hermas fait partie d'une tradition; elles ne prouvent pas qu'il n'a pas rassemblé lui-même ces éléments. D'ailleurs, dans une étude récente sur les morceaux hymniques de l'Apocalypse, Jörns arrive à une conclusion analogue ${ }^{2}$.

Un certain nombre de particularités qui nous sont connues par le reste du Pasteur confirment que le texte est bien d'Hermas. Par exemple, si l'insistance lourde, pour ne pas dire excessive, sur la création divine est frappante, elle surprend moins dans le cas d'Hermas, car ailleurs aussi il revient sans ctsse sur Dieu en tant que Créateur ${ }^{3}$. Nous retrouvons ailleurs dans le Pasteur le lien entre l'Église et la création au lieu de la rédemption* Nous avons déjà signalé la tendance qu'a Hermas de faire suivre une condition, suivie d'une promesse, d'une autre condition (voir supra, pp.25 et 27). Nous ne pouvions pas citer le passage en question à ce propos, car ici la promesse n'est pas précédée d'une condition. La phrase avec éóv, qui termine la révélation de la vieille femme, rappelle pourtant irrésistiblement cette tendance. De même dans le choix des mots nous retrouvons les préférences

I. Surtout des réminiscences des Psaumes. Cf. Giet, Les Pasteurs, pp.82-83 et les commentaires ad loc.

2. K.-P. Jörns, Das hymnische Evangelium, Untersuchungen zu Aufbau, Funktion und Herkunft der hymnischen Stücke in der Johannesoffenbarung, Gütersloh $197 \mathrm{I}$, Pp. $17^{8-1} 79$.

3. Voir Snyder, Shepherd, ad 1,6; G. Delling, Partizipiale Gottesprädikationen in den Briefen des Neuen Testaments, Studia Theologica 17, 1963, Pp.(I-59)25-26, 45. C'est un trait typiquement juif, cf. M. Philonenko, Joseph et A séneth. Leiden 1968, PP.58-59.

4. Voir Pernveden, Church, chap. I. 


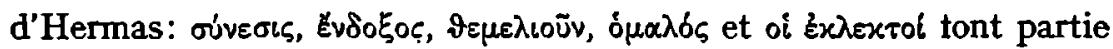
de son vocabulaire favori ${ }^{1}$. Tout cela amène à conclure ou bien que le texte a été écrit spécialement pour cette occasion ou bien qu'il a été si bien retravaillé qu'Hermas en est tout de même moralement propriétaire.

Pour le passage que nous venons d'examiner, nous avons eu quelque difficulté à voir clair du fait qu'en dehors du Pasteur, nous ne disposions pas de textes comparables. Mais pour les deux exemples qui suivent nous trouvons d'autres textes chrétiens à peu près contemporains qui permettent une comparaison.

Dans 35,2-4, Hermas utilise le motif, certainement traditionnel, de la double voie ${ }^{2}$. Dans sa version-standard, ce motif, pour citer le

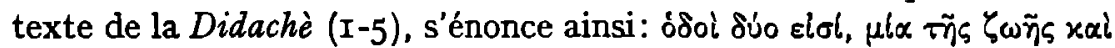

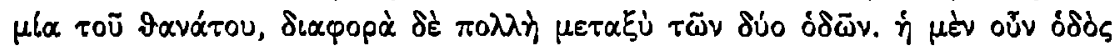

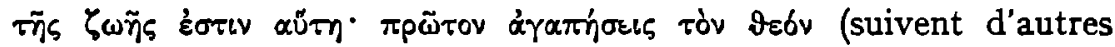

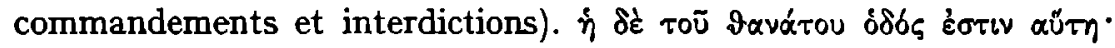

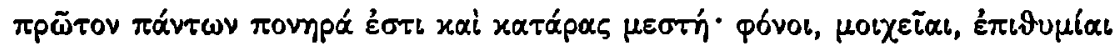
(suivent d'autres péchés et pécheurs, avec l'exhortation à les fuir). Ce morceau se retrouve en grande partie sous la même forme chez le Ps.-Barnabé I 8-20 et dans d'autres textes. Chez Hermas, il n'est cependant pas question de citation. $\Pi$ se borne à glisser l'idée maîtresse,

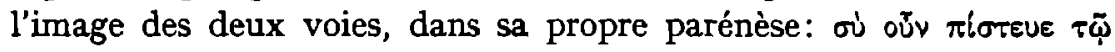

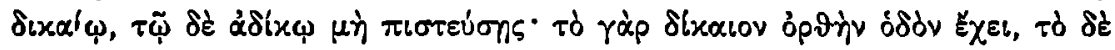

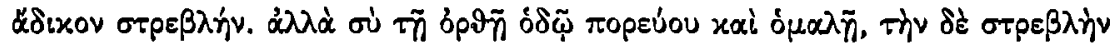

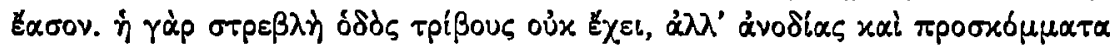

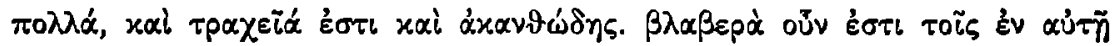

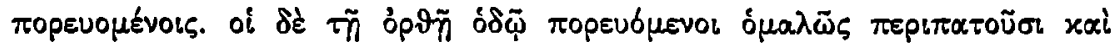

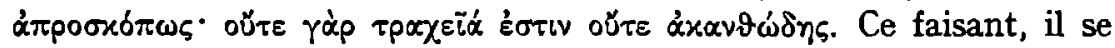
permet un écart remarquable par rapport à la forme-standard. La Didachè et le Ps.-Barnabé ne se prononcent pas sur la viabilité des deux routes ${ }^{3}$. Dans l'évangile de Matthieu, qui puise aussi dans la

I. Súveals I $3 \times$ Hermas, $7 \times$ Nouveau Testament, un rapport de 9 à I, Evoo $44 \times, 4 \times, 55$ à I, $\theta \varepsilon \mu \varepsilon \lambda$ เoũv $7 \times, 5 \times, 7$ à $I, \delta \mu \alpha \lambda 656 \times$ Hermas, aucun dans le Nouveau Testament, ol Ex $x$ rextol $9 \times$ dans les Visions.

2. Voir W. Rordorf, Un chapitre d'ethique judé-chretienne: les deux voies, Recherches de Science Religieuse 60, 1972, Pp.Io9-128; Suggs, Two ways, pass. et la littérature citée dans ces études.

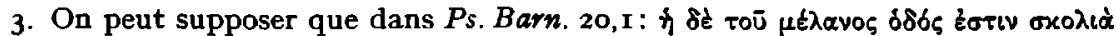

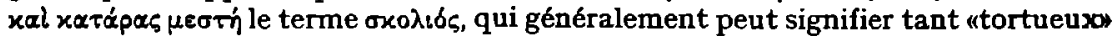
que «mauvais», n'a d'autre signification que le terme novnpós du passage cor-

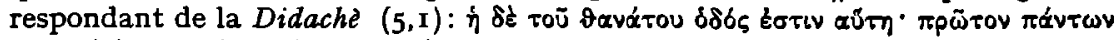

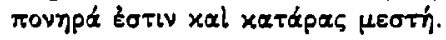


tradition des deux voies, nous voyons même que le mauvais chemin est représenté comme plus aisé et le bon plus difficile à pratiqueı (Mt. 7,I3-I4). Hermas pourtant affirme le contraire: le bon chemin est plus aisé, le mauvais plus difficile ${ }^{1}$. Nous retrouvons la manière

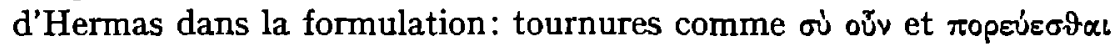

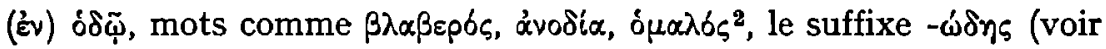
supra p. 25), tendance à exprimer chaque chose à la fois positivement et négativement ${ }^{3}$. Dans l'ensemble, le passage en question se présente comme un exemple classique de l'intégration d'une source.

Dans 27,4-6, le document original est laissé en grande partie intact:

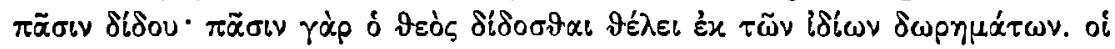

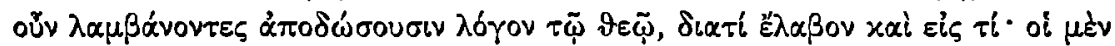

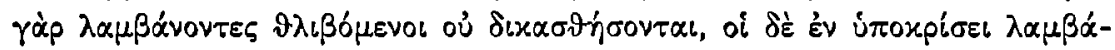

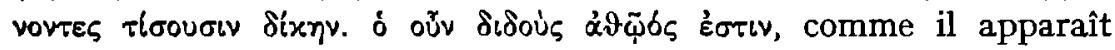

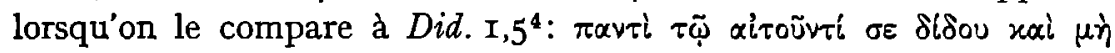

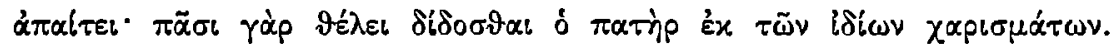

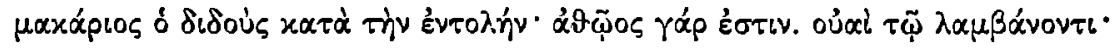

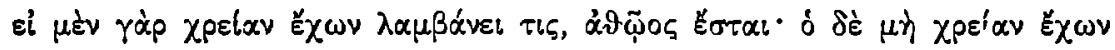

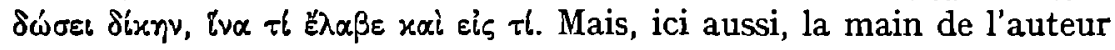
semble se trahir: $\delta \iota \alpha \tau i$ au lieu de iva $\tau i$ (nulle part on ne trouve ivo $\tau i$

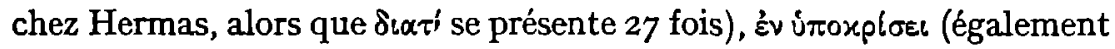
dans I4,I et 72,2), ỡv maladroit.

Nous pouvons conclure que les matériaux étrangers repris dans le Pasteur y sont intégrés de telle sorte qu'ils ne détonnent pas du point de vue stylistique. Dans son ensemble, nous pensons être en droit de traiter le Pasteur comme un document qui nous donne la langue d'un seul écrivain.

\section{LIEU ET DATE D'ORIGINE}

On admet généralement que le Pasteur a été écrit à Rome. Certains détails topographiques $(I, I-3 ; 5, I ; 22,2)$ l'indiquent; de plus, Origène, Comm. in Rom. Io,3I et le Canon de Muratori 73-75, s'ils divergent sur

I. Cf Dibelius et Joly ad loc et Giet, Les Pasteurs, p 202

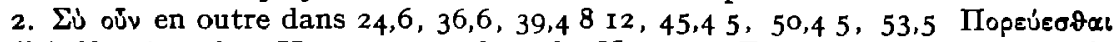
(Év) $\delta \delta \bar{\omega} 6 \times$ chez Hermas, $2 \times$ dans le Nouveau Testament, un rapport de

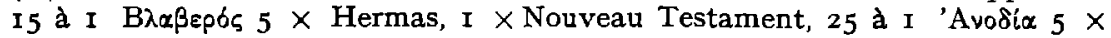
Hermas, aucun dans le Nouveau Testament Pour $\delta \mu \alpha \lambda b_{\zeta}$ vorr supra p 30 n I 3 En outre I 5,6, 25,7, 33,5, 77,3, 90,9

4 Cf A Resch, Agrapha, Leipzig I9062, pp 194-I96, E Peterson, Guudaısmo $e$ Cristianesimo culto gutaico e culto cristiano, Rivista di Stona e Letteratura Religiosa I, I965, PP (367-39I) 388-39I 
la date, concordent sur ce point'. Il est plus difficile de savoir si l'auteur est né et a été élevé à Rome. On lui a prêté comme lieu d'origine Aquilée, Cumes, Ephèse, l'Arcadie, la Palestine et l'Egypte, mais aucun argument valable n'a pu être produit à ce sujet. Souvent

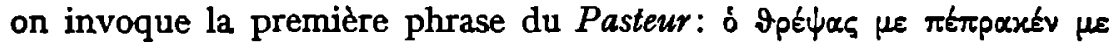

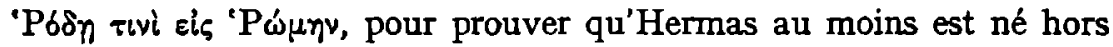
de Rome, mais ici non plus l'argument n'est pas assez solide. Ce qui fait question est le sens de elc. Si le mot signifie "vers", Hermas vient d'ailleurs. Si, au contraire, il signifie «à", comme c'est souvent le cas chez Hermas (cf. supra, pp.25 et 27) et comme le veulent aussi les deux versions latines, il n'est pas question de lieu d'origine. On ne peut pas dire non plus que, si Hermas habitait déjà à Rome, ajouter «à Romew serait superflu ${ }^{2}$, car la mention du Tibre dans I,2 sans qu'au préalable la scène ait été localisée à Rome, aurait rendu le récit encore

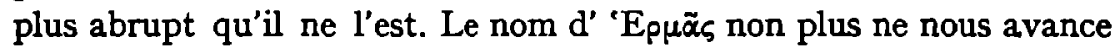
guère; car, à cette époque, à Rome, un nom grec ne certifiait pas l'origine ethnique du porteur de ce nom ${ }^{3}$. La seule chose sur laquelle nous puissions tabler, c'est qu'Hermas était un Chrétien. Comme Chrétien, il appartenait, en effet, selon toute vraisemblance, au groupe de la population qui venait de la moitié orientale de l'Empire. Mais on ne saura jamais si c'est lui qui est venu en Occident ou si sa famille y était déjà établie depuis une ou plusieurs générations.

Comme pour tant d'autres œuvres de la plus ancienne littérature paléochrétienne et de la littérature juive apocryphe, il est difficile de dater avec précision le Pasteur. Trois données qui remontent à l'Antiquité jouent un rôle dans la discussion: une phrase d'Origène, une mention dans le Pasteur lui-même et une information dans le Canon de Muratori. Dans Comm. in Rom. Io,3I, Origène formule l'hypothèse que le Hermas salué par saint Paul dans Rom. I6, I4 est l'auteur du Pasteur. Etant donné que l'Épitre aux Romains date de 57 ou $5^{8}$ après J.-C., le Pasteur remonterait à la deuxième moitié du Ior siècle. Bien qu'actuellement le témoignage d'Origène ne soit plus pris au sérieux, il s'accorde

I. Voir intra pp.32-33. E. Peterson, Frühkirche, Judentum und Gnosis, Freiburg 1959, Pp.274-275, 282-283, 284 et $308 \mathrm{n} .93$ dénie l'historicité de la localisation à Rome. Selon lui, les détails topographiques ne servent qu'à déguiser le milieu palestinien juif d'Hermas, et le Canon de Muratori reflète une polémique contre les apocalypses qui devrait nous rendre méfiants à l'égard de ses déclarations. Mais dans son Giudaismo e Cristianesimo, p.381, l'auteur le situe, lui aussi, a Rome.

2. Ainsi Harnack ad loc.

3. H. Solin, Beiträge zur Kenntnis der griechischen Personennamen in Rom. I, Helsinki 1971, P.I $5^{8}$. 
avec la deuxième donnée, que nous avons trouvée dans le Pasteur

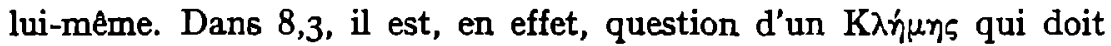

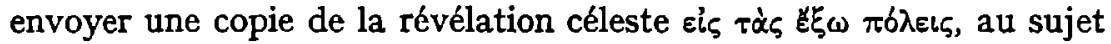

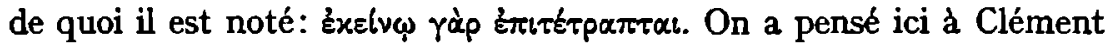
de Rome, qui a été évêque de Rome pendant la dernière décennie du Ier siècle, mais cette identification est très loin d'être sûre ${ }^{1}$. Elle ne peut donc être un argument pour dater le Pasteur d'environ 95 après J.-C. Le Canon de Muratori enfin, nous fait savoir, 1.73-77, qu'Hermas a écrit le Pasteur sous l'épiscopat de Pie, c'est-à-dire dans les années quarante du $\mathrm{II}^{\mathbf{e}}$ siècle. Mais cette affirmation n'est peut-être pas tout à fait désintéressée, car l'auteur du Canon tient à présenter le Pasteur comme un ouvrage non-canonique (1.77-80). Une date tardive devait plaider en faveur de cette idée 2 . On ne peut donc guère se fier à ces indications sur la date. En outre, les deux premières excluent la dernière et vice versa. Des théories ont pourtant été échafaudées pour essayer de les réconcilier.

Ceux qui estiment que le Pasteur a été écrit par plus d'un auteur ont moins de peine que les autres à y réussir. C'est ainsi que Giet, pour ne nommer que lui, n'applique ce que dit le Canon de Muratori qu'à l'auteur de 78-iro et, sans se laisser embarrasser par le Canon, donne pour ses deux autres auteurs les dates qui lui semblent les plus appropriées ${ }^{3}$. À notre avis, ce n'est pas de ce côté que nous pouvons trouvet la solution: $\mathrm{ni}$ la tradition antique ni la langue et le style du Pasteur ne justifient l'hypothèse de plusieurs auteurs. Mais il $\mathrm{y}$ a d'autres théories qui cherchent à combiner les indications sur la date sans renoncer à l'idée d'un auteur unique.

La première de ces théories veut que le Pasteur ait été conçu graduellement à une époque qui va de l'épiscopat de Clément à celui de $\mathrm{Pie}^{4}$. Mais il est difficile d'imaginer une genèse d'un demi-siècle, et ceux qui défendent cette thèse ne la rendent pas plus vraisemblable lorsqu'ils raccourcissent cette période en reportant l'année de la mort de Clément à r ro ou plus tard.

La deuxième théorie consiste à dire que le Pasteur a été écrit à l'époque de Pie, mais veut se donner un cachet de respectabilité en se

I. Joly, Pasteur, p.14; Giet, Les Pasteurs, p.283; Pernveden, Church, p.I45.

2. Cf. Snyder, Shepherd, pp.22-23.

3. Giet, Les Pasteurs, p.280-305.

4. Ainsi par exemple A. Harnack, Geschichte der altchristlichen Litteratur bis Eusebius, II I, Leipzig 1897, Pp.257-267; R. van Deemter, Der Hirt des Hermas, Delft I929, pp.58-59. 
faisant passer pour une cuvre de l'époque de Clément ${ }^{1}$. Cette théorie, elle aussi, suscite des objections. Tout d'abord, elle vaut ce que vaut l'identification de $\mathrm{K} \lambda \hat{n} \mu \eta \bar{s}$ de 8,3 avec Clément de Rome, identification qui est parfaitement douteuse. En outre, si déjà le Pasteur est une mystification, pourquoi néglige-t-il quelques subterfuges élémentaires: le livre n'est pas mis au nom d'un personnage célèbre des premiers temps du christianisme, au début de l'ouvrage rien n'est tenté pour l'antidater (mais on y trouve toutes les données personnelles sur Hermas), de même, après 8,3 , il n'y a aucune référence évidente à l'époque de Clément de Rome. Est-ce naiveté de la part de l'écrivain ou raffinement? Pour notre part, nous trouvons qu'il est plus simple d'admettre qu'il n'est pas question de mystification. L'hypothèse devient d'ailleurs encore plus discutable si elle doit aussi prendre en compte le témoignage d'Origène, mais il n'est pas nécessaire de le prouver $^{2}$.

Les seules données vraiment solides sont qu'Hermas parle de persécutions de la part des autorités romaines ${ }^{3}$ et qu'Irénée, Haer. 4,20,2, cite le Pasteur. L'ouvrage doit donc avoir été écrit entre 65 et I80. Mais, selon toute probabilité, on peut écourter cette période, tant au début qu'à la fin, de 30 à 35 ans. Nous fixons le terminus post quem d'après ce qu'Hermas dit des persécutions. Si, traditionnellement, on considère la persécution des Chrétiens par Néron comme la première, les fidèles d'alors ne virent probablement pas les choses de la même façon. C'est seulement lorsque le phénomène se reproduisit et cette fois sous des empereurs plus responsables - ce fut pour la première fois, pour autant que nous sachions, en 95, sous Domitien -, que l'idée d'une "persécution des Chrétiens" se sera formée dans leur esprit. Or, de toute évidence, pour les lecteurs du Pasteur, les persécutions étaient une notion familière et, en outre, Hermas les met en garde contre une nouvelle persécution imminente. Il est donc raisonnable de ne pas situer le Pasteur avant la fin du Ier siècle. Le terminus ante quem est la citation qu'on trouve chez Irénée. Le respect avec lequel celui-ci parle du Pasteur nous laisse supposer que ce n'est pas un ouvrage qui date de peu avant l'Adversus Haereses. Mais, chose plus importante encore,

I. Ainsi déjá, par exemple, Fr. Lücke, Versuch einer vollständigen Einleitung in die Offenbarung Johannis und in die gesammte apokalyptische Litteratur, Bonn 1832, pp.I42-I 43 , et beaucoup d'autres après lui. A l'époque moderne, par exemple, Mohrmann, Origines, pp.77-78.

2. Cf. Van Deemter, Hirt, pp.57-58.

3. Les passages chez O. de Gebhardt-A.Harnack, Hermae Pastor graece, Lipsiae I877, pp.LXXVII-LXXVIII. 
le Canon de Muratori, qui est contre une datation trop précoce du Pasteur, le situe pourtant sous l'épiscopat de Pie, donc au plus tard vers le milieu du IIe siècle. Il n'est pas possible, à notre avis, de préciser davantage. Ce qu'Hermas dit des persécutions est trop vague pour que nous puissions le tapprocher des persécutions que nous connaissons, par exemple celle de Trajan ${ }^{1}$; le développement des fonctions ecclésiastiques au temps des premières générations chrétiennes est si peu connu que les données du Pasteur à ce sujet ne peuvent servir à une datation, et le silence sur Marcion, qui, vers $I_{45}$ après J.-C., a dû susciter tant de remous à Rome, est un argument ex silentio et de ce seul fait trop faible. Tout ce que nous pouvons affirmer - et telle sera notre conclusion - c'est donc que le Pasteur a été écrit dans la première moitié du II ${ }^{\mathrm{e}}$ siècle après J.-C.

1. W. H. C. Frend, Martyrdom and persecution in the early Church, Oxford I965,

p. 194 . 


\section{SEMITISMES ET LATINISMES}

\section{PHÉNOMÈNES D'INTERFÉRENCE}

Quand on parle deux ou plusieurs langues on a parfois de la peine à garder les cloisons étanches entre les systèmes de ces langues. Au moment où l'on utilise une d'entre elles, on est porté à s'écarter de ses normes à cause de la connaissance qu'on a des autres. Ces écarts sont ce qu'on appelle des phénomènes d'interférence'. Les sémitismes et les latinismes sont les phénomènes d'interférence qui se présentent en grec sous l'influence soit de langues sémitiques soit du latin. L'interférence peut aussi être produite par l'influence de plusieurs langues à la fois ${ }^{2}$. C'est ainsi qu'il faudra peut-être considérer certains phénomènes comme étant à la fois des sémitismes et des latinismes.

On peut diviser les phénomènes d'interférence en deux grands groupes qui relèvent l'un de la substitution, l'autre de l'importation's. Il y a substitution lorsque des éléments d'une langue, qui sont en partie identiques à ceux d'une autre, s'identifient à eux entièrement ou du moins dans une plus large -mesure. Souvent l'interférence consiste seulement en ce que, sous l'influence stimulante d'une autre langue, un phénomène devient plus fréquent. La substitution se présente dans tous les domaines de la langue: phonétique, morphologie, syntaxe et vocabulaire. Il y a importation lorsque, au lieu que les éléments existants reçoivent une fonction nouvelle, ce sont des éléments nouveaux qui se trouvent tout simplement importés. La forme la plus fréquente est le mot d'emprunt.

Les problèmes qui se présentent pour l'étude des sémitismes et des latinismes sont évidemment ceux qui sont inhérents à l'étude de l'interférence. Mais à cela s'ajoute que ce sont des phénomènes qui ont

I. U. Weinreich, Languages in contact, New York 1953, p.I.

2. Cf. V. Vildomec, Multilingualism, Leyden 1963, Pp.79-80, 85, I46, I60, I 7 I, 200, 2I2-2I3, 232.

3. E. Haugen, Bilingualism in the Americas, Alabama 1956, p.50. 
eu lieu dans un lointain passé. Nous ne connaissons vraiment bien aucune des langues en cause, le grec, l'hébreu, l'araméen, le latin, et pour compléter nos connaissances nous ne disposons que de corpus de textes qui sont clos ${ }^{1}$. On ne peut, comme il le faudrait, consulter ceux dont c'est la langue maternelle. Aussi, les résultats seront-ils forcément plus hypothétiques que lorsqu'on étudie les interférences dans les langues modernes. Dans la plupart des cas, nous devrons nous contenter de raisonnements fondés sur des probabilités. Nous nous trouvons à chaque fois confrontés à deux questions: I. un phénomène donné est-il un phénomène d'interférence ou le résultat d'une évolution interne de la langue? 2. s'il s'agit bien d'un phénomène d'interférence, est-ce, dans un texte donné, le résultat d'une influence directe de la langue donnante ou bien s'agit-il d'un élément déjà intégré dans la langue réceptrice? Les deux questions demandent quelques éclaircissements.

Ad I. Dans le cas d'importation, l'interférence est évidemment facile à reconnaître: les emprunts de mots, de suffixes, etc. sont généralement repérables, en grec, avec certitude. En revanche, il est souvent difficile de savoir si l'on a affaire à des substitutions. En effet, il ne suffit pas de montrer que, dans deux langues, certains éléments se mettent à présenter une ressemblance plus grande qu'avant, mais aussi que cette ressemblance accrue est due à l'influence d'une langue sur l'autre. Voilà une difficulté parfois insoluble. Tout d'abord, il arrive que, par manque d'information, nous nous fassions une idée fausse de l'époque à laquelle un phénomène linguistique a pris naissance et s'est répandu. C'est ainsi que, dans une langue donnée, on pourrait en venir à imputer à de l'interférence certains phénomènes linguistiques que cette langue présentait en réalité bien avant la prétendue langue donnante. Mais, même si le phénomène est postérieur à son pendant dans l'autre langue, rien ne nous autorise, sans plus, à parler d'interférence. En principe, on peut poser que des phénomènes qui sont contraires au système de la langue sont probablement dus à l'interférence et que ceux qui s'y accordent sont probablement le résultat d'un développement autonome. Mais il n'est pas toujours facile de savoir si un phénomène s'accorde bien ou moins bien au système et, en tout cas, le principe ne nous dispense pas de tenir compte, autant que possible, à chaque fois des circonstances particulières dans lesquelles le phénomène se produit.

I. Cf. pour ce problème H. Pinkster, On Latin adverbs, Amsterdam-London 1972, Pp.9-13. 
Ad 2. En général, il nous semble raisonnable d'écarter l'intertérence directe lorsqu'on peut supposer que l'auteur utilise des éléments déjà admis. Il est possible aussi que les renseignements disponibles sur un auteur nous permettent de supposer que ce qui, chez d'autres, n'est qu'éléments plus ou moins adoptés, soit dû, chez lui, à son polyglottisme, ou bien que ce qui chez d'autres était possible, mais peu usuel, soit stimulé par sa connaissance d'une autre langue. Voilà ce que nous nous proposons d'examiner chez Hermas. Nous commencelons par voir dans quelle mesure, à l'époque d'Hermas, l'influence sémitique et latine s'est exercée, en grec, sur la langue courante ou éventuellement sur la ou les langues de groupe que connaissait Hermas. Ensuite, nous chercherons à voir s'il connaissait lui-même les langues sémitiques et le latin.

\section{SEMITISMES}

Bien que, de tout temps, les peuples sémitiques aient été en rapport avec les Grecs, les traces que leurs langues ont laissées sur le grec sont infimes. C'est à peine si quelques emprunts sémitiques sont passés dans la langue courante, méme quand, après Alexandre, les contacts entre Grecs et Sémites s'intensifièrent. Il y a pourtant eu, à n'en pas douter, des milieux - îlots de langue grecque dans un entourage sémitique et îlots de langue sémitique dans un entourage grec - où l'on parlait un grec beaucoup plus sémitisé. En général, nous connaissons si mal leurs conditions de vie que pour étudier leur langue il ne nous reste que les conjectures. Pour un groupe cependant nous disposons d'une documentation abondante: les Juifs de Palestine et ceux de la diaspora occidentale, y compris les premières générations de Chrétiens, dans la mesure où ils étaient issus du judaïsme. Ce groupe nous a laissé des textes si nombreux et si variés que nous ne manquons pas d'informations, du moins pour la langue écrite.

Les textes dont il est question peuvent se diviser en trois groupes. Tout d'abord la Bible grecque et les écrits de Juifs et de Judéochrétiens qui y sont apparentés. Les œuvres de Philon, Flavius Josèphe et autres auteurs juifs hellénistiques forment un deuxième groupe. Et troisièmement, nous disposons d'une quantité de documents non littéraires: inscriptions et papyrus. Le giec vıaiment sémitisant se 
trouve seulement dans le premier groupe; c'est pourquoi nous nous occuperons plus spécialement de celui-ci. Dans une certaine mesure, ce groupe forme un tout. A l'époque où ces écrits ont vu le jour, la ligne de démarcation entre ce qui était juif et chrétien, canonique et non canonique n'était pas encore très marquée. ${ }^{1} \mathrm{Si}$ nous voulons comprendre la démarche linguistique des auteurs de ce groupe, nous devrons, nous aussi, nous tenir à cette idée d'unité. C'est pourquoi, dorénavant, nous traiterons comme faisant partie d'un tout relativement un les livres proto- et deutérocanoniques de la Septante, le Nouveau Testament et les apocryphes paléo- et néotestamentaires jusqu'à environ 200 après J.-C. ${ }^{2}$.

L'ensemble de ces textes doit avoir fait sur le Grec moyen une impression d'exotisme ${ }^{3}$. Les idées fondamentales de ces écrits venaient d'un tout autre monde culturel et les procédés de présentation étaient, eux aussi, très différents des normes littéraires habituelles. Mais il y avait autre chose encore. Le fondement de cette littérature se trouvait dans des traductions. En général, on le sait, traduire favorise les phénomènes d'interférence, mais ici l'interférence eut un terrain d'autant plus favorable que les traducteurs croyaient devoir rendre le plus fıdèlement possible non seulement le fond, mass aussi la forme4. Aussi, leur méthode se distingue-t-elle par un littéralisme qui, au cours des temps, alla croissant plutôt que décroissant. Elle consistait à rendre un mot du texte par un mot grec; a toujours traduire autant que pos-

I Pensons, par exemple, à des citations de textes non canoniques dans le Nouveau Testament, of $\mathbf{M}$ Phllonenko, La litterature intertestamentarve et le Nouveau Testament, Revue des Sciences Religleuses 47, 1973, pp (270-279) 272-273

2 Par «apocryphes», nous entendons les écrits quı ne figurent pas dans la Bıble grecque, cf infra p 53 Nous n'utilisons donc pas le terme pour les livres deutérocanoniques de la Septante La limite de 200 après $\mathrm{J}-\mathrm{C}$ a été choisie parce que, d'une part, le canon des livres bibliques commence à prendre forme et que d'autre part, l'époque d'Illermas est défınitıvement passée Sur la nécessité d'étudier aussı le grec biblique dans les apocryphes, voir $\mathrm{N}$ Turner, The 'Testament of Abraham' problems in biblical Greek, New Testament Studies I, 1954-1955, Pp 219-223 et surtout Phlonenko, Littérature intertestamentane

3 Cf Christine Mohrmann, Problemes stylistiques dans la litterature latine chrétzenne, Etudes III. pp (I47-I 70) 148-I49, E J Bickerman, The Septuagint as a translation, Proceedings of the Amencan Academy for Jewish Research 28, 1959, PP (I-39) II-12, 36-37, G J M Bartelink, Observations stylistiques et lingursiqques chez Isidore de Peluse, Vigliae Chrstianae I8, 1964, pp (163-180) I69-1 70

4 Le littéralısme des traducteurs peut cependant être do aussi, en partie, à une connaissance insuffisante de l'hébreu, vorr $\mathrm{Ch}$ Rabın, The translation process and the character of the Septuagint, Textus 6, I968, pp (I-26)23-24 
sible un mot de l'original, quelqu'en fût la nuance, par le même équivalent grec; à tendre à choisir des mots grecs dont la consonance rappelât l'équivalent sémitique; à s'en tenir scrupuleusement à l'ordre des mots de l'original' ${ }^{1}$. Ce littéralisme a fortement accentué le caractère exotique de la Bible grecque. Les phénomènes d'interférence qui en résultèrent, en l'occurrence les sémitismes, devinrent les signes distinctifs de la littérature biblique naissante.

Les traducteurs du Pentateuque avaient fait ouvre de pionniers. Ils avaient été les premiers à s'attaquer aux difficultés que présente la traduction de textes sémitiques. Ils avalent aussi été les premiers à mettre le grec au service des besoins d'une littérature religieuse juive. On comprend donc que les traducteurs ultérieurs aussi bien que les auteurs d'œuvres originales ne se privèrent pas de profiter de ces acquisitions. L'autorité dont jouissait la traduction de la Bible juive était une raison de plus pour l'imiter. Chez les Juifs de langue grecque et chez les Chrétiens, la Septante prenait, en effet, la place que le texte original avait chez les Juifs de langue sémitique et passait généralement chez eux pour un texte inspiré2. En adoptant la forme de la Bible grecque, on pouvait manifester une conformité d'esprit avec elle et avoir part à l'autorité qui s'attachait aux livres traduits de l'hébreus. Or, étant donné que le style de ces livres était particulièrement marqué par les sémitismes, les sémitismes passèrent, eux aussi, dans ce qui s'y ajouta de textes écrits directement en grec.

Mais les sémitismes qui s'étaient glissés dans les traductions ne furent pas tous conservés. Pour le comprendre, il faut considérer la fonction que les sémitismes ont successivement remplie dans les traductions et dans les ouvrages ultérieurs écrits en grec. Dans le premier groupe, ils furent pour ainsi dire dictés par les exigences du texte à traduire; dans le deuxième, ils étaient utiles dans la mesure où

I. Sommaire chez H. St. J. Thackeray, A grammar of the Old Testament in Greek, Cambridge I909, pp.25-55. Voir aussi, entre autres, Bickerman, Septuagint, pp.16-26; Rabin, Septuagint; S. P. Brock, The phenomenon of the Septuagint, Oud-testamentische Studiën 17, 1972, pp. I r-36.

2. Voir D. Tabachovitz, Die Septuaginta und das Neue Testament, Lund 1956, Pp.7-8; V. A. Tcherikover, Corpus Papyrorum Judaicarum, I. Cambridge Massachusetts 1957, Pp.42-43; P. Benoit, L'inspiration des Septante d'apres les Pères, L'homme devant Dieu, Mélanges offerts au Père Henri de Lubac, I, Paris 1963. pp.169-187. Chez les Juifs, on ne juge inspiré que la Septante au sens étroit. donc le Pentateuque, chez les Chrétiens, la Septante au sens plus large, voir Bickerman, Septuagint, pp.5-6.

3. Cf. Tabachovitz, Septuaginta, pp. 14, 16-23; Brock, Septuagint, p.36 (avec des parallèles dans d'autres littératures). 
ils pouvaient servir de signes distinctifs de la littérature biblique ${ }^{1}$. Car, ce que cherchaient les auteurs de cette deuxième catégorie d'ouvrages, c'étaient des "signes distinctifs", non des "sémitismes». D'une part, certains sémitismes qu'on trouve dans les traductions font défaut, soit qu'ils aient passé inaperçus, soit qu'ils aient été jugés inutilisables ou qu'ils aient été évités parce qu'incompréhensibles². D'autre part, partout dans la Bible grecque et dans des ouvrages apparentés, nous trouvons des particularités linguistiques qui n'ont rien en soi de sémitique et qu'une influence sémitique n'explique d'aucune manière, mais dont la fréquence extrême tient à ce qu'on les considérait comme typiques du style de la Bible. Nous pensons à des phénomènes comme l'attraction du relatif, le présent avec une valeur de futur avec des verbes signifiant aller, le présent historique avec $\lambda \varepsilon \dot{\gamma} \gamma \varepsilon \iota v$ et ópãv.

C'est ainsi que le grec biblique devint une langue de groupe qui, d'une part, s'écartait de la langue de tous les jours - nous en reparlerons -, d'autre part, se rattachait à un genre littéraire. Elle prit, pour employer un terme de la philologie classique, les traits d'une langue stylisée (Kunstsprache). Une comparaison avec ce qu'on pourrait appeler le prototype d'une langue stylisée, le grec de la poésie hexamétrique, est instructive ${ }^{3}$. Ces langues stylisées sont toutes deux traditionnelles. Toutes deux s'inspirent d'une grande ouvre littéraire (Homère, le Pentateuque grec) qui, du moins pour les générations ultérieures, est à l'origine du développement. Toutes deux utilisent des éléments hétérogènes, des formes artificielles, des tournures stéréotypées. Toutes deux passent par un développement qui, tout comme la langue courante, est influencé pat la communauté où elles remplissent leur fonction. Mais le parallèle le plus intéressant, c'est que les deux langues stylisées ne s'écartent que plus ou moins de la langue de la vie

I. Dans des cas pareils, on parle de "septuagintismes» ou de "biblismes». Il serait intéressant de suivre leur histoire ultérieure, histoire qui, pour certains d'entre eux, se poursuit jusqu' à nos jours. Du moins faudrait-il établir d'abord quelles anomalies sont des sémitismes: c'est ce que nous nous proposons de faire dans cette étude.

2. Sur l'embarras des commentateurs grecs devant ces sémitismes ancompréhensibles", voir M. Harl, $Y$ a-t-il une influence du "grec bibliquen sur la langue spurituelle des chrétiens? Exemples tivés du psaume 118 et de ses commentateurs d'Origene d Theodoret, La Bible et les Pères, Paris I971, pp 243-262.

3. Christine Mohrmann, Le dualisme de la latintté médiévale, Revue des Études Latines 29, 1952, pp.(330-348)336-337 fait une comparaison entre cette langue stylisée et le latin médiéval. Mais l'analogie du grec épique avec le grec biblique est encore plus grande dans la mesure où l'un et l'autre se sont développés dans un milieu grec, tandis que le latin médiéval a pris forme dans un milieu où le latin n'était la langue maternelle de personne. 
quotidienne. Du fait que la langue hexamétrique est liée au mètre des vers, il est plus facile de déterminer la frontière qui la sépare du grec courant que pour le grec biblique, où les caractéristiques se présentent à tous les degıés de dilution. Mais, dans les textes hexamétriques aussi, l'écart par rapport à la langue courante est très variable.

Une langue stylisée s'écarte de la langue courante. Qu'il en soit ainsi pour le grec biblique, c'est, malgré les efforts de Deissmann et son école, chose facile à démontrer si nous le comparons à la langue commune. Mais le problème se situe ailleurs. Ne se peut-il pas que ce que nous considérons comme une langue stylisée ait été en réalité la langue courante des milieux qui ont produit la littérature biblique? Les sémitismes n'auraient-ils pas existé déjà dans le grec que parlaient les Juifs? Grec biblique ne serait-il pas synonyme de grec des Juifs? Malheureusement, la langue parlée s'est tue à jamais. Mais, des données indirectes peuvent nous aider à nous faire une certaine idée. Que peuvent-elles nous apprendre?

En Palestine, le grec a pénétré à une date très précoce et, comme cela apparaît de plus en plus clairement, des groupes importants de Juifs l'utilisaient comme langue véhiculaire. D'autre part, les langues sémitiques s'y conservèrent fortement, l'araméen dans la vie quotidienne, l'hébreu dans la religion et, à certains endroits, comme langue parlée ${ }^{1}$. Il y a indubitablement eu de nombreux milieux bi- et trilingues, et les phénomènes d'interférence dans le grec des Juifs palestiniens ont dû être chose courante. Il se peut que des traces de cette interférence aient pénétré aussi les produits littéraires des Juifs palestiniens. Mais pour nous, ce ne sont là que choses d'une importance secondaire. Une petite partie seulement de la littérature biblique a été écrite en Palestine ${ }^{2}$, guère plus sans doute qu'Esther, I Maccabées, les évangiles synoptiques et l'Epître de Jacques, écrits dont une partie remonte elle-même a des originaux sémitiques, de sorte que les sémitismes qu'on y rencontre peuvent s'expliquer par le littéralisme.

Dans la diaspora ${ }^{3}$, la masse des Juifs, dès le début de l'époque

I. Voir J. A. Fitzmyer, The languages of Palestine in the first century A.D., The Catholic Biblical Quarterly 32, I97o, pp 50I-53I. (2b. pp.528-53 I sur l'hébreu comme langue parlée).

2. Voir Hengel, Judentum und Hellenismus, pp.r86-19o; Fitzmyer, Languages of Palestıne, pp.503 (n.6), 5 Io. Il est très probable qu'une revision du texte de la Septante d'après l'hébreu a été faite par des Juifs palestiniens.

3. Pour ce qui suit, voir V. Colorni, L'uso del greco nella liturgia del giudaismo ellenistico e la Novella 146 di Giustinnano, Annali di Storia del Diritto 8, 1964, pp. I9-80; J. N. Sevenster, Do you know Greek?, Leiden I968, pp 82-87; K. Treu, 
hellénistique, ne parlait plus que le grec'. Les papyrus et les inscriptions en donnent une preuve éloquente: à côté de quelques restes insignifiants de textes sémitiques, on trouve des textes grecs en abondance ${ }^{2}$. Pour continuer à remplir sa fonction, il faut alors que la Bible soit traduite. Les services dans la synagogue se font en grec. On est encore loin de la réhébraīsation, qui commença à se dessiner dans les derniers siècles de l'Antiquité. Au contraire, on assiste, par rapport à la culture profane, à un rapprochement qui allait devenir inconcevable plus tard. Pour ne parler que de littérature, il suffit de penser à la prose de Philon, aux cuvres du poète Ézéchiel et du PseudoPhocylide, aux Livres Sıbyllins écrits par des Juifs, aux épitaphes métriques ${ }^{3}$.

Pour les Juifs, le choix du grec était déjà, en soi, un acte d'assimilation. $\mathrm{Et}$, à en juger d'après l'abandon des langues sémitiques qui s'ensuivit et le rapprochement de la culture profane, les circonstances étaient peu favorables pour le développement d'une forme de grec sémitisanté ${ }^{4}$ D'un autre côté, il est difficile d'admettre que la langue des Juifs ait été en tout point identique à celle de leur entourage paien. Il est probable que, bien avant que les traducteurs de la Septante ne commencent leur travail, il s'est au moins formé un vocabulaire religieux inspiré sans nul doute du modèle sémitique. Pour la terminologie, les traducteurs ont donc certainement pu puiser dans un vocabulaire déjà constitué. Mais, pour les sémitismes syntaxiques, l'explication est beaucoup moins recevable; elle ne l'est pas notamment pour les phénomènes qui étaient des hébraīsmes sans être en même temps des aramaiismes. Les Juifs de la diaspora étaient passés, pour la langue courante, non pas de l'hébreu au grec, mais de

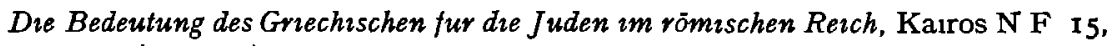
I973, PP (123-I44) I 25-I3 I

I Selon certains, les Juifs d'Égypte faisaient aussı un grand usage de l'égyptien et un certain nombre de sémitismes supposés de la Septante seraient aussi, pour une part du moins, des égyptianismes, vorr U Rapallo, Per una definızıone diacronica e tipologica der calchr ebraicr nelle antiche version del Levitico, Rendconti dell' Istituto Lombardo, Classe di Lettere, Scienze moralı e storiche I03, 1969, pp (369-437) 377-378, Brock, Septuagint, pp 33-35 Sur la connassance des langues sémitıques chez les Juifs d'Égypte, voir Thackeray, Grammar, p 28, L Delekat, Ein Septuagintatargum, Vetus Testamentum 8, I958, pp (225-252) 225-228

2 Hengel, Judentum und Hellenısmus, p Io9, Treu, Bedeutung des Grnechischen, PP 128-1 29

3 Volr aussı Tchenkover, Corpus Papyrorum Judatcarum, I, pp 25-47

4 Cf Christine Mohrmann, Quelques observations sur l'ornginalnte de la littérature latıne chrétienne, Études I, PP (139-I50) 143 
l'araméen. Le passage de l'hébreu à l'araméen avait eu lieu beaucoup plus tôt, encore en Palestine. Il est vrai qu'en Palestine, comme nous l'avons déjà dit, l'hébreu s'était perpétué dans le culte et, à certains endroits, comme langue parlée, mais pas au point qu'il faille tenir compte, chez les Juifs de la diaspora, d'une minorité de langue hébraique de quelque importance. Nous pouvons donc poser en principe que tout sémitisme dû au bilinguisme général dans la diaspora devrait être un aramaisme ${ }^{1}$. Plus tard, lorsque la Septante eut une fois pour toutes conquis sa place dans le judaīsme de la diaspora, certaines interactions se produisirent peut-être, qui firent que la traduction biblique influença la langue courante et qu'alors celle-ci agit à son tour sur la langue de la littérature biblique ultérieure. Mais on peut se demander si cela concerna plus qu'une série de termes et d'expressions.

En fin de compte, le grec parlé ne semble avoir contribué que pour une part modeste aux sémitismes qu'on trouve dans la littérature biblique. A cet égard, il est intéressant de voir qu'il n'y a pas de sémitismes dans la littérature judéo-hellénistique dite apologétique. Autrefois, on expliquait la chose par la destination de ces écrits: ceux à qui ils s'adressaient auraient été des non-Juifs qu'il valait mieux ne pas irriter d'avance par des singularités de langue. Or, Tcherikover a montré de façon plausible que les auteurs destinaient cette littérature à leur propre communauté juive ${ }^{2}$. Il n'allait donc pas tellement de soi que les Juifs, quand dans leurs écrits ils s'adressaient à des Juifs, le fissent en grec sémitisant. Lorsqu'ils le faisaient, c'était parce qu'ils tenaient à donner une teinte biblique à ce qu'ils écrivaient plutôt que parce que le grec qu'ils parlaient les y portait.

En résumé: l'influence sémitique en grec se réduit, dans la langue commune, à quelques emprunts. Nous pouvons supposer que, dans certains milieux bilingues, on parlait une langue plus sémitisante, mais il ne nous est plus possible de la ressaisir. Enfin, un grec sémitisant s'est développé pour devenir la langue d'un genre littéraire, la littérature biblique. Cette langue a pu s'appuyer sur la langue commune des milieux bilingues dont nous avons parlé; mais elle devait essentiellement son existence tout d'abord au littéralisme des traductions et ensuite à l'imitation de ce grec de traduction dans des auvres écrites en grec. C'est ainsi qu'elle a pu se maintenir chez des auteurs

I. Voir Brock, Septuagint, pp.3r-33.

2. V. Tcherikover, Jewish apologetic literature reconsidered, Eos $4^{8}, 195^{5}$. Pp. (169-193) I 7 I-I 83 . 
qui ignoraient les langues sémitiques et n'avaient pas non plus de contact, dans leur entourage quotidien, avec une forme quelconque de grec sémitisant.

\section{LATINISMES}

Aux premiers siècles de l'Empire, il y eut des rapports extrêmement suivis entre le monde de langue grecque et celui de langue latine. D'une façon générale, les Romains furent sensibles aux stimulants culturels qui leur venaient du monde grec. En outre, à certains endroits, en Occident, les Grecs et les Orientaux de langue grecque formaient une minorité importante de la population. L'attitude des Grecs à l'égard de l'influence romaine était moins bienveillante, surtout chez les gens cultivés, mais il était impossible de l'ignorer complètement, même en Orient. Les Romains n'étaient-ils pas les maîtres du monde? Partout on rencontrait des marchands, des soldats, des fonctionnaires romains. Le latin était la langue officielle du droit, de l'armée et de l'administration.

Nous trouvons des exemples frappants d'interférence latine dans des textes traduits, surtout les textes juridiques: traités, décrets, testaments. Mais selon toute vraisemblance, ces latinismes n'ont pas dépassé le stade du grec de traduction. Ils n'invitaient pas à l'imitation dans des documents grecs originaux. Ils n'intéressent guère notre recherche. On peut considérer comme indubitable que les interférences étaient nombreuses dans la langue parlée; mais nous n'avons guère de documents où nous pourrions, pour ainsi dire, les prendre sur le fait. Quoi qu'il en soit, des latinismes se sont introduits dans le grec post-classique. Il faut néanmoins distinguer l'importation et la substitution. L'importation se vérifie par la présence d'emprunts latins en matière de mots et de suffixes dans le grec de l'époque impériale. Il est vrai qu'il y avait une forte tendance à refuser ces latinismes en grec $^{\mathbf{I}}$, mais cette résistance met le fait d'autant mieux en évidence. La substitution est déjà, en soi, plus difficile à déceler, mais nous confronte ici à des problèmes supplémentaires.

Le grec post-classique présente, en effet, une série de particularités qui, d'une part, n'existent pas en grec classique et qui, d'autre part, sont usuelles dans le latin classique contemporain. Ici se pose donc la question de savoir s'il s'agit d'un développement interne ou d'une

I. Voir V. Reichmann, Römische Literatur in grechischer Ubersetzung, Leipzig 1943. Pp.r-r6. 
influence externe, question dont nous avons parlé plus haut, p.37. Plusieurs hypothèses sont possibles: les particularités en question seraient dues à une influence latine, ou bien, au contraire, le latin aurait été influencé par le grec post-classique, ou bien la même évolution aurait eu lieu indépendamment dans les deux langues ${ }^{1}$. Il nous semble que souvent on a opté un peu trop prématurément pour la première solution. Il y a, en tout cas, quelques remarques à faire qui nous incitent à la prudence avant de conclure aux latinismes.

Tout d'abord, il faut se rappeler qu'au cours d'un processus qui a duré de nombreux siècles, le grec était devenu un moyen de communication d'une extrême souplesse, capable de s'adapter à toute circonstance nouvelle. Le latin, au contraire, pour suivre l'exemple du grec précisément, faisait des efforts laborieux pour acquérir la même malléabilité, efforts qui, selon des Romains compétents en la matière, n'aboutissaient que partiellement. Vu sous ce jour, le rôle de fournisseur revient plutôt au grec. Ensuite, nous devons tenir compte des tendances classicistes qui se manifestaient de plus en plus dans le grec post-classique. Les auteurs grecs étaient beaucoup plus que les latins entravés dans le recours à la langue de leur temps, car les moyens d'expression à employer devaient, si possible, exister déjà dans les modèles littéraires vieux de trois à six siècles. Il faut ajouter que, quand ils n'ont pas assez tenu compte des normes classicisantes, l'oubli des siècles les en a le plus souvent punis. Ce n'est pas sans raison que nous sommes obligés d'élaborer en grande partie l'image que nous avons du grec post-classique à partir de documents qui, ou bien ont été retrouvés par hasard, comme des papyrus et des inscriptions, ou bien ont été conservés pour l'importance du fond, malgré la forme, comme la Bible. La littérature officielle est peu représentative de la langue du temps. Enfin, il se peut aussi que l'état actuel de nos connaissances nous amène à conclure trop vite aux latinismes. Les anciens manuels portent encore souvent les traces de l'idée que le grec post-classique normal est à peu près identique au grec classique. Tout ce qui n'est pas classique et présente des analogies avec le latin est tout naturellement ramené à une influence latine. Mais, même indépendamment de ce malentendu, on pense trop facilement au latin parce que, jusqu'à aujourd'hui, on connaît beaucoup mieux le latin r. Cf. E. Löfstedt, Late Latin, Oslo 1959, pp.88-1 19; E. Coseriu, Das Problem des griechischen Einflusses aut das Vulgärlatein, Sprache und Geschichte, Festschrift für Harri Meier zum 65. Geburtstag, München 1971, pp. 135-1 47. Pour des développements parallèles dans les langues sémitiques et le grec cf. K. Beyer, Semitische Syntax im Neuen Testament, I, Göttingen r968², P.I I. 
classique que le grec post-classique qui lui était contemporain. Des recherches plus poussées pourraient montrer que ce qu'on tient jusqu'icl pour latinisme se trouve dans des sources grecques pour lesquelles il ne peut être question d'influence latıne.

\section{HERMAS}

Lorsque nous étudions des phénomènes d'interférence dans la langue de nos contemporains, nous tenons compte de leur milieu, de leur culture et autres données de ce genre sans oublier, bien entendu, leur connaissance des langues. Nous obtenons ces données simplement en nous informant. Mais s'il s'agit, comme dans cette étude, de personnes qui ont vécu dans un lointain passé, nous sommes obligés de nous livrer à une laborieuse reconstitution. C'est ce que nous allons essayer de faire pour Hermas.

Il existe un certain lien entre degré de culture et interférence. Plus un locuteur est illettré, moins châtiée sera sa langue. Il ne se rend pas bien compte des influences qui agissent sur son langage et son écriture. Ainsi un terrain favorable s'offre aux phénomènes d'interférence. Une personne plus cultivée connaît généralement mieux sa langue, et son sens plus raffiné des normes s'oppose au libre jeu des interférences. On fait bien de se le rappeler dans le cas d'Hermas, dont on taxe en général le style de simple et naîf ${ }^{1}$. Car quoi qu'on pense de ses talents d'écrivain, il n'est pas tout a fait sans avoir des lettres. Dibelius a signalé des passages qui s'inspirent de romans grecs, Joly a montré les analogies avec le Tableau de Cébès et Reiling a prouvé que les éléments hellénistiques dans le passage sur la prophétie chrétienne sont plus qu'une forme de s'exprimer ${ }^{2}$.

Un des passages où se révèlent certains rapports avec la littérature profane, le chapitre $5 \mathrm{I}$, nous amène d'emblée à la question de sa connaissance des langues. En effet, l'image qu'il y emploie, la comparaison de l'orme et de la vigne, revient régulièrement chez les auteurs latins, mais, pour autant que nous sachions, est étrangère à la littérature grecque ${ }^{3}$. On pourrait supposer qu'il l'a rencontrée dans ses

I Plus nuancé chez Joly, Pasteur, pp 55-57

2 Dibelus, Hirt. pp 429-430, 618-619, R Joly, Judaisme, christianisme et hellénisme dans le Pasteur d'Hermas, La Nouvelle Clio 5, I953, pp (394-406) 402405, le même, Pasteur, Pp 5I-53, le même, Le Tableau de Cébès et la philosophre relıgreuse, Bruxelles I963, pP 46, 49, 60, 81-83, Reiling, Hermas, pass

3 Voir P Demetz, The elm and the vine, Notes toward the history of a marriage topos, Publications of the Modern Language Association of America 73, 1958, PP 521-532, Alfons1, La vite, pp 83-85, cf supra P I3 
lectures des auteurs latins; donc qu'il connaissait le latin. Mais cette supposition n'est pas contraignante. Tout d'abord, il peut avoir emprunté lui-même directement l'image à la réalité vivante. Mais même s'il utilise là un motif existant, il ne l'a pas nécessairement rencontré dans des textes latins, l'image a pu s'introduire dans le grec, même si les textes dont nous disposons n'en témoignent pas. Pour savoir si notre auteur n'aurait pas connu d'autres langues nous aurions besoin de plus de renseignements.

Or, ni le Pasteur ni les témoignages de l'Antiquité ne nous disent la langue ou les langues qu'Hermas connaissait outre le grec. Nous devons laisser hors de cause ici les indications données par sa langue ellemême. C'est pourquoi nous devons ramener la question à celle de son milieu. Mars pour commencer, quel était le milieu d'Hermas? Comme nous l'avons vu, Hermas écrit son œuvre à Rome, dans la première moitié du IIe siècle après J.-C. Il était, à n'en pas douter, Chrétien. Mais son œuvre abonde en idées et en façons de penser juives ${ }^{1}$, à tel point, qu'il est un de nos pnncipaux témoins du judéo-christianisme non hétérodoxe. Les liens entre Juifs et Chrétiens sont vraisemblablement si étroits encore que les données que nous possédons sur les Juifs de Rome peuvent s'appliquer grosso modo au mllieu d'Hermas.

La langue que parlaient les Juifs et les Chrétiens de Rome était le grec. Les Juifs étaient originaires de la moitié orientale de l'Empire, soit de naissance, soit de descendance. Il en était de même pour la plupart des Chrétiens, qu'ils fussent d'origine paienne ou juive. De nombreux autres groupes de Grecs et d'Orıentaux vivaient également à Rome. A eux tous, ils tormaient une fraction importante de la population et la langue de cette fraction était le grec ${ }^{2}$. Leur fidélité au grec ne s'explique d'ailleurs pas seulement par leur nombre. Les communautés orientales se distinguaient aussi par une grande solidarité

I Voir, par exemple, J Daniélou, Théologıe du Judé-chrıstianısme, Toumaı 1958, pass, le même, Les symboles chrétıens primutıfs, Pans I96I, pass, Pernveden, Church, pp 282-291, L W Barnard, Studies in the Apostolic Fathers and thetr background, Oxford 1966, pp I57-I63. Snyder, Shepherd, pp 16-17, J Massingberd Ford, $A$ possible liturgical background to the Shepherd of Hermas, Revue de Qumran 64,24 1969, pp 531-552, A T Hanson, Hodayoth vi and vin and Hermas Sim VIII, Studia Patristica 10, Berlin 1969, pp 105-108 Joly, Judaisme, pp 394-406 et Relling, Hermas, pp 25-26, I73-174 mettent en garde contre la tentation de ne penser qu'à l'influence juive

2 Vour G La Plana, Forengn groups in Rome during the first centurzes of the Empire, The Harvard Theological Review 20, I927, pp I83-403 
et un certain isolement par rapport à la population autochtone. En outre, à Rome, on faisait grand cas du grec et nombreux étaient ceux qui, ayant le latin comme langue maternelle, le parlaient couramment ${ }^{1}$. Et enfin, n'oublions pas que le grec, comme nous l'avons déjà remarqué, était un moyen de communication qui s'adaptait facilement à des circonstances nouvelles. En conséquence, nous pouvons présumer que parmi les Orientaux de Rome beaucoup n'ont parlé que grec jusqu'a la fin de leurs jours ${ }^{2}$.

Cela ne signifie pas que, dans ces milieux, on ignorait complètement les langues sémitiques et le latin. Chez les Chrétiens, on connaissait sans doute peu les langues sémitiques. Mais loin de nous l'idée d'être apodictique. C'était là un monde mouvant, où l'on voyageait beaucoup. C'est ainsi qu'Hégésippe, Juif converti, qui connaissait l'hébreu et l'araméen (Eusèbe, H.E. 4,22,8), habita longtemps à Romes. Chez les Juifs, ces langues, comme on pouvait s'y attendre, avaient été mieux conservées. Un certain nombre d'inscriptions juives sont entièrement ou partiellement en araméen et en hébreu et ce, en lettres hébraĩques. Après la chute de Jérusalem et à différentes autres occasions, l'élément de langue sémitique parmi les Juifs de Rome a probablement été renforcé par l'arrivée de prisonniers qu'on vendait comme esclaves. Le Talmud babylonien parle du séjour à Rome, au premier quart du IIe siècle après J.-C., du tanna palestinien R. Mattiah ben Heresh, qui y aurait même fondé une école4.

En outre, le latin entrait de plus en plus dans l'usage. Pour les Chrétiens, il n'en est encore que peu de traces au début du IIe siècle. Les données fournies par le Pasteur à cet égard sont discutables (cf. nos conclusions infra, p.I85). Mais si on peut dater la version latine de la Prima Clementis d'environ I6o après J.-C., l'emploi du latin parmi les Chrétiens de Rome a certainement dû commencer à l'époque

I. Voir P. Boyancé, La connaissance du grec d Rome, Revue des Études Latines 34. 1956, pp.III-131; H.-I. Marrou, Histoire de l'education dans l'antiquite, Paris I965, Pp. $362-363,378-388$.

2. Cf. Coseriu, Vulgärlatein, p.r39. Pour le grec des Juifs et des Chrétiens de Rome voir H. J. Leon, The Jews of ancient Rome, Philadelphia I960, pp.75-92 et Caspari, Quellen, III, Pp.267-466; Mohrmann, Origines, pp.67-73.

3. Voir aussi G. Bardy, La question des langues dans l'Église ancienne, I, Paris 1948, Pp.88-9o.

4. Sur lui Colorni, Uso del greco, p.30 n.52, cf. ib. pp.63-64. Sur la solidarité des Juifs de Rome avec le judaïsme de langue sémitique M. Hengel, Die Synagogeninschrift von Stobi, Zeitschrift für die neutestamentliche Wissenschaft 57 , I966, pp.(145-183) I81-182. Au temps de saint Jérôme, les Juifs de Rome disposent de manuscrits hébreux de la Bible, cf. Jérôme, $E p .32,1 ; 36,1$. 
d'Hermas ${ }^{1}$. Sur les Juifs cependant nous sommes mieux renseignés. Un quart de leurs inscriptions est en latin; mais il faut reconnaître qu'il est impossible de les dater avec précision et que les inscriptions latines sont probablement en grande partie du III ${ }^{\mathbf{e}}$ et IVe siècles. Des auteurs romains décrivent les Juifs dans des situations où ils auraient difficilement pu parleı autre chose que le latin. Plusieurs Juifs se risquèrent à la poésie métrique traditionnelle. Martial déjà s'attaque à un rival "né au cour de Jérusalem» (II,94). Dans la catacombe juive de Monteverde, on a trouvé une épitaphe hexamétrique de treize vers (CIJud $\mathrm{I}, 476)$ qui, selon certains, date du début du IIe siècle après J.-C.

Tous ces faits confirment l'usage de plusieurs langues parmi les Juifs et les Chrétiens de Rome. Mais ce qu'on peut constater dans le groupe ne s'applique pas nécessairement à Hermas lui-même. En effet, certaines choses nous amènent à penser qu'il ne faut pas trop se hater de croire qu'il connaissait les langues sémitiques et le latin. Pour ce qui est des langues sémitiques, langues d'ailleurs peu répandues chez les Chrétiens, la langue du Pasteur est, de l'avis général, étroitement apparentée à celle de la Bible grecque, ce qui n'étonne guère pour un ouvrage qui s'était presque fait une place dans le canon. Il n'y a donc aucune raison d'imputer à une connaissance personnelle des langues sémitiques des sémitismes qui peuvent remonter au grec biblique. Est-ce l'explication de tous ces sémitismes? C'est ce que la suite devra nous apprendre.

Pour les latinismes d'Hermas, le problème se pose différemment. Ici, point de modèles littéraires. Qu'il ait lu des écrivains latins, nous semble peu probable. Pour autant que nous le sachions, il n'existe pas, à son époque, de textes latins d'auteurs juifs ou chrétiens². Ce qu'on suppose être des réminiscences d'auteurs profanes latins peut fort bien être en réalité des réminiscences d'auteurs grecs, si réminiscences il y $a^{3}$. S'il est vrai que, comme nous l'avons dit plus haut, p.3I, les détails topographiques qu'il fournit permettent de le localiser à Rome, il y en a parmi eux qui surprennent: ainsi les "promenades" à Cumes $(\mathrm{I}, 3 ; 5, \mathrm{I})$, le voyage aérien en Arcadie $(78,4$, cf. supra p.24). Un autre détail est encore plus frappant: alors qu'un texte comme l'évangile de

I. Cf. Mohrmann, Origines, pp.78-ro6.

2. Du moins, l'existence d'une traduction latine juive de la Bible est parfaitement hypothétique, voir Colorn, Uso del greco, pp.75-77. La date précoce que J. Daniélou, La litterature latine avant Terhullien, Revue des Études Latines 48, 1970, pp.357-375 propose pour 5 Esdras et autres textes latino-chrétiens n'est pas convaincante.

3. Cf. supra p.rz. 
Matthieu, qui a pourtant certainement vu le jour en Orient, utilise une mesure de longueur romaine, $\mu$ i $\lambda$ iov $(5,4 \mathrm{I})$, Hermas, en pleine Italie, s'exprime en employant une mesure de longueur grecque,

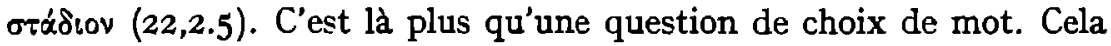
indique un manque d'intégration dans la communauté romaine. Nous soupçonnions déjà que les habitants de Rome d'origine orientale, surtout dans les communautés plus ou moins étanches, se tiraient fort bien d'affaire avtc comme seule langue le grec. Si nous combinons tous ces éléments nous sommes amenés à la conclusion suivante: bien qu'il ait vécu à Rome, nous ne pouvons pas supposer qu'Hermas ait été plus influencé par le latin que n'importe quel autre auteur grec de son temps. Il est possible que l'étude de sa langue nous forcera à reviseı ce jugement. Mais a priori, on n'a pas le droit, nous semble-t-il, du seul fait qu'il habitait Rome, de voir des latinismes dans ce qu'en d'autres textes nous jugerions être du grec courant't.

\section{METHODE À SUIVRE}

Pour autant que nous sachions, personne jusqu'à présent n'a attribué les phénomènes d'interférence dans le Pasteur à d'autres langues que l'hébreu, l'araméen et le latin. Nous aussi, nous partons du principe que tout ce qui n'est pas grec dans le Pasteur est dû à l'influence d'une ou de plusieurs de ces langues. La question se pose maintenant de savoir comment procéder pour dépister ces éléments non grecs. En théorie, la réponse devrait être qu'une comparaison du système de la langue grecque avec celui des trois autres langues (toutes trois au degré de développement qu'elles avaient dans la première moitié du $\mathrm{II}^{\mathrm{e}}$ siècle après J.-C.) nous permettrait de prévoir quels sémitismes et latinismes sont possibles en grec: il ne resterait plus qu'à vérifier ceux qui se trouvent chez Hermas. Mais la chose est impossible par définition; car, comme nous l'avons montré plus haut, pp.36-37, nous ne connaissons pas - et nous ne connaîtrons jamais - suffisamment les systèmes en question. Nous ne devons pas pour autant renoncer à l'étude des sémitismes et des latinismes. Même pour les langues aujourd'hui vivantes il n'existe pas encore de descriptions complètes. Cela n'a pas été une raison pour renoncer à étudier les interférences dans le présent.

I. Nous nous opposons ici à une habitude de Blass-Debrunner entre autres,

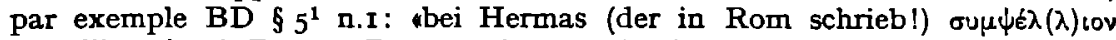
subsellium (auch Pap., s. Bauer s.v.)"; $\S 4^{62^{1}}$ : "Auch sonst erzählt Hermas gern asyndetisch, wohl unter lat. Einfluss»; voir aussi $i b . \$ 660^{1}, 347^{3}$. 
Et puis nous n'ignorons pas les langues en question ici au point de ne pouvoir porter un jugement valable sur les sémitismes et les latinismes, comme on le suggère parfois ${ }^{1}$.

La méthode que nous avons suivie est la suivante. En lisant et relisant le Pasteur, nous avons noté toutes les particularités linguistiques qui nous semblaient s'écarter des normes du grec de l'époque. En présumant que tous les phénomènes non grecs sont dus ici à une influence de l'hébreu, de l'araméen ou du latin ou des trois combinés, nous avons obtenu un premier inventaire des sémitismes et des latinismes dans le Pasteur. Nous avons complété cette liste avec les données fournies par les grammaires, les dictionnaires et les monographies. Nous avons ensuite soigneusement examiné la collection ainsi constituée en éliminant ce qui, à y regarder de plus près, s'avérait conforme aux normes du grec contemporain: ce qui est resté est ce que nous supposons constituer les sémitismes et les latinismes du Pasteur d'Hermas.

Nous avons alors entrepris une comparaison avec un certain nombre d'autres textes grecs, classiques et post-classiques. Tant qu'il s'agissait d'un seul phénomène linguistique, il n'était pas difficile de trouver de bonnes sources. Mais le choix diminuait considérablement lorsqu'il s'agissait de trouver des textes d'une certaine longueur, qui devaient donner une image-type du grec tel qu'on l'écrivait au temps d'Hermas. Papyrus, inscriptions et auteurs d'une date incertaine étaient à écarter ${ }^{2}$; certaines tendances classicisantes diminuaient l'utilité de tel ou tel texte; et le reste des auteurs, d'après ce que nous savions de leur vie, éveillait le soupçon de n'avoir pas échappé, dans leur langue, à l'interférence soit sémitique soit latine. C'est ainsi que nous n'avons pas cru pouvoir considérer comme représentative la prose du contemporain d'Hermas, Lucien de Samosate, Syrien de naissance, qui dit lui-même qu'il n'a appris le grec que comme seconde langue (Bis Acc. 27). Au II siècle après J.-C., tout sujet de l'Empire romain avait fatalement affaire au latin; en outre, la plupart des auteurs de ce

I. Cf. I. Soisalon-Soininen, Die Infinitive in der Septuaginta, Helsinki 1965.p.9; I. Bettini, Tendenze e problemi nello studio della lingua dei Settanta, Atene e Roma N.S. 16, I971, pp.(I-I2) 9-10.

2. Des textes comme la Vie d'Ésope et l'Historia Alexandri Magni, qu'on cite dans des grammaires du Nouveau Testament pour contester les sémitismes, sont suspects pour deux raisons: ils n'ont pris la forme que nous leur connaissons qu'à la fin de l'Antiquité et la Bible grecque leur a déjà servi de modèle. On ne peut evidemment pas non plus invoquer les apocryphes néotestamentaires plus tardifs pour contester les sémitismes. 
temps ont séjourné plus ou moins longtemps à Rome. Du reste, si cela est un inconvénient décisif, il n'y a plus qu'à renoncer à trouver un texte représentatif.

Mais nous n'avons pas été jusque là. Pour savoir ce qu'il en était du grec courant à l'époque où le Pasteur a été écrit, nous avons choisi quelques textes pour $\mathrm{y}$ dépister tous les phénomènes qui nous intéressent. Ce sont: Épictète, livre I, Plutarque, De sera numinis vindicta et Vie de Lycurgue, Appien, livre I2, Pausanias, livre 2 et Achille Tatius, livres I-4. Les auteurs de ces textes ne sont ni des Juifs ou des Chrétiens, ni des Romains. Abstraction faite des phénomènes d'importation, leur langue ne présentera donc généralement pas de sémitismes et de latinismes. Ce groupe de textes a donc une fonction négative: les phénomènes qui, dans le Pasteur, donnent l'impression d'être des sémitismes et des latinismes devront être considérés comme du grec courant s'ils se rencontrent dans les textes en question.

En outre, nous avons fait une étude systématique de la Septante, du Nouveau Testament et des apocryphes paléo- et néotestamentaires jusqu'à la fin du II ${ }^{\theta}$ siècle environ. Parmi les apocryphes paléotestamentaires nous comptons l'Apocalypse de Moise, I Henoch, le Testament d'Abraham (A et B), Joseph et Aséneth, les Testaments des Douze Patriarches, les Paralipomènes de Jérémie, l'A pocalypse grecque de Baruch, les Vies des Prophètes, l'Apocalypse grecque d'Esdras et le Testament de Job. Parmi les apocryphes néotestamentaires, nous comptons le Protévangile de Jacques, l'Evangile de Pierre, 3 Corinthiens, les Actes de Paul et Thècle, les Actes de Paul, le Martyre de Paul, l'E pitre du Pseudo-Barnabé, l'A pocalypse de Pierre, la Didachè et la II Ode Oe Salomon. Pour les latinismes, cet ensemble de textes, à quelques exceptions près, remplit la même fonction que le groupe de textes profanes. Quant aux sémitismes, il faudra vérifier dans ces textes dans quelle mesure les phénomènes qui se présentent chez Hermas étaient d'un usage courant en grec biblique.

Il est évident que, dans l'unité relative de la Bible grecque et des écrits apparentés, on peut remarquer une grande diversité, suivant l'époque et le lieu d'origine, suivant le fond et suivant la forme. La différence essentielle dans cette étude est celle qui existe entre les traductions et les livres originairement grecs. Dans la première catégorie, les sémitismes sont dus surtout au littéralisme, dans la deuxième à l'imitation du style des traductions (cf. supra, pp. 40-4I). Aussi, dans les chapitres suivants, nous comparerons continuellement entre elles ces catégories. Font partie de la première catégorie tous les livres 
dont nous possédons l'original sémitique complet. Elle comprend donc en réalité les livres protocanoniques de la Septante. La deuxième catégorie embrasse tous les autres textes. Il est vrai qu'une partie de ces textes - des livres entiers comme I Maccabées et des passages de certains livres comme probablement les évangiles synoptiques -, remontent d'une manière ou d'une autre à des documents sémitiques. Mais, premièrement, il n'est plus possible, en général, de savoir avec exactitude quelle a été leur relation à ces documents; deuxièmement, on peut présumer que, dans ces textes aussi, l'imitation du style des plus anciens livres de la Septante a été d'une importance décisive. Dans les chapitres suivants, lorsque nous citerons des passages des livres dont il existe deux versions: les Juges, Tobie, Suzanne, Daniel et $\mathrm{Bel}$, nous indiquerons a chaque fois les passages de l'une et l'autre version, comme si chacune d'elles était un livre à part. Compte tenu de cela, d'après nos calculs, les livres protocanoniques de la Septante représentent environ $54 \%$ de l'ensemble des textes formé par la Bible grecque et les apocryphes. Le reste comporte donc $46 \%$, dont les livres deutérocanoniques constituent $13 \%$, le Nouveau Testament $\mathbf{I} 8 \%$, les apocryphes paléotestamentaires $9 \%$ et les apocryphes néotestamentaires $6 \%$. Ces chiffres montrent en même temps que le gros du corpus en question, à savoir $85 \%$, est formé par la Bible grecque.

La plupart des sémitismes et des latinismes ont trouvé accès dans le grec grâce au fait qu'ils se raccrochaient à un usage qui existait ou semblait exister déjà'. S'ils s'écartent du grec usuel, c'est seulement à cause de leur fréquence beaucoup plus grande. En pareil cas, nous parlons de sémitismes et latinismes "secondaires" ou "partiels». Ceux-ci, évidemment nous mettent devant de grands problèmes: où placer la limite? On a remarqué que, dans des textes traduits, la fréquence ne peut être un critère, puisqu'elle y est dictée en une certaine mesure par le texte de base ${ }^{2}$. Mais, quand il s'agit des sémitismes de la littérature biblique, la fréquence nous permet souvent de mesurer le degré auquel un élément est étranger, et ce, en étudiant l'imitation dans les livres écrits originairement en grec: ces livres, en effet, utilisent un certain nombre de sémitismes avec une fréquence qui se situe entre celle des livres traduits et celle du grec profane. De toute évidence leur exotisme

I. C'est ainsi qu'on peut penser que les traducteurs de la Septante se sentirent autorisés à employer le év instrumental du fait qu'ils entendaient les tournures

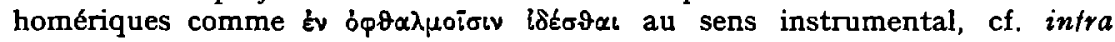
pp.82-83.

2. Soisalon-Soininen, Infinitive, pp.r3,208. 
a frappé les auteurs, qui les jugèrent typiquement piopres au grec biblique, et c'est bien pourquoi ils invitaient à l'imitation; d'un autre côté, le grec profane contemporain pouvait exercer là un rôle modérateur plus que dans le cas des textes traduits. Nous aurons l'occasion, dans les chapitres suivants, de revenir à plusieurs reprises sur ce rapport de fréquence caractéristique.

Pour certains sémitismes, nous disposons d'un deuxième moyen de détection: les déclarations faites à cet égard par les auteurs paléochrétiens. A plusieurs reprises, surtout dans la littérature exégétique, nous voyons faire la remarque que tel phénomène linguistique est particulier au grec biblique ou est inusité en grec profane. De pareils témoignages ne doivent évidemment pas être exploités sans circonspection; mais ils peuvent être utiles, en tout cas comme argument d'appoint. Il n'en existe pas de relevé systématique ${ }^{\mathbf{1}}$; mais nous ne manquerons pas d'invoquer ceux que nous pourrions découvrir, dans la mesure où ils s'appliquent aux phénomènes à examiner.

Après avoir inventorié les sémitismes et les latinismes isolés, il nous restera à en expliquer la présence dans le Pasteur. Dans ce qui précède, nous avons déjà parlé de plusieurs raisons possibles: le texte a été écrit dans un milieu où l'on parlait plusieurs langues; il peut avoir été soumis à des conventions littéraires où certains éléments exotiques étaient de mise; il peut avoir été écrit par une personne plurilingue; il peut être une traduction. A chacune de ces raisons correspondra une situation particulière. Pouvons-nous la prévoir? C'est ce qu'il faut essayer de faire, non sans les réserves nécessaires et la claire conscience que de pareilles remarques restent forcément vagues.

Si les textes ont vu le jour dans un milieu polyglotte ou ont été écrits selon certaines conventions stylistiques, ils seront, en général, d'une aisance raisonnable dans le maniement de la langue. Ce ne sera pas par incapacité que les auteurs retomberont dans des phénomènes d'interférence: ils possèdent le jargon et font intervenir les phénomènes d'interférence de telle sorte que même les lecteurs unilingues puissent comprendre. L'interférence se limitera surtout au lexique. Dans les cas de conventions littéraires, il peut s'y ajouter des particularités stylistiques; mais, pour la syntaxe, l'interférence n'interviendra que dans une série d'expressions stéréotypées et ne deviendra pas productive.

Les traductions littérales, au contraire, donneront des entorses à la 
syntaxe sans faire acception de personne et, tant qu'on ne saura pas que ce sont des traductions, elles donneront l'illusion que l'interférence est bel et bien devenue productive. $\Pi$ en résultera souvent une certaine obscurité. Le traducteur, en effet, cherche à se plier à la langue du texte original, non à celle de ses lecteurs; et ceux-ci, à leur tour, sont prêts à montrer une tolérance remarquable parce qu'ils partagent le respect du tıaducteur pour le texte original' 1 .

Quand l'interférence est due au fait que l'auteur utilise une langue qui lui est moins familière, elle se manifeste d'une autre façon encore. L'auteur alors s'exprime avec précision dans un domaine restreint, mais avec incertitude et maladresse aussitôt qu'il le quitte; il a du mal à sentir la valeur stylistique des mots et des expressions, à manier les particules et autres finesses de la langue ${ }^{2}$; il se sert peu de mots empruntés à la langue qu'il connaît le mieux de peur de se trahir aussitôt ${ }^{2}$.

A la fin de notre étude, nous reviendrons sur ces observations pour les confronter au texte du Pasteur. Il s'agit maintenant d'examiner les différents phénomènes qui ont l'apparence de sémitismes ou de latinismes.

I. Cf Brock, Septuagint, pp I6-3r, 32 On trouve un essal pour fixer des critères du grec traduit de l'hebreu et l'araméen chez R A Martin, Syntactical evidence of Semitic sources in Greek documents, Cambndge, Massachusetts 1974, pass

2. Cf Chnstine Mohrmann, The Latın of Saint Patrzck, Dublin 196I, PP 9-12

3. Cf. Vildomec, Multilingualism, pp 96, 103, 157, 208, 213, 231 


\section{LE VERBE}

\section{LE PARFAIT}

Dans le Pasteur nous rencontrons un certain nombre de fois le parfait là où, selon les règles du grec classique, on s'attendrait à trouver plutôt

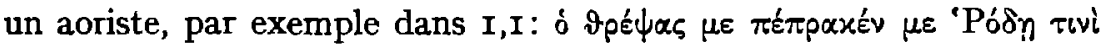

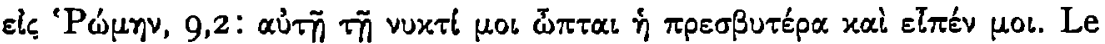
partait est employé ici de la même façon que le parfait latin. S'agit-il là d'une influence latine? ${ }^{1}$ Pour répondre à cette question, il nous faudrait nous arrêter un moment à l'histoire du parfait dans le grec post-classique.

Dès la période classique, le parfait avait subi une évolution qui le rapprochait de l'aoriste. $\mathrm{Si}$, anciennement, il indiquait un état qui était le résultat du processus exprimé par la racine du verbe, on se mit de plus en plus à l'employer comme l'aoriste, pour désigner le processus lui-même, à cette différence près que l'aoriste s'employait pour un processus accompli sans plus et le parfait pour un processus accompli qu'on désirait représenter comme concernant encore le présent. Il ne nous est plus guère possible aujourd'hui, en bien des cas, de percevoir pourquoi un auteur ancien choisit le parfait plutôt que l'aoriste. Cela ne nous autorise pas pour autant à conclure que le parfait est devenu un doublet de l'aoriste. Pour arriver à une telle conclusion il faut des preuves positives ${ }^{2}$.

Or ces preuves, nous pouvons effectivement les donner, au moins

I. Comme le présume, entre autres, L. Radermacher, Neutestamentliche Grammatik, Tübingen $1925^{2}$, p.I5o, pas spécialement pour Hermas d'ailleurs.

2. Ainsi, a bon droit, K. L. McKay, The use of the ancient Greek perfect down to the second century $A . D$., Bulletin of the Institute of Classical Studies of the University of London I2, I965, Pp.(I-2I)4-5, 2 I n.I. Mais son zèle à réfuter les preuves des chercheurs qui l'ont précédé fait craindre qu'il ne fasse parfois violence aux textes. C'est ainsi qu'il considère comme fautes des graveurs de pierre les parfaits qui l'embarrassent (p.8), ou qu'il choisit les lectiones faciliores (p.3, cf. p. I 5). 
pour le grec post-classique. Pour ce faire, il faut distinguer entre un contexte narratif et un contexte non narratif, disons un contexte de constatation. Dans le premier cas, le présent des faits rapportés est évoqué comme se situant avant le présent du narrateur; dans le second cas, ce présent coincide avec celui du narrateur ${ }^{1}$. Or, l'essentiel est qu'en grec classique, pour autant que nous sachions, le parfait ne s'emploie pas dans un contexte narratif ${ }^{2}$. Le parfait se réfère à un état dans le présent et, bien qu'a priori on ne puisse en inférer qu'il ne peut donc se présenter dans un contexte narratif, qui se situe forcément dans le passé du narrateur, c'est bien ce qui se produit en fait. Il n'a pas d'emploi en ce cas: l'aoriste sert à désigner des actions accomplies purement et simplement, le plus-que-parfait des actions qui, par leurs conséquences, continuent à exister dans le présent du récit. Or, lorsque, dans des textes post-classiques, nous trouvons des verbes au parfait dans un contexte manifestement narratif, c'est la preuve que le parfait a pris une valeur d'aoriste.

On trouve en fait des parfaits de ce genre. Ils sont rares dans les textes littéraires, où la norme attique, même chez les auteurs non atticistes, continue à être respectée à cet égard ${ }^{3}$. On trouve pourtant

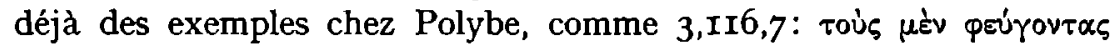

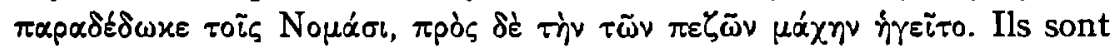
plus nombreux dans les textes sans prétentions stylistiques, comme les papyrus non-littéraires et la Bible grecque. On en trouve déjà des

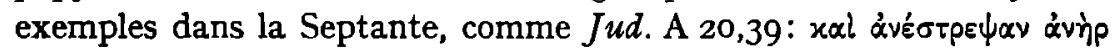

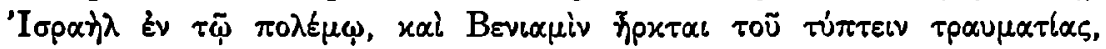

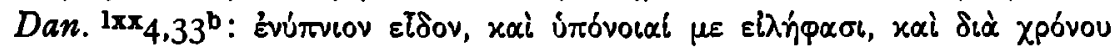

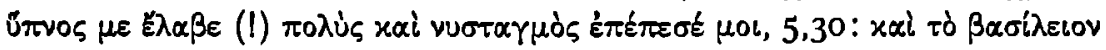

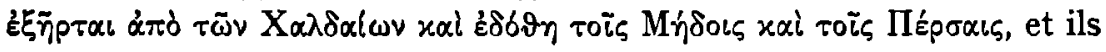
deviennent de plus en plus fréquents dans le Nouveau Testament, mais surtout dans les apocryphes.

Dans un contexte narratif, le parfait peut donc prendre une valeur d'aoriste. Ce résultat obtenu, nous pouvons faire un pas de plus. Il

I Sur cette matière voir l'étude de $\mathrm{H}$. Weinrich, Tempus, Stuttgart 1971 ${ }^{2}$, pass.

2. Cf. R. Kühner-B. Gerth, Ausführliche Grammatik der griechischen Sprache, II I, Hannover-Leipzig 18989, p.I67; J. Wackernagel, Vorlesungen über Syntax, I, Basel I9262, p. I7o; SD II, p.287; Weinrich, Tempus, pp.289-29o.

3. P. Chantraine, Histoire du parjait grec, Paris 1927, p. 214.

4. Par exemple Mt. 13.46; A poc. 5.7; 7,14; 8,5; 19,3; Apoc. Mos. I; I Hen. 13.3; 21,2-3.7; 22,5;24,4;26,2;32,1; I07.I; Test. Abr. A 6.16.20; Jos. et As. 2, I; 9, I; Test. Jud. 12,4; Test. Gad 2,3; Test. Beni. 1,3; Par. Jer. 3,16; 7, 1 7; Vit. Proph. Is. 3; Jer. 7.13; Ez. 19; Test. Job 7,1.3; 16,4.5;18,2.8; 28,4; 40,6; $44,2.5 ; 46.9 ; 50,2 ;$ Ev. Petri 23.31. 
est extrêmement improbable qu'un parfait indiquant un état dans le présent soit devenu un parfait tel qu'il fonctionne dans un contexte narratif, sans passer par une phase intermédiaire. Il est quasi certain que cet emploi a été préparé par une adaptation du parfait à l'aoriste dans un contexte de constatation. Cela confirme l'idée que, dans des textes post-classiques et peut-être déjà dans des textes classiques tardifs où s'énonce une constatation, pour les passages où le choix d'un parfait plutôt qu'un aoriste ne s'expliquait pas d'après les règles classiques, nous pouvons parler en fait d'un parfait aoristique. De tels passages ont été inventoriés depuis longtemps dans toutes sortes d'études', et il est superflu d'en citer ici de nouveau des exemples. Une chose est certaine, c'est que, si notre raisonnement est juste, le parfait aoristique est employé si tôt et si fréquemment qu'il n'est plus nécessaire d'envisager une influence latine.

Peut-être avons-nous suggéré dans ce qui précède que le parfait, dans sa valeur traditionnelle, tomba en désuétude. Ce serait là une erreur. Vers les débuts de notre èıe, la situation, à notre avis, est plutôt la suivante: dans la plupart des cas, le parfait est conforme aux règles du grec classique; dans des cas moins nombreux, il peut à la rigueur s'expliquer d'après les normes classiques et, dans une minorité de cas, il a pris une valeur purement aoristique.

La langue du Pasteur correspond exactement à ce tableau. Dans la grande majorité des cas les parfaits répondent aux règles classiques.

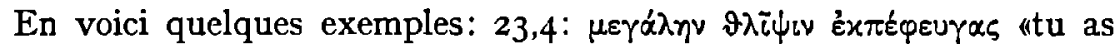

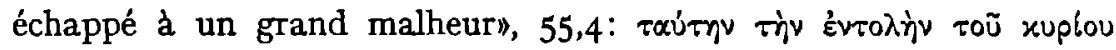

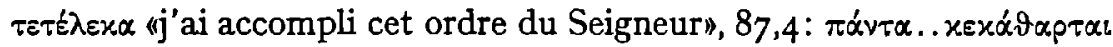

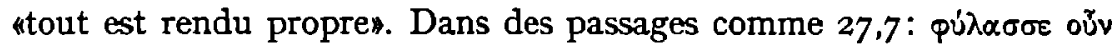

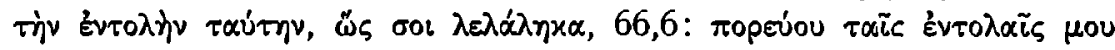

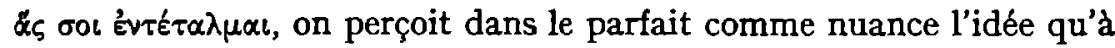
partir du moment où ils ont été donnés, les commandements restent définitivement valables. Un bel exemple de l'emploi fonctionnel d'un

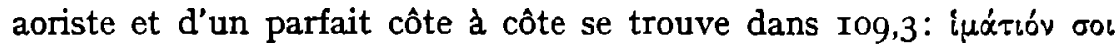

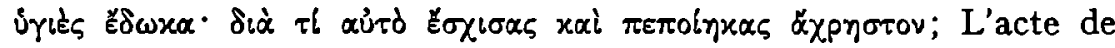
déchirer est un événement qui se produit dans l'instant, mais le fait d'être inutilisable est un état permanent.

I. Par exemple A P M Meuwese, De rerum gestarum divi August versione graeca, Buscoduci 1920, pp 87-92, N Turner, A grammar of New Testament Greek, III, Syntax, Edinburgh 1963, pp 69-7I; B G Mandilaras, The verb in the Greek non-literary papyr, Athens 1973, P 226; A D Papanikolaou, ChartonStudien, Gottingen 1973, pp 72-74. 
Hermas connaît, en outre, le parfait aoristique. Les passages que nous avons cités au début de cette analyse en étaient des exemples. Il est intéressant aussi de comparer les deux passages exactement parallèles,

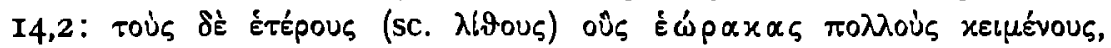

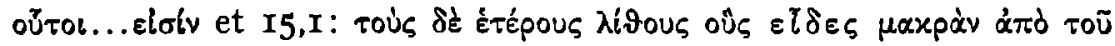

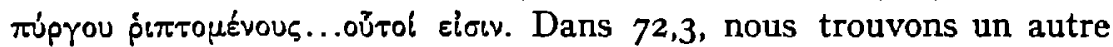
bon exemple d'emploi mêlé dans le cas de participes: oi $\pi \iota \sigma \tau \varepsilon u ́ \sigma \alpha v \tau \varepsilon \varsigma$

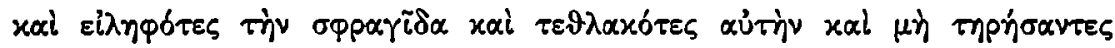
ípin. Signalons aussi que le Codex Athoüs, qui a tendance à donner au grec du Pasteur un tour plus classique ${ }^{1}$, met à plusieurs reprises un aoriste là où le Codex Sinaiticus ou le Michigan Codex a un parfait²,

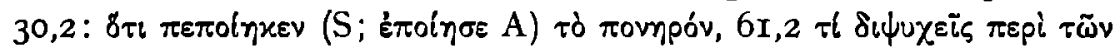

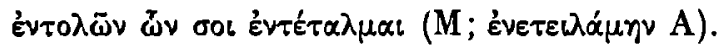

Que l'évolution du parfait dans le sens d'un aoriste se soit faite indépendamment du latin, le grec du Pasteur nous porte aussi à le penser. Hermas utilise, en effet, le parfait aoristique non seulement là où le latin utiliserait un parfait - nous en avons déjà cité des exemples-, mais aussi là où ce serait impossible en latin, à savoir pour exprimer

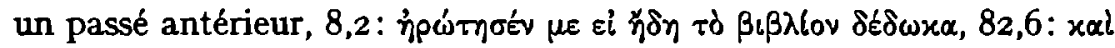

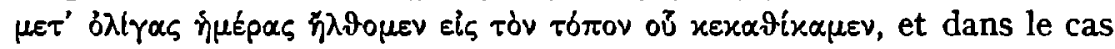

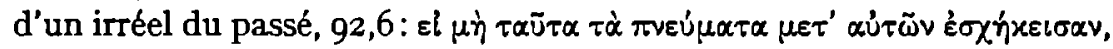

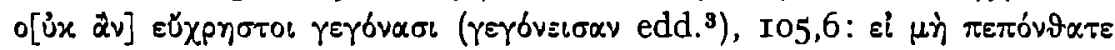

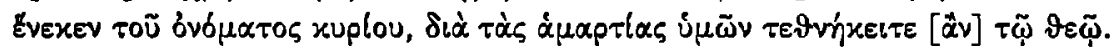
Nous croyons donc pouvoir conclure en fin de compte que ce n'est pas l'influence du latin qui a donné naissance au parfait avec valeur d'aoriste classique, que ce soit dans le grec post-classique en général ou chez Hermas en particulier.

\section{LE PLUS-QUE-PARFAIT}

Ce que nous avons remarqué en grec post-classique au sujet du parfait s'applique aussi au plus-que-parfait: dans la plupart des cas, il s'emploie selon les règles du grec classique; à certains autres endroits, il peut à la rigueur s'expliquer selon ces règles et, à d'autres endroits

I. Voir supra pp. I 5 et I6-I 7.

2. Outre les passages cités, il y a aussi 8,$2 ; 9,2 ; 69.5$.

3. L'émendation est superflue, voir A. N. Jannaris, $A n$ historical Greek grammar, London I 897, p.439; W. J. Aerts, Periphrastica, Amsterdam 1965, p.180. C'est a bon droit que Joly et Whittaker n'ont pas donné suite a la suggestion de

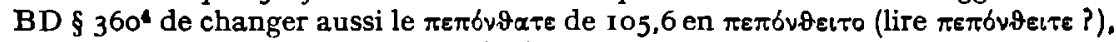
à l'exemple du үøүóveı $\sigma \alpha v$ de 92,6 (qui résulte déjà d'une émendation). 
encore, la valeur classique a manifestement disparu. L'évolution s'est faite parallèlement à celle du parfait. Ici aussi, on a supposé une influence latine ${ }^{1}$, et apparemment avec des raisons plus valables. Car, alors que le parfait se mit à partager sa valeur aoristique avec le parfait latin, le plus-que-parfait donna l'impression de se mettre à partager avec le plus-que-parfait latin non seulement sa valeur aoristique, mais aussi l'expression du passé antérieur. Cette impression était cependant erronée. De même que le parfait indiquait une action accomplie dont les conséquences persistaient dans le présent, le plus-que-partait en faisait autant par rapport au passé. En réalité, il se référait donc à un passé, antérieur à un point donné dans le passé; en d'autres termes: le plus-que-parfait grec classique, lui aussi, impliquait une antériorité dans le passé. Mais c'était un phénomène concomitant dans l'expression des conséquences d'une action. Or, lorsque plus tard la valeur classique du plus-que-parfait s'affaiblit ou disparut, l'élément d'antériorité dans le passé resta pour ainsi dire isolé et ne pouvait donc manquer d'attirer l'attention. Mais ce n'était pas nouveau.

De même que pour le parfait, l'ancienneté de l'évolution plaide contre une influence latine. Cette évolution a été moins étudiée que celle du parfait. C'est pourquoi nous citons quelques exemples anciens. Xénophon, $A n \cdot 5,4,18$, à propos d'une défaite des Grecs, raconte

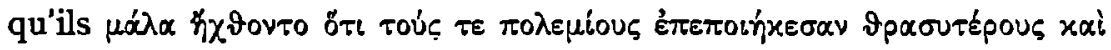

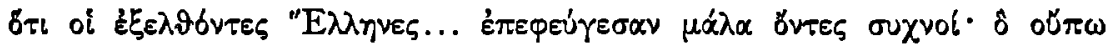

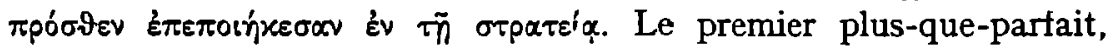
ह่ $\pi \varepsilon \pi 0 เ \hat{n} \varkappa \varepsilon \sigma \alpha v$, s'explique facilement à partir du grec classique: l'audace accrue des ennemis est un facteur dont ils devront tenir compte

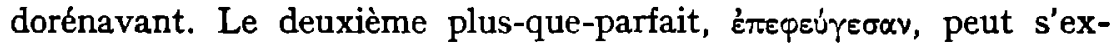
pliquer comme une manière d'insister sur leur responsabilité: à partir de maintenant ils avaient une fuite à leur nom ${ }^{2}$. Mais on ne voit guère

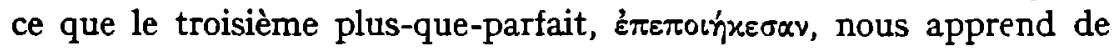
plus que ne l'aurait fait un aoriste. On peut penser ici qu'il s'agit d'un cas de persévération: les plus-que-parfaits qui précèdent immédiatement entraînent l'auteur à continuer à employer ce temps alors que le sens ne l'exige plus. Mais cette explication ne tient plus pour des

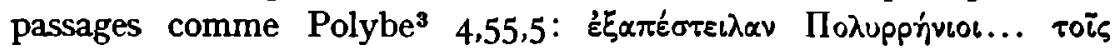

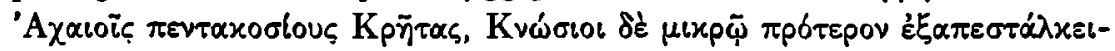

1. Par exemple J. Hering, Lateinisches bei Appian, Weida i. Thür. 1935, pp. 59-66; cf. Meuwese, De rerum gestarum divi A ugusti versione graeca, Pp.92-94.

2. Chantraine, Parfant, Pp.I64-I65.

3. Cf. J.-A. de Foucault, Recherches sur la langue et le style de Polybe, Paris $197^{2}$, P.I 43. 


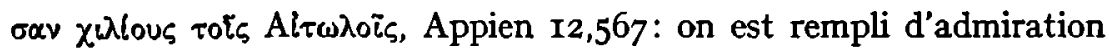

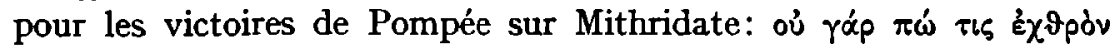

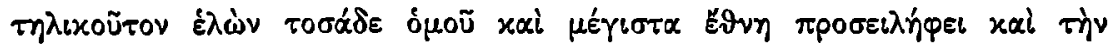

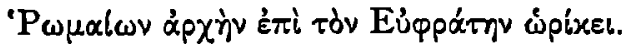

A propos de l'usage dans le Pasteur, Blass-Debrunner remarquent: "Hermas setzt das Ppf. in Nachahmung des Lat. oft für Vorvergange-

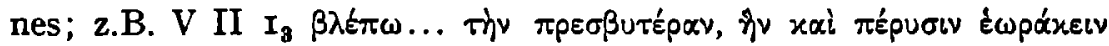

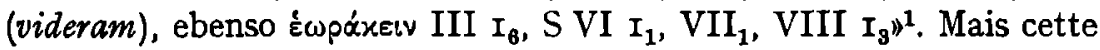
remarque est contestable. L' rimitation du latin n'est pas prouvée; comme nous l'avons montré plus haut, il ne va pas de soi qu'on puisse considérer comme un latinisme le parfait post-classique, qui a cessé d'indiquer la permanence des effets d'une action passée. "Souvent» est chose relative; en tout cas, sur un total de six passages, cinq sont

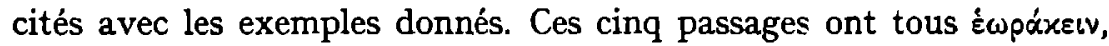

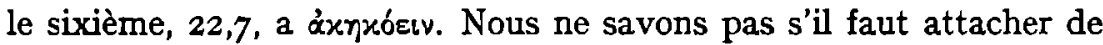
l'importance au fait que ce soient précisément ces formes-là qui aient été utilisées ${ }^{2}$; en tout cas, pour des actions qui se situent dans un passé antérieur, Hermas emploie généralement, c'est-à-dire plus de 30 fois, et pour toutes sortes de verbes, l'aoriste, comme c'était l'usage depuis

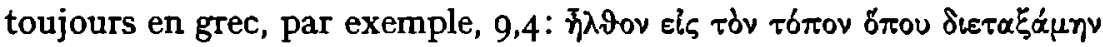

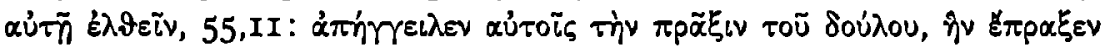

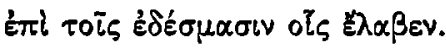

Outre les sept passages que nous venons de citer, le plus-que-parfait se présente encore ${ }_{5} 5$ fois chez Hermas $^{3}$, et, dans tous ces cas, il est

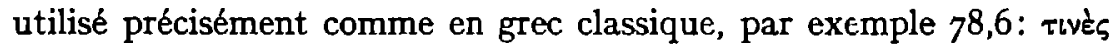

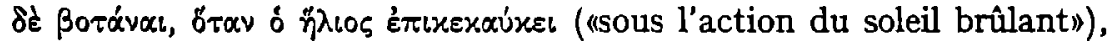

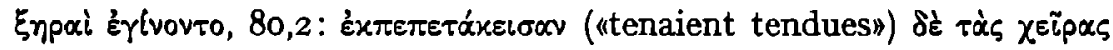

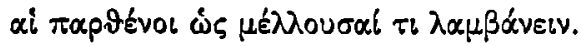

\section{L'ABSENCE D'OPTATIFS}

Dans les manuscrits du Pasteur, on rencontre cinq fois un optatif:

I. BD $\S 347^{2}$. De même Coleborne, Linguistic approach, pp.372, 444, 592; G. Mussies, The morphology of koine Greek as used in the Apocalypse of St. John, Leiden 197 I, p.277.

2. Turner, Syntax, p.84, remarque que the resultative pf. was becoming lifeless, fossilized in a few verbs only". Parmi ceux-ci les parfaits de ópãv et de dxxoúrı. Voir aussi Mandilaras, Papyri, pp.218, 226-227.

3. 54,$2 ; 67,2 ; 78,6 ; 79,3$ bis.5; 80,$2 ; 81,1 ; 82,7 ; 84,3$ bis; 88,$5 ; 96,6 b$ is; 105,6 . 


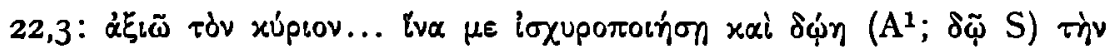

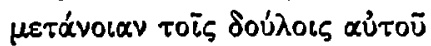

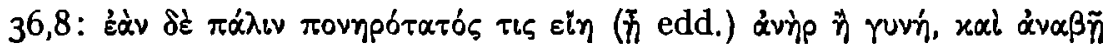

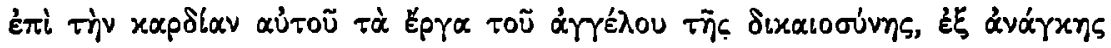

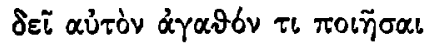

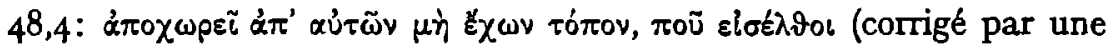
autre main en $\left.\varepsilon i \sigma \varepsilon \lambda \eta_{n}\right)$

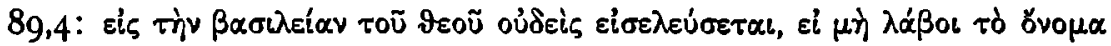

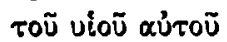

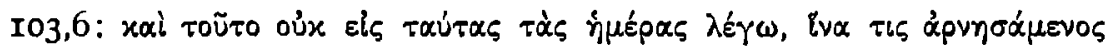

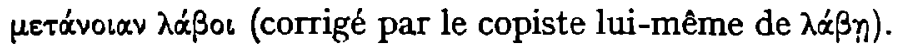

On peut se demander combien de ces passages remontent à Hermas. Quatre de ces optatifs se trouvent dans les parties du Pasteur que seul le Codex Athoiis nous a conservées et le cinquième passage 22,3 comporte chez les autres témoins le subjonctif au lieu de l'optatif. Il faudra donc avoir une grande confiance dans la qualité de $\mathrm{A}$ pour croire que les optatifs sont d'origine. Cette confiance ne va pas sans dire. Tout d'abord, en deux des cinq passages, 48,4 et ro3,6, A montre lui-même une certaine hésitation. Ensuite, à l'époque où le Codex Athoüs fut écrit, il n'y avait plus de différence depuis longtemps entre la prononci-

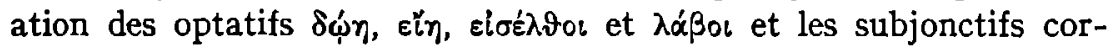

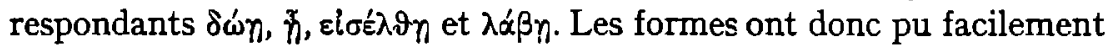
se substituer les unes aux autres au cours de la transmission. Et enfin, le Codex Athoüs se caractérise par de petites retouches données au texte pour lui donner un air plus classique et l'optatif était un excellent moyen de le faire.

Mais il est vrai qu'en revanche - nous en parlerons plus loin - on continue à trouver des optatifs dans les textes post-classiques, même pour les formes phonétiquement différentes des subjonctifs correspondants; souvent, en pareil cas, les deux s'emploient indifféremment. Dès lors, les optatifs du Pasteur que nous venons de citer ont des chances sérieuses d'être d'origine ${ }^{2}$.

Mais cinq optatifs dans un ouvrage qui a les dimensions du Pasteur, c'est peu, et, à plusieurs reprises, on y a vu le signe qu'Hermas connaissait mal le grec et se sentait plus à l'aise dans une langue qui ne

I. Ainsi chez les éditeurs. Mais d'après la transcription de K. Lake, Facsimiles of the Athos fragments of the Shepherd of Hermas, Oxford 1907, A a le subjonctif $\delta$ ín. Cf. Reinhold, Quaestiones grammaticae, pp.90-9I; BD $§ 95^{2}$.

2. Les éditeurs ne gardent en général l'optatif que dans 89,4 . Chez Joly et Whittaker les variantes dans 48,4 et I03,6 ne sont même pas enregistrées. 
disposait pas d'une catégorie comme l'optatif grec: une langue sémitique ou le latin ${ }^{1}$. C'est là une idée acceptable dans la mesure où on juge la langue du Pasteur d'après les critères du grec attique. Mais si on la compare au grec en usage à l'époque d'Hermas, on voit les choses tout autrement.

En grec post-classique, l'optatif se perd de plus en plus'2. C'est dans l'expression du souhait qu'll résiste le mieux; les autres fonctions passent à différents autres moyens d'expression, substitutions ou périphrases. A partir du Ier siècle avant J.-C. son emploi connaît une recrudescence sous l'influence du mouvement atticiste, influence qui s'exerce surtout sur les textes littéraires, mais qui atteint aussi, par exemple, la langue des papyrus non-littéraires. Mais 1 ne s'agit là que d'une floraison artificielle. $A$ voir que les fautes contre les règles attiques sont nombreuses, même chez les Atticistes, qu'on se limite souvent à des formules stéréotypées et que des formes d'expression concurrentes coexustent, on s'aperçoit que l'emploı de l'optatif n'était plus soutenu par un sens vivant de la langue.

Cette histoire de l'optatif se reflète dans le grec de la Bible ${ }^{3}$. Dans la Septante, ce mode s'emploie encore régulièrement pour exprimer un vœu; mais, à part cela, il est rare, sauf dans le Quatrième livre des Maccabées, dont on sait les tendances littéraires. Dans le Nouveau Testament, il fait complètement défaut chez Matthieu, chez Jean (évangile et épîtres) et dans l'A pocalypse, et on ne le rencontre qu'une seule fois chez Marc et dans l'Épitre aux Hébreux. La grande majorité des optatifs se rencontre chez Luc (II fois dans son évangile, I7 fois dans les Actes) et chez Paul (3I fois), mais la plupart du temps, il s'agit là aussi de formules stéréotypées. Cette régression de l'optatif

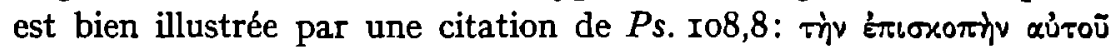

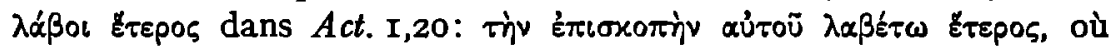
l'optatif de la Septante est remplacé par un impératif.

I Une langue sémitique selon Zahn, Hirt, pp 495-496 (cf supra pp 9-1o Le latın selon Mohrmann, Origines, pp 75-76, G J M Bartelınk, Lexicologischsemantische studie over de taal van de Apos'olische Vaders, Utrecht [1952], p 66, Giet, Les Pasteurs, p 284 (si l'on peut en croire ces auteurs, Hermas n'emploie pas d'optatif du tout) Dibelius, Hivt, p 424 et Demaray, Hermas, p 76, au contraure, estıment superflu de supposer une influence étrangère

2. Vour, entre autres, A Mellet, Aperf̧u d'une historre de la langue grecque, Pans 19657, pp 289-294, G Anlauf, Standard late Greek oder Attzzismus? Eine Studie zum Optativgebrauch im nachklassischen Griechisch, Koln 1960, pass: A Debrunner-A Scherer, Geschichte der griechischen Sprache, II, Berlun 1969", Pp II7-122, De Foucault, Polybe, pp I45-I 55, Mandilaras, Papyri, Pp 271-287 3 Voir Turner, Syntax, Pp I I9-133, Anlauf, Optatzvgebrauch, pp 75-94, Reinhold, Quaestiones grammaticae, PP Iog-II 3 
A la lumière de ces données, il n'y a aucune raison de supposer que l'emploi parcimonieux qu'Hermas fait de l'optatif soit dû à l'influence de langues étrangères. Cet usage est parfaitement conforme au grec de l'époque. Il nous faut insister sur les points suivants:

I. selon les règles attiques, la construction d'éáv avec l'optatif, comme dans 36,8 , est impossible, et l'optatif après une proposition principale avec un temps primaire, comme dans $48,4,89,4$ et 103,6 , est pour le moins inhabituel. Mais en grec post-classique cela l'est moins ${ }^{1}$; on peut même citer des parallèles pour éáv + l'optatif².

2. l'emploi de l'optatif post-classique se distingue aussi par le flottement. Constamment on trouve un mélange d'optatifs et de subjonctifs ${ }^{3}$. C'est ainsi que, dans 22,3 et 36,8 , Hermas emploie indistinctement l'un après l'autre un subjonctif et un optatif et conclut le chapitre où

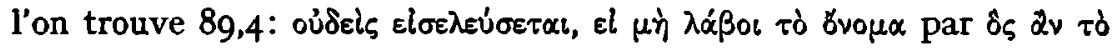

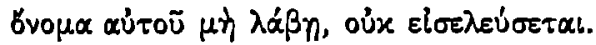

3. Hermas fait un usage abondant des moyens d'expression qui remplacent l'optatif en grec post-classique ; ainsi, par exemple, dans

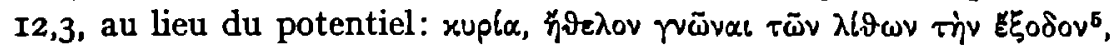

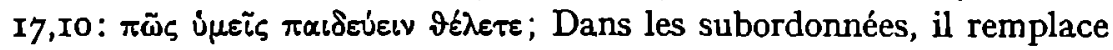
l'optatif, qui, même en attique, n'était pas obligatoire dans tous les cas, par l'indicatif ou le subjonctif. L'optatif de souhait ne se rencontre pas, mais il n'est pas non plus remplacé, étant donné qu'aucun souhait n'est formulé dans le Pasteur.

4. le petit nombre d'optatifs dans un ouvrage qui a les dimensions du Pasteur n'est pas chose anormale à cette époque. Nous avons déjà donné quelques chiffres pour le Nouveau Testament; pour les Pères Apostoliques signalons, par exemple, que la Didachè, l'Epitre du Ps. Barnabé et la Deuxiéme Epitre de Clément n'ont chacun qu'un seul optatif*.

Enfin, il est peut-être intéressant de signaler que la conclusion que nous tirons pour le Pasteur, d'autres l'ont tirée pour des textes qu'on s'attendrait beaucoup plus à voir touchés par l'interférence, c'est-àdire les documents romains officiels rédigés en grec jusqu'a l'époque

I. Voir Radermacher, Grammatik, pp.I63-I64; SD II, pp.331-333.

2. Voir Radermacher, Grammatik, p.200; Anlauf, Optativgebrauch, PP.9, III, I18, I19, 141, 146-147, 156.

3. Cf. Anlauf, Optativgebrauch, pp.74-75.

4. Voir Debrunner-Scherer, Geschichte, II, Pp.rig-r20; Anlauf, Optativgebrauch, pass.; cf. aussi Adrien 98.

5. Cf. supra pp.25 et 27.

6. Did. 5,$2 ;$ Ps. Barn. 21,5; 2 Clem. 12,3. 
d'Auguste inclusivement. La rareté de l'optatif fut, ici aussi, attribuée à une influence étrangère, celle du latin en l'occurrence; mais c'était à une époque où l'on n'avait pas encore pu se faire une idée très nette du caractère particulier du grec post-classique'.

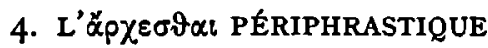

Le verbe áp $\chi \varepsilon \sigma \vartheta \alpha$, "commencer", construit avec un infinitit se présente toujours chez Hermas avec un sens plus ou moins affaibli². Bien que, formellement, il indique le début d'une action, il ne sert pas à opposer le début à la suite, mais il inclut le suite de l'action dans la formulation. La construction n'est guère plus qu'une périphrase du verbe qui se trouve à l'infinitif. C'est ainsi que le traducteur de la version Palatine

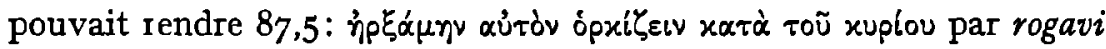

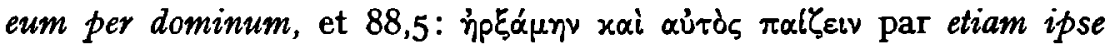
ludebam. Cette traduction est d'autant plus frappante que le latin, qui ne disposait pas d'un équivalent de l'aoriste ingressif grec, avait, d'une façon générale, plus besoin que le grec d'une périphrase avec un verbe marquant un commencement. Dans la suite, nous désignerons

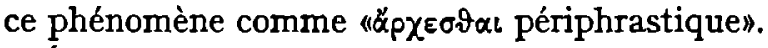

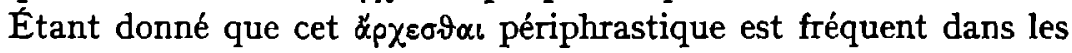
évangiles synoptiques, il a depuis longtemps attiré l'attention dans l'étude philologique du Nouveau Testament. En I898, Dalman, se fondant sur une forme d'expression analogue dans la littérature rabbinique, déclara que c'était un aramaissme ${ }^{3}$ et, depuis, on est allé répétant cette affirmation, sans fournir de nouvelles preuves. Un article d'Hunkin, de I924, qui contenait des données qui auraient dû mener à une revision de la communis opinio, n'y changea rien ${ }^{4}$. On

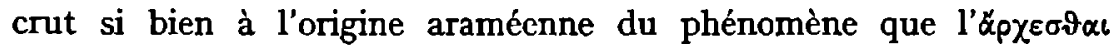
périphrastique devint de son côté un critère qui permettait d'établir les origines araméennes de certains textes de tradition grecque. C'est ainsi que Black et Wilcox s'en servent pour déceler d'éventuelles sources araméennes chez les Synoptiques et dans les Actes, et que Thackeray l'utilise pour dépister l'origine araméenne de Flavius

I. Cf. A. Thumb, Die griechische Sprache im Zeitalter des Hellenismus, Strassburg 1901, P.153.

2. I, I. $3 ; 5,2 ; 22,4.5 .7 ; 47,2 ; 55,10 ; 83,2 ; 87,5 ; 88,4$ bis.5.

3. G. Dalman, Die Worte Jesu, Leipzig I8981, 19302, Pp.21-22, 29, cf. ib. p.37o.

4. J. W. Hunkin, Pleonastic \& $\rho \chi 0 \mu \alpha$ in the New Testament, The Journal of

Theological Studies 25, 1924, Pp.390-402. 
Josèphe ${ }^{1}$. Donc, même sans compter le Pasteur, les raisons d'examiner le phénomène ne manquent pas. Dans ce qui suit, nous donnons les résultats de nos recherches, qui nous condusent à des conclusions très différentes de celles de Dalman et de ses disciples. Nous pouvons résumer de la façon suivante ce que nous espérons démontrer: il y a fort peu de raisons de parler d'un aramaisme; il y en a plus, mais pas beaucoup plus, pour parler d'un hébraisme; il y a, au contraire, de bonnes raisons de penser à un latinisme; mais toutes ces hypothèses sont superflues, car le phénomène peut s'expliquer comme une évolution interne du grec.

En araméen, nous n'avons rencontré jusqu'à présent que trois exemples convaincants de notre périphrase: deux dans l'ouvrage appelé Apocryphe de la Genèse et un autre dans un fragment d'un Testament de Lévi en araméen ${ }^{2}$. Le phénomène fait défaut dans les parties araméennes de l'Ancien Testament et dans les Targums; les trois passages du Talmud de Jérusalem, dont Dalman se 1éclamait, ne peuvent, eux non plus, servir de référence ${ }^{3}$. Dalman signalait en outre la grande fréquence de la périphrase dans la version éthiopienne du texte de base supposé araméen de I Hénoch 85-90. Mais ce sont là des preuves peu solides, car la périphrase a pu être introduite par le traducteur. C'est souvent le cas, par exemple, dans le latin des Vitae Patrum $^{4}$, qui ont été traduites du grec à peu près à la même époque que le texte éthiopien d'Hénoch.

En hebreu, le nombre de passages où l'on trouve la périphrase est un peu plus grand. Bien que le plus souvent - de nouveau à la suite de Dalman - on prétende qu'on n'en trouve pas d'exemples dans l'Ancien Testament, nous croyons pouvoir parler avec quelque raison d'une

I Black, Aramaic approach, Pp I25-I26; M Wilcox, The semitisms of Acts, Oxford 1965, pp I25-127, $\mathrm{H}$ St J. Thackeray, An unvecorded "aramaism" in Josephus, The Journal of Theological Studies 30, 1929, Pp (36I-370)364-370 L Radermacher, compte-rendu de J H Moulton, Einlentung in die Sprache des Neuen Testaments, Indogermanische Forschungen 3r, I9I2, Anzeiger P (6-1o) ro, avait déjà mis en garde contre pareille argumentation

2. IQapGn 19,18, Test Levz araméen I3 25-26 Nombreux sont ceux qui pensent d'ailleurs qu'un modèle hébreu est à la base du Testament de Lévi araméen

3 Voir Hunkin, "Pleonastic" \&p $p \circ \mu \alpha \mathrm{L}$, pp 395-399

4 Vour J B Hofmann, Bettrage zur Kenntnis des Vulgārlateins, Indogermanische Forschungen 43, 1926, pp (80-1 22)95 On sart moins que, dans les deux versions latines d'Hermas, elles aussi, on a souvent introduit un coepi pér1phrastique Les deux versions l'ont dans $55,4,63,6,74,2,75,4,86,67,109,4$, en outre $\mathrm{L}_{1} 9,35,75,4,80, \mathrm{I} 5,90,7$ et $\mathrm{L}_{2} \mathrm{I}, 2,9,5,32, \mathrm{I}, 55,5,88, \mathrm{~T}, 89,4 \mathrm{Le}$ contraire, c'est-à-dire la non-traduction de la périphrase de l'ongınal du Pasteur, est chose beaucoup plus rare, cf supra $\mathrm{P} 66$ 
périphrase dans une douzaine de cas ${ }^{1}$. Dans l'hébreu des textes de Qumran, nous n'en avons pas trouvé d'exemples; mais trois des quatre passages que Dalman signale dans la littérature rabbinique nous semblent, au contraire, convaincants ${ }^{2}$.

Mais, en latin, la fréquence est considérablement plus grande qu'en hébreu. On peut citer des centaines d'exemples, dans des textes de tous genres, à partir du $\mathrm{II}^{\mathrm{e}}$ siècle avant J.-C. ${ }^{3}$. A ce propos, il taut mentionner un aspect stylistique qui requerra aussi notre attention pour le grec. La périphrase, en effet, semble s'employer de préférence dans le style narratif et, en particulier, pour décrire des actions qui sont en liaison avec des émotions. A titre d'exemple, citons Lucrèce, 1,125-126: commemorat speciem lacrimas effundere salsas coepisse et rerum naturam expandere dictis

César, Gal. I,20,I: Diviciacus multis cum lacrimis Caesarem complexus obsecrare coepit ne quid gravius in fratrem statueret

Aulu-Gelle 1,26,7: postremo vociferari inter vapulandum incipit.

Par commodité, nous nommerons la périphrase, dans ce genre de contexte, périphrase dramatique.

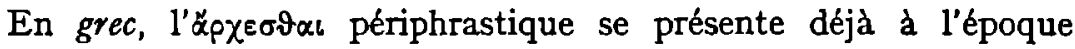
classique屯, surtout chez Xénophon, par exemple dans $C y r$. I,I,5: ö $\pi 0$ ○

1. Pour יאל hi Deut. 1,5, pour חלל hi Gen. 9,20; 41,54; Num. 25,1; Deut. 2, 24.25.3Ibis; Jud. 10,18; I6,19; 2 Regn. (LXX 4 Regn.) I 5,37; Jon. 3,4. Partout

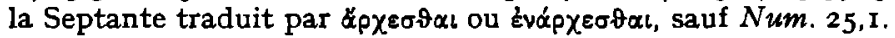

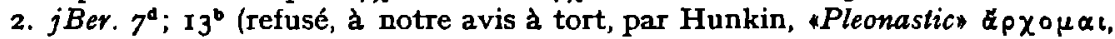
p.397); jPes. 33*. Dalman cite aussi Koh. R. 5, Io, mais ce passage ne date pas d'avant le VIIIe siecle après J.-C.

3. Voir, par exemple, Thesaurus Linguae Latinae s.vv. coepi II A I, incipio

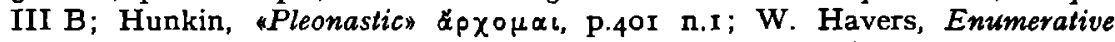
Redewerse, Indogermanische Forschungen 45, 1927, pp.(229-25I)238, 240; A. Szantyr, Lateinische Syntax und Stilistik, München 1965, p.319, cf. $2 b$. pp. 308, 313 .

4. Outre le passage cité, par exemple Xénophon, $A n .7,2,24 ; 7,6,15 ; H G ~ 2,1,32$; Cyr. 8, 1,2; plus tard, par exemple Polybe $18,21,3$; Longus 3, I8,3. Pour Flavius Josèphe voir Thackeray, Aramaism, pp.366-369, pour les Synoptiques Hunkin, "Pleonastic" \& $\rho \chi 0 \mu \alpha \iota$, pp. 391-395; J. C. Doudna, The Greek of the gospel of Mark, Philadelphia 196r, pp.52-53. Dans la Septante, un certain nombre de cas sont dus à une traduction incorrecte de l'hébreu: Gen. 2,3; 18,27; $2 \operatorname{Regn.~7,29;}$ I Par. 17,27; 2 Par. 3,3; Os. 5, I I; 6,1 1; 7,5; Mich. 6,13; Ez. 13,6. Ensuite,

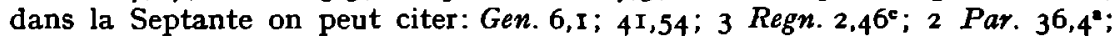
I Esdr. 2,25; 4,I.1 3; Tob. BA 8,5; S 8,5; I Macc. 9,66; 1 3,42; I 5,40; 4 Macc. 5, I 5 ; Prov. I9, Io; cf. Tabachovitz, Septuaginta, Pp. 25-29; Doudna, Mark, Pp. I I2116. Dans le Nouveau Testament: $A c t .24,2 ; 2$ Cor. 3,1, dans les apocryphes Apoc. Mos. 21; Test. Abr. A 7; Test. Naphth. 1,5; Apoc. Esdr. Pp. 24.32bis; Test. Job 28,5 et dans les textes chrétiens ultérieurs, par exemple Pass. Perp. 4, I0; 9, I.2; 10,7.II.I2.13; I I,2; 13.8; Acta Thomae 27.52. Voir aussi les passages cités supra n. I et infra p. 69 n. I et 3 . 


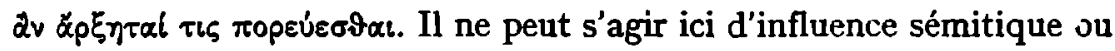
latine. Il en va autrement pour la sous-catégorie, la périphrase dramatique. Nous ne la trouvons qu'à partir du IIe siècle avant J.-C., donc à une époque où l'influence sémitique et laține ne peut être exclue a priori. Nous en trouvons des exemples chez Polybe, dans le livre de Tobie, chez les Synoptiques, Flavius Josèphe, Hermas etc. ${ }^{1}$. Mais l'influence éventuelle d'une langue étrangère sur cet usage ne peut être que celle du latin; car, dans les langues sémitiques, nous n'avons pas trouvé d'exemples de la périphrase dramatique. D'autre part, nous pouvons limiter le dépistage d'éventuelles influences étrangères au plus ancien auteur chez qui on en trouve, c'est-à-dire Polybe. En effet, si, chez lui, elle n'est pas due à une influence étrangère, on peut admettre que, dans les textes ultérieurs, cette influence n'existe pas non plus.

Chez Polybe, on ne peut exclure sans plus une éventuelle influence latine, car il a fréquenté des Romains et il a habité à Rome². Mais si nous considérons que Polybe est un auteur hautement cultivé, qui passa les trente premières années de sa vie en Grèce, qu'au II' siècle avant J.-C., le latin n'avait pas encore produit de modèles de style auxquels un historien grec pût se reférer, et que la périphrase dramatique, considérée comme phénomène d'interférence, fait partie de l'substitution, qui s'insinue beaucoup moins facilement dans une langue que la importation, alors il est difficile de croire que Polybe ait emprunté la périphrase dramatique au latin.

À cela s'ajoute l'existence d'indices suivant lesquels la périphrase dramatique s'est organiquement développée, dans le cadre du grec,

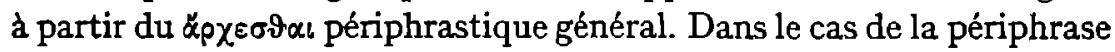
dramatique, les actions liées à des émotions sont souvent des actions

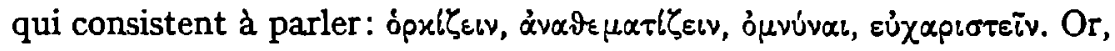

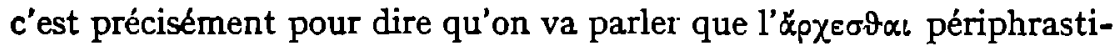
que, fût-ce sans contexte dramatique, est déjà en usage à l'époque classi-

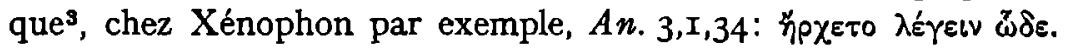

I. Polybe $28,27,4 ;$ Tob. S 3.1; 8.5; 10,3; Mt. 26,22.74;Mc. 8,32; 10,4I; II,15;

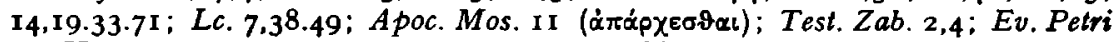
25: Hermas 1,$3 ; 5,2 ; 22,4.5 ; 55,10 ; 87,5 ; 88,4$ bis. 5 .

2. Cf. à ce sujet L. Hahn, Rom und Romanismus im griechisch-rōmischen Osten, Leipzig 1906, pp.40-50; De Foucault, Polybe, Pp.57-62.

3. Sauf les passages cités, par exemple Xénophon, $A$ n. 5,6,28; plus tard, par exemple Polybe 15,6,4; Flavius Josèphe, $A J$ 4,133; 7,289; 8,3.276.295; 9,129; I0,237; II,38.43.55.300; 13,289; Chariton I,3,I; 3,4,I3; 4,3,I; Achille Tatius 8,9,1; Longus 3,22,4; 4,19,3. Cf. E. Rodenbusch, Präsentia in perfektischer Bedeutung, Indogermanische Forschungen 28, I9I I, p.(252-285) 277. 
Nous aboutissons donc à la conclusion que l' $\alpha p \chi \varepsilon \sigma \vartheta \alpha \iota$ périphrastique sous toutes ses formes est issu du grec lui-même; ce n'est ni un sémitisme ni un latinisme. Que ce phénomène ait fort bien pu être engendré par un développement interne se trouve confirmé par le fait que des périphrases analogues se sont élaborées dans d'autres langues à d'autres époques'. Si, en grec, la périphrase dramatique fut beaucoup moins fréquente qu'en latin, c'est que le grec post-classique était prisonnier d'une tradition classique, qui ignorait cette façon de s'exprimer. L'œuvre de Flavius Josèphe nous en donne une illustration. Dans les parties où des collaborateurs formés à l'école atticiste l'ont assisté, il évite la périphrase: quand il est laissé à lui-même, elle revient au galop ${ }^{2}$. Puis, les temps qui suivirent contıibuèrent à faire disparaitre la périphrase dramatique de notre horizon: les textes qui ne se souciaient pas des prescriptions atticistes ne faisaient généralement pas partie de ceux que l'on conservait. Ce n'est donc pas par hasard si le phénomène est plus fréquent dans des textes qui ont été recopiés non pour leur style, mais, malgré leur style, pour leur contenu: Flavius Josèphe et la littérature bibliques. Enfin, en ce qui concerne Hermas: aucune raison ne nous force à considérer chez

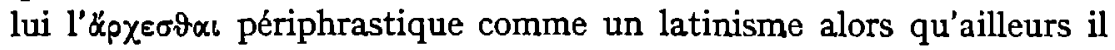
peut être pris pour du grec usuel. Dans le Pasteur non plus, ce n'est ni un sémitisme ni un latinisme.

\section{PERIPHRASE AVEC Elval + PARTICIPE PRESENT}

Les constructions périphrastiques avec elvoc font, depuis quelques décennies, l'objet d'une attention particulièré. Néanmoins, on est

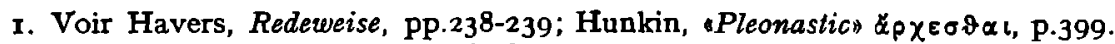
Explication psychologique chez Rodenbusch, Präsentia, p.277; Havers, Syntax, Pp.I68-I69.

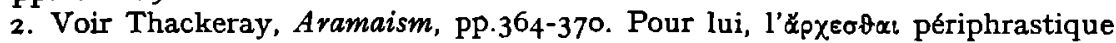
est un aramaïsme; il suffit pourtant de supposer que les collaborateurs de Flavius Josèphe considéraient la chose comme non classique. Les théories de Thackeray sur ces collaborateurs n'ont d'ailleurs pas été généralement admises, voir, par exemple, Sevenster, Greek, p. 75.

3. Tabachovitz, Septuaginta, pp.24-29, en explique la présence chez les Synoptiques par la seule imitation du style de la Septante. Il ne faut pas négliger ce facteur, mais il perd beaucoup de son importance du fait qu'on trouve la périphrase en grec profane et chez Flavius Josèphe.

4. Citons seulement G. Björck, ${ }^{\top} H \nu \delta 1 \delta \& \sigma \times \omega v$, Uppsala 1940, pass.; Aerts, Periphrastica, pass.; H. B. Rosén, ' $\mathrm{H} v \delta_{1} \delta \dot{\alpha} \sigma \times \omega \nu$ et questions apparentes, Bulletin de la Société de Linguistique de Paris 62, 1967, Pp.XXI-XXVI; L. Rydbeck, Bemerkungen zu Periphrasen mil elvai + Präsens Partizip bei 
encore loin d'être d'accord sur leur origine et leurs fonctions; et les conditions d'une communis opinio, c'est-à-dire la prospection relativement exhaustive des textes à ce sujet, sont, elles aussi, loin d'être remplies. Pour notre part, nous examinerons la périphrase avec elvxı + un participe présent dans le Pasteur. En guise d'introduction, voici quelques remarques générales.

La grande difficulté en cette matière ce sont les limites. On s'accorde en général à penser qu'on ne peut parler de périphrase partout où हiva et un participe présent se trouvent réunis; mais il n'est pas facile de distinguer entre les différents cas, étant donné que nous ne disposons pas de moyens formels pour le faire. Seule l'interprétation du sens d'une phrase peut nous apprendre de quelle catégorie relève le passage en question.

Lorsque stvaı est construit avec un participe, on peut se trouver, grosso modo, en présence de deux possibilités:

I. Elvar est une copule; le participe est adjectivé; à eux deux, ils

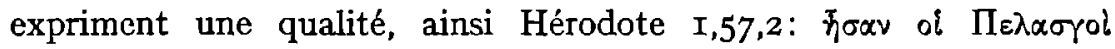

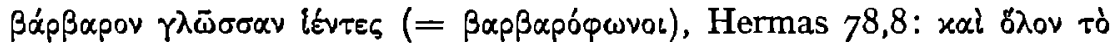

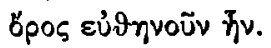

II. elvar est un verbe auxiliaire; le participe est purement verbal; à eux deux, ils expriment un état ou une action: Hérodote $9,15,4: \bar{\eta} v \delta \varepsilon$

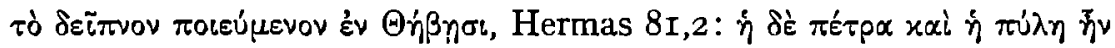

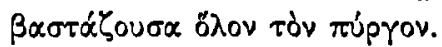

Dans le premier groupe, on peut faire la distinction entre, d'une part, des participes devenus définitivement adjectifs: dérivés d'im-

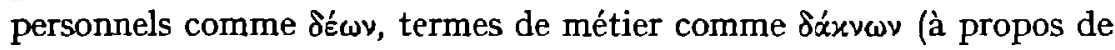
médicaments) et d'autres participes devenus termes stéréotypés,

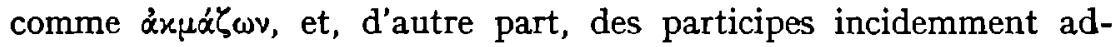
jectivés, tels qu'ils sont utilisés dans les citations du groupe $\mathrm{I}^{\mathbf{1}}$. C'est surtout la frontière entre les participes incidemment adjectivés et les participes "verbaux» qui est difficile à déterminer. En effet, posséder une qualité peut être équivalent à se trouver dans un état. Il faudrait examiner la question en détail, en faisant attention au temps d'eivou, à la diathèse du participe, au sens du verbe dont le participe dérive et au sujet de la phrase. Dans le cadre de cette étude ce n'est malheureusement pas possible. Mais nous tenons à dire que nous nous rangeons à

Herodot und in der Koine, Glotta 47, 1969, pp.r86-200; Mussies, Koine Greek, pp.302-308.

I. Björck, ${ }^{\gamma} \mathrm{H}_{v} \delta \imath \delta \alpha \sigma \times \omega v$, parle successivement de Daueradjektivierung (p.I7) et de Gelegenheitsadjektivierung (p.23). 
l'avis des auteurs qui ne reconnaissent comme périphrases que les cas du groupe II ${ }^{1}$.

L'emploi de participes adjectivés a toujours été courant en grec². Il n'y a aucune raison d'attribuer leur présence en grec biblique à l'influence d'autre langues. La périphrase authentique est moins fréquente ${ }^{3}$. En grec classique, elle sert le plus souvent à insister sur la notion que renferme le verbe (périphrase expressive), comme, par exemple, chez

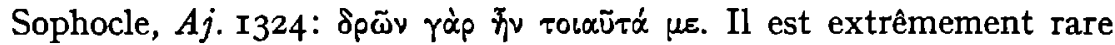
qu'elle exprime une durée (périphrase durative), comme, par exemple,

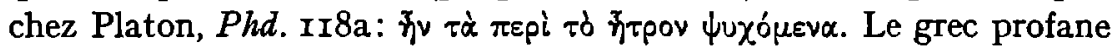
post-classique n'a pas encore été l'objet d'études poussées, mais, à en juger d'après les données disponibles, la périphrase durative reste un moyen d'expression extrêmement rare4.

La Septante, au contraire, utilise la périphrase durative à de nom-

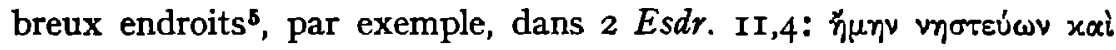

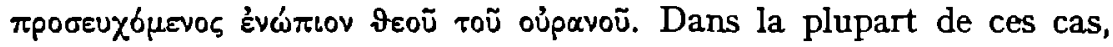
c'est la traduction de היה ou + participe; parfois l'hébreu n'a que le participe. Certains passages ne peuvent s'expliquer par un texte original hébreu, ce qui veut dire que la construction commence à acquérir droit de citéb. Dans le Nouveau Testament, elle se présente surtout chez Luc (évangile et Actes) ${ }^{7}$, ce qui peut s'expliquer comme une imitation du style de la Septante plutôt que comme une influence de l'araméen ${ }^{8}$. Après le Nouveau Testament, nous trouvons abondamment la périphrase durative dans les actes apocryphes ${ }^{\theta}$, ce qui prouve, une fois de plus, que la construction était perçue comme caractéristique du grec biblique.

Dans le Pasteur, le participe adjectivé est remarquablement fréquent $^{10}$. Au contraire, il n'y a qu'un seul exemple incontestable de

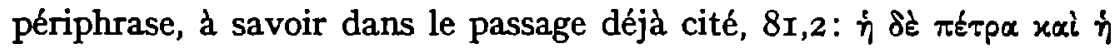

I. Cf. Björck, 'Hv $\delta\llcorner\delta\{\sigma \times \omega v$, pp. 9-10, 28-29, 63; Aerts, Periphrastica, pp.12-17.

2. Cf. Björck, "Hv $\delta \imath \delta \alpha \sigma \kappa \omega v$, pp. I7-40; Aerts, Periphrastica, pp.I 2-I 7.

3. Cf. Aerts, Periphrastica, p. 26.

4. Voir BD § 353; Aerts, Periphrastica, P.56; Rydbeck, Periphrasen, p.196; Papanikolaou, Chariton-Studien, p.79-8I.

5. Voir Tabachovitz, Septuaginta, pp.42-45; Doudna, Mark, pp.ro6-ıog; Aerts, Periphrastica, pp.62-69.

6. Cf. Aerts, Periphrastica, pp.66-67.

7. Voir Björck, 'Hv $\delta\llcorner\delta \& \alpha \sigma \times \omega v$, Pp.44-49.

8. Cf. Tabachovitz, Septuaginta, Pp.4I-47; Aerts, Periphrastica, Pp.57-58, 62. Autrement Mussies, Koine Greek, pp.305-306.

9. Voir Aerts, Periphrastica, PP.54-55.

10. Voir supra p.25. 


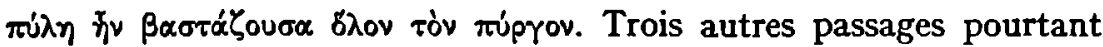
demandent qu'on s'y arrête: l'un avec l'imparfait d'elvaı et les deux autres avec le futur. Le passage avec l'imparfait, 6r,6, s'énonce ainsi :

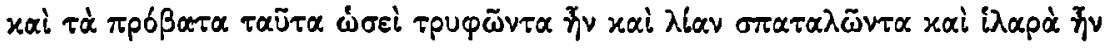

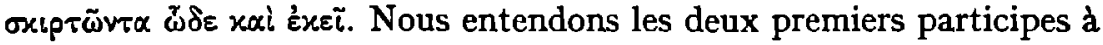
la façon d'adjectifs: «ces brebis étaient comme folâtres et très gourmandes». On peut comprendre le reste de la citation comme: «elles

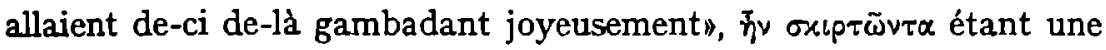

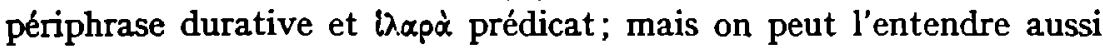
comme ket elles étaient joyeuses, à en juger par le fait qu'elles gambadaient de-ci de-là». Nous aurions tendance à préférer la premiète interprétation. L'i $\alpha$ pá prédicatil est quelque peu inhabituel' ${ }^{1}$, mais

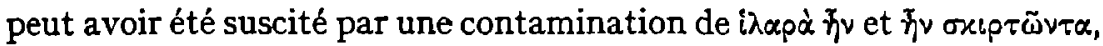

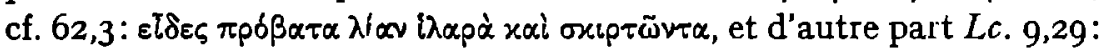

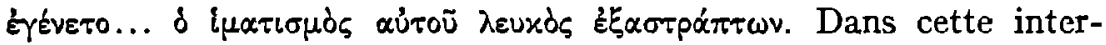
prétation, notre passage présente un cas de la loi des membres croissants:

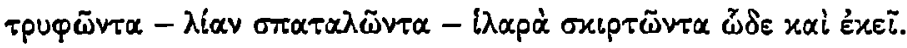

Le futur d'eivaı s'accompagne dans deux passages de participes, dont il est difficile de dire s'ils sont adjectivaux ou "verbaux»:

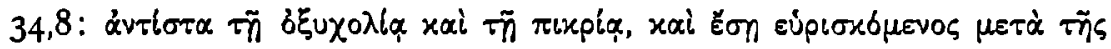

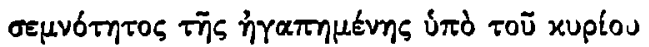

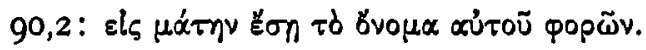

Nous inclinons à croire qu'il s'agit, dans 34,8 , d'une valeur verbale et, dans 90,2, d'une valeur adjectivale; mais, il nous semble que la frontière entre valeur adjectivale et valeur verbale est ici encore plus difficile à définir du fait que les choses qu'on dit concernent l'avenir.

En résumé, nous pouvons dire qu'Hermas connaît la périphrase Eivaı + participe présent, mais qu'il ne l'emploie que très incidemment. Quel jugement porter sur ces faits? Hermas emploie cette construction si parcimonieusement que, sous ce rapport, sa langue ne diffère pas du grec profane. Sachant d'un autre côté que le grec biblique, en définitive sous l'influence sémitique, fait un emploi beaucoup plus fréquent de cette périphrase, qu'elle a été imitée dans les livres ultérieurs de la Septante, dans le Nouveau Testament et dans les apocryphes, et qu'on connaît chez Hermas d'autres exemples de sémitismes utilisés incidemment, il y a quelque raison de parler d'un sémitisme dans son cas. Nous signalons les deux hypothèses sans pouvoir fournir un seul adjectifs de temps et leurs pareils, cf. SD II, pp.r78-179. 
argument qui rende l'une plus vraisemblable que l'autre. Ici le non liquet s'impose.

\section{Elvar eis}

Le verbe hébreu היה, comme copule, signifie à la fois «être» et "devenir». II serait naturel que la Septante rende ces sens l'un par हlvaь

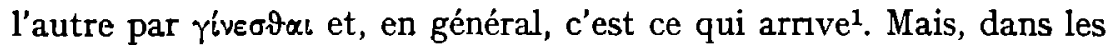
passages qui parlent de l'avenir, on utilise toujours elvo ı ${ }^{2}$. Si l'on considère que, généralement, la différence entre «ils seront» et cils deviendront" est négligeable, c'est parfaitement admissible. Dans

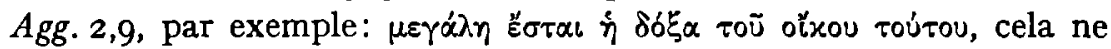

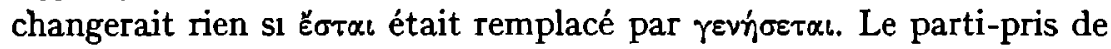
traduire toujours un mot par le même mot correspond d'allleurs à la tendance générale des auteurs de la Septante à unıformiser leur traduction.

Au lieu de la seule copule היה, l'hébreu emploie souvent la combinaison $\}$ ציה $^{3}$. Les traducteurs de la Septante ont généralement rendu ce ל היה ליה ליה littéralement: on traita même manière que le היה simple, et ? fut traduit par eic, qui en est l'équivalent à peu de chose près. C'est ainsi qu'à de nombreux endroits de la Septante on

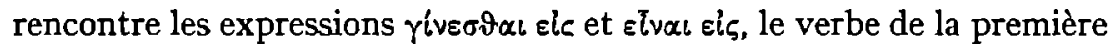
étant généralement à l'aoriste et celui de la deuxième au futur ${ }^{4}$, par

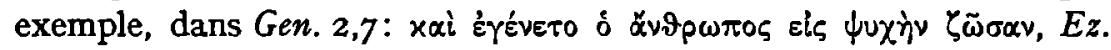

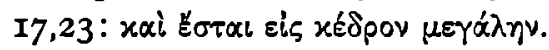

En grec, ces expressions n'étaient pas d'un usage courant. Els peut

I Cf J Bar, The semantics of biblical language, Oxford I96I, p 59 n I; Beyer, Semitische Syntax, pp 31, 64 Nous n'avons pas pu consulter l'étude annoncée de Barr sur le verbe «êttre" en hébreu et grec biblıques

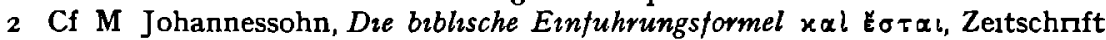
fur die alttestamentliche Wissenschaft N F I8, 1942-I943, pp (I 29-I84)137-138,

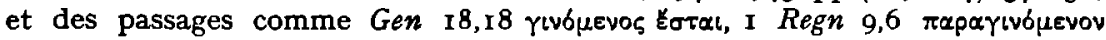

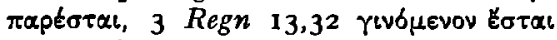

3 Le plus souvent l'expression signifie «devenir», mass pas toujours, cf par exemple $E z \quad 37,22$ היה peut avoir d'autres significations encore, volr $L$ Koehler-W Baumgartner, Hebraisches und aramaisches Lexikon zum Alten Testament, Leiden $1967 \mathrm{sq}$, $\leqslant v$ היה qal 7 Nous ne parlerons pas $1 \mathrm{cl}$ de ces significations et leur traduction éventuelle par Eivou sls Chez Hermas, elvaı elis ne se présente pas dans ces significations

4 Cf M Johannessohn, Der Gebrauch der Kasus und der Präpostionen in der Septuagınta, I, Berlin I910, pp 2-5, R Helbing, Die Kasussyntax der Verba ber den Septuaginta, Gottingen I928, pp 64.65 Voir aussi intra p 75 n 5 
s'employer pour indiquer une destination ${ }^{1}$, par exemple Hérodote

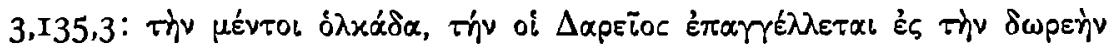
$\tau о \tau \sigma \sigma \iota \dot{\alpha} \delta \lambda_{\varphi \varepsilon \circ \tilde{\tau} \sigma \iota .}$ Par analogie, on le trouve parfois aussi accompagnant

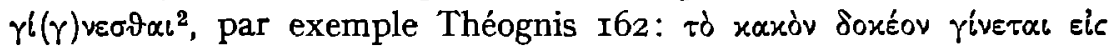

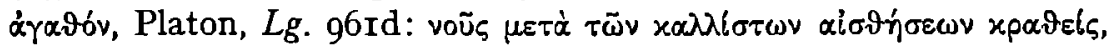

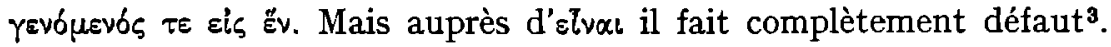
Il faut croire que le sens d'sivas, qui indique l'existence d'un état, et le sens d'elc, dans la mesure où il indique le passage à un autre état, étaient incompatibles. C'est ainsi qu'eivaı eic est un des cas relativement peu nombreux de sémitisme primaire.

On ne peut cependant prétendre que l'expression ait réellement acquis droit de cité. Il est vrai qu'on trouve plusieurs fois, dans la Septante, Eĩvos ziç là où l'hébreu, du moins dans notre texte, a seulement היה, mais le contraire, Elvab pour ליה ל, est plus fréquent ${ }^{4}$ On trouve encore l'expression surtout dans les livres traduits de la Septante (plus de roo fois) ${ }^{\mathbf{5}}$; hors de là, on ne peut citer de la Septante que Sap. 4,I9. Dans le Nouveau Testament, on ne la trouve que dans des citations de la Septante ${ }^{6}: M t .19,5=M c \cdot 10,8=\mathrm{I}$ Cor. 6, $16=$

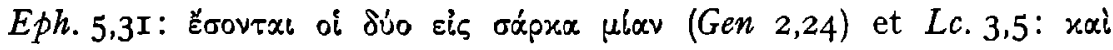

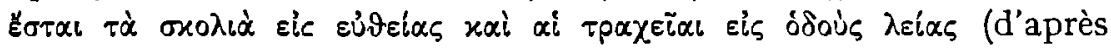
Is. 40,4). Dans les apocryphes paléotestamentaires, nous ne trouvons plus que trois cas (dans les Testaments des Douze Patrarches ${ }^{7}$ ), dans les apocryphes néotestamentaires nous n'en trouvons plus.

Dans notre texte grec d'Hermas, eivaı zis se présente une fois, dans

I Voir Radermacher, Grammatık, p 2I, Helbing, Kasussyntax, pp 6I-64, Mayser, Grammatzk, II 2, pp 362-366

2 D'autres passages chez Helbing, Kasussyntax, p 64

3 Du morns, 1 ne se présente nulle part dans le groupe de textes profanes que nous avons cités plus haut, p 53 Nous n'en avons trouvé d'exemple nı dans d'autres textes, ni non plus dans la littérature que nous avons consultée à ce sujet

4 Johannessohn, Kasus, pp 3-5

5 Gen 2,24, 9, I 5, 18, 18, 48, I 9 brs, Num 5,27, 14,3, Jud I0, 18, I 1,6 8 9, I Regn 4,9, 14,40bis, $2 \operatorname{Regn} 5,2,7,8,14,17,15,33,3 \operatorname{Regn} 1,35,2,2,9,7,4 \operatorname{Regn} 22,19$. I Par I I,2 6, I 7,7, 2 Par 6,5, I 2,8, 16,8, 2 Esdr I 5, I 4, Judth 5,2 I 24, 7, I8 27, 8,22, I Macc I,41, Ps I8, 15, 36,26, Job 27, I4, Mich 1, 2, 5, I , 7, IO I3, Joel 4, I9bis, Abd I, 18, Zach 2,9, 8, 10, 14,9, Is 4,6, 5,5bis 9, 8, 18, I0,17, 14,23 25, I7, I, 22,$23 ; 31,8,34,12,35,7$ brs $, 40,4,55,13,59,6,60,22,66,24$, Jer $5,13,7,34,9,21$, $16,4,21,9,22,5,24,9,25,11,26,19,27,13,28,2637,30,7$ I $827,31,926,32,33$, $37, \mathrm{I} 6,45,2,46, \mathrm{I} 8,49,5 \mathrm{I} 8,5 \mathrm{I}, \mathrm{I} 2, \mathrm{Bar} 2,23,4,34, \mathrm{Ez} 5, \mathrm{I} 6, \mathrm{I} 2,20, \mathrm{I} 4, \mathrm{I} 5 \mathrm{I} 6$, I 7,8 23, 20, 12 20,34,22,37, I9 22, 47, I 2, $\operatorname{Dan} \mathbf{T h}_{2,5}, 3,96$

6 Dans les passages Act 8,23, I Thess 3,5, I Joh 5,8, qu'on cite parfors, l'expression a une autre fonction

7 Test Sim 5,6, Test Levi 10,4, 16,5 


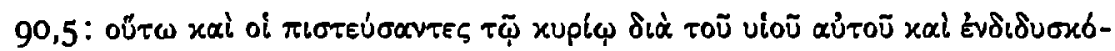

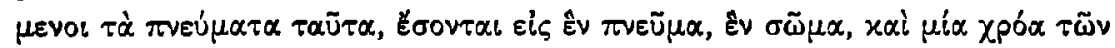
i $\mu \alpha \tau i \omega \nu \alpha \dot{\tau} \tau \tilde{\omega} v$. C'est indubitablement l'influence du style de la Septante

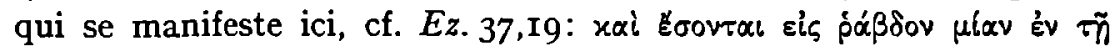

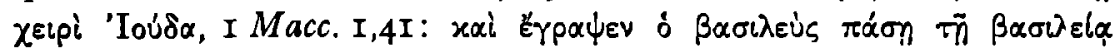

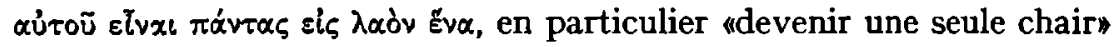
de Gen. 2,24, texte qui, comme nous venons de le voir, est cité quatre fois dans le Nouveau Testament'. Chose remarquable d'ailleurs, lorsque Hermas, dans 95,4, s'exprime en des termes semblables ${ }^{2}$, il

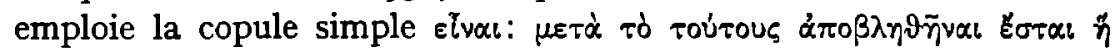

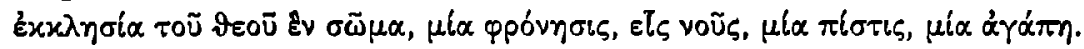

Il est possible que I08,4 comportait aussi Elval eic. Dans le texte qui

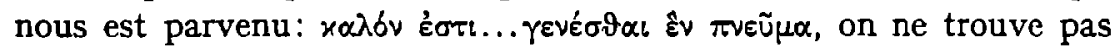
d' $\varepsilon \zeta_{\zeta}$, il est vrai, mais nous ne connaissons ce texte que comme citation chez Antiochus et souvent Antiochus cite assez librement. En revanche, les deux versions latines et la version éthiopienne comportent la préposition: dıco autem omnibus vobıs... in unum quoque spiritum fieri $\mathrm{L}_{1}$, in uno spiritu ${ }^{3}$ efficiemini $\mathrm{L}_{2}$, estote in uno spiritu $\mathrm{E}$ (traduction d'Abbadie).

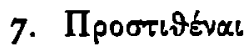

Le verbe hébreu "סי, "ajouter», peut être suivi d'un infinitif ou de $\zeta+$ infinitif, et exprime alors que l'acte de l'infinitif se continue. Les

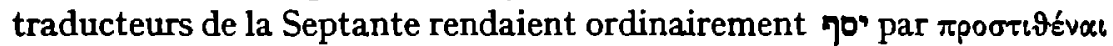

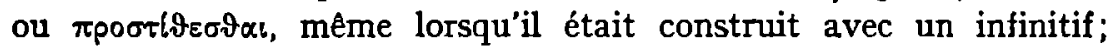

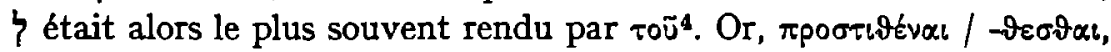

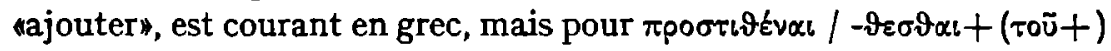
infinitif "continuer à", "de nouveau commencer à", on ne trouve pas de parallèles profanes ${ }^{5}$. C'est donc un sémitisme ou, étant donné qu'une

r. Voir aussi Daniélou, Judéo-christianisme, pp.331-334.

2. Cf. aussi 90,7; 94,4.5; 95,3; Test. Jud. 25,3 et Reiling. Hermas, pp. I 31 1-132.

3. Pour l'ablatif au lieu d'un accusatif voir Szantyr, Syntax, pp.276-278. Dans 90.5 $\mathrm{L}_{2}$ a même erunt in uno spiritu et in unum corpus.

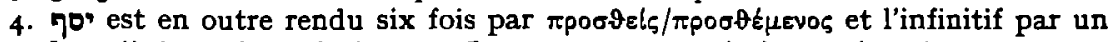
verbum finitum, formulation que Luc, entre autres, imite, mais qui ne se trouve pas chez Hermas. Pour la traduction de $\eta 0$ dans la Septante, voir aussi Helbing, Kasussyntax, Pp.300-302; Soisalon-Soininen, Intinitive, pp.23-24, 44-45, 47-48, 136, I 40, 158; pour le latin, J. H. Moulton-W. F. Howard, A grammar of New Testament Greek, II, Accidence and word-formation, Edinburgh 1919-1029, P.445; U. Rapallo, Calchi ebraici nelle antiche versioni del «Levitico», Roma 197I, p.r3o; pour le copte, Brock, Septuagint, p.35.

5. Cf. $\mathrm{BD} \& 435^{\circ}$. 
construction analogue fait défaut en araméen, un hébraïsme. Dans la Septante, il est très fréquent, Iog fois; mais, dans les textes grecs originaux, cet emploi n'a guère été repris. A part un passage du Pasteur que nous aurons à citer tout à l'heure, nous ne connaissons que

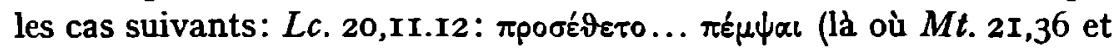

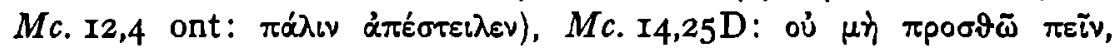

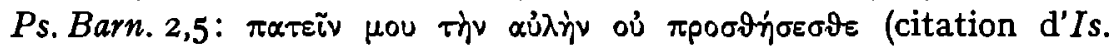
I,I2-I3), $\epsilon$ t deux variantes de manuscrits dans des apocryphes plus

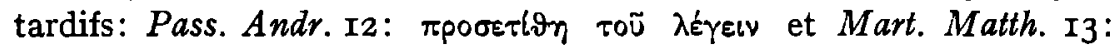

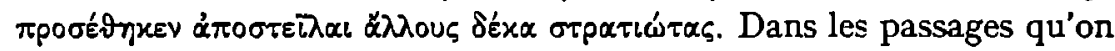
donne souvent aussi comme exemples de cette signification, Act. 12,3:

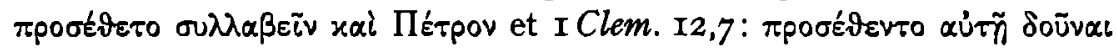
on ${ }^{2 i o v}$, le verbe pourrait cependant signifier aussi "charger de". La forme active de $\pi p o \sigma \tau \imath$ véval a déjà ce sens chez Hérodote, 5,30,6:

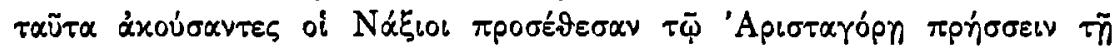

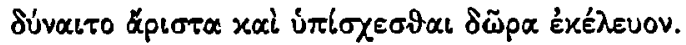

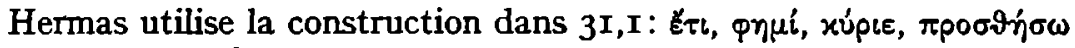

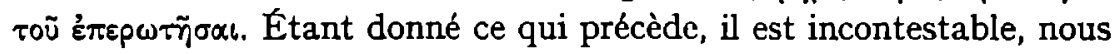
semble-t-il, qu'Hermas ait emprunté l'expression à la Septante. Il y a même différents endroits de la Septante où se retrouvent non

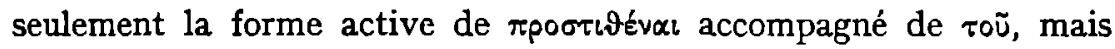

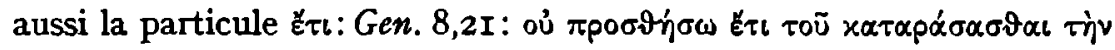

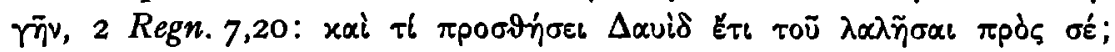

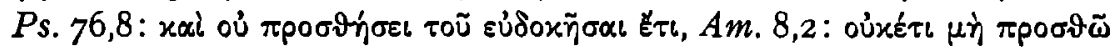

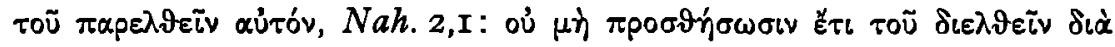
бoũ. Comme la construction n'a pas de pendant en araméen, l'emploi que nous examinons ici indiquerait qu'Hermas doit ses sémitismes à la lecture de la Septante et non à la pratique d'une langue sémitique parlée de son époque. A cet égard, il rappelle Luc qui, dans le Nouveau Testament, est le seul chez qui l'hébraisme en question se rencontre ${ }^{1}$.

\section{FORMULES AVEC DES VERBES SIGNIFIANT DIRE}

Lorsque nous citons littéralement les paroles de quelqu'un nous les annonçons par une pause, et souvent nous marquons la citation par l'intonation. Dans les textes écrits, nous nous servons de deux points et de guillemets. Il faut que les citations soient mises en évidence. Cela dérouterait complètement l'auditeur ou le lecteur s'il prenait les pa- 
roles citées pour le récit du narrateur lui-même. Pour éviter cette confusion, on se sert parfois de moyens supplémentaires pour attirer l'attention sur la modification qui intervient dans le texte ${ }^{\mathbf{1}}$. C'est ainsi que le narrateur, au lieu d'un simple "il dit», peut introduire le discours direct par une combinaison de verbes signifiant dire. Chez

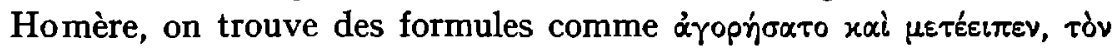

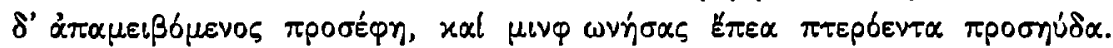
On pourrait citer aussi quantité de combinaisons utilisées en grec classique et en grec post-classique. D'un autre côté, les langues sémitiques connaissent aussi cet emploi. Dans ce qui suit, nous nous occuperons de deux formules du grec biblique qui remontent à des originaux sémitiques.

Dans l'Ancien Testament, un discours direct est souvent annoncé en ajoutant aux mots (il parle» l'expression ראל "לen disant", "disant" 2 . Dans la Scptante, cette expression est parfois négligée dans la traduction, mais, habituellement, on la rendait par le participe $\lambda \varepsilon ́ \gamma \omega v$,

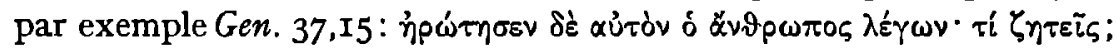
Dans les livres traduits, on trouve des centaines d'exemples de ce $\lambda \hat{\varepsilon}^{\prime} \omega_{\omega}{ }^{3}$. On ne s'étonne donc pas de voir que ce $\lambda \varepsilon^{\prime} \gamma \omega v$ est passé dans le style des livres de la littérature biblique qui ont été écrits en grec*. Dans les textes protanes, on ne le rencontre que rarement, seul Hérodote en fait un usage assez fréquent ${ }^{5}$. C'est donc un sémitisme en vertu de sa fréquence: en grec profane, cette tournure n'est pas impossible, mais elle est peu fréquente; en grec biblique, au contraire, elle est très fréquente non seulement dans les traductions, où son emploi était stimulé par le texte original sémitique, mais aussi dans les ouvrages originairement grecs, sous l'influence des traductions ${ }^{6}$.

Chez Hermas, ce $\lambda \varepsilon ́ \gamma \omega \nu$ se présente huit fois", par exemple dans

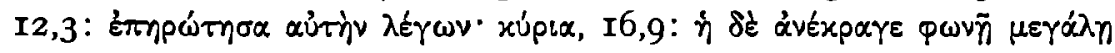

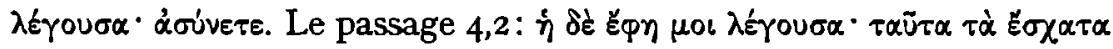

I Cf pour de tels moyens Weinrıch, Tempus, pp. I 72-1 76 .

2 Volr E Kieckers, $Z$ ur oratio recta in den indogermanischen Sprachen, I, Indogermanische Forschungen 35, I9I 5, pp (I-93) 37-40, Koehler-Baumgartner, Lexikon, $s v$. I אמר qal I.

3 Vorr Soisalon-Soininen, Infınztive, pp 68-75

4 Pour le Nouveau Testament, voir Kieckers, Oratio recta, pp 36-37

5 Voir Kieckers, Oralıo recta, PP 34-35; BD § 420

6 En réalité, la même chose vaut pour le dicens analogue en latın paléochrétıen, cf Chnstine Mohrmann, Quelques tratts caractéristıques du latın des chrétiens, Études I, P (2I-50) 49

7 I,2, 4,2;9,3, I2,3, 16,9; 30, I; 32, I; 55,4 Comme v l. 57,3 


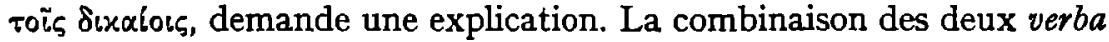

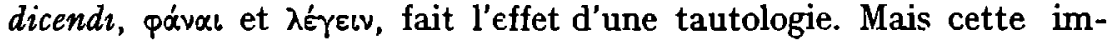

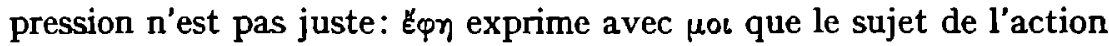
s'adresse en parole à celui qui dit je, $\lambda$ é youox annonce le texte littéral des mots. Hermas a pu trouver des modèles de cette formulation dans

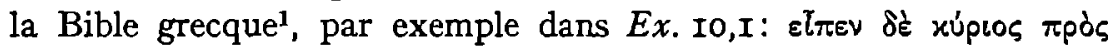

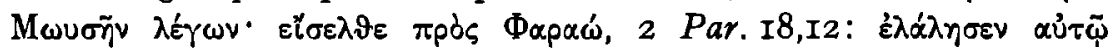

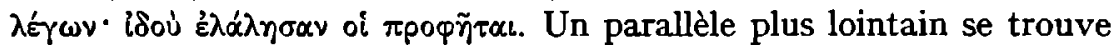

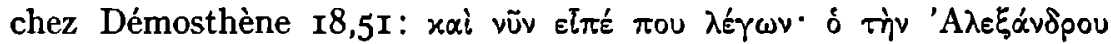

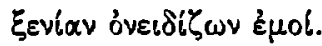

Une deuxième expression très en faveur pour introduire une citation est, dans l'Ancien Testament, רמא' parole) et parla». Dans la Septante, elle se traduit de trois manières:

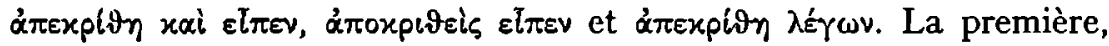
la traduction la plus littérale, est usuelle dans I-4 Rois, la deuxième dans la Genèse, la troisième est plus répandue ${ }^{2}$. Nous trouvons des expressions analogues dans des textes profanes ${ }^{3}$, Homère, Od. 15,434:

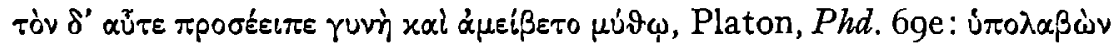

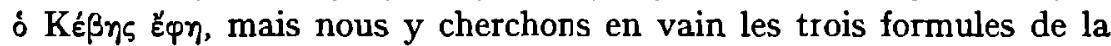
Septante. C'est pourquoi notre conclusion est que ces formules, bien qu'elles ne soient pas contraires aux normes du grec, doivent être considérées comme des sémitismes. Deux considérations étayent cette conclusion. Tout d'abord, ces formules ont été abondamment imitées dans les originaux grecs de la littérature biblique. C'est ainsi qu'on les trouve tous trois en grand nombre dans le Nouveau Testament:

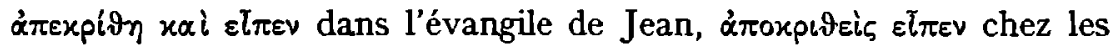

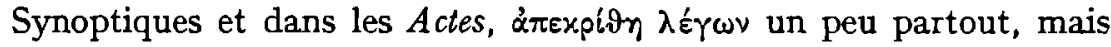
surtout chez Matthieu. La fréquence avec laquelle sont ainsi reproduites des formules très courantes dans la Septante, mais inconnues en grec profane, montre à elle seule que cet emploi était perçu comme

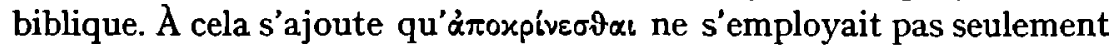
dans le sens normal de "répondre», mais aussi dans le sens sémitisant

I. Cf Kieckers, Oratio recta, p 39; Moulton-Howard, Acctdence, p 454; W. Suss, Studien zur laternischen Bibel, I, Tartu 1932, Pp I 16-II7, W Bauer, GriechischDeutsches Worterbuch zu den Schriften des Neuen Testaments und der ubrigen urchristlichen Literatur, Berlin $195^{\circ}$, sv $\lambda \alpha \lambda \in \omega$

2 Vour P Jouon, Respondit et d2xit, Biblica 13, 1932, pp (309-314) 309-312; Doudna, Mark, pp 123-126

3 Vour, par exemple, S Trenkner, Le style xoi dans le réctt attıque oral, Assen $1960^{2}$, pp 42,44

4 Voir Jouon, Respondit et dixit, Pp 313-314. 
de uprendre la parole», et cela aussi bien dans les traductions que dans les ouvrages grecs originaux ${ }^{1}$.

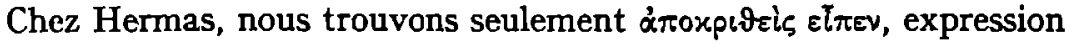
qui, chez lui, ne se présente pas moins de 24 fois ${ }^{2}$. Une chose intéressante est le temps qu'il emploie alors. Dans la Septante, où le présent historique est relativement rare parce que le texte original n'en

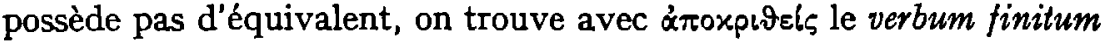
à l'aoriste, ou parfois au futur; une fois seulement, dans $\operatorname{Dan}$. 18x 7,I6,

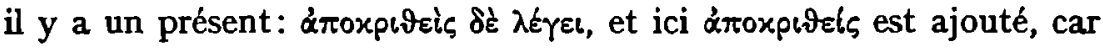
l'original araméen ne le comporte pas. Dans le Nouveau Testament, le présent historique gagne du terrain: la formule comporte ir fois le

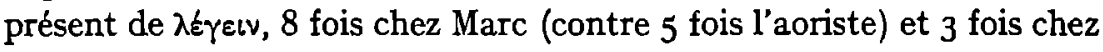
Luc (contre 29 fois l'aoriste). Or, chez Hermas, le présent a presque

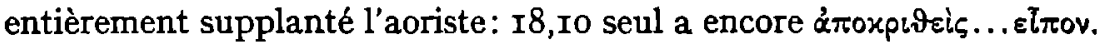
Ce fait confirme, nous semble-t-il, que les sémitismes de sa langue ne lui viennent pas d'une langue maternelle sémitique, mais qu'il les a empruntés à la Bible grecque en les adaptant à ses habitudes linguistiques, car, ailleurs aussi, il utilise volontiers le présent historique, notamment avec des verbes signifiant dire ${ }^{3}$.

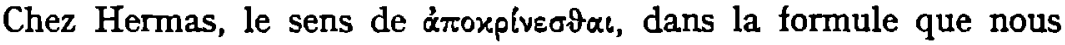
examinons ici, est le plus souvent urépondre». Dans 25.5 cependant, on peut hésiter. Le Pasteur y apparaît à Hermas et lui explique le but

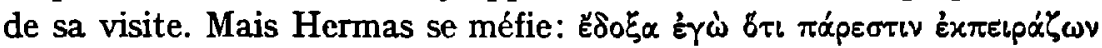

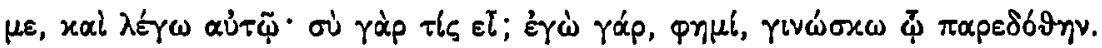

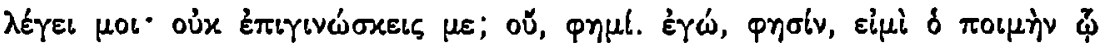

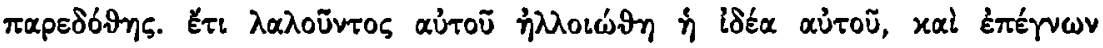

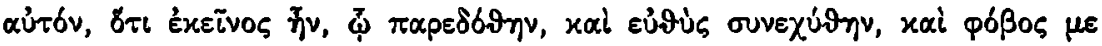

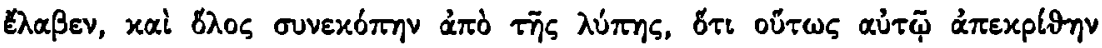

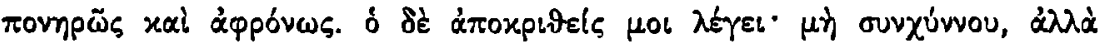

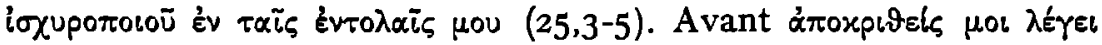
c'était le Pasteur qui avait la parole: en toute logique, il ne faut donc pas répondre. Mais l'explication ne serait-elle pas que le Pasteur "répond" à la réaction angoissée d'Hermas? C'est ce qu'indiquent aussi, au début de la phrase, les mots ó $\delta \dot{\varepsilon}$, qui s'opposent nettement à

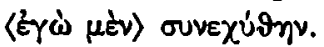

Il y a d'ailleurs un passage où, selon toute vraisemblance, $\alpha$ zoxpl-

I. Voir Bauer, Wörterbuch, s.v. ḋroxplvouaı 2.

2. I,7; I I,I ; I $2,1.3 ; 14,5.6 ; 18,2.9$ bis. $10 ; 23.3 ; 24,1 ; 25,5 ; 30,2 ; 46,5 ; 52,1 ; 57,2$; 65,$2 ; 68,7 ; 69,6 ; 77.3 ; 84,4.5 ; 88,2$.

3. Voir, par exemple, supra p.25. 


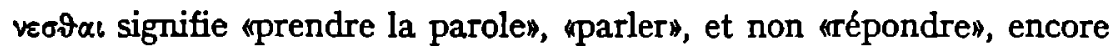

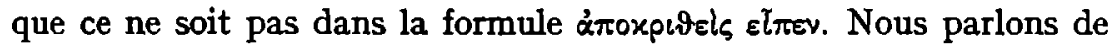
22,4. Hermas prie le Seigneur de bien vouloir achever les visions qu'il lui a données, de le fortifier et de permettre aux fidèles qui sont tombés dans le péché de se convertir. Lorsqu'ensuite il glorifie le Seigneur et

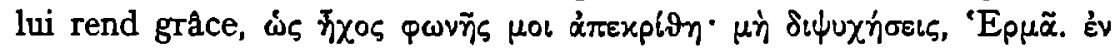

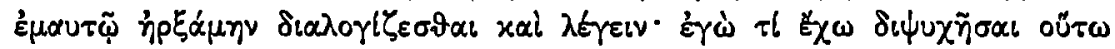

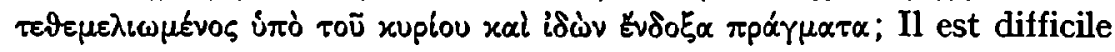
d'interpréter ici ámexpîn comme une réponse à la prière d'Hermas. Cette prière ne témoigne guère de $\delta \iota \psi u \chi \iota \alpha$, et la réaction d'Hermas montre de toute évidence qu'il ne considère pas les paroles de la "voix" comme une réponse. Plus loin aussi, dans $22,6-7$, on voit que $\mu \eta \dot{~ \delta \iota \psi u \chi \eta ் ~}$ encore dans le futur. Bref, ici le sens de $\alpha \pi \circ x p i v \in \sigma \vartheta \alpha \iota$ n'est pas tellement "répondre", mais plutôt oparler à». 


\section{PREPOSITIONS}

\section{LE Év INSTRUMENTAL}

Le év instrumental est un phénomène difficile à saisir. Son histoire n'a jamais été décrite en détail; nous sommes réduits à ce que nous oftrent les données tıès incomplètes des grammaires, dictionnaires et monographies. Dans ces publications - et c'est là une deuxième difficulté le mot instrumental n'est pas toujours pris dans le même sens. De notre côté, nous le réservons aux cas où év indique un moyen ou une cause; nous excluons donc le év modal et le év limitatif, c'est-à-dire celui qui prend la place d'un datif de manière, comme dans Sophocle,

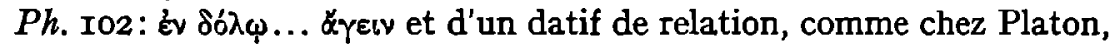

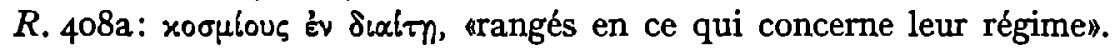
Mais alors nous tombons dans une troisième difficulté, celle que présentent toutes sortes de cas intermédiaires qu'on pourrait tout aussi bien faire entrer dans une catégorie que dans l'autre.

La question cruciale est à chaque fois: dans ce cas précis, év est-il encore local ou faut-il le comprendre comme instrumental, modal ou limitatif? Selon la réponse qu'on y donne, on pourra - pour citer seulement quelques extrêmes - faire commencer le év instrumental chez Homère ou à l'époque hellénistique, souligner son caractère sémitisant en grec biblique ou le rayer de la liste des sémitismes. La manière dont on comprend le sens en chaque cas a donc une influence décisive sur la place qu'on reconnaît au év instrumental dans l'histoire du grec. Chez Hermas aussi, on se trouve confronté à des difficultés

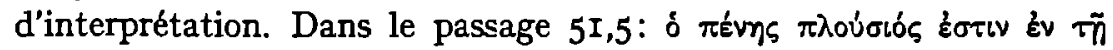

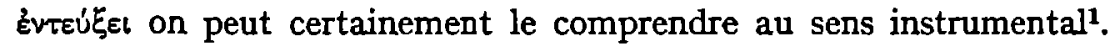
Mais, en raison du contexte, nous sommes porté à croire que le sens

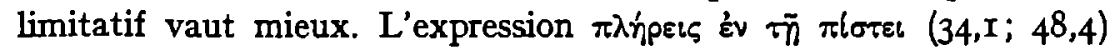

I. Ainsi J. Humbert, La disparition du datif en grec (du ror au $X \bullet$ siecle), Paris r930, P.I29. 
s'explique bien, elle aussi, dans le sens instrumental ${ }^{1}$; mais là encore nous préférons le sens limitatif, d'autant plus qu'il existe des ex-

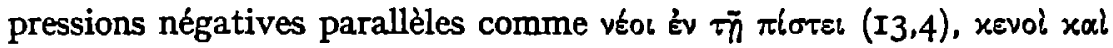

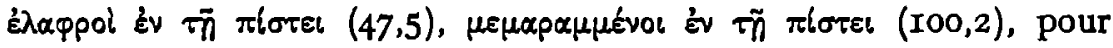
lesquelles le sens instrumental serait absurde. Après ces quelques remarques, qui ont pour but d'appeler l'attention sur le caractère forcément fragile des considérations qui suivent, nous nous proposons de voir la question en détail.

A toute époque, la fonction locale de év est la plus fréquente. A partir du Ve siècle avant J.-C., nous trouvons incidemment cette préposition dans un sens instrumental ${ }^{2}$, par exemple chez Euripide, $B a$. 277:

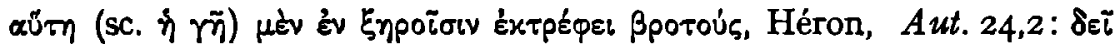

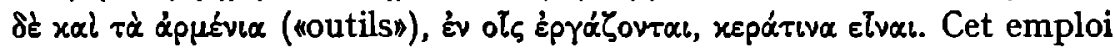
instrumental devient plus ou moins courant lorsqu'il indique la cause auprès des verbes exprimant des sentiments ${ }^{3}$ et dans la construction Ev $\tau \bar{\varphi}+$ inf. 4 .

Les traducteurs de la Septante ont profité de cette fonction instrumentale pour rendre la préposition hébraique ว̧, qui pouvait avoir une fonction aussi bien locale qu'instrumentale, par une seule préposition aussi, ह́v, dans ces deux fonctions, par exemple Deut. 22,10:

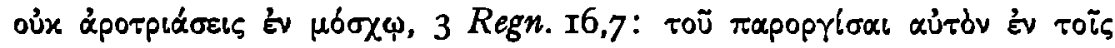

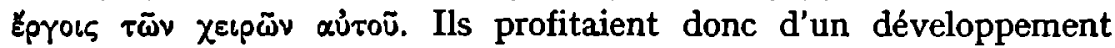
interne du grec; mais, à l'exemple sémitique, ils ont fait un usage si excessif de la fonction instrumentale ${ }^{5}$ que c'était là dépasser de beaucoup les normes du grec de leur temps ${ }^{6}$. Dans les livres de la littérature biblique écrits en grec, la fréquence est bien moins grande que dans les

I. Humbert, Datif, p.129; Demaray, Hermas, p.ro7.

2. Passages chez Helbing, Kasussyntax, pp.I46-147.

3. Passages chez Helbing, Kasussyntax, pp.267-268.

4. Voir F. Krapp, Der substantivierte Infinitiv abhangig von Präpositionen und Prapositionsadverbien in der historischen Gräcitäl (Herodot bis Zosimus). Heidelberg I892, Pp.21,69-70.

5. Cf. Rapallo, Calchi ebraici r969, pp.417-42I. Pour une influence éventuelle de l'égyptien, voir J.Vergote, Grec biblique, Dictionnaire de la Bible, Supplément III, Paris I938, col.(1320-1 369) r 357 ; Brock, Septuagint, P.35.

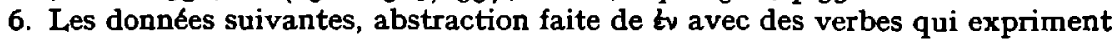
des sentiments et dans $\varepsilon^{2} \tau \tilde{\varphi} \vec{\varphi}+$ inf., peuvent en donner une impression. C'est à peine si, a l'aide des études qui ont été faites, par exemple Helbing, Kasussyntax, Pp.146-I47, on peut trouver 50 passages dans des textes profanes de 500 avant jusqu'à 300 après J.-C. Dans le corpus de textes profanes que nous avons constitué (voir supra p.53) nous n'en avons pas trouvé d'exemples. Dans les livres protocanoniques de la Septante, au contraire, on en rencontre près de i roo. 
livres traduits, mais bien plus grande que dans la littérature profane ${ }^{1}$. Le év instrumental était vraisemblablement devenu un procédé stylistique: l'emploi remarquable qui en est fait dans les livres traduits invitait à l'imitation, mais on n'allait pas jusqu'à atteindre la même fréquence faute d'un texte sémitique de base pour y pousser. Le fait qu'en plusieurs cas des expressions entières sont reprises confirme bien

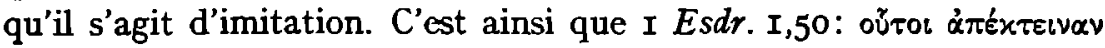

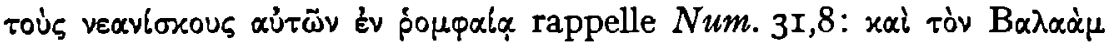

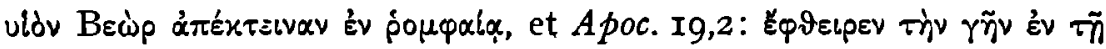

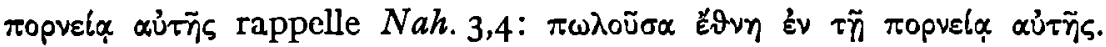
Dans la littérature patristique, l'emploi instrumental de cette préposition est plusieurs fois l'objet de l'attention. Celui qui en parle avec le plus de précision, - pour le latin il est vrai, mais la situation y était la même ${ }^{2}$-, est Augustin, Locut. Hept. 2,9I: Et virgam, in qua percussisti flumen, accipe in manu tua: "in qua percussisti» dixit pro eo, quod dicimus «de qua percussisti》, et est ista locutio creberrima in scripturis $^{\mathbf{3}}$. Chez les Grecs, citons au moins Jean Chrysostome, qui remarque que le żv biblique doit être entendu comme $\delta$ ‘́, Hom. 4,2 in 2 Thess.:

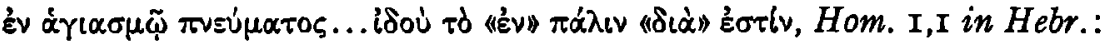

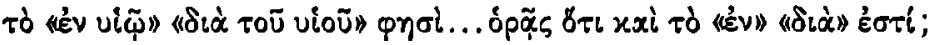

Dans l'édition d'Hermas par Whittaker, on compte 18 passages où la préposition est susceptible d'une explication instrumentale. Dans trois d'entre eux, c'est à tort, croyons-nous, qu'elle a trouvé place dans le texte ( $\mathrm{r})$. Les autres cas se divisent en deux groupes: dans l'un, il s'agit probablement d'une façon de parler sémitisante (2); dans l'autre, la tournure correspond à l'usage du grec profane (3). Examinons chacune de ces catégories.

(I). Les passages où év nous semble devoir être supprimé sont les suivants:

I. Dans les livres deutérocanonıques de la Septante, le Nouveau Testament et les apocryphes, la moyenne de la fréquence est de moitıé moins grande que dans les livres protocanonıques de la Septante, mais la répartıtion est très ırégulıère les cas de loin les plus nombreux se trouvent dans Judith, l'Ecclesiastique, les Psaumes de Salomon, les Testaments des Douze Patrarches, œuvres dont nous savons qu'elles remontent à un orıgınal sémitıque, et dans l'A pocalypse de Jean, dont la langue présente un caractère fortement sémitısant Dans les apocryphes du Nouveau Testament, au contraure, on ne peut, par exemple, citer en tout que 9 passages

2 Voir G A Beckmann, Die Nachfolgekonstruktionen des instrumentalen Ablativs im Spatlatein und im Französischen, Tubingen 1963, pp 9-205

3 Au sujet de ce passage voir J Schnjnen-Chr Mohrmann, Studien zur Syntax der Briefe des hl Cyprian, I, Nijmegen 1936, pp I 13-I I 7, Beckmann, Nachfolgekonstruktionen, Pp 97-98. 


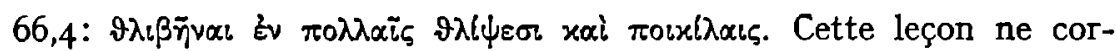
respond complètement à aucun des trois textes témoins grecs.

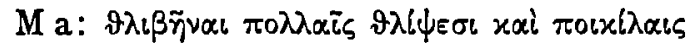

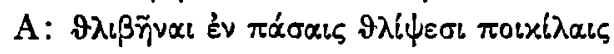

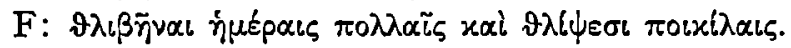

Rien ne s'oppose à ce qu'on s'en tienne, comme Joly, à la leçon de M, qui est le meilleur des trois manuscrits. Cette leçon correspond au

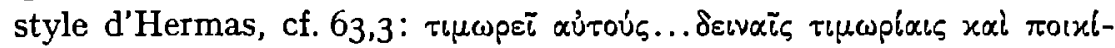

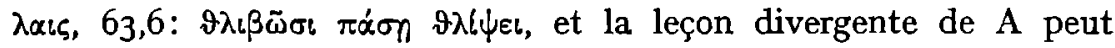

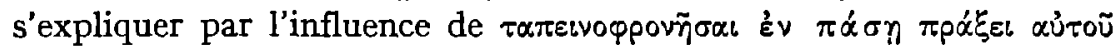
iøxupãs, qui précède immédiatement.

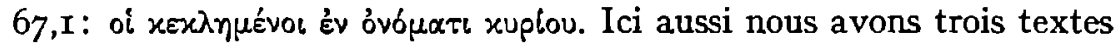
grecs comme témoins: un fragment de papyrus du VIe siècle a la leçon de Whittaker (et Joly), mais A et M, au lieu de Év, ont l'article $\tau \tilde{\varphi}$. Il est beaucoup plus vraisemblable que $\tau \tilde{\varphi}$ óvó $\mu \alpha \tau \iota$ xupiou ait été

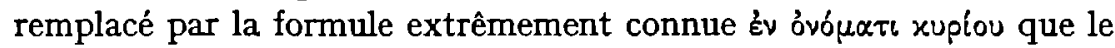
contraire.

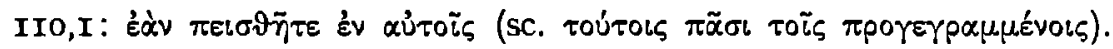
La préposition est une conjecture, le seul témoin grec, $F$, a une lacune

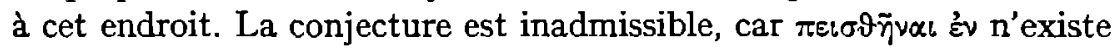
pas ou n'existe guère dans le grec qui nous a été transmis; oũv est plus probable, à notre avis (cf. ergo $\mathrm{L}_{1}$ ). D'ailleurs, il n'est pas nécessaire de donner ici à un év éventuel un sens instrumental.

(2). Dans les passages suivants, la langue nous semble sémitisante:

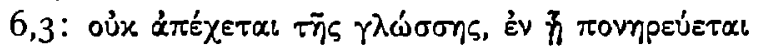

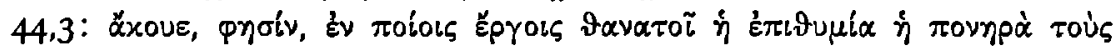

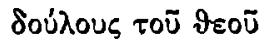

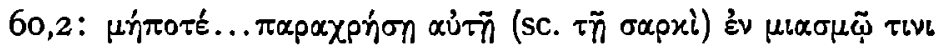

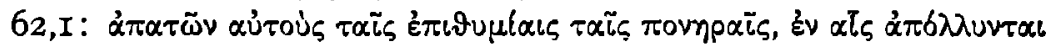

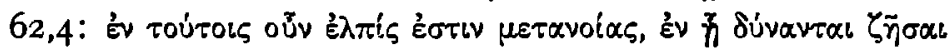

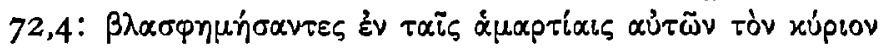

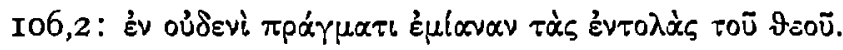

Nous interprétons ces passages au sens instrumental ${ }^{1}$, sans exclure cependant la possibilité de ressentir plus ou moins tel ou tel au sens local. Mais c'est surtout 62,4 qui montre à quel point la métaphore locale a pâli: à strictement parler, dans la proposition principale,

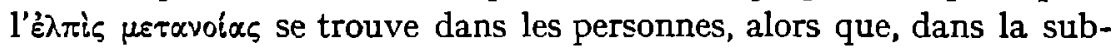

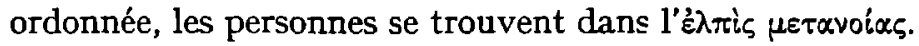


D'après Humbert, on a affaire à un développement autochtone dans la langue d'Hermas: l'affaiblissement progressif du datif, qui nécessite l'emploi de prépositions là où auparavant le simple cas suffisait. Chez Hermas, dit Humbert, tla vie commence à se retirer visiblement du

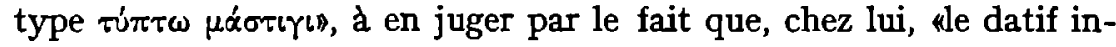
strumental soit relativement peu employén et par les Io endroits où

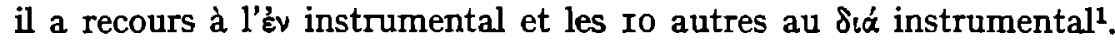
Pour le nombre de passages nous arrivons à des chiffres un peu différents: respectivement $\mathrm{I}_{4}$ et $6^{2}$; mais cet écart tient en partie à des différences d'interprétation, en partie aux différences entre les éditions utilisées; en tout cas, il ne tire pas à conséquence. Mais l'objection décisive contre l'explication d'Humbert, c'est que, chez Hermas, on ne compte pas moins de 42 exemples de pur datif instrumental; si nous laissons de côté les datifs avec $\chi \chi_{\rho} \tilde{\alpha} \sigma \vartheta \alpha_{\ell}{ }^{3}$ et les datifs qui entrent dans une figure étymologique, il en reste encore $28^{5}$. En outre, l'expression

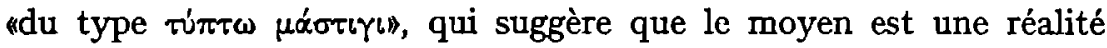
concrète, n'est guère appropriée, puisqu'Hermas emploie év presque exclusivement avec des abstraits; le simple datif, au contraire, se présente aussi bien avec des abstraits qu'avec des concrets.

Si donc Hermas utilise beaucoup le év instrumental, ce n'est pas parce que le datif instrumental pur serait trop usé. Ce n'est le cas ni chez Hermas ni en général à son époque ${ }^{6}$. A notre avis, c'est parce qu'il utilise le grec biblique. La fréquence avec laquelle il emploie le Év instrumental fait que le Pasteur ainsi que la plupart des livres de la Bible grecque tranchent sur les textes du grec profane. En outre, presque toutes les formulations citées font écho à des passages bibliques. C'est

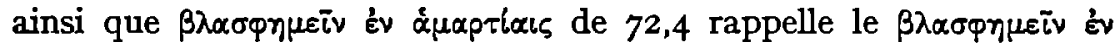

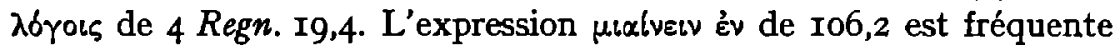
dans le Lévitique et Ezéchiel, mais se trouve aussi ailleurs dans la Septante et les apocryphes paléotestamentaires?.

I. Humbert, Datif, p.I28, cf. ib. p.132.

2. Nous parlons dans le texte des passages avec év. Les passages avec óć́ sont: I 1,$5 ; 21,3 ; 24,4 ; 81,8 ; 90,4.6$.

3. I0,8; $50,3.4 .6 ; 55,10 ; 93.4$.

4. 7,$1 ; 63.3 .6 ; 66,4$ (voir p.85);73,3;74,4;78,2;95,3.

5. 2,$1 ; 3.4 ; 9,9 ; 10,4 ; 11,5 ; 19,3 ; 23.3 ; 34,1.7 ; 43,3.9 ; 44,2 ; 45,4 ; 51,7 ; 62,1$; 63,$4 ; 65.7 ; 67,1 ; 68,9 ; 72,5 ; 75,3 ; 78,10 ; 83,3 ; 89,6 ; 90,9 ; 103.7 ; 104,2 ;$ 105, 1.

6. Voir L. Rydbeck, Fachprosa, vermeintliche Volkssprache und Neues Testament, Uppsala 1967, pp.57, 59.

7. A savoir 2 Par. 29,19; Ps. 105,39; Ps. Sal. 8,12; Jer. 3,2; Dan.1xx 11,32;

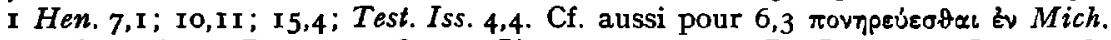

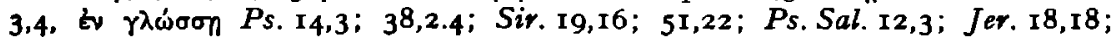


(3). Le év instrumental avec des verbes exprimant des sentiments et dans la construction $\dot{e} v \tau \tilde{\varphi}+$ inf. est chose normale en grec. Les passages comportant des verbes de sentiment sont:

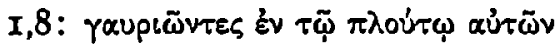

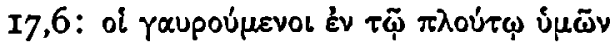

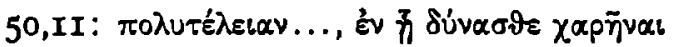

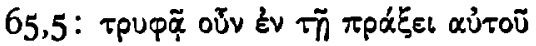

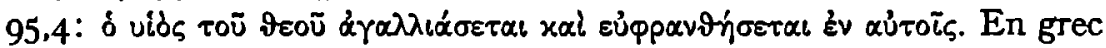
profane, la plupart de ces verbes s'emploient aussi avec év1.

Le év $\tau \bar{\varphi}$ instrumental + infinitif ne se présente qu'une fois chez

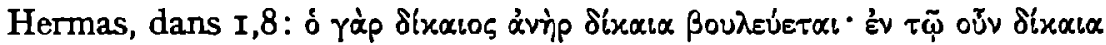

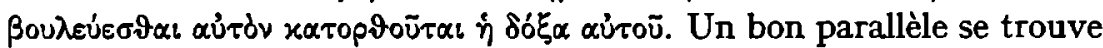

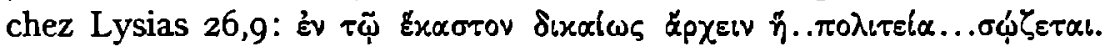
Dans la Septante, où l'on rencontre un nombre considérable de év $\tau \bar{\varphi}+$ inf., la fonction instrumentale ne se présente que rarement; dans le plus grand nombre de cas, la valeur est temporelle, conformément à l'hébreu².

Examinons enfin un passage où, si l'on n'a pas affaire à du grec usuel, ce n'est pourtant pas à du grec sémitisant; il s'agit de 78,10:

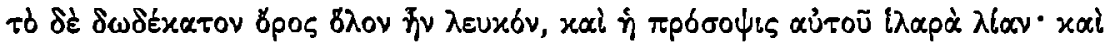

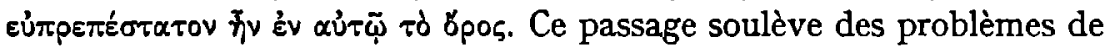
critique textuelle aussi bien que de sémantique. D'abord de critique textuelle: le év $\propto u \dot{\tau} \bar{\varphi}$ des manuscrits (du moins A; M comporte $\varepsilon v \alpha u \tau \omega$ ) est compris en général comme réfléchi. C'est pourquoi les éditeurs le

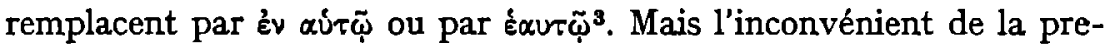
mière émendation, aussi élégante qu'elle soit, c'est qu'à cette époque, du moins chez les auteurs sans prétentions littéraires, les formes

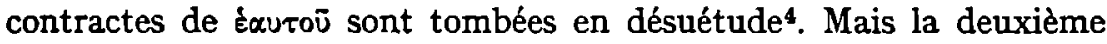

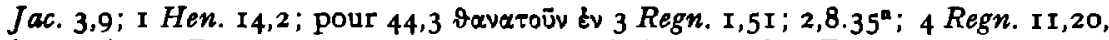

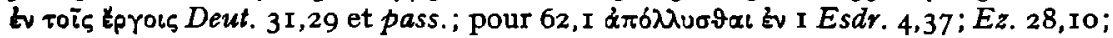

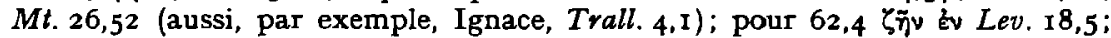
2 Esdr. 19,29; Ps. I18,40.93; Ez. 18,22; 20,11.13.21.25; 33,16.19; Rom. 10,5;

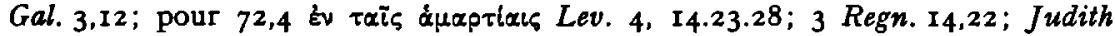
I I,I I : Is. 59,3; Ez. I6,52; Dan. 18x I I,32.

1. Voir Helbing, Kasussyntax, pp.267-268. Pour la construction rpupãv év $(65,5)$ nous n'avons trouvé des parallèles ni dans la littérature profane ni dans la littérature biblique.

2. Voir Soisalon-Soininen, Infinitive, pp.80-83, 192. Pour $\epsilon^{-1} \tau \bar{\varphi}+$ inf. en grec profane voir supra p.83 n.4.

3. 'Ev $\alpha u ̛ \tau \bar{~}$ Anger, Tischendorf, Hilgenfeld, Funk', Lelong, Lake, Whittaker,

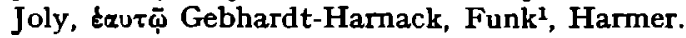

4. Cf. BD $\$ 64^{1}$. 
émendation est peut-être, elle aussi, superflue. Les cas obliques d' đủróc ont aussi depuis toujours un emploi réfléchi ${ }^{1}$. C'est le cas chez Hermas, comme en témoigne, par exemple, la comparaison entre les deux

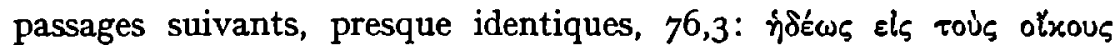

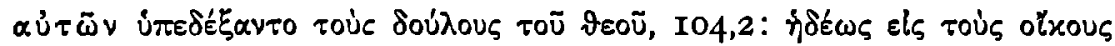

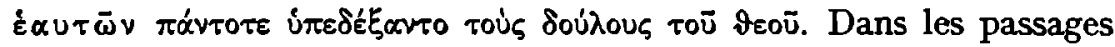

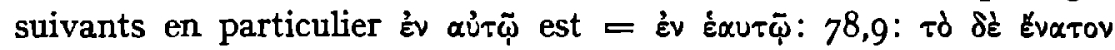

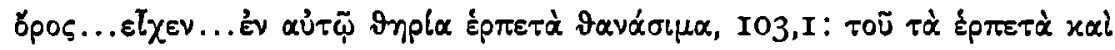

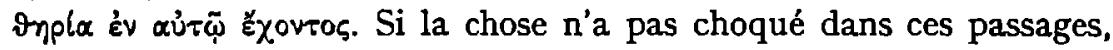
mais bien dans 78,10 , c'est sûrement parce que le pronom précède, au lieu de suivre, le sujet de la proposition. Cependant, à la lumière des rapprochements que nous avons faits, nous croyons pouvoir laisser

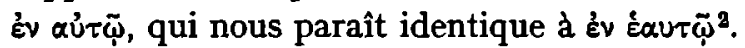

Dans ce contexte, la préposition èv nous semble instrumentale et

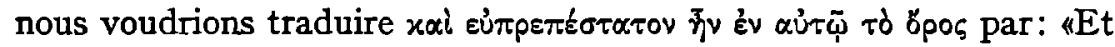
la montagne était très belle par elle-mêmen, c'est-à-dire par ce qu'elle avait elle-même à offrir sans que, comme pour d'autres montagnes, il $\mathrm{y}$ ait eu besoin de végétation. La construction év aủrọ rappelle alors

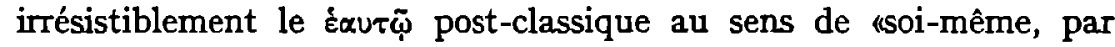
soi-même: ${ }^{3}$ et pourrait en être une variante. Cet emploi n'est pas un cas tout à fait isolé si on peut présumer qu'il se présente aussi dans

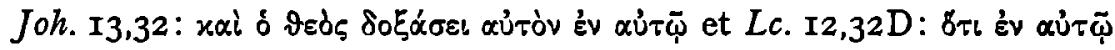

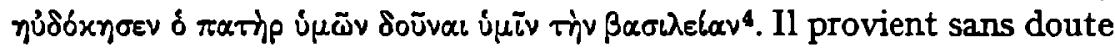
d'une évolution interne du grec; du moins ne trouve-t-on pas d'expressions sémitiques ou latines qui auraient pu servir de modèle pour Év $\alpha u ̛ \tau \overline{~ a u ~ s e n s ~ d e ~ " m e ̂ m e » ~} 5$.

I. Voir SD II, p.196; BD $\S 283^{2}$; Mussies, Koine Greek, p.I64.

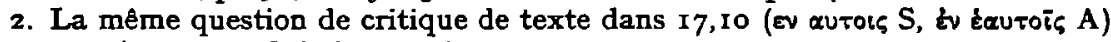
et 75.4 (ev autors $\mathrm{M}$, Èv éautois A).

3. A ce sujet Rydbeck, Fachprosa, pp.51-6I; H. Ljungvik, Aus der Sprache des Neuen Testaments, Eranos 66, r968, pp.(24-5I) 40-42.

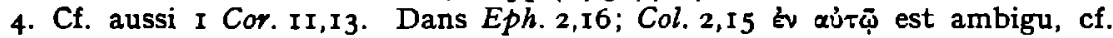
Turner, Syntax, p.43 et 265. L. Radermacher, Griechischer Sprachbrauch, Philologus 68, 1909, p. (449-455) 450 cite trois passages où, à son avis, tv tau s'emploie ainsi, à savoir: Sir. prol. 22; Tab. Defix. Aud. 242,16; Cornutus, $N D$ 2I, mais aucun des trois cas n'est tout à fait convaincant.

5. Les passages de la Septante pour kquī̄ "soi-même", "de soi-même" chez Rydbeck, Fachprosa, pp.53-54 (ajouter Cant. 2,1 I) correspondent en hébreu

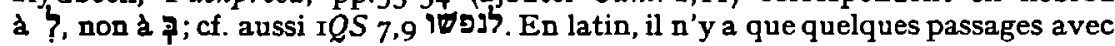
$s i b i$ sans préposition, voir $i b$. p.61; on pourrait y ajouter les deux traductions latines d'Hermas 78, 10: $\mathrm{L}_{1}$ et ipse sibi summum praestabat decorem, $\mathrm{L}_{2}$ sibique mons ille summum praebebat decorem. 


\section{LE Uं $\pi \hat{p} \rho$ COMPARATIF}

La préposition $\delta$ đép peut indiquer le dépassement d'une limite et ainsi prendre plus ou moins le sens de "plus que". En grec classique, il s'emploie déjà ainsi avec des chiffres et dans la locution elvau úrtép "surpasser". A priori, cette préposition se prêtait à un tel développement sémantique aussi bien avec le génitif qu'avec l'accusatif; mais en attique et en grec post-classique, c'est exclusivement avec l'accusatif qu'elle se présente dans ce sens. Nous trouvons le génitif un certain nombre de fois chez Pindare et, beaucoup plus tard, quelquefois aussi dans des apocryphes juifs et une fois dans une inscription chrétienne. $n$ n'y a certainement pas eu de lien particulier entre Pindare et les textes ultérieurs; mais si, avec útép "plus que" + gén., Pindare utilise à titre personnel une des ressources que le grec lui offrait encore à son époque, plus tard, il s'agit plutôt d'une réaction des auteurs contre l'emploi de plus en plus répandu de l'accusatif après les prépositions ${ }^{2}$.

Voyons maintenant de plus près ce comparatif úxép + gén./acc. avec des verbes (abstraction faite de $\varepsilon$ ivœr) et avec des adjectifs. Pour la première catégorie, nous trouvons les plus anciens exemples chez Pindare, exemples auxquels nous faisions allusion tout à l'heure,

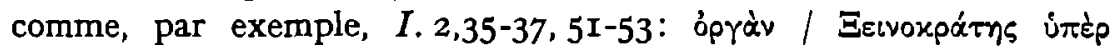

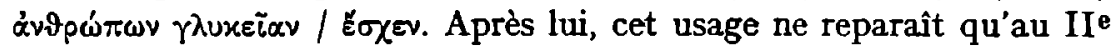

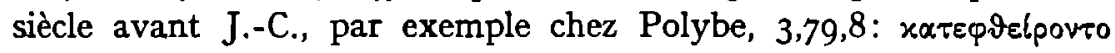

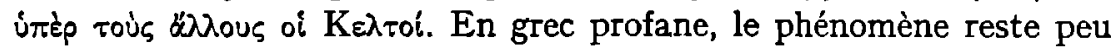
répandu ${ }^{3}$. Avec des adjectifs, nous ne trouvons íx́́p qu'à partir du IIe siècle après J.-C., par exemple chez Philostrate, Im. I,26,3:

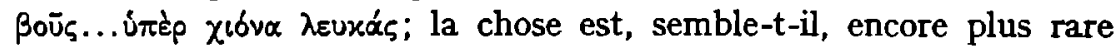
qu'avec des verbes4. L'adjectif se trouve alors au positif. En grec profane, le comparatif ne s'emploie pas de cette manière, bien qu'on puisse citer des exemples qui se rapprochent de cette construction ${ }^{5}$.

\section{Cf. les passages de SD II pp.519-52I.}

2. A ce sujet, voir Wackernagel, Vorlesungen, II, Pp. 2 I 5-2I6. Les passages pour ürtép + gén. sont les passages de Pindare cités dans la note 3 , ainsi que $A p o c$. Esdr. pp.24-25 Tisch.; Apoc. Mos. 16; MAMA 8,252b; dans les apocryphes ultérieurs Acta Phil. 68.

3. Outre les passages cités, nous ne connaissons que Pindare, $N .9,54$, I 29-1 30 ; O. I0,72,87; fr.6I Sn.; Ep. Arist. 19.295; Diodore 1,90,2; 24,5,2; Achille Tatius 4, 19, I; Herodien, Hist. 3,I 4,2; Ps. Lucien, Enc. Demosth. 25.

4. Sauf le passagc cité, nous ne connaissons que Lucien, Peregr. 34 (cas limite) et Ps. Lucien, Macr. 5.

5. Ainsi Thucydide 6,12,2; Philostrate, V.A. 2,15. 
On imagine facilement pourquoi le ú $\pi \dot{\rho}$ comparatif n'a pas été utilisé davantage avec des verbes et des adjectifs: il était loin d'être indispensable. Le grec exprimait habituellement la comparaison par le génitif de comparaison ou par la particule $\eta_{\text {; }}$ il disposait en outre de

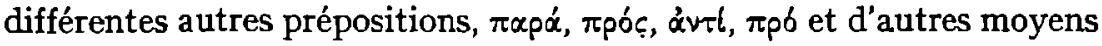
encore pour remplir la même fonction. 'Y $\pi_{\text {ép }}$ "plus que», n'en était, pour ainsi dire, qu'un parmi de nombreux autres.

L'hébreu et l'araméen ne possèdent pas de forme comparative distincte. Dans une comparaison, ils utilisent la forme positive + ip "à partir de", "plus que", par exemple 'מר "sort plus qu'un lion", "plus fort qu'un lion". Il est impossible de traduire cette construction en grec d'une façon qui soit à la fois littérale et grammaticalement correcte. Les traducteurs de la Bible s'en sont tirés de différentes manières. Parfois ils ont choisi de traduire d'une façon littérale:

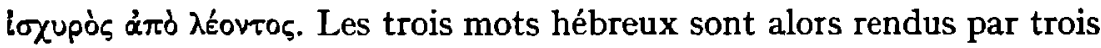
mots grecs, la forme positive est conservée et $\eta_{\text {po }}$ est traduit par une préposition grecque qui en était l'équivalent le plus employé. Mais le résultat n'était évidemment pas du grec idiomatique ${ }^{1}$. A d'autres

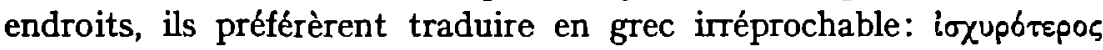

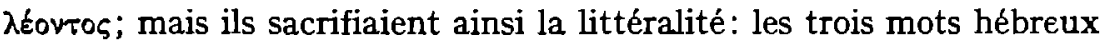
se sont réduits à deux en grec, la forme positive a été sacrifiée au comparatif et la préposition a fait place à une toute autre manière de s'exprimer. Enfin, on a aussi cherché des compromis. Le úmép comparatif en était un, l'adjectif sémitique étant rendu, tantôt par le

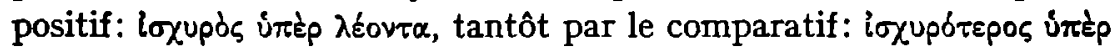

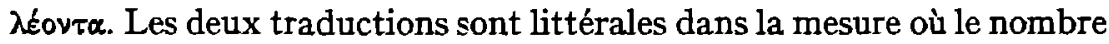
de mots reste trois et où à la préposition correspond une préposition et elles répondent aux exigences du grec dans la mesure où úmép traduit mieux

On trouvait la préposition ip "plus que" non seulement avec des adjectifs, mais aussi avec des verbes. Dans ce cas, les traducteurs optaient aussi, assez souvent, pour íftép. La liste qui suit donnera une idée de la fréquence du comparatif dans la Bible grecque, les apocryphes et le grec profane.

r. Différent en cela du $a b$ latin qui, en tant que préposition comparative, a connu une certaine vogue, cf. Szantyr, Syntax, pp. III-II2, \& $\pi 6$ ne s'est mis à remplir cette fonction qu'en grec moderne. Voir aussi Rapallo, Calchi ebraici I969. pp. $37^{8-382}$.

2. La préposition hébraique 68 , comparable à útép, pouvait d'ailleurs, elle aussi, prendre un sens comparatif. Dans 4 passages de la Septante: Gen. 48,22; Deut. 25,3; Ps. 65,5; Dan.1xx1,20 útép au sens de "plus que" remonte à ce 79. 


\begin{tabular}{lccc}
\hline & $\begin{array}{l}\text { livres protoca- } \\
\text { noniques de la } \\
\text { LXX }\end{array}$ & $\begin{array}{l}\text { livres deutéroca- } \\
\text { noniques de la LXX, } \\
\text { N.T., apocryphes }\end{array}$ & $\begin{array}{l}\text { textes profanes } \\
\text { (pour autant que } \\
\text { nous sachions) }\end{array}$ \\
\hline positif & $5 \mathrm{I} \times$ & $\mathrm{I} 7 \times$ & $3 \times$ \\
comparatif & $\mathrm{I} \times$ & $6 \times$ & - \\
verbe & $90 \times$ & $46 \times$ & $\mathrm{I2} \times$ \\
\hline
\end{tabular}

Il ressort de ces chiffres que le úrép comparatif en grec biblique doit être compris comme un sémitisme. Il est remarquable que ce íćp se présente avec des comparatifs, contrairement, d'une part, à l'hébreu et a l'araméen, qui n'ont pas la forme comparative, d'autre part, au grec profane, où ú $\pi \hat{\text { p }}$ avec le comparatif, pour autant que nous avons pu le vérifier, fait complètement défaut. C'est là un nouvel exemple du caractère particulier du grec biblique.

Dans le Pasteur, le írép comparatif se présente deux fois. Dans

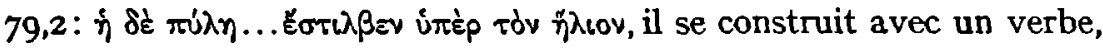
construction dont nous avons déjà parlé plus haut. On a toute raison de croire que c'est la langue de la littérature biblique qui a inspiré cette

I. Positif Gen. 48,22; Deut. 30,5;Ruth 4,I5; I Regn. 1,8; 9,2; I 5,22bis.28; 24,18; 2 Regn. 1,23; I3,I5.16; I 7,14; 19,8; 3 Regn. 2,22.32; 20,2; 4 Regn. 5,12; I Par. 4,9; I I,21.25; 2 Par. 21, I3; Ps. I8, I I; 65,5; I I8,72.103; Eccl. 2,7; 4.3.6. 9.13; 6,3.9.10; 7,1bis.3.5.8bis.10; 9.4.16.18; 10,1; Cant. 1,2; Agg. 2,9; Lam. 4,19; Ez. 5,1; Dan. ThI, 15; I 1,13. Comparatif Deut. 25,3; Jud. A 16,30; B 3,9; 1 1,25; I5,2; 18,26; Ruth 3,12; 3 Regn. 12,24' 19.4; 4 Regn. 6, 16; Ps. I8, I I; 36, 16; 62,4;83, I I; Prov. 16, 16; Eccl. 7,26; Hab. 1,8; Dan.11x 1,20. Verbe Jud. A 2, 19; B 2,19; Ruth I,13; 3,10; I Regn. I,5; 2,29; 10,23; 2 Regn. 1,23.26; 6,21bis; I0,I Ibis; I3,I 4 ; 18,8; 19,44;20,6;22,18; 3 Regn. I,37.47bis; 2,35bbis; 5, 1obis. I Ibis; 10,23; 16,25.30.33; 21,23bis.25; 4 Regn.3,26; I 7,14; 11,9 ; I Par. I9, I 2bis; 2 Par. 9,22; I1,21; 33,9; Ps. 17.18; 37,20; 39,6.13; 50,9; 51,5bis; 54,$22 ; 68,5.32$; 86,2; 104,24; 118,98.99.100.127; 138, 18; 141,7; Prov. 8, 10; Eccl. 4,2; 7,19.24; 9,17; Cant. 1,4; Job 28,18; Joel 1,8; Nah. 2,10; 3,14.16; Hab. 1,8; Jer. 5,3; 7,26; I5,8; I6, I2; I7,23; 26,23; Lam. 4,6.7ter.8; Ez. 16,51.52bis; 23, I Ibis; Dan.1xх3, $22 ; 6,4$.

2. Positif I Macc. I2,24; Sap. I 5,14; Sir. 22,11; 24,20bis; 26,5; Ps. Sal. 4,2; BelTh $;$ Eph. 3,20; I Hen. 98,2; Test. Abr. A 16; Jos. et As. 1,6; 23,3; Test. Lev. 8,12; 14,3; Apoc. Esdr. pp.24-25 Tisch. (ce passage + gén. au lieu de + acc., cf. supra p.89); Acta Pauli 7,7. Comparatif Sir. 30,17; Lc. 16,8; Hebr. 4, 2 ;

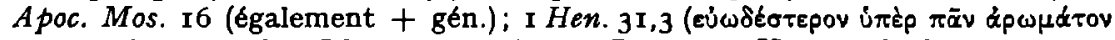
ms.; conjectures chez Black app. cr.); Ps. Barn. 5,9. Verbe I Esdr. 1,47; 3,12; I Macc. 3,30; 7,23; 2 Macc. 8,35; 4 Macc. 9,3; Or. Man. 9; Sap. 7,10; 16,19; Sir. 8,13; 22,14; 29,13bis; 36,22; 40,19.24.25.26.27; Ps. Sal. 2,26; 8,13; 17,43; Suz.Th 39 ; Mt. 10,37bis; Act. 26,13; I Cor. 10, 13; 2 Cor. 1,8; 12,6.13; Gal. 1, 14; Philem. 2 ; Test. Abr. A 4.7.8bis; Jos. et As. 13, I I Test. Rub. 5,3; Test. Sim. 4,6; Test. Dan. 1,5; Test. Gad 7,1; Ps. Barn. 1,2.4; 4,6;19.5; Did. 2,7 .

3. Voir supra p.89. 
formulation à Hermas. Dans cette littérature, on trouve d'ailleurs plusieurs autres passages où úrép "plus que» est construit avec un verbe signifiant rayonner ${ }^{\mathbf{1}}$.

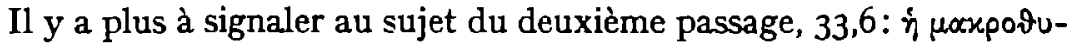

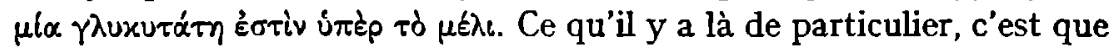
i $\pi \hat{e} p$ se présente avec un superlatif, combinaison que nous n'avions pas encore rencontrée. Si nous prenons la phrase en soi, il semble

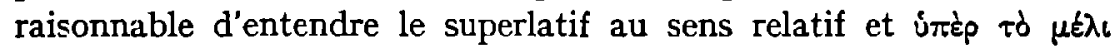
comme une précision épexégétique du superlatif. C'est ainsi que Snyder traduit: "patience is very sweet, even more than the honey". Mais, dans le contexte, cette interprétation perd de sa pertinence. Dans ce qui précède immédiatement, il est question de miel dans une comparaison. La phrase met la comparaison en rapport avec le propre sujet du chap. 33, l'excellence de la vertu de patience. C'est pourquoi il vaut mieux traduire: "la patience est encore plus douce que le miel (dont nous parlions dans la comparaison)». Les traducteurs anciens

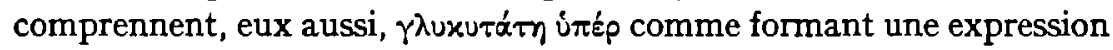
comparative globale: dulcior melle $\left(\mathrm{L}_{1}\right.$, et $\mathrm{E}$ dans la traduction $\mathrm{d} \cdot \mathrm{Ab}-$ badie), melle dulcior $\left(\mathrm{L}_{1}\right)$. Le Codex Vaticanus et le Codex Dresdensis de $\mathrm{L}_{1}$ traduisent par dulcissima plus quam mel. Nous avons là un exemple de littéralisme qui a le mérite de confirmer l'exactitude de la tradition grecque, mais qui ne doit pas nécessairement être pris comme une contestation de l'interprétation comparative que nous venons de donner.

Nous n'avons pas pu trouver de parallèles de ce superlatif avec ứćp en grec profane, mais bien dans un texte dont la langue, comme celle du Pasteur, se conforme à la Bible grecque, à savoir dans Test.

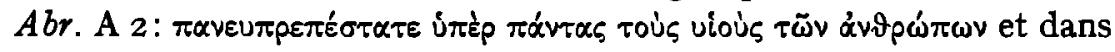
une inscription chrétienne du IVe siècle, MAMA 8, 252b: Гopóıavds

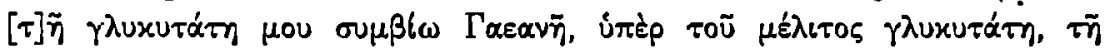

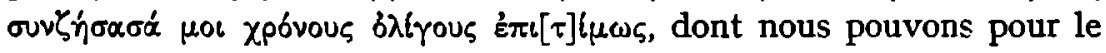
moins supposer qu'elle est influencée par le grec biblique 2 .

Deux causes peuvent être à l'origine de ce superlatif remplaçant un comparatif, et sans doute elles ont agi toutes deux. D'une part, ce peut être l'analogie du superlatif remplaçant un comparatif avec le génitif de comparaison, phénomène que nous rencontrons de temps en

r. Lam. 4,7; Act. 26,13; Test. Abr. A 7; cf. Ignace, Eph. 19,2; Acta Philippi 20; Apoc. Pauli 23.

2. Pour le génitif après intép voir supra p.89. 
temps à partir d'Homère $\mathrm{e}^{1}$. D'autre part, il peut s'agir d'une réaction suscitée par la tendance post-classique à abandonner l'emploi du superlatif au profit du comparatif ${ }^{2}$; lorsqu'on remplace régulièrement une tournure par une autre il arrive souvent qu'incidemment le remplacement se fasse dans l'autre sens ${ }^{3}$. Quoi qu'il en soit, il est évident que le remplacement du comparatif par le superlatif avec úrép était dû au dynamisme du grec lui-même. Comme nous l'avons dit, nous n'en avons pas trouvé d'exemples en grec profane, mais on n'y trouve pas non plus úiép avec le comparatif. Пхpó "plus que», au contraire, qui en grec profane, se rencontre très légitimement avec le comparatif, se présente aussi incidemment avec le superlatif, par exemple chez Oppien, $H .2,200$ :

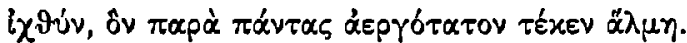

En latin, nous trouvons d'ailleurs un cas où super "plus que» dépend d'un superlatif, et ce, chez un contemporain d'Hermas, Suétone: Vit. 13: Famosissima super ceteras tuit cena data ei adventicia a fratre. Cela ne prouve évidemment pas une influence latine sur la langue d'Hermas, mais cela montre bien que les mêmes tendances étaient à l'œuvre en même temps dans le latin et dans le grec.

Parmi les diverses tournures comparatives utilisées dans le Pasteur,

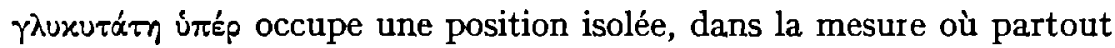
ailleurs Hermas utilise des constructions bien grecques. Le procédé habituel est le comparatif + gén. comp.; à côté de quoi on rencontre incidemment le comparatif $+\not \not$, le comparatif $+\pi \alpha \rho \alpha \dot{\alpha}+$ acc. et

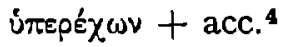

On pourrait penser que ces remarques nous ont fait consacrer une attention démesurée à un seul passage d'Hermas. Mais, si notre raisonnement est correct, ce passage peut nous apprendre beaucoup de choses sur l'origine des sémitismes d'Hermas. On peut y trouver trois indices qu'Hermas a pris ses sémitismes dans la langue grecque, en l'occurrence dans la littérature biblique grecque, et ne les a donc pas

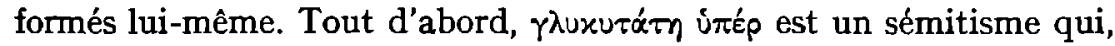
en grec, du fait de forces intérieures à la langue, a déjà subi une

I. Voir SD II, p.Ioo.

2. Voir Mayser, Grammatik, II I, Pp.49-5I; BD $\S 60,244$; Turner, Syntax, pp.29-3o.

3. Voir Wackernagel, Vorlesungen, II, Pp.2I 5-2I6; Havers, Syntax, Pp.182-I83, 264.

4. Gén. comp. 12,3bis; 18,$8 ; 38,9.10 ; 40,2 ; 43,8 ; 47,5 ; 51,4 ; 56,3 ; 89,2 ; 106,3$.

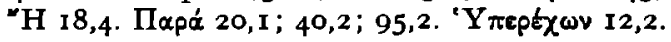


déformation: le comparatif initial a été remplacé par le superlatif à une époque qui se situe entre le début de la Septante et la rédaction du Pasteur. Ensuite, un locuteur à qui une langue sémitique serait plus familière que le grec n'utiliserait probablement pas íxép sous l'influence de cette langue sémitique, mais $\alpha \dot{\pi} \delta b$ et certainement pas un superlatif ou un comparatif, mais un positif, étant donné qu'excepté en arabe, les formes comparatives et superlatives n'existent pas dans les langues sémitiques. Enfin, il serait surprenant qu'un écrivain laissât échapper un sémitisme de sa langue maternelle dans un domaine où il montre qu'il sait utiliser correctement un grand choix de constructions bien grecques.

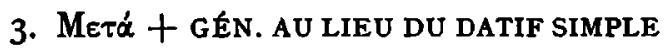

Des trois prépositions plus ou moins synonymes oúv, $\mu \varepsilon \tau \dot{\alpha}$ ( + gén.) et $\not \alpha \mu \alpha$, c'est seulement $\mu \varepsilon \tau \dot{\alpha}$ que nous trouvons chez Hermas'. Cette préférence pour $\mu \varepsilon \tau \dot{\alpha}$ est générale en prose grecque, avec Xénophon comme seule exception importante ${ }^{2}$. De même, les fonctions de $\mu \varepsilon \tau \dot{\alpha}+$ gén. sont en grand, chez Hermas, ce qu'elles sont ailleurs en grec. Mais, dans certains cas, il emploie cette construction là où l'on s'attendrait à un simple datif, soit le datif sociatif, soit le datif proprement dit.

\section{A. Metá + GEN. AU LieU du datif Soclatif}

Avec des expressions sociatives, c'est-à-dire qui expriment des actions qui, en vertu de leur caractère, se font au contact d'autres personnes ou choses, comme une association, une communauté, une relation d'amitié ou d'inimitié, le grec indique la personne ou la chose au contact de laquelle l'action a lieu, par le datif, le datif dit sociatif. Parfois aussi il fait usage d'une expression avec une préposition: dans

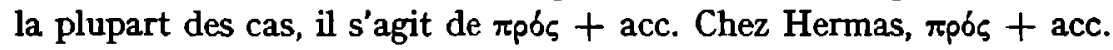
ne se présente pas dans cette fonction, mais on trouve, chez lui,

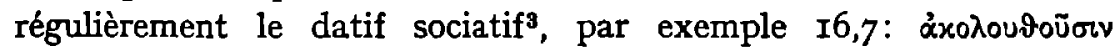

1. Whittaker et Joly ont une fois, 67,18 , oúv, mais c'est une reconstruction de Bonner.

2. Comme nous le savons par les recherches de T. Mommsen, Beitrage zu der Lehre von den griechischen Präpositionen, Berlin 1895, pass., qui a compulsé toute la littérature antique et byzantine au sujet de oúv, \&ua et $\mu \varepsilon \tau \dot{x}$.

3. Sauf les passages cités 10,$6 ; 13,1 ; 14,2 ; 15,6 ; 16,4 ; 17,7 ; 29.9 ; 33.6 ; 36,5.9$; $38,5.10 ; 40,6 ; 4 I, 3 ; 43.4 .13$ bis; 46,$1 ; 51,1 ; 55.7 .8$ bis; 59,$6 ; 65.3 .5 ; 74,1 ; 83.4$; 84,$2 ; 96,2 ; 97,2 b i s ; 98,2 ; 103,3$. 


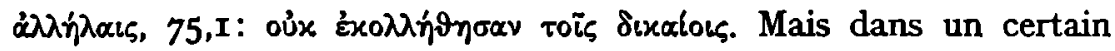

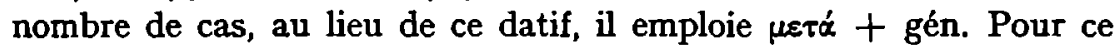
$\mu \varepsilon \tau \dot{\alpha}$ sociatif, comme nous le nommerons ici, il faut tenir compte de trois facteurs: une tendance du grec, une intluence sémitique et une influence latine. Inutile de dire que ces trois facteurs ont pu agir à la

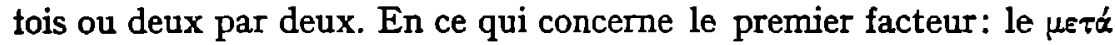
sociatif s'insinue déjà dans le grec classique (de même que parfois oúv et $\alpha{ }^{\prime}(\alpha)$ ), mais il semble ne se présenter qu'avec des composés comportant ó $\mu$-, ouv- et $\mu \varepsilon \tau \alpha-$, avec des verbes signifiant accompagner, comme $\varepsilon \pi \varepsilon \sigma \vartheta \alpha \iota$ et $\delta \pi \eta \delta \varepsilon i \nu$, et avec des verbes signifiant mélanger; avec tous ces verbes, le datif simple est également possible ${ }^{1}$. A partir du III ${ }^{\theta}$ siècle avant J.-C. nous trouvons aussi le $\mu \varepsilon \tau \dot{\alpha}$ sociatif avec des verbes signifiant combattre ${ }^{2}$, et, au cours des siècles suivants, il fait lentement mais sûrement d'autres conquêtes. Chez un auteur comme Malalas, il se présente avec toutes sortes d'expressions sociatives ${ }^{3}$. En grec moderne, le $\mu$ é issu de $\mu \varepsilon \tau \alpha \dot{~ e s t ~ f i n a l e m e n t ~ d e v e n u ~ l a ~ p r e ́ p o s i t i o n ~}$ normale avec les expressions sociatives. $\Pi$ est difficile de savoir dans quelle mesure la préférence pour le $\mu \varepsilon \tau \dot{\alpha}$ sociatif s'était répandue à l'époque d'Hermas. En tout cas, elle peut avoir contribué à déterminer l'emploi qu'il en fait. D'autre part, en hébreu, en araméen et en latın, des expressions sociatives s'accompagnent régulièrement d'une pré-

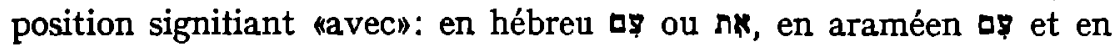
latin cum. Chez les auteurs qui ont subi une influence directe ou indirecte d'une de ces trois langues, on peut donc aussi expliquer le $\mu \varepsilon \tau \dot{\alpha}$ sociatif comme un sémitisme ou un latinisme.

Chez Hermas, le $\mu \varepsilon \tau \dot{\alpha}$ sociatif se présente dans les constructions

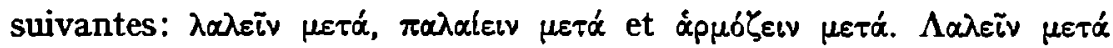

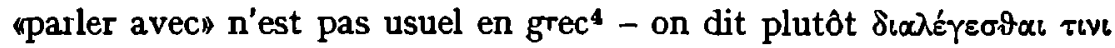

I. Voir SD II, p I60; J S. Lasso de la Vega, Sintaxıs griega, I, Madnd I968;

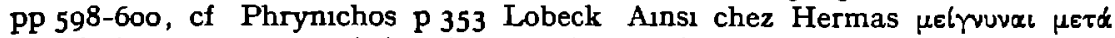

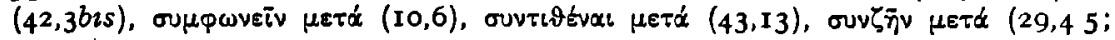
$75, \mathrm{I} .3)$.

2. Les seuls passages que nous connaissions sont Antigone de Caryste 35 ( $2 b .39$ $\pi p 6 \varsigma_{\text {) }}$, PPar 18, 10 (cf Helbing, Kasussyntax, p $233 \mathrm{n} \mathrm{I}$ ), Wilcken Chr 1,23, 9-Io (VP), OGI 1,20I,3 I3 I6 I8 I9 (VIp, l'inscription dite de Silco) Cf. Mommsen, Präpositzonen, P 2 I I n 5I, ses passages Athénée 7,2964 et Apollodore, Poliorc p $4 \mathrm{YC}$ sont incertains.

3. Cf K. Wolf, Studien zur Sprache des Malalas, II, Munchen I9I 2, pp 43, 45, 46; E. Trapp, Der Dativ und der Ersatz seiner Funktionen in der byzantinischen Vulgärdichtung bis zur Mitte des I5 Jahrhunderts, Jahrbuch der Osterreichischen Byzantinischen Gesellschaft I4, I965, p (21-34)28

4 Mommsen, Präposttionen, pp 251, 252, 261 parle d'un hébraisme Helbing, Kasussyntax, Pp 238-239 non plus ne peut citer des passages. Les parallèles les 


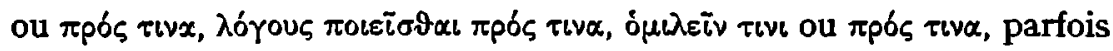

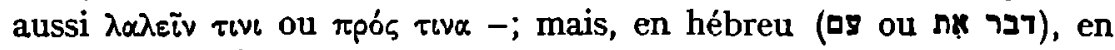
araméen (מל (ס) et en latin (loqui cum), on s'exprime d'une manière qui correspond à $\lambda \alpha \lambda \varepsilon i \bar{v} \mu \varepsilon \tau \alpha$. C'est donc probablement un sémitisme ou un latinisme, ou les deux. Comme sémitisme, nous le tıouvons 32 fois dans les livres protocanoniques de la Septante et I 5 fois dans les autres livies de la Septante et le Nouveau Testament ${ }^{1}$. Il fait défaut dans les apocryphes paléo- et néotestamentaires, mais réapparaît dans des textes chrétiens ultérieurs, surtout apocryphes ${ }^{2}$. Dans tous ces textes, à côté de $\lambda \alpha \lambda \varepsilon i \bar{\nu} \mu \varepsilon \tau \dot{\alpha}$, nous trouvons également $\lambda \alpha \lambda \varepsilon i \bar{\nu} \tau i v \iota$,

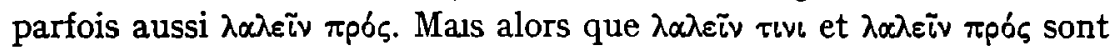
des expressions à valeur générale, utilisées aussi bien pour des personnes parlant entre elles que pour Dieu ou son émissaire parlant avec les hommes, nous voyons qu'à la longue on réserve $\lambda \alpha \lambda \varepsilon \tilde{c}_{\text {iv }} \mu \varepsilon \tau \dot{\alpha}$ quand c'est Dieu, Jésus ou un envoyé céleste qui parlent. Dans la Septante, on ne perçoit pas encore très bien cette spécialisation: dans I7 cas, il s'agit d'hommes qui parlent et dans I $7_{7}$ cas de Dieu ou de son ange, mais dans le Nouveau Testament, dans tous les cas, sauf Eph. 4,25, c'est déjà Jésus, une "voix" ou un ange qui parlent". Dans les textes ultérieurs aussi, sauf $A$ pophth. Patr. PG 65, 205A et la version grecque

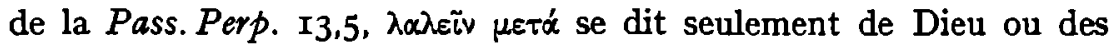
anges. C'est ainsi que $A c t$. Andr. et Matth. I5 marquent de la façon suivante la différence entre le cas où c'est une statue qui parle et le

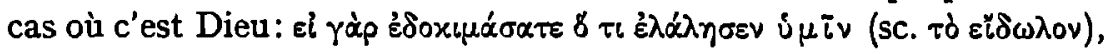

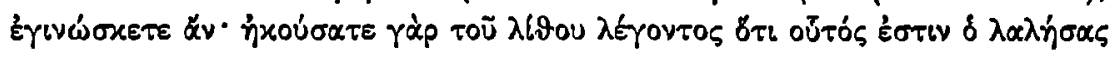

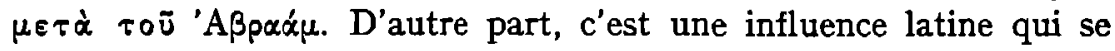
manifeste dans un papyrus du II ${ }^{\mathrm{e}}$ siècle après J.-C., où l'expression est employée deux fois à propos d'un préfet romain qui, se prononçant

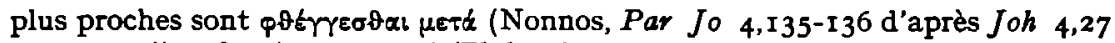

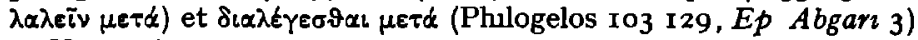

I Vour infra $\mathrm{n} 3$

2 Hegemonius I2, Apoc Paul 47 48, Act Andr et Matth 15 17; Mart Petri et Pauli 5, Act Petri et Pauli 26, Apophth Patr PG 65, 205A, Pass Perp 13,5: Act Thom 32.

3 Dans la Bible grecque, l'expression s'emplose lorsque Dieu, Jésus, un ange ou une "voux" parle Gen 35, I3 I4 I5, Num I1,17, Jud A 6, I7; Jud B 6, 17; Ez 3,10, 44,5, Dan 1x88, I8, 9,22, I0, I I I 5 19, Dan Th8, 18; 9, 22; I0, 15 I9, Mc 6,50, Joh 4,27bis, 9.37, 14,30, Apoc I,12, 4, I; 10,8, 17, I; 21,9 15; lorsque des hommes parlent Gen 31,24 29, 2 Regn 13,22; 3 Regn 1,14 22; 4 Regn 6,33, 18,26, 25,28, Judith 7,24, 10,9, 15,8, I Macc 7,15, Ps 27,3, Dan 1xx 4,32, 10, 17 , Dan $\mathrm{Th}_{1,19}, 10,17, E p h$ 4,25 Nous ne comptons pas Hermas 11, vorr note suivante La construction ne se présente pas dans les apocryphes. 
sur le statut d'enfants adultérins de parents romains, donne son

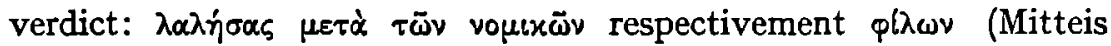
Chr. 372,III,I8; IV,I2-I3).

Hermas emploie II fois $\lambda \alpha \lambda \varepsilon i \bar{\tau} \mu \varepsilon \tau \dot{\alpha}^{1}$. Partout ceux qui parlent sont

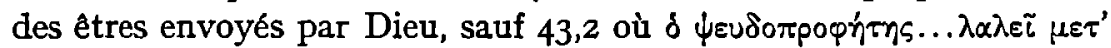

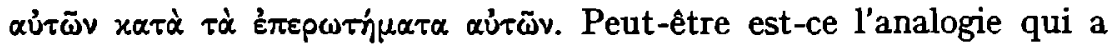
joué ici. Mais il se peut aussi que ce soit la leçon qui soit inexacte et

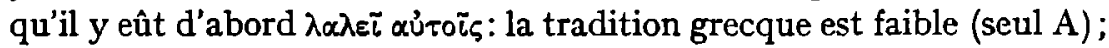
parmi les versions latines, $\mathrm{L}_{1}$, qui passe pour être la meilleure, $a$ : loquitur illis ( $\mathrm{L}_{2}$ loquitur cum illis). Quoi qu'il en soit, la tendance que nous avons remarquée dans la Bible et dans les textes ultérieurs à

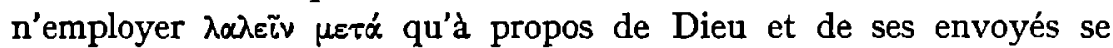
retrouve incontestablement chez Hermas. Cela indiquerait qu'Hermas a pris l'expression dans la littérature biblique, impression qui se confirme lorsqu'on sait que les livres apocalyptiques Daniel et l'Apocalypse de Jean, qui, de tous les livres de la Bible grecque sont ceux qui utilisent le plus souvent $\lambda \alpha \lambda \varepsilon i v ~ \mu \varepsilon \tau \alpha ́$, partagent le goût du Pasteur apocalyptique pour cette expression. D'un autre côté, naturellement, le loqui cum latin a pu influencer Hermas, mais alors ce serait tout au plus en créant des conditions favorables.

Zahn considère, semble-t-il, que l'emploi de $\lambda \alpha \lambda \varepsilon i ̃ \nu ~ \mu \varepsilon \tau \alpha \dot{\alpha}$ au sens de "dire quelque chose à quelqu'un" et pas seulement de "converser avec quelqu'un" soit une spécialité d'Hєrmas ${ }^{2}$. En réalité, le premier sens est à retenir aussi pour plusieurs passages de la Septante et du Nouveau Testament ${ }^{3}$. Souvent le contexte ne permet pas de savoir si un dialogue s'établit ou si les choses en restent à un monologue. Il est évident que, dans le deuxième cas, il n'est plus question d'un datif sociatif. Le latin aussi connaît ce phénomène. Chez Ovide, Met. 6,205:

\section{talibus est dictis gemina cum prole locuta}

ce n'est pas seulement le contexte général, mais talibus dictis à lui seul qui prouve qu'un monologue s'annonce.

I. 4,$3 ; 18,1 ; 36,3 ; 43,2 ; 50,1 ; 56,2 ; 57,5 ; 61,5 ; 63,2 ; 78,1 ; 88,1$. Dans 28,3 , Whittaker et Joly, a bon droit, ont remplacé le é $\lambda \dot{\alpha} \lambda \eta \sigma \alpha \mu \varepsilon \tau \dot{\alpha}$ de la tradition grecque (in casu A) par ह

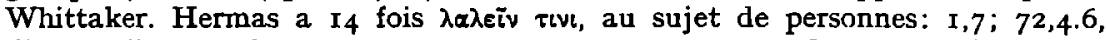
d'êtres célestes: 18,$4 ; 27,7 ; 32,3 ; 47,1.2 ; 57,2 ; 61,4 ; 77,5 ; 84,6 ; 110,1$ bis.

2. Zahn, Hirt, p. 493 .

3. Par exemple Gen. 35, I3-I5; Num. II,I7 (cf. ib. II,25); 4 Regn. 6,33 (cf. ib.

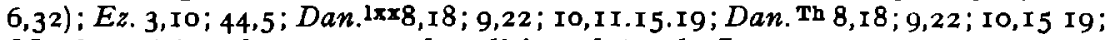
Mc. 6,50 et tous les passages dans l'A pocalypse de Jean. 


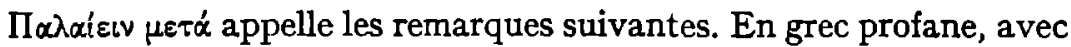
les verbes signifiant combattre, l'adversaire est désigné ordinairement

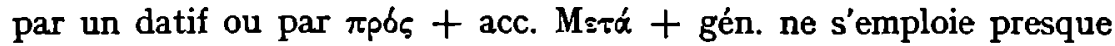
jamais (cf. supra, p.95, note 2) et en tout cas jamais avec $\pi \alpha \lambda \alpha\left\{\varepsilon \omega \nu^{1}\right.$. En grec biblique, au contraire, l'influence sémitique fait qu'on rencontre 1égulièrement la construction d'un verbe de combat avec $\mu \varepsilon \tau \alpha^{2}$. C'est ainsi que $\pi \alpha \lambda \alpha \hat{\alpha} \varepsilon \iota v \mu \varepsilon \tau \alpha \dot{~ s e ~ p r e ́ s e n t e ~ d a n s ~ G e n . ~ 32,25.26 ~ e t ~}$ Test. Job 27,3. Hermas lui-même emploie cette expression une fois,

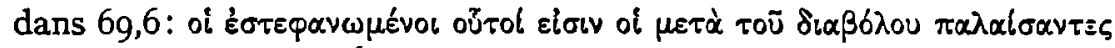

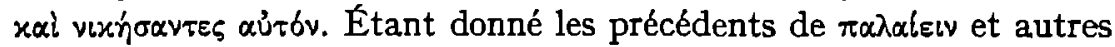
verbes signifiant combattre avec $\mu \equiv \tau \dot{\alpha}$ en grec biblique, nous pouvons présumer que, chez Hermas, l'expression est un sémitisme. Le contexte dans lequel il l'emploie plaide en ce sens. En effet, nous avons affaire ici à l'idée du combat contre le diable, idée qu'on trouve non seulement dans la littérature des martyrs et des moines, mais qui existait déjà dans le judaīsme hellénistique ${ }^{9}$ et qu'on retrouve ailleurs dans le Pasteur4. Il se pourrait fort bien que $\pi \alpha \lambda \alpha l \varepsilon t v \mu \varepsilon \tau \alpha$ vienne de l'arsenal d'expressions qui servaient à formuler ce lieu commun. Nous disposons, en tout cas, d'un parallèle: Test. Job 27,3, dont nous avons déjà

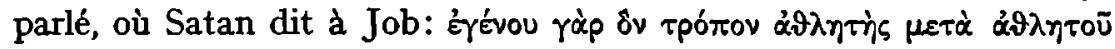

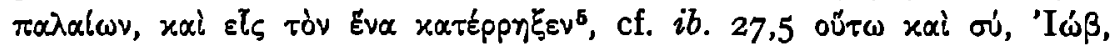

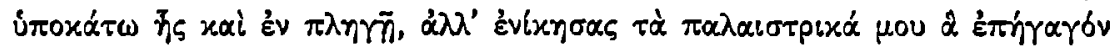
бot. Pour compléter ces remarques, disons encore que le latin parle de luctari cum, expression qui correspond exactement à $\pi \alpha \lambda \alpha i \varepsilon t v \mu \varepsilon \tau \alpha ́$. Rien n'indique cependant que c'est le latin qui a inicté Hermas à choisir cette expression.

Un troisième cas de $\mu \varepsilon \tau \dot{\alpha}$ sociatif est l'expression $\dot{\alpha} \rho \mu b \zeta \varepsilon เ \nu \mu \varepsilon \tau \dot{\alpha}$,

I. Helbing, Kasussyntax, p.238 cite le passage tardif Ps. Callisthène 2,20 Meusel, mais la langue de ce texte semble avoir subi l'influence du grec biblique.

2. Gen. I4,2; 26,20;32,25.26.29; Deut. $25,1 \mathrm{I} ;$ Jud. A 5,20; I I,4.5.20.25; 20,14 . I8.20bis.23.28; Jud. B 5,20; II.5.25; I Regn. 17,32.33; 28,1; $2 \operatorname{Regn}$. I0,17; I1,17; 21,15bis. I8.19; 3 Regn. I2,24; 4 Regn. 8,29; 9,15.16; 14,I5; 19,9; I Par. 5,19; 20,4.5; 2 Esdr. 23,25; Sir. 8,1.2.3.16; Os. 12,5; Dan.1xх10,20; II,1I; Dan.Th7,21; 10,20; 11,11; A poc. 2,16; 11,7; 12,7.17; 13,4; 17,14; 19,19; Par. Jer. 7. I2; Test. Job 27.3. Nous avons laissé hors de cause ici les expressions de lutte non violente, de caractère juridique ou analogue. A ce sujet et au sujet des constructions des verba pugnandi en général, voir Helbing, Kasussyntax, Pp.233238.

3. Voir, par exemple, V. C. Pfitzner, Paul and the agon motif, Leiden 1967, pass. 4. 47,7; 48,2; cf. 45,2-5. Voir Pfitzner, Agon motif, pp.198, 201 n.3.

5. Ainsi deux des trois manuscrits grecs et la version paléo-slave. Dans le

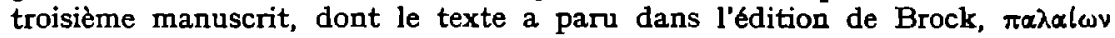

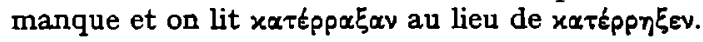




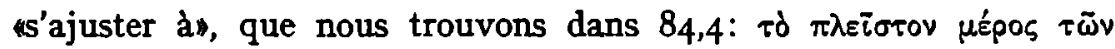

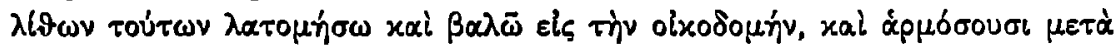
$\tau \bar{\omega} \nu \lambda$ a le sens d' «avec", c'est-à-dire «de même que». Mais quelques phrases plus haut, dans 84,2 , Hermas parle dans exactement le même contexte de $\dot{\alpha} \rho \zeta_{\zeta \varepsilon \iota \nu}+$ dat., expression qui ne peut signifier autre chose que «s'ajuster d̀. A ce propos, il ne faut pas oublier que la langue d'Hermas est souvent capricieuse (cf. supra, pp.Ig et 23). C'est ainsi que, dans un contexte identique à celui qui nous occupe ici, il emploie ces deux

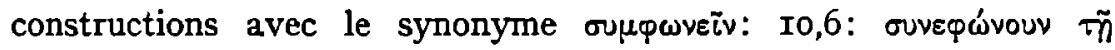

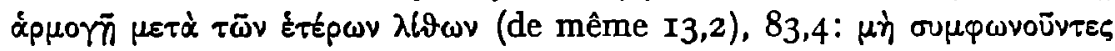

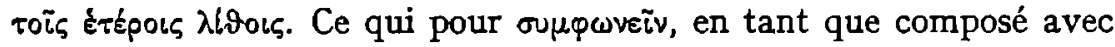
ouv-, était déjà d'un usage courant à l'époque classique (cf. supra, p.95), peut avoir servi d'exemple pour des constructions analogues avec des verbes synonymes formés d'une façon différente. Si c'est le cas, $\dot{\alpha} \rho \mu b \zeta \varepsilon \varepsilon \nu \mu \varepsilon \tau \dot{\alpha}$ est le résultat d'une évolution interne du grec, et point n'est besoin de supposer une influence sémitique ou latine. C'est d'autant plus vrai que la construction ne se présente nulle part ailleurs dans la littérature biblique, et qu'on ne trouve pas de parallèle exact en latin (cum s'emploie seulement avec des composés avec con-, tels convenire et congruere), et que, d'autre part, en grec post-classique, il

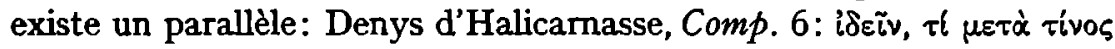

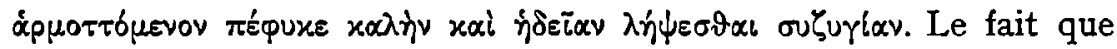
$\dot{\alpha} \rho \mu \delta \tau \tau \varepsilon เ v \mu \varepsilon \tau \dot{\alpha}$ ne signifie pas ici "s'ajuster à" mais «joindre à" n'enlève rien au sens sociatif de $\mu \varepsilon \tau \dot{\alpha}$.

Reste à parler de deux cas douteux. Dans 59,6, dans un contexte qui compte parmi les plus difficiles du Pasteur, certains ont peut-être tort d'entendre $\mu \varepsilon \tau \dot{\alpha}$ dans un sens sociatif. Le passage est le suivant:

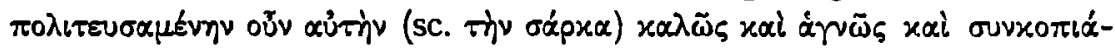

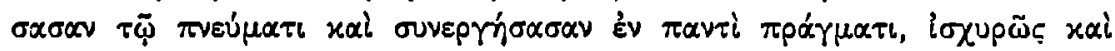

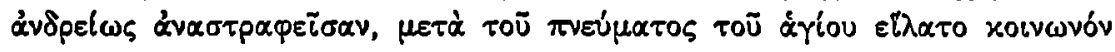
sc. $\delta$ xúplos. Le sens, à notre avis, n'est pas que le Seigneur associe la chair à l'esprit ${ }^{1}$, mais que, comme l'esprit, il en fait son associée. C'est

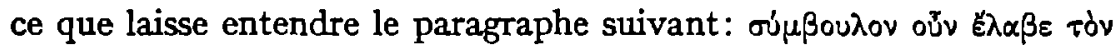

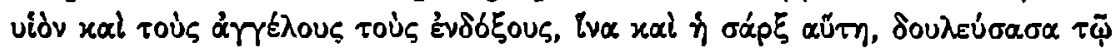

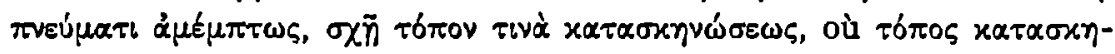

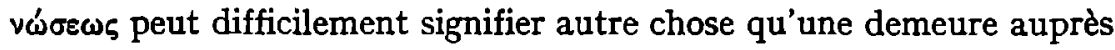
du Seigneur. Il est évident que cette relation avec le Seigneur comporte

I. Ainsi, entre autres, Dibelius et Joly dans leurs traductions et Bauer, Wörterbuch, s.v. xoเvwvbs ray. 
une relation avec l'esprit - en termes grecs xouvwvòs zoũ xupiou et

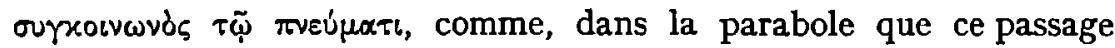

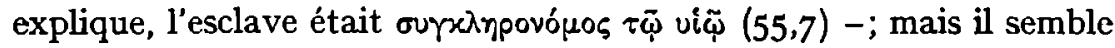
bien qu'il s'agisse ici de la relation entre la chair et le Seigneur. Dans

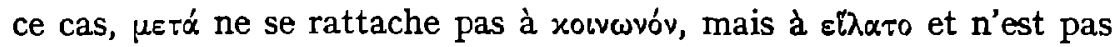
sociatif. Les deux traductions latines s'écartent assez considérablement du grec, mais semblent pourtant confirmer l'interprétation que nous donnons: cum spiritu sancto comprobatum Deo receptum est $\mathrm{L}_{1}$, probatum a Deo cum spiritu sancto receptum est $\mathrm{L}_{2}$.

Dans 90,5, le texte est incertain. Whittaker et la plupart de ses

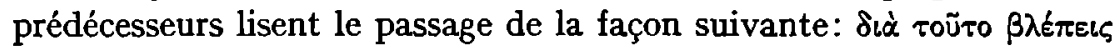

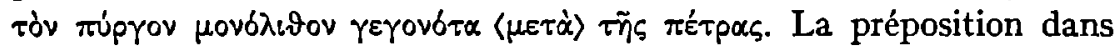
cette phrase a été ajoutée par Hilgenfeld, qui s'inspira du cum des deux versions latines: $\mathrm{L}_{1}$ : ideoque totam turrim (vv.ll.: + concolorem ou concordem ou consimilem) vides cum petra (v.l.: + et) velut ex uno lapide factam, $\mathrm{L}_{2}$ ideoque vidi (= vides?) totam turrem monolitham cum petra factam. En faveur de l'émendation d'Hilgenfeld on peut alléguer que $\mu \varepsilon \tau \dot{\alpha}$ après - $v \delta \tau \alpha$ a pu disparaître par haplographie. Le texte souligne alors l'unité entre la tour et le rocher, c'est-à-dire entre l'Église et le Fils de Dieu, et a été utilisé dans ce sens par Daniélou dans ses recherches sur l'ecclésiologie du judéo-christianisme ${ }^{1}$. Mais

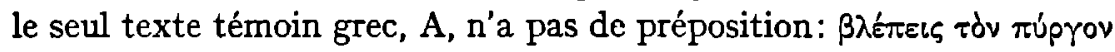

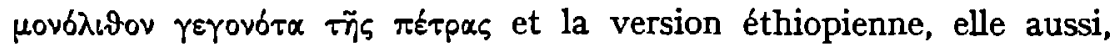
semble avoir utilisé un texte sans $\mu \varepsilon \tau \alpha ́$; elle comporte, dans la traduction latine d'Abbadie: et idcirco vides hanc turrim quasi uno lapide

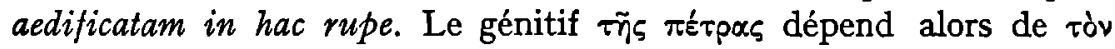
Túpyov dont il est séparé par deux mots, exactement comme on a,

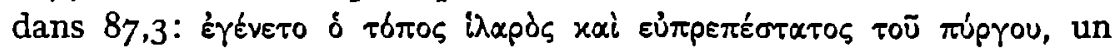
génitif séparé par trois mots de ó тóros dont il dépend. Le texte indique alors l'unité que les fidèles forment entre eux, ce qui, étant donné le contexte, n'offre aucun inconvénient, car aussitôt après, nous

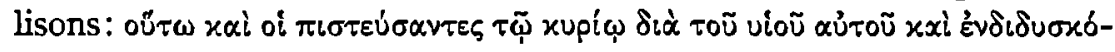

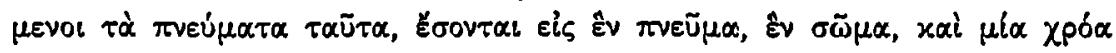

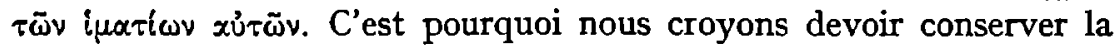
leçon manuscrite.

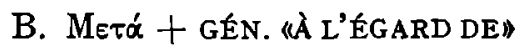

Différent en cela du grec $\mu \varepsilon \tau \dot{\alpha}$ le $\square$ hébreu et araméen, parfois n’indique pas tant la communauté que l'action de l'agent sur l'objet. C'est,

I. Daniélou, Judé-christianisme, p.333. 


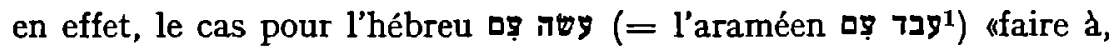

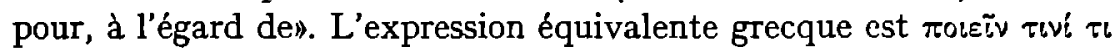

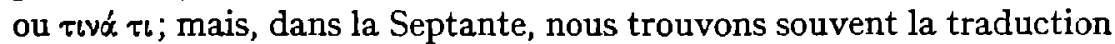

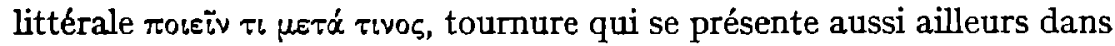

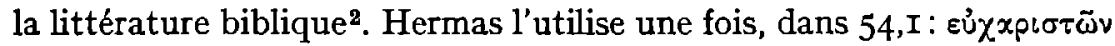

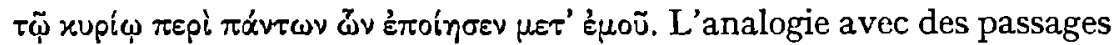

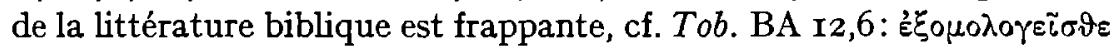

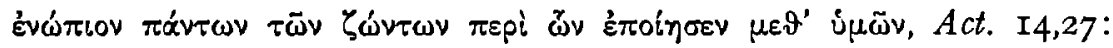

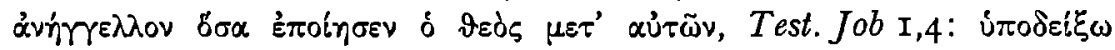

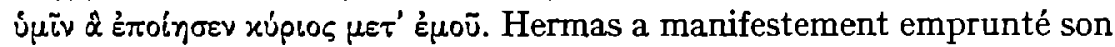
expression à cette littérature.

Meqx́ a également le sens de "à l'égard de» dans deux passages où il est question de la relation d'un homme avec ses semblables. Dans

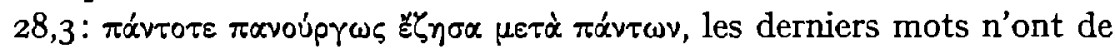
sens que s'ils signifient «à l'égard de tous»; "au milieu de tous» n'aurait

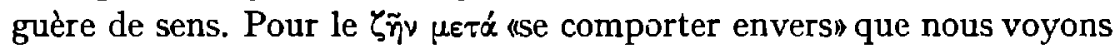
ici, nous pouvons citer deux parallèles dans des épitaphes, l'une

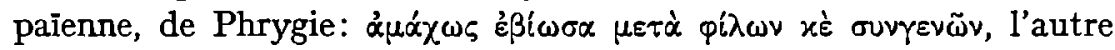
juive, de Rome: $x \alpha \lambda \omega \omega_{\varsigma} \beta \iota \omega \dot{\omega} \sigma \varsigma \mu \varepsilon \tau \dot{\alpha} \pi \dot{\alpha} v \tau \omega v$, toutes deux de l'époque impériale $^{3}$. En latin, le vivere cum correspondant est beaucoup plus habituel.

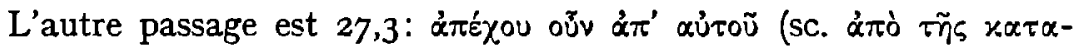

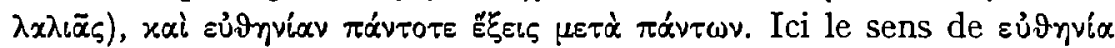
est un problème. Le sens courant, tant en grec profane que dans la Bible, est "bonheur", "prospérité", "abondance», et c'est de toute évidence le sens que lui donne $\mathrm{L}_{2}$ : et eris semper quietus inter omnes. Dans ce cas, $\mu \varepsilon \tau \alpha$ ' n'est pas sociatif, mais local. $\mathrm{Si}$, au contraire, comme

I. Pour l'araméen, voir C. C. Torrey, The composition and date of Acts, Cambridge 1916, p.38; Wilcox, Acts, p.85.

2. Avec Ėגeos comme objet: Gen. 24,12; Jud. A 1,24; 8,35; Jud. B 8,35; Ruth I,8ter; I Regn. I5,6; 20,8.14; 2 Regn. 2,6; 3,8; 9, I.3.7; 10,2bis; 15,20; 3 Regn. 3.6; I Par. 19,2bis; 2 Par. 1,$8 ; 24,22 ;$ Ps. 108,2 I;Lc. 1,72; 10,37; Jos. et As. 23,4; Test. Job I I,3; avec d'autres objets: Gen. 21,23; 26,29; Deut. 33,21; Jud. A 8,35; I I, 27; 15,3; Jud. B 8,35; 9. I6bis; I I,27; I 5,3; 2 Regn. 2,6bis; I3, I6; 4 Regn. I8,31; 2 Par. 24, I6bis.24; 2 Esdr. 6,8; 12, I2; 19, I7; Judith 8,26; I1,6.16; 13.16; 15,10; Tob. BA I2,6; 13,7; Tob. S 12,6; 13,7; I Mace. 6,49.58bis; 10,27; Ps. 85, I7;

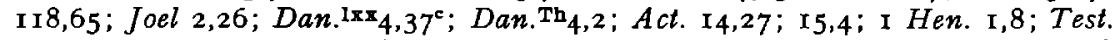
Jud. 7,7; 9, 1bis; Test. Benj. I0,3; Test. Job 1,4; emploi absolu: Gen. 21,23; Jud. B 9,19bis; 2 Par. 2,2; Tob. BA 3,6; 8, 16; Tob. S 3,6; 8, 16; Ps. 118, I24; I 25,2.3; Sir. 50,22; Ez. 23,25; Dan. ${ }^{1 \times x} 3,4^{2} ; 4,37^{\text {b }}$; $\operatorname{Dan}^{\text {th }}{ }^{1}{ }_{13} ; 3,4^{2}$. Cf. Helbing, Kasussyntax, pp. 3-9.

3. W. M. Ramsay, The cities and bishoprics of Phrygia, I 2, Oxford $I 897, \mathrm{n}^{\circ} .343,6$; CIJud. I,353. 
on le fait généralement, on entend eủinví dans le sens de «bonne entente) (comme le fait $\mathrm{L}_{\mathbf{1}}$ : et semper pacem habe cum fratre tuo), alors $\mu \varepsilon \tau \dot{\alpha}$ est sociatif. Nous ne connaissons aucun autre texte grec où zủinvic ait ce sens. Mais si c'est bien ainsi qu'il faut le comprendre ici,

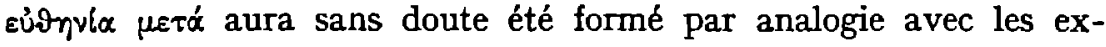

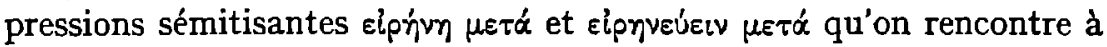
plusieurs reprises en grec biblique ${ }^{1}$.

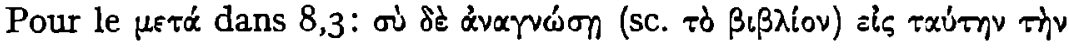

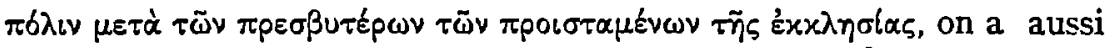
présumé qu'll s'agit de l'action de l'agent sur l'objet. A notre avis, le terme a ici la signification courante de «avec», et nous n'en parlerions pas si une opinion divergente émise par Zahn n'avait eu l'oreille de différents commentateurs ultérieurs. Il croit, en effet, que c'est

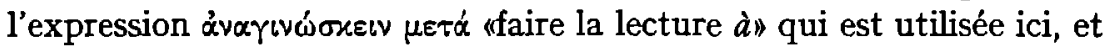
que cette expression est un sémitisme dans lequel $\mu \varepsilon \tau \dot{\alpha}$ est une traduction servile de $a^{2}$. Ceci est aussi contestable que cela. Commençons par la deuxième affirmation: avec un «faire la lecture», l'hébreu utulise pour la personne à qui on fait la lecture נגֶר ou ou

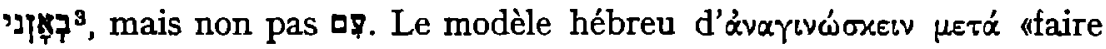
la lecture à" n'existe donc pas; ce n'est donc pas un sémitisme. Mais, qui plus est, l'interprétation du texte est erronée. Hermas ne doit pas faire la lecture aux presbytres, mais avec les presbytres aux fidèles rassemblés. La chose était préparée dans ce qui précède, 5,3: Súvn

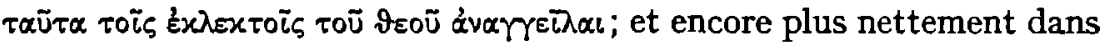

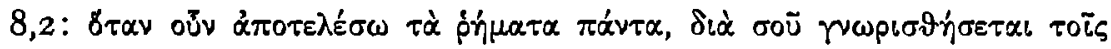

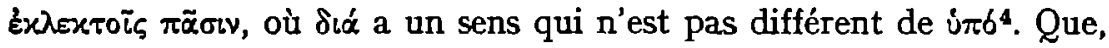
dans le même paragraphe, il soit aussi question du' don du livre des révélations aux presbytres ne fait pas contradiction; car, si l'on veut en faire la lecture aux fidèles $\mu \varepsilon \tau \dot{\alpha} \tau \tilde{\omega} \nu \pi p \varepsilon \sigma \beta u \tau \varepsilon \rho \omega \nu$, il est raisonnable

I. 3 Regn 22,45, I Macc 6,49 58bis, 10,4bis, Rom 12, 18, Hebr 12, I4, I Hen. I,8, Test Jud 7,7, 9, Ibis, aussi, par exemple, I Clem 54,2, Pass Perp

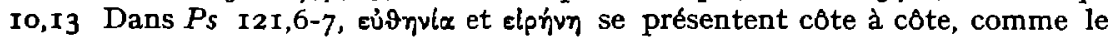

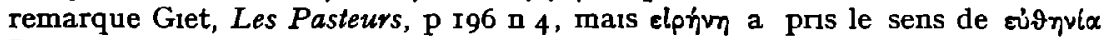
Ic1 nous avons le cas contraire

2 Zahn, Hivt, p 493, ef pp 42, 309 n 2, 376, 380 (mais $2 b$ p roo il parle de (Mittheilung desselben an die Gemeinde durch Hermas und die Presbyter») Harnack ad loc et Lelong et Dibehus dans leurs traductions, s'accordent sur ce sens, bien que Lelong, dans une note, donne comme autre sens possible ade concert avec les presbytres.

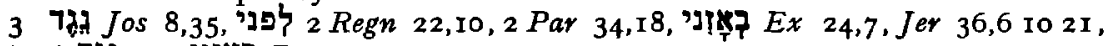

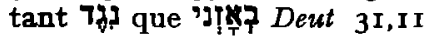

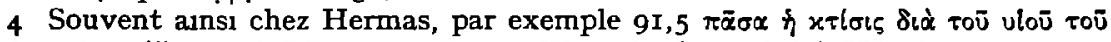

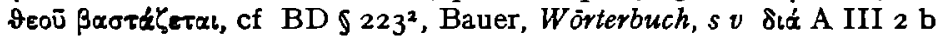


qu'ils en prennent d'abord connaissance. Nulle part non plus dans la suite du Pasteur, quand Hermas reçoit mission de divulguer la révélation $(23,5 ; 24,6 ; 1 I 2,2)$, il n'est dit que cela doit se faire par le truchement des presbytres. C'est à bon droit que Funk ad loc. compare la tâche d'Hermas à celle du prophète qui, dans Jer. 43,6 , reçoit

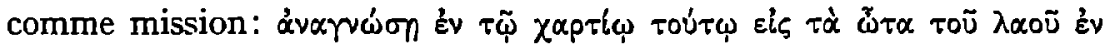

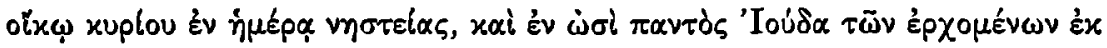

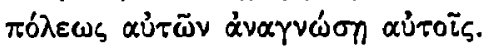

Il semble bien que personne ne se soit aperçu que l'explication de Zahn, outre qu'elle est inexacte, résulte d'une stupide bévue. Il expose ses raisons en disant que les termes ne permettent aucune autre interprétation et qu'en conséquence on les entend généralement dans ce sens; il se réfère ensuite à des publications de I832, I857, I856 et $1853^{1}$. Laissons la première remarque pour ce qu'elle est; mais en ce qui concerne la référence à des auteurs antérieurs, disons tout de suite qu'à l'exception de celui de 1857 , ceux-ci devaient encore se contenter des versions latines du Pasteur et, çà et là, de citations grecques chez les auteurs patristiques. Il se trouve qu'Origène, dans Princ. 4,2,4, nous a précisément transmis le passage qui nous occupe ici, et ce sous

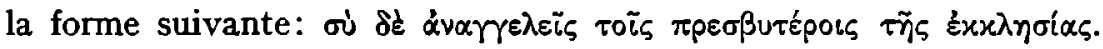
Les autorités dont Zahn s'autorise ne parlent donc pas de $\mu \varepsilon \tau \dot{\alpha}$, mais

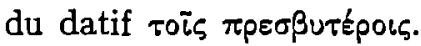

Il n'empêche que la traduction de $\mu \varepsilon \tau \alpha$ par "avec» est susceptible, elle aussi, de plusieurs interprétations. Celle de Funk, ad loc., selon laquelle il s'agit d'une lecture commune simultanée d'Hermas et des presbytres, «ita ut presbyteri quoque legentes esse videantur», ne s'accorde pas avec le fait qu'il n'y a de disponible qu'un seul exemplaire du livre de la révélation, les autres étant destinés à être utilisés ailleurs. D'aucuns ont pensé qu'Hermas et les presbytres devaient faire la lecture à tour de rôle, ou que chacun des presbytres devait lire le message à une réunion d'une partie de la communauté ${ }^{2}$. I nous semble qu'un simple $\mu \varepsilon \tau \alpha ́$ serait par trop sommaire pour indiquer de telles intentions. $\AA$ notre avis, la tâche de la lecture incombe au seul Hermas: oi $\delta \dot{e}$ ávarvwón et les presbytres sont présents à la lecture apportant ainsi leur autorité à ce que fait Hermas. Le sens de $\mu \varepsilon \tau \alpha$. prend ainsi une double nuance: "en présence de» (Joly) et «d'accord avec". Pour la première nuance, nous trouvons déjà des parallèles

I. Zahn, Hirt, p.493 n.2.

2. Voir H. Urner, Die ausserbiblische Lesung im christlichen Gottesdienst, Göttingen 1952, p.16. 
chez les auteurs classiques: Homère, $O d$. I6,I40-I4I: $\mu \varepsilon \tau \grave{\alpha} \delta \mu \omega ́ \omega \nu ~ \tau^{\prime}$

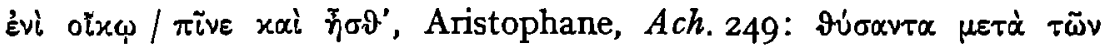

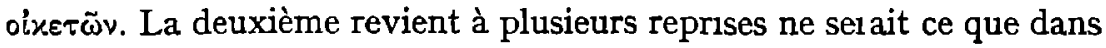
des inscriptions et des papyrus'.

\section{PREPOSITIONS IMPROPRES}

Comme on le sait, l'usage des prépositions dites impropres augmente à l'époque hellénistique ${ }^{2}$. On perçoit la même tendance dans la littérature biblique ${ }^{3}$; mais, en plus, on y trouve quelques prépositions impropres qui, en grec profane, sont rares. Dans les livres protocanoniques de la Septante, elle sont beaucoup plus fréquentes que dans le reste de la littérature biblique: c'est donc que leur vogue fut le résultat d'un procédé de traduction. Ce procédé est cependant différent de celui que nous avons vu pour les «vraies» prépositions. Si là il s'agissait de mots grecs tréquents qui prirent un sens secondaire d'après un modèle sémitique, ici ce sont des mots grecs peu fréquents qui, sans changer de sens, devinrent fréquents en grec biblique. Ce ne peut donc être qu'en raison de leurs propriétés formelles que les traducteurs de la Septante eurent tant de goût pour eux. Les propriétés qui expliquaient ce goût ont pu être les suivantes: de même que, en général, leurs équivalents sémitiques, $\mathrm{I}^{\circ}$ ces mots ont au moins trois syllabes, $2^{\circ}$ on les trouve à la fois comme adverbes et comme prépositions, $3^{\circ}$ ils sont (le plus souvent) composés. Examinons maintenant quelques-unes de ces prépositions impropres qui se présentent dans le Pasteur.

A. 'O $\pi i \sigma \omega+$ GÉN.

'O Mais comme préposition, auquel cas il se construit avec le génitif, il est rarissime. Nous n'en connaissons que six exemples: une inscription dorico-sicilienne de date inconnue, une inscription égyptienne de 239/8 avant J.-C., trois passages d'auteurs profanes de l'époque impériale et un papyrus du III'e siècle après J.-C. ${ }^{4}$, on pourrait ajouter

I Voir $\mathrm{R}$ Gunther, Die Präpositionen in den griechischen Dialektinschriften, Indogermanische Forschungen 20, 1906-I907, P (I-I63) I23, F-M Abel, Grammarre du grec biblique survie d'un chorx de papyrus, Pans 1927, p 226, Mayser, Grammatık, II 2, Pp 442-443

2 Vorr, par exemple Wackernagel, Vorlesungen, II, p 158

3 C'est ainsi que le Nouveau Testament a 42 et Hermas ${ }_{7} 7$ différentes prépositions impropres, voir Demaray, Hermas, pp I25-I 27

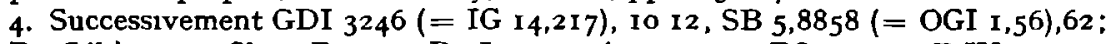

Ps Cébès 10, 1, Chio, Ep 4,3, Ps Lucien, $A \sin$ 17,29, POxy 1,43, B,IV,3 
v Homère, Il. I7,357 et Sophocle, fr.534 Pearson, où l'on trouve la forme

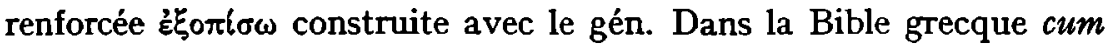
annexıs, au contraire, ó $\pi \delta \omega \omega+$ gén. se présente 34I fors: 300 fois dans les livres protocanoniques de la Septante et $4 \mathrm{I}$ fors dans les autres livres de la Septante, le Nouveau Testament et les apocryphes ${ }^{1}$. Chez les traducteurs de la Septante, ómi $\sigma \omega$ traduit presque toujours רox ou 'רצx; il se peut qu'en plus des motifs que nous avons dits, qui étaient les mêmes pour d'autres prépositions impropres, ce terme fut choisi parce qu'il ne présentait pas d'ambiguité. Il aurait été plus naturel de traduire par $\mu \varepsilon \tau \alpha \dot{\alpha}$; mais cette préposition avait l'inconvénient de prendre des sens tout à fait différents suivant le cas avec lequel on la construisait. On peut d'ailleurs se demander pourquoi, dans ce cas, le grec profane n'a pas cherché également à se prémunir contre cette éventuelle ambiguité.

Hermas emploie óríw + gén. deux fois. Du premier passage,

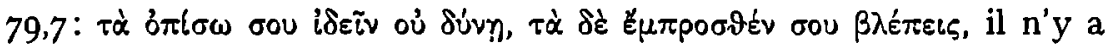

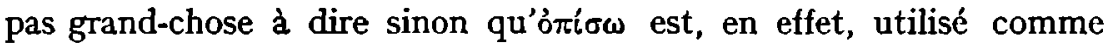
préposition; $\tau \grave{\alpha}$ ne substantive pas ó $\pi i \sigma \omega$, mais ó $\pi i \sigma \omega \sigma o v$, de même

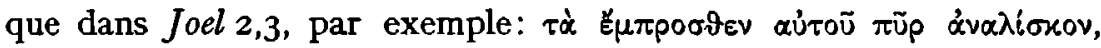

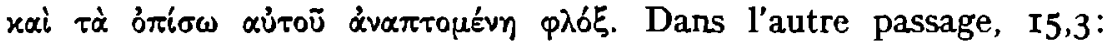

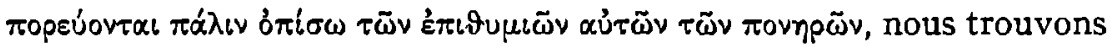
l'usage typiquement biblique d'un verbe signifiant aller avec óriow, usage que le grec profane ne connaît pas du tout². Dans la Septante, cette locution s'emploie en particulier lorsqu'il est question de suivre Dieu, des idoles ou de mauvais penchants. Comme exemple de ces

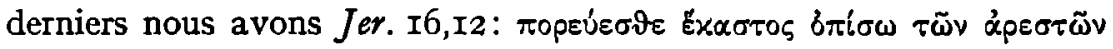

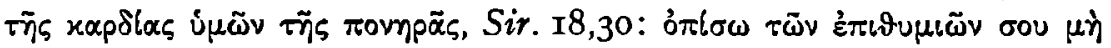
$\pi o p=u_{0} u^{3}$. L'analogie entre ces termes et ceux qu'emploie Hermas est si frappante qu'on ne peut douter qu'il se soit inspiré de la Septante.

Signalons en passant qu'ò $\pi \dot{\sigma} \sigma \omega$ se présente une fois aussi, chez

I Pour les livres protocanonıques, voir E Hatch-H A Redpath, $A$ concordance to the Septuagint and the other Greek versions of the Old Testament, Oxford 1897I 906, s v, cf M Johannessohn, Der Gebrauch der Präpositionen in der Septua-

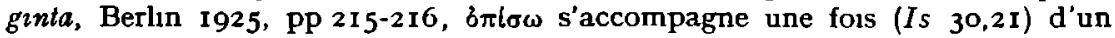
accusatif Dans le reste de la Bible grecque et les apocryphes óriow + gén se présente $10 \times$ dans I Maccabées, $3 \times$ dans Tobre, $9 \times$ dans l'Ecclésıasinque, $26 \times$ dans le Nouveau Testament et $4 \times$ dans l'ensemble des apocryphes (Jos et $A s$ $26,7,27,6$, Test Jud 13,2, Test Zab 2,6)

2 Pour le modèle sémitıque vorr, entre autres, F -J Helfmeyer, "Gott nachfolgen" in den Qumrantexten, Revue de Qumran 72,25, 1969, pp 81-104

3. Aussi, par exemple, 4 Regn I3,2, Ez 20,16,33,31, cf 2 Petr 2,10, Test Jud. 13,2, Macaruus, Elev 3 
Hermas, comme adverbe, et ce dans l'expression $\varepsilon l_{\zeta} \tau \dot{\alpha}$ ó $\pi i \sigma \omega(24,7)$. Cette expression, pour autant que nous sachions, n'existe pas en grec

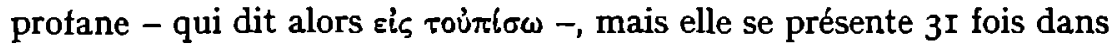
la Bible grecque ${ }^{1}$. Ici encore Hermas se conforme donc de toute évidence à la langue du grec biblique.

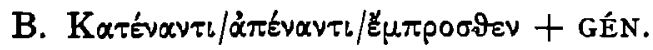

La Septante présente un ensemble de sémitismes qui est constitué par les prépositions impropres signifiant "devant», "envers»; ce sont:

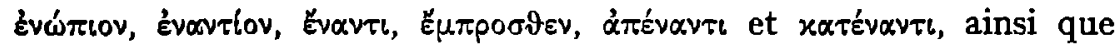
quelques autres moins tréquents, toutes construites avec le génitif. Ce sont des sémitismes par leur tréquence: on les trouve comme prépositions en grec profane, mais notablement moins que dans la Septante. Ce groupe de sémitismes a ceci de remarquable que chacun d'eux sert à rendre non pas un seul mot du texte sémitique, mais

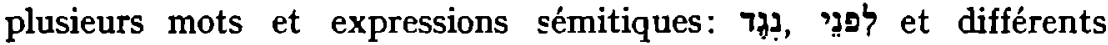
autres $^{2}$. Ils sont donc, dans une certaine mesure, interchangeables, d'où le grand nombre de variantes dans les manuscrits. Ce sont là aussi des points sur lesquels les livres protocanoniques ont été imités dans la littérature biblique ultérieure et tout ce qui en dépend. C'est ainsi que,

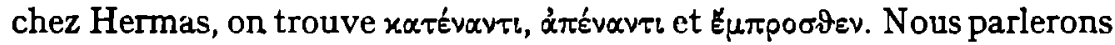
des deux premiers ensemble; le dernier demande à être expliqué séparément.

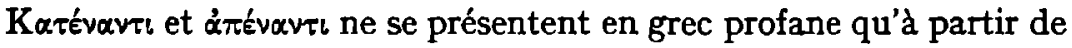
l'époque hellénistique, tant dans la fonction d'adverbe que de pré-

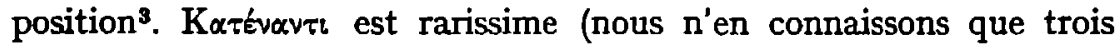
cas), ג'évavrı est plus fréquent (Ig cas) ${ }^{4}$. Mais dans les livres proto-

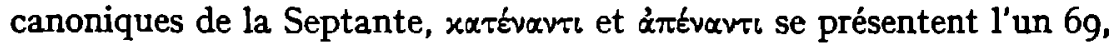
l'autre 79 fois; dans les autres livres de la Septante, le Nouveau I. Gen. 19,17.26; 49,17; Jos. 8,2; 2 Regn. I,22; 4 Regn. 20, IO.I I; I Macc. 9,47; Ps. 9.4; 34,4; 39,15; 43,11.19; 49,17; 55,10; 69,3; 77,66; 113,3.5; 128,5; Prov. 25,9; Is. 28, 13; 42, 17; 44,25; Lam. 1, 13; Mc. 13,16; Lc. 9,62; 17,31; Joh. 6,66; 18,6; 20,14. Dans les apocryphes seulement Protev. Jac. 7,2.

2. Voir J. Waldis, Die Präpositions-Adverbien mit der Bedeutung wor" in der Septuaginta, Luzern 1922, pass.; Tabachovitz, Septuaginta, pp.21-22, 52-53; Rapollo, Calchi ebraici 197I, pp.208-213.

3. Au sujet de ces mots, voir J. Wackernagel, Hellenistica, Gottingae 1907, pp. 3-6.

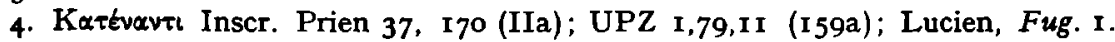
'A Évavit 4 fois dans des inscriptions, voir Wackernagel, Hellenistica, p. 3; 9 fois dans des papyrus, voir Mayser, Grammatik, I 3, p. I 20; II 2, p.538; 6 fois dans des textes littéraires (Polybe $1,86,3 ; 4,56,7 ; 4,70,5$; Ps. Scymnus 587; Diodore de Sicile $13.54,2 ; 18,34,6)$. 
Testament et les apocryphes, on le trouve respectivement $3^{8}$ et $\mathbf{2 8}$ fois ${ }^{1}$. Ils sont parfois adverbes, mais le plus souvent prépositions, et signifient généralement «en face (de)». C'est également le cas dans le Pasteur où ils se présentent en tout quatre fois, et cela uniquement dans les trois premières Visions. Voici les passages:

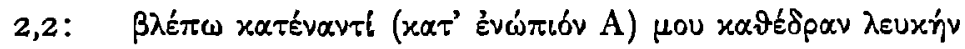

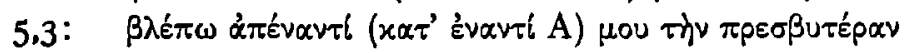

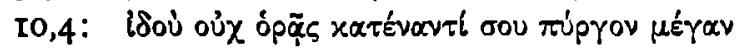

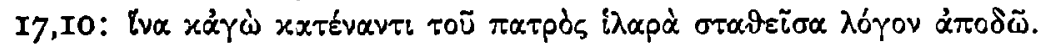

En grec profane, है $\mu \pi \rho \sigma \sigma \vartheta \varepsilon v$ s'emploie le plus souvent comme adverbe ("par devant", "devant», "en avant"; "avant», «auparavant»), moins souvent comme préposition (adevant»; "avant»). Il y est cependant moins fréquent que dans la Bible grecque et les apocryphes, où il se présente 226 fois, le plus souvent comme préposition ${ }^{2}$. Hermas l'utilise deux fois, les deux fois comme préposition. Nous avons déjà

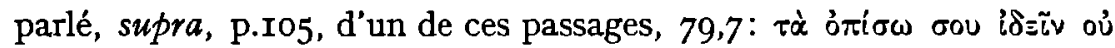

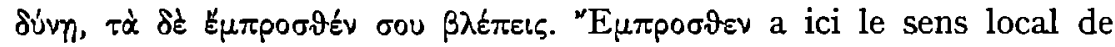
*devant qu'il a toujours eu en grec.

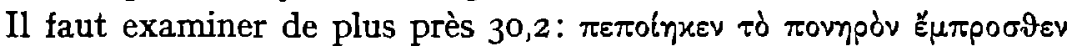

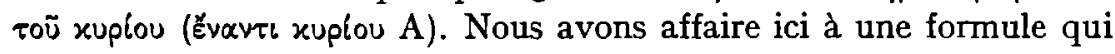
vient de l'Ancien Testament et qui, dans l'original se dit: הָדָ

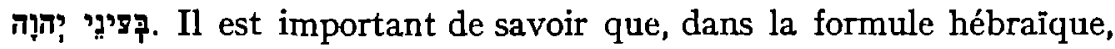

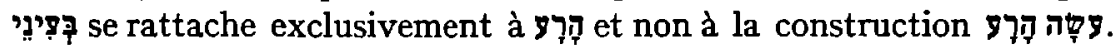
En hébreu, la formule signifie donc «faire ce qui, aux yeux du Seigneur, est mal», et non "faire le mal devant les yeux du Seigneur»" ${ }^{3}$ C'est ce qu'à certains endroits les traducteurs de la Septante ont exprimé sans ambiguité en traduisant

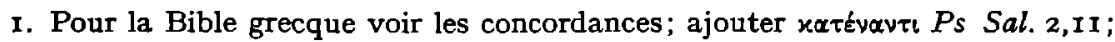
4,19, a $\pi$ évavil Ps. Sal. 2,12; 17.4. Dans les apocryphes nous trouvons 7 fois xattravti (1 Hen. 14,15; Jos. et As. 28,3.5; Test.Dan 6,2; Test. As. 1,4; 5,1; Par. Jer. 7,15) et árévavtı 6 fois (Apoc. Mos. 29; Jos.et As. 24, 19; Test. Jud. I 2, I; Acta Pauli 3, I I.30; 10,2 I).

2. Voici quelques chiffres pour le grec profane: Herodote a $\varepsilon_{\mu \pi p o \sigma \theta \varepsilon v} 9 \times$ comme adverbe et 1o $\times$ comme préposition, Polybe $23 \times$ et $2 \times$; chez Épictète le mot fait complètement défaut. Dans le Septante ces chiffres sont \pm 37 et \pm I ro, dans le Nouveau Testament 4 et 44 , dans les apocryphes 7 et 24 (les passages dans les apocryphes sont: adverbe Jos. et As. 18,5; 24,17.21; Test. Jud. 3.3; Apoc. Bar. 7,3; 8, I Test. Job 27, I; préposition Apoc. Mos. I9.29; i Hen. 89,47; Ioo, 13; Test. Abr. A I 2bis.16.19; B 2; Jos. et As. 3,2; 25,4; Test. Zab. 3,6.7bis; Apoc. Bar. 6,2; 9.3; I I,9; Ev. Petri I2.48; Ps. Barn. 3,4; 4, I2; II,4; I2, II; Apoc. Petri 6).

3. Voir Dalman, Worte Jesu, p. I74; cf. C. F. Burney, The Aramaic origin of the fourth gospel, Oxford 1922, Pp.I4-I5. 


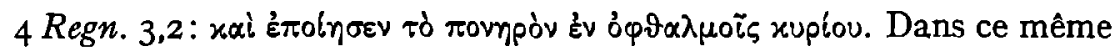
livre 4 Regn., où nous avons pris cette citation et où cette traduction

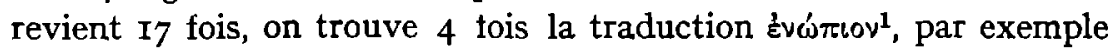

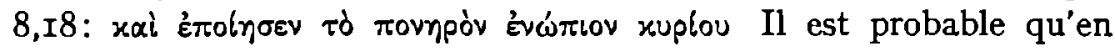
employant źvómเov le traducteur n'avait pas d'autres intentions qu'avec

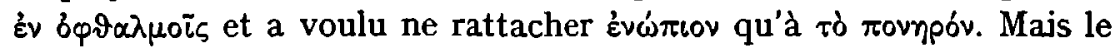
lecteur qui ne voyait que le grec a dû être tenté de rattacher évóriov

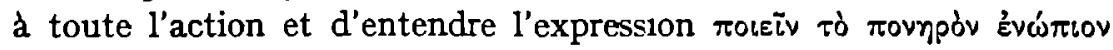
xuplou dans le sens de "pécher devant le Selgneur"². C'était d'autant plus facile que, dans le texte hébreu de l'Ancien Testament, il y avait déjà un passage qu'il fallat entendre ainsi, à savoir $N e h .9,28$, où il n'y a pas '7צ, mais לפיני "devant la face de" et où la Septante

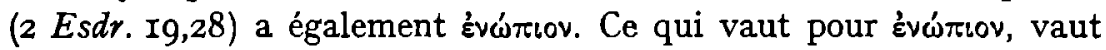
aussi pour les deux autres traductions de 9 'צ्

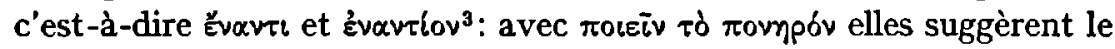
sens de "pécher devant» non pas "faire ce qui est mal aux yeux de».

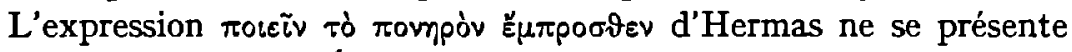
pas dans la Septante ${ }^{4}$. Étant donné qu'É une de ces prépositions impropres qui ont le sens de "devant" et dont nous avons déjà dit qu'elles sont plus ou moins interchangeables, Hermas pouvait facilement l'employer par analogie avec les expressions de la Septante. Ici aussi, la préposition se rapporte probablement à toute l'action, comme les traducteurs latins l'ont manifestement cru: sentit enim qui peccavit, quia fecit nequiter ante conspectum Domini $\left(\mathrm{L}_{1}\right)$, intelligit enim qui peccavit malitiam suam et delicta quae coram Deo fecit $\left(\mathrm{L}_{2}\right)$.

Nous avons affaire dès lors à une de ces expressions qui prennent leur source dans un sentiment de crainte révérencielle devant ce qui est puissant et sacré. Cette crainte a comme conséquence qu'on fait se

I Dans les livres protocanoniques de la Septante nous trouvons la traduction

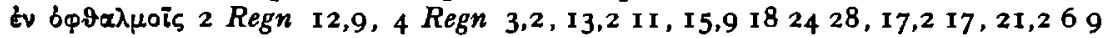
I5 I6 20; $23.3237,24,9$, Evíriov Jud B 2, I I, 3.12, 4, I, 6, I, 10,6, I3, I, I Regn 15,19, $3 \operatorname{Regn} 1 \mathrm{I}, 8,14,22,15,2634,16,192530,20,2025,4 \operatorname{Regn} 8$, I $827,14,24,24,19,2$ Par 33,22 36,9 12, Ps 50,6

2 Sur le cas où le lecteur ignorant du texte orıginal interprète la traduction dans un autre sens que celui du traducteur, voir Rabin, Septuagint, p Io n 32 3 "Evavti Num 32,13, Jud A 3,12bis, 4, I, 6, I, 10,6, Évavtiov Deut 4,25, 9, 18 , 1 7,2, 31,29, Jud A 2, I 1, 3,7, 6, I, 13, I, B 3,7, 3 Regn 22,53, 2 Par 21,6, 22,4, $29,6,33,26,36,5$, Is $65,12,66,4$, Jer 7,30 , I8, so Une fols, Jer 39,30 , une

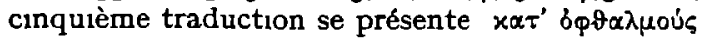

4 Même en dehors de celle-cı, elle est, pour autant que nous sachions, unique

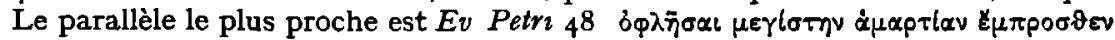
รับิ ษยoũ 
jouer "devant» le maître ce qui, en réalité, est fait par lui ou s'adresse

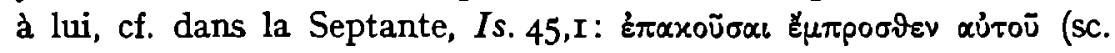

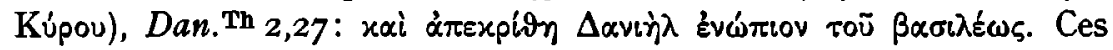
formules servent surtout à exprimer la conscience qu'on prend de la transcendance de Dieu ${ }^{1}$; mais la force première n'en est probablement plus toujours ressentie avec la même intensité. Chez Hermas, en tout cas, les formules directes et celles plus voilées alțernent ${ }^{2}$.

I. Cf. Dalman, Worte Jesu, pp.171-174, 381-382; Havers, Syntax, pp.106-1o7, $184,186,265$; Joüon, Respondit et dixit, P.312; C. T. Fritsch, The anti-anthropomorphisms of the Greek Pentateuch, Princeton 1943. Pp. I0, I2-1 3, 40-41, 47, 48, 59.

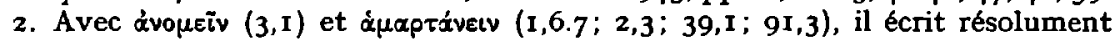

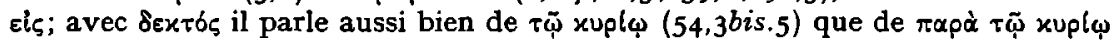
$(51,7 ; 56,8)$. Cette tendance s'affirme encore chez le traducteur de $\mathrm{L}_{\mathrm{g}}$, qui rend

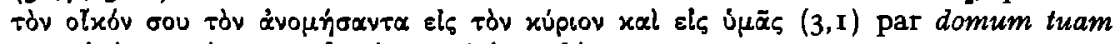
quae iniqua egit ante dominum et in vobis. 


\section{AUTRES PHÉNOMENES SYNTAXIQUES}

\section{LE "GENITIF HEBRAÏQUE*}

En grec classique, il est rare qu'on emploie un simple génitif dépendant d'un nom pour exprimer une qualités. On trouve quelques exemples,

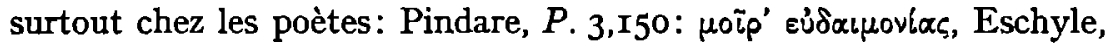

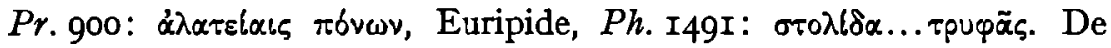
même, en grec post-classique, on ne rencontre cet usage qu'incidem-

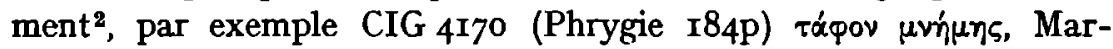

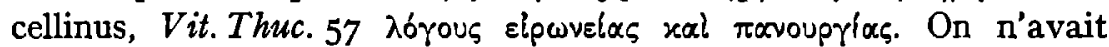
guère besoin d'un génitif de ce genre, étant donné que pour désigner des qualités, le grec possédait l'adjectif. Aussi le génitif ne s'employaitil que lorsqu'on voulait faire porter tout l'accent sur la qualité.

Mais en hébreu, où l'adjectif est peu développé, le nomen rectum, équivalent du génitif grec, est devenu le moyen usuel pour désigner des qualités. C'est ainsi que Lev. I,9 רים־ signifie un parfum agréable,

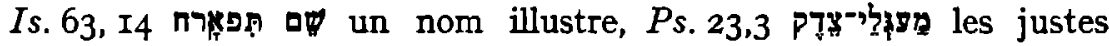

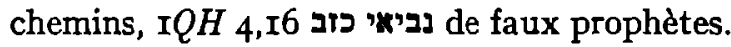

Les traducteurs de la Septante optèrent le plus souvent pour la traduction littérale de ces constructions ${ }^{3}$. C'est ainsi que les expressions paléotestamentaires que nous venons de citer furent traduites

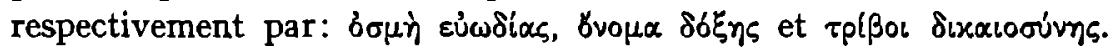
Même là où le texte sémitique original est perdu, nous pouvons souvent presque à coup sûr parler d'une traduction littérale, comme Ps. Sal.

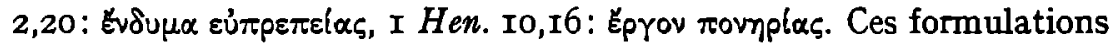

I. Voir Kühner-Gerth, Grammath, II 1, p 264; SD II pp 122, I 29; Lasso de la Vega, Sintaxıs, I, pp. 473-475 En latın non plus le génıtif de qualité sans adjectif épithète n'est pas très courant, cf Szantyr, Syntax, p 70.

2 Passages chez E Nachmanson, Syntaktzsche Inschriftenstudien, Eranos 9, 1909, pp (30-8I)63-66, Radermacher, Grammatzk, pp Io8-ro9, i I I.

3. On attend encore une étude complète de cette matière complexe. Passages chez Johannessohn, Kasus, pp 27-28; E Percy, Die Probleme der Kolosser- und Epheserbriefe, Lund 1946, p 252. 
n'ont pas tardé à faire école dans la littérature judéo-grecque et

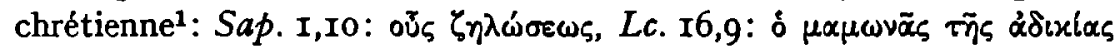

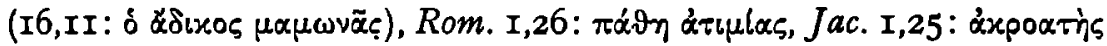

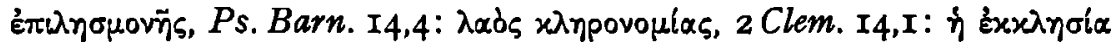
$\tilde{r}_{j} \zeta_{\omega} \tilde{\eta}_{5}$. Dans la littérature biblique, cet usage a été manifestement stimulé par les exemples sémitiques, à tel point même qu'on a parlé de "génitif hébraĩque». Mais il pouvait prolonger ce qui avait commencé en grec classique. Comme dans cette langue, il met l'accent sur la qualité2. C'est ainsi qu'il se prête particulièrement à la formation d'expressions à contenu théologique ou moral.

Le grec a repris la particularité hébraique qui consiste à placer auprès du nomen rectum un possessif ou un démonstratif qui, logique-

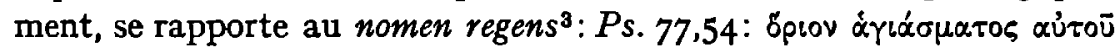

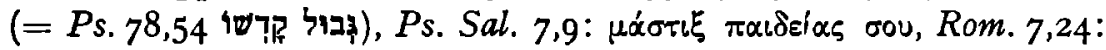

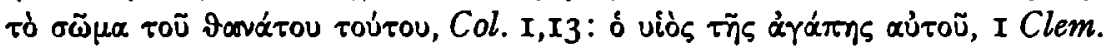

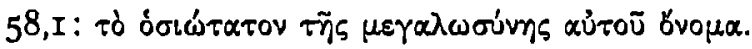

Chez Hermas aussi, l'on trouve des exemples du "génitif hébraĩque et de l'hypallage du pronom auquel ce génitif donne lieu parfois". Voici groupe par groupe les exemples que l'on trouve chez lui:

\section{a. avec é $\pi \iota \vartheta u \mu l \alpha$}

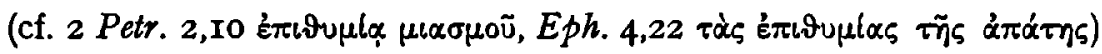

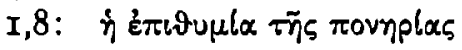

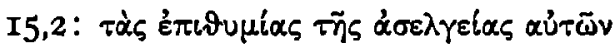

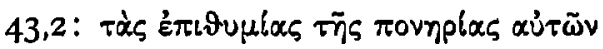

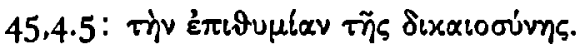

En ce qui concerne $I, 8$ et $45,4.5$, on peut se demander si le génitif indique une qualité ou un objet. Dans $r, 8$, nous penchons pour la première interprétation à cause de l'analogie avec 43,2 , où $\pi$ oviplac désigne sûrement une qualité et à cause du contexte qui fait de

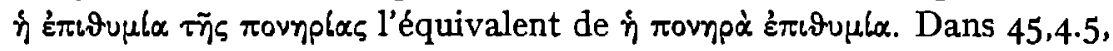
le choix est plus difficile, mais les arguments ne manquent pas non plus en faveur de la même interprétation: Hermas utilise parfois

I. Passages chez Percy, Probleme, pp.197-199, 250-252; Tumer, Syntax, Pp.212214.

2. Cf. E. Pax, Die syntaktischen Semitismen im Neuen Testament, Studii Biblici Franciscani Liber Annuus 13, 1962-1963, pp.(136-162) 145-146.

3. Cf. W. Gesenius-E. Kautzsch, Hebräische Grammatik, Leipzig 190928, p.46r; Tumer, Syntax, P.2I4.

4. Déjà examiné en partie par Zahn, Hirt, pp.493-494; Percy, Probleme, P.25I;

G. Harder, art. $\pi$ ov $\eta p l \alpha$, ThWNT VI, Pp. (562-566) 565-566. 


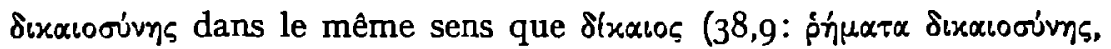

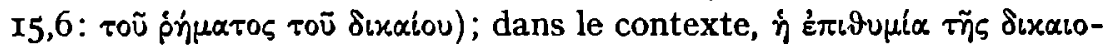

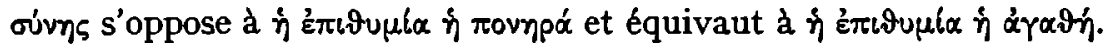

b. avec ג'pern

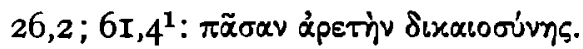

On peut entendre $\delta \iota x \alpha 10 \sigma i v \eta n$ comme indiquant une perfection spécifiquement chrétienne et considérer alors le génitif comme le substitut d'un adjectif. C'est ainsi que Bauer interprète: rjede Tugend d. Gerechtigkeit $=$ jede christliche Tugend $\|^{2}$. Mais en tenant compte

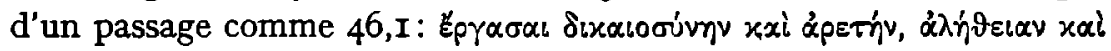

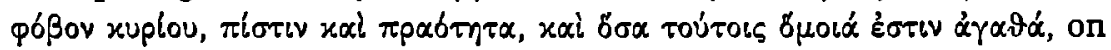

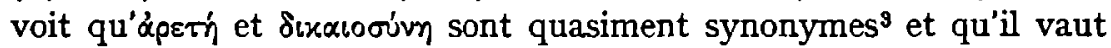
mieux interpréter le génitif comme un gentivus inhaerentiae 4 . Ici $\delta$ ¿xacooúrns joue presque le rôle d'épithète d'ornement qui échoit à

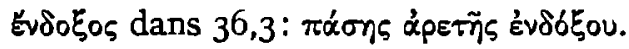

\section{c. avec $\pi v \varepsilon \tilde{\mu} \mu \alpha$}

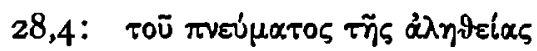

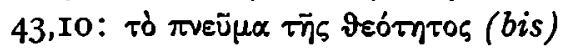

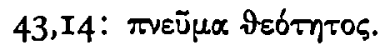

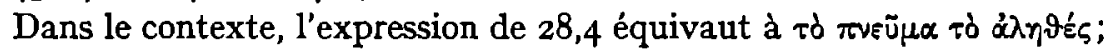

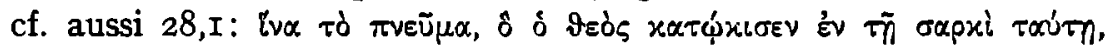

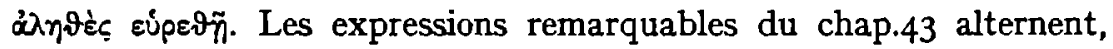

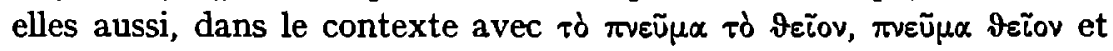
VEīov $\pi v E \bar{u} \mu \alpha$.

\section{d. avec Épy $\alpha$}

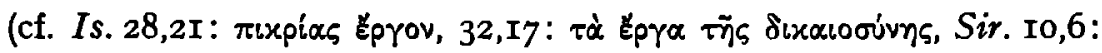

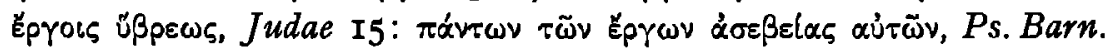

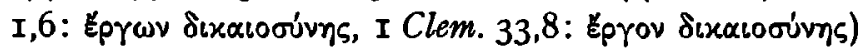

I. A la suite d'une conjecture de Bonner, Whittaker et Joly lisent aussi cette

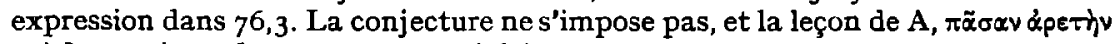

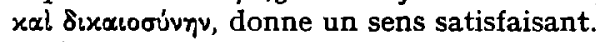

2. Ainsi Bauer, Wörterbuch, s.v. גperń $\mathrm{I}$.

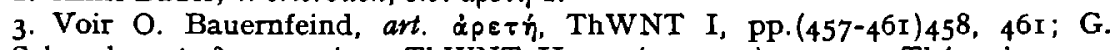

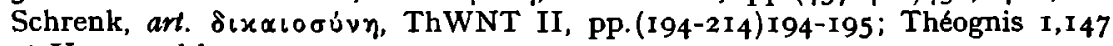
et Young ad loc.

4. On trouve des exemples de ce génitif dans la Bible chez P. A. H. J. Merkx, Zur Syntax der Kasus und Tempora in den Traktaten des hl. Cyprian, Nijmegen 1939, pp.I2-13; Percy, Probleme, pp.r94-I97. Pour le grec classique voir Lasso

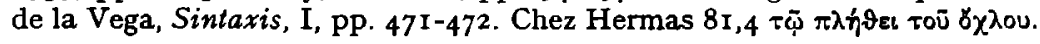




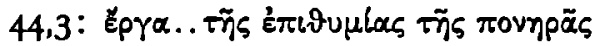

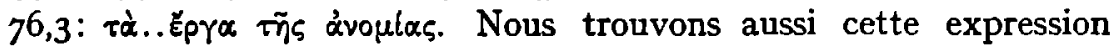
dans Is. 59,6; Ps. Barn. 4,I; cf. 2 Petr. 2,8 ávónous Épyous.

e. autres cas

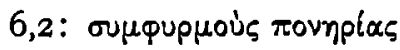

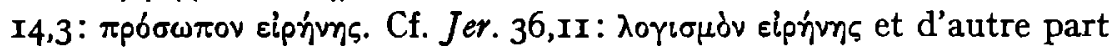

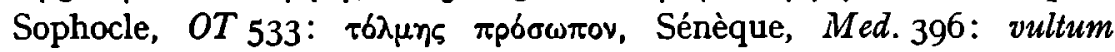
furoris.

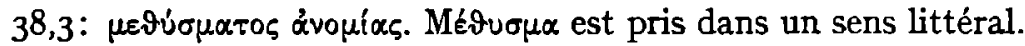

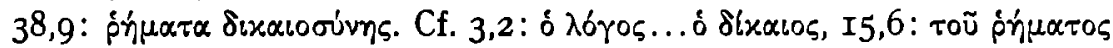

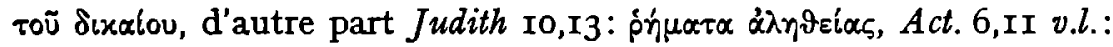

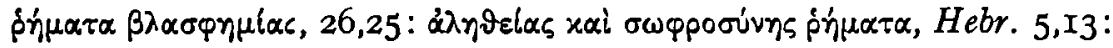

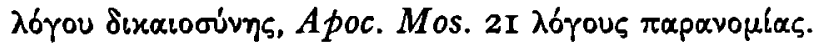

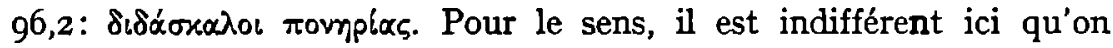
entende le génitif comme désignant une qualité ("böse Lehrer» Dibelius $^{1}$ ) ou un objet ("des docteurs du vice" Joly).

La conclusion que nous pouvons tirer de ces données, c'est qu'ici Hermas emploie une construction qui n'était pas tout à fait étrangère au grec profane, mais qu'il a probablement apprise dans la Bible grecque, où l'usage a été stimulé par l'influence de l'hébreu.

\section{LE REDOUBLEMENT DISTRIBUTIF}

Pour indiquer qu'une affirmation vaut pour chaque partie d'un tout,

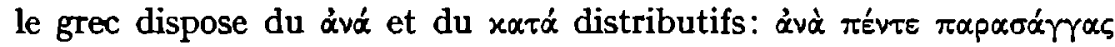

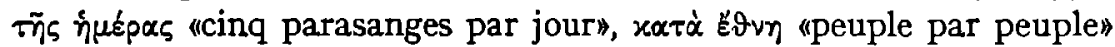
(Xénophon, $A n, 4,6,4$ et $\mathrm{r}, 8,9$ ). Hermas aussi connaît cet usage:

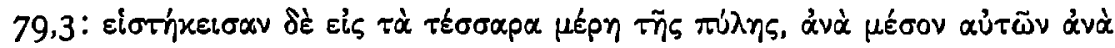

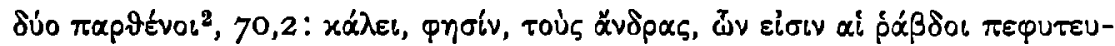

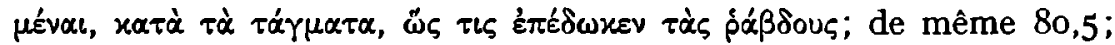
83,3 bis. En outre, il utilise une fois ${ }^{3}$ le redoublement dit distributif:

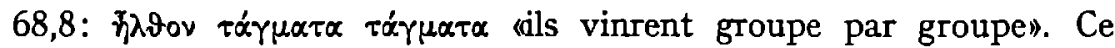
procédé est très inusuel en grec, mais c'est la manière normale de

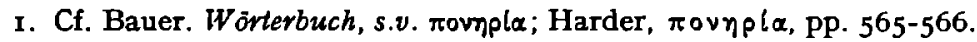

2. Ici, ג'vá est devenu adverbe, cf. Radermacher, Grammatik, p. 30; Mayser, Grammatik, II 2, Pp. 44, 402-403; Liddell-Scott-Jones, Lexicon, s.v. ávó́ D; $\mathrm{BD} \S 305$.

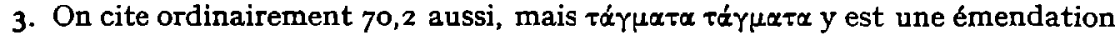
superflue. C'est à bon droit que Joly lit, avec les manuscrits, $\pi \dot{\alpha} v \tau \alpha \tau \tau_{\alpha} \tau \alpha \dot{\gamma} \gamma \mu \alpha \tau \alpha$. 
s'exprimer dans les langues sémitiques ${ }^{1}$. On peut donc se demander si, chez Hermas, ce n'est pas un sémitisme. C'est ce que nous voulons voir de plus près.

Tout d'abord, il est bon de se rappeler que des langues qui n'ont aucun contact généalogique ou autre avec les langues sémitiques possèdent aussi le redoublement distributif ${ }^{2}$. En principe, cette forme d'expression a donc pu s'introduire aussi dans la langue grecque indépendamment d'une influence sémitique. Si c'est bien le cas, nous pouvons comprendre aussi pourquoi elle n'a pas pu se faire une place dans la langue écrite à côté du ává et du $x \alpha \tau \alpha ́$ distributifs. Le redoublement, en effet, a souvent une valeur expressive et affective, et une telle valeur convient mal à la sobriété et l'intellectualité de la prose littéraire $^{3}$. Il va sans dire que ce n'est là qu'une supposition, mais il est assez remarquable que les seuls redoublements distributifs que nous ayons pu recueillir dans les textes grecs profanes, se trouvent soit dans des textes poétıques soit dans des textes non-littéraires.

C'est ainsi que nous trouvons des redoublements de substantifs employés comme compléments de temps dans deux anciens textes

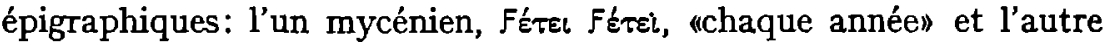

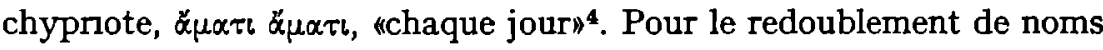
de nombre, cette fois comme complément attributif, nous avons trois exemples, dont deux nous viennent de la tragédie et le troisième d'un papyrus non-littéraire:

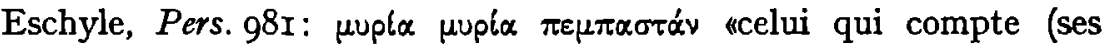
sujets) par groupes de dix mille» ${ }^{5}$.

Sophocle, fr.zor Pearson, transmis par l'Antiatticiste dans Bekker

I Voir H Reckendorf, Uber Paronomasie in den semitzschen Sprachen, Giessen 1909, pp I46-I50, Johannessohn, Kasus, P 3, Gesenius-Kautzsch, Grammatzk, pP 413-4I 4, 456-457, A J Wensinck, Un groupe d'aramarsmes dans le texte grec des évangiles, Amsterdam 1936, pass En latin, le redoublement distributif des substantıfs et des noms de nombres fait quasıment défaut, cf Szantyr, Syntax, p r97. Rapallo, Calchn ebrarcr 1969. pp 396-398

2 Vorr, par exemple, J Gonda, The functions of word duplication in Indonesian languages, Lingua 2, I949, Pp (170-197) I 71, 176-177, 178, 180, 193

3 Cf Gonda, Duplication, p I70, J B Hofmann, Laternische Umgangssprache, Herdelberg $195^{3}$, Pp 60-6I

4 Volr $O$ Masson, $A$ propos de deux formules redoublées au locatzf mycénzen weterweter "chaque année", chyprote amatramatı "chaque jour", Żiva Antika 15 , I966, pp 257-266 L'inscnption chypnote date de \pm 600 avant J -C ( $2 b$ p 258) 5 Ce passage peut aussı être interprété d'une manière autre que distributive, vour Radermacher, compte-rendu de J $\mathrm{H}$ Moulton, Einleitung in die Sprache des Neuen Testaments, p (6-ro)8, Mussies, Koine Greek, p 218 


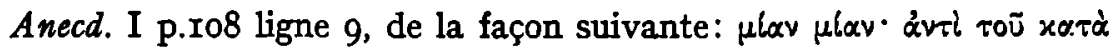

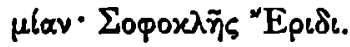

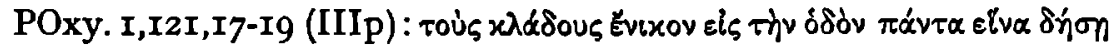

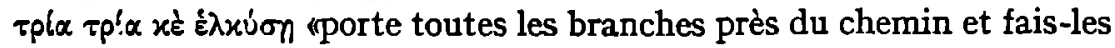
attacher trois par trois et enlever".

En grec post-classique, nous trouvons aussi des contaminations comme

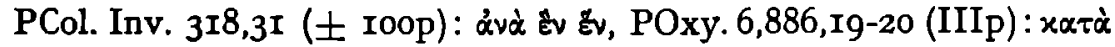

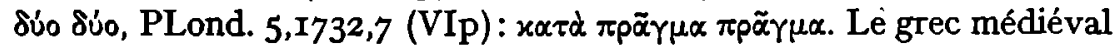
et le grec moderne connaissent également le redoublement distributif de substantifs'.

Dans la Septante, le redoublement de l'original sémitique est parfois escamoté, signe que les traducteurs, malgré leur tendance au littéralisme, n'étaient pas sûrs de pouvoir s'exprimer ainsi en grec. Les passages où il est employé ne manquent pourtant pas ${ }^{2}$, par exemple,

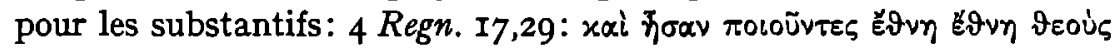

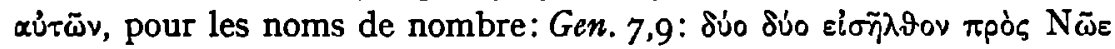

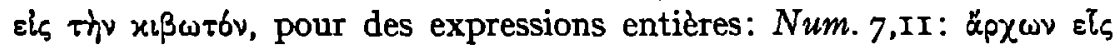

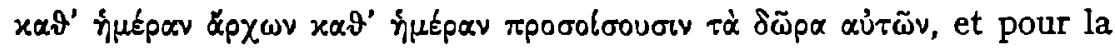

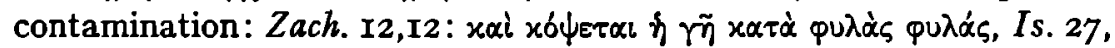

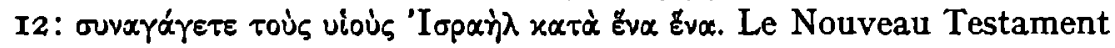
et les apocryphes connaissent aussi le phénomène. Citons seulement les passages qui comportent un redoublement de substantifs:

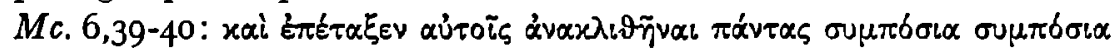

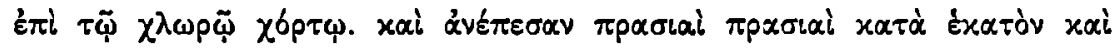

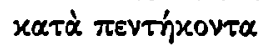

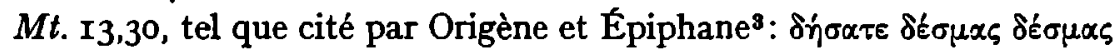

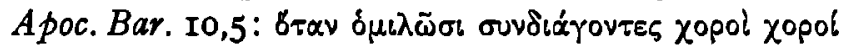

I. Voir J. Psichari, Essai sur le grec de la Septante, Revue des Etudes Juives 55, 1908, P. (I6I-208) I 84; BD $\$ 24^{1}$.

2. Voir Johannessohn, Präpositionen, pp.25I-253; Bickerman, Septuagint, p.28; Doudna, Mark, pp.96-98; Rapallo, Calchi ebraici 1969, pp.388, 393-398. Pour avoir une impression exacte, il est nécessaire de connaître la répartition et la fréquence du phénomène. C'est pourquoi nous donnons ici la liste complète des passages dans la Bible grecque et les apocryphes. Redoublement des substantifs: Lev. I 5,2; 17,3.8.10.1 $3 ; 18,6 ; 20,9 ; 22,18 ;$ Num. 5,$12 ; 7,11 ; 9,10 ; 17,17 ; 28,13 v . l$. 21.29; 29.4.I0.15; 30.3; 4 Regn. 17,29.32; 2 Par. 31,6; Jer. 50,11; Ez. 14,4.7; Mt. 13,30v.l.; Mc. 6,39,40; Apoc. Bar. 10,5; Hermas 68,8; redoublement des noms de nombres: Gen. 6, 19.20; 7,2.3.9.15; Num. 31,4.6; I Par. 24,6; Sir. 33, 15; Mc. 6,7; Test. As. 1,4; préposition + redoublement des substantifs: r Regn. 7,16; 3 Regn. 10,25; 2 Par. 9,24; Zach. 12,12; préposition + redoublement des noms de nombres: 3 Regn. 18,13v.l.; Is. 27, 12; Lc. 10, 1v.l.; Ev. Petri 35.

3. Origène, Hom. in Jer. I, I5; Épiphane, Haer. 66,65,3. BD $\$ 493^{2}$ estiment qu'il s'agit de la leçon originale, parce que lectio difficilior. 


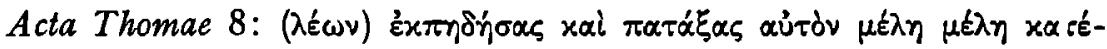

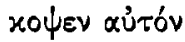

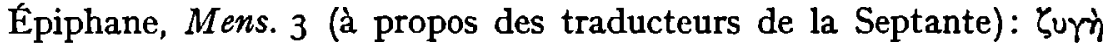

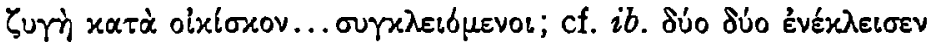

Dial. Tim. et Aquil. p.90-gI Conybeare (également à propos des

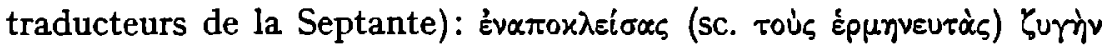
కurív.

$\mathrm{Si}$ on considère ces données, on fait les constatations suivantes:

I. le grec profane connaissait dans une certaine mesure le redoublement distributif.

2. ce redoublement est beaucoup plus fréquent dans le grec de la Septante sous l'influence de l'original sémitique; il s'y présente aussi sous une forme que nous ne retrouvons pas dans nos textes profanes: comme redoublement de substantifs avec la fonction de complément attributif.

3. nous retrouvons aussi cette forme dans les textes écrits en grec dans la ligne de la Septante, entre autres chez Hermas.

Devant cet état de choses, on serait tenté d'expliquer l'emploi $\mathrm{n}^{\circ} 3$ à partir de l'emploi $n^{\circ} 2$, donc d'admettre qu'il s'agit d'un sémitisme. Mais il n'est pas impossible que de nouvelles trouvailles nous amènent à reviser notre jugement. $D^{\prime}$ abord, dans les exemples que nous avons donnés pour l'emploi $n^{\circ} 3$, ni le choix des mots ni le contexte ne rappellent la Septante ${ }^{1}$. Il se pourrait d'ailleurs (cf. supra, p. II4) qu'on ait évité le redoublement distributif dans la prose littéraire, c'est-à-dire dans la grande majorité des textes qui nous sont parvenus; à cet égard, ces textes nous donneraient alors une image erronée du grec de l'époque à laquelle ils ont été écrits ${ }^{2}$. Si ce redoublement se présente dans la littérature qui dépend de la Septante, ce pourrait être parce que les auteurs ne se croyaient pas tenus par les normes de la prose littéraire.

\section{LA PARATAXE DANS LES SUBORDONNÉES}

C'est une erreur d'opposer les langues sémitiques au grec et au latin en disant que les premières ne connaissent guère l'hypotaxe, alors que les dernières l'utilisent presque toujours lorsqu'il faut exprimer des choses de valeur inégale dans une seule phrase. En grec et en latin

I. Cf. pour Hermas 68,8 Zahn, Hirt, p.490.

2. G. Schulze, Graeca Latina, Göttingen IgoI, p. I3 suppose une évolution interne du grec; de même, semble-t-il, Abel, Grammaire, P.I53. 
aussi, les exemples de parataxe sont légion pour la liaison de grandeurs inégales. C'est le style qui détermine le choix. Dans un style proche de la langue parlée, l'auteur ne se donnera souvent pas la peine d'exprimer au moyen de subordonnées et de participes les rapports logiques entre ses idées'. S'il s'exprime d'une façon plus intellectualiste, les subordonnées et les participes seront nombreux. Il est évident que l'hypotaxe ne sert pas seulement à exprimer le rapport entre les choses principales et les choses secondaires, mais aussi le rapport entre les choses secondaires et d'autres choses secondaires qui en dépendent. Les auteurs qui s'expriment de façon hypotactique le font d'une façon conséquente. C'est pourquoi, en grec, nous ne trouvons presque pas d'exemples de subordonnées, de participes ou d'infinitifs paratactiques lorsque ne sont pas désignées par là des choses égales entre elles.

Il y a une exception pourtant: c'est quand l'auteur veut exprimer une opposition plutôt qu'une inégalité dans l'importance ${ }^{2}$; nous trouvons alors une construction paratactique à l'aide des particules

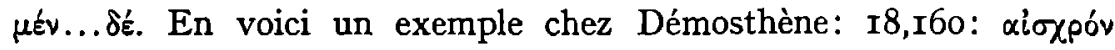

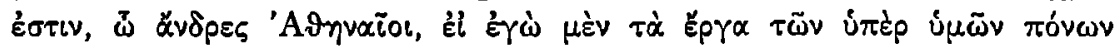

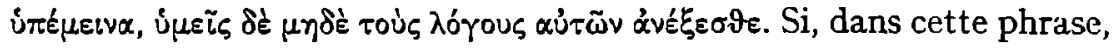
qui comporte une principale et une subordonnée à deux membres, nous examinons le rapport de la principale avec chacun des deux

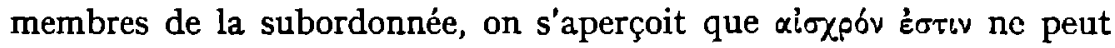
s'appliquer au premier membre, mais bien au deuxième. Il va sans dire que ce n'est pas ainsi qu'il faut lire ce passage. La subordonnée à deux membres torme un tout indivisible qui dépeint la situation paradoxale que la principale condamne. Il n'empêche que, dans les textes profanes, cette formulation est extremement rare. Les circonstances indiquées par une subordonnée à deux membres ont beau former un tout, si leur valeur par rapport au contenu de la principale est inégale, cette inégalité s'exprime habituellement d'une façon syntaxique. Inversement, là où des subordonnées ont un lien paratactique, c'est qu'elles ont le même genre de relation à la principale.

Pour exprimer le rapport des idées par la parataxe et l'hypotaxe, il faut une ordonnance de la pensée qui est plus facile à réaliser dans la langue écrite que dans la langue parlée. Dans la langue parlée, il sera bien souvent arrivé, même en grec, qu'une parataxe logiquement in-

I. Voir Trenkner, Style xal, pass.

2. Cf. Kühner-Gerth, Grammatik, II, pp.232-234; Beyer, Semitische Syntax, pp.265,27l. 
correcte se présente dans les subordonnées, les participes et les infinitifs. A supposer que la chose ait passé dans les textes écrits, il n'en est rien parvenu jusqu'à nous - du moins, pour notre part, n'en connaissons-nous pas d'exemples -; c'est sans doute parce que, du point de vue du style, on voyait là trop d'incorrection. Mais dans les papyrus non-littéraires, qui nous sont parvenus par hasard et non grâce au choix fait par les générations ultérieures, nous trouvons quelques passages, par exemple BGU 2,424,I0-I2 (II-IIIp): $\mu \varepsilon \mu q о \mu \alpha \iota$

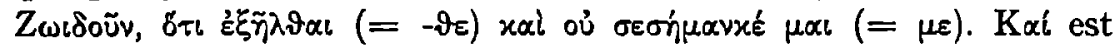
essentiel ici: il indique un lien, mais ce lien est une opposition: ket pourtant». Cela n'empêche d'ailleurs pas que le premier membre de la subordonnée, si on le considère isolément, ne se rapporte pas directement à la principale. Dans les textes littéraires qui nous sont parvenus, on évite presque toujours cette façon de s'exprimer. Mais, dans les papyrus, on en trouve plus d'exemples. Le Gnomon de l'Idiologue, recueil de règles de droit d'environ $\mathrm{I}_{50}$ après J.-C., en offre plusieurs

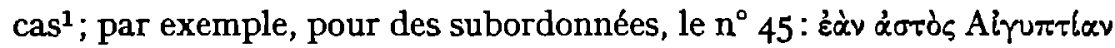

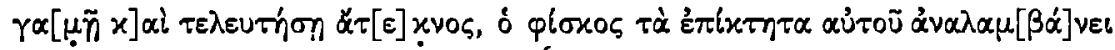
qquand un bourgeois épouse une Egyptienne et meurt sans enfants, le fisc recueille ses acquêts"; et, pour des participes, le $\Omega^{\circ} 2 I$ : ó ż $\lambda \varepsilon v \vartheta \varepsilon-$

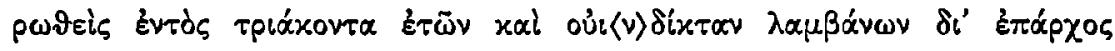

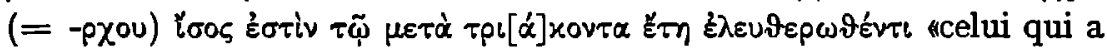
été affranchi avant trente ans et reçoit la vindicta du préfet est comme l'affranchi de trente ans*.

A propos des deux dernières citations remarquons deux choses. D'abord, qu'il s'agit de textes juridiques. Or, c'est précisément dans des textes juridiques, où il est souvent question d'un ensemble de conditions qui ne sont pas toujours égales, que le locuteur se sera souvent trouvé devant le choix de l'hypotaxe ou de la parataxe. Les critères de la langue littéraire ne valaient pas pour les textes techniques comme les textes législatifs; là où la clarté n'en souffrait pas, on aura souvent eu recours à la parataxe pour ne pas compliquer inutilement l'énoncé.

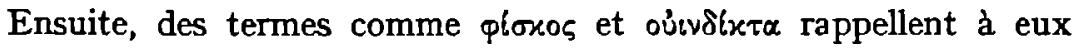
seuls l'influence romaine dans le Gnomon. Faut-il également attribuer à cette influence la parataxe dans des subordonnées et des participes? Sur ce point, la situation du latin ne nous semble pas différer de celle du grec. Dans les deux langues, le style littéraire exigeait une correction 
rigoureuse dans l'emploi de la parataxe et de l'hypotaxe' et, dans l'une et dans l'autre, on se laissait probablement entraîner plus facilement à renoncer, dans les subordonnées, à l'hypotaxe au profit de la parataxe lorsqu'il s'agissait de textes juridiques. On a des exemples de ce phénomène en latin: Pline, Ep. Tra. 10,97,I : si deferantur et arguantur, puniendi sunt, Gaius, Inst. I,67: si civis Romanus... peregrnam uxorem duxerit... et filium procreaverit, hic non est in potestate eius. Or, étant donné qu'à l'époque impériale la pratique du droit était, même en Orient, entièrement calquée sur le droit 1omain, beaucoup de formules grecques, y comprs celles du Gnomon, ont sans doute été inspirées par les modèles latins. Mais si nous avons raison de supposer que, dans les documents juridiques, le grec employait aussi volontiers que le latin la parataxe dans les subordonnées, nous ne pouvons voir là des latinismes.

Les langues sémitiques contrastent fortement avec le grec et le latin. En hébreu et en araméen, qui ne connaissent guère la double subordination et le participium coniunctum, l'emploi de la parataxe dans les subordonnées est un phénomène des plus fréquents ${ }^{2}$. C'est ainsi que Lev. 18,5 et $E z .20,13$ parlent de commandements רשุ

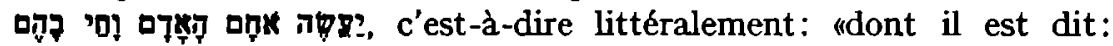
l'homme les accomplira et par là il vivra", mais cela signifie: "si l'homme les accomplit, il vivra par là».

En général, la Septante traduit littéralement, bien qu'on trouve aussi des formules plus grecques ${ }^{3}$. C'est ainsi que le texte hébreu que

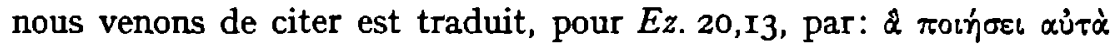

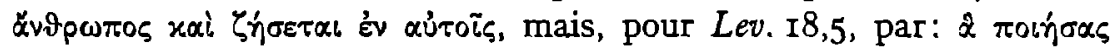

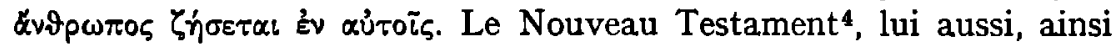
que les écrits grecs qui lui sont apparentés, n'hésitent pas à utiliser cette manière de parler. A chaque fois, la conjonction ou le relatif commun n'a de rapport qu'avec la dernière subordonnée. La conjonction est toujours xal. Voici quelques exemples: Test. Job 9,8: Éxé $\lambda \varepsilon v o v$

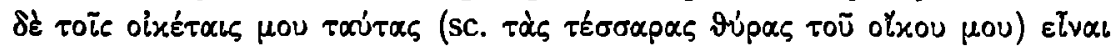

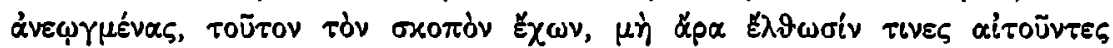

r. En latın aussı on s'écarte rarement de cette règle Voıcı un exemple avec des infınıtıfs Phèdre I,9, I-2.

sibi non cavere et alirs consilium dare

stultum esse paucis ostendamus versibus

2 Vour P Jouon, Grammarre de l'hebreu brblıque, Rome 1947², PP 520, 524; Beyer, Semitische Syntax, pp 259-265

3 Volr Beyer, Semitzsche Syntax, p 260

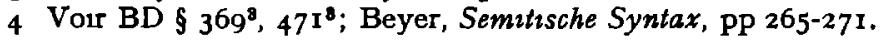




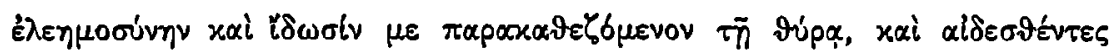

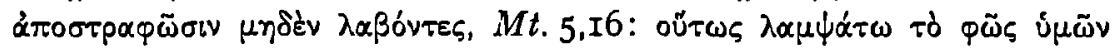

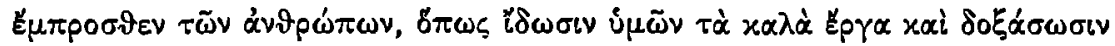

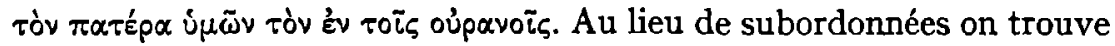
parfois aussi des participes et, rasement, des infinitifs: Sir. 34,25:

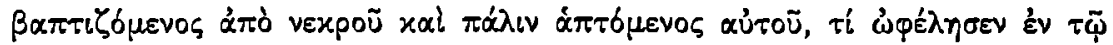

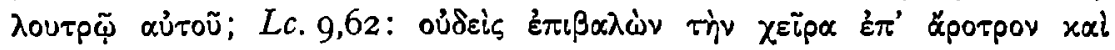

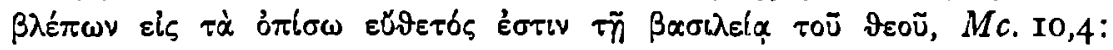

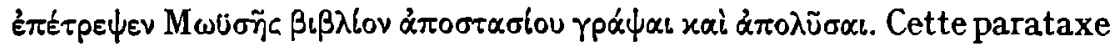
était particulièrement en faveur pour donner des prescriptions dé-

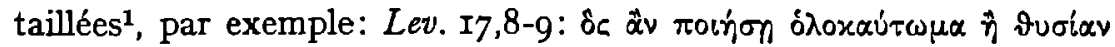

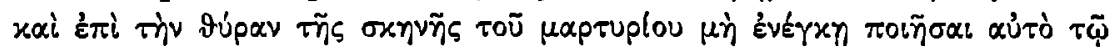

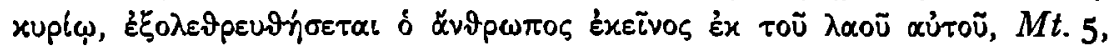

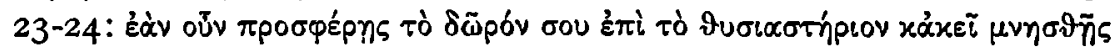

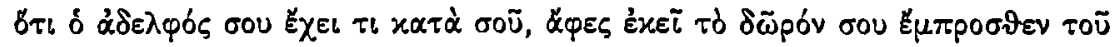

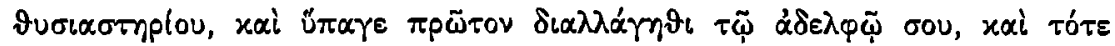

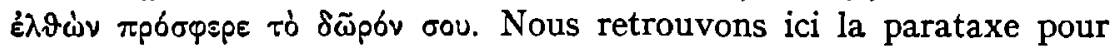
ainsi dire juridique dans des subordonnées, telle que nous l'avons décrite plus haut.

En ce qui concerne le Pasteur, nous avons parlé ci-dessus pp. 23-24 du peu de rigueur logique dont témoigne l'usage d'Hermas. Un exemple, dans le domaine qui nous occupe ici, est fourni par 48,3, où, malgré la différence de valeur pour le contenu, les principales comme les sub-

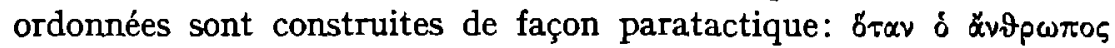

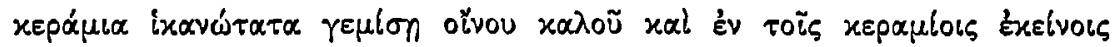

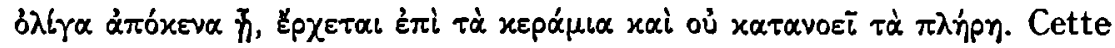
citation montre en même temps à quel point une phrase peut devenir obscure lorsque l'hypotaxe est appliquée à demi; l'auteur aurait pu être clair en n'utilisant que des phrases principales ou en ordonnant systématiquement la phrase d'une manière hypotactique, par exemple

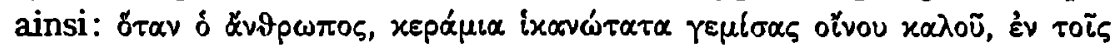

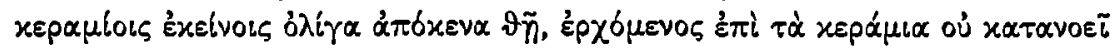
$\tau \dot{\alpha} \pi \lambda \eta \dot{p} \eta^{2}$. Hermas ne dominait pas sa langue au point d'utiliser l'hypotaxe et la parataxe avec logique. Et, étant donné que ses exemples littéraires, les livres de la littérature biblique, présentaient à

I. Cf. P. Fiebig, Der Erzählungsstil der Evangelien im Lichte des rabbinischen Erzählungsstils untersucht, Leipzig I925, p.5.

2. Les versions latines ont façonné cette phrase de manière qu'elle soit une avec les phrases suivantes jusqu'au $\S 4$. $L_{1}$ le fait avec une certaine virtuosité; c'est a cette version que nous avons pris le $\vartheta \bar{n}$ de notre métaphrase. 
profusion la parataxe dans des subordonnées, des participes et des infinitifs, on ne peut s'étonner de leur fréquence chez lui. Nous avons compté 25 passages pour des subordonnées', par exemple 36,7: Ėàv

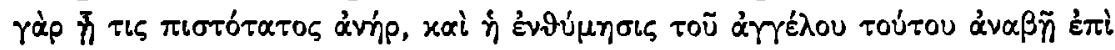

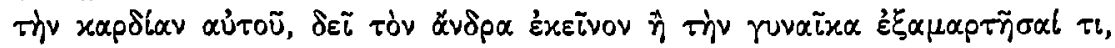

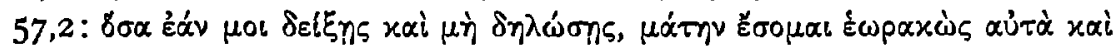

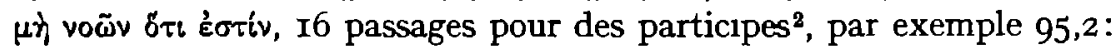

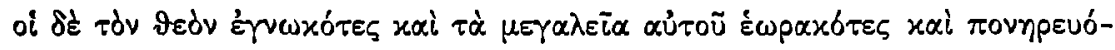

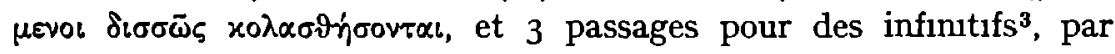

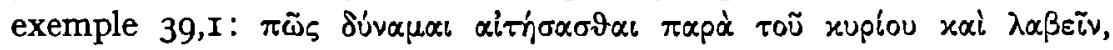

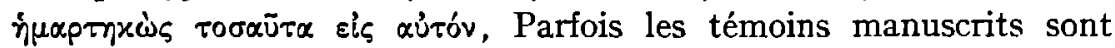

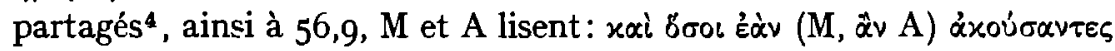

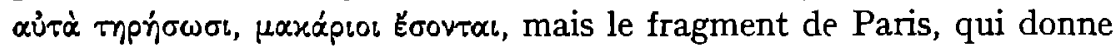
une leçon maladroite, donc probablement plus authentique, comporte:

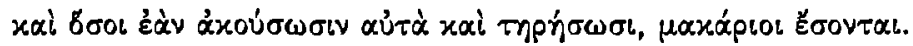

La parenté avec la Bible grecque ressort aussi de l'usage qu'Hermas fait souvent de cette parataxe dans le même contexte que la Bible grecque. Nous pensons à des formules avec áxoúeuv xai + un verbe

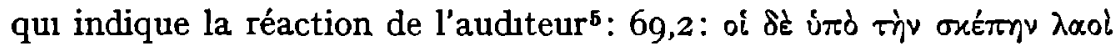

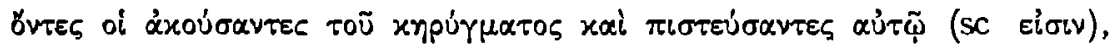

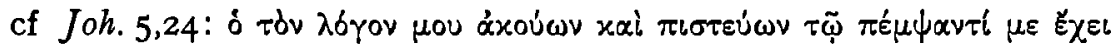

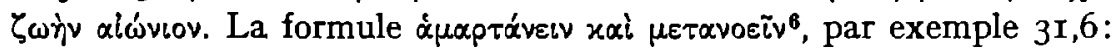

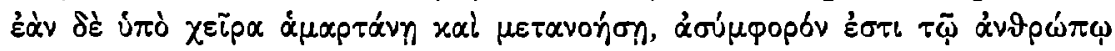

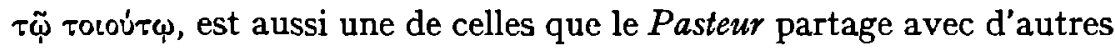

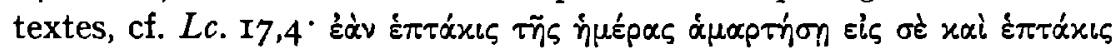

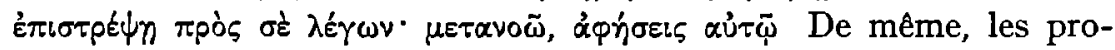
blèmes du divorce et du remariage s'expriment chez Hermas d'une

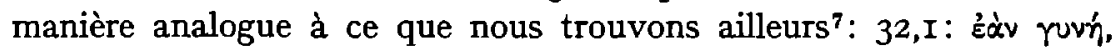

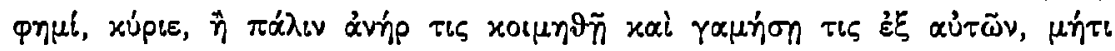

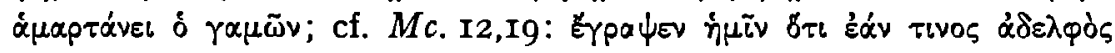

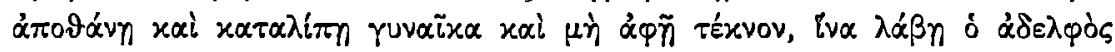

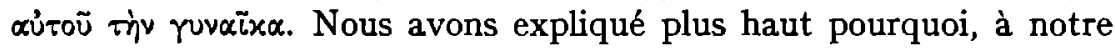

I II, 2, 29,4 5, 3I,6, 32, I, 33,5, 36,7 8, 40,3, 4I, 2, 43,9 I4 I5 20,44,2, 48,3, $53,4,57,2 b \imath s, 68,7,82,2,84,6$, 105,4bis, rog, 3

$2 \mathrm{II}, \mathrm{I}, \mathrm{I} 5,3$ bis, I6,II, I7,4, 23,6, 29,8,40,5, 5I, 10, 69,26,72,3, 95, I 2 . I09, 4 , IIO, 3

$339,1,43,12,53,6$

$477, \mathrm{I}$ auss1

5 II,2, I5,3, I6, I I, 23,6, 69,2 Cf $M t$ 7,24 26, 13,1923, Mc 4,12 20, Lc 6, $4749,8,21$, II 28, Joh $5,24,12,47$

$629,58,31,6,53,4$

7 29,4 5, 32,1 Cf $M t$ 19,9, Mc 10,4 II, 12,19, Le 16,18, I Cor 7,12 13 
avis, la parataxe qui sert à relier des grandeurs inégales dans des subordonnées, des participes et des infinitifs ne peut être un latinisme. Une conclusion analogue s'impose, nous semble-t-il, pour l'hypothèse d'un sémitisme. Pourtant l'emploi qu'Hermas fait de la parataxe en ces cas-là remonte en dernier ressort à une influence sémitique: courante en grec parlé, mais évitée dans la langue littéraire, elle pénètre en fin de compte, par suite du littéralisme des traducteurs de la Septante, dans la langue écrite de la littérature biblique, langue qu'Hermas prend comme modèle.

Enfin, au sujet de la parataxe, nous tenons à attirer l'attention sur une particularité qu'Hermas partage avec la Bible grecque et la littérature rabbinique. Elle se présente lorsque, dans des récits racontés en vue d'illustrer des propos parénétiques, on représente un épisode du récit comme fortuit, alors qu'en réalité il est une condition nécessaire pour le déroulement des événements ultérieurs ${ }^{1}$. Chez

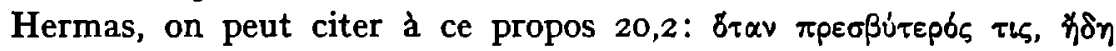

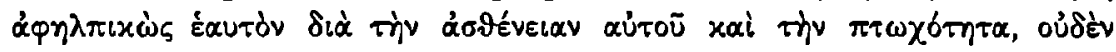

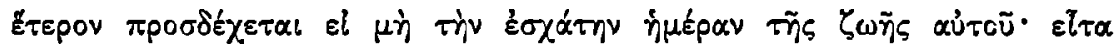

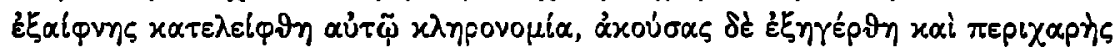

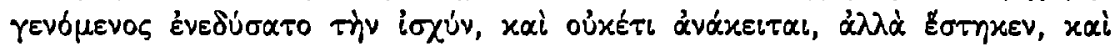

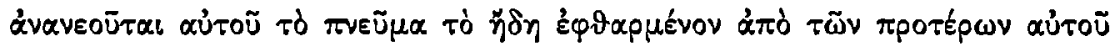

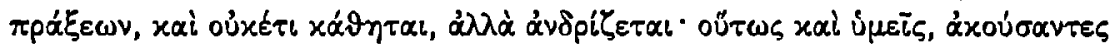

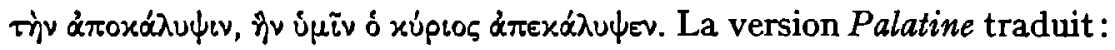

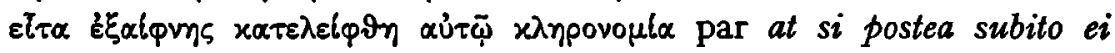
relinquatur haereditas. Le passage 48,3 , que nous avons déjà cité plus haut, p.I2o, peut, lui aussi, être considéré sous cet angle. Là aussi, la version Palatine prend la liberté d'intervenir en traduisant la principale

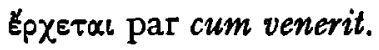

\section{QUELQUES LATINISMES SUPPOSES}

Dans le chapitre III, nous avons signalé que, chez Hermas, du fait qu'il a habité à Rome, on a tendance à attribuer à une influence latine ce que, chez d'autres, on considère comme d'un usage courant. Nous avons objecté que la preuve d'une influence latine particulière sur le grec du Pasteur n'a pas encore été donnée et qu'en attendant, il ne faut nommer latinismes chez Hermas que les seuls phénomènes linguistiques qui, non seulement semblent être formés sur un exemple latin, mais qui 
ne se présentent pas ailleurs dans des sources grecques plus anciennes ou contemporaines (à moins que ces sources elles-mêmes n'aient subi une influence latine évidente). Si, dans les textes qui n'ont subr aucune influence latine, il arrivait qu'on trouve des exemples de phénomènes considérés jusqu'alors comme latınismes dans le Pasteur, alors il faudrait, d'une façon générale, reviser la thèse des latinismes. C'est suivant ce principe que, dans le chap. IV surtout, nous avons essayé de réfuter l'existence d'un certain nombre de prétendus latinismes du Pasteur. Mais allons parler maintenant succinctement des principaux autres cas syntaxiques ${ }^{1}$. On ne sera pas sans remarquer qu'à plusieurs reprises nous empruntons nos pièces à conviction à des auteurs qui, eux, parlent de latinismes, surtout Blass et Debrunner dans leur grammaire ${ }^{2}$.

C'est ainsi que, dans cette grammaire, les auteurs présument que la vaste diffusion du datif de relation en grec post-classique est due à l'influence de l'ablatif de limitation latin ${ }^{3}$. Mais cette diffusion commence à une époque où il ne pouvait pas encore être question d'une influence latine sur le grec. D'alleurs, ce qui change, c'est seulement la fréquence par rapport à l'accusatif de relation: en grec homénque et classique, le datif de relation est déjà fréquent en soi ${ }^{4}$, par exemple,

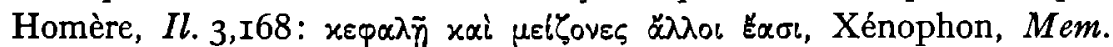

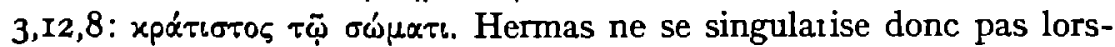

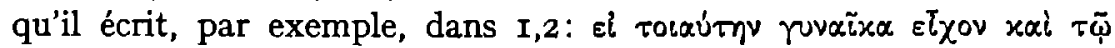

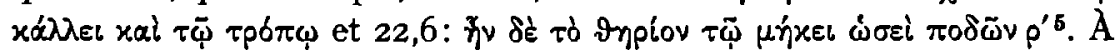
son époque et en grec post-classique en général, ce qui paraît insolite, c'est plutôt l'accusatif, modèle d'emploi classicisant.

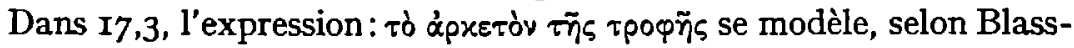
Debrunner, sur satis cibi ${ }^{6}$. S'il en est ainsi, il est étrange que l'adverbe

I Pour une liste de publications, complète pour autant que nous sachions, où l'on signale des latinismes chez Hermas, voir supra p $6 \mathrm{n}$ I

2 N'oublions pas que ces assertions au sujet de latinismes proviennent le plus souvent des premıères éditıons du livre et que les preuves qui les infirment n'ont été apportées que peu à peu Logiquement, ll faudrait buffer les véntés dépassées, mass on comprend que la chose n'alt pas encore pu toujours être faite Dans le Ergänzungsheft de Tabachovitz, on perçoıt déjà plus de réserve envers les latınismes (cf $\imath b$ pP 24, 26, 40), comme d'ailleurs envers les sémitısmes 3 BD $\$ 197$, de même Mohrmann, Ongines, p 76, Bartelınk, Apostolısche Vaders, p 66, Giet, Les Pasteurs, p 284 A H Salonius, Zur Sprache der greechischen Papyrusbriefe, Helsinkı I927, p 3 I hésite Voir aussı supra pp 9, Io, I I

4 SD II pp 86, I68

5 Voic1 les passages $1,2,2,3,10,6,13,12,18,5,22,7,25,1,38,10,62,5,63,5$, $78,2(ग), 80,1 ; 83,1,86,57,94,2$

$6 \mathrm{BD} \S 13 \mathrm{I}$ 


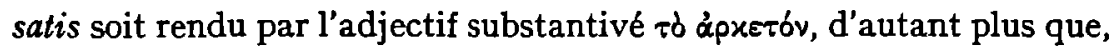

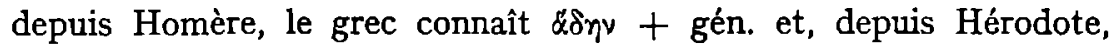
$\not \lambda \iota s+$ gén. En outre, un génitif partitif dépendant d'un adjectif de quantité substantivé est chose normale en grec: cf. Sophocle, $E l$. 532-

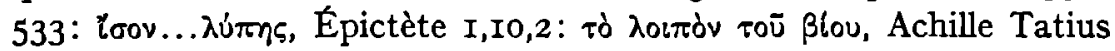

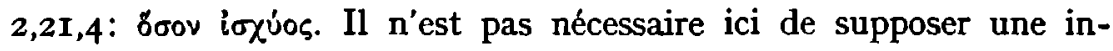
fluence latine. La génitif partitif dans 56,3 est plus remarquable:

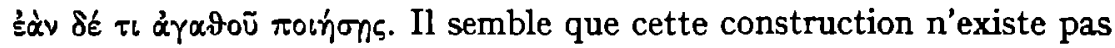
en grec $^{1}$. Mais le passage n'est pas sûr du point de vue de la critique

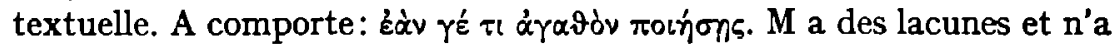
que ] Эou $\pi$ oin[. $\mathrm{L}_{\mathbf{2}}$ traduit par aliquid boni, qui est aussi une traduction

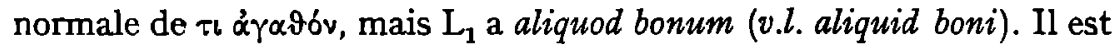
donc douteux que le texte original ait eu un génitif. Si c'était le cas, il pourrait, en effet, s'agir ici d'un latinisme.

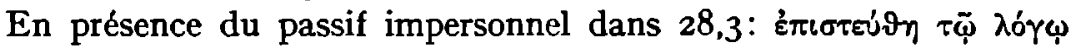

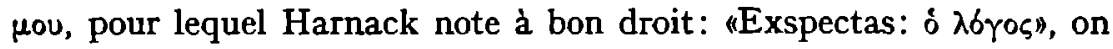
pourrait également être tenté de parler d'un latinisme. Ailleurs, Hermas lui-même emploie aussi la construction personnelle, cf. 51,6: ó $\pi \varepsilon ́ v \eta s$

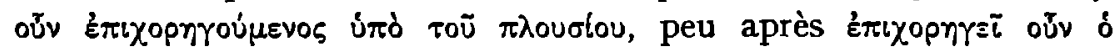

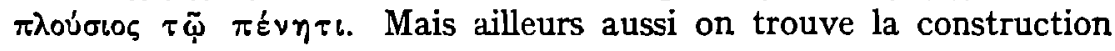

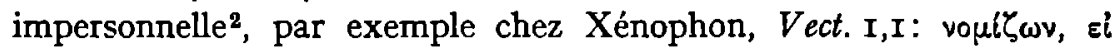

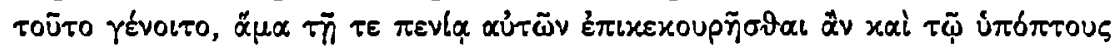

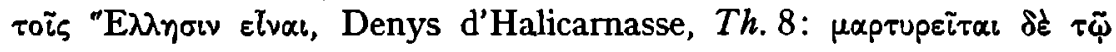

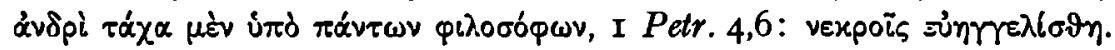
Elle s'explique donc tacilement sans qu'il soit question d'influence latine.

A plusieurs reprises, Hermas utilise une proposition infinitive, là

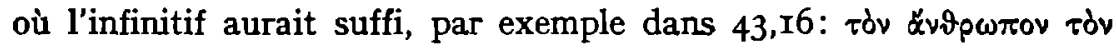

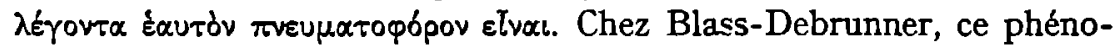
mène est rangé parmi les latinismes ${ }^{3}$. Mais on trouve cette construction aussi bien en grec classique qu'en grec tardif, même si le pronom réfléchi n'est pas particulièrement souligné, par exemple chez Platon,

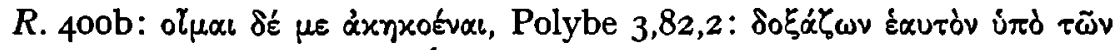

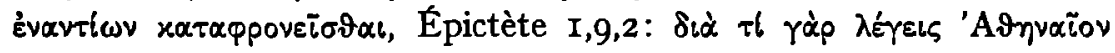

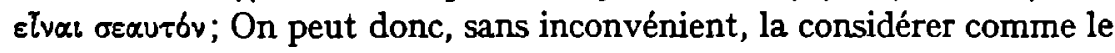

I. H. W. Smyth-G. M. Messing, Greek grammar, Cambridge Massachusetts 1956, p.3r6. Kühner-Gerth et Schwyzer-Debrunner ne se prononcent pas sur la question.

2. Voir BD $\$ 13^{1}$, 312 ; Rapallo, Calchi ebraici 1969, pp.432-434.

3. BD $\S 406$ (cf. ib. Sachregister s.v. Latinismen). Chez Hermas également 49,$4 ; 63,5$. 
résultat d'une évolution interne du $\mathrm{grec}^{1}$. Nous trouvons d'ailleurs cette proposition infinitive dans des cas où, en latin, il ne pourrait s'employer, à savoir après $\omega \sigma \tau \varepsilon$ et $\pi p l v$ et après des prépositions ${ }^{2}$, par

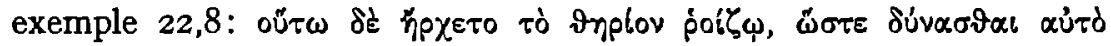

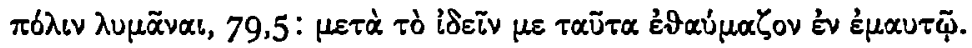

Le verbe $\varkappa \varepsilon \lambda \varepsilon u ́ \varepsilon เ v$ enfin, chez Hermas, s'accompagne d'un infinitif passif lorsque, avec cet infinitif, on ne nomme pas de personne comme

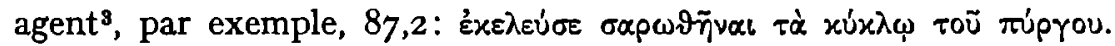
Il est vrai que cette construction ne se présente pas en grec classique et correspond à celle de iubere en latin, mais elle est courante aussi en grec post-classique, tant dans la langue profane littéraire et nonlittéraire qu'en grec bibliquex.

I. Voir J. H. Moulton, Einleitung in die Sprache des Neuen Testaments, Heidelberg I9I I, p. 337 .

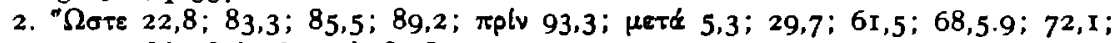

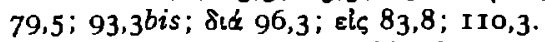

3. 66,$2 ; 68,1 ; 81,7 ; 83,5.6 b i s ; 85,1.2 .3 .4$ bis.5; 87,$2 ; 107,2 ; 110,3$.

4. Voir BD $\$ 392^{4}$. Egalement dans Test Jos. I3,1.4.9; 14,2. 


\section{MOTS ET EXPRESSIONS}

\section{LE PLURIEL oúpavol}

Dans $90 \%$ des cas, la Septante traduit le plurale tantum a "ciel" par oúpavóc et dans $10 \%$ des cas par oúpavol. Cette dernière traduction est une dérogation à l'usage courant, car, dans le grec de l'époque classique et des siècles qui ont vu naître la Septante, on ne connaissait pas le pluriel oúpavo! dans le sens du singulier "ciel»: dans les quelques passages où oúpavol se présente, il a le sens d'un pluriel' Ce n'est qu'à partir du IIe siècle après J.-C. qu'il figure cà et là avec le sens d'un singulier. Ainsi, dans CMRDM I,83, inscription de la Lydre, probablement du II $^{\mathbf{e}}$ ou III ${ }^{\mathrm{e}}$ siècle après J.-C., on lit: $\varepsilon \boldsymbol{\tau}_{\zeta}$

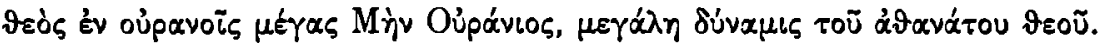
Ensuite, chez quelques auteurs tardifs, nous trouvons l'expression, sis oujpavoús "au ciel"». Mais tout cela ne prouve naturellement pas qu'aux siècles où fut traduite la Septante, oúpavol au sens du singulier était en usage en grec profane. Et même plus tard, le terme semble avoir été si inhabituel que l'oủpavol biblique signifiant "ciel" donna lieu à des malentendus. C'est ainsi que certains auteurs patristiques prennent soin d'expliquer qu'il faut entendre le pluriel à partir de

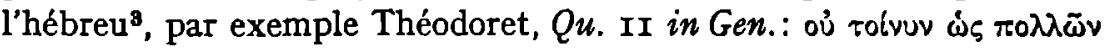

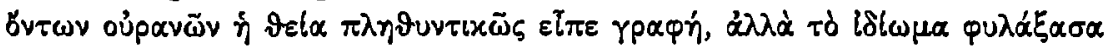

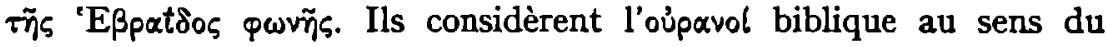

I Passages chez H Traub, art oúpar6́, ThWNT V, p (496-50I, 509-536) 497 n 2, Bauer, Wörterbuch, sv aujpavbs I e De même en latın caeli, par exemple Lucrèce 2,1097 Le caelı paléochrétıen avec le sens d'un singuler remonte à l'hébreu, of Schrijnen-Mohrmann, Cyprian, I, pp 64-65, G Q A Meershoek, Le latın bıblique d'après Saint Jéróme, Nijmegen-Utrecht 1966, pp I88-19 I

2 Achille Tatıus 2,36,4, 2,37,2, Himénos 66,4 et dans une partie de la tradition Apollodore, $B \imath b l$ 1,6, I. Thémistios 2,36 Julien, dans $E p$ 115, épitre sur les

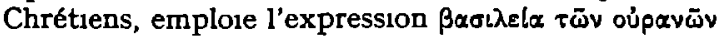

3 Vour Meershoek, Latın biblique, pp 188-197, à ses citations on peut ajouter Adnen 72. 
singulier comme un sémitisme, et cette opinion s'est perpétuée jusqu' aux temps modernes.

En r934, Torm contesta la qualification de "sémitisme». Les arguments qu'il avança furent qu'oúpavoí se présentait aussi dans des textes classiques, que, dans la plupart des passages de la Septante, le terme est au singulier et qu'avec la traduction oủpavòs roũ oúpavoũ les traducteurs n'ont pas profité de l'occasion la plus évidente d'employer le pluriel ${ }^{1}$. Ces arguments ne sont pas impressionnants. Pour les passages profanes qu'il cite comme ayant oupavol, Torm omet de dire qu'il s'agit aussi du sens du pluriel. L'argument statistique peut, lui aussi, s'utiliser d'une toute autre façon: oupavoí "ciel» qui, au pluriel dans le sens d'un singulier, correspond à l'hébreu, se présente 50 fois dans la Septante, mais fait défaut en grec classique et dans le grec contemporain de la Septante. D’une façon plus générale, n'oublions pas que, dans la Septante, se trouvent représentées différentes techniques de traduction et qu'un traducteur est parfois, lui aussi, inconséquent.

Une explication acceptable, qui fait droit au caractère sémitisant d'oúpavol "ciel», a été donnée en I950 par Katz². D'après lui, les premiers traducteurs traduisaient invariablement שִ̣ par oủpavós $\varsigma^{3}$. Ce n'est que dans les textes poétiques qu'on en vint à traduire par oúpavol. Lorsque, dans ces textes, était précédé par un prédicat au pluriel, il était fort tentant de rendre les pluriels hébraiques par un pluriel. Souvent aussi, la traduction au pluriel avait son origine dans le parallélisme de la phrase, lorsque le membre correspondant était au pluriel. En grec, on percevait le pluriel oúpavol, même indépendamment du contexte, comme un pluriel poétique ${ }^{4}$. C'est ainsi que tant le contexte que la valeur affective ont déterminé la fonction du pluriel: oúpavol s'emploie dans le style élevé de la poésie, de la prophétie, de la prière, lorsque le ciel forme l'idée centrale, donc surtout au nominatif et au vocatif.

Le pluriel pénètre aussi dans les livres de la Septante écrits en grec $^{5}$. Dans le Nouveau Testament et les apocryphes, il devient même très

I. F Torm, Der Pluralıs oủ pavol, Zeitschnft fur die neutestamentliche Wissenschaft 33,1934, pp 48-50 Sa théone fut contestée par P. Katz, Philo's Bible, Cambridge I950, Pp I4I-I46, $2 b$ PP I4I-I 42 sut oúpards toū oúpavoũ

2 Vour note précédente

3 Cf Katz, Philo's Bible, pp I42, I45 Le Pentateuque a le plunel seulement dans Deut 32,43, qui a été ajouté plus tard, cf $\imath b$ P $\mathrm{I}_{44}$

4. Cf Katz, Philo's Bıble, P I 45, SD II, P 44

5. Cf Traub, oúpavos, P,510 
en vogue. L'évangile de Matthieu est le texte qui compte de loin le plus de pluriels - 55 fois, contre 27 singuliers - , mais, même en faisant abstraction de ce texte, le nombre de pluriels est proportionnellement plus grand que dans la Septante: dans le Nouveau Testament (moins Matthieu), dans les apocryphes paléotestamentaires et néotestamentaires, le rapport du singulier au pluriel est de 3 à I. Mais la répartition sur les différents livres est très irrégulier ${ }^{1}$. Quant au sens, il y a tendance à réserver le pluriel, plus solennel, pour le ciel en tant qu'habitation de Dieu, et le singulier pour le ciel en tant que «air» ou "firmament $\|^{2}$. Mais cette tendance n'est pas devenue une règle rigide et n'est pas également perceptible dans tous les textes.

Hermas utilise oúpavós I5 fois, dont 4 au pluriel. Ces quatre cas, tous dans la première Vision, sont les suivants:

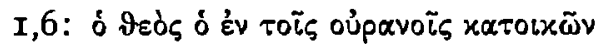

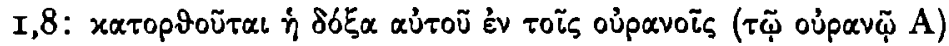

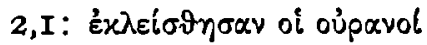

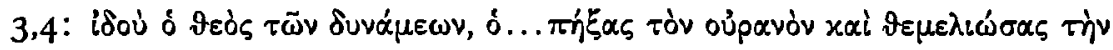

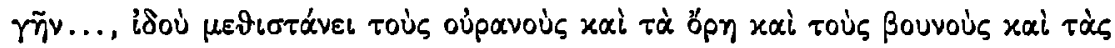
$\vartheta \alpha \lambda a ́ \sigma \sigma \alpha s$.

Dans I,6, le plunel correspond au texte du Pater tel qu'll a été

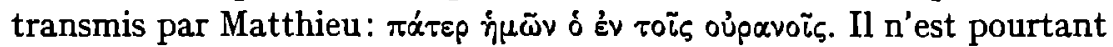
pas sûr du tout que le pluriel était de règle dans ce genre de formules servant à désigner Dieu. Dans la Didachè $(8,2)$, le Pater commence par

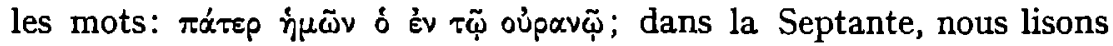

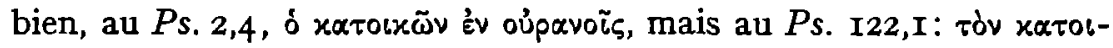

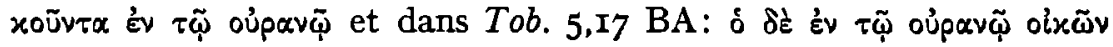

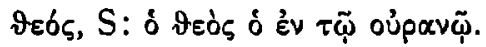

I Voic1, respectivement pour le singulier et le pluriel, le nombre de cas dans le Nouveau Testament $M t$ 26/55, $M c$ 12/5, Lc 31/4, Joh 18/o, Act 24/2, Paul II/Io, Hebr 3/7, Jac 2/0, I Petr 2/I, 2 Petr I/5, Apoc 5I/I, cf Traub, oúpav6s, pp 512-513 Ces proportions, à notre avis, ne s'accordent pas avec l'opinion de Meershoek, Latın bıblique, p 190, selon laquelle le plurnel s'est maintenu à côté du sıngulıer aussı longtemps que les liens restèrent étroits avec le judaisme et que, lorsque l'auteur se fut hellénısé, le plunel disparut Pour les apocryphes paléotestamentaires, les chiffres sont $O$ r Man 2/I, Ps Sal 5/r, Apoc Mos 5/0, I Hen 46/4, Test Abr A 17/2, Test $\mathrm{Abr}$ B 4/4, Jos et As 12/0, Test XII Patr 1/14, Par Jer 7/o, Apoc Bar 16/1, Vit Proph I/o, Apoc Esdr 10/2, Test Job 5/4, pour les apocryphes néotestamentarres Protev Jac 6/o, Ev Petri 2/4, 3 Cor 3/o, Acta Pauli et Th 2/2, Acta Pauln 1/2, Mart Pauln 1/o, Ps Barn 6/I, Did $4 / 0$

2 Cf Torm, oúpavol, pp 49-50, $\mathrm{H}$ Bietenhard, Die hrmmlische Welt im Urchristentum und Spätjudentum, Tubingen 195I, PP 6-7, BD § 141 ${ }^{1}$, Turner, Syntax, P 25 
Dans I,8, de même que dans I,6, "ciel" signifie l'habitation de Dieu, mais les textes-témoins ne sont pas d'accord sur le nombre. De même, dans 2,I, le ciel est l'habitation de Dieu, mais il faut remarquer que cette partie de la phrase forme la fin de la description de la vision qui commence, dans I,4, par des singuliers: ḣvoím ó oủpavós, xal $\beta \lambda \dot{\varepsilon} \pi \omega$

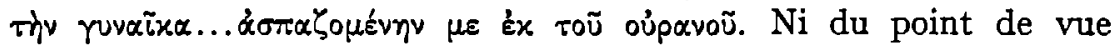
sémantique, ni du point de vue stylistique on ne peut voir de différence entre le singulier de I,4 et le pluriel de 2,I. Dans 3,4, le singulier et le pluriel se présentent presque coup sur coup dans le sens de «firmament"; mais ici le choix a pu être déterminé par le fait que le mot correspond d'abord au singulier $\tilde{\eta}^{\nu}$ et ensuite aux pluriels bo

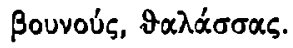

Sur les onze passages où Hermas emploie le singulier d'oủpavós nous en avons déjà vu trois: $1,4 b i s ; 3,4$. Dans les autres passages, le mot a chaque fois le sens d' "air", "firmament". Dans l'un d'eux, il emploie une expression qui est fréquente dans la Septante et ailleurs dans la

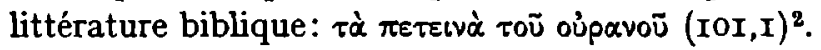

En résumé, nous pouvons dire qu'Hermas reflète les tendances du grec biblique: pour le sens de "séjour divin" il y a concurrence entre le singulier et le pluriel; pour le sens d' «air", "firmament", on trouve régulièrement le singulier, mais alors il ne s'emploie pas d'une façon assez conséquente pour qu'un pluriel ne le remplace lorsque, dans le contexte, les idées qui l'entourent appellent un pluriel.

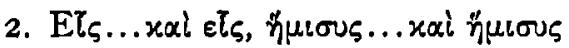

Pour nous modernes, il n'y a rien de surprenant dans une formule

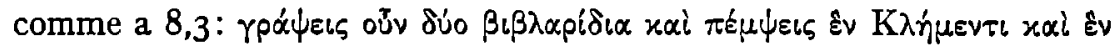
$\Gamma \rho \alpha \pi \tau \tilde{n}$, au contraire, dans sa simplicité, elle nous paraît aller de soi. Mais, pour juger des phénomènes linguistiques du grec ancien, il est dangereux de se fier à notre sens moderne de la langue. Ainsi, rien que dans ce cas, il y a deux remarques à faire pour la construction Ề... xai $\varepsilon v$, une au sujet du nom de nombre et l'autre au sujet de la conjonction xol.

I. $22.4 ; 43,18$ ler; 47,$2 ; 79.5 ; 94,4 ;$ ror, 1 .

2. Cf. Katz, Philo's Bible, p.r43; Tabachovitz, Septuaginta, pp.r Io-r I I Bauer,

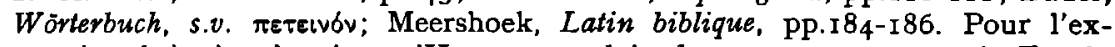

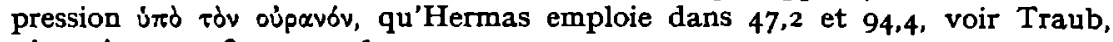

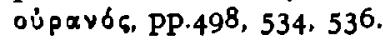


L'emploi du nom de nombre dans cette formule est du grec postclassique. En grec classique, pour énumérer les deux parties dont se compose un tout donné, on aurait utilisé non pas l'adjectif numéral

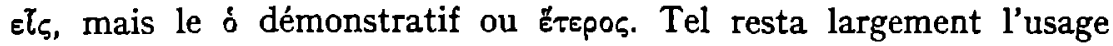
jusqu'à une époque tardive. C'est ainsi que le contemporain d'Hermas,

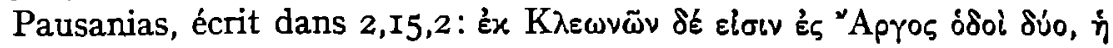

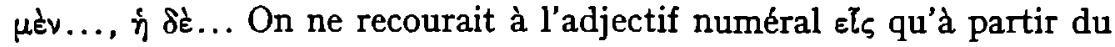
moment où le nombre des parties composantes était de trois ou plus,

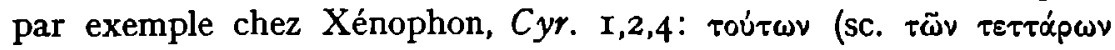

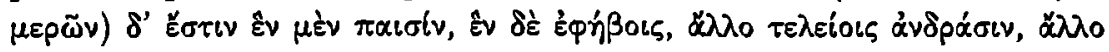

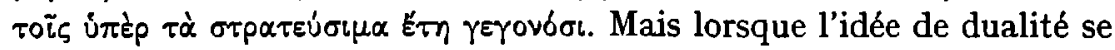
fut affaiblie, cette règle ne fut plus strictement suivie ${ }^{1}$. C'est ainsi

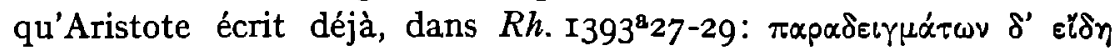

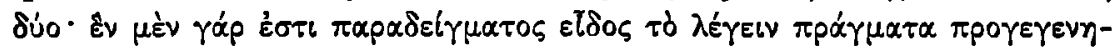

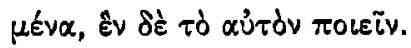

Mais c'était une chose tout à fait inhabituelle, tant en grec classique qu'en grec post-classique, que de relier les parties composantes par kol. La construction habituelle est la construction avec $\mu \hat{e}^{\prime} . . . \delta^{\prime}$, parfois avec suppression d'une des particules ou des deux (asyndète) ${ }^{2}$. Nulle part dans les textes profanes, pour autant que nous sachions, xoí ne s'emploie seul; le parallèle le plus proche est POxy.8, I I53,I3-I4 (Ip), qui

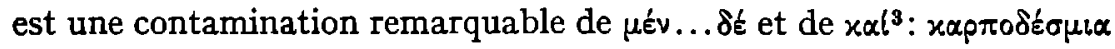

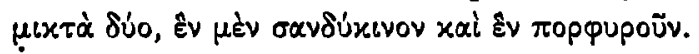

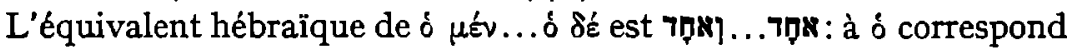

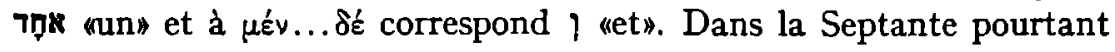

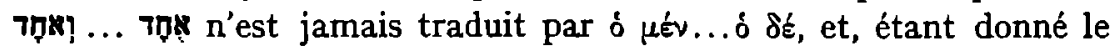
littéralisme des traducteurs, on le comprend facilement. Ces traducteurs ne pensaient pas par membres de phrase et par expressions, mais par mots pris à part. Chaque mot hébreu devait toujours, autant que possible, être rendu par un même mot grec. C'est surtout en présence de mots très fréquents et sans équivoque comme 7 sk "un" et ? "uet" que cette tendance a dû être forte. C'est ainsi qu'on en vint à traduire

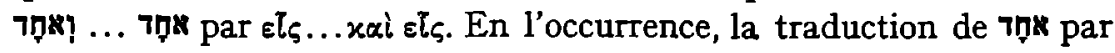
Eโs était parfaitement valable à l'époque hellénistique. La traduction

I. Voir J. H. Moulton, Grammatical notes from the papyri, The Classical Review 15. I901, Pp (31-38, 434-442) 439-440; BD § $64^{\circ}$.

2. Exemples dans BD $\S \S 247^{\mathrm{a}}, 25^{\circ}$; Bauer, Wörterbuch, s.v. $\varepsilon l_{\zeta} 5$ abd. Asyndète dans la Septante I Regn. 6, I7.

3. Cf. Moulton, Notes, p.440; Bauer, Wörterbuch, s.v. $\mu$ tev 2 d. L'emploi de $\mu \varepsilon_{v} . . . x \alpha l$ pour lier des phrases entières est très ancien, voir J. D Denniston, The Greek particles, Oxford, $1954^{2}$, P.374. 
de 1 par $x \alpha i$, au contraire, comme nous l'avons vu, divergeait nettement du grec courant. La combinaison $\varepsilon \tau_{\zeta} \ldots$.. xai $\varepsilon \tau_{\zeta}$ est donc manifestement un sémitisme, dû à une traduction littérale. Elle a cependant dépassé le stade de grec de traduction et s'est frayé un chemin dans les livres grecs de la littérature biblique 1 . Comme on pouvait s'y attendre, la combinaison $\mu\{v \ldots . . \delta \hat{\varepsilon}$ qui, du fait de l'absence d'un équivalent exact dans le texte original, est extrêmement rare dans les livres traduits,

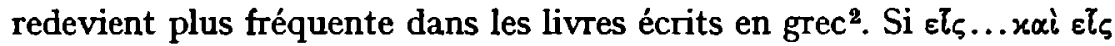
s'y perpétue, c'est par imitation voulue du grec des livres traduits.

Chez Hermas, l'usage biblique a l'air d'une survivance. En plus du passage déjà cité, il en est encore un autre où il emploie la combinaison

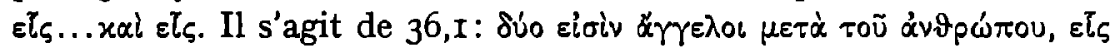

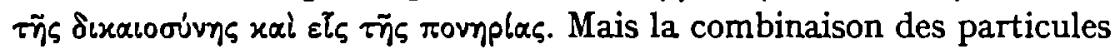
$\mu \hat{\varepsilon} \nu$ et $\delta \dot{\varepsilon}$ se présente 55 fois chez lui ${ }^{3}$, pour relier non seulement des phrases ou des parties de phrases, mais aussi des mots isolés, par

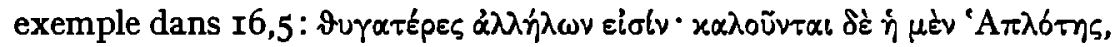

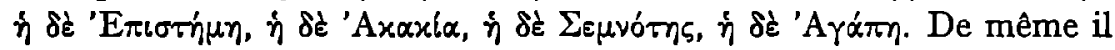

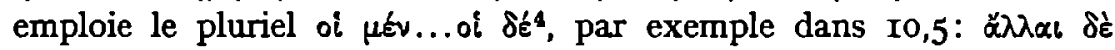

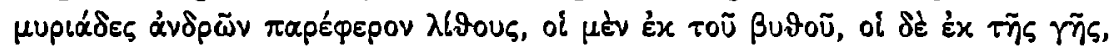
ou la substitution avec le pronom relatif ${ }^{5}$, par exemple $53, \mathrm{I}$ : ह̌ $\delta e เ \xi \varepsilon$

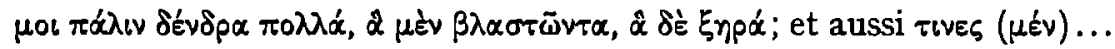

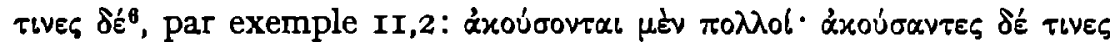

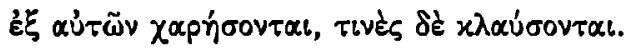

Le $x a l$ qui, à l'encontre du sens de la langue, remplace $\mu e_{v} . . . \delta \dot{\varepsilon}$, se

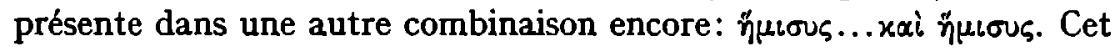
usage est parfaitement analogue à celui de $\varepsilon \tau_{\zeta} \ldots$... $\alpha i_{\text {e }} \boldsymbol{I}_{\zeta}$ : exactement

1. Les passages de la Bible grecque et des apocryphes sont: Ex. 17,12; 25,19; 38,7; Lev. 5,7; 12,8; 14,22.31; 15,15.30; 16,8; Num. 6, I1; 8, I2; Deut. 21,15; Jud. A 16,29; 20,31; Jud. B 16,29; 20,31; I Regn. 10,3; 13,17-I8; 2 Regn. 12, I; I Par. 24,6; 2 Par. 3,17; 2 Esdr. I4, I1; Esth. 10,3"; Sir. 34,23.24; Zach. 4,3; Is. 6,2; Ez. 40,44; 48,31.32.33.34; Dan.1אx 12,5; Dan.Th12,5;Mt. 17,4; 20,21; 24,40.41; 27,38; Mc.9.5; 10,37; 15,27; Lc. 9,33; Joh. 20,12; Gal. 4,22; Test. Abr. A 4.1 r; Jos. et As. 14, I6; Protev. Jac. 17,2; Did. I, I.

2. Cf. BD $\S 447^{2}$; Bickerman, Septuagint, p.23; Turner, Syntax, pp.331-332, et pour des textes traduits du latin: A. P. M. Meuwese, De versione graeca Monumenti Ancyrani quaestiones, Mnemosyne N.S. 54, 1926, pp.(224-233)229-231.

3. $10,5.7$; I1,2; 13,1bis.3; $14,2.4 .5 ; 15,1 ; 16,3.5 ; 17,7 ; 18,3.4 ; 19,2 ; 24,2 ; 27.5$; 36,$10 ; 38,6 ; 41,4 ; 42,2 ; 53,1 b i s .4 ; 62,2.4 ; 63,4 ; 67,10 ; 69,5 ; 70,6 ; 71,1 ; 75,1$ bis; 78,2 bis.6.7; $83.6 ; 88,5 ; 89,2 ; 90,3 ; 92,2.3 .4 ; 93.6 ; 96,2 ; 97$. Ibis; 98,1 bis; 99,1 ; 103,$2 ; 105,3 ; 113,2$.

4. I0,5; I3,I;63,4;70,6;88,5;97,I;98,I.

5. Io,7A; 53, Ibis; 69,5; 78,7. Cf. BD § 250; De Foucault, Polybe, Pp.89-9o.

6. 11,$2 ; 62,2 ; 70,5$. 
comme ce dernier, il est dû, dans la Septante, au littéralisme', et ce n'est pas parce que le grec manquait de moyens linguistiques ordinaires, mais parce qu'on imitait le style biblique qu'il s'est perpétué dans des textes ultérieurs. Chez Hermas, on trouve cette expression dans 67, II et 74,I; le premier passage, 67,II s'énonce en ces termes:

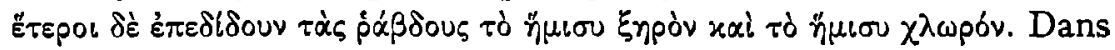

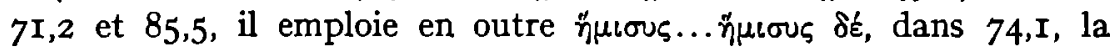

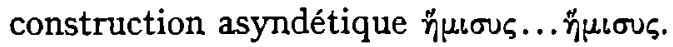

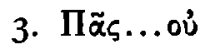

Le grec, tout comme le latin et d'autres langues, dispose d'une série de mots qui expriment à la fois une négation et une généralité, tels

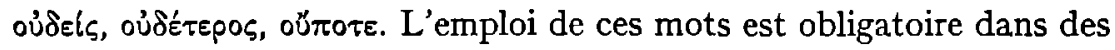
propositions où l'on porte une affirmation générale de sens négatif.

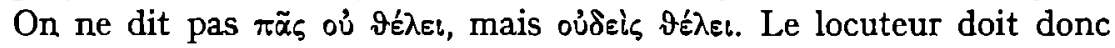
savoir d'avance s'il veut s'exprimer d'une manière positive ou négative. Dans les textes qui nous sont parvenus, les auteurs y sont presque toujours arrivés. Nous ne connaissons que quatre passages où des auteurs étaient tellement absorbés par l'idée de la généralité qu'ils commencèrent la phrase par $\pi \tilde{\alpha}_{5}$ et ajoutèrent ensuite une négation ${ }^{2}$;

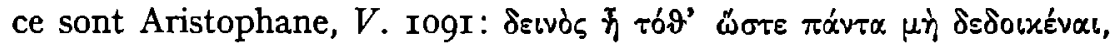

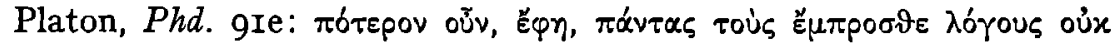

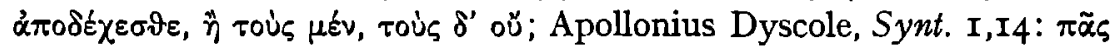

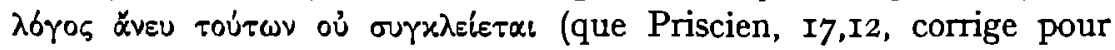
traduire par nulla oratio sine iis completur), BGU 2, 632, II-I2 (IIP):

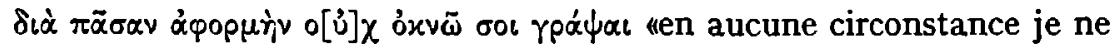
néglige de t'écrire». En latin aussi, de tels cas sont rares. Parmi les textes profanes, nous ne connaissons que César, Gal. 6,11,4: suos enim quisque opprimi et circumvenuri non patitur, Properce 2,28,13: semper, formosae, non nostis parcere verbis. On pourrait d'ailleurs interpréter tous ces cas en considérant que la négation porte sur le verbe et non sur la proposition comme tout: $\mu \dot{\eta} \delta \in \delta o$ óeval "être sans crainte", oủx

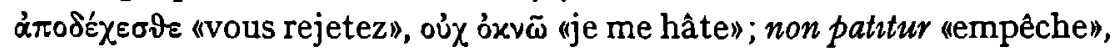

I. Les passages sont: Lev 6,13, 3 Regn. 3,25, 2 Esdr. I4, ro; Zach 14,4brs.8. Dans le Nouveau Testament le h̆j

2 H Ljungvik, Beiträge zur Syntax der spatgriechischen Volkssprache, Uppsala 1932, p 2 I cite encore un passage de la lettre POxy. I6, I831, papyrus du Ve siècle, mais le texte est trop incertain pour qu'on le compte Selon certains, le $\pi \alpha ́ v \tau \alpha$ du passage d'Anstophane, que nous citons cr-après, est un adverbe; dans ce cas, 1 s'agit de l'usage dont nous parlons à la fin de ce paragraphe. 
non nostis $=$ nescitis. Aussi se justifieraient-ils aussi selon les règles de la grammaire normative.

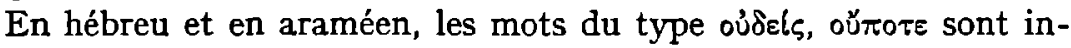
connus. La généralité et la négation s'expriment chacune par un mot propre. C'est ainsi qu'à l'oúdeís grec correspond une combinaison de "chacun" et "ל "לnon pas", et chacun des deux mots peut se mettre en tête: expressions mot à mot. C'est ainsi que $\boldsymbol{x}^{\prime}$.... ל , auquel nous nous bornerons, se traduit par $\pi \tilde{\alpha} \varsigma \ldots \circ 0^{2}$, par exemple Ex. 22,2I: $\pi \tilde{\alpha} \sigma \alpha \nu$

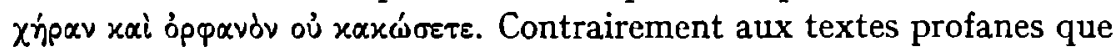
nous avons cités, on ne peut en général faire porter la négation seulement sur le verbe. Nous trouvons aussi $\pi \tilde{a} c . .$. oủ dans le Nouveau Testament et les apocryphes ${ }^{3}$, par exemple dans I Joh. 2,2I: $\pi \tilde{\alpha} v$

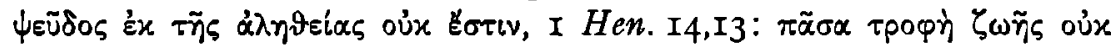

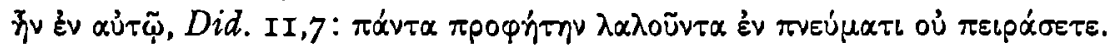
Dans certains cas, comme dans le passage i $H e n$. 14, I3 que nous venons de citer, $\pi \tilde{\alpha} \varsigma \ldots$ ou pourrait comme dans la Septante, provenir de la traduction littérale d'un texte original sémitique. Ailleurs, l'imitation du style de la Septante est évidente ${ }^{4}$. Cette forme d'expression avait d'ailleurs d'autant plus de chances de succès que, dans un certain sens, elle facilitait les choses pour les auteurs plus que ne le faisait le grec profane. Car on pouvait maintenant commencer à formuler une idée générale sans avoir à se demander d'avance si on lui donnerait une forme positive ou négative.

Hermas utilise cette forme d'expression dans les trois passages suivants:

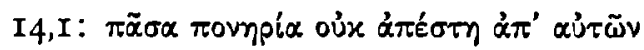

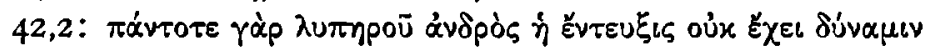

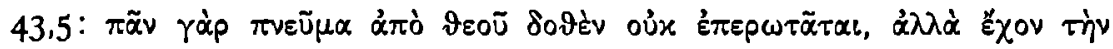

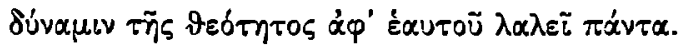

La formulation du dernier passage est d'autant moins frappante que

I Vo1r Gesenius-Kautzsch, Grammatik, pp 501, 503-504; Burney, Aramaic origin, p 98, Jouon, Grammatre, PP 492-493, Beyer, Semitisehe Syntax, pp I 89 (n 4), 213

2 Cf Tabachovitz, Septuaginta, pp 89-9r, $2 b$ p 90 n 3, qui donne un choix de passages de la Septante Pour les traductions latines de la Bible cf Rapallo, Calchi ebraici r969, pp 398-400

3 La seule liste complète de passages dont nous disposions est du Nouveau Testament; elle comporte' $M t$ i 2,25, $M c$ 7,18; $L c$ 14,33. Joh 3,16; 6,39, I I, 26; 1 2,46; Act 5,42, Rom 9,33v l, I0, I I, Eph 4,29, 5,5, 2 Petr I, 20, I Joh 2, 16 21; 3,6bis 9 10 I 5, 4,3, 5,18; Joh 9; Apoc 18,22, 22,3

4 Cf Tabachovitz, Septuaginta, pp 90-9I 
la négation ne porte que sur la première partie de l'énoncé, la deuxième partie est positive. Nous voyons la même chose dans Joh. 3,I6: ivo

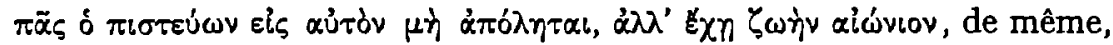
dans I Joh. 2, I6.

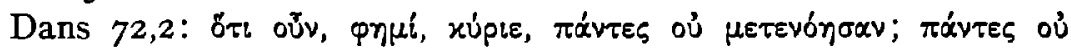
signifie "pas tous", oủ nie la généralité, non pas la phrase dans l'ensemble. Nous retrouvons cet usage dans les textes profanes ${ }^{1}$.

Hermas ne connaît pas le sémitisme oủ... $\pi \tilde{a} \varsigma$. Nous ne pouvons compter comme tels des cas comme 65,I: oủ...6 $6 \lambda \omega \varsigma$ et 66,4 : oú $\pi \alpha \vee \tau \varepsilon \lambda \tilde{\omega} \varsigma^{2}$. Ic1 la négation ne nie pas l'adverbe, mais l'adverbe renforce la négation: «absolument pas». Ce procédé a toujours existé en grec ${ }^{3}$.

\section{LE @́ COMPARATIF}

En hébreu et en araméen, pour comparer deux personnes ou deux choses, on utilise le préfıxe ?̧ «comme». Ce préfixe correspond, dans la plupart de ses emplois, au $\dot{s}$ grec, "comme», et les traducteurs de la Septante ont pensé devoir le traduire par ce terme lorsqu'ils comparaient, eux aussi, des personnes ou des choses", par exemple Gen.

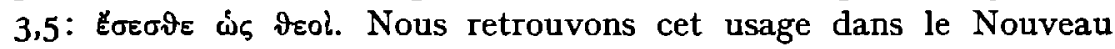

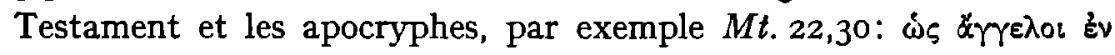

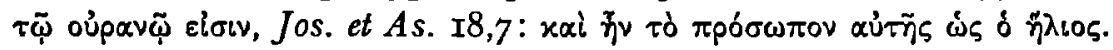
En grec profane, cet $\varepsilon$ lvaı $\dot{\omega} c$ "être comme", "ressembler à", n'est pas usuel: on utilise des adjectifs indiquant une équivalence entière ou partielle comme ioos ou ónoĩos, par exemple chez Epictète $1,12,27$ :

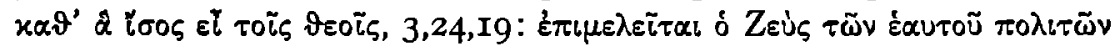

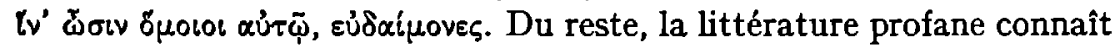
un emploi qui se rapproche beaucoup de l'usage biblique en question ${ }^{5}$.

I Volr Wackernagel, Vorlesungen, II, PP 274-275 Cf encore chez Hermas

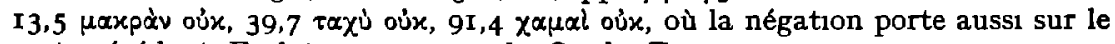
mot précédent En latın, par exemple, Ovide, $\operatorname{Tr} 4,10,130$ protınus non

2 Ainsı $A$ et $F$ Whittaker et Joly se conforment à $M$ oú $\pi \dot{x} v \tau \omega s$, ma1s, chez

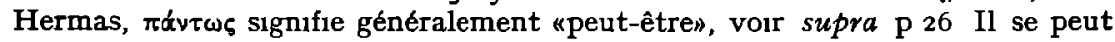

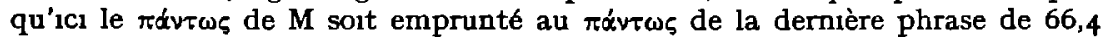
Cf aussi Bonner, Papyrus codex, $\mathrm{p} 8 \mathrm{o}$

3 Vour Wackernagel, Vorlesungen, II, P 274 et cf Abel, Grammarre, p 335,

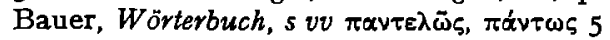

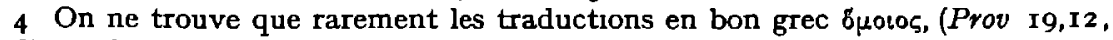

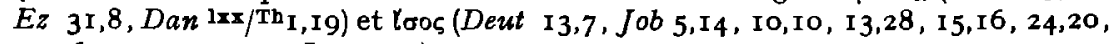
$27,16,29,14,40,15$, Is 51,23$)$

5 Cf aussı l'emploı déjà classique d'adverbes comme ós au heu de $\tau o i ̄ o s$, voir H Ljungvik, Zum Gebrauch einiger Adverbien im Neuen Testament, Eranos 62, 1964, PP 26-39, et des expressions comme 8polos is, vour P Montell, La phrase relative en grec ancien, Pans $1963, \mathrm{P} 337$ 


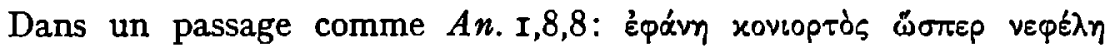

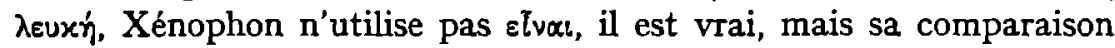
s'établit directement entre la poussière et le nuage et non pas entre l'apparition de l'une et celle de l'autre. On pourrait traduire en expliquant «il apparut de la poussière qui était comme un nuage blanc». On pourrait faire une remarque du même genre sur Lucien, Philops. 25:

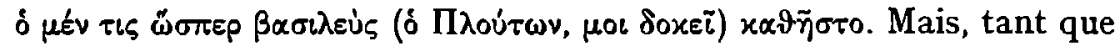
nous ne trouvons pas de parallèle exact dans des textes profanes, nous devrons tenir l'elvar $\dot{\omega} \varsigma$ du grec biblique pour un sémitisme. Dans le Pasteur, il se présente quatre fois ${ }^{1}$, par exemple, dans $27, \mathrm{I}$ : हैon $\boldsymbol{\omega}_{\varsigma}$ $\tau \dot{\alpha} v \dot{\eta} \pi\llcorner\alpha$.

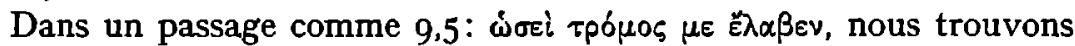
un usage dont l'analogie n'est qu'apparente. A première vue, nous avons affaire, ici aussi, à une comparaison. On pourrait, par exemple, traduire par «cela me saisit comme un frisson». Dans ce cas, le sujet que ren-

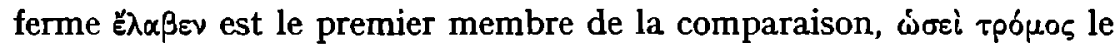
second. Mais le contexte montre clairement que ce n'est pas ce qu'entend l'auteur. Ici, la fonction d'iøei n'est pas syntaxique, mais sémantique; le mot ne sert pas à amener une comparaison, mais à atténuer le sens du mot suivant: «une espèce de frisson me saisit». Hermas s'exprime souvent de la sorte, par exemple encore dans 9,5:

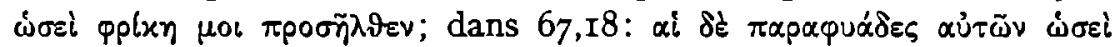

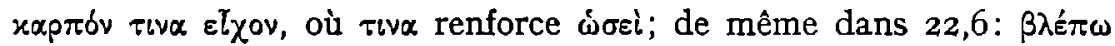

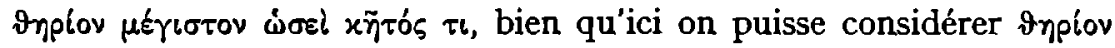

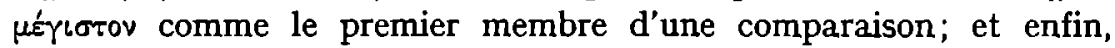

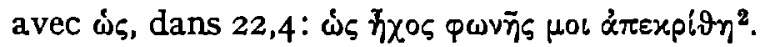

Ce $\dot{\omega}_{s}$ ou $\dot{\omega} \sigma \varepsilon l$ d'atténuation se présente aussi ailleurs dans la littérature biblique ${ }^{3}$. Dans la Septante, on peut constater qu'il rend

I 27,I, 39,3, I06,I 3 ' $\Omega \xi$ devant des adjectıfs et des partıcıpes n'est pas traité 1 c1

2 L'hésıtation de BD $\S 435^{\circ}$ entre etwas wie eıne schallende Stımme" et uer wie eıne schallende Stımme" nous semble osseuse La premıère traduction est incontestablement la bonne, cette "vorx" est un élément qui revient contınuellement lorsqu'ıl s'agit d'événements épiphanıques, voir, par exemple, Peterson,

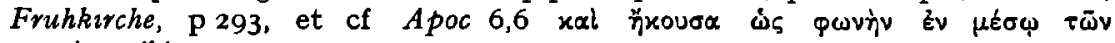

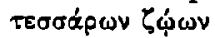

3 La liste qui suit donne tous les passages de la Bible grecque et des apocryphes où le sujet ou l'objet de la phrase s'estompe de la manière que nous avons dite Lev 14,35, Num 9, I 5; Job 28,5, Ez 1,4 526 27bus, 8,2, I0, I, 40,2, Dan 1xx 7,6. I 3 bis, 8, I5, 10,16bis I8; Dan Th7,6 13, 8,15, 10, 16 I8, Act 9, I8; Apoc 4,6, 6,6; 8,8, 9,7, 15,2, 19, I 6ter, Jos et As 17,6, Test Stm 7,2bis, Test Jos 19,8 Cf $\mathrm{R} H$ Charles, $A$ critical and exegetical commentary on the Revelation of St John, I, Edinburgh I920, pp 35-37 
littéralement le Э̦ hébraiqque et araméen¹. Est-ce bien un sémitisme? Les rares auteurs qui se posent la question l'affirment ${ }^{2}$. Mais il existe des parallèles profanes qui nous obligent à penser que les traducteurs de la Septante sont restés ici dans les limites du grec courant. C'est

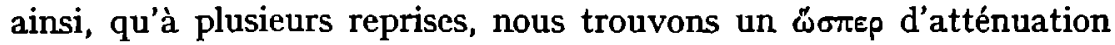
devant le verbe, sans que la construction en soit influencée du tout,

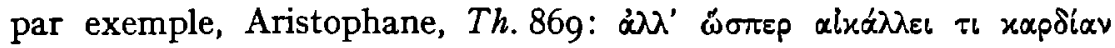

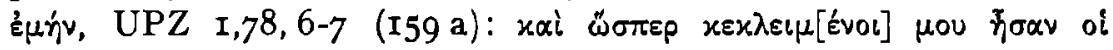
o $Q \vartheta \alpha \lambda \mu o l$ rov. Mais nous trouvons aussi de telles atténuations avec le sujet et l'objet de la phrase ${ }^{8}$, par exemple, Ps. Hésiode, $S c .72: \pi \tilde{p} \delta^{\prime}$

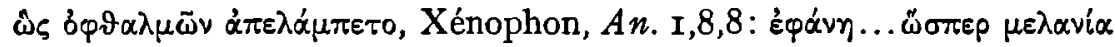
$\tau \iota \varsigma$, Galien, De atra bile 4,5: olov $\lambda \varepsilon \pi i \varsigma$ á $\pi \dot{\varepsilon} \pi \iota \pi \tau \varepsilon$. Ce genre d'expressions se rencontre en latin: César, Gal.6,26,2: ab eius summo sicut palmae ramique late diffunduntur, Pline, $E p .8,16,1$ : permitto servis quoque quasi testamenta facere.

Comme nous l'avons dit, nous ne croyons pas que les $\dot{\omega} \zeta$, $\dot{\omega} \sigma l$,

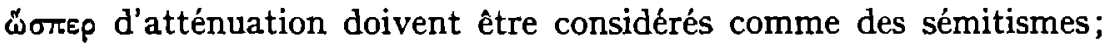
encore moins d'ailleurs comme des latinismes, ainsi que les dernières citations pourraient l'avoir suggéré. Nous avons pourtant quelques raisons pour nous arrêter encore un moment sur cet emploi. On constate, en effet, qu'on y recourait avec une certaine prédilection dans

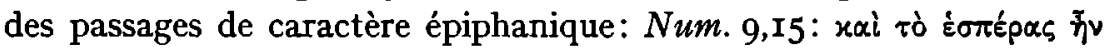

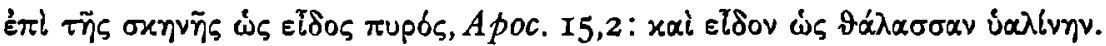
On retrouve la même chose dans des textes ultérieurs ${ }^{4}$, par exemple

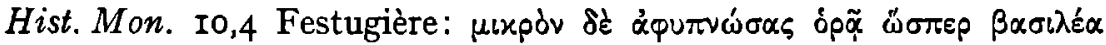

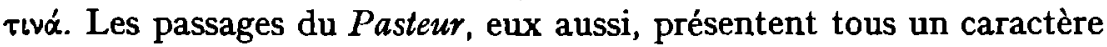
de vision. De toute évidence, ís est un des moyens qui servent à indiquer que le prophète a accès à une autre réalité (cf. Act. II,5:

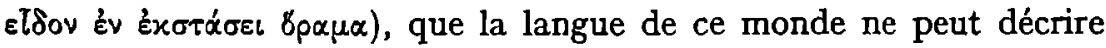
que par approximation. La citation de UPZ I,78,6-7 montre d'ailleurs que ce moyen était aussi utilisé hors des cercles juifs et chrétiens.

I. Pour l'hébreu cf. Koehler-Baumgartner, Lexikon, s.v. ๆ 6.

2. Bauer, Worterbuch, s.v. \&s II 3 a; T. Muraoka, The use of is in the Greek Bible, Novum Testamentum 7, 1964-1965, p.(51-72)57.

3. Cf. Monteil, Phrase relative, pp.343-344.

4. Aux passages cités, nous pouvons ajouter: Ev. Evae (chez Epiphane, Haer. 26,3,1); Pass. Perp. 12,3; Acta Pil. 15,6; 18,1; Mart. Matth. 22; Acta Phil. 97. 106.124; Acta Joh. 94. 


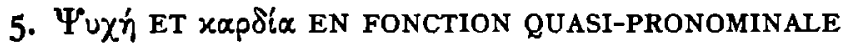

Dans beaucoup de langues, on peut employer des mots signifiant "esprit", "cœur", "âme" pour désigner la personne qui possède cet esprit, ce couur, cette âme. C'est annsi qu'ils peuvent servir aussi de paraphrases au pronom personnel et au pronom réfléchi. Parmi les innombrables exemples que nous pourrions citer, en voici trois:

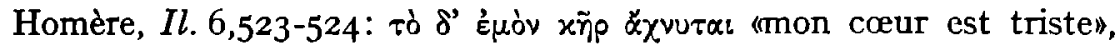

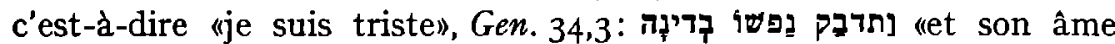
s'attacha à Dina", c'est-à-dire «il s'attacha à Dina», Plaute, Men. ro82: nisi me animus fallıt, "si mon esprit ne m'abuse», c'est-à-dire «si je ne me trompe». Parfois l'auteur emploie les pronoms et ces équivalents

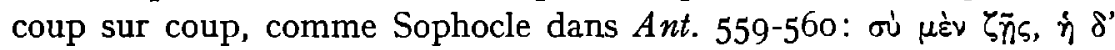

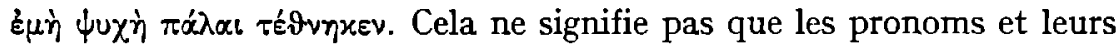
paraphrases sont simplement interchangeables. L'emploi de ces paraphrases est soumis à certaines restrictions. Pour commencer, elles conservent généralement assez de leur sens propre pour ne pouvoir servir à désigner que des actions et des situations spirituelles ou psychiques. Ensuite, elles ont souvent une valeur d'ordre stylistique, de sorte qu'on ne peut les utiliser dans n'importe quel contexte elles caractérisent fréquemment l'usage poétique ${ }^{1}$. A la lumière de ces remarques, examinons chez Hermas quelques expressions avec $\psi u \chi \dot{n}$ et $x \alpha \rho \delta i \alpha$.

Dans la Septante, $\psi u \chi \eta \dot{~ e s t ~ g e ́ n e ́ r a l e m e n t ~ l a ~ t r a d u c t i o n ~ d e ~}$ Cette traduction a souvent été critiquée parce qu'elle suggérerait la dualité de l'homme en âme et corps, idée étrangère au terme hébreu et à la pensée de l'Ancien Testament en général. Bratsiotis a remarqué que c'est prendre le terme $\psi u \chi \eta \dot{~ d a n s ~ s o n ~ s e n s ~ p h i l o s o p h i q u e ~ e t, ~ e n ~}$ particulier, dans son sens platonicien, sens que $\psi u \times \chi_{n}^{\prime}$ n'avait pas à l'origine. Il montre que dans le grec d'avant Platon, les différents sens de $\psi u x h ́$ correspondent d'une façon étonnante à ceux de $\nabla$ g, et il en conclut que la critique faite aux traducteurs de la Septante est injustifiée ${ }^{3}$. Son argumentation aurait pourtant eu beaucoup plus de force si ses investigations s'étaient étendues au grec ultérieur, c'est-àdire celui des siècles qui ont vu naître la Septante Car, on a l'impression

I Pour cet emplo1 quasi-pronominal, vorr, par exemple, Havers, Syntax, Pp IIo-III, SD II p I92 n I, A Dihle e a, art $\psi v \chi \dot{\eta}$. ThWNT IX, pp 604-66r. 2 Voir $\mathrm{D}$ Lys, The Israelite soul according to the $L X X$, Vetus Testamentum 16 ,

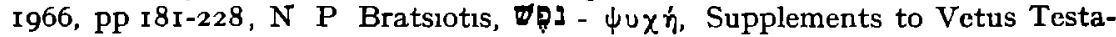

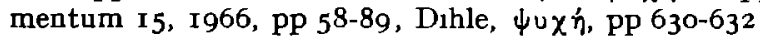

3 Bratsiotis, $\psi \cup \chi \dot{\eta}$, pass Vour aussi Dihle, $\psi \cup \chi \dot{\eta}, \mathrm{p} 630$ 
qu'à cette époque, le sens platonicien a prédominé et que les sens plus anciens, sauf le sens de "vie» sont tombés en désuétude 1 . Sans aucun doute, Bratsiotis a raison de défendre les traducteurs de la Septante contre l'accusation qu'avec leur traduction, ils auraient déformé le message de l'Ancien Testament. D'un autre côté, il faut reconnaître que la langue de la Septante devait bien avoir ici quelque chose de bizarre pour des oreilles grecques: plusieurs auteurs patristiques signalent qu'une des particularités de la langue biblique c'est que

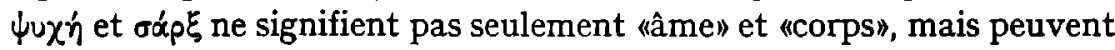

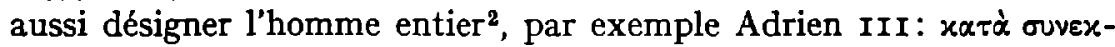

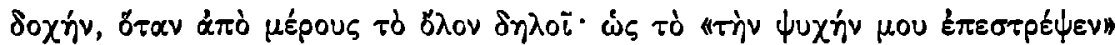

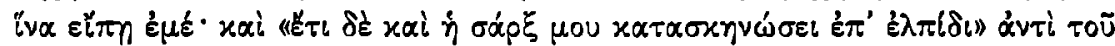
É$\gamma \omega \dot{\omega}$. En examinant les significations que $\psi v \chi \hat{\eta}$ peut avoir dans la littérature biblique, il faut donc tenir compte de sémitismes possibles. L'emploi quasi-pronominal de ce mot, entre autres, est considéré comme un sémitisme, même par ceux qui sont peu enclins à en supposer. Le fait que l'hébreu ne disposait pas d'un pronom réfléchi et se trouvait donc réduit à utiliser de telles périphrases ${ }^{3}$, alors que le

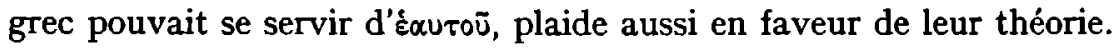
Signalons pourtant, en grec, une ébauche de cet emploi dans un

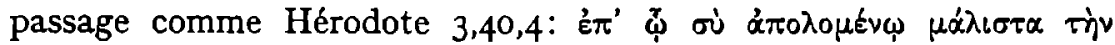

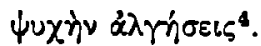

Dans le Pasteur, dès le chap. I,9, nous pouvons relever une for-

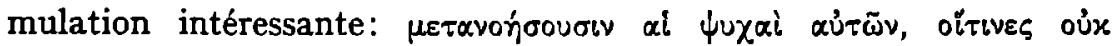

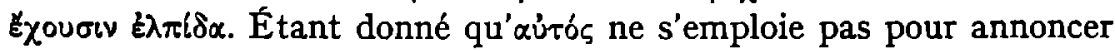
une proposition relative, l'antécédent du masculin oîtıves est le féminin Wuxal, construction ad sensum, qui trahit que, dans la pensée de l'auteur,

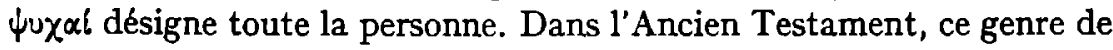
constructions ad sensum se présentent régulièrement avec অọ Ensuite, il faut signaler, dans le chap. 39, l'emploi alternatif de tó

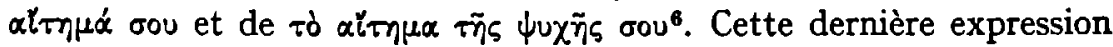

I. Cf. Lys, Soul, p.227; G. Dautzenberg, Sein Leben bewahren, $\Psi$ $\cup(\dot{\eta}$ in den Herrenworten der Evangelien, München 1966, pp.31, 39; Dihle, $\psi \cup \chi \dot{\eta}$, pp.608-614.

2. Théodore de Mopsueste ad Ps. 15,9.10; 33,3; Adrien 1 7.49. I I I.

3. Passages chez J. H. Becker, Het begrip nefesj in het Oude Testament, Amsterdam 1942, pp.58-76; cf. ib. pp.I, I IO-II 2; Dautzenberg, Leben, pp.20, Ior n.26.

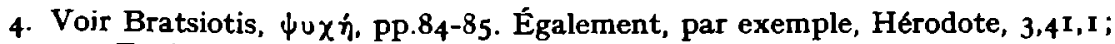
3.43.2 ; Euripide, Ba.75.

5. Voir Becker, Nefesj, pp.82-85.

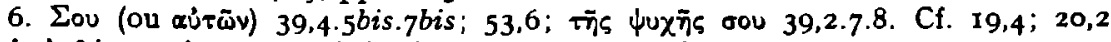

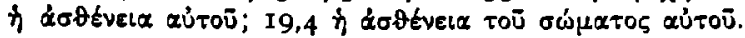




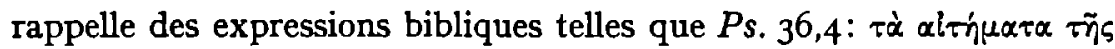

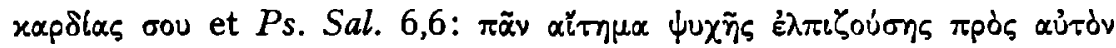

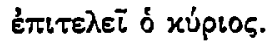

Quatre fois Hermas emploie le mot $u ̛ x$ ń pour désigner l'âme en tant que siège de la faim et de la soif ou, d'une façon plus générale, des besoins matériels. Cette fonction n'est pas inconnue non plus dans le

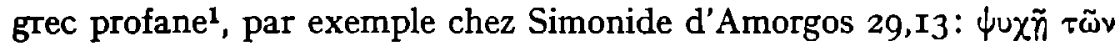

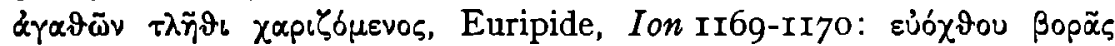

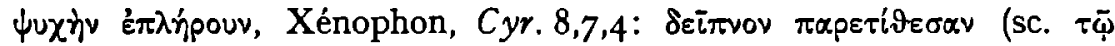

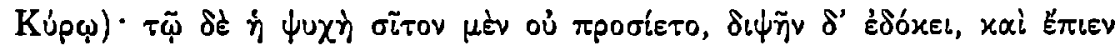

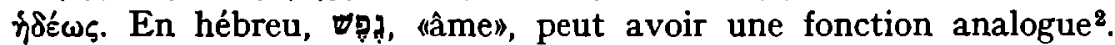
C'est pourquoi les traducteurs de la Septante étaient parfaitement fondés de traduire ici par $\psi u x \hat{n}$, par exemple $N u m$. II,6: vuvì $\delta \dot{\varepsilon}$ (à savoir, maintenant que nous sommes partis d'Egypte) $\dot{\eta} \psi u \chi \dot{\eta} \dot{\eta} \mu \tilde{\omega} v$

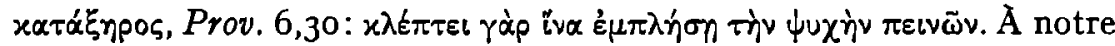
avis, Hermas se conforme à cet usage. Mais étant donné que, dans un article de I952, Van Unnik interprète différemment les passages que nous visons ${ }^{8}$, il nous faut nous y arrêter un moment.

Dans 56,7, Hermas recommande au Pasteur de manger moins et de

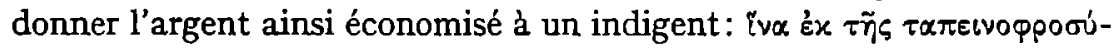

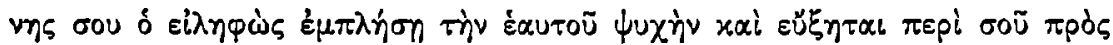

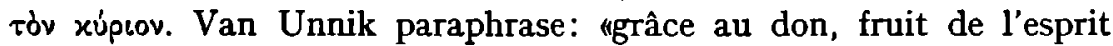
d'humilité, le pauvre est porté à la reconnaissance et à la prière, parce

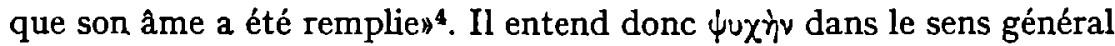
d' «âme». On peut faire deux objections à cette explication. Premièrement, au sujet du bénéficiaire, il n'est pas dit que son âme se remplit, mais qu'il la remplit; par conséquent, selon l'interprétation de Van Unnik, il faudrait dire qu'au moyen du don, le pauvre se porte à la reconnaissance et à la prière, ce qui manque tout à fait de naturel.

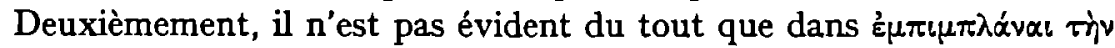

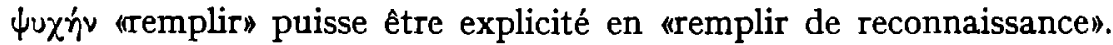
En revanche, pour le complément wavec de la nourriture» on trouve des

I. Voir Bratsiotis, $\psi u \chi \eta \dot{n}$, p.67. Également, par exemple, Euripide, $C y c$. 340-34I; Ramsay, Phrygia, I 2, p.477, inscr.343,5.

2. Voir, par exemple, Becker, Nefesj, pp.31-36, 42-43, 70, ro4-ro5. Dans le même sens $\psi u x \dot{\eta}$ dans la Septante, voir Bratsiotis, $\psi u \chi \eta^{\prime}$, p.66; Lys, Soul, pass. Dans le Nouveau Testament $M t .6,25 ; L c$. 12,19.22-23, voir Dautzenberg, Leben, pp.92-97 et 85; dans les apocryphes Par. Jer. 9, I 8 .

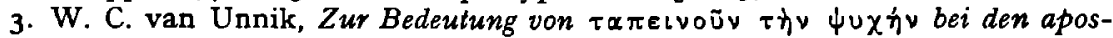
tolischen Vätern, Zeitschrift für die neutestamentliche Wissenschaft 44, 19521953, pp. 250-255.

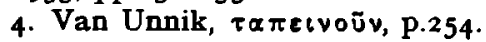


parallèles tant en grec profane que dans le grec de traduction de la Septante, ainsi que nous l'avons vu plus haut, et le simple verbe

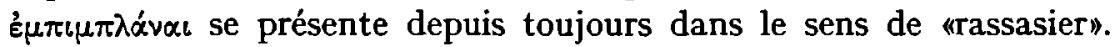
C'est pourquoi nous pensons qu'ici il faut entendre é $\mu \pi \iota \mu \pi \lambda \alpha^{\prime} v \alpha \iota$ in่

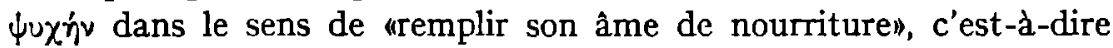
"se rassasier".

Nous trouvons plus de difficultés dans 5I,8: oi $\pi \varepsilon ́ v \eta \tau \varepsilon s ~ u ̇ \pi \varepsilon ̀ p ~ \tau \tilde{\omega} v$

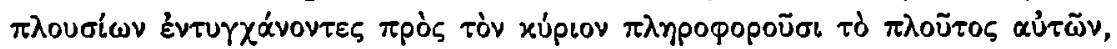

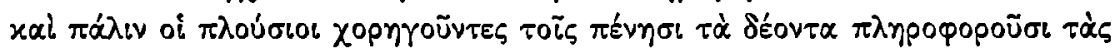

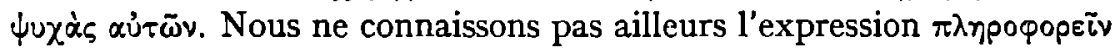
Triv $\psi$ uxh̆v et ne verrions pas d'inconvénient à la considérer comme un

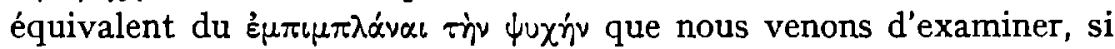

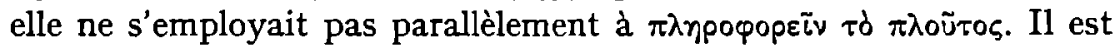
évident, en effet, que là on ne peut traduire $\pi \lambda \eta$ рофорвĩ par «remplir». Mais, dans les deux locutions, on peut interpréter $\pi \lambda$ गрофореī comme "compléter ce qui manque à. Ce qui manque chez les riches, c'est la bienveillance de Dieu, et chez les pauvres, c'est la nourriture. Le parallélisme est forcé, mais cela ne peut nous étonner chez Hermas. Quant à $\psi v \chi \eta^{\prime}$, le mot peut désigner ici le siège de la faim et de la soif ou, d'une manière plus générale, le siège des besoins matériels.

Les autres passages peuvent être traités ensemble. Ce sont:

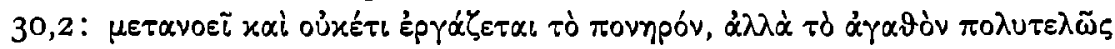

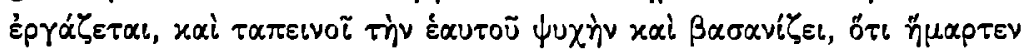

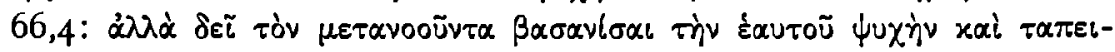

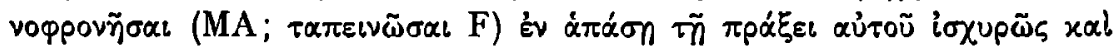

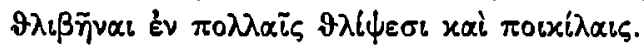

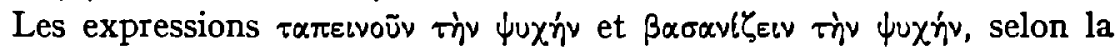
plupart de ceux qui ont traité cette question, visent le jeûne ${ }^{1}$. C'est là

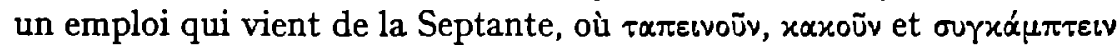
construits avec $\psi v \chi_{n j} \eta^{\prime}$ désignent le jeûne ou la pénitence qui s'y rattache, comme s'asseoir dans le sac et la cendre. Nous le retrouvons dans les Testaments des Douze Patriarches, dans une inscription juive du II siècle avant J.-C. et dans le Protévangile de Jacques ${ }^{2}$; comme

I. W. Grundmann, art. $\tau \alpha \pi \varepsilon$ เvós $x \tau \lambda$, ThWNT VIII, Pp.(I-27)25, 26; Bauer,

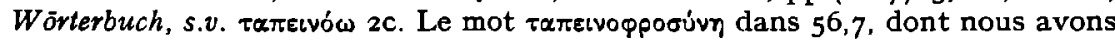
parlé ci-dessus, est d'ailleurs souvent compris comme un terme désignant le jeûne.

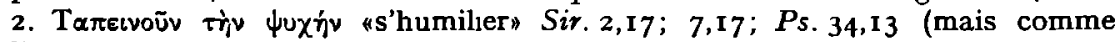

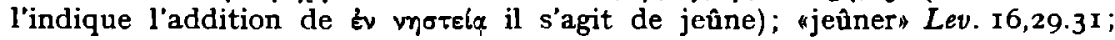
23.27.32; "se mettre dans le sac et la cendre" (?) Judith 4.9; Is. 58,3.5; Protev.

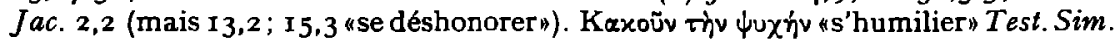

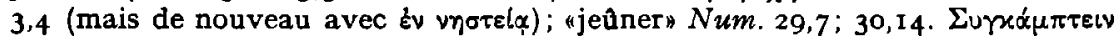


citation de la Septante, on le trouve chez Philon, Poster. I3,48 et le Ps. Barnabé 3,I.

Van Unnik croit que, dans le cas d'Hermas, il faut penser au sens plus général de "s'humilier", "se mortifier"1, mais, bien que, dans les deux citations, ce sens soit très valable, cette interprétation suscite

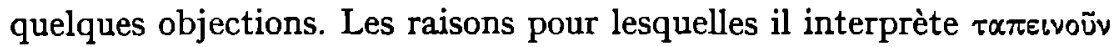

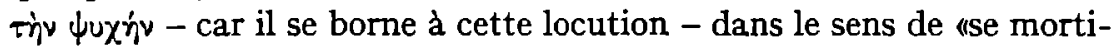
fier", semblent être $I^{\circ}$ qu'il s'agit, chez Hermas, de la disposition intérieure et non de l'acte extérieur, $2^{\circ}$ qu'ainsi, pour des Grecs, l'expression était compréhensible et ne constituait pas un spécimen de jargon biblique inintelligible.

Au sujet du premier argument nous voudrions dire qu'en effet, il ne fait aucun doute que, pour Hermas, le jeûne est subordonné à un sentiment d'humilité et n'en est qu'une expression ${ }^{2}$. Mais cela ne

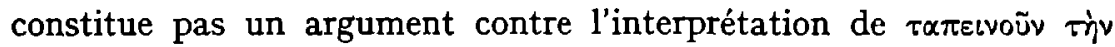
$\psi u x$ ńv au sens de jeûne. A plusieurs reprises Hermas dit de lui-même qu'il jeûne $(6,1 ; 9,2 ; 18,7)$ et même que l'Église le lui prescrit ex-

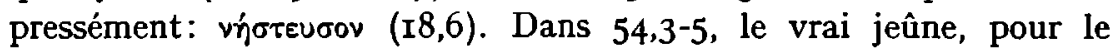
Pasteur, c'est sans doute de faire le bien et d'éviter le mal, et il semble rejeter le jeûne ordinaire comme inutile. Mais c'est à une forme très particulière de jeûne qu'il pense là, comme on le constate dans $56,7-8$, où il recommande le jeûne afin de donner aux pauvres ce qu'on a ainsi épargné; cf. infra, p.I73. Le deuxième argument pèche par la base, étant donné qu'Hermas n'écrit pas pour un public paien, mais pour des lecteurs qui sont habitués au grec de la Bible. Aussi est-il difficile d'admettre, pour ne pas dire de comprendre, que Van Unnik écrive: uje ne crois pas qu'il soit nécessaire d'examiner à cet égard les données fournies par la LXX, car on n'a pas affaire là à une expression spécifiquement grecque» ${ }^{3}$. La conclusion que nous croyons pouvoir tirer, c'est que le mieux sera de continuer à interpréter ces expressions

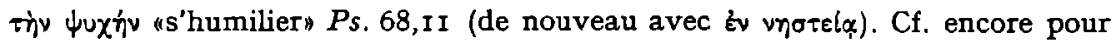

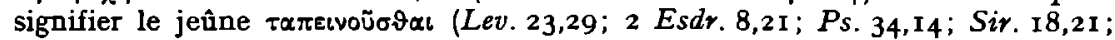

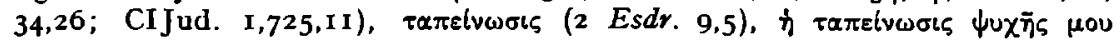

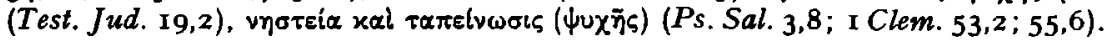
Voir aussi Grundmann, $\tau \alpha \pi \varepsilon i v \delta s$, pp. 7-8, I I, I3. 15, 23, 25-27, et, pour dater CIJud. 1, 725, A. Deissmann, Licht vom Osten, Tübingen I $923^{4}$, p.360.

I. Van Unnik, $\tau \alpha \pi \varepsilon เ$ voūv, p.254, cf. p.25I. Philon, Poster. 13.48 avait déjà donné la même interprétation pour Lev. 23,27.

2. Cf. Pernveden, Church, pp. 255-257.

3. Van Unnik, $\tau \alpha \pi \varepsilon เ$ ขอบัข, p.254. 
comme désignant le jeûne ou des pratiques de pénitence qui s'y rattachent.

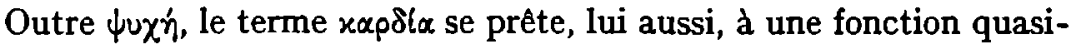
pronominale. Chez Hermas, on peut signaler tout d'abord l'expression

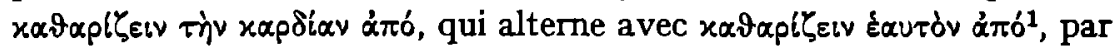

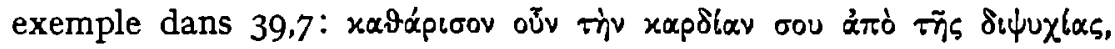

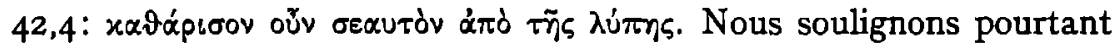
de nouveau que, si les deux expressions se rejoignent pour le sens, cela n'implique pas que rapঠí ait perdu à peu près toute signification. $\mathrm{K} \alpha p \delta l \alpha$ désigne ici le siège de la conscience, la vie morale (cf. Hebr.

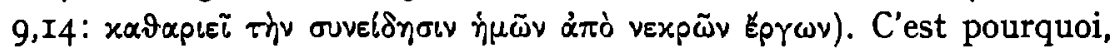

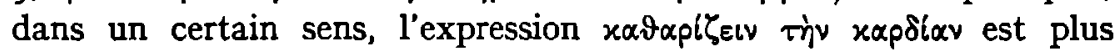

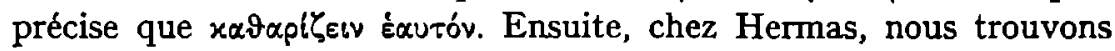

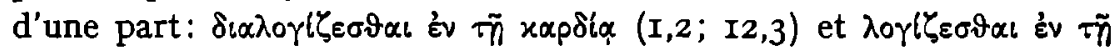

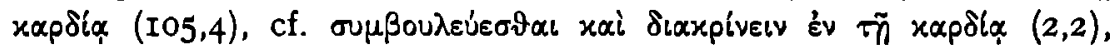

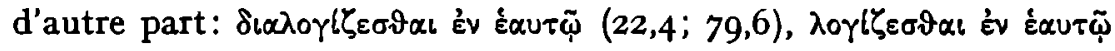

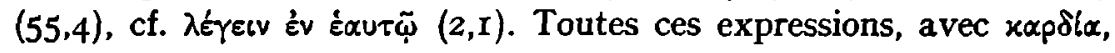
remontent directement ou indirectement à l'hébreu ou à l'araméen, ou aux deux.

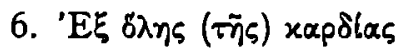

Dans le texte du Pasteur qui est parvenu jusqu'à nous, nous trouvons

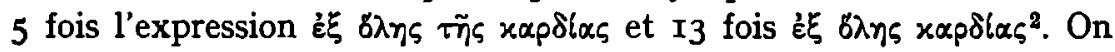
peut se demander si la variabilité dans l'emploi de l'article est le fait de l'auteur, d'autant plus qu'à certains endroits la tradition manuscrite est divisée $(6,4 ; 20,3 ; 39,2)$. Mais, si nous tenons compte du fait que ces variations se produisent aussi ailleurs, par exemple dans

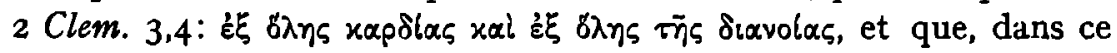
genre de petites choses aussi, Hermas se montre peu logique ${ }^{3}$, nous

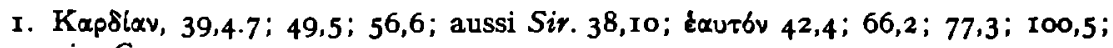
aussi 2 Cor. 7,1 .

2. Successivement 6,$4 ; 33,7 ; 39,2 ; 49,2 ; 105,2$ et 3,$2 ; 20,3 ; 21,4 ; 23,5 ; 35,5$; 48,$2 ; 49,1 ; 66,4$ bis; 72,$2 ; 77,3 ; 91,6 ; 110,3$.

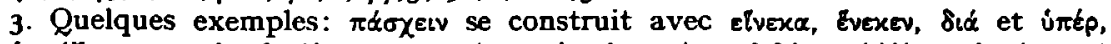

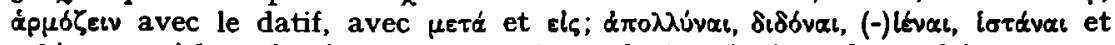

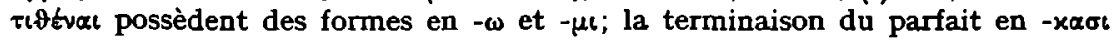
alterne avec $-x a v$; les verbes en $-a \omega$ a la deuxième personne du singulier du

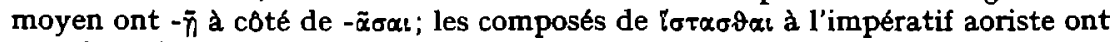

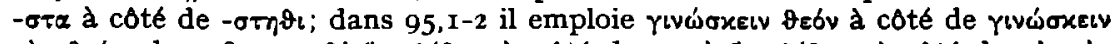

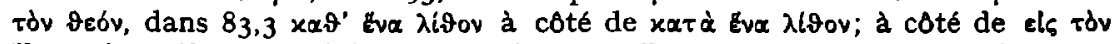

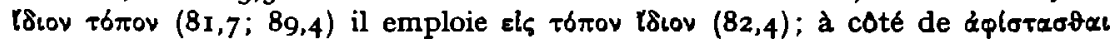


pouvons à bon droit, nous semble-t-il, partir du texte tel qu'il nous a été transmis.

Les formules d'Hermas n'existent pas en grec profane, mais font partie de l'idiome de la Bible grecque. Pour déterminer leur place dans celle-ci, considérons d'abord la Septante. Nous y trouvons cinq expressions étroitement apparentées, qui toutes tirent leur origine de

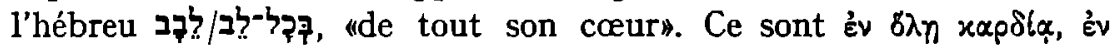

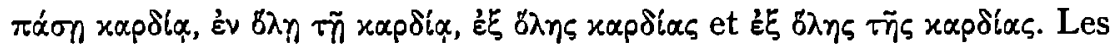
deux premières sont les traductions les plus littérales de l'hébreu; les deux autres s'écartent de l'original par l'article et la préposition éł; la cinquième, qui combine article et $\mathfrak{g} \xi$ est donc la traduction la moins littérale. Du point de vue du grec, l'article n'est pas nécessairement une

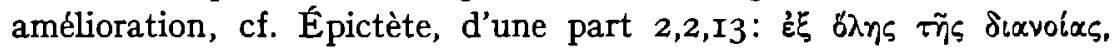

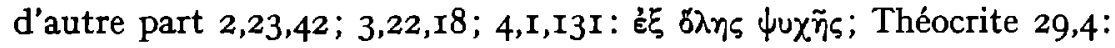

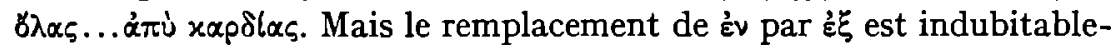
ment une concession au grec, qui connaît de nombreuses expressions synonymes avec éx et $\alpha \dot{\alpha} b^{1}$, mais aucune avec év.

La fréquence des cinq expressions présente une répartition re-

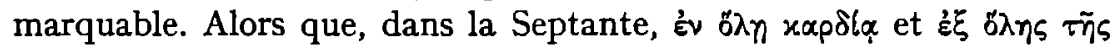

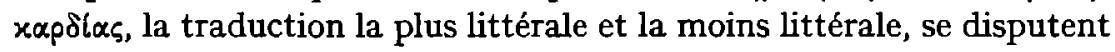
la première place et que les trois autres ne sont que rarement utilisées, plus tard, en grec biblique ultérieur, les expressions en év sont tombées

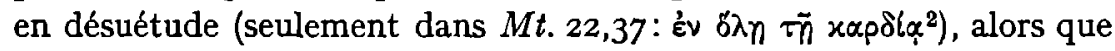
se maintiennent, fût-ce sur une échelle restreinte, les deux expressions en $\dot{\varepsilon}_{\xi}$. Des deux dernières, c'est celle avec l'article qui est la plus répandue, alors qu'Hermas emploie de loin le plus souvent celle sans l'article $^{3}$. Le schéma suivant donne un aperçu de la fréquence.

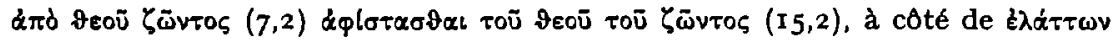

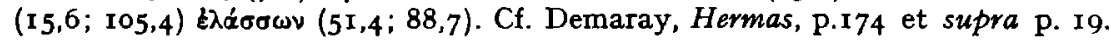

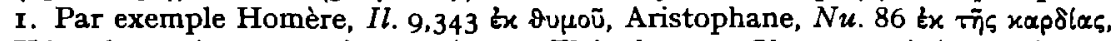

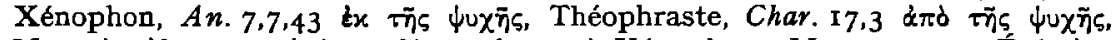
Marc-Aurèle 2,3,3 ámò xapolas; cf. aussi Xénophon, Mem. 3,11,10; Épictète

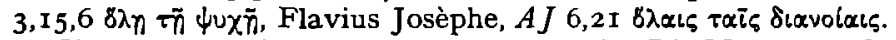

2. C'est un texte du shema'. Selon J. Jeremias Die Muttersprache des Evangelisten Matthäus, Zeitschrift für die neutestamentliche Wissenschaft 50, I959, pp.27o274, ce texte prouve pour Matthieu que "comme on peut s'y attendre d'un Palestinien, sa langue maternelle était l'araméen, sa langue de prière l'hébreu». Cf. aussi Dautzenberg, Leben, pp. I I4-1 23.

3. Les passages pour év $5 \lambda \eta$ xapdia sont: I Regn. 7,3; 12,20.24; 3 Regn. 2,4; 8,48; 4 Regn. 10,31; 23,25; 2 Par. 6,14.38; 22,9; 34,31; 35,19'; Tob. BA I3,6; S 2,2; Ps. 9,2; 85, I2; I I0,I; I I8,2.10.34.58.69.145; 137,I; Prov. 3,5; Sir. 7,27;

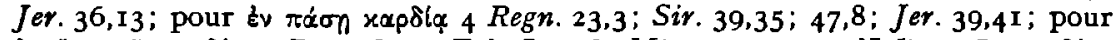

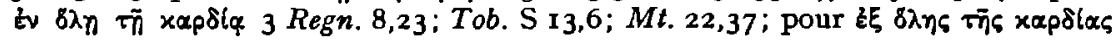




\section{Autres textes juifs \\ et chrétiens \\ jusqu'au IIIe siècle}

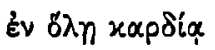

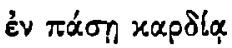

Ev $6 \lambda \eta n$ in xapdía

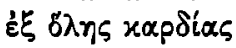

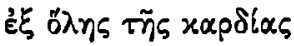

$27 \times$

$4 \times$

$2 \times$

$\mathrm{I} \times$

I6 $X$

-
-
$I X$
$6 \times$

Io $X$
$-$

$-$

-

$13 X$

$5 \times$

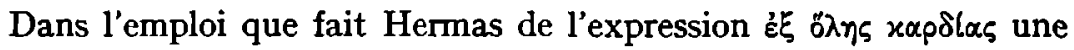
particularité syntaxique mérite, elle aussi, notre attention. Il pourvoit,

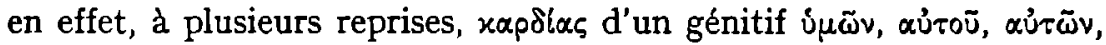
qui détermine le mot de telle sorte que, selon l'usage grec, il faudrait

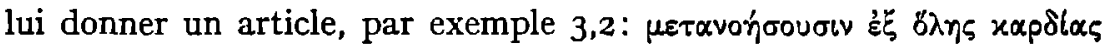

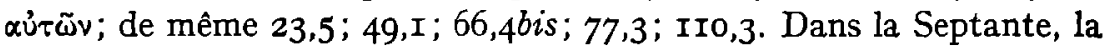

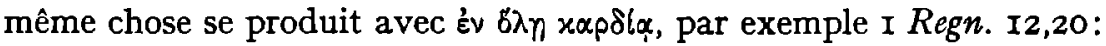

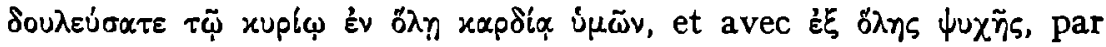

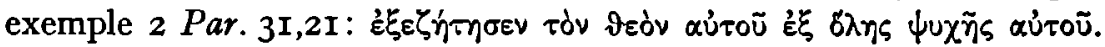
Ici, on peut attribuer la suppression de l'article à l'exemple hébreu qui, du fait qu'il était déterminé par un suffixe, ne pouvait avoir d'article. Hermas peut avoir, consciemment ou non, imité l'usage de la Septante. D'un autre côté, l'article, en pareil cas, fait souvent défaut aussi dans les inscriptions et les papyrus grecs. Et enfin, une éventuelle influence latine a aussi pu stimuler la suppression de l'article'.

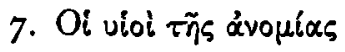

Dans I4,I, nous voyons des pécheurs impénitents caractérisés par l'expression oi vioi tĩs ávoulac. Nous avons là un exemple de métaphore généalogique, manière de s'exprimer qui représente le lien entre deux choses ou entre une chose et une personne, comme une relation entre parent et enfant. Pareille métaphore se retrouve dans beaucoup de langues, en grec aussi. Néanmoins, nous voulons montrer que l'expression d'Hermas est un sémitisme.

4 Macc. 7,18; Test. Levi 13,1; 2 Clem. 3.4; 17,1.7; 19,1; Clément d'Alexandrie,

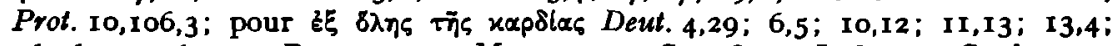
26,16; 30,2.6.10; 2 Par. 15,12; 4 Macc. 13,13; Sap. 8, 21 ; Joel 2,12; Soph. 3,14; Jer. 3,10; 24.7; MC. 12,30.33; LC. 10,27; (Act. 8.37); I Clem. 8,3; 34,4; 2 Clem. 8,2; Justin, I A pol. 16,6; Dial. 93,2bis.3. Pour Hermas voir supra p. I42 n. 2. I. Cf. G. B. Winer-P. W. Schmiedel, Grammatik des neutestamentlichen Sprachidioms, Göttingen I894-I898, Pp.I67-168; Meuwese, De rerum gestarum divi A ugusti versione graeca, Pp.36-39; SD II Pp.24-26; BD $\$ 275^{4}$. 
Nous ne pourrons pas en prendre à notre aise en disant comme Turner qu'viós, employé avec un génitif pour désigner une certaine qualité, ne se présente que rarement dans le grec pré-biblique et se limite à des expressions comme vids 'E $E \lambda \alpha \alpha \delta \delta \varsigma$ '. Au contraire, le grec dispose d'un grand nombre de métaphores généalogiques. Dans

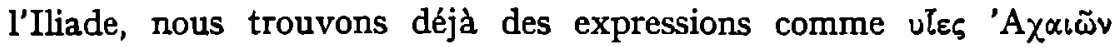

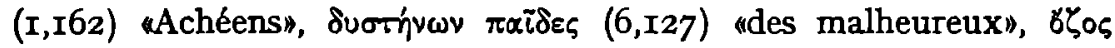
"Apnos (I2,I88) "combattant courageux". On pourrait citer de nombreux exemples très variés dans des périodes ultérieures; nous ne

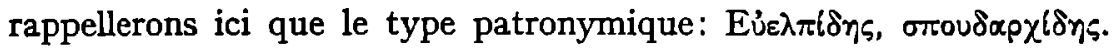
Dans la poésie dramatique, on trouve quelques passages où les génıtıfs

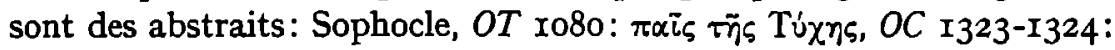

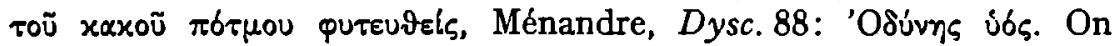
remarque que ces abstraits indiquent le sort auquel est soumis la personne en question. Des constructions où l'abstrait indique une qualité dont dispose la personne, comme c'est le cas chez Hermas, ne se présentent pas en grec profane pour autant que nous sachions. D'une façon plus générale, on peut remarquer qu'en grec, la métaphore généalogique a un caractère expressif qui la réduit à se confiner dans certains genres littéraires, surtout poétiques.

En hébreu et en araméen, la métaphore généalogique est plus fréquente qu'en grec, et, dans ces langues, elle n'a pas non plus la valeur stylistique qu'elle a en grec. Nous devons en chercher la raison dans le nombre restreint d'adjectifs dont disposent ces langues. Là où les Grecs pouvaient désigner des qualités avec des adjectifs, l'hébreu et l'araméen devaient avoir recours à des substantifs. Il a déjà été question, supra p.Iıo, du procédé le plus courant, qui consiste à joindre au nomen regens le substantif comme génitif. Mais les métaphores généalogiques sont, elles aussi, nombreuses, tant dans l'Ancien Testament et les textes de Qumran que dans la littérature rabbinique ${ }^{2}$,

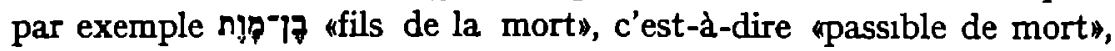

I. Turner, Syntax, pp 207-208. Les grands lexıques, aux mots desıgnant -fils", "flllew, "enfant", donnent une meilleure idée de ce que la littérature grecque offre dans ce domaine Vour aussi Kuhner-Gerth, Grammatı, I, p 28I, Winer-

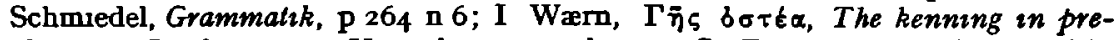
christian Greek poetry, Uppsala 1951, chap 7, S Boschennı, 'O súvis is s (Menandri, Dysc, $v$ 88), Studi Italiani di Filologia Classica N S 31, 1959, pp 247-253 (pour le latin aussi) Cf aussı l'emploı métaphonque non sémitisant de ouýrinp chez Hermas $16,4-5,39,9,45,2$.

2 Vour J Zobel, Der bildliche Gebrauch der Verwandtschaftsnamen im Hebrärsehen mit Berucksichtigung der übrigen semitischen Sprachen, Halle 1932, pp 22-35; G Fohrer, E. Lohse et aln, art. ul6,, ThWNT VIII, P (334-400)347, 359-360 
"fils d'une rançon», c'est-à-dire «otages». Des qualités, en particulier des qualités morales, s'indiquent aussi de cette façon, par

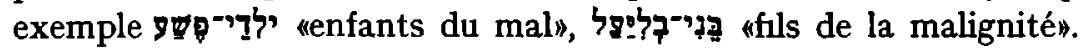

Les traducteurs de la Septante ont vu que la plupart de ces images avaient totalement pâli et ont traduit en conséquence, par exemple

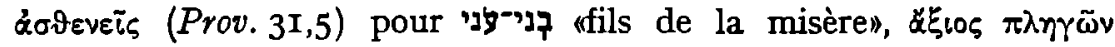

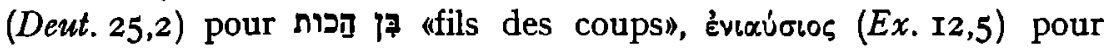

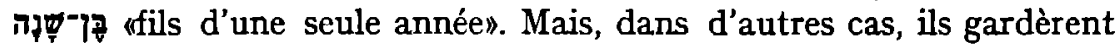
les métaphores ${ }^{1}$. C'est ainsi que virent le jour des expressions comme

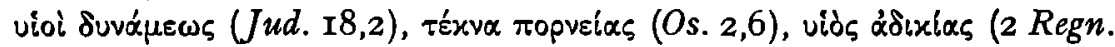
$7, \mathrm{IO})$. Ces expressions et d'autres de ce genre invitaient à l'imitation dans les textes écrits en grec $^{2}$, par exemple vids sipñvns (Lc. I0,6),

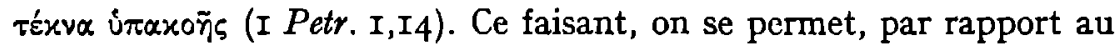
modèle sémitique, des écarts qui améliorent le grec sans tout à fait oblitérer l'origine sémitıque. C'est ainsi qu'on intervertıt l'ordre du nomen regens et du nomen rectum: óprñs víbs (Apoc. Mos. 3), qu'on met

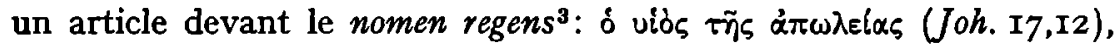

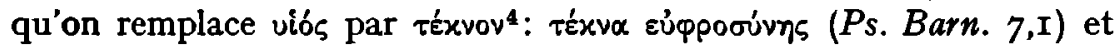

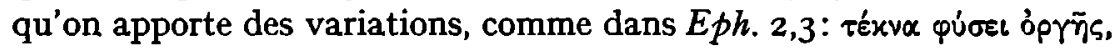

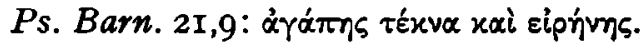

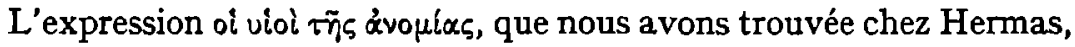
se présente aussi dans $P s .88,23$ (uìs ávoulas) et Apoc. Petrı 3. Dans le Nouveau Testament, elle fait défaut, mais cf. 2 Thess. 2,3: ó \&v 9 pwros

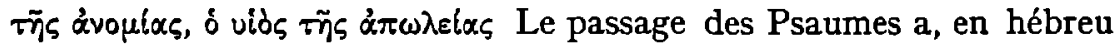

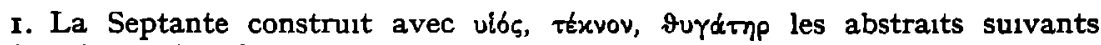

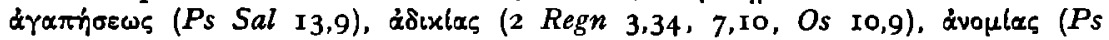

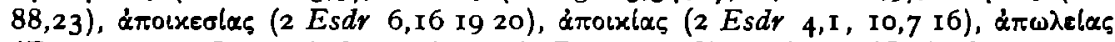

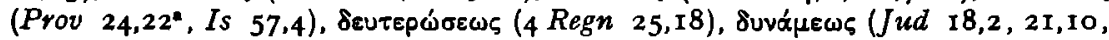
I Regn 10,26, 14,52, 18,17, 2 Regn 13,28, 17, 10bis, 3 Regn 1,52, 21, 15, 4 Regn

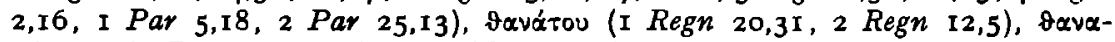

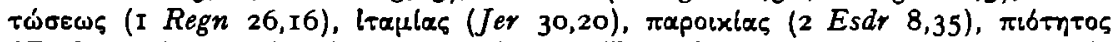

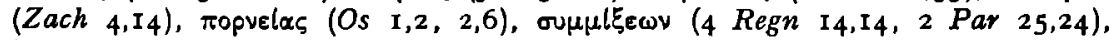

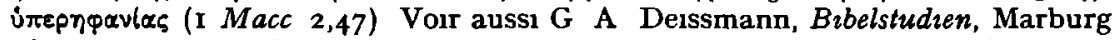
I895, Pp I64-165. Johannessohn, Kasus, pp 25-26, 32-33. Thackeray, Grammar, Pp 4I-42

2 Pour le Nouveau Testament vour Moulton-Howard, Accidence, p 44I (ajouter Joh 12,36, Act 3,25) Des apocryphes, outre les passages indiqués dans le texte, nous pouvons citer I Hen 10,9, Test Job 43,6 vl, Ps Barn 9,7, 3 Cor. 19 3 Déjà dans la Septante

4 Dans la Septante $O s$ ro,9 est le seul passage où 'jọ soit traduit par Téruva plus littéraire L'autre passage avec téxva, Is 57.4, comporte déjà 'Tl' en hébreu 
(Ps. 89,23) ) l'Ancien Testament et dans les écrits de Qumran'.

Notre conclusion sera donc que l'expression d'Hermas est étrangère au grec profane, qui n'utilisait pas la métaphore généalogique pour des qualités morales et qui ne l'utilisait pas dans la prose littéraire: elle correspond à l'usage sémitisant de la Bible grecque. Dans ce sens, c'est un sémitisme. D'autre part, l'adjonction d'un article devant vioi, qui en hébreu et en araméen ferait défaut, montre qu'Hermas développe ce qu'il a trouvé dans le grec et que le passage où cette expression se présente n'est pas la traduction d'un document sémitique.

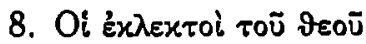

Dans le Pasteur, nous trouvons à plusieurs reprises les constructions

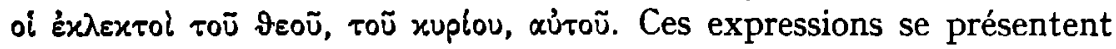
uniquement dans les Visions et d'aucuns en ont tiré argument pour supposer que cette partie de l'ouvrage est d'un auteur différent ${ }^{2}$. C'est comme sémitismes qu'elles nous intéressent ici. De ce point de vue, elles sont importantes, aussi bien prises globalement qu'envisagées pour la fonction que le génitif y remplit. Nous verrons d'abord ce dernier aspect.

En hébreu et en araméen, un participe passif et un adjectif verbal peuvent être suivi d'un nomen rectum pour indiquer l'agent de l'action:

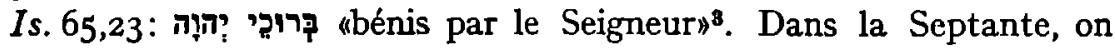
traduisait généralement le participe ou l'adjectif verbal de façon adéquate par un participe passif. Mais le nomen rectum présentait une difficulté. Une traduction littérale exigeait un génitif. Cette traduction était habituelle lorsque le nomen rectum dépendait d'un substantif, et elle aurait pu être employée d'une manière analogue dans le cas où il dépendait d'un participe passif ou d'un adjectif verbal. Mais en grec, avec un participe passif, on n'avait guère l'habitude de désigner l'agent par un génitif. Il y a pourtant quelques exemples, comme Euripide,

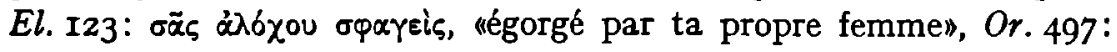

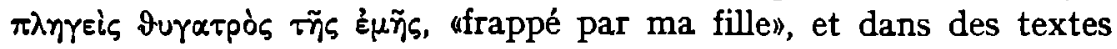

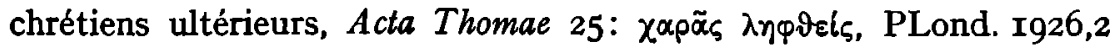

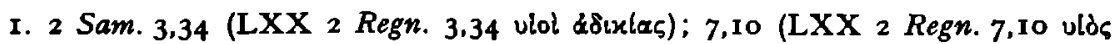

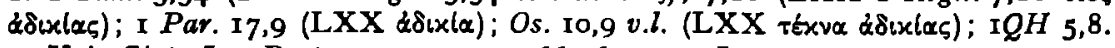
2. Voir Giet, Les Pasteurs, pp. 179, 266-267, 271. Les passages sont: 3.4; 5.3; 6,$5 ; 13,1 ; 16,3 ; 17,10 ; 23.5 ; 24.5$.

3. Voir Gesenius-Kautzsch, Grammatik, pp. 374, 438; Joüon, Grammaire, pp. 345, 389. 


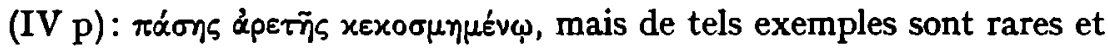
sans doute susceptibles d'être expliqués d'une autre manière'. Pour les traducteurs de la Septante, ce genitivus auctoris, comme on dit, était manifestement du grec si impossible qu'ils ont tout fait pour l'éviter en le remplaçant par exemple par úxó + gén. ${ }^{2}$; c'est ainsi qu'Is. 65,23 ,

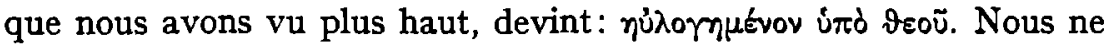
connaissons que deux cas où ils ont admis le simple génitif: 2 Par.

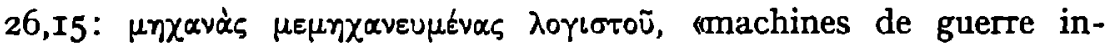

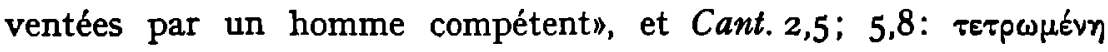

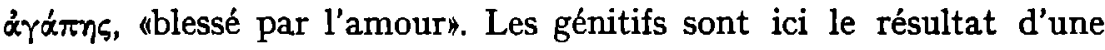
traduction littérale; mais, dans 2 Par.26,I5, le participe du grec correspond à un substantif de l'hébreu.

Dans un certain nombre de cas, on trouve les expressions sémitiques dont il était question plus haut, en fonction de substantifs, par

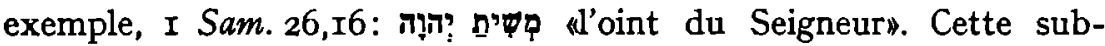
stantivation n'est pas formellement caractérłsée. Mais les traducteurs de la Septante ont cherché à marquer une différence. Outre que, dans la plupart des cas, la substantivation était marquée par l'article, ici, au lieu de leur traduction habituelle: participe passif $+\dot{u} \pi \delta+$ génitif,

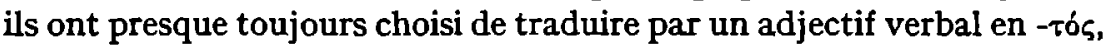
suivi d'un simple génitif. C'est ainsi que I Sam. 26, I6, dans la Septante,

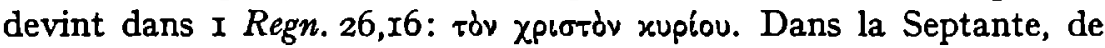

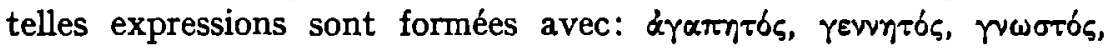
$\delta เ \delta \alpha x \tau \delta \zeta, \dot{\varepsilon} x \lambda \varepsilon x \tau \delta \zeta, x \lambda \eta \tau \delta \zeta, \mu\left\llcorner\sigma \vartheta \omega \tau \delta \varsigma^{3}\right.$.

Au sujet du génitif dans ces expressions, on peut remarquer que, si on entend les adjectifs verbaux substantivés comme des noms, le génitif est un génitif possessif, ce qui est du grec parfaitement usuel. Mais il nous paraît plus juste de supposer que, dans le cas de ces expressions, le chemin d'une fonction verbale à une fonction nominale n'a été parcouru qu'à demi. D'une part, elles présentent un caractère nominal et s'accompagnent d'un article; d'autre part, elles continuent à évoquer l'idée d'une action. Or, à l'égard de cette action, le génitif se charge d'indiquer la personne qui agit, comme un genitivus auctoris. La

I. Cf. SD II p.I I9, et d'un autre côté Lasso de la Vega, Sintaxis, I, pp.46r-465. 2. Voici quelques passages: 2 Regn. 3.39; Judith 9,4; Ps. 106,2; Prov. 22, 14; Sir. 46,13; Is. 62,12; Bar. 3.37; Dan. 3,35. Quelquefois un adjectif verbal au

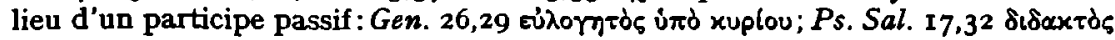
í $\pi \delta$ froū.

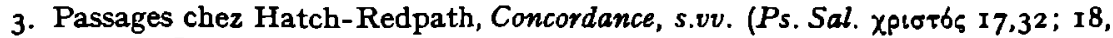
inscr. 5.7). Ici nous avons laissé de côté les substantivations neutres comme dans $I s .46,1 \tau \dot{\alpha} \gamma \lambda u \pi \tau \dot{\alpha} \alpha u \dot{\tau} \tau \bar{\omega}$. 
question se pose dès lors de savoir si, avec des adjectifs verbaux substantivés, où l'idée d'une action est encore présente, le grec peut utiliser le génitif pour la personne qui accomplit l'action. Nous avons, en effet, l'impression qu'on peut s'exprimer ainsi en grec, mais qu'on a rarement recours à cette possibilité. Voici des exemples: Démosthène 18,52 :

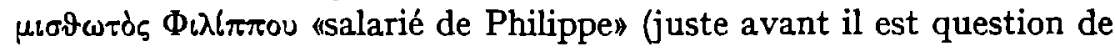

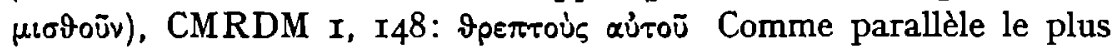

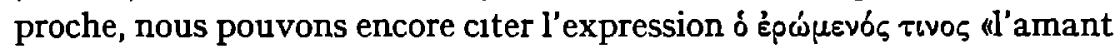

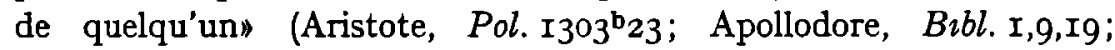
$2,5,8 ; E$ prt. 2,3$)$.

Le Nouveau Testament et les apocryphes se bornent en général à reprendre les expressions qui avaient été formées dans la Septante ${ }^{1}$. Mais ce qui est nouveau, c'est, chez Matthieu et le Ps. Barnabé, l'emploi du participe passif, par analogie avec l'adjectif verbal: of

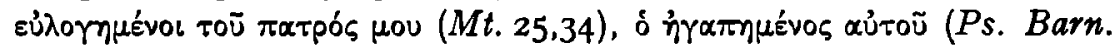
$3,6 ; 4,3)$. Dans des textes ultérieurs, l'adjectif verbal sert aussi à

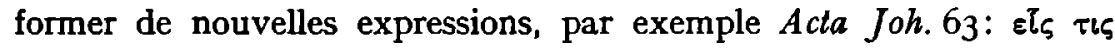

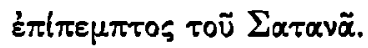

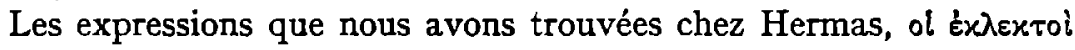

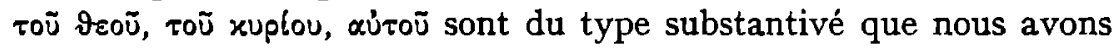
décrit ci-dessus. La substantivation, qui est indiquée extérieurement par l'addition de l'article, ressort aussi, plus profondément, du contexte. Partout ces expressions sont considérées comme des touts indivisibles, comme des termes techniques à l'usage des Chrétiens, et ce, dans un contexte où l'attention se porte sur autre chose que sur le choix des élus par Dieu. Cela déconcerterast le lecteur s'il lisait ces expressions de telle sorte qu'elles appellent toute son attention sur le choix de Dieu. En conséquence, on peut couramment supprimer le

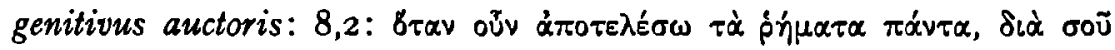

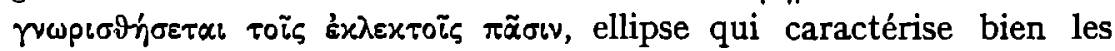
langues de groupe ${ }^{2}$. Que, d'autre part, on puisse encore faire transparaître la nuance verbale, c'est ce que montre 24,5: Tò $\delta \varepsilon \dot{\varepsilon} \lambda \varepsilon u x \delta े v$

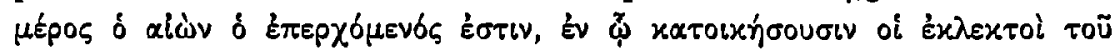

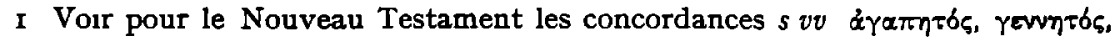

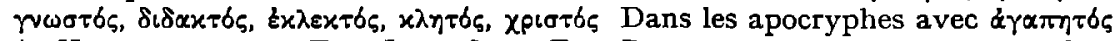
(I Hen 12,6, 100,2, Test Levi 18,13, Test Benj 11,2, Apoc Esdr pp 24 32bis,

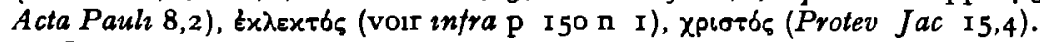
2 Voir G. Bartelınk, Ellipse und Bedeutungsverdichtung in der christlachen griechischen Literatur, Vigiliae Chnstianae 10, 1956, pP I-I3 Ol Exגextot également absolu 1 Clem 6,1 , 58,2, Mart Pol 16,1 


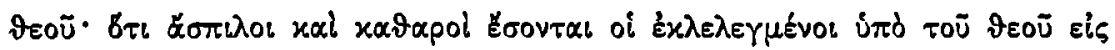

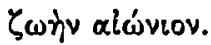

Ces expressions trouvent leur origine dans la Septante. Mais on continua à les employer plus tard: dans le Nouveau Testament, les apocryphes, les Pères Apostoliques et les auteurs chrétiens ulté-

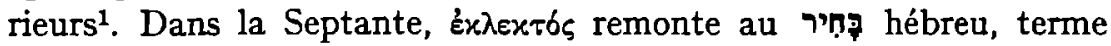
que, dans les textes de Qumran, nous retrouvons accompagné d'un mot signifiant Dieu ${ }^{2}$. Ces expressions étaient donc d'un usage courant dans des milieux divers. Mais le sens de "Chrétiens", chez Hermas, montre bien que, dans son cas, nous n'avons pas besoin de chercher à dépister des contacts avec Qumran ou d'autres cercles juifs au sujet de ces expressions. Ce sens s'est développé à partir de passages paléotestamentaires, comme Ps. 104,43:

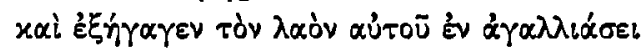

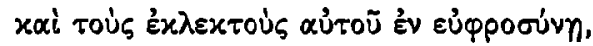

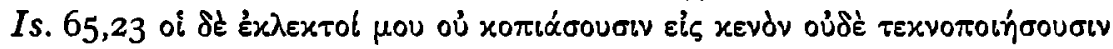

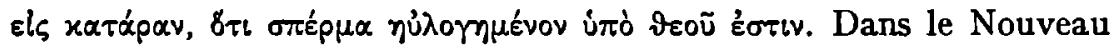
Testament, l'expression s'applique aux Chrétiens: $M t .24,31$ : xai

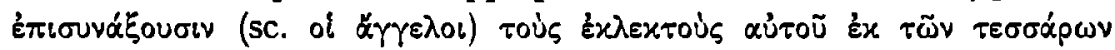

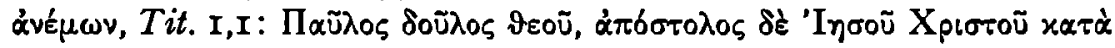

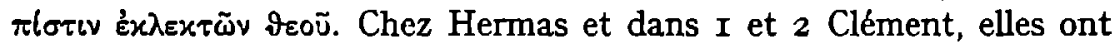
atteint le stade de terme technique.

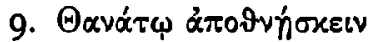

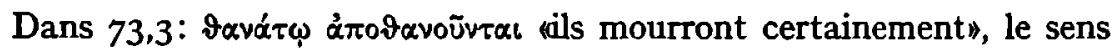
du verbe est renforcé par un nom étymologiquement apparenté, au datif. Il est important de remarquer que le datif, de son côté, n'est pas pourvu d'un adjectif qualificatif. Lorsque c'est le cas, comme, par

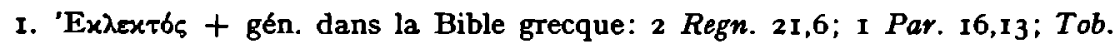
BA 8,15; Ps. 88,4; 104,6.43; 105,5.23; Sap. 3,9; 4,15; Sir. 46,1; 47,22; Agg. 2,22 v.l.; Is. 42,I; 45,4; 65,9.15.23; Lam. 1,15; Dan. $\mathrm{Th}_{\mathrm{I} I, 15 ;}$ Mt. 24,31; Mc. 13,27; Lc. 18,7; Joh. 1,34 v.l.; Rom. 8,33; Col. 3,12 (?); Tit. 1,1; dans les apocryphes paléotestamentaires: Test. Abr. A 2; Jos. et As. 8, I ; 13, 10; Test. Benj. I I,4: Par. Jer. I, 1.4; 3.4.5; 7,I5; Vit. Proph. Jer. II; A poc. Esdr. pp.24.32; Test. Job 4, I I. Il n'y en a pas dans les apocryphes néotestamentaires, mais bien chez les Pères Apostoliques I Clem. I, I ; 2,4; 46,4.8bis; 49,5; 59,2; 2 Clem. 14,5. Cf. G. Schrenk, art. éx $\lambda \in x \tau \delta$ s. ThWNT 1V, pp.I86-197.

2. I $Q p H a b$ 5,4; 9,12; 10,13; $1 Q M$ 1 2,4; $1 Q H$ I4,15; $4 Q p J s^{4}$ 1,3; $1 Q p P s 37$ 2,5; 3,$5 ; 4,12 ; 4 Q M e s s$ ar 1,10 . Dans les livres protocanoniques de la Septante le

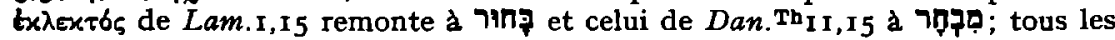
autres passages correspondent a ר. ThWNT IV, Pp. (I 47-I8I) 148-I 49 . 


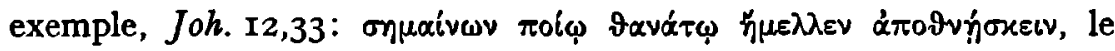
datif a une tout autre fonction. Il ne sert pas alors à renforcer le verbe, mais à ne soutenir que formellement l'adjectif qui, dès lors, devient le

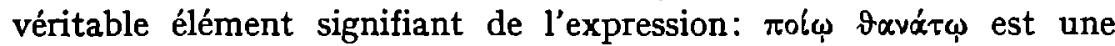
périphrase plus sonore de l'adverbe $\pi \bar{\omega} \varsigma$. Ce dont nous parlons ici, c'est le datif sans adjectif.

L'emploi de ce datif n'est pas tout à fait inconnu en grec classique ${ }^{1}$.

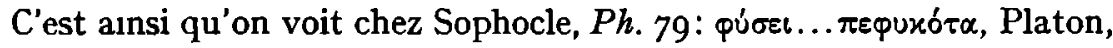

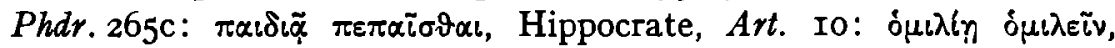

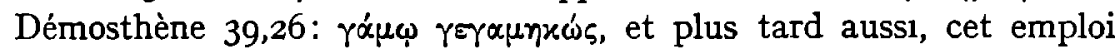

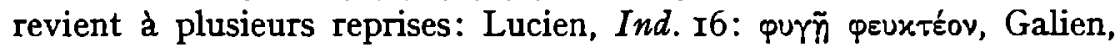

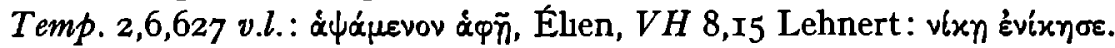
Mais il est si rare qu'on ne peut parler d'un procédé productif. Toute différente est la situation dans la Septante, où le nombre d'expressions aussi bien que le nombre de passages où elles se présentent est plusieurs fors ce qu'on trouve en grec profane.

Cette fréquence n'est pas sans raisons particulières ${ }^{2}$. Les traducteurs, en effet, étaient confrontés à la difficulté de trouver une traduction adéquate pour l'infinitif absolu hébreu servant à renforcer une forme

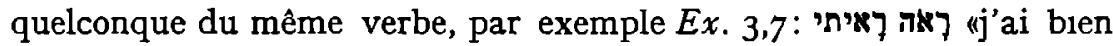
vu $\|^{3}$. Une traduction strictement littérale aurait été incompréhensible en grec; aussi n'en trouve-t-on, dans la Septante, qu'un seul exemple:

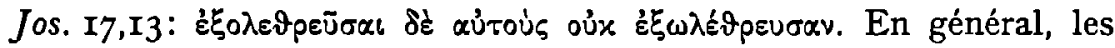
traducteurs ont cherché un compromis en traduisant l'infinitif par un mot étymologiquement apparenté au verbe, soit un nom au datif,

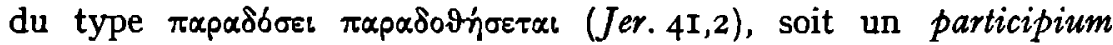

I Voir Chr A Lobeck, Paralipomena grammaticae graecae, Lipsiae 1837, pp 523-527, Lasso de la Vega, Siniaxis, I, Pp 61 I-61 2 Pour le latin voir Szantyr, Syntax, p 125; le phénomène y est sı peu fréquent, qu'il faut exclure toute influence latine sur Hermas 73,3

2 Cf G R Hauschild, Die Verbindung finiter und infinter Verbalformen desselben Stammes in einzgen Bibelsprachen, Bernchte des Freien Deutschen Hochstiftes zu Frankfurt am Main NF 9, I893, pp 99-I 27 (également pour la Bible latine), $\mathrm{H}$ St $\mathrm{J}$ Thackeray, Renderings of the infinitive absolute in the $L X X$, The Journal of Theological Studies 9, 1908, pp 597-601, 1d , Grammar, pp 47-50; Suss, Studien, pp I Io-I I6 (également pour la Bible latıne et le jugement d'Augustin), H Kaupel, Beobachtungen zur Ubersetzung des Infinutivus absolutus in der Septuaginta $(G)$. Zeitschrift fur die alttestamentliche Wissenschaft N F 20, I945-I948, PP I9I-I 92

3 Voir à ce sujet J M Solá-Solé, L'ınfınıtıf sémitıque, Paris I96I, pP 96-104, 201-204, G Goldenberg, Tautological infinitive, Israel Oriental Studies I, I97I, pp $(36-85) 63-73$ 


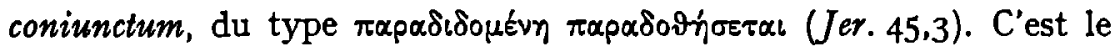
premier type de construction qui nous intéresse ici.

En s'autorisant des parallèles profanes que nous avons cités plus haut, on a parfois cru ne pas devoir considérer cette construction comme un sémitisme. Mais rien que du point de vue fréquence - plus de 200 fois dans la Septante - elle mérite le nom de sémitisme secondaire. A cela s'ajoute que les nombreuses formules avec des composés,

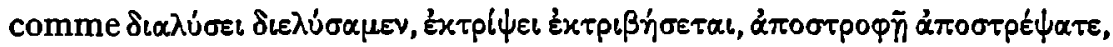
n'ont pas de pendant, en grec profane, à notre connaissance.

Au sujet de la répartition de la construction avec le datif et avec le participe, remarquons encore que, dans le Pentateuque, on trouve surtout le datif, dans les autres livres historiques, presque toujours le participe; ailleurs, les constructions participiales priment, sauf dans Isaie, Ezéchiel, Michée et le Codex Vaticanus de Josué et des Juges. En grec biblique ultérieur, on ne trouve plus les constructions participiales que dans les citations de la Septante. La construction avec le datif continue cependant à être imitée ${ }^{1}$. C'est ainsi que les expressions de la Septante non seulement sont de nouveau utilisées, par exemple $L c$.

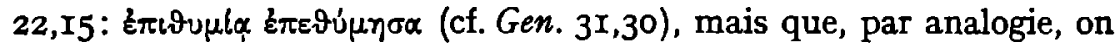
forme des expressions qui n'existent pas dans la Septante, par exemple

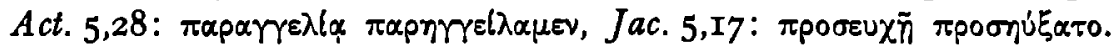
On introduit même, après coup, des constructions avec le datif dans

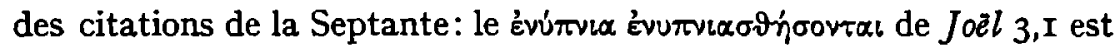

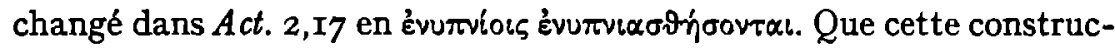
tion, dans le Nouveau Testament et la littérature apparentée, soit due à l'imitation de la Septante, cela ressort du fait qu'en hébreu et en araméen contemporains cette construction n'existe guère ou pas du tout ${ }^{2}$. A cet argument, tiré de l'absence, on peut ajouter un argument positif: la construction est surtout fréquente dans les œuvres de Luc (Lc. 22,I5;Act. 2,17; 4,I7 v.l.; 5,28; 23,14; 28,26), auteur dont les sémitismes, comme on l'admet généralement, ne viennent pas d'une langue maternelle sémitique, mais tiennent à une imitation intentionnelle du style de la Septante ${ }^{a}$.

I. Cf. Thackeray, Renderings, pp.600-60I. Outre les endroits que nous citons dans le texte, la construction avec le datif se présente dans $M t$. 13,14 (cit.); Joh. 3.29; Test. Abr. A 15; Test. Levi 9, 14; Test. Zab. 3,3; Test. Jos. 12,2; Par. Jer. 8,7; 9,21; Ps. Barn. 9, I (cit.). 2 (cit.); cf. Mt. 15,4 (cit.); Mc. 7, 10 (cit.); 2 Petr. 3.3; A poc. 2, 23; Test. Zab. 2,3. Quelques passages tardifs: $A$ cta Prl. A 4,3; 15,5.6; Callinicos I24,43,9; POxy. 8, I I62,5 (IVP); Jean Chrysostome, Ep. I4,3. 2. Voir Solá-Solé, Infmntit, pp.103-104, I5I-153; cf. Mussies, Koine Greek, pp.99. 323-324.

3. Voir supra p.72 n.8. 


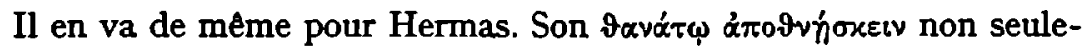
ment s'inspire de la Septante, mais en reproduit la langue. On l'y retrouve dans différents livres, comme le Pentateuque, l'Ecclésiastique et Jérémie et, dans les Livres des Rois, cette expression se maintient comme quasiment la seule traduction avec le datif au milieu de traductions avec le participe'. Hors de la Bible, elle est utilisée par Apoc. Mos. 17, dans le récit du péché originel, qui la comporte aussi dans Gen. 2,I7; 3,4. En grec profane, l'expression fait complètement défaut.

Remarquons enfin qu'Hermas a peut-être utilisé plusieurs fois

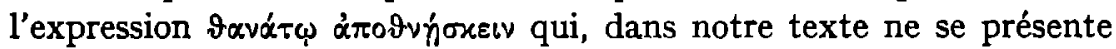
que dans 73,3. C'est ainsi que, dans 45,3 , là où nous lisons $\alpha \pi \circ \vartheta \alpha v o u ̃ v \tau \alpha \iota$

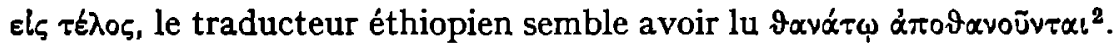

\section{Io. Kai isoú}

A partir du VIe siècle avant J.-C., à côté de l'impératif aoriste moyen

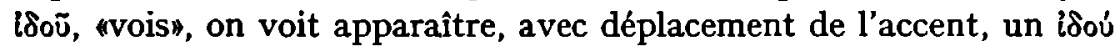
interjection, qui s'emploie pour attirer l'attention sur une chose. De son origine vient la propriété qu'il conserve de s'adresser à une deuxième personne. Aussi, sa place naturelle est-elle dans la conversation: théâtre, dialogue, littérature épistolaire. Hermas l'utilise plusieurs fois dans un contexte de ce genres. Mais il l'emploie aussi à plusieurs reprises d'une manière qui ne se présente pas en grec profane, à savoir dans un contexte narratif. C'est le cas dans les passages suivants:

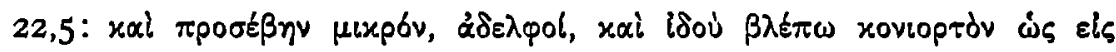
Tòv oủpavóv

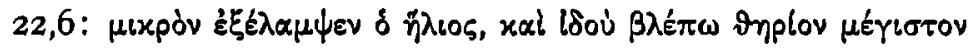

I. Voici les passages: Gen. 2,17; 3,4; Num. 26,65; Jud. AB 13,22; A 21,5; I Regn. 14,39.44; 20,14; 22,16; 2 Regn. 12,14; 14,14; 3 Regn. 2,37.42; 4 Regn. 1,4.6.16; 8,10; Sir. 14,17; Jer. 33,8, et en outre dans le codex Alexandrinus

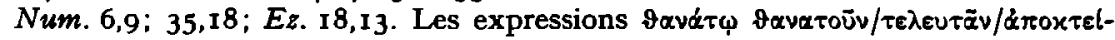

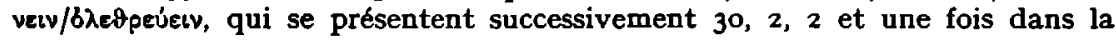
Septante, sont apparentées.

2. Dans la traduction d'Abbadie morte morientur.

3. 3,4 bis; 7,$4 ; 10,4 ; 66,4$. Dans ces passages, nous ne trouvons pas de différence marquée avec l'usage profane, comme c'est le cas dans les citations chez P. Fiedler, Die Formel uund siehe" im Neuen Testament, München 1969, Pp. 17-2I. Mais un contemporain aurait peut-être remarqué une nuance hébraique dans 3,4 et 7,4, cf. Théodore de Mopsueste ad Ps. 26,6; 48,16; 50,7; 53.6; 54,8; 58,8. 


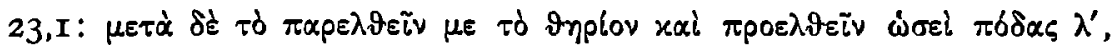

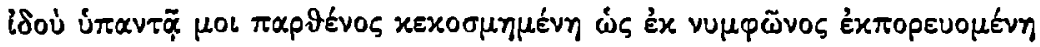

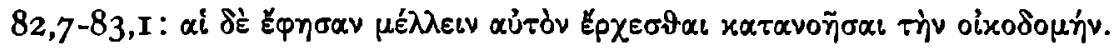

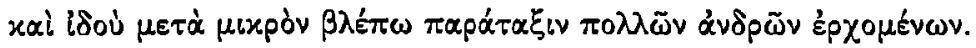

Sauf 23,1 , ces passages ont en outre la particularité qu'íoú soit précédé de xai, combinaison qui n'existe pas non plus en grec profane'.

Or, en grec biblique, nous retrouvons aussi bien l'emploi du mot dans

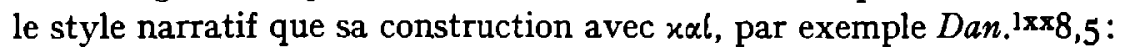

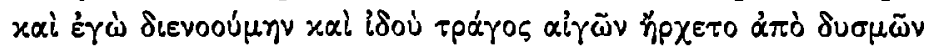

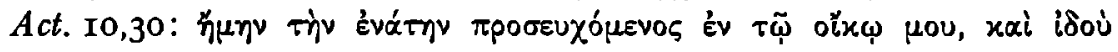

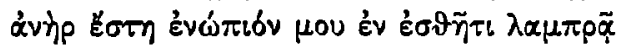

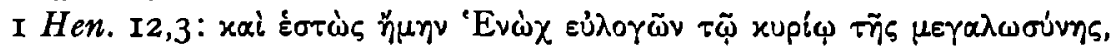

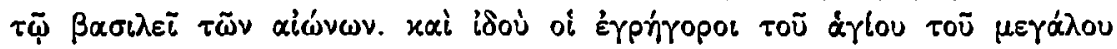
Éxádiouv $\mu \varepsilon$.

Dans ces passages, l'emploi d'íoú est sémitisant; il remonte à celui du הạ hébreu ${ }^{2}$. Mais avant de conclure qu'Hermas se conforme ici à l'emploi de la Bible grecque, il faut signaler que le ecce latin peut s'employer d'une manière analogue, par exemple Virgile, $A$. 2,268-27 I :

tempus erat quo prima quies mortalibus aegris

incipit et dono divom gratissima serpit:

in somnis ecce ante oculos maestissimus Hector

visus adesse mihi largosque effundere fletus

Ovide, Met. 7,863-864:

flentibus haec lacrimas heros memorabat: et ecce Aeacus ingreditur duplici cum prole

Apulée, Met. II,3: necdum satis coniveram, et ecce pelago medio venerandos diis etiam vultus attollens emergit divina facies.

I. Selon Fiedler, Und siehe, pp.21, 25, 45, 46. Il ne cite pas Epictète 3, 22, 107

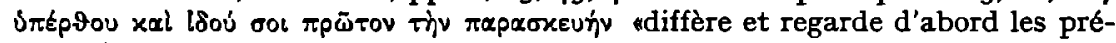
paratifsı, mais là isoú a encore une valeur verbale; le passage n'est d'ailleurs pas narratif.

2. Voir M. Johannessohn, Das biblische xai $1 \delta 00$ in der Erzählung samt seiner hebräischen Vorlage, Zeitschrift für vergleichende Sprachforschung 66, 1939. Pp.145-195; 67, 1942, pp.30-84; Bickerman, Septuagint, Pp.25-26, 28 n.6o. Pour l'araméen voir Beyer, Semitische Syntax, p.69. La fréquence dans les apocryphes égale celle de la Bible grecque; les passages narratifs sont: isoú Apoc. Mos. 32; I Hen. 14,8; Test. Abr. B 9; Jos. et As. I 4, I; 18, 1; Test. Jud. 3,5; Par. Jer. 4, I; 6,II; Apoc. Bar. 12,1; xal tsoú Apoc. Mos. 33; I Hen. 12,3; 13,8; 14,15; Test. Abr. A 2,1 2 bis.14. 16; Test. Abr. B 10; Jos. et As. 5, I; 10, I8; 14,8; 26,8; 28,8; Test. Levi 2,6; Test. Naphth. 5,2.6.8; 6,2.10; Par. Jer. 6, I ; 9, I ; A poc. Bar. 1,3; 6,2; I I.5; Protev. Jac. 4, I.2.4; 8.3bis; 9, I; I I,I.2.3; 13. I; 14,2; 20,3.4; $21,1.3$. 
Ces citations montrent tout d'abord que ecce admet aussi la combinaison avec et. Ensuite, on constate que ecce se présente aussi dans le style narratif, et plus particulièrement lorsqu'il est question de visions décrites par un témoin oculaire. Enfin, un trait commun à tous

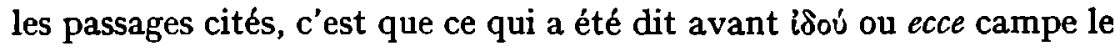
décor dans lequel l'action introduite par idoú/ecce se déroule'.

Avec l'emploi qu'Hermas fait d'ioou, nous sommes donc en présence d'un phénomène dont, en théorie, l'explication peut être aussi bien sémitique que latine. Étant donné que les deux influences devraient mener au même résultat, la formulation grecque en soi ne permet pas de savoir si nous avons affaire à un sémitisme, à un latinisme ou aux deux à la fois. Dans le cas d'Hermas, on ne peut défendre une éven-

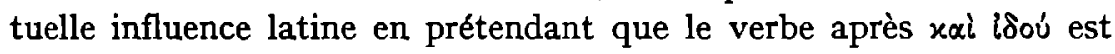
un présent historique. Il est vrai qu'une chose similaire est impossible en hébreu, et normale en latin; mais nous avons affaire ici à une adaptation à l'usage grec, qui s'explique fort bien sans influence étrangère, et que déjà l'on constate à plusieurs reprises dans la Septante ${ }^{2}$. Reste donc la question de savoir à quelle influence il faut attribuer le cas d'Hermas. Attendu que $I^{\circ}$ cet usage est très fréquent en grec biblique, alors qu'il est relativement et absolument beaucoup plus rare en latin, $2^{\circ}$ que le cachet sémitique de la langue du Pasteur, pour autant que nous ayons pu le constater jusqu'ici, est beaucoup plus fort, en étendue et en profondeur, que le cachet latin, nous nous croyons autorisé, dans le cas d'Hermas, à ne penser qu'à un sémitisme. Mais la convergence de l'influence sémitique et de l'influence latine éventuelle a pour conséquence qu'on ne peut prouver qu'il n'y a pas eu d'influence latine.

\section{L'APPORT SEMITIQUE DANS LE VOCABULAIRE}

Hermas n'utilise pas d'emprunts sémitiques. Mais on trouve chez lui un grand nombre de termes dont le sens remonte à l'hébreu et à l'araméen. C'est par la Bible grecque et le milieu chrétien où on la lisait

I. Hermas 83, I est le seul à introduire un nouvel épisode. Pour ecce voir Thesaurus Linguae Latinae s.v.; M. Johannessohn, Et ecce und sein Ersatz in der Vulgata, Glotta 33, I954, Pp.125-156.

2. Gen. I 5,12; Jud. A 21,9; B 3,25;Ruth 3,8; 4 Regn. 7,5.10; Dan.1xx 3,92 . Dans le Nouveau Testament Mt. 2,13.19, dans les apocryphes Test. Naphth. 5,2; Apoc. Bar. 1,3; Protev. Jac. 4,4; 14,2. La Vulgate change d'ailleurs le présent historique dans Mt. 2,13.19 ldoù aryelos xuplou qaivetal en parfait: ecce angelus Domini apparuit. Ce n'est là qu'une des nombreuses adaptations au grec qu'il faut remarquer dans l'looś biblique, cf. Johannessohn, xai $i \delta \circ u ́$, pass. 
que ces mots lui sont parvenus; ils ne sont autre chose que le vocabulaire de base que le jeune christianisme emprunte au judaīsme. Aussi leur présence chez Hermas ne signifie-t-elle en aucun cas qu'il ait été en contact direct avec l'hébreu et l'araméen. Au contraire, plus clairement encore que pour les sémitismes syntaxiques, ils suggèrent que la tradition biblique est à l'origine de l'élément sémitique dans sa langue. Mais avant d'examiner ces sémitismes dans le Pasteur quelques problèmes préliminaires demandent notre attention.

Comment identifier des sémitismes dans le domaine du vocabulaire? Une réponse satisfaisante à première vue, c'est que nous considérons comme sémitismes les mots qui ou bien désignent une notion typiquement biblique, ou bien se présentent uniquement dans la Bible grecque et les textes qui en dépendent: dans le premier cas nous pouvons parler de sémitisme sémantique, dans le second de sémitisme lexicologique. Mais cette classification s'avère peu satisfaisante. Pour les sémitismes sémantiques, elle est trop étroite, pour les sémitismes lexicologiques, trop large. Précisons notre affirmation.

En ce qui concerne les sémitismes sémantiques, il ne fait aucun doute que les mots désignant des notions typiquement bibliques doivent être considérés comme des sémitismes. On constate cependant que le grec biblique comporte un groupe important de mots dont le sens objectif ne contient rien de spécifiquement biblique, mais que les traducteurs de la Septante ont vraisemblablement introduits en grec pour rendre le caractère hiératique du texte qu'ils traduisaient, tel qu'ils le ressentaient. Ces sémitismes ont généralement été formés malgré l'existence de termes adéquats en grec profane. Il est certain que reconnaître ce type de sémitismes est chose délicate, car il s'agit ici de nuances affectives qu'il est difficile d'établir objectivement. Mais l'envergure du phénomène est telle qu'on ne peut l'ignorer.

L'autre catégorie aussi pose parfois des problèmes: celle des notions typiquement bibliques. C'est ainsi qu'on ne peut pas toujours bien discerner dans quelle mesure un mot a pris un sens technique. Chez Hermas, il est par exemple question, dans 43,9 , de la venue d'un

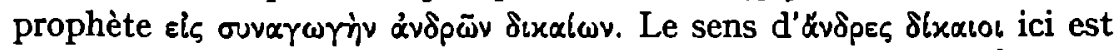

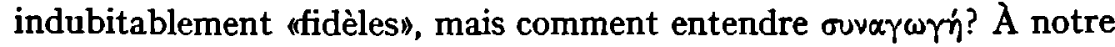
avis, le mot ne signifie guère plus ici qu' «assemblée», et encore faut-il voir le contexte pour savoir qu'il s'agit d'une assemblée religieuse de Chrétiens. Mais d'autres songeront ici à un terme technique d'abord juif, puis chrétien, qui remonte au הִ̣ hébreu' ${ }^{1}$. Ensuite, il est bon de

I. Cf. Reiling, Hermas, p.122 n.2. 
se rappeler que toutes sortes de termes religieux s'employaient en un sens analogue dans des milieux religieux paiens, comme \&ryøios,

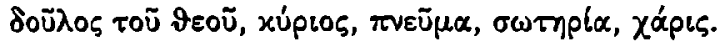

Quant aux sémitismes lexicologiques, l'absence de certains termes dans les textes profanes n'est guère qu'un critère superficiel qui, en soi, ne prouve pas que le mot en question remonte à un original sémitique. En outre, le rôle du hasard est ici trop grand pour être laissé de côté. En effet, si nous ne disposons pas de passages profanes à cet égard,

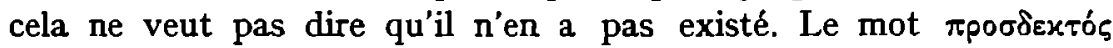
"agréable", par exemple, ne se trouve que dans la Bible et la littérature

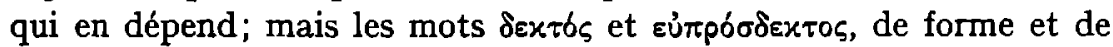
sens semblables, se présentent aussi dans des textes profanes. Depuis les recherches de Deissmann et consorts, l'impressionnante collection de voces mere biblicae lexicologiques dans les dictionnaires du Nouveau Testament n'a pas cessé de se restreindre, et le processus n'est pas terminé. De même, la liste de néologismes que Demaray proposa en I940 pour le Pasteur a, elle aussi, besoin d'être revisée' ${ }^{1}$. Ainsi, pour le mot $\dot{\sigma \tau t e ́ p \eta \mu \alpha, ~ d o n t ~ i l ~ n e ~ c o n n a i s s a i t ~ p a s ~ d ' e x e m p l e s ~ p r o f a n e s, ~ l e ~}$ Supplement du Liddell-Scott cite maintenant le texte d'un papyrus du III' siècle avant J.-C. Rien ne dit donc que ce qui aujourd'hui est un sémitisme lexicologique, ne devra pas demain, grâce à une découverte nouvelle, s'appeler sémitisme sémantique ou ne cessera pas tout à fait de mériter le nom de sémitisme.

C'est pourquoi nous croyons qu'il vaut mieux renoncer à distinguer lexicologique et sémantique et que, pour les sémitismes dans le domaine du vocabulaire, qu'ils soient lexicologiques ou sémantiques, nous pouvons utiliser les critères que nous indiquions pour ces derniers: ce sont donc des mots qui désignent des notions spécifiquement bibliques ou du moins qui comportent une valeur affective d'ordre hiératique caractéristique du grec de la Bible. A cela s'ajoute qu'un certain nombre de ces mots sont rares en grec profane ou font même complètement défaut.

Dans ces conditions, nous voudrions, pour le vocabulaire du Pasteur, compter au nombre des sémitismes les mots suivants: $\alpha \gamma \alpha \lambda \lambda \iota \tilde{\alpha} \sigma \vartheta \alpha_{\iota}$

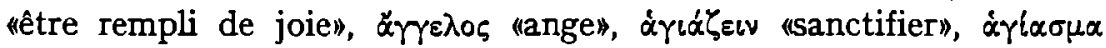

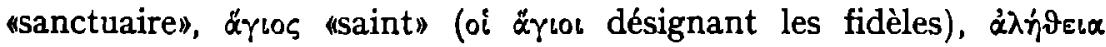

r. Demaray, Hermas, pp.r39-157. C'est une liste de termes post-classiques dans le Pasteur avec l'indication de leur présence dans la Septante, dans d'autres sources juives, dans le Nouveau Testament, dans d'autres sources chrétiennes, dans d'autres textes profanes littéraires et non-littéraires. 


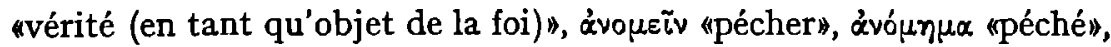

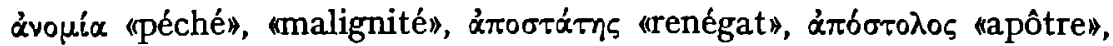

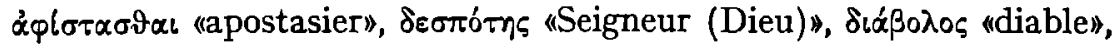

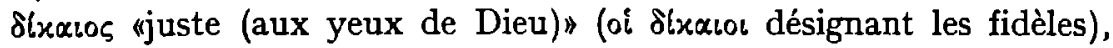

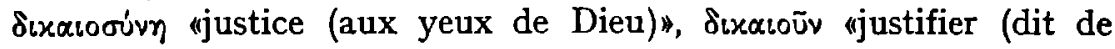
Dieu)», $\delta 6 \xi \alpha$ "gloire (de Dieu)», $\delta \circ \xi \alpha \zeta \varepsilon \iota v$ «glorifier (Dieu et les œuvres

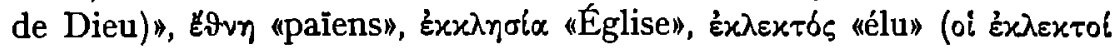

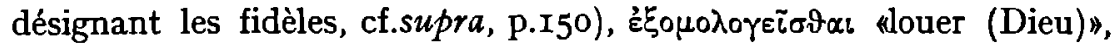

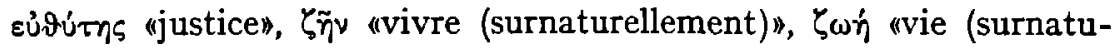

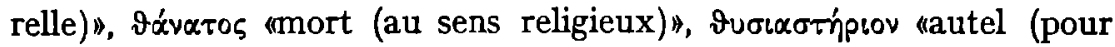

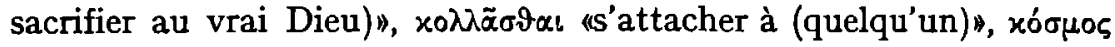

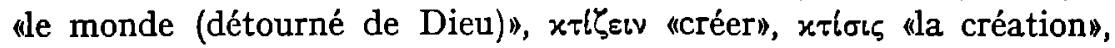

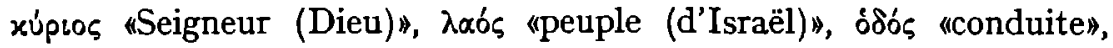

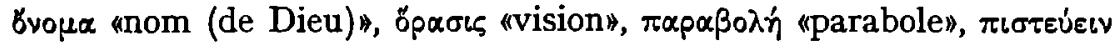

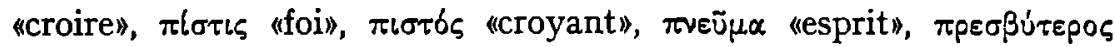

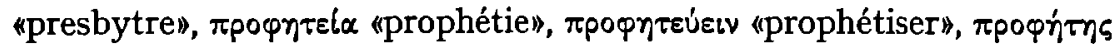

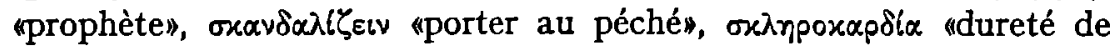

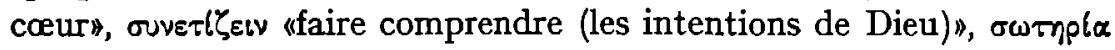

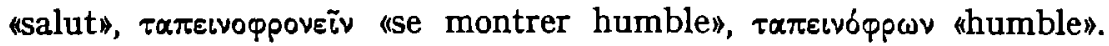

$\mathrm{Si}$, une fois encore, nous reprenons la distinction entre les sémitismes sémantiques et lexicologiques, nous pouvons constater que la grande majorité des mots que nous venons d'énumérer sont des sémitismes sémantiques, c'est-à-dire, abstraction faite du sens, se présentent aussi dans des textes profanes. Il n'y a guère que les sept mots suivants qui soient des sémitismes lexicologiques, c'est-à-dire qui ne se présentent pas dans les textes grecs profanes qui nous sont parvenus (pour autant

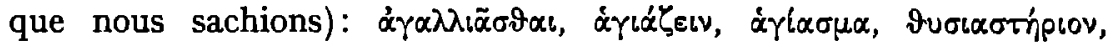
$\sigma x \alpha v \delta \alpha \lambda \zeta \zeta \varepsilon เ v, \sigma x \lambda \eta p \circ \alpha \alpha \rho \delta l \alpha, \sigma u v \varepsilon \tau l \zeta \varepsilon i v$.

Les sémitismes cités jusqu'ici se présentent déjà dans la Septante ${ }^{1}$. Mais, depuis lors, un grand nombre d'entre eux ont continué à se développer dans les communautés juives et chrétiennes. C'est ainsi

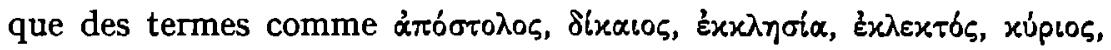
$\pi i \sigma \tau\llcorner\varsigma, \pi \rho \varepsilon \sigma \beta u ́ \tau \varepsilon \rho \circ \varsigma, \pi \rho \circ \varphi \dot{r} \tau \varsigma$ se trouvent chez Hermas dans un sens spécifiquement chrétien, sens qu'ils ne pouvaient pas encore avoir dans la Septante. En outre, à l'aide des premiers sémitismes, on forma des mots nouveaux, qui ainsi ont, eux aussi, indirectement une origine sémitique. Dans le Pasteur, on peut citer à ce sujet: éqvix́b "paien», 


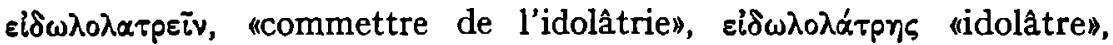

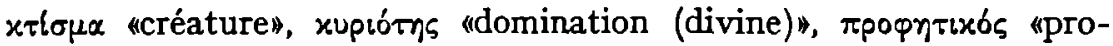

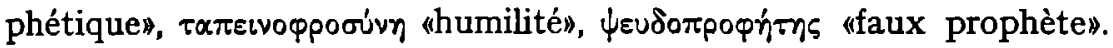

Non seulement des mots isolés, mais aussi des combinaisons de mots témoignent de l'apport sémitique dans le vocabulaire d'Hermas. Il

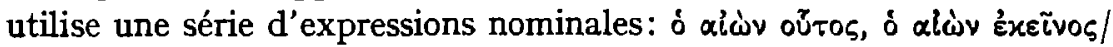

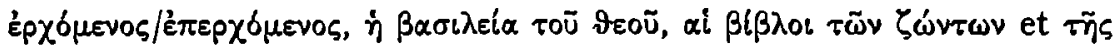

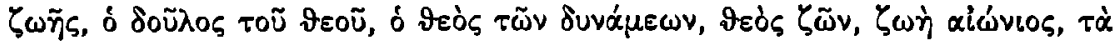

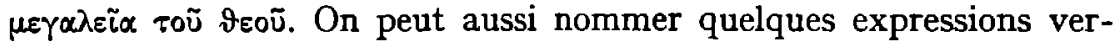

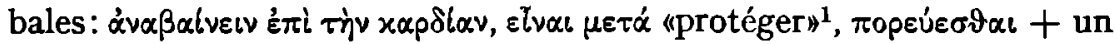

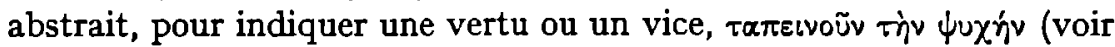
supra pp. I40-I42). Comme expressions adverbiales, nous avons है $\omega \varsigma$

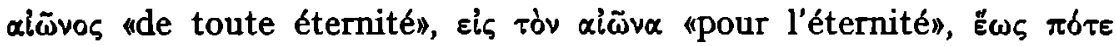
«jusqu'à quand». Les expressions de ce genre continuèrent, elles aussi, à

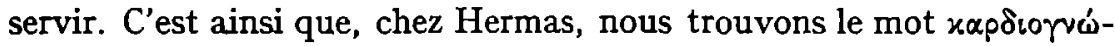

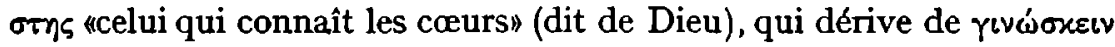

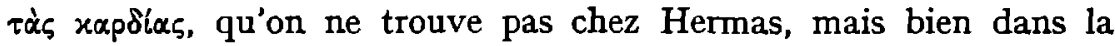
Septante (3 Regn. 8,39; 2 Par. 6,30; Prov. 24,12).

Comme nous l'avons déjà vu, les sémitismes qu'Hermas utilise dans le domaine du vocabulaire, se trouvarent déjà, pour la plupart, dans la Septante. La terminologie des deux aī̄ves fait exception, mais, elle aussi, on la trouve bien avant le Pasteur dans des écrits de langue grecque $^{2}$. Cela correspond à ce que nous avons constaté au sujet des sémitismes syntaxiques. Mais une autre constatation s'impose ici, constatation qui ne pouvait naturellement être faite là, à savoir qu'un grand nombre de sémitismes, après avoir été introduits dans le grec biblique par les traducteurs de la Septante, se sont mis à vivre in-

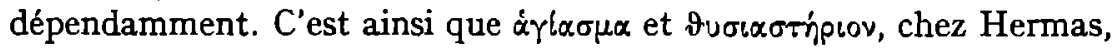
ne sont plus, comme c'est le cas habitucllement dans la Septante, le sanctuaire et l'autel du Temple de Jérusalem, mais, par analogie, le sanctuaire et l'autel dans le ciel $^{3}$. Ensuite, nous avons déjà cité le sens chrétien donné à des termes d'origine judaïque et les mots nouveaux

I. Pour cette expression voir W C van Unnik, Dominus vobiscum The background of a liturgical formula, New Testament Essays, Studies in memory of Thomas Walter Manson, Manchester 1959, pp 270-305, sur Hermas $\imath b$ pp 292, 295-297, 300 n 2.

2 Vour Pernveden, Church, pP 206-209; \& P. Orbán, Les dénominations du monde chez les premiers auteurs chrétiens, N1jmegen I970, Pp I I 4-I 20, I 26-I 32

3. Cf. Dibelius ad 10, I; 42,2. Pour cette ıdée du sanctuaıre céleste, voir Bietenhard, Himmlische Welt, pp 123-137, 192-193, 263; M de Jonge, The Testaments of the Twelve Patriarchs, Assen 1953, P 48 
formés, dérivés ou composés à partir de mots sémitisants. Voilà différentes choses qui prouvent que, dans le vocabulaire d'Hermas, rien n'indique qu'il connaissait l'hébreu ou l'araméen.

\section{I2. QUELQUES LATINISMES SUPPOSES}

Pour terminer, nous examinerons encore quelques termes et expressions que certains spécialistes considèrent comme des latinismes, mais qui, à notre avis, s'expliquent fort bien sans influence latine.

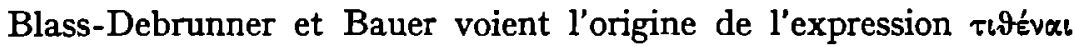

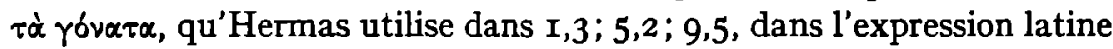
genua ponere ${ }^{1}$. Pourtant, eux-mêmes disent qu'on la trouve aussi chez Euripide et chez Marc, Luc et dans les Actes. En outre, pour autant que nous sachions, il n'y a pas tellement moins de passages avec

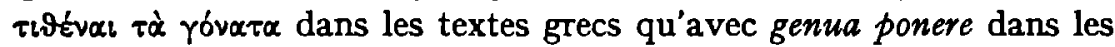
textes latins ${ }^{2}$. C'est pourquoi la thèse de l'influence latine ne nous semble pas prouvée.

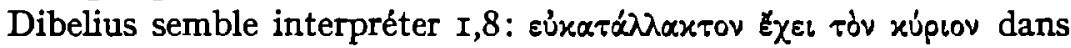

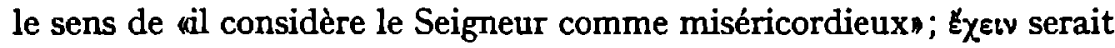
alors utilisé d'une manière non-grecque, comme le habere latin "considérer comme ${ }^{3}$. Mais le sens n'est pas ici subjectivement «tenir pour», mais objectivement "avoir comme», sens qui se présente aussi ailleurs

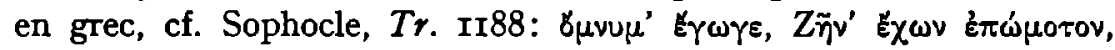

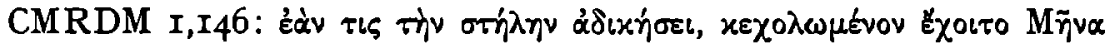

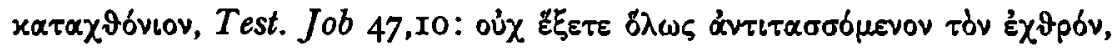

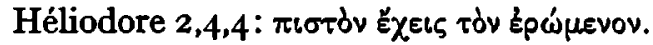

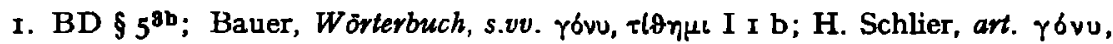
ThWNT I, pp.738-740.

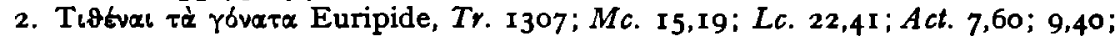
20,36; 21,5; Acta Thom. 167; Mart. Andr. prius 8; Mart. Petri el Pauli 52; Eusèbe, H.E. 5,5, 1. Ponere genua: Ovide, Met. 1,729-730; Fast. 2,438; Sénèque, Suas. 1,2; Quinte-Curce 4,6,28; 8,7,13; Quintilien 9,4,11; Pétrone 73,4; 133,2; Sénèque, Her. F. 546; Tro. 715; fr. I20 Haase; Tertullien, Or. 23, I; Marc. 3,13,8; Ps. Tertullien, Iud. 9,12; 10,10; Justin 12,9,12; Végèce, Epit. 1,20; Claudien 28,71-72; Pass. Petr. Paul. long. 52; Mirac. Thom. p. II7,3 Bonnet = Pass. Thom. p. 157,20 Bonnet; Acta Pauli et Th. A et C 5.24; Ass. Mos. 4, 1. Pour le latin, nous avons pu consulter le Thesaurus; c'est pourquoi la liste, probablement, est beaucoup plus complète pour le latin que pour le grec. En outre, dans beaucoup de cas, les apocryphes latins remontent sans doute à des textes grecs.

3. Il remarque du moins ad loc.: Zu der wahrscheinlich latinisierenden Kon-

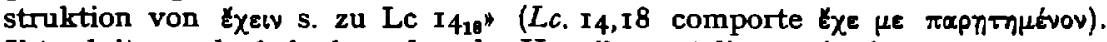
Il traduit: «er darf glauben, dass der Herr ihm gnädig geneigt ist». 
C. H. Turner crut trouver dans 7,4: żáv ool $\varphi_{\alpha} \bar{n}$, un latinisme qui aurait été formé d'après si tibi videtur, et d'autres après lui furent de cet avis ${ }^{1}$. Mais cette expression est très fréquente dès le III ${ }^{e}$ siècle avant J.-C. dans les papyrus non-littéraires. Dans les sources littéraires aussi, elle revient à plusieurs reprises ${ }^{2}$, par exemple Ep. Arist. 32 : Éàv

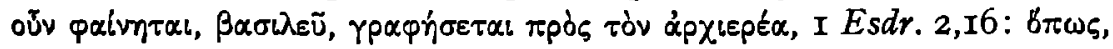

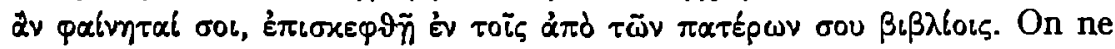
peut opposer que, dans les traductions grecques de décrets du sénat

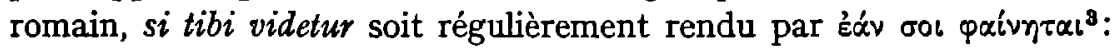
dans ce cas, les traducteurs préfèrent justement une traduction idiomatique, donc moins littérale.

Comme symptôme d'influence latine, Christine Mohrmann cite 9,9:

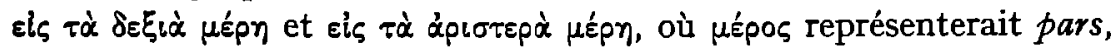
"côté". Mais nous trouvons aussi ces expressions dans des textes où il ne peut pas être, ou guère être question d'une influence latine, Platon,

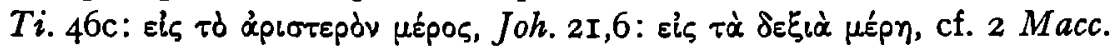

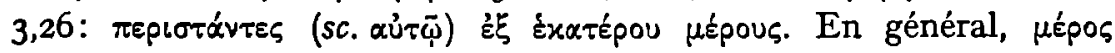
"côté", est assez fréquent dans le grec ultérieur".

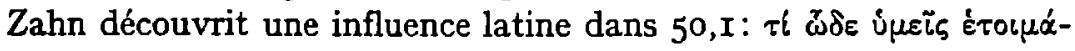

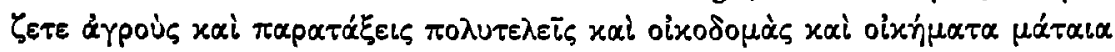

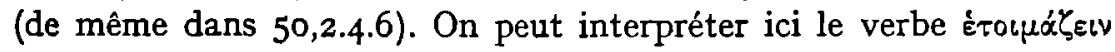

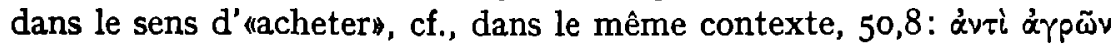

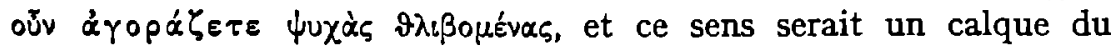

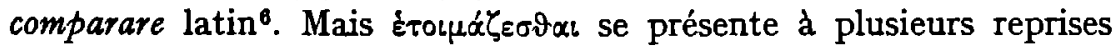
dans le sens de "se procurer (pour de l'argent)": Démosthène 23,209:

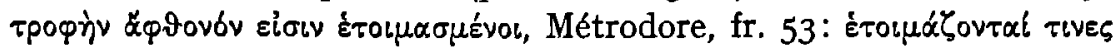

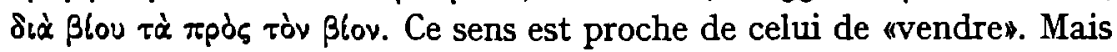

I. Turner, Shepherd, p.198 n.I; également Mohrmann, Origines, p.75; Giet, Les Pasteurs, p.284; Tabachovitz, Evgänzungsheft, p.7.

2. Par exemple Denys d'Halicarnasse, $A R$ 2, I 4,4; 2 Esdr. 7,20; Épictète I, I 2, I9; Eusèbe, $H . E .3,25,4$. Pour les papyrus non-littéraires voir C.Harsing, De optativi in chartis Aegyptiis usu. Bonnae I9Io, pp.50-51; H. A. Steen, Les clichés épistolaires dans les lettres sur papyrus grecques, Classica et Mediaevalia I, 1938, PP.(I I9-I 76) I 50-I 52 .

3. Voir R. K. Sherk, Roman documents from the Greek East, Baltimore, Maryland, 1969, p.I5 (ajouter: nr.12,I7-18). D'ailleurs il ne reste que deux passages de l'original latin; dans ces passages, il est fait usage d'abréviations: 22,24 $s(e i) e(i s) v($ ideveturr), 22,29 sei v(ideantur) $e(i s)$.

4. Mohmann, Origines, p.75; Giet, Les Pasteurs, p.284.

5. Par exemple Polybe 1,42,8; Diodore 2,9,3; Achille Tatius 2,9,2; Pausanias 2,32,3. Voir aussi J. A. L. Lee, $A$ neglected sense of $\mu t p \circ 5$, Antichthon 6, 1972, Pp.39-42.

6. Zahn, Hirt, p.487 n.2; Mohrmann, Origines, P.75; Giet, Les Pasteurs, p.284. 
il y a aussi des passages où, comme chez Hermas, on est tout aussi bien

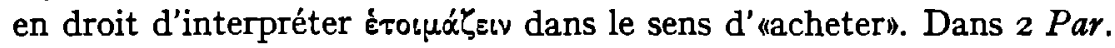
3,I, il est dit à propos de l'endroit où le Temple devait être bâti :

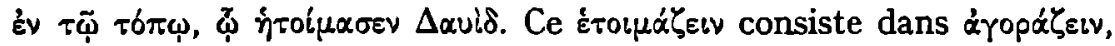
comme il ressort de I Par. 21,22-25, notamment dans 21,24: áyopá̧

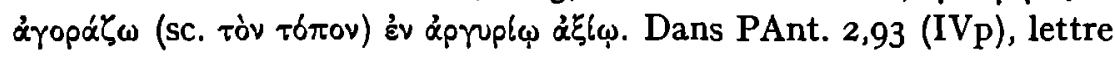
où il est question d'une maison à acquérir, nous lisons 1.r6-I8:

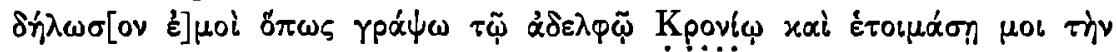
oixlav.

Un latinisme supposé qu'Hermas partage avec le Nouveau Testa-

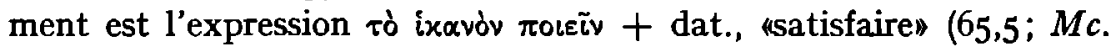
I5,I5), qui s'inspirerait du latin satisfacere. Ici aussi tout dépend de la façon dont on interprète les faits, car Blass-Debrunner, qui parlent d'un latinisme, citent tant de passages de textes post-classiques à partir de Polybe, que nous serions porté à croire que l'expression peut avoir son origine dans le grec même'.

r. BD $\S 5^{3 \mathrm{~b}}$. Le considèrent aussi comme latinisme Harnack, Funk et Lelong ad loc.; Moulton, Einleitung, p.29; Turner, Shepherd, p.198 n.1; Salonius, Papyrusbriefe, p.25; Mohrmann, Origines, p.75: Giet, Les Pasteurs, p.284. 


\section{EMPRUNTS}

\section{INTRODUCTION}

Chacun sait que, notamment dans le domaine du vocabulaire, le grec classique était une langue jalouse de son autarcie. "Ill n'y a pas de langue plus rebelle à l'emprunt à des langues étrangères que le grec de l'époque classique, parce qu'il n'y a eu nulle part civilisation plus évidemment supérieure aux civilisations voisines que n'était, dans l'antiquité, la civilisation hellénique»'A A l'époque hellénistique, lorsque les échanges avec les peuples non-grecs s'intensifient, la situation change. D'une part, la langue grecque s'impose avec beaucoup plus de force dans le monde non-grec; d'autre part, elle ne peut plus, à la longue, se défendre contre l'infiltration d'éléments étrangers. L'expansion politique des Romains notamment a eu des conséquences considérables. Des soldats, des fonctionnaires et des marchands romains font leur entrée dans le monde hellénistique, apportant avec eux en grand nombre objets et usages nouveaux. Calendrier romain, monnaie romaine, mesures et poids romains se répandent partout. Et avec les choses s'imposèrent aussi les mots latins.

Mais ce processus ne s'accomplit pas sans heurts. Les Grecs cultivés, ceux qui avaient conscience d'écrire une prose d'art et de devoir sauvegarder une tradition littéraire, s'opposaient autant que possible à l'importation de mots latins. Les Atticistes étaient en tête. Libanios réussit encore, dans son œuvre considérable, à ne pas employer un seul mot latin². Même les noms propres latins étaient intolérables aux yeux des Atticistes ${ }^{2}$.

Mais un purisme intégral était irréalisable. Dion Cassius $(55,3)$ 1. Meillet, Apercu, p.320.

2. R. Foerster-K. Münscher, Libanios, Paulys Real-Encyclopädie der Classischen Alterturnswissenschaft, Neue Bearbeitung XII, Stuttgart I925, col. $\left(24^{8} 5-255 \mathrm{I}\right) 2535$.

3. Voir E. Norden, Die antike Kunstprosa vom VI. Jahrhundert v. Chr. bis in die Zeit der Renaissance, Leipzig-Berlin $1909^{2}$, p.60 n.2. 
reconnaît qu'il ne trouve pas de mot en bon grec pour senatus auctoritas. Les écrivains qui s'occupaient du passé romain, comme Polybe, Denys d'Halicarnasse et Plutarque, se voyaient bien forcés d'emprunter les termes techniques des institutions politiques romaines. D'autres, comme Épictète, ne se souciaient pas d'écrire un grec littéraire et n'avaient aucun scrupule à utiliser des mots latins. Les auteurs techniques, comme Dioscuride, Erotianos et l'écrivain du Periple de la Mer Erythrée ne se croyaient pas tenus aux règles littéraires' Dans les inscriptions et les papyrus non-littéraires, on trouve des emprunts à foison ${ }^{2}$.

La Bible grecque donne bien la mesure dans laquelle les emprunts latins ont pénétré la langue. Le grec biblique, en effet, qui garda largement sa liberté à l'égard des normes de la littérature profane, était beaucoup moins réfractaire à l'importation que les auteurs profanes, comme en témoignent les emprunts sémitiques dans la Septante et le Nouveau Testament. Mais il n'existe absolument pas d'emprunts latins dans la Septantes. A l'époque où ces textes ont été écrits, ils n'étaient évidemment pas encore d'un usage courant dans la langue commune. Les traductions grecques ultérieures de la Bible, au contraire, contiennent déjà différents emprunts latins4.

Dans le Nouveau Testament, le nombre de mots latins - vingt-sept mots différents - est déjà relativement grand. Les emprunts désignent en général des choses concrètes. C'est pourquoi on les trouve surtout dans des livres narratifs, les évangiles et les Actes. Ce sont des termes venant du monde des autorités romaines (justice, administration, armée) et de celui du commerce et du trafic (mesures, monnaies, objets, noms de vents). Chez Luc, nous voyons une certaine tendance à écarter les mots étrangers, kpreuve indirecte de la popularité dont jouissaient les emprunts latins ${ }^{5}$. Les apocryphes présentent la même image que le Nouveau Testament; ils contiennent en tout vingt-

I. Voir Hahn, Romanismus, pp.235-236, 253-257.

2. Voir A. Cameron, Latin words in the Greek inscriptions of Asia Minor. The American Journal of Philology 52, 1931, Pp.232-262; S. Daris, Il lessico latino nel greco d'Egitto, Barcelona I97I, pass.

3. Les mots d'origine celtique yaioos (Jos. 8, 18bis; Judith 9,7), xáppov (I Esdr.

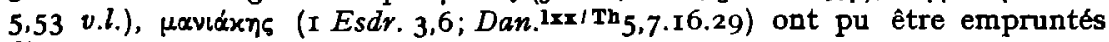
directement au celtique de Galatie.

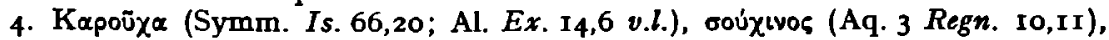

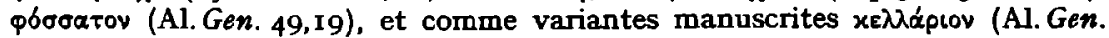

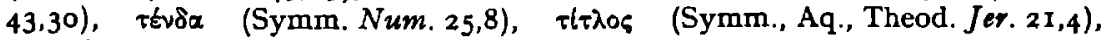
poupxlseiv (Symm. Num. 25,4).

5. $\mathrm{BD} \S 5^{1}$. Ib. énumeration des mots latins dans le Nouveau Testament. 
deux emprunts latins différents ${ }^{1}$. Dans la littérature paléochrétienne ultérieure, on trouve surtout des mots latins dans des écrits plus populaires. Mais même certains auteurs à prétentions littéraires ne poussent pas le purisme au point où le font les Atticistes profanes ${ }^{2}$.

Jusqu'ici nous n'avons parlé que des emprunts latins. Mais dans les milieux qui avaient des rapports avec le monde sémitique, à savoir le judaīsme de langue grecque et le christianisme le plus ancien, les emprunts hébreux et araméens pénètrent d'une manière analogue. Leur nombre pourtant resta limité. Même dans le Nouveau Testament, les emprunts sémitiques ne sont pas plus nombreux que les emprunts latins. Chez Hermas, ils font complètement défaut.

\section{HERMAS}

Dans le Pasteur, nous trouvons cinq noms propres latins, deux noms de

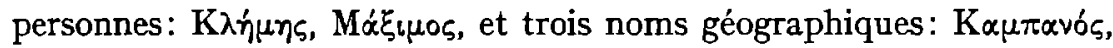

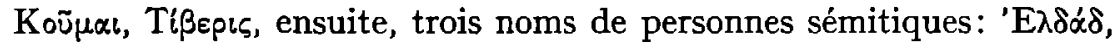

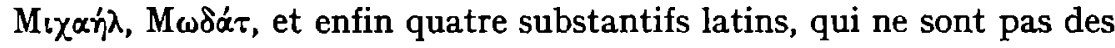

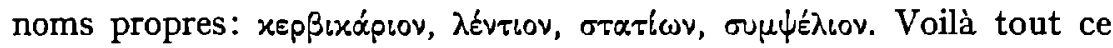
que notre texte comporte en fait d'importation. Devantce nombre relativement restreint, on pourrait être tenté de conclure qu'en réalité, Hermas s'en tire fort bien sans l'aide d'autres langues, qu'il se sert du grec comme on le fait d'une langue matemelle. D'autre part, c'est précisément quand on utilise une langue moins familière, qu'on s'abstient en général autant que possible d'avoir recours à de l'importation ${ }^{3}$. Les données numériques ne conduisent donc pas à des

r. Mots qui se présentent dans le Nouveau Testament: Kaĩoap (Acta Pauli et Th. 36; Acta Pauli 9,4.14.26; 10,32; I1,4; Mart. Pauli Iter. 2quinquies. 3), xevtupluv (Ev. Petri 31.32.38.45.47.49; Acta Pauli 9,18; 11,4.12; Mart. Pauli

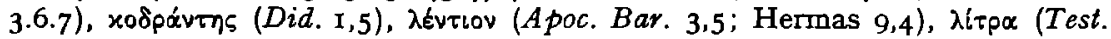

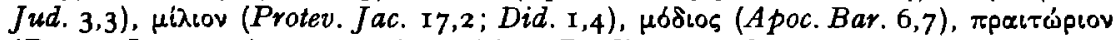

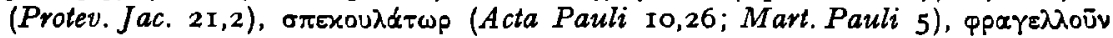
(Test. Benj. 2,3; Acta Pauli et Th. 21; Acta Pauli 1,30), ainsi que dxtov (Acta

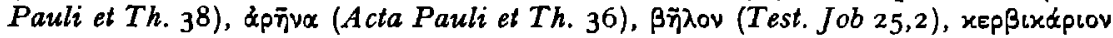

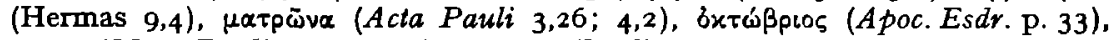

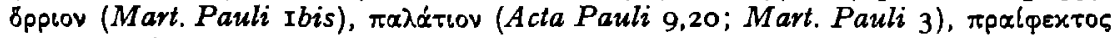

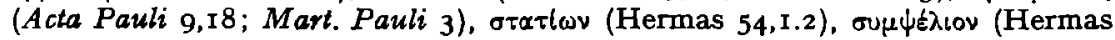
9,4 bis.7; 10,4; 18, 1.5; 21,3 bis; 43, Ibis), $\sigma \omega \lambda \alpha$ prov (Jos. et $A s .7,2$ ).

2. Voir, par exemple, la liste d'emprunts latins dans l'cuvre d'Athanase chez Bardy, Question des langues, p.I23 n.4; il y en a aussi un grand nombre dans un texte comme Jean Chrysostome, Ep. I4.

3. Voir supra p.56. Nous employons ici le terme importation, tout comme ailleurs, dans le sens technique que nous avons indiqué supra, p.36. 
conclusions sûres: il faudra examiner les matériaux de plus près. Nous nous arrêterons tout d'abord à la forme des termes empruntés.

Au sujet des noms latins qui ne sont pas des noms propres, il faut remarquer qu'en passant dans la langue grecque, ils ont subi les modifications habituclles'. C'est ainsi que les neutres de la deuxième déclinaison linteum et subsellium furent recueillis comme neutres en -ov dans la deuxième déclinaison grecque, et qu'à statio au nominatif on ajouta un $-\nu$ final, comme c'était la règle pour les mots en -io. Cervical fut traité comme l'étaient normalement les neutres en -al et

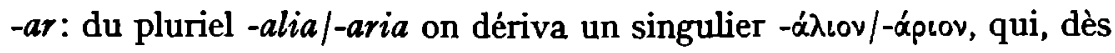
lors, entrait dans la deuxième déclinaison. On retrouve ici l'assimilation du $-l$ - au - $r$ - précédent que plusieurs emprunts présentent. Il est courant, à cette époque, de rendre $c$ et $v$ par $x$ et $\beta$. Dans $\lambda e^{\prime} v \tau$ iov

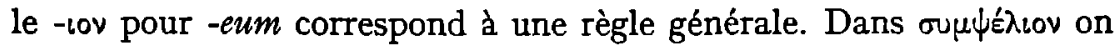
remarque que $-\breve{u}$ - est rendu par $-v-,-b s$ - par $-\psi$ - et la simplification de $-l l$ - en $-\lambda-$, modifications normales dans ces positions, et aussi l'adjonction du $-\mu-$, qu'on peut attribuer à l'étymologie populaire, qui assimilait la première syllabe avec le préfixe ouv-, mais qui peut aussi se fonder sur des lois phonétiques ${ }^{2}$.

Pour les noms propres latins, les choses se passent de façon analogue: signalons, par exemple, la disparition du $-n$-devant $-s$ dans $\mathrm{K} \lambda \dot{n} \mu \eta s_{\text {, }}$ disparition qui s'était produite aussi en latin, mais qui ne s'y manifestait généralement pas dans l'orthographe. Pour les noms géographiques, une deuxième remarque s'impose. A l'époque d'Hermas, le grec littéraire se distinguait de la langue courante, entre autres, par le fait que, pour une série de villes et de rivières, il utilisait des noms différents de ceux employés dans la vie quotidienne. C'étaient pour la plupart des noms qui avaient été courants en grec classique ou des noms qui avaient été expressément hellénisés. Hermas est loin de

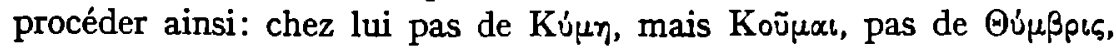
mais $T l \beta \varepsilon p l \varsigma^{3}$.

Dans le cas des noms propres et des mots sémitiques en général, la

I. Cf. Daris, Lessico latino, pp.r8-2r et la bibliographie $i b$. p.18.

2. Cf. H. B. Rosén, Palestinian xoเv' in rabbinic illustration, Journal of Semitic Studies 8, r963, p. $(56-72) 68$.

3. Une tendance contraire amène les poètes romains à utiliser les noms franchement grecs: Cyme (Stace, Silv. 4,3,65), Thybris (Virgile, A., pass.). La forme Koü $\mu$ al chez Hermas est une conjecture acceptable de Tischendorf, app. crit. ad. 1,3, et W. Dindorf, Nachträgliche Bemerkungen zu Hermas, II, Leipziger Repertorium der deutschen und ausländischen Literatur I 5 1 I 857, pp. (65-79)

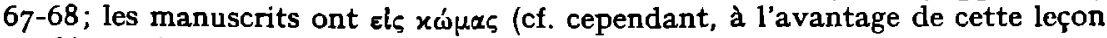

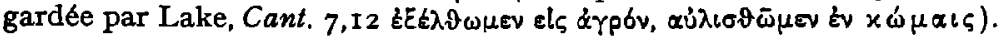


situation est différente en ce que ces mots se terminaient souvent par des consonnes qui, en grec, ne pouvaient se trouver à la fin d'un mot. Les noms qui n'étaient pas des noms propres étaient généralement transformés de telle sorte que les règles grecques leur fussent appli-

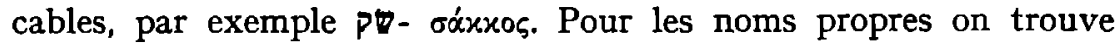
parfois des doublets: une forme littéraire, adaptée au grec, et une forme non littéraire, contraire aux règles grecques, par exemple ๆọi:

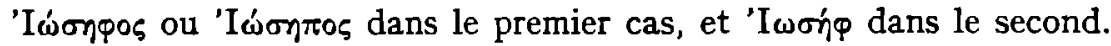
Les formes qu'utilise Hermas sont ici encore celles du deuxième groupe, le groupe non-littéraire. Il se conforme d'ailleurs en cela à l'usage de la Bible et des apocryphes'.

Si nous résumons nos constatations, nous voyons, qu'en ce qui concerne la forme extérieure des emprunts, Hermas se conforme entièrement à l'usage qui, avant lui, était déjà celui de la langue commune et du grec biblique. À voir la forme de ses emprunts, rien ne permet de les considérer comme une preuve de sa connaissance du latin, de l'araméen ou de l'hébreu.

Nous arrivons à la même conclusion si nous considérons ce que deviennent en grec les noms latins qui ne sont pas des noms propres. Les quatre mots dont il s'agit nous ont été transmis dans des textes littéraires, mais surtout dans des textes non-littéraires. Pour le moment nous connaissons au total, datant du $\mathrm{II}^{\mathrm{e}}$ et du IIIe siècle après J. -C.,

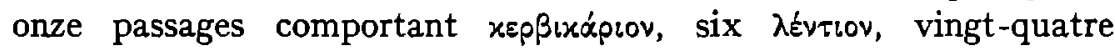
$\sigma \tau \alpha \tau i \omega v$, et six $\sigma u \mu \psi \varepsilon \lambda \iota_{c o v}^{2}$. Nous retrouvons en outre les trois derniers

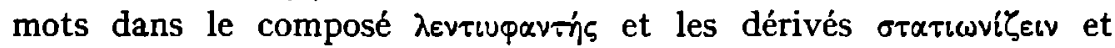

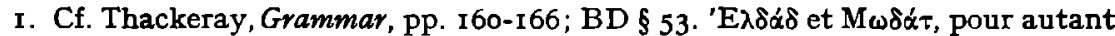
que nous sachions, ne se présentent nulle part sous une forme plus hellénisée,

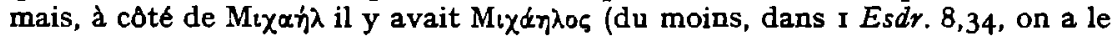

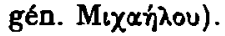

2. Dans les inscriptions, ostraca et papyrus, nous avons compté un mot pour

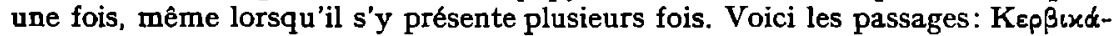
prov Hérodien le grammairien dans Lex. Vind.p.312 (cf. intra p.r68 n.2); papyrus IIP POxy.10, 1269,37; PFay.347; PHeid. 237, 10; II/IIIP POxy. 24, 2424,39; Stud. Pal. 20,46,19; 20,67,29; IIIp POxy. 6,921,8.20; BGU 3,814, I I;

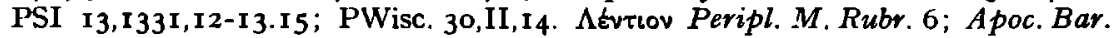
3.5; Joh. 13,4-5; inscriptions IIp Inscr. Magn. 1 16,34-35; BSA 27, 1925-1926,

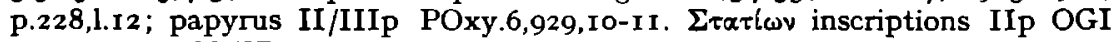
2,595, pass.; IGUR 1,84,4; II/IIIp IGUR I,83,2-3; 2,4I 3,4; IIIP SEG 2,666,3; ostraca IIp Ostr. I45,6;146,6; 147,$5 ; 273,4 ; 278,6 ; 287,4 ; 293,6$; Ostr. Wilbour 20,3; II/IIIP Ostr. Bodl. 2,873,5; IIIP Ostr. Bodl. 2,872,4; papyrus IIp PCol. 2 pass.; BGU I, 326,II, 10; 9, I89I pass.; PAberd. 78,4; SB I,436I,6; 5,7630,24;

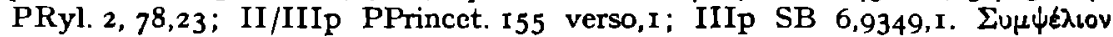
Acta Thom. A 49; inscriptions Ip Ephes. 3,65,12; ostraca IIIP SB 1,4292,4; papyrus IIP PPetaus 34,17; IIIP PMichael. I8,III, 10; POxy. 6,921. 


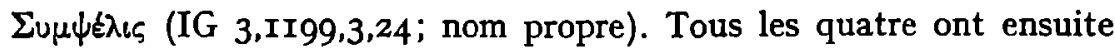
pénétré dans d'autres langues par le grec'. Il est intéressant aussi de songer à la remarque d'un contemporain plus jeune qu'Hermas, le grammairien Hérodien, qui cite comme exemple de "barbarisme"

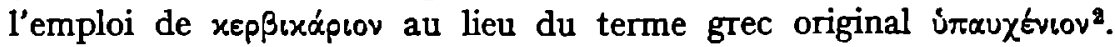
Tout cela montre bien que les quatre emprunts en question s'étaient bien ancrés dans la langue grecque.

Mais il ne suffit pas de considérer la forme et la fréquence, il nous faut examiner aussi le sens. A cet égard, il y a peu de choses à signaler

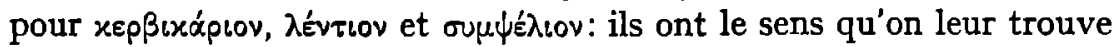
ailleurs dans les textes grecs. En revanche, l'interprétation de $\sigma \tau \alpha \tau i \omega \nu$ demande un examen détaillé, car le terme a, chez Hermas, un sens qu'on ne trouve nulle part ailleurs en grec $^{3}$.

\section{3. $\Sigma \tau \alpha \tau i \omega \nu$}

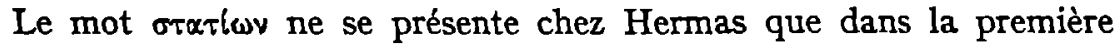

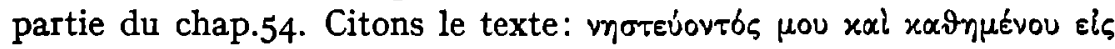

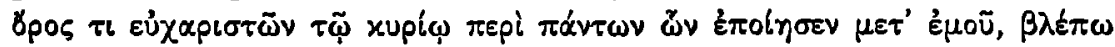

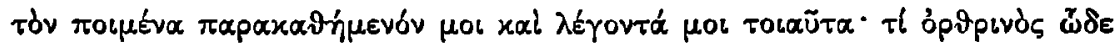

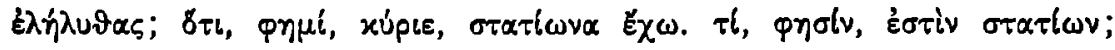

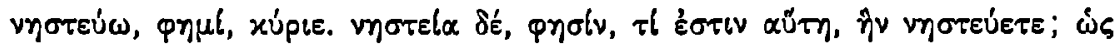

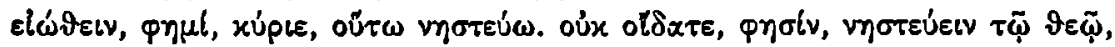

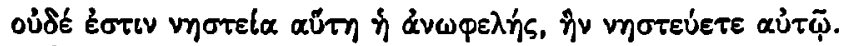

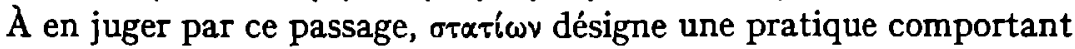

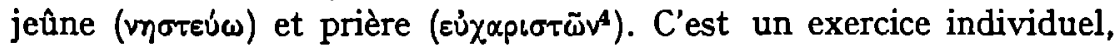
car Hermas est seuls, et il est facultatif, car s'il avait été prescrit par

I. Voir Bauer, Wörterbuch, s.vv. et cf. Cameron, Latin words, p.233.

2. Lex. Vind. p.3 2. Mais l'attribution à Hérodien du De soloecismo et barbarismo, où se trouve la parole, est contestée.

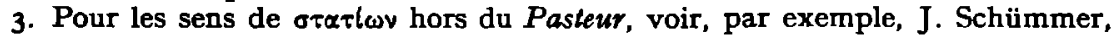
Die altchristliche Fastenpraxis mit besonderer Berücksichtigung der Schriften Tertullians, Münster in Westf. 1933, pp.129-131, 135, 137-138; L. Moretti, Inscriptiones graecae urbis Romae, I, Romae I968, p. 70.

4. Lorsqu'il s'agit de jeûne, on s'attend surtout à des prières de contrition et d'imploration, comme c'est le cas chez Hermas 6,$1 ; 9,2 ; 18,6-7$, mais la louange de Dieu est un pendant naturel de ces types de prière, cf. 2 Esdr. 19; Ps. 106; Sir. 39,5-6 et la prière dite shemone 'esre. Dans 5I,5, Hermas met sur le même

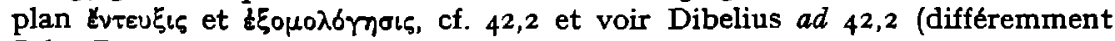
Joly, Pasteur, p. 433).

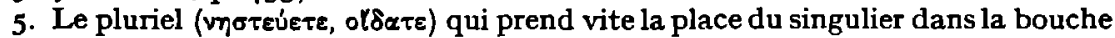
du Pasteur, ne signifie pas qu'Hermas est en compagnie, mais est un signe que la parénèse, indépendamment de la situation, prend le dessus; de même, par exemple, 6r,4. Voir Reiling, Hermas, pp. I65-I69. 
l'Église on comprendrait mal la réprobation du Pasteur. Si nous comparons les éléments que nous avons rassemblés ici avec ceux que nous trouvons chez Tertullien, le premier auteur qui, après Hermas, parle de la statio, nous trouvons beaucoup d'analogies': le jeûne, la prière, le caractère facultatif. Ce qu'Hermas ne dit pas, c'est que la statio se faisait le mercredi et le vendredi et qu'elle se terminait à la troisième heure de l'après-midi. En outre, à l'époque de Tertullien, la statio n'a pas encore de forme très fixe. C'est ainsi que Tertullien s'en prend à ceux qui, le jour de la statio, ne participent pas à l'eucharistie. La synaxe eucharistique des jours de statio a manifestement été instaurée plus tard et n'était pas encore généralisée ${ }^{2}$. Dans une phase antérieure, pour autant que nous puissions l'inférer de la description chez Hermas, il n'est même pas encore question de célébrations communes. A tous égards, la statio semble y être un exercice individuel ${ }^{3}$.

On a donné de nombreuses explications à l'origine du terme statio4. On peut les diviser en trois groupes suivant qu'on part d'un sens profane de statıo, d'une origine juive ou d'une combinaison d'éléments profanes et juifs Dans le premier groupe, il faut surtout mentionner l'idée qu'on a affaire à une métaphore mulitaire. Stationem habere signifıerait à peu près umonter la garde devant Dieu*. Chez Tertullien, on trouve déjà plusieurs témoignages en faveur de cette interprétation, par exemple Or. 19,5: si statio de militari exemplo nomen acceput (nam et militia Dei sumus), Ieiun. 10,6-7: impressius tamen tum ei secundum ipsum stationis vocabulum addicimur. nam et milites numquam immemores sacramenti magis stationibus parent Mais ces formules nous sont de peu d'utilité; car nous avons affaire ici à une explication de circonstance, à wun de ces artifices stylistiques dont Tertullien est coutumier et qui devraient nous détourner de prendre au sérieux ses étymologies et ses explications sémasiologiquesı) ${ }^{5}$. Une autre possibilité d'explication est celle que, sous toute réserve, Thörnell a ouverte en partant de statio au sens d' "arrêt". Le terme indiquerait alors l'arrêt des occupations quotidiennes, et serait devenu ainsi un terme technique pour les pratiques religieuses en question'. Cette explication n'a guère

I Cf Schummer, Fastenpraxts, p 83, Mohrmann, Statio, pp 3i I-3I4

2 Cf $\mathrm{E}$ Dekkers, Tertullıanus en de geschiedems der liturgıe, Brussel-Amsterdam 1947, P 133, Mohrmann, Statio, PP 31 2-313

3 Cf Mohrmann, Statio, pP 312, 318, 327

4 Vorr Schummer, Fastenpraxis, pp I23-I29, Mohrmann, Statio, pp.307-308

5 Mohrmann, Statio, P 313, ef $\imath b$ PP 313-314, 319 et Dekkers, Tertullianus, P I4O n I

6 G Thörnell, compte-rendu de St W J Teeuwen, Sprachlıcher Bedeutungswandel ber Tertullian, Gnomon 3, 1927, pp (48-50)49-50 
eu de succès; un des inconvénients est d'insister sur ce qui est omis plutôt que sur ce qui est fait.

Ceux qui cherchent l'origine du terme dans le judaisme ont depuis longtemps appelé l'attention sur le terme hébraīque Tp̣pge, qui est un nomen actionis formé sur עמד "être debout", exactement comme statio l'est pour stare. On s'est fondé, en particulicr, sur le passage suivant du traité $T a^{\prime}$ anit de la Mishna $(4,2)$ : "Les premiers prophètes créèrent vingt-quatre sections (de prêtres et de lévites), et à chaque section correspondait une représentation (מ̣g) ${ }^{1}$ à Jérusalem, composée de prêtres, de lévites et d'Israélites. Lorsque le temps était venu pour une section d'avoir son tour, les prêtres et les lévites de la section allaient à Jérusalem, et les Israélites de la même section (s'ils étaient empêchés d'aller, eux aussi, à Jérusalem) se rassemblaient dans leurs demeures et lisaient le récit de la création, et les hommes de la représentation (7pge) jeûnaient pendant quatre jours de la semaine, du deuxième au cinquième jour». Lors de ces réunions locales, il $\mathrm{y}$ avait aussi des prières communes. Il $\mathrm{y}$ a donc des points de ressemblance entre les réunions locales des "représentations", d'une part, et la statio chrétienne, d'autre part: le jeûne, la prière, les jours fixes, ce qui incita certains spécıalistes à considérer la statio comme la continuation de la «représentation" ${ }^{2}$. Mais il y a aussi de grandes différences: la pratique juive est collective et obligatoire, elle a lieu chaque semaine dans un des vingt-quatre districts du pays, donc deux fois par an dans chaque district; la pratique chrétienne est individuelle et facultative, peut se faire chaque semaine et n'est pas liée à un pays ou un district. A cela s'ajoute que le judaĩsme connaît un autre jeûne, qui ressemble beaucoup plus à la statio chrétienne et qui se fait également pendant deux jours de la semaine (à ce sujet, voir infra, p.172). Dans ces conditions,

I A partir de maintenant nous désıgnons l'institution du ma'amad par areprésentation" En allemand, on parle ordinairement de Standmannschaft ou de Opferberstände. l'avantage de ces traductions est que le rapport avec עמש "être debout" continue à transparaître Certains ne tradussent pas le terme, mais ıci, où 1 peut être question d'autres sens de 7 g encore, cela prêterait à confusion Il y a aussi des inconvénients à parler de istation", comme le font $\mathrm{J}$. Bonsirven, Notre statio liturgique est-elle empruntée au culte junt', Recherches de Science Religieuse 15, 1925, pp 258-266, et Peterson, Giudaismo e Cristıanesımo, P 378: cela suggérerait un rapport avec la statıo chrétıenne, ce qui n'est pas encore démontré Sur la représentation", vorr Bonsırven, $2 b$ pp 260-266 et I Elbogen, Der judische Gottesdienst ins einer geschichtlichen Entwicklung, Frankfurt am Main 1931 ${ }^{3}$, PP 237-239, 553

2 Voir Schummer, Fastenpraxis, pp 125-1 27, Mohrmann, Statıo, pp 324-325. Aussi par exemple, Volter, Die Apostolischen Vater, I, PP 293-294, Peterson, Giudaismo e Cristianesimo, PP 377-379 
il est difficile de penser que la statio puisse venir de la «représentation». Schümmer a proposé une autre théorie, qui avait également le judaīsme pour point de départ, mais qui voulait échapper aux inconvénients que nous venons de signaler' ${ }^{1}$. Il remarque que, selon le Talmud, les Juifs, aussi bien individuellement que collectivement, priaient souvent en plein air et qu'ils attachaient une grande importance à l'endroit où se faisait la prière. Bien que les textes ne le disent pas, cet endroit se serait appelé 7p̧p. En grec, ce terme aurait été traduit par $\sigma \tau \alpha \tau i \omega v$, qui était également dans cette langue la désignation d'un endroit (attesté en tout cas pour uinstallation d'un

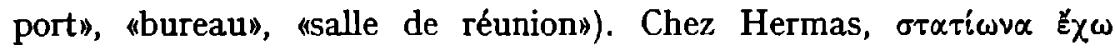
signifierait d'abord "j'ai occupé une place pour prier»; mais, étant donné que cette occupation d'une place fait partie d'une pratique de jeûne, l'expression équivaudrait à uje jeûne». Cette théoric n'est pas satisfaisante. Car, outre le caractère hypothétique de d' «endroit pour prier» et la faible probabilité que le grec ait traduit

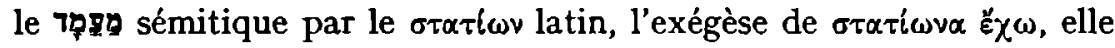
aussi, semble bien forcée. Il suffit de rappeler qu'Hermas lui-même

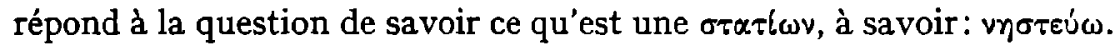
$\Sigma \tau \alpha \tau i \omega v$ n'indique donc pas un endrott, mais une actıon.

Reste la théorie de ceux qui combinent les données latines profanes et les données judaiques. Teeuwen propose l'idée d'une évolution du sens de statıo qui, de "réunion" serait devenu "réunion liturgique le mercredi et le vendredi, jours de jeûne», et ensuite «jeûne»" ${ }^{2}$. Il faudrait alors attacher le sens de "réunion liturgique» à la "représentation" juive. La difficulté cependant, et elle est considérable, c'est que statio "réunion liturgique" apparaît pour la première fois chez Cyprien, au milieu du IIIe siècle ${ }^{3}$, et que, chez Tertullien et plus encore chez Hermas, l'accent tombe entièrement sur le jeûne. Si l'on voulait en croire Teeuwen, l'évolution du sens de statio se serait accomplie dans l'ordre contraire à celui qu'on trouve dans les textes.

Mohrmann part d'un sens de statio qui a sa source dans la pensée grecque, à savoir: la place, le poste qu'on occupe dans la vie ${ }^{4}$. Ce sens métaphorique initial s'élargit en celui de «service». Il finit par désigner surtout la tâche du "premier serviteur de l'État", l'empereur. Les

I Schummer, Fastenpraxis, pp I35-150

2 St W J Teeuwen, Sprachlicher Bedeutungswandel bei Tertullian, Paderborn I 926, pp Ior-I 20

3 Vour Mohrmann, Statıo, pp 328-330

4 Mohrmann, Statio, pp 318-327 
Chrétiens lui donnent alors le sens spécial de "service de Dieu" et indiquent par là le jeûne stational. Statıo, dans le sens de "service", était beaucoup moins usuel que ses synonymes munus et officium. Mais s'il a été choisi, c'est à cause de l'influence du mot hébreu qui avait également évolué à partir du sens plus général de uservice», "fonction", pour en venir à indiquer une fonction liturgique, celle notamment de la "représentation", tant à Jérusalem que dans les services correspondants des districts. Mais, comme nous l'avons dit plus haut, pp. I70-I7I, il y a certains inconvénients à mettre en rapport le jeûne de la "représentation" avec la statıo chrétienne, alors que d'autres formes judaiques de jeûne conviennent mieux à cet égard. Cette théorie ne résout donc pas non plus tous les problèmes.

Avant de chercher une explication à notre tour, nous voudrions faire deux remarques d'un ordre plus général. Pour commencer, un terme abstrait comme statio, qui indique une chose aussi universelle que l'action de se tenir debout, est prédestiné, tant dans la langue commune que dans les langues de groupe, à développer une multitude de sens, sens qui peuvent exister côte à côte' ${ }^{1}$ C'est pourquoi, lorsqu'on cherche à reconstituer une évolution sémantique, il ne faut pas se laisser hypnotiser par un seul sens, qui semble dominer, comme l'ont fait ceux qui supposaient dans statıo une métaphore militaire. Il en va exactement de même pour le מפֵ hébreu, qui trop souvent a fait penser au seul phénomène de la représentation.

Deuxièmement, on peut tenir pour probable que l'origine de la

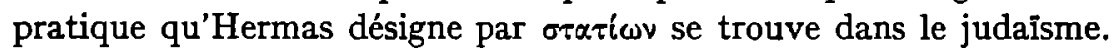
La Didachè $(8, I)$ recommande aux Chrétiens de faire leur v pas le lundi et le jeudi, jours de jeûne juifs ${ }^{2}$, mais le mercredi et le vendredi. Cette recommandation nous apprend donc que l'habitude de jeûner les jours de jeûne juifs était encore vivace dans les milieux chrétiens et aussi que les objections ne concernaient pas le maintien des pratiques de jeûne juives, mais l'observance des jours juifs alors que l'Église en avait fixé d'autres. L'exhortation de la Didachè et le fait que, d'une façon générale, le jeûne est, chez les Chrétiens, un héritage du judaīsme, nous engagent à chercher les origines du terme statio, et non pas seulement la pratique qu'il désigne, dans le milieu juif.

I Cf Mohrmann, Statio, pp 318-319. 327

2 Voir Elbogen, Gottesdrenst, pp 76-77, 524 Il pouvalt s'agr d'un jeûne in-

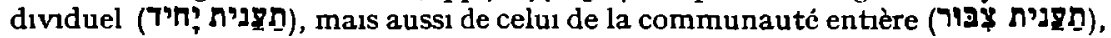
voir Schummer, Fastenpraxis, pp 84-90, J Behm, art vĩ $\sigma \iota \zeta$, ThWNT IV, pp (925-935) $930,931-932$ 
Nous constatons d'abord que l'Ancien Testament et, pour autant que nous sachions, les textes de Qumran et la littérature rabbinique ne possèdent pas de mot signifiant kla station debout pour désigner

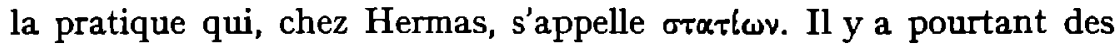
indications que, dans le milieu juif, les temps étaient mûrs pour un tel terme. Cette affirmation demande à être précisée.

Pour commencer, nous voudrions signaler que les éléments qui, à en juger par les passages du Pasteur que nous avons cités, paraissaient caractériser la $\sigma \tau \alpha \tau i \omega v$ d'Hermas, se trouvent aussi dans les sources juives, tant dans l'Ancien Testament que plus tard ${ }^{1}$. Ce sont la combinaison du jeûne et de la prière, mais aussi des aspects comme le

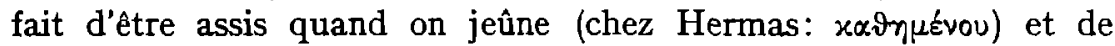
commencer tôt le matin (chez Hermas: ópきpıvós). En particulier, nous

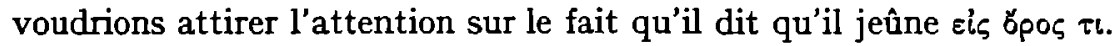
La plupart des commentateurs négligent cet élément comme sans importance. Seul Schümmer dit que «le départ matinal pour la montagne fait partie de sa pratique; car, c'est seulement après avoir demandé la raison de cette sortie matinale qu'il est question de la station ${ }^{2}$. Partant de cette idée, on comprend mieux aussi le passage qui suit la partie du chap.54 dont nous avons parlé. Le Pasteur considère que la pratique d'Hermas est sans intérêt et déclare que le vrai jeûne consiste à éviter le mal et à obéir aux commandements de Dieu (54,3-5). Il ne veut pourtant pas supprimer le jeûne au sens littéral: dans 56,7 , il engage Hermas, le jour où il jeûne, à ne se nourrir que d'eau et de pain et de donner à un indigent ce qu'il a économisé ; ainsi son jeûne plaira au Seigneur. Ce que le Pasteur condamne, c'est évidemment le jeûne considéré comme ayant un but en lui-même, conception que souligne l'isolement sur la montagne.

Or, on peut difficilement admettre que la statio, pour mériter son nom, devait absolument se faire sur une montagne. Mais si on prend l'élément "montagne» dans un sens large et qu'on entende par là un "endroit isolé, qui se situe habituellement quelque part dans la nature»,

I. Voir, par exemple, H. L. Strack-P. Billerbeck, Kommentar zum Neuen Testament aus Talmud und Midrasch, II, Munchen 1924, Pp.24I-244; IV I, München 1928, pp.94-Ioo; Schümmer, Fastenpraxis, pp.84-88, 91, 136; Behm,

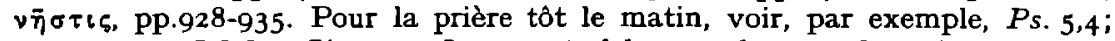
$62,2.7 ; 91,3 ; \mathrm{Job} 8,5$; Sir. 39,$5 ;$ Os. 5,15 et cf. les grands exemples paléotestamentaires comme Gen. 19,27; 22,3; Ex. 24,4; 34,2.4; Num. 14,40.

2. Schümmer, Fastenpraxis, p.83. Peterson, Giudaismo e Cristianesimo, p.379, lui aussi, prend la mention de la montagne au sérieux, et établit un rapport avec le jeâne individuel chez les Juifs. 
nous trouvons toute une série de parallèles dans les sources juives d'avant et d'après le Pasteur. L'exemple le plus célèbre est sans doute le jeûne de Moỉse sur le mont Sinaï: «Moīse fut là avec Yahvé quarante jours et quarante nuits; il ne mangea pas de pain et ne but pas d'eau" $(E x .34,28)$. Daniel jeûne près de la rivière: «En ces jours-là, moi Daniel, je fus dans le deuil trois semaines entières; je ne mangeai aucun met agréable, ni viande ni vin n'entrèrent dans ma bouche et je ne me fis aucune onction, jusqu'à ce que fussent accomplies trois semaines entières. Et le vingt-quatrième jour du premier mois, j'étais sur le bord du Grand Fleuve, c'est-à-dire le Tigre" (Dan. I0,2-4; suit une vision). Jésus aussi jeûne pendant quarante jours dans le désert (Mt. 4,I-2; Lc. 4, I-2) et le Protévangile de Jacques raconte la même chose à propos de Joachim, ce qui lui permet de souligner la combi-

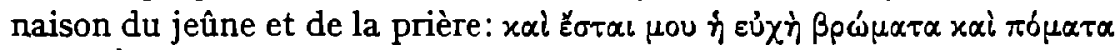
$(r, 3)$. A propos des pratiques juives de son temps, Tertullien, Ieiun. I6,6, déclare: Iudaicum certe ieiunium ubique celebratur, cum omissis templis per omne litus quocumque in aperto aliquando iam precem ad caelum mittunt. La littérature rabbinique, elle aussi, parle du jeûne et de la prière en plein air. On s'y réfère volontiers aux exemples bibliques. C'est ainsi que le Talmud babylonien, dans la gemara sur $T a^{\prime}$ anit 3,I-9, raconte du fameux jeûneur Honi: "Alors (c'est-à-dire, après que d'abord sa prière pour la pluie n'eut pas été exaucée), il traça un cercle et se mit dedans, ainsi qu'autrefois l'avait fait le prophète Habacuc, ainsi qu'il est dit: 'A mon poste de garde je veux me tenir et rester debout sur mon rempart' (Hab. 2,I) $)$.

On s'isolait pour entrer en contact avec Dieu dans le jeûne et la prière. Pour dire l'acte de se mettre ainsi en présence de Dieu à un endroit isolé, l'Ancien Testament emploie à plusieurs reprises l'ex-

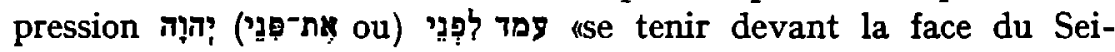

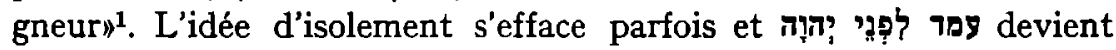
une manière de dire "prier»². Dans le judaïsme ultérieur, cette expression semble même être devenue plus ou moins technique, comme nous croyons pouvoir le constater dans un passage du Talmud Babylonien, dans la gemara sur Berakot I,I: «Et où (prend-on) que notre père Abraham ait fixé un endroit? Car il est dit: 'Et Abraham se rendit de

1. Gen. 18,22; 19,27; I Regn. I9,II; cf. Ex. 34,2-3; Deut. 5,31; 10,10; Ps. $24,3$. Cf. pour l'expression J. Reindl, Das Angesicht Gottes im Sprachgebrauch des Alten Testaments, Leipzig 1970, pp 20, 35-36, 48 .

2. Jer. I5,1; I8,20; avec la métaphore «être sur la brèche» Ps. I06,23; Ez. 22,30. Cf. aussi I Hen. I2,3. 
grand matin à l'endroit où il s'était tenu debout' (Gen. 19,27); et se tenir debout ne signifie autre chose que prier, car il est dit: 'Et Pinhas se tint debout et pria' (Ps. 106,30)". "Se tenir debout» est interprété ici très exactement comme "prier". Mais on cite en outre Gen. I9,27, sous une forme abrégée. Là où la Genèse a: «à où il était debout devant Dieu», le Talmud dit simplement: "où il était debout». La citation présente donc une ellipse qui caractérise bien la terminologie technique des langues de groupe ${ }^{1}$.

Peut-on chercher dans cet usage l'origine du terme statio pour désigner une pratique de prière et de jeûne? On pourrait objecter que "se tenir debout devant la face du Seigneur" signifie surtout "prier", alors que dans statio c'est le jeûne qui prime. Il ne faut pourtant pas trop dissocier les deux éléments. Il est frappant que régulièrement, dans la Bible et dans les textes juifs et chrétiens ultérieurs, la prière et le jeûne vont de pair, surtout lors que la prière est uneconfession de fautes ou une supplication ${ }^{2}$. C'est pourquoi nous croyons que cette objection n'est pas insurmontable et que l'hypothèse mérite d'être examinée.

La question est donc de savoir d'où peut venir le terme statio. Elle peut se poser en termes un peu plus larges: dans quel milieu un mot signifiant «être debout» se mit-il pour la première fois à signifier le jeûne stational? Non pas dans un milieu de langue grecque, pour autant que des conclusions puissent se tirer de l'absence d'un terme de ce genre dans les nombreux textes qui parlent de la chose ${ }^{3}$. Mais il reste trois autres possibilités qui méritent d'être sérieusement prises en considération. On peut penser qu'il y eut à l'origine: $I^{\circ}$ un substantif

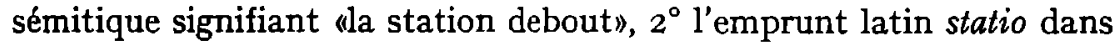
un milieu sémitique, $3^{\circ}$ statio dans un milieu latin.

Le premier cas, comme nous l'avons dit, ne se présente pas dans nos

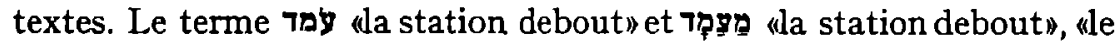
posten, s'emploient plusieurs fois de telle sorte qu'on pourrait les prendre pour le point de départ d'une évolution vers le sens technique. C'est ainsi que, dans $\operatorname{Dan} .8,17$, lorsque Daniel a une vision sur la rive de l'Ulaĩ (probablement après avoir jeûné et prié, cf. Dan. 9,3), il est dit au sujet de Gabriel: «et il vint près de ma 'place' ('pְq)"), (c'est-à-

I Cf. supra p.149.

2 Vour les textes chez Behm, vĩo $\tau \iota \varsigma$, pp 93I, 933,934 Cf aussı supra p I68 n 4

3 Did 8,1, Clément d'Alexandre, Str 7,12,75,2; Ongène, Hom ro in Lev 2; Pierre d'Alexandrie, Ep. Can 15, Epiphane, Haer 75,3,7, 75,6,2 4, Exp Fid 22,1; Const App 5,14,20; 5,20,18,7,23,2; 8,47,69, Ps Ignace, Phil 13,cf Socrate, $H E{ }_{7,22,5}$ Cf Mohrmann, Statio. pp 309-3 1o 
dire jusque là où je me tenais debout). Et, dans $\mathrm{I} Q H$ II,I3, il est dit que Dieu purifie un homme des péchés «afin qu'il aille se placer à la place devant ta face (במצמד לפניכה)".

Mais il est une règle linguistique qui rend peu acceptable l'idée qu'on en soit venu à la formation d'un terme technique. D'après cette règle, une langue qui emprunte tend à rendre les mots concrets par un emprunt (importation) et les abstraits par une traduction (substitution). C'est ainsi que, dans la Septante, des mots concrets comme $\pi \dot{\alpha} \sigma \chi \alpha$, $\sigma \alpha \dot{\beta} \beta \alpha \tau o v, \chi \varepsilon p o u ́ \beta$ ont été empruntés à l'araméen et à l'hébreu, alors que

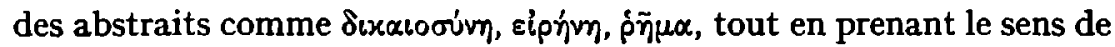
leur équivalent sémitique, sont formés par la langue à laquelle ils appartiennent. De même en latin paléochrétien, on importe sans les traduire des termes concrets comme baptisma, catechumenus, ecclesia, alors que des termes abstraits comme gloria, revelatio, salus sont des mots d'origine latine ${ }^{1}$. Le jeûne stational doit être considéré comme une institution concrète. C'est pourquoi, en passant du latin au grec,

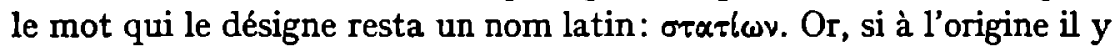
avait eu un terme sémitique, la règle en question ferait, qu'avec la chose, le terme serait passé dans la langue qui emprunte, ce qui n'est pas le cas. Il va sans dire qu'il ne s'agit ici que d'une vraisemblance. On pourrait facilement trouver nombre de cas de mots concrets qui, en passant de l'hébreu au grec, ont été traduits, mais qui, en passant du grec au latin, furent adoptés comme emprunts, ainsi רip $\psi \alpha \lambda \mu \delta \zeta$ - psalmus.

La deuxième possibilité, à première vue, semble encore moins vraisemblable. Des Juifs de langue sémitique auraient-ils besoin de mots latins pour une chose qui leur appartenait autant que le jeûne et la prière? Il ne faut pourtant pas sous-estimer l'importance des emprunts latins dans la langue sémitique. L'esprit impur, dans l'évangile, se nomme lui-même $\lambda \varepsilon \gamma\llcorner\omega ́ v ~(M c .5,9 ; L c .8,30)$ et Jésus, lui, utilise ce mot latin pour désigner les légions d'anges ( $M t .26,53)$; ces passages ont toute chance de remonter à des originaux sémitiques. Un grand nombre de mots latins ont pénétré dans la littérature rabbinique. Deux d'entre eux se trouvent dans des textes qui sont d'une époque plus récente, mais qui pourraient bien témoigner pourtant d'un usage qui remonte aux Ier et $\mathrm{II}^{\mathrm{e}}$ siècles après J.-C.; ils méritent plus spécialement notre attention. Dans $j$ Demai $26^{b}$, on lit qu'un maître d'école est

I. Voir, par exemple, chez Mohmann, Traits caractéristiques, pp. 22-23, 41-45, 47-48. 
trouvé ieiunus ${ }^{1}$ (affaibli); il s'avère que la raison en est le jeûne excessif. Ce passage nous apprend qu'on utilisait bien en fait des mots latins pour désigner certains aspects du jeûne. Quelques midrashim emploient le mot statio ${ }^{2}$. Il est vrai qu'il est utilisé dans un sens beaucoup plus général, «'existence», mais ils prouvent du moins que le mot circulait dans le judaîsme de langue sémitique. Mais il y a une autre objection contre l'emprunt statio au sens de pratique du jeûne et de la prière. En effet, s'il est exact que le mot s'est développé à partir de

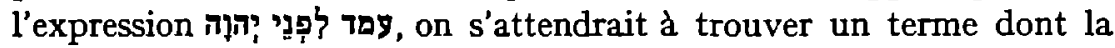
parenté avec עמ serait perceptible pour l'oreille sémitique: donc un mot sémitique et non un emprunt.

La troisième possibilité suppose une communauté de langue latine qui reproduisit en latin l'expression le simple Tay, pour désigner le jeûne stational: stare ante faciem Domini, et par la même occasion forma même un terme, statio. En présence des données dont nous disposons, cette dernière possibilité nous semble la plus acceptable. Mais la question se pose dès lors de savoir si le mot a été formé par les Juifs ou seulement par les Chrétiens. Jusqu'à présent, on semble généralement admettre que statio est un néologisme chrétien. Pourtant l'hypothèse d'une origine juive nous paraît mériter la préférence. D'une part, à l'époque d'Hermas, la communauté juive de Rome était beaucoup plus importante que la communauté chrétienne, et le latin y avait fait aussi plus de progrès ${ }^{3}$. D'autre part, comme nous avons essayé de le montrer, la pratique qu'Hermas appelle $\sigma \tau \alpha \tau i \omega v$ est d'origine judaĩque et toutes les étapes préparatoires pour la création du terme avaient été franchies. En même temps que l'institution, les Chrétiens d'Occident ont fort bien pu reprendre aussi le nom donné par les Juifs.

Le terme, nous apprend-il quelque chose sur l'emploi du latin chez les Chrétiens de Rome? Dans son étude sur les origines du latin chrétien à Rome, Christine Mohrmann partait de l'idée que le terme statio avait vu le jour chez les Chrétiens. Elle en tirait la conclusion qu'à côté du grec, les Chrétiens de Rome, et Hermas en particulier, utilisaient le latin`. Il n'y a guère d'objections à cette conclusion si la

1. ייני On. On identifie parfois ce mot avec un mot grec, voir S. Krauss, Griechische und lateinische Lehnwörter im Talmud, Midrasch und Targum, II, Berlin 1899, p.33, mais cf. Rosén, Palestinian xoเvทं, p.6o.

2. ןlivook. Cf. Krauss, Lehnwörter, II, p.79.

3. Cf. supra pp.49-5I. Sur la possibilité que le latin ait été employé même dans les services synagogaux à Rome, voir Colomi, Uso del greco, p.77.

4. Mohrmann, Origines, pp.76-78. 
prémisse est juste, mais comme nous l'avons expliqué, une autre prémisse nous semble plus vraisemblable. Mlle Mohrmann trouvait un

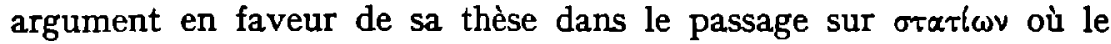
Pasteur demande qu'Hermas lui explique le sens du mot $(54,1-2)$ :

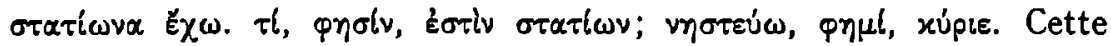
explication aurait été ajoutée à l'usage des lecteurs grecs qu'Hermas soupçonne de ne pas comprendre ce terme technique latin. Il est clair, en effet, que le passage en question demande à être expliqué, car l'ignorance dont le Pasteur fait preuve n'est pas tout à fait ce qu'on attendrait d'un envoyé de Dieu venu pour donner une révélation. Nous doutons cependant qu'il faille chercher la solution dans ce sens. Le Pasteur, en effet, n'est pas entièrement satisfait par la réponse

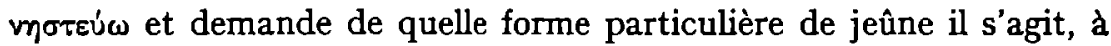
quoi Hermas répond simplement: celle dont j'avais (jusqu'à présent)

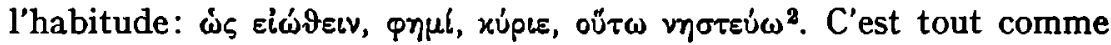
explication, et cette réponse laisse le lecteur dans son ignorance. Et cela, alors que le contexte, mise à part cette explication, rend suffisamment évident, même pour nous, ce qu'il faut se représenter par une $\sigma \tau \alpha \tau \hat{\tau} \omega v^{3}$.

A notre avis, il faut chercher l'explication des questions posées par le Pasteur dans un procédé narratif que nous retrouvons ailleurs chez Hermas. Dans le chap.2, alors qu'il s'afflige d'avoir été accusé de "désir mauvais" par son ancienne maitresse, Rhodè, Hermas a la vision d'une vieille femme. Celle-ci, avec une certaine compassion, s'informe de la raison de son chagrin; mais, il ne lui a pas plus tôt répondu à demi-mots qu'elle paraît bien savoir parfaitement la raison et même qu'elle le réprimande sérieusement. Pour illogique qu'elle soit, cette manière d'écrire a l'avantage de définir d'une manière concrète et avec force la situation à propos de laquelle la révélation de l'envoyé de Dieu sera faite. Il s'agit ici d'une condamnation du jeûne stational. Il nous paraît utile qu'Hermas insiste pour le faire com-

I. Mohrmann, Origines, p.76; id., Statio, pp.309-3ro. Cf. aussi Harnack ad loc.

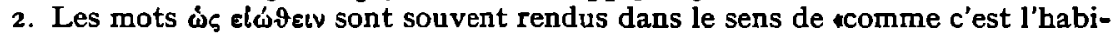
tude», ce qui permet une interprétation plus commode, mais qui, à notre avis,

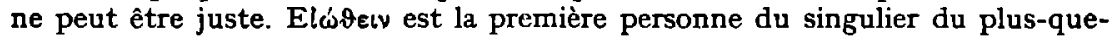
parfait; $\mathbf{L}_{1}$ et $\mathbf{L}_{2}$ traduisent littéralement solebam. Le plus-que-parfait a-t-il ici

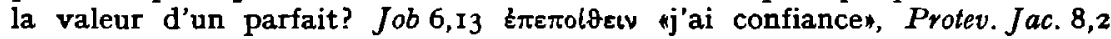

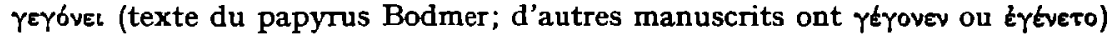
sont peut-être des parallèles.

3. Parfois on explique la question du Pasteur en supposant qu'il s'agit d'un terme récent. On signale alors que Did. 8, I ne le connaît "pas encore». Mais c'est le cas pour tous les textes qui ont été écrits en Orient, cf. supra p.175. 
prendre à ses lecteurs (qui sans doute connaissaient déjà et la chose et le nom) en appelant la chose par son nom².

Nous pouvons résumer l'hypothèse que nous venons de développer en disant qu'il faut chercher l'origine du terme statio dans l'expression biblique "se tenir devant la face du Seigneur" en tant qu'elle désigne une manière de prier et de jeûner en se retirant dans la solitude et en se mettant en plein air. De cette expression, sans doute sous sa forme abrégée de "se tenir debout", on dériva, dans les communautés juives de langue latine, notamment à Rome, un terme technique, statio. Les Chrétiens, tant ceux qui parlaient habituellement le grec que ceux qui parlaient habituellement le latin, reprirent le terme. La présence de

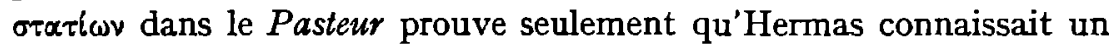
terme technique emprunté au latin, mais non qu'il connaissait luimême le latin.

\section{Oủal}

Dans le Pasteur, l'interjection ov่al se présente deux fois:

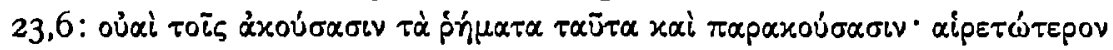

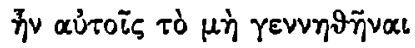

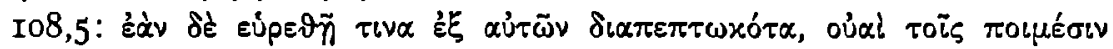
छ $\sigma \tau \alpha$. Le dernier passage n'a été transmis en grec que par Antiochus, mais il est étayé par $\mathrm{L}_{1}$ : vae erit pastoribus.

En grec profane, oúaí ne se présente que relativement tard et reste rare dans nos textes. Nous ne connaissons qu'un passage, dans un mime, qui probablement date du Ier siècle avant J.-C. ${ }^{2}$, deux chez Epictète $(3,19,1 ; 3,22,33)$, un dans une fable ésopique (I90II Hausr.), deux dans la Vita Aesopi Westermanniana (2.37) et une mention dans

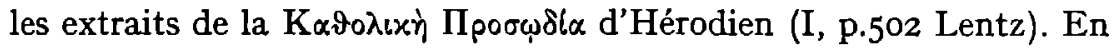
grec biblique, au contraire, oủal est fréquent. Le mot se présente 73 fois dans la Septante (surtout Is. et Jer.), 45 dans le Nouveau Testament (surtout $M t$., Lc. et $A$ poc.) et 30 fois dans les apocryphes (surtout I Hen.) ${ }^{3}$.

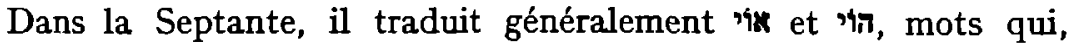
pour le son aussi, correspondaient plus ou moins au mot grec. C'est

I. Dans le même esprit Dibelius ad loc.

2. Transmis par POxy.3,423,I84-I86 (I/IIp). Pour la datation du mime, voir H. Wiemken, Der griechische Mimus, Bremen I972, p.43; le texte $\imath b$. p.88.

3. Les passages dans les apocryphes sont: I Hen. 97-100; 103,8; Test. Abr. B 9; Apoc. Esdr. pp.24.25.28.30; Test. Job 22,2; 53,2bis; Protev. Jac. 20, 1; Ev. Petri 25; Ps. Barn. 4, I ; 6,2.7; Did. 1,5. 
pourquoi il a été souvent considéré comme un emprunt à l'hébreu. Mais la différence de prononciation entre le mot grec et les mots hébreux en question est pourtant telle qu'il faut écarter cette hypothèse'. Si nous tenions à voir dans oúaí un emprunt, c'est plutôt au vae latin qu'il faudrait penser, car il en est le pendant phonétique exact. Mais il est peu probable que les traducteurs de la Septante, dans l'Alexandrie du IIe siècle avant J.-C., aient déjà eu recours à des mots latins ${ }^{2}$. Enfin, il y a le' ' araméen, que nous trouvons dans la littérature rabbinique. On peut faire à son sujet des réserves du même genre. Il est difficile de croire, en effet, qu'un mot apparaisse comme emprunt (ici dans le grec de la Septante) plusieurs siècles avant qu'on le rencontre dans sa langue d'origine. C'est pourquoi il nous semble plus raisonnable de croire que c'est le grec lui-méme qui a donné naissance à oủal. Étant donné que le mot manque en grec classique et est fréquent dans la Septante, il a dû se répandre au début de l'époque hellénistique ${ }^{3}$.

A cette hypothèse on pourrait objecter que les mots d'origine grecque ne commencent pas par oú- suivi d'une voyelle. Mais une particularité des interjections, qu'on observe aussi dans beaucoup d'autres langues, c'est que, pour elles, les règles phonétiques ordinaires n'ont pas cours4. La fréquence minime d'oủal dans les textes profanes s'explique facilement aussi: en tant que mot post-classique il est banni de la langue littéraire, d'inspiration attique. Ainsi, pour traduire le vae victis proverbial du chef gaulois Brennus (Tite-Live 5,48,9;

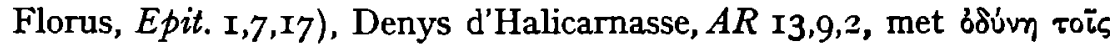

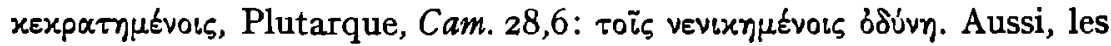

1. Cf. A. D. Lowe, The origin of o ial, Hermathena 105, 1967, p.(34-39)34. En plus des auteurs qu'il cite, Turner, Syntax, p. 308 (cf. Pp.296, 309), parle aussi de "Hebraic oúal».

2. Cf. Lowe, ov่ $\propto$, p.34. Ceux qui supposaient une origine latine sont Wackernagel, Vorlesungen, II, p.257 et Hofmann, Umgangssprache, p.I3. Pour BD $\S 4^{20}$ c'est aussi bien un hébraisme qu'un latinisme.

3. Voir Lowe, oủxl, p. $3^{8}$ n. I. De l'existence d'interjections indoe-européennes, apparentées selon lui, (le vae latin, le wai gothique, le woe anglais) il tire, pour sa part, la conclusion que oval existed in early Greek and reached the Koine from some pre-Koine dialect*; ce serait une forme secondaire du $6 \alpha$ thelas" qu'on rencontre chez Eschyle (ib. p.34, cf. p.36). Mais supposer des formes secondaires pour des interjections est chose hasardeuse (c'est ainsi que le oủ de $M c .15,29$, par exemple, a une tout autre valeur qu'oúal); et les termes apparentés en question ne se présentent pas avant (pour vae) ou beaucoup plus tard (pour wai, woe) qu'oúal dans les sources.

4. Cf. Mussies, Koine Greek, p.62 (et pp.54, 59). Pour la question de savoir si une interjection construite avec un datif mérite encore ce nom, cf. Pinkster, Latin adverbs, Pp.I4r-I43. 
passages que nous avons cités à propos d'oúal viennent-ils d'auteurs qui ne se soucient pas des règles atticistes. Une remarque du Ps. Basile nous autorise peut-être aussi à penser qu'oủa est d'origine grecque,

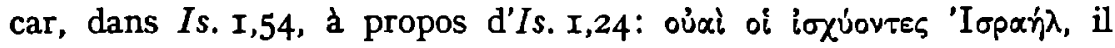

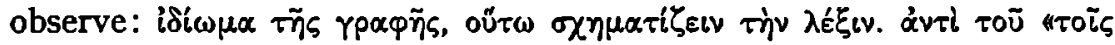

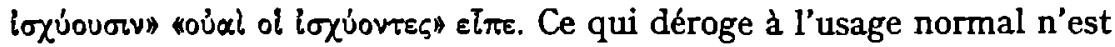
manifestement pas oúxh, mais le nominatif dont il s'accompagne. Dans les passages que nous avons tirés des extraits de la $\mathrm{K} \alpha \vartheta \circ \lambda(x) \eta \Pi_{p \circ \sigma \omega} \delta i \alpha$ d'Hérodien, oủal est également traité comme une interjection normale.

L'hypothèse d'un oủal d'origine grecque existant dès le début de l'hellénisme se confirme peut-être d'une autre manière encore. Après avoir supposé, dans son Lateinische Umgangssprache, que oủaí est emprunté au latin, Hofmann signale un peu plus loin que le latin archaique a emprunté une grande quantité d'interjections au grec. peut-être même plus qu'on ne l'avait supposé jusqu'ici, et que le zèle des auteurs dramatiques romains joua un grand rôle à cet égard ${ }^{1}$. Se peut-il que vae ait été emprunté au grec? Dans les textes, le oúal grec est plus ancien (Num. 2I,29) que le vae latin (à partir de Plaute). Et le passage le plus ancien en grec profane provient du monde du théatre. Le 'J araméen pourrait fort bien, lui aussi, être interprété comme un emprunt au grec ${ }^{2}$.

L'hypothèse suivant laquelle, à l'époque des premiers traducteurs de la Septante, oúá était un mot récent, mais d'origine grecque, explique aussi la faveur dont cette interjection jouissait dans la Septante. Du point de vue phonétique, les traducteurs pouvaient, sans devoir recourir al a transcription, trouver ainsi un équivalent qui, pour le son, offrait une certaine similitude avec les mots du texte originals $^{3}$ Du point de vue sémantique, ils ont trouvé un mot qui, parce qu'il était encore plus ou moins vierge de charge affective, pouvait se charger de ce qu'il y avait de sublime et souvent de prophétique dans le mot hébreu, si bien qu'on pouvait éviter des cris comme $\varphi \in \bar{u}$ ou l'́, qui auraient pu évoquer des associations indésirables avec, par exemple, la tragédie classique. Ensuite, le Nouveau Testament reprend oúai avec cette charge. C'est ainsi que, dans les évangiles,

r. Hofmann, Umgangssprache, pp. 13, 23. Les interjections s'introduisent facilement dans d'autres langues, cf. Weinreich, Languages in contact, pp.35-36, 47; Lowe, où l, Pp.34, 36.

2. Cf. Lowe, oủal, p.36. M. Schwab, Transcription de mots grecs et latins en hebreu, aux premiers siecles de J.-C., Joumal Asiatique IX 10, 1897. Pp.(4I4444) 443, 444 pense à un emprunt au latin.

3. Cf. Thackeray, Grammar, Pp.36-38; Lowe, oủal, Pp.36-37. 
le mot se présente uniquement dans les paroles du Seigneur et qu'ailleurs on le trouve surtout dans l'A pocalypse.

Chez Hermas aussi, il est revêtu d'une gravité prophétique qui rappelle fort la Bible. Dans le cas de 23,6, on pourrait même penser - ce qui a été fait - à une citation libre de $M t$. 26,24 (qui est à peu près

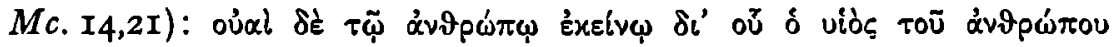

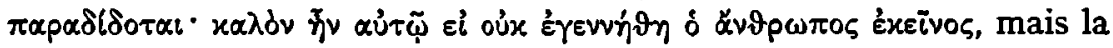
formule qui est commune à ces passages se présente aussi dans d'autres textes juifs et chrétiens ${ }^{1}$. II n'empêche qu'on a l'impression que, dans son emploi de oúal, Hermas s'inspire moins de la langue courante que du grec biblique. Ce n'est d'ailleurs pas une raison pour mettre en doute que oúal soit d'origine grecque. Sur la question de savoir quelles autres langues que le grec Hermas a éventuellement pu connaître, notre interprétation ne nous apprend rien.

I. Voir H. Köster, Synoptische Uberlieferung bei den Apostolischen Vätern Berlin I957, p.246; Reiling, Hermas, pp.166-167. Cf. aussi Fab. Aesop. I90II Hausrath. 
Nous avons donc considéré dans le Pasteur d'Hermas une série de phénomènes linguistiques qui donnaient l'impression d'être des sémitismes ou des latinismes. Notre propos était avant tout d'examiner s'il s'agissait bien de sémitismes et de latinismes ou si, en réalité, nous n'avions rien là qui ne fût conforme au grec de l'époque d'Hermas. Deux principes ont présidé à nos recherches: $\mathrm{I}^{\circ}$ Le grec post-classique connaît une évolution propre qui peut être parallèle à celle des langues sémitiques ou du latin sans en dépendre: quand un phénomène qui n'existe pas en grec classique a un équivalent en langue sémitique ou en latin, on ne peut pas pour autant affirmer a priori qu'il soit un sémitisme ou un latinisme. $2^{\circ}$ On ne peut pas non plus, sous prétexte que le Pasteur comporte de nombreux éléments juifs et que son auteur est d'un milieu romain, considérer comme sémitismes ou latinismes des phénomènes qui, chez d'autres auteurs de l'époque impériale, sont acceptés comme du grec normal. On doit, au contraire, voir d'abord, grâce à une étude impartiale des faits linguistiques, s'il y a lieu, chez Hermas, de tenir compte de la connaissance d'une langue sémitique ou du latin. Notre propos était, en deuxième lieu, de préciser ce que nos constatations pourraient nous apprendre sur la connaissance qu'Hermas avait éventuellement des langues. Pour les sémitismes, nous sommes parti du principe que les phénomènes linguistiques qui se présentent déjà dans la Septante ne peuvent en général servir de preuve pour la connaissance de l'hébreu et de l'araméen.

Voici donc le bilan de nos recherches sur la présence de sémitismes et de latinismes dans le Pasteur. Peuvent valoir comme sémitismes, dans le chapitre IV: slvaı zis "devenir", peut-être la périphrase au

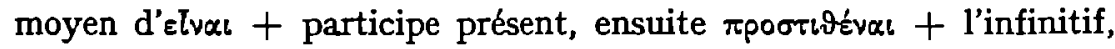

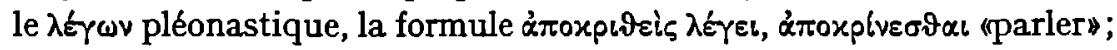

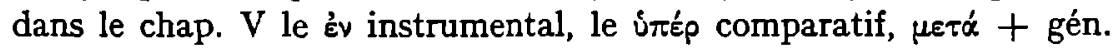

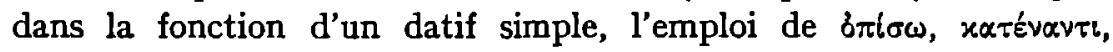




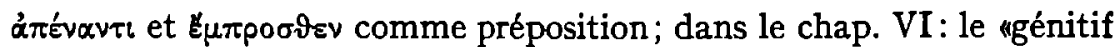
hébraiqque», le redoublement distributif des substantifs, la parataxe dans des subordonnées; dans le chap. VII: le pluriel oúpavol, les tour-

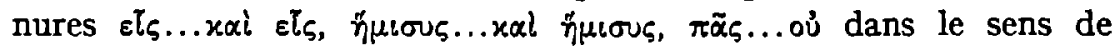

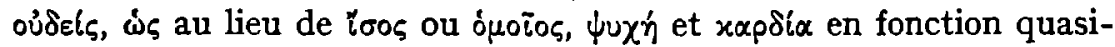

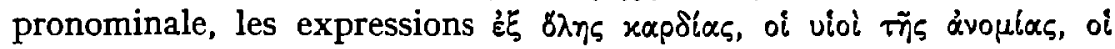

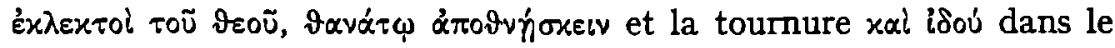
style narratif; enfin, une série de mots et d'expressions qui devaient être considérés comme des sémitismes non pas pour des raisons syntaxiques, mais sémantiques. Tous ces sémitismes pouvaient être ramenés à l'hébreu, la plupart en même temps à l'araméen (sauf:

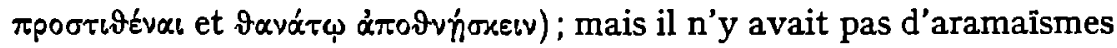
qui ne fussent pas des hébraîsmes.

Contrairement aux sémitismes, les latinismes étaient peu nombreux. Le génitif dans la combinaison $\tau \iota \dot{\alpha} \gamma \propto \vartheta \circ \tilde{u}$ semblait bien être latinisant, mais le passage où il se présente est si peu sûr qu'il vaut mieux ne pas s'en servir pour échafauder des théories. Le $\mu \varepsilon \tau \alpha ́$ sociatif et la tournure

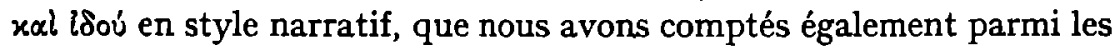
sémitismes, pouvaient, en principe, être dûs au latin; mais, chez Hermas, ils sont suffisamment élucidés si nous les entendons comme des sémitismes. Ensuite, le latin n'est représenté que par les quatre

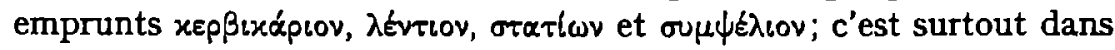
le grec non-littéraire que ces mots nous ont paru être abondamment

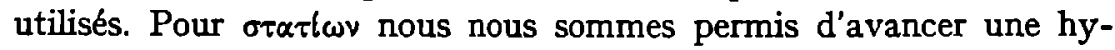
pothèse qui, si elle s'avérait juste, ferait de ce terme en même temps un sémitisme. Il y a un grand nombre de latinismes supposés que nous avons cru pouvoir expliquer comme étant du grec courant. Le plus fondamental parmi eux est l'emploi aoristique du parfait, et le plusque-parfait pour signifier le passé antérieur. Dans quatre cas nous avons contesté la qualification de sémitisme comme de latinisme:

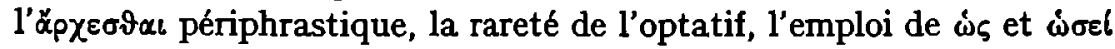
pour adoucir et l'interjection oúal.

Nous avons laissé de côté l'emploi de constructions qui sont impossibles dans les langues sémitiques et le latin. On cite souvent ce genre de constructions pour prouver que le texte où ils se présentent est d'origine grecque. Mais nous les avons rencontrées tant de fois, même dans les livres de la Septante qui sont littéralement traduits, que nous ne croyons guère à leur force probante. Ils ne manquent pas d'ailleurs dans le Pasteur. Citons le phénomène de deux négations qui

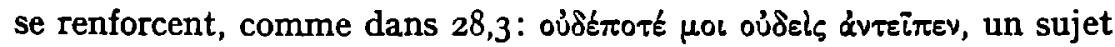


neutre au pluriel avec un verbe au singulier, comme 7,2: $\tau \alpha \tilde{\tau} \tau \alpha$ $\sigma \varepsilon \sigma \omega x \in \hat{v} v \varepsilon$, l'ordre des mots plus libre que dans les langues sémitiques.

En interprétant ces constatations, nous sommes amenés à nous faire des choses une image nette. Presque tous les sémitismes se trouvaient déjà dans la Septante. La seule exception, constituée par la terminologie des deux aĩ̄ves, s'était développée néanmoins assez longtemps avant que le Pasteur ne vît le jour. Pour les sémitismes syntaxiques, nous avons pu observer que, dans certains cas, ils avaient évolué depuis leur emploi dans la Septante. C'est ainsi que, chez Hermas, on trouve maintenant ú $\pi \varepsilon_{p}$ iplus que», construit avec le superlatif au lieu du positif ou du comparatif, et que, dans la formule ámoxpıəzis

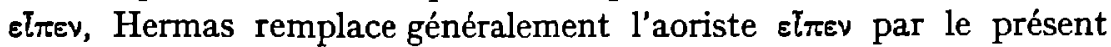
historique $\lambda \varepsilon^{\prime}{ }^{\prime} \iota$ si en faveur chez lui. Pour les sémitismes sémantiques, les cas d'évolution que nous avons pu relever sont beaucoup plus nombreux, notamment lorsque des termes d'origine juive ont pris un sens spécifiquement chrétien. Cette évolution était d'ailleurs chose faite bien avant le Pasteur. Dans l'ensemble, nous pouvons conclure qu'Hermas a pris ses sémitismes dans la Bible grecque et dans le milieu chrétien où on lisait cette Bible. Ils ne prouvent nullement qu'il connaissait des langues sémitiques.

Il y a d'autres manières de montrer que ce n'est pas une soi-disante langue maternelle sémitique qui joue des tours à Hermas. Ses sémitismes, en effet, consistent surtout en mots et en formules: le système de la langue n'est pas entamé. Le fait qu'il s'arrange pour que ses sémitismes n'obscurcissent pas son texte montre également qu'il est un écrivain d'expression grecque. Aussi bien, tous les sémitismes du Pasteur sont-ils de ces sémitismes qui se sont formés dans la ligne de ce que le grec profane avait amorcé, ou bien de ceux qui ne s'écartent de la langue profane que par la fréquence de leur emploi.

$\mathrm{Au}$ sujet de l'élément latin, nous pouvons être bref. Il n'est représenté que par quatre emprunts dont ni la forme ni le sens ne permettent de conclure qu'Hermas connaissait le latin.

Notre conclusion finale sera donc que, dans les matériaux linguistiques, nous ne trouvons aucune preuve qu'Hermas était familiarisé avec l'hébreu, l'araméen ou le latin. Ses sémitismes sont des éléments traditionnels du grec biblique, ses latinismes sont des emprunts déjà intégrés dans le grec de son temps.

Enfin, nous tenons à faire encore quelques remarques sur des sujets qui ont été abordés dans les chapitres d'introduction II et III. Les diverses hypothèses d'une pluralité d'auteurs ne se sont pas confirmées 
au cours de la recherche linguistique. Au contraire, les phénomènes que nous avons examinés se présentaient toujours des deux côtés des frontières tracées par Giet et d'autres. La question de la composition reste un point difficile; mais il est trop commode d'en chercher l'explication dans une pluralité d'auteurs. Cette solution soulève plus de questions qu'elle n'en résout.

Les sémitismes et les latinismes ne permettent pas de déterminer la date avec plus de précision que les moyens auxquels nous avons eu recours dans le chap. II. Mais il est remarquable de voir combien de fois Hermas partage avec la Septante des phénomènes qui manquent dans le Nouveau Testament. Dans la mesure où l'étude de la langue peut nous renseigner sur ce point, Hermas montre une indépendance assez grande à l'égard des livres du Nouveau Testament, même les plus anciens. De même qu'un auteur comme Clément de Rome, il diffère sensiblement à cet égard des auteurs des évangiles et actes apocryphes, qui imitaient d'une manière beaucoup plus servile le Nouveau Testament non seulement pour le contenu, mais aussi pour la langue et le style. Cette question mériterait d'être examinée de plus près. Mais dès maintenant nous pouvons dire que l'indépendance relative d'Hermas à l'égard du Nouveau Testament suggère une date précoce.

Nous avons parlé assez longuement de la question du grec biblique comme étant la langue non seulement de la Septante et du Nouveau Testament, mais aussi des apocryphes qui s'y rattachent. Les matériaux que nous avons rassemblés dans les derniers chapitres ont montré plus clairement encore l'existence de cette langue. Notre connaissance du grec biblique présente d'ailleurs encore beaucoup de lacunes, et pour un grand nombre de sémitismes nous avons été réduit à nos propres recherches. Il serait souhaitable d'avoir une connaissance plus complète en premier lieu du comportement des différents traducteurs de la Septante. L'importance du grec biblique, qui a eu une influence incalculable non seulement sur le grec chrétien ultérieur, mais aussi sur les innombrables langues où, par le truchement des traductions bibliques, cette influence se prolongea, justifierait pleinement les efforts indispensables. 


\section{MOTS YRECS}

$\dot{\alpha} \gamma\{\varepsilon \sigma \mu \alpha \quad 157,158,159$

cióv 159, 185

¿่Xov́eเV 62, 121

- $\dot{a} \lambda$ lov 166

\&ँux 94

duxpráveเv els IO9

đ̇uá II3, II4, II 5

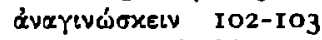

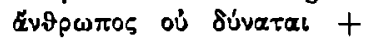
inf. 25

drvodice $3 \mathrm{I}$

¿́vopeiv I09, I57

¿́vopla II3, I44, I46, I57, 184

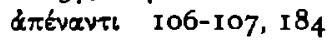

¿̇ं $90,94,143$

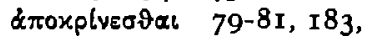
185

\&pern் III

-áprov 166

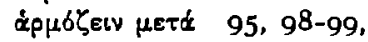
${ }^{4} 42$

PXeofar $\quad 66-70,184$

Łửंt่s $87,88,138$

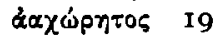

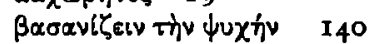

Bגaßßepós 3I

$\beta \lambda \alpha \sigma \varphi \eta \mu \varepsilon i v$ Ev 86

Ylverqal els 74-76

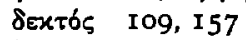

$\delta$ \&́x 86, 102, I25, 142

Sext 31

Sixalo5 II2, 156, 158

Sixarooivi IIIIII3, I58, I 76

cáv 65

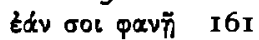

Exutou $87-88, I_{3} 8$

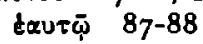

elval Els 74-76, 183

$\mu \varepsilon \tau \dot{\alpha} \quad 159$

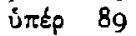

is 134-135

+ partic. prés.

$70-74,183$

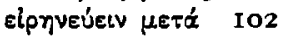

Elprivn 102, 176

els 9, 10, 25, 27, 32, 74-

$76,109,125,142$

Els...xal els I29-I32,

184

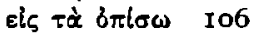

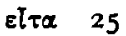

$E x$, ÉF I42-I 44

Eัx入еxтós 30, I47-I50, 158,182

'E

है/

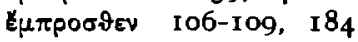

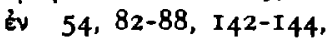
183

Eัขavтเ $106-108$

Évavilov 106,108

Èv $\alpha \dot{\tau} \bar{\varphi} \quad 87-88$

Evô̧०५ 30, II2

Ev $\delta \lambda_{n}(\bar{n})$ xapdía 143144

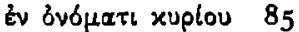

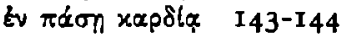

EVTEuELS 168

ㄴ $\tau \ddot{\varphi}+$ inf. $9,83,87$

Ev ப́roxplaer $3 I$

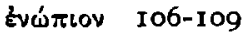

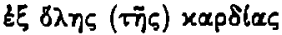

$142-144,184$

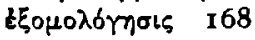

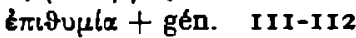

Epye + gén. II2-II3
'Eppāc 32

- Ec acc. 18

Ẽepos 130

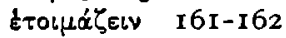

Eủ७nvia IOI-IO2

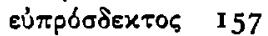

E้ $\chi$ ELV 160

$\tilde{\varepsilon} \omega \varsigma \pi \delta \tau \varepsilon \quad I 59$

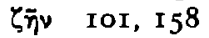

मे 90,93

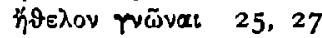

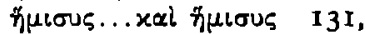
I 84

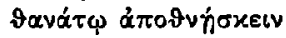
150-1 53, I 84

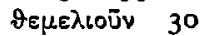

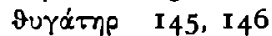

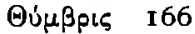

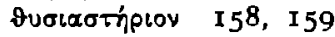

İoú 29, 153-155, 184

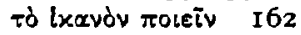

loos I34, 182

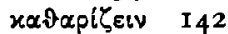

xal 9, 25, 27, I 18, I I9, I 29-I 32, I 53-I 55

xal l8oú I53-155, I 84

xapdla I 37, I42-144,

I 59, 184

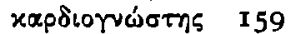

xard II3-II5

xarevvavil 106-107, 183

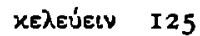

xepßlxkpiov $165,167-$ 168,184

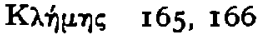

xoเvovós 100

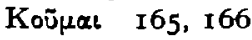

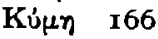

xஸ́na! 166 


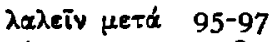

$\lambda$ EүEเV 4I, 79-80, I42, 185

$\lambda E \gamma \omega v \quad 78-79,183$

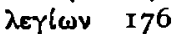

$\lambda$ Évilov I65, I67-I68, 184

$\lambda l a v 25$

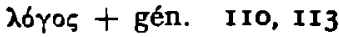

$\mu \varepsilon \hat{\imath} \cup \sigma \mu \alpha \quad I I 3$

$\mu t v \ldots \delta \varepsilon$ II 7, I 29-1 32

मÉpos $16 \mathrm{I}$

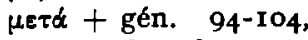

$142,183,184$

+ acc. 9, 25, 105, I 25

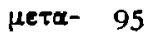

H:alvetv है 86

$\mu(\lambda$ iov 51,165

Mเxanj入 I65, I67

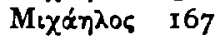

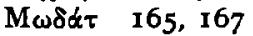

$8 \lambda \omega s \quad 25$

$\delta \mu-95$

$\delta \mu \alpha \lambda \delta \zeta$ 30, 31

8 Hotos 134,184

bilow 104-106, 183

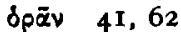

oủal 179-182, 184

oúbels I32-133, I 84

Oป๋ $25-26,31,85$

ớ... $\pi \bar{a}_{\zeta} \quad 134$ oujpavol I26-129, 184

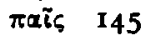

$\pi \alpha \lambda \alpha$ leLV $\mu \varepsilon \tau \dot{\alpha} \quad 95,98$

$\pi \dot{\alpha} \tau \omega_{5} \quad 25,26,134$

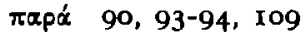

$\pi \bar{a}_{5} \ldots$ oủ 132-134, I 84

$\pi \dot{\alpha} \sigma x E$ ELV $1_{42}$

$\pi \varepsilon เ \sigma \theta \bar{\eta} v a \iota$ tै 85

$\pi$ tuns Io

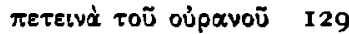

$\pi \lambda$ пророреiv $\mathbf{I} 40$

$\pi v E \tilde{\mu \alpha}$ I I 2, 157,158

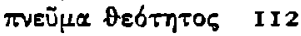

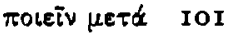

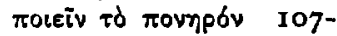
I08

Tounpla III, II 3

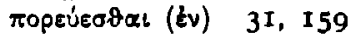

$\pi \rho l v \quad I 25$

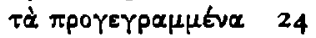

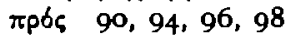

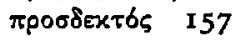

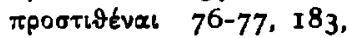
184

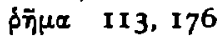

बर्यद 138

oxoגiss 30

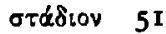

$\sigma \tau \alpha=k \omega \nu$ 6, I65, 167 ,

168-1 79, 184

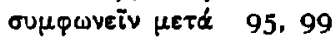

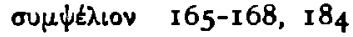

oúv 95

ouv- 95. 99

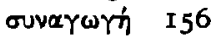

ớveols 30

oì oũv 31

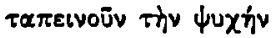

I40-I 4 I, I 59

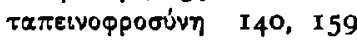

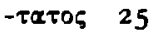

Téxvov I 46

Tißepis I65, I66

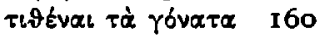

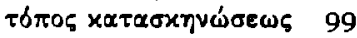

$-\tau b_{6} \quad 148$

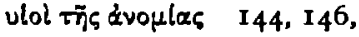
184

vitos I 44-I 47

Üंत्रe xal 25, 27

írauxéviov 168

ú $\pi \varepsilon_{p}$ 89-94, 142, I83, 185

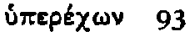

บं $\pi 6$ I02, 148

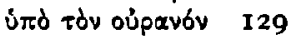

qúvar $5,25,79$

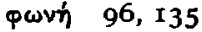

廿uxh́ 137-142, 184

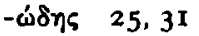

is $134-136,184$

कoel 135-136, 184

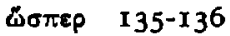

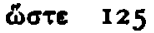

\section{MOTS LATINS}

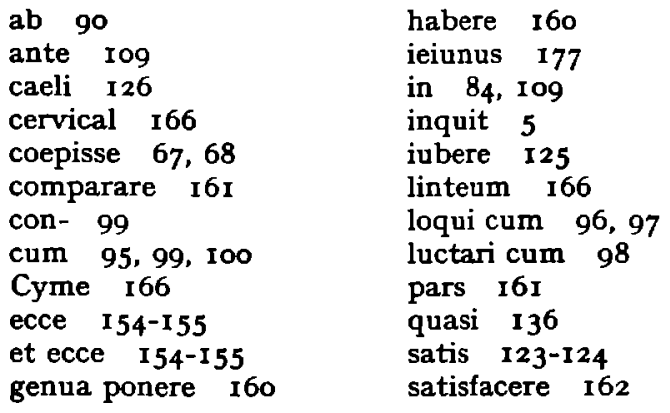

senatus auctoritas $\quad 164$

sicut 136

si tibi videtur I6I

stare ante faciem Domini

I 77

statio I66, I68-I 79

subsellium $\mathbf{I} 66$

super 93

Thybris 166

vae 179-18I

vivere cum IOI 
III. MOTS HÉBREUX ET ARAMEENS

\begin{tabular}{|c|c|c|c|}
\hline rix & 179 & $\mathfrak{9}$ & 134,136 \\
\hline טֶֶ & Iзо & לל & 133 \\
\hline אָָָד & I30 & לל .... לא & 133 \\
\hline רחֵ & 105 & ? & $74-75,76,88$ \\
\hline 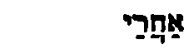 & 105 & לא & 133 \\
\hline אייתים & 177 & לאג ... מל & I 33 \\
\hline$\Gamma x$ & 72 & לִאממר & $7^{8}$ \\
\hline אסטשיח & 177 & לנסשר & 88 \\
\hline 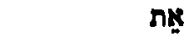 & 95 & לִיְנַי & $102,106,108,174$ \\
\hline$\frac{9}{1}$ & 83,88 & מִבְחָר & 150 \\
\hline 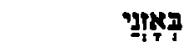 & 102 & מִזְממר & 176 \\
\hline בדחוּר & 150 & מלל עִם & 96 \\
\hline בדתחיר & 150 & מִן & 90 \\
\hline 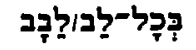 & 143 & מַוּעָד & $170-172,175$ \\
\hline 翟 & $145-147$ & 羿 & 102, 106 \\
\hline 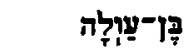 & 147 & נֶטֶט & $137-139$ \\
\hline 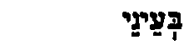 & $107-108$ & עבד עִם & IOI \\
\hline דבר אֵתוצִם & 96 & עַרְה & 156 \\
\hline הּז & 179 & עַל & 90 \\
\hline היה & $72,74-75$ & צִ & $94-95$, IO0-102 \\
\hline היה ל? & $74-75$ & עמד - - - עמ & $170,174,177$ \\
\hline 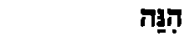 & I 54 & עמָד & 175 \\
\hline$?$ & I 30-I 3 I & 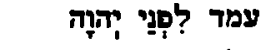 & 174,177 \\
\hline$\eta$ & 180,181 & צטשה צִם & IOI \\
\hline בָעצון & 79 & 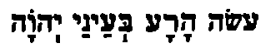 & 107 \\
\hline חלל & 68 & קרא & 102 \\
\hline 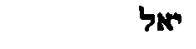 & 68 & 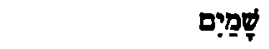 & $126-127$ \\
\hline זיָלד & 146 & 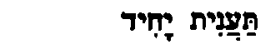 & 172 \\
\hline יסי & 76 & תַתָנִית צִבּוּר & 172 \\
\hline
\end{tabular}




\section{HERMAS}

L'astérisque dénote des questions de critique textuelle

\begin{tabular}{|c|c|c|c|}
\hline I, I-3 & $3 \mathrm{I}$ & 9,4 & 62,165 \\
\hline $\mathbf{I}, \mathbf{I}$ & $23,27,32,57,66$ & 9,5 & $67,135,160$ \\
\hline 1,2 & $26,32,67,78,123,142$ & 9.7 & 165 \\
\hline 1,3 & $25,26,50,66,69,160,166^{*}$ & 9,8 & 26 \\
\hline 1,4 & 129 & 9,9 & $26,86,161$ \\
\hline 1,6 & $19,109,128,129$ & $10, \mathbf{I}$ & I 59 \\
\hline 1,7 & $80,97,109$ & 10,4 & $86,107,153,165$ \\
\hline 1,8 & $26,27,87,111,128,129,160$ & 10,5 & I 3 I \\
\hline $\mathbf{I}, 9$ & $\mathrm{r}_{38}$ & 10,6 & $94,95,99,123$ \\
\hline 2 & 178 & 10,7 & I8*, I3I \\
\hline $2, \mathrm{I}$ & $26,86,128,142$ & 10,8 & 26,86 \\
\hline 2,2 & $26,107,142$ & II,I & 80,121 \\
\hline 2,3 & $26,109,123$ & II, 2 & I 2 I, I3I \\
\hline 2,4 & 26 & II,5 & $3-4^{*}, 5,86$ \\
\hline 3,I & 23, 109 & $12, I$ & \\
\hline 3,2 & $113,142,144$ & 12,2 & 26,93 \\
\hline 3.3 & 26,29 & 12,3 & $26,65,78,80,93,142$ \\
\hline 3,4 & $28,86,128,129,147,153$ & 13,1 & $18^{*}, 94,123,131,147$ \\
\hline 4,2 & 29,78 & 13,2 & 99,123 \\
\hline 4,3 & I8*, 97 & 13.3 & I3I \\
\hline 5,1 & 31,50 & 13,4 & 83 \\
\hline 5,2 & $66,69, \mathbf{I} 60$ & 13,5 & 26,134 \\
\hline 5.3 & $26,62,102,107,125,147$ & I4, I & 31,133, I 44 \\
\hline $6, \mathrm{I}$ & $I 4 I, I 68$ & I4,2 & $60,94, I_{3} I$ \\
\hline $6,2-7,4$ & 24 & I4,3 & II3 \\
\hline 6,2 & II3 & 14,4 & I3I \\
\hline 6,3 & 85,86 & 14,5 & 80, 131 \\
\hline 6,4 & 26,142 & 14,6 & 80 \\
\hline 6,5 & $\mathbf{1 4 7}$ & I 5,1 & 60,131 \\
\hline 6,8 & II* & 15,2 & III, 143 \\
\hline 7,1 & 86 & I 5.3 & 105,121 \\
\hline 7,2 & $26,143,185$ & 15,5 & 26 \\
\hline 7,4 & $24,153,16 \mathrm{I}$ & I5, 6 & $31,94, I I 2, I I 3, I 43$ \\
\hline 8,1 & 26 & 16,3 & I3I, I47 \\
\hline 8,2 & $60,102,149$ & $16,4-5$ & I45 \\
\hline 8,3 & $26,33,34,102,129$ & 16,4 & 94 \\
\hline 9,2 & $57,60, I 4 I, I 68$ & 16,5 & I3I \\
\hline 9,3 & $67.7^{8}$ & I6,6 & 26 \\
\hline
\end{tabular}




\begin{tabular}{|c|c|c|c|}
\hline $\begin{array}{l}16,7 \\
16,9 \\
I 6,1 \text { I }\end{array}$ & $\begin{array}{l}94 \\
78 \\
\text { 1 21 }\end{array}$ & $\begin{array}{l}25,3-5 \\
25,5 \\
25,7\end{array}$ & $\begin{array}{l}80 \\
80 \\
\text { I8, } 31\end{array}$ \\
\hline 17,3 & 8,123 & 26,1 & I9 \\
\hline 17,4 & I2I & 26,2 & 26,112 \\
\hline 17,5 & 26 & 27 & 7 \\
\hline $\mathrm{I} 7,6$ & 87 & 27,1 & I35 \\
\hline 17.7 & $26,94,131$ & 27.3 & $8,18^{*}$, IOI \\
\hline 17,10 & $65,88^{*}, 107$ & $27,4-6$ & $3 I$ \\
\hline I 7, I I & 147 & 27,5 & I $3 \mathbf{I}$ \\
\hline $\begin{array}{l}18,1 \\
18,2\end{array}$ & $\begin{array}{l}97,165 \\
80\end{array}$ & $\begin{array}{l}27,7 \\
28,1\end{array}$ & $\begin{array}{l}59,97 \\
\text { I } 2\end{array}$ \\
\hline 18,3 & I3I & 28,3 & $97^{*}$, IоI, I 24, I 84 \\
\hline 18,4 & $93,97,131$ & 28,4 & II 2 \\
\hline I8,5 & $26,123,165$ & 28,5 & 26 \\
\hline I8,6-7 & I68 & 29,4 & 95, I 21 \\
\hline I8,6 & $27,14 I$ & 29,5 & 95,121 \\
\hline I8,7 & $14 I$ & 29,7 & 26,125 \\
\hline I8,8 & I 4 I & 29,8 & 12 I \\
\hline I8,9 & 80 & 29,9 & 94 \\
\hline I8, IO & 80 & 30,1 & $7^{8}$ \\
\hline 19,2 & 26, I3I & 30,2 & $26,60^{*}, 80,107,108,140$ \\
\hline 19,3 & 86 & 30,4 & 27 \\
\hline I 9,4 & $26,27, I_{3} 8$ & $31, I$ & 77 \\
\hline 20,1 & 93 & $3 I, 6$ & I 2 I \\
\hline 20,2 & $\mathbf{1 2 2}, \mathbf{I}^{\mathbf{8}}$ & 32,1 & $67,78,121$ \\
\hline 20,3 & 142 & 32,3 & 26,97 \\
\hline $2 I, 3$ & $18 *, 86,165$ & 33 & 92 \\
\hline 21,4 & 26,142 & 33.5 & $3 I, I 2 I$ \\
\hline 22,2 & $3 I, 5 I$ & 33,6 & $26,92,94$ \\
\hline 22,3 & $63^{*}, 65$ & 33,7 & 26,142 \\
\hline 22,4 & $66,69,81,129,135,142$ & $34, I$ & 82,86 \\
\hline 22,5 & $51,66,69,153$ & 34.3 & 26 \\
\hline 22,6-7 & 81 & 34,6 & 7 \\
\hline 22,6 & $123,135, I 53$ & 34,7 & 86 \\
\hline 22,7 & $62,66,123$ & 34,8 & 26,73 \\
\hline 22,8 & 125 & $35,2-4$ & 30 \\
\hline 22,10 & 25 & $35,2-3$ & 7 \\
\hline 23, I & 24,26, I 54 & 35.3 & 25 \\
\hline 23,2 & I8 & 35,4 & 25 \\
\hline 23,3 & 80,86 & 35,5 & 142 \\
\hline 23,4 & 9,59 & 36,1 & I3I \\
\hline $\begin{array}{l}23,5 \\
23,6\end{array}$ & $\begin{array}{l}26,103,142,144,147 \\
121,179,182\end{array}$ & $\begin{array}{l}36,3 \\
36,4\end{array}$ & $\begin{array}{l}97,112 \\
26\end{array}$ \\
\hline 24,1 & 26,80 & 36,5 & 94 \\
\hline 24,2 & I 3 I & 36,6 & 31 \\
\hline 24,3 & 25 & 36,7 & 26,121 \\
\hline 24,4 & 86 & 36,8 & $26,63^{*}, 65$, I 21 \\
\hline 24.5 & I 47,149 & 36,9 & 94 \\
\hline 24,6 & 31,103 & 36,10 & $\mathbf{1 3 I}$ \\
\hline 24,7 & I06 & 37.5 & $18 *$ \\
\hline $25, I$ & $18,26,123$ & $3^{8,2}$ & 26 \\
\hline 25,2 & 26 & $3^{8,3}$ & II3 \\
\hline
\end{tabular}


$38,4 \quad 26$

$38,5 \quad 94$

$3^{8,6} \quad$ I3I

38,9 93, II 2, II 3

38 , $1093,94,123$

$39 \quad 138$

39, I I09, 121

$39,2 \quad 138,14^{2}$

$39,3 \quad 135$

$39,426,31,138,142$

$39,5 \quad 138$

$39,726,134,138,142$

$39,8 \quad 31,138$

$39,9 \quad 145$

39,1231

$4^{0,2} 26,93$

$40,3 \quad 121$

$40,5 \quad 121$

$40,6 \quad 94$

4 I, 2 I2I

$4 \mathrm{I}, 3 \quad 94$

$4 I, 4$ I3I

42,2 131, 133, I59, 168

$42,3 \quad 26,95$

$42,4 \quad 142$

$43 \quad 112$

$43, I \quad 165$

$43,2 \quad 97$, I I I

43.386

$43,4 \quad 94$

43,5 133

$43,6 \quad 26$

$43,8 \quad 26,93$

$43,986,121,156$

$43,10 \quad 112$

$43,1226,27,121$

$43,1394,95$

43,14 II, I 2 I

43,15 12 I

$43,16 \quad 124$

$43,18 \quad 129$

$43,19 \quad 27$

$43,20 \quad 121$

$44,2 \quad 86,121$

$44,326,85,87$, II 3

$45,2-5 \quad 98$

$45,2 \quad I 45$

$45,3 \quad 25,153^{*}$

$45,4 \quad 31,86$, I I I

45.5 3I, I I I

46 , I 26, 94, I I 2

$46,4 \quad 26$

$46,5 \quad 26,80$
$46,6 \quad 26$

$47, \mathrm{I} \quad 26,97$

$47,2 \quad 19,66,97,129$

$47,5 \quad 83,93$

$47,7 \quad 98$

$48,2 \quad 98,142$

48,326, I 20, I 2I, I 22

$48,4 \quad 63 *, 65,82$

49, I I 42,144

$49,2 \quad I_{42}$

$49,4 \quad 124$

$49,5 \quad 142$

$50, \mathrm{I} \quad 97,161$

$50,2 \quad 26,161$

$50,3 \quad 26,86$

$50,4 \quad 3 I, 86$, I6I

$50,5 \quad 3 \mathrm{I}$

$50,6 \quad 86$, I $6 I$

$50,7 \quad 26$

$50,8 \quad 16 \mathrm{I}$

50,1026

$50,11 \quad 87$

5 I IO, I3, 47

5I,I 26, 94

$51,2 \quad 26$

$51,4 \quad 93,143$

$51,5 \quad 26,82,168$

$51,6 \quad 124$

$51,7 \quad 86,109$

$51,8 \quad 140$

51,10 I2I

$52, \mathrm{I} \quad 80$

$53, I \quad 26,13 I$

$53,2 \quad 26$

53.4 I2 I, I3I

$53,53 \mathrm{I}$

53.6 I2I, 138

54, I-3 I68, I 73

$54, \mathrm{I}-2 \quad 178$

54 , I 26, Ior, I65

$54,2 \quad 62,165$

54,3-5 I 4I, I 73

$54,3 \quad 109$

$54,5 \quad 109$

$55.2 \quad 26$

$55,426,59,67,78,142$

$55,5 \quad 67$

$55.794,100$

$55,8 \quad 94$

55, 10 $66,69,86$

55 , I I 62

$56,2 \quad 97$

$56,3 \quad 26,93,124^{*}$ 


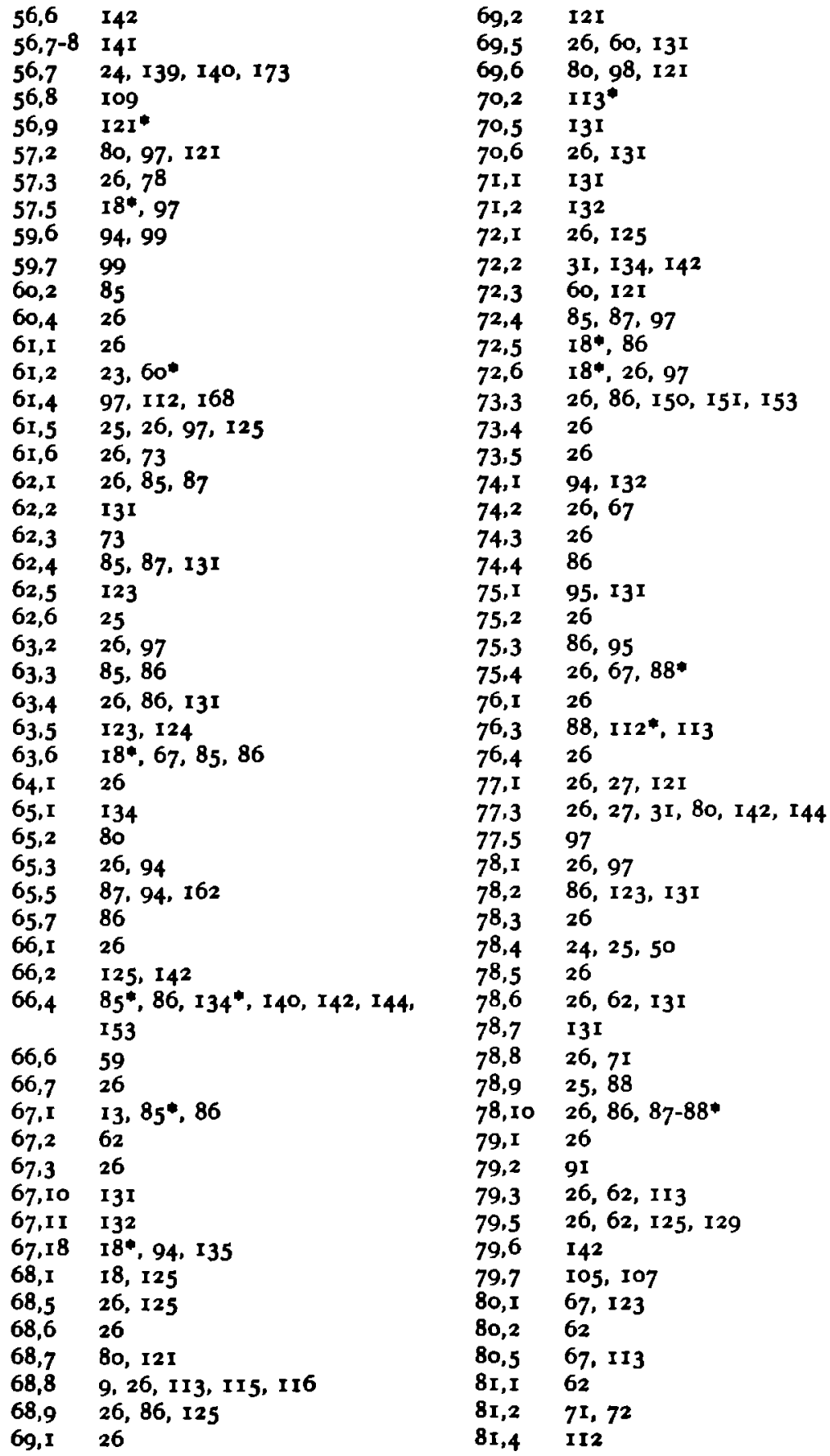




\begin{tabular}{|c|c|c|c|}
\hline 81,7 & I25. 142 & 92,1 & 26 \\
\hline 81,8 & 26,86 & 92,2 & I3I \\
\hline 82,1 & 23 & 92,3 & I3I \\
\hline 82,2 & 26,121 & 92,4 & I3I \\
\hline 82,3 & 26 & 92,6 & $60^{*}$ \\
\hline 82,4 & 26,142 & 93.3 & 125 \\
\hline 82,6 & & 93,4 & 86 \\
\hline $82,7-83$ & $1 \quad 154$ & 93,6 & I3I \\
\hline 82,7 & 62 & 94,2 & 123 \\
\hline $83, \mathrm{I}$ & 26, I23, I 55 & 94,4 & 76,129 \\
\hline 83,2 & 26,66 & 94,5 & $26,7^{6}$ \\
\hline 83,3 & $86,113,125,142$ & $95, I-2$ & 142 \\
\hline 83,4 & $26,94,99$ & $95, I$ & I2I \\
\hline 83,5 & 125 & 95,2 & 93,121 \\
\hline 83,6 & $125, \mathbf{I}^{1} 1$ & 95,3 & $26,76,86$ \\
\hline 83,8 & 125 & 95.4 & $26,76,87$ \\
\hline 84,2 & 94,99 & 96,2 & $94, I_{3}, I_{3}$ I \\
\hline 84,3 & 62 & 96,3 & 125 \\
\hline 84,4 & $26,80,99$ & 96,6 & 62 \\
\hline 84,5 & 80 & 97,1 & I3I \\
\hline 84,6 & 97,121 & 97,2 & 94 \\
\hline 85,1 & 125 & 98,1 & 26,131 \\
\hline 85,2 & I25 & 98,2 & 26,94 \\
\hline 85,3 & 125 & 99,1 & 26,131 \\
\hline 85.4 & 125 & 100,2 & 83 \\
\hline 85,5 & 125,132 & 100,5 & 26,142 \\
\hline 86,5 & 26,123 & IOI,I & 26,129 \\
\hline 86,6 & 26,67 & $103, I$ & 25,88 \\
\hline 86,7 & 67,123 & 103.2 & I 31 \\
\hline 87,2 & I 25 & 103,3 & 25,94 \\
\hline 87.3 & 26,100 & 103,6 & $63^{*}, 65$ \\
\hline 87.4 & 26,59 & 103.7 & 26,86 \\
\hline 87,5 & 66,69 & 104,2 & 86,88 \\
\hline 88,1 & 67,97 & 105,1 & 86 \\
\hline 88,2 & 24.80 & 105,2 & 142 \\
\hline 88,4 & 66,69 & 105,3 & 26,131 \\
\hline 88,5 & $62,66,69$, r $31^{1}$ & 105,4 & I 21,143 \\
\hline 88.7 & I 43 & 105,6 & $60^{*}, 62$ \\
\hline $\begin{array}{l}89,2 \\
89,4\end{array}$ & $93,125,13 \mathrm{I}$ & $\begin{array}{l}\text { I06, I } \\
106,2\end{array}$ & \\
\hline $\begin{array}{l}89,4 \\
89,5\end{array}$ & $\begin{array}{l}63^{4}, 65,67,142 \\
26\end{array}$ & $\begin{array}{l}106,2 \\
106,3\end{array}$ & $\begin{array}{l}05,00 \\
93,135\end{array}$ \\
\hline 89,6 & 86 & 106,4 & 26 \\
\hline 90,2 & 26,73 & 107,2 & I 25 \\
\hline 90,3 & I31 & 108,4 & $76^{*}$ \\
\hline 90,4 & 26,86 & 108,5 & ro, 179 \\
\hline 90,5 & $26,76,100^{*}$ & 109,3 & 59,121 \\
\hline 90,6 & 86 & $109,4-5$ & 27 \\
\hline 90,7 & 67,76 & 109,4 & $67, \mathrm{I} 2 \mathrm{I}$ \\
\hline 90,9 & 31,86 & $110, \mathrm{I}$ & $85 *, 97$ \\
\hline 91,3 & 109 & IIo,3 & I $21, I 25, x_{42}, I_{44}$ \\
\hline $9 \pi, 4$ & 134 & II I, I-2 & 26 \\
\hline 91,5 & I9, 102 & $\operatorname{II2,2}$ & 103 \\
\hline 91,6 & 142 & II 3,2 & I3I \\
\hline
\end{tabular}


II. ANCIEN TESTAMENT

Gen.

$9,20 \quad 68$

I8, $22 \quad \mathrm{I} 74$

$19,27 \quad 174$

$34,3 \quad 137$

$41,54 \quad 68$

Ex.

$3.7 \quad 151$

$12,5 \quad 146$

$24.7 \quad 102$

34, 2-3 I74

Lev.

I,9 IIO

18,5 II9

Num.

25, I 68

Deut.

I,5 68

$2,24 \quad 68$

$2,25 \quad 68$

$2,31 \quad 68$

$5,31 \quad 174$

I0, $10 \quad 174$

$25,2 \quad 146$

3I, I I 102

Jos.

8,35102
Jud.

$10,18 \quad 68$

$16,19 \quad 68$

I Sam.

$26,16 \quad 148$

2 Sam.

$3,34 \quad 147$

$7,10 \quad 147$

I Regn.

I9, II $\quad 174$

2 Regn.

I5,37 68

22,10 IO2

I Par.

I6, $13 \quad 150$

2 Par.

34,18 IO2

Neh.

9,28 108

Ps.

23,3 110

$24,3 \quad 174$

78,54 II I

$89,23 \quad 147$
9I, 3 I73

$106,23 \quad \mathrm{I} 74$

Prov.

$31.5 \quad 146$

Os.

I0,9 I46, I47

Jon.

$3,4 \quad 68$

Is.

$57.4 \quad 146$

63,14 I I0

$65,23 \quad 147$

Jer.

36,6 I02

$36,10 \quad 102$

36,21 IO2

Lam.

I, $15 \quad 150$

Ez.

20,13 II9

$37,22 \quad 74$

Dan.

8,17 I75

$9,3 \quad \mathrm{I} 75$

\section{SEPTANTE}

Gen.

$2,3 \quad 68$

$2,7 \quad 74$

2,17 I 53

$2,24 \quad 75,76$

$3.4 \quad 153$

$3,5 \mathrm{I} 34$

6,168

6,19 II5

$6,20 \quad 115$

$7,2 \quad 115$

$7,3 \quad 115$

$7,9 \quad 115$

7,15 II5

8,21 77

$9,15 \quad 75$
$9,20 \quad 68$

$14,2 \quad 98$

$15,12 \quad 155$

I8, $18 \quad 74,75$

$18,22 \quad 174$

$18,27 \quad 68$

$19,17 \quad 106$

$19,26 \quad 106$

19,27 I73, I74, I75

21,23 IOI

$22,3 \quad 173$

24, I 2 IOI

$26,20 \quad 98$

26,29 IOI, 148

31,2496

$31,29 \quad 96$
$31,30 \quad 152$

$32,25 \quad 98$

$32,26 \quad 98$

$32,29 \quad 98$

$35,13 \quad 96$

$35,14 \quad 96$

35.1596

35. $13-1597$

$37.15 \quad 78$

$4 \mathrm{I} .54 \quad 68$

$48,19 \quad 75$

$48,22 \quad 90,91$

$49.17 \quad 106$

Ex.

IO, I 79 


$\begin{array}{ll}12,5 & 146 \\ 17,12 & 131 \\ 22,21 & 133 \\ 24,4 & 173 \\ 25.19 & 131 \\ 34,2 & 173 \\ 34,2-3 & 174 \\ 34,4 & 173 \\ 34,28 & 174 \\ 38,7 & 131 \\ & \end{array}$

Lev.

I,9 I IO

$4,14 \quad 87$

$4,23 \quad 87$

$4,28 \quad 87$

$5,7 \quad \mathrm{I3}$

$6,13 \quad 132$

$12,8 \quad I 3 I$

I4,22 I3I

14,3I I3I

$14,35 \quad 135$

$15,2 \quad 115$

15,15 I3I

I5.30 I3I

16,8 I3I

$16,29 \quad 14^{\circ}$

$16,31 \quad 14^{\circ}$

$17,3 \quad 115$

$17,8 \quad 115$

I 7,8-9 120

$17,10 \quad 115$

$17,13 \quad 115$

$18,5 \quad 87,119$

I8,6 II5

20,9 II5

22,18 II5

$23,27 \quad 140,14 I$

$23.29 \quad 14 \mathrm{I}$

$23,32 \quad 140$

Num.

$5,12 \quad 115$

$5,27 \quad 75$

$6,9 \quad 153$

6,II I3I

$7,11 \quad 115$

$8,12 \quad 131$

9,10 115

9, I5 I35, 136

$I I, 6 \quad I 39$

I I, $17 \quad 96,97$

II,25 97

$\begin{array}{ll}14,3 & 75 \\ 14,40 & 173 \\ 17,17 & 115 \\ 21,29 & 181 \\ 25,1 & 68 \\ 26,65 & 153 \\ 28,13 & 115 \\ 28,21 & 115 \\ 28,29 & 115 \\ 29,4 & 115 \\ 29,7 & 140 \\ 29,10 & 115 \\ 29,15 & 115 \\ 30,3 & 115 \\ 30,14 & 140 \\ 31,4 & 115 \\ 31,6 & 115 \\ 31,8 & 84 \\ 32,13 & 108 \\ 35,18 & 153\end{array}$

Deut.

I,5 68

$2,24 \quad 68$

$2,25 \quad 68$

$2,31 \quad 68$

$4,25 \quad 108$

$4,29 \quad I_{44}$

$5,31 \quad 174$

$6,5 \quad 144$

$9,18 \quad 108$

10,10 $\quad 174$

$10,12 \quad 144$

II, I3 144

$13,4 \quad 144$

$13.7 \quad 134$

$17.2 \quad 108$

21,15 I3I

$22,10 \quad 83$

$25,2 \quad 146$

$25,3 \quad 90,91$

25, II 98

$26,16 \quad 144$

30,2 144

$30,5 \quad 9 I$

30,6 144

30, $10 \quad 144$

$31,29 \quad 87,108$

$32,43 \quad 127$

33, 2 I IOI

Jos.

8,2 106
$8,18 \quad 164$

$17,13 \quad 151$

Jud.

$2,19 \quad 91$

$3.7 \quad 108$

$5,20 \quad 98$

$6,17 \quad 96$

8,35 IOI

$10,18 \quad 68,75$

$11,5 \quad 98$

$11,6 \quad 75$

II, $8 \quad 75$

II,9 75

II, $25 \quad 98$

II, 27 IOI

$13,22 \quad 153$

I5,3 IOI

16,1968

$16,29 \quad 131$

$18,2 \quad 146$

$20,31 \quad 131$

21,10 146

Jud. A

I,24 IOI

2,I I 108

3.12 108

4, I $\quad 108$

$6,1 \quad 108$

8,35 IOI

10,6 108

I 1,498

I $1,20 \quad 98$

13, I 108

I6,30 9I

$20,14 \quad 98$

$20,18 \quad 98$

$20,20 \quad 98$

$20,23 \quad 98$

$20,28 \quad 98$

$20,39 \quad 58$

$21,5 \quad 153$

$21,9 \quad 155$

Jud. B

2, I I 108

3,991

$3,12 \quad 108$

$3,25 \quad 155$

4,I 108

6,I 108

9, 16 I0I 


\begin{tabular}{|c|c|c|c|c|c|}
\hline $\begin{array}{c}9,19 \\
10,6\end{array}$ & $\begin{array}{l}\text { I0I } \\
108\end{array}$ & $\begin{array}{l}I, 23 \\
1,26\end{array}$ & $\begin{array}{l}91 \\
91\end{array}$ & $\begin{array}{l}2,4 \\
2,8\end{array}$ & $\begin{array}{l}143 \\
87\end{array}$ \\
\hline I 1,25 & 91 & 2,6 & IOI & 2,22 & 91 \\
\hline 13,1 & 108 & 3,8 & IOI & 2,32 & $9 I$ \\
\hline I 5,2 & $9 I$ & 3,34 & 146,147 & $2,35^{b}$ & 91 \\
\hline I 8,26 & 91 & 3,39 & 148 & $2,35^{n}$ & 87 \\
\hline & & 5,2 & 75 & 2,37 & 153 \\
\hline Ruth & & 6,21 & $9 I$ & 2,42 & I 53 \\
\hline$I, 8$ & IOI & 7,8 & 75 & $2,4^{6 c}$ & 68 \\
\hline I, I 3 & 9I & 7,10 & 146,147 & 3,6 & IOI \\
\hline 3,8 & I55 & 7,20 & 77 & 3,25 & 132 \\
\hline 3,10 & $9 I$ & 7,29 & 68 & 5,10 & 91 \\
\hline 3,12 & 9I & $9, \mathrm{I}$ & IOI & $5, \mathrm{II}$ & 91 \\
\hline 4,15 & 91 & 9,3 & IOI & 8,23 & 143 \\
\hline & & 9.7 & IOI & 8,39 & I59 \\
\hline I Regn. & & 10,2 & IOI & $8,4^{8}$ & $I_{43}$ \\
\hline 1,5 & 91 & IO, I I & $9 I$ & 9.7 & 75 \\
\hline 1,8 & $9 I$ & $10, I_{7}$ & 98 & 10,23 & 91 \\
\hline 2,29 & $9 I$ & I I, I7 & 98 & 10,25 & I I 5 \\
\hline 4.9 & 75 & 12,1 & I3I & I 1,8 & 108 \\
\hline 6,17 & I30 & I2,5 & 146 & I 2,24 & 98 \\
\hline 7.3 & I43 & 12,9 & 108 & $12,24^{r}$ & $9 I$ \\
\hline 7,16 & I I 5 & 12,14 & 153 & 13,32 & 74 \\
\hline 9,2 & 91 & I3. I4 & 91 & $I_{4,22}$ & 87,108 \\
\hline 9,6 & 74 & 13,15 & 91 & 15,26 & 108 \\
\hline 10,3 & I3I & I 3, I6 & 9I, IOI & I 5,34 & 108 \\
\hline 10,23 & 91 & I 3,22 & 96 & 16,7 & 83 \\
\hline 10,26 & $I_{4} 6$ & 13.28 & 146 & 16,19 & 108 \\
\hline 12,20 & $I_{43}, I_{44}$ & 14,14 & 153 & 16,25 & 91,108 \\
\hline 12,24 & $I_{43}$ & 14,17 & 75 & $16,3^{\circ}$ & 91,108 \\
\hline 13,17 & I 3I & I 5,20 & IOI & 16,33 & $9 \mathrm{I}$ \\
\hline 13,18 & I 31 & 15.33 & 75 & I $8, I_{3}$ & I I 5 \\
\hline 14,39 & I53 & 17,10 & 146 & 19.4 & 91 \\
\hline $14,4^{\circ}$ & 75 & 17,14 & $9 I$ & 20,2 & 91 \\
\hline$I_{4,44}$ & 153 & 18,8 & $9 I$ & 20,20 & 108 \\
\hline 14,52 & 146 & 19,8 & $9 I$ & 20,25 & 108 \\
\hline I 5,6 & IOI & 19,44 & 91 & $21, I 5$ & 146 \\
\hline I5, I9 & 108 & 20,6 & $9 \mathrm{I}$ & 21,23 & $9 I$ \\
\hline 15,22 & 91 & 21,6 & 150 & 21,25 & $9 I$ \\
\hline I 5,28 & 91 & 21, I 5 & 98 & 22,45 & 102 \\
\hline 17,32 & 98 & 21,18 & 98 & 22,53 & 108 \\
\hline 17.33 & 98 & 21,19 & 98 & & \\
\hline 18,17 & 146 & 22,18 & 91 & 4 Regn. & \\
\hline 20,8 & IOI & & & $I, 4$ & I 53 \\
\hline $20, I_{4}$ & IOI, I53 & 3 Regn. & & 1,6 & I53 \\
\hline 20,31 & 146 & 1,14 & 96 & 1,16 & 153 \\
\hline 22,16 & 153 & 1,22 & 96 & 2,16 & 146 \\
\hline 24,18 & 91 & 1,35 & 75 & 3,2 & 108 \\
\hline 26,16 & $I_{4} 6, I_{4} 8$ & 1,37 & $9 I$ & 3,26 & 9r \\
\hline 28,1 & 98 & $I, 47$ & 9I & 5,12 & 91 \\
\hline & & $I, 51$ & 87 & 6,16 & 91 \\
\hline 2 Regn. & & 1,52 & 146 & 6,33 & 96 \\
\hline 1,22 & 106 & 2,2 & 75 & 7.5 & I55 \\
\hline
\end{tabular}




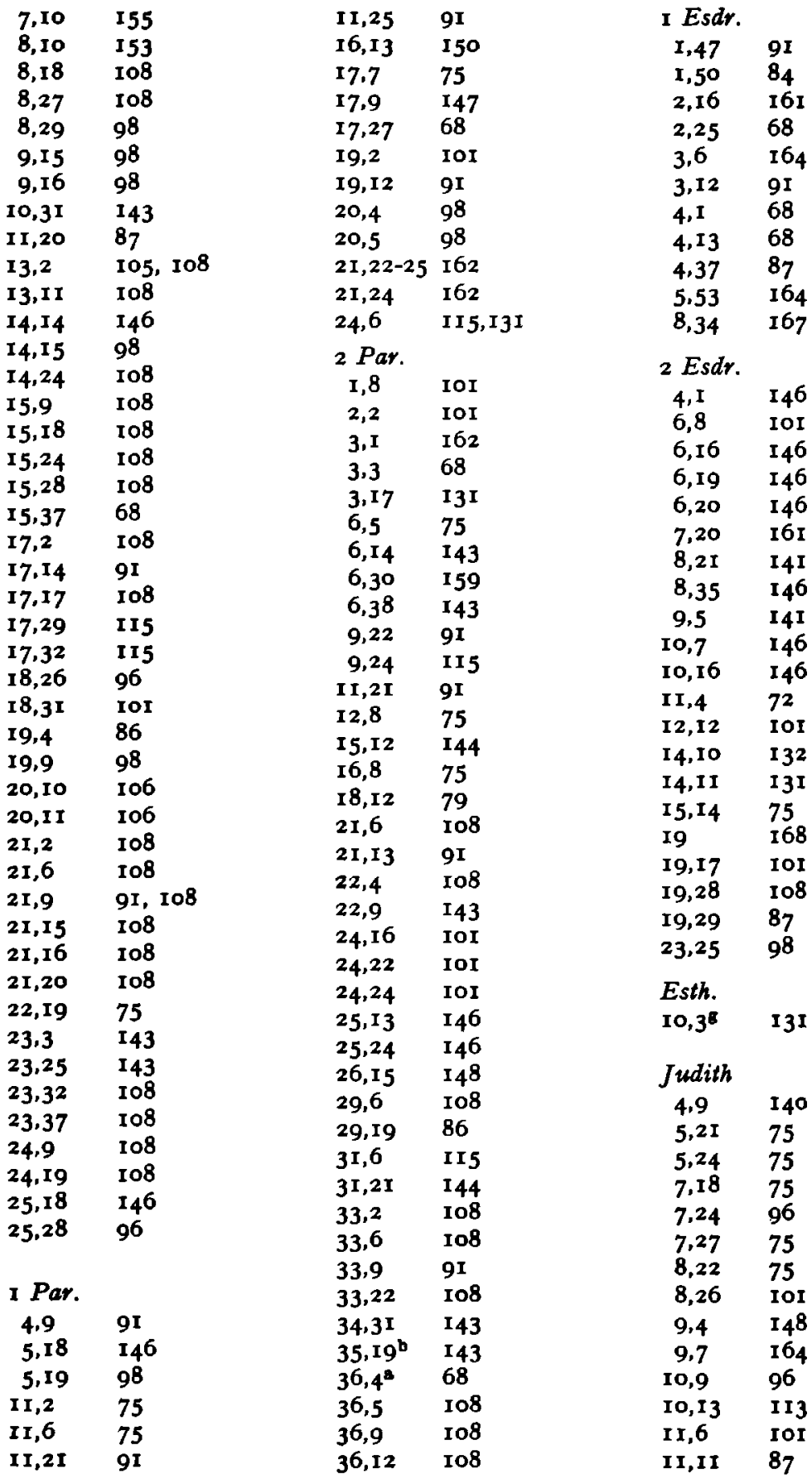




\begin{tabular}{|c|c|c|c|c|c|}
\hline $\begin{array}{l}\text { I I, I6 } \\
13,16\end{array}$ & $\begin{array}{l}\text { IOI } \\
\text { IOI }\end{array}$ & 9,4 & $\begin{array}{l}106 \\
86\end{array}$ & $\begin{array}{l}\text { I06,30 } \\
108,8\end{array}$ & $\begin{array}{l}\text { I75 } \\
64\end{array}$ \\
\hline $\begin{array}{l}\text { I3, I6 } \\
\text { I5,8 }\end{array}$ & $\begin{array}{l}\text { IOI } \\
96\end{array}$ & $\begin{array}{l}14,3 \\
17,18\end{array}$ & $\begin{array}{l}80 \\
91\end{array}$ & $\begin{array}{l}100,0 \\
108,21\end{array}$ & $\begin{array}{l}4 \\
\text { IOI }\end{array}$ \\
\hline \multirow[t]{2}{*}{15,10} & IOI & I8, I I & 91 & I IO, I & $I_{43}$ \\
\hline & & 18,15 & 75 & I 13,3 & 106 \\
\hline Tob. & & 23,3 & IIO & 113,5 & I06 \\
\hline 3,6 & IOI & 27,3 & 96 & I 18,2 & I 43 \\
\hline 5,17 & 128 & 34,4 & 106 & I I 8, 10 & 143 \\
\hline 8,5 & 68 & 34,13 & $I_{40}$ & I 18,34 & $I_{43}$ \\
\hline 8,16 & IOI & 34,14 & I 4 I & I I $8,4^{\circ}$ & 87 \\
\hline 12,6 & IOI & 36,4 & I 39 & I $18,5^{8}$ & 143 \\
\hline 13,6 & I43 & 36,16 & $9 I$ & 118,65 & IOI \\
\hline 13,7 & IOI & 36,26 & 75 & 1 I 8,69 & 143 \\
\hline \multicolumn{2}{|c|}{ Tob. BA } & 37,20 & $9 I$ & 118,72 & $9 I$ \\
\hline 8,15 & I5O & 38,2 & 86 & I I 8,93 & 87 \\
\hline 12,6 & IOI & 38,4 & 86 & I 18,98 & 91 \\
\hline & & 39,6 & $9 \mathbf{I}$ & I I 8,99 & $9 I$ \\
\hline \multicolumn{2}{|l|}{ Tob. S } & 39,13 & $9 I$ & I I 8, 100 & $9 \mathrm{I}$ \\
\hline 2,2 & 143 & 39,15 & 106 & 118,103 & $9 I$ \\
\hline $3, I$ & 69 & 43, I I & I06 & I I 8, I 24 & IOI \\
\hline 8,5 & 69 & 43.19 & 106 & I I 8, I 27 & $9 I$ \\
\hline 10,3 & 69 & 49,17 & 106 & I I 8, I 45 & 143 \\
\hline \multicolumn{2}{|l|}{ I Mace. } & 50,6 & 108 & I $21,6-7$ & IO2 \\
\hline$I, 4 I$ & 75,76 & 50,9 & 91 & I 22, I & 128 \\
\hline 2,47 & $\begin{array}{l}75,70 \\
146\end{array}$ & 51,5 & 91 & I 25, 2 & IOI \\
\hline 3.30 & 91 & 54,22 & 9I & 125.3 & IOI \\
\hline 6,49 & IOI, IO2 & 55,10 & 106 & I 28,5 & 106 \\
\hline 6,58 & 101, 102 & 62,2 & 173 & I 37, I & I43 \\
\hline 7,15 & 96 & 62,4 & 91 & I 38,18 & 91 \\
\hline 7.23 & 91 & 62,7 & 173 & 141,7 & $9 I$ \\
\hline 9,47 & 106 & 65,5 & 90,91 & Or. Man. & \\
\hline 9,66 & 68 & $\begin{array}{l}08,5 \\
68,11\end{array}$ & $\begin{array}{l}91 \\
141\end{array}$ & 9 & $9 I$ \\
\hline 10,4 & 102 & 68,32 & $9 I$ & & \\
\hline I0, 27 & IOI & 69,3 & 106 & Prov. & \\
\hline I 2,24 & 9I & 76,8 & 77 & 3.5 & I 43 \\
\hline I 3,42 & 68 & 77,54 & I I I & 6,30 & I 39 \\
\hline \multirow[t]{2}{*}{ I $5.4^{\circ}$} & 68 & 77,66 & 106 & 8,10 & 91 \\
\hline & & 79,1 I & $I_{3}$ & I6, I6 & $9 I$ \\
\hline \multicolumn{2}{|l|}{2 Mace. } & $8_{3}, 11$ & 91 & I9,IO & 68 \\
\hline 3,26 & $16 I$ & 85,12 & $I_{43}$ & 19,12 & I34 \\
\hline \multirow[t]{2}{*}{8,35} & 91 & 85,17 & IOI & $22, I_{4}$ & 148 \\
\hline & & 86,2 & $9 I$ & 24,12 & I 59 \\
\hline \multicolumn{2}{|l|}{4 Mace. } & 88,4 & I 50 & $24,22^{8}$ & 146 \\
\hline 5,15 & 68 & 88,23 & 146 & 25.9 & 106 \\
\hline 7,18 & I 44 & 104,6 & I5o & $3 I, 5$ & 146 \\
\hline 9.3 & 91 & 104,24 & 91 & & \\
\hline \multirow[t]{2}{*}{ I 3, I 3} & 144 & 104,43 & I 50 & Eccl. & \\
\hline & & 105.5 & I 50 & 2,7 & $9 I$ \\
\hline Ps. & & 105,23 & 150 & 4,2 & 9 I \\
\hline 2,4 & 128 & 105,39 & 86 & 4,3 & 91 \\
\hline 5,4 & 173 & 106 & I68 & 4,6 & $9 I$ \\
\hline 9,2 & 143 & 106,2 & 148 & 4,9 & $9 I$ \\
\hline
\end{tabular}




$\begin{array}{ll}4,13 & 91 \\ 6,3 & 91 \\ 6,9 & 91 \\ 6,10 & 91 \\ 7,1 & 91 \\ 7,3 & 91 \\ 7,5 & 91 \\ 7,8 & 91 \\ 7,10 & 91 \\ 7,19 & 91 \\ 7,24 & 91 \\ 7,26 & 91 \\ 9,4 & 91 \\ 9,16 & 91 \\ 9,17 & 91 \\ 9,18 & 91\end{array}$

Cant.

$\begin{array}{ll}I, 2 & 9 I \\ I, 4 & 9 I \\ 2,5 & I_{4} 8 \\ 2, I 1 & 88 \\ 5,8 & 1_{4} 8 \\ 7, I 2 & 166\end{array}$

$J o b$

$5,14 \quad 134$

$6,13 \quad 178$

$8,5 \quad I 73$

I0, I0 134

I3,28 I34

$15,16 \quad 134$

$24,20 \quad 134$

$27,14 \quad 75$

$27,16 \quad 134$

28,5 I35

$28,18 \quad 91$

$29.14 \quad 134$

$40,15 \quad I 34$

Sap.

I,IO III

$3,9 \quad I 50$

$4,15 \quad 150$

$4,19 \quad 75$

$7,10 \quad 91$

$8,21 \quad I_{44}$

I5, I 49 I

I6,I9 91

Sir.

prol. 2288

$2,17 \quad 140$

$7,17 \quad 140$

$\begin{array}{llll}7,27 & 143 & 17,4 & 107 \\ 8,1 & 98 & 17,32 & 148 \\ 8,2 & 98 & 17,43 & 91 \\ 8,3 & 98 & 18, i n s c r .148 \\ 8,13 & 91 & 18,5 & 148 \\ 8,16 & 98 & 18,7 & 148 \\ 10,6 & 112 & & \\ 14,17 & 153 & 0 s . & \\ 18,21 & 41 & 1,2 & 146 \\ 18,30 & 105 & 2,6 & 146 \\ 19,16 & 86 & 5,11 & 68 \\ 22,11 & 91 & 5,15 & 173 \\ 22,14 & 91 & 6,11 & 68 \\ 24,20 & 91 & 7,5 & 68 \\ 26,5 & 91 & 10,9 & 146,147 \\ 29,13 & 91 & 12,5 & 98 \\ 30,17 & 91 & & \\ 33,15 & 115 & A m . & \\ 34,23 & 131 & 8,2 & 77\end{array}$

Mich.

I,2 75

$3.4 \quad 86$

$5,1 \quad 75$

$6,13 \quad 68$

$7,10 \quad 75$

$7.13 \quad 75$

$39,35 \quad I 43$

$40,19 \quad 91$

$40,24 \quad 9 I$

$40,25 \quad 91$

$40,26 \quad 9 I$

$40,27 \quad 9 I$

$46, I \quad I 50$

$46, I_{3} \quad I_{4}^{8}$

$47,8 \quad 143$

$47,22 \quad 150$

50,22 IOI

$51,22 \quad 86$

Ps. Sal.

2,II 107

$2,12 \quad 107$

$2,20 \quad 110$

2,26 9I

$3,8 \quad 141$

4,291

$4,19 \quad 107$

$6,6 \quad 139$

7,9 I I I

$8,12 \quad 86$

8,1391

$12,3 \quad 86$

Joel

I, $8 \quad 91$

$2,3 \quad 105$

$2,12 \quad 144$

2,26 IOI

$3.1 \quad I 52$

$4,19 \quad 75$

Abd.

I, $18 \quad 75$

Jon.

$3,4 \quad 68$

Nah.

2, I 77

2,10 91

$3,4 \quad B_{4}$

3,1491

$3,16 \quad 91$

Hab.

I,8 9I

$2,1 \quad 174$

Soph.

$3,14 \quad 144$ 


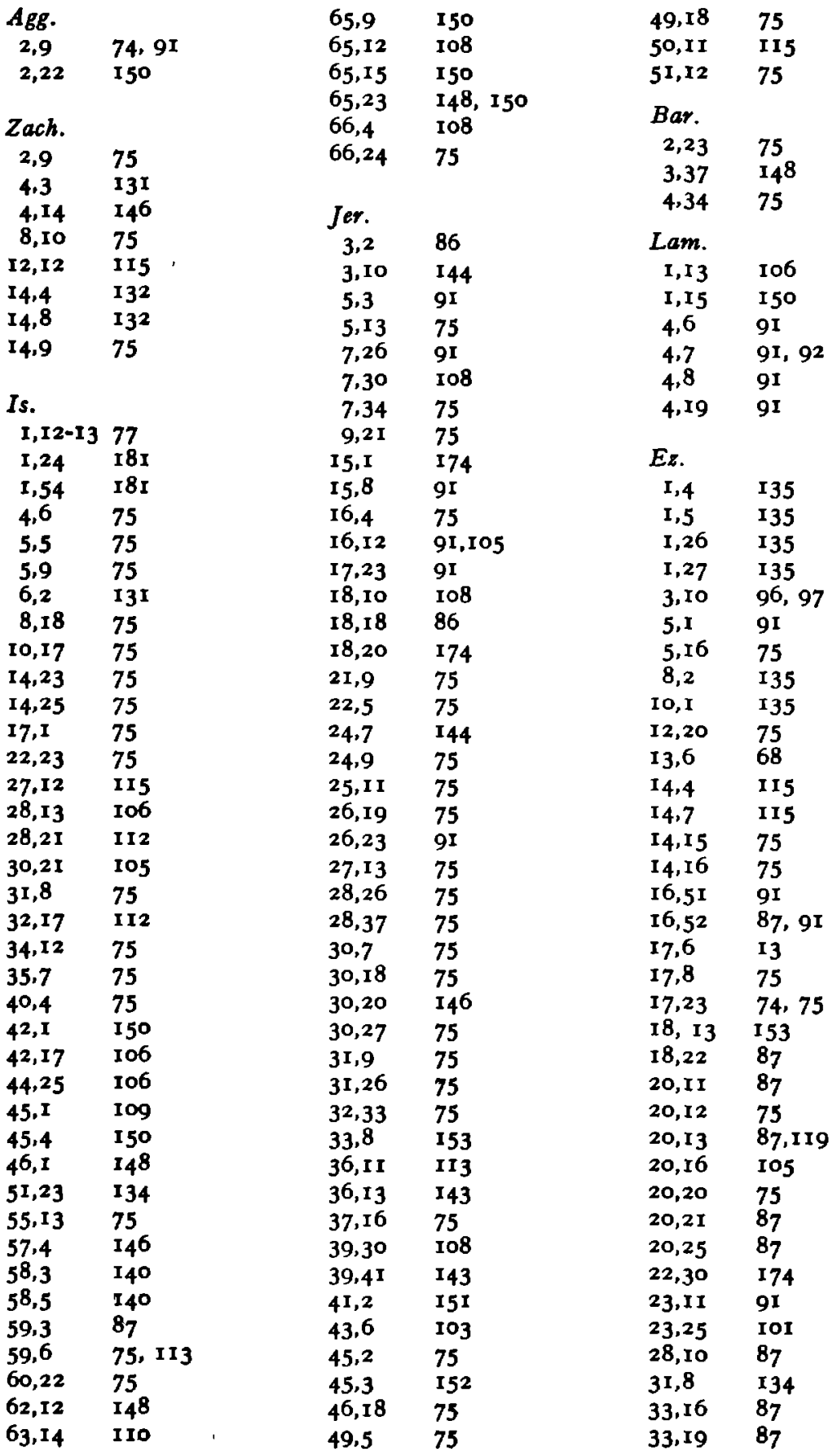




\begin{tabular}{|c|c|c|c|c|c|}
\hline 33,31 & 105 & 5,29 & 164 & $4,37^{b}$ & IOI \\
\hline 34,22 & 75 & 7,6 & 135 & $4,37^{\mathrm{c}}$ & IOI \\
\hline 37,19 & $75,7^{6}$ & $7, \mathbf{1} 3$ & 135 & 5,30 & $5^{8}$ \\
\hline 37,22 & 75 & 8,15 & 135 & 6,4 & $9 I$ \\
\hline 40,2 & I 35 & 8,18 & 96,97 & 7,16 & 80 \\
\hline 40,44 & I3I & 9,22 & 96,97 & 8,5 & I54 \\
\hline 44,5 & 96,97 & $10,2-4$ & 174 & IO, I I & 96,97 \\
\hline 47.12 & 75 & 10,15 & 96,97 & 11,32 & 86,87 \\
\hline $4^{8,31}$ & I 3 I & 10,16 & I 35 & Dan.Th & \\
\hline 48,32 & I3I & 10,17 & 96 & I, I 3 & IOI \\
\hline $4^{8,33}$ & 131 & 10,18 & I35 & I, I 5 & $9 I$ \\
\hline 48,34 & I3I & I0, I9 & 96,97 & 1,19 & 96 \\
\hline Suz. Th & & $\begin{array}{l}\text { IO, } 20 \\
\text { I I, I I }\end{array}$ & $\begin{array}{l}98 \\
98\end{array}$ & $\begin{array}{l}2,5 \\
2,27\end{array}$ & $\begin{array}{l}75 \\
109\end{array}$ \\
\hline 39 & $9 I$ & 12,5 & I3I & 3.96 & 75 \\
\hline Dan. & & $\operatorname{Dan} .1$. & & 4,2 & IOI \\
\hline I,I9 & 134 & 1,20 & 90,91 & 7,21 & 98 \\
\hline 3,35 & 148 & 3,22 & 91 & I I, I 3 & 91 \\
\hline 3,42 & IOI & 3,92 & I 55 & I I, I 5 & 150 \\
\hline 5.7 & 164 & 4,32 & 96 & $\mathrm{Bel}$ Th & \\
\hline 5,16 & 164 & $4,33^{b}$ & $5^{8}$ & 2 & 91 \\
\hline
\end{tabular}

IV. AUTRES VERSIONS GRECQUES DE L'ANCIEN TESTAMENT 164

V. NOUVEAU TESTAMENT

\begin{tabular}{|c|c|c|c|c|c|}
\hline$M t$ & & 17,4 & I3I & $I, 44$ & 27 \\
\hline $2, I_{3}$ & I 55 & I9.5 & 75 & 4,5 & 26 \\
\hline 2,19 & I 55 & 19,9 & I2I & 4,12 & I 2 I \\
\hline 3,15 & 26 & 19,21 & 27 & 4,16 & 26 \\
\hline $4, I-2$ & 174 & 19.22 & 26 & 4.20 & I 2 I \\
\hline 5,16 & 120 & 20,21 & I3I & 5,9 & I 76 \\
\hline $5,23-24$ & 120 & 21,28 & 27 & 5,34 & 26 \\
\hline 5,24 & 27 & 21,36 & 77 & 6,7 & I 15 \\
\hline 5,25 & 26 & 22,30 & I34 & $6,3^{8}$ & 27 \\
\hline $5,4^{1}$ & 51 & 22,37 & 143 & 6,39 & II5 \\
\hline 6,25 & 139 & 24,31 & 150 & $6,4^{\circ}$ & I 5 \\
\hline $7, I_{3}-I_{4}$ & $3 I$ & 24,40 & 131 & 6,50 & 96,97 \\
\hline 7,24 & I 21 & 24,41 & I3I & 7,10 & 152 \\
\hline 7,26 & I 2 I & 25.34 & 149 & 7,18 & 133 \\
\hline 8,4 & 27 & 26,22 & 69 & 8,32 & 69 \\
\hline $8, I_{3}$ & 27 & 26,24 & 182 & 9,3 & 26 \\
\hline I0,37 & 91 & $26,5^{2}$ & 87 & 9.5 & I3I \\
\hline I 2,4 & 26 & 26,53 & 176 & 10,2 & I2I \\
\hline 12,25 & 133 & 26,74 & 69 & Io, 4 & 120 \\
\hline I3, 5 & 26 & 27,33 & 26 & 10,8 & 75 \\
\hline $13, I_{4}$ & 152 & $27,3^{8}$ & I 3I & ro, Io & 26 \\
\hline 13,19 & I 21 & 27,65 & 27 & IO, I I & I2I \\
\hline 13,20 & 26 & 28,10 & 27 & 10,21 & 27 \\
\hline 13,23 & 121 & & & 10,22 & 26 \\
\hline 13,30 & I 5 & $M c$. & & 10,37 & I JI \\
\hline 13,46 & $5^{8}$ & 1,9 & 26 & 10,41 & 69 \\
\hline I 5,4 & 152 & 1,39 & 27 & I I, I 5 & 69 \\
\hline
\end{tabular}




\begin{tabular}{|c|c|c|c|c|c|}
\hline $\begin{array}{l}12,4 \\
12,19\end{array}$ & $\begin{array}{l}77 \\
\text { 121 }\end{array}$ & $\begin{array}{l}16,11 \\
16,18\end{array}$ & $\begin{array}{l}\text { I I I } \\
\text { I } 2 \text { I }\end{array}$ & $\begin{array}{l}5,42 \\
6,11\end{array}$ & $\begin{array}{l}133 \\
113\end{array}$ \\
\hline$I 2,30$ & I 44 & 17,4 & I 21 & 7.4 & 26 \\
\hline I2,33 & I 44 & 17,31 & 106 & 7,12 & 26 \\
\hline 13,3 & 26 & I 8,7 & 150 & 7,60 & 160 \\
\hline г 3,9 & 26 & 19,17 & 26 & 8,23 & 26,75 \\
\hline 13,16 & 26,106 & 20,11 & 77 & 8,37 & I 44 \\
\hline I 3,27 & 150 & 20,12 & 77 & 8,40 & 26 \\
\hline$I_{4}, 19$ & 69 & 21,37 & 26 & 9,9 & 26 \\
\hline$I_{4}, 2 \mathrm{I}$ & 182 & 22,15 & 152 & 9,18 & 135 \\
\hline$I_{4,25}$ & 77 & 22,4 I & 160 & 9,21 & 26 \\
\hline$I_{4,33}$ & 69 & & & $9,4^{\circ}$ & 160 \\
\hline$I_{4,7}$ & 69 & Joh. & & 10,30 & I 54 \\
\hline I5, I 5 & 162 & 1,18 & 26 & I I, 5 & 136 \\
\hline 15,19 & 160 & 1,34 & I5O & I 2,3 & 77 \\
\hline 15,27 & I3I & 3,16 & I33, I34 & I 3, I I & 26 \\
\hline I 5,29 & I 80 & 3,27 & 26 & 14,25 & 26 \\
\hline \multirow{2}{*}{16.7} & 27 & 3,29 & 152 & I 4,27 & IOI \\
\hline & & 4,16 & 27 & I 5,4 & IOI \\
\hline$L c$. & & 4,27 & 96 & I8,7 & 26 \\
\hline 1,20 & 26 & 5,24 & 121 & 19,22 & 26 \\
\hline 1,72 & IOI & 6,39 & I 33 & 19,36 & 26 \\
\hline 3,5 & 75 & 6,66 & 106 & 20,36 & I6o \\
\hline $4,1-2$ & I 74 & 9.7 & 27 & 21,5 & 160 \\
\hline 4,23 & 26 & 9.37 & 96 & 21,13 & 26 \\
\hline 4,44 & 26 & 10,12 & 122 & $23,1 \mathrm{I}$ & 26 \\
\hline 6,47 & I 21 & I I , 26 & I 33 & 23,14 & I 52 \\
\hline 6,49 & I2I & I 2,33 & I5I & 24,2 & 68 \\
\hline $7,3^{8}$ & 69 & 12,36 & 146 & 25,4 & 26 \\
\hline 7,49 & 69 & $12,4^{6}$ & I 33 & 26,13 & 91,92 \\
\hline 7,50 & 26 & 12,47 & I $2 I$ & 26,25 & 113 \\
\hline 8,21 & I 2 I & I $3.4-5$ & I67 & 28,26 & 152 \\
\hline 8,30 & I 76 & I $3, I_{7}$ & 26 & & \\
\hline $8,4^{8}$ & 26 & 13,32 & 88 & Rom. & \\
\hline 9,29 & 26,73 & 14,30 & 96 & 1,26 & I I I \\
\hline 9.33 & $\begin{array}{l}\text { I3I } \\
26\end{array}$ & 17,12 & $I_{4} 6$ & 7,24 & I I I \\
\hline $9,6 \mathbf{r}$ & $\begin{array}{l}26 \\
106,120\end{array}$ & 18,6 & 106 & 8,33 & I $5^{\circ}$ \\
\hline 9,62 & 106,120 & 20,12 & I $3 I$ & 9,33 & 133 \\
\hline IO, I & I 5 & $20, I_{4}$ & 106 & 10,5 & 87 \\
\hline 10,6 & 146 & $2 I, 6$ & $I 6 I$ & IO, I I & 133 \\
\hline 10,27 & 144 & & & 12,18 & 102 \\
\hline 10,37 & IOI & Act. & & 15,14 & 26 \\
\hline II, 7 & 26 & $I-I 5$ & 12 & 16,14 & 32 \\
\hline I 1,28 & I2I & 1,20 & 64 & & \\
\hline 12,19 & I 39 & 2,5 & 26 & I Cor. & \\
\hline 12,22 & I 39 & 2,17 & 152 & 6,16 & 75 \\
\hline 12,23 & 139 & 2,27 & 26 & 7,12 & 121 \\
\hline 12,32 & 88 & 2,29 & 26 & 7,13 & 121 \\
\hline I 3, I I & 26 & 2,39 & 26 & 10,13 & 91 \\
\hline 14,18 & 160 & 3,25 & 146 & $I T, I 3$ & 26,88 \\
\hline 14,33 & 133 & 4,5 & 26 & & \\
\hline 16,8 & 91 & 4,17 & 152 & 2 Cor. & \\
\hline 16,9 & I II & 5,28 & 152 & $I, 8$ & 91 \\
\hline
\end{tabular}




$\begin{array}{ll}3,1 & 68 \\ 6,14 & 26 \\ 7,1 & 142 \\ 9,12 & 26 \\ 12,1 & 26 \\ 12,4 & 26 \\ 12,6 & 91 \\ 12,13 & 91\end{array}$

Gal.

$\begin{array}{ll}1,14 & 91 \\ 1,22 & 26 \\ 3,12 & 87 \\ 4,22 & 131 \\ 4,24 & 26\end{array}$

Eph.

$\begin{array}{ll}2,3 & 146 \\ 2,16 & 88 \\ 3,20 & 91 \\ 4,22 & \text { I I I } \\ 4,25 & 96 \\ 4,29 & 133 \\ 5,5 & \text { I 33 } \\ 5,31 & 75\end{array}$

Col.

$$
\begin{array}{ll}
\text { I,I3 } & \text { III } \\
2,15 & 88 \\
2,23 & 26 \\
3,12 & 150
\end{array}
$$

I Thess.

3.5 75

2 Thess.

$2,3 \quad 146$

I Tim.

$4,7 \quad 26$
Tit.

I,I I 50

2 Joh.

9

133

Philem.

2 I

9I

Judao

I5

I 2

Hebr.

$4,12 \quad 9 I$

$5,12 \quad 26$

$5, I_{3}$ II 3

$9,14 \quad 142$

I2, I4 II3

Jac.

1,25 I I I

$2,15 \quad 26$

$3.9 \quad 87$

$3,15 \quad 26$

5,17 I52

I Petr.

$I, 14 \quad 146$

$4,6 \quad 124$

$5,12 \quad 26$

2 Petr.

I,20 I 33

$2,8 \quad I I 3$

2,10 I05, III

$3,3 \quad 152$

I Joh.

2, I6 I33, I34

2,2 I $\quad$ I33

$3,6 \quad 133$

$3,9 \quad I 33$

3,10 $\quad 133$

$3,15 \quad I 33$

$4.3 \quad 133$

$5.8 \quad 75$

$5,18 \quad 133$

$2,23 \quad 152$

I I, I $7 \quad 98$
Apoc.

I,8 26

I,I2 96,97

$2,5 \quad 26$

2,16 98

$3,2 \quad 26$

$4,1 \quad 96,97$

$4,6 \quad 135$

$5,7 \quad 5^{8}$

$6,6 \quad 135$

$7.14 \quad 5^{8}$

$8,5 \quad 5^{8}$

$8,8 \quad 135$

$9.7 \quad 135$

$9,17 \quad 26$

I0,8 27, 86,97

12,7 98

12,1798

$13,4 \quad 98$

$15,2 \quad 135, I_{3} 6$

I 7,I $\quad 96,97$

$17,14 \quad 98$

$18,22 \quad I 33$

I9, I I35

$19,2 \quad 84$

$19.3 \quad 5^{8}$

I9,6 I 65

I9, $19 \quad 98$

$21,9 \quad 96,97$

2I, I5 96,97

22,3 I33

\section{APOCRYPHES}

cf. P. 53

Apoc. Mos. 58, 68, 69, 89, 91, 107. $146,153,154$

I Hen. 58, 67, 86, 87, 91, 101, 102. I07, I I0, I33, 146, 149, I54, 174, 179

Test. Abr. A 58, 68, 91, 92, 107, 131, 150, 152, 154

Test. Abr. B I07, I54, 179

Jos. et As. 58, 91, 101, 105, 107, 131, I34, 135, I50, I54, I65
Test. XII Patr. 58, 68, 69, 75, 76, 84, 86, 9I, IOI, I02, 105, 107, I15, I35, 140, I4I, I44, I49, I50, I52, I54, 155, 165

Par. Jer. 58, 98, 107, I50, I52, 154 Apoc. Bar. 107, $115,154,155,165$, 167

Vit. Proph. 58,150

Apoc. Esdr. 68, 89, 91, 149, 150, 165, 179 
Test. Job 58, 68, 98, I01, 107, 119, Acta Pauli et Th. 160, 165 $146,150,160,165,179$

Acta Pauli 91, 107, 149, 165

Mart. Pauli 165

Protev. Jac. 106, I31, 140, I49, I54, I 55, 165, 174, 178, I 79

Ps. Barn. I0, 30, 65, 77, 91, 107, III, I I 2, I I 3, I4 I, I46, I49, I 52, I79

Ev. Petri 58, 69, I07, I08, II5, I65, I 79

3 Cor. $I_{46}$

Apoc. Petri 107, 146

Did. 10, 30, 31, 65, 91, 128, I31, 133, $165,172,175,178,179$

\section{AUTRES TEXTES}

Abgar, Épitre d' 96

Achille Tatius 53,69,89, 124, 126, I6I

Adrien $65,126,138$

André, Martyre d' 160

André, Passion d' 77

André et Matthieu, Actes d' 92, 96

Antigone de Caryste 95

Apollodore 95, I 49

Apollonius Dyscole 132

Apophthegmata Patrum 96

Appien 53.62

Apulée 154

Aristee, Lettre d' 89, I6I

Aristophane 104, 132, 136, 143

Aristote 130, 149

Athénée 95

Augustin 84

Aulu-Gelle 68

Callinicos 152

Canon de Muratori 31-35

César 68, 132

Chariton 69

Chio 104

Claudien I6o

Clément d'Alexandrie 144,175

Clément de Rome 49, 77, IO2, III, I I 2, I 4I, I44, I49, I5

2 Clément 65, I I I, 142, 144, 150

Constitutions Apostoliques I 75

Cornutus 88

Cyprien I7I

Defixionum Tabellae 88

Démosthène 79, I $17,149,151,161$

Denys d'Halicamasse 99, I24, I6I, I80

Diodore de Sicile 86, 106, I61

Diognète, Épitre a 16

Dion Cassius 163

Dioscuride 164

Élien I5I
Épictète 53, 107, 124, 134, 143, I 54 , I6I, 179

Epiphane II5, I16, I36, I75

Erotianos 164

Eschyle I10, II4

5 Esdras 50

Ésope 179

Ésope, Vie d' 52, 179

Euripide 84, 110, 139, I38, 147, 160

Eusèbe 49, I60, 16r

Ézéchiel le tragique 43

Flavius Josèphe 68, 69, 143

Flore I80

Gaius II9

Galien I 36, I 5 I

Gnomon de l'Idiologue $\quad$ I18

Hegemonius 96

Hégésippe 49

Hérodien le grammairien 167,168 , I 79

Hérodien l'historien $\mathbf{8 9}$

Hérodote $71,75,77,107,138$

Héron 83

Himérios I 26

Hippocrate $15 \mathrm{I}$

Historia Alexandri Magni 52

Historia Monachorum 136

Homère 79, 82, 104, 105, I23, I37. I 43,145

Horace 8, 13

Ignace d'Antioche 87.92

inscriptions 50,89, 92, 95, 101, 104, 106, $110,114,126,139,141,149$. I $60,167,168$

Irénée 34

Jean, Actes de 136,149

Jean Chrysostome 84, 152, 165

Jérôme 49

Julien I 26

Justin l'apologiste $\quad \mathbf{4 4}$ 
Justin l'historien 160

Lévi, Testament de 67

Libanios 163

Longus 68,69

Lucien de Samosate 52, 89, I06, 135. I5I

Lucrèce 68, 126

Lysias $\mathbf{8 7}$

Macarius 105

Malalas 95

Marcellinus 1 Io

Marc-Aurèle 143

Martial 50

Matthieu, Martyre de 77,136

Ménandre 145

Métrodore I6I

midrashim 68, 177

Mishna I70

Moïse, Assomption de 160

Nonnos 96

Oppien 93

Origène 31, 32, 34, I03, I I 5, I 75

ostraca I67

Ovide $97,134,154,160$

papyrus 95, 97, 104, I06, II5, I18, I 30, 132, 136, I47-148, 152, 162, 167 . 179

Paul, Apocalypse de 92, 96

Pausanias 53, 130, $16 \mathrm{I}$

Périple de la Mer Érythrée 164, 167

Perpétue, Passion de 68, 96, I02, 136

Pétrone I6o

Phèdre II 9

Philippe, Actes de 89, 92, 136

Philogelos 96

Philon 43, I4I

Philostrate 89

Phrynichos 95

Pierre d'Alexandrie $\quad 175$

Pierre et Paul, Actes de 96

Pierre et Paul, Martyre de 92, 160

Pierre et Paul, Passion de 160

Pilate, Actes de 136,152

Pindare 89, 1 10

Platon 72, 75, 79, 82, 124, 132, 151 , I6I

Plaute I37

Pline le Jeune 119, 136

Plutarque 53, 180
Polybe 58, 6I, 68, 69, 89, 106, 107, I24, 161

Polycarpe Io

Polycarpe, Martyre de 149

Priscien 132

Properce 132

Ps. Basile I8I

Ps. Callisthène 98

Ps. Cébès 47, 104

Ps. Hésiode 136

Ps. Ignace I75

Ps. Lucien 89, 104

Ps. Phocylide 43

Ps. Scymnus 106

Ps. Tertullien $\mathrm{I} 60$

Quinte-Curce I60

Quintilien I60

Qumran I2, I6, 67, 88, I10, I47, I50, 176

Sénèque le philosophe II3, I60

Sénèque le rhéteur 160

Sibyllins, Livres 43

Simonide d'Amorgos 139

Socrate l'historien 175

Sophocle 72, 82, I05, II3, II4, I24, I37, 145,151

Stace 166

Suétone 93

Talmud de Babylone 49, 174

Talmud de Jérusalem $67,68,176$

Targums 67

Tertullien $160,169,171,174$

Thémistios 126

Théocrite 143

Théodore de Mopsueste I38, 153

Théodoret 126

Théognis 75

Théophraste I43

Thomas, Actes de 68, 96, I16, I47, I6o, 167

Thomas, Miracles de 160

Thomas, Passion de 160

Thucydide 89

Timothée et Aquila, Dialogue de II 6

Tite-Live I80

Végèce 160

Virgile I3, I54, I66

Vitae Patrum 67

Xénophon 6r, 68, 69, 94, 113,123 , I 24, I 30, I 35, I 36, I 39, I43 
ablatif latin II, 76, I23

accusatif prépositionnel 89

accusatif de relation $9,11,123$

adjectif remplacé par le génitif I IoII3, I45

- substantivé 124, 148-150

- verbal I47-150

aoriste $22,57-60,61,66,74,80,185$ Apocalypse 28-29, 64, 84, 97, I28, I 79, 182

apocryphes $38-39,53-54,5^{8}$ et pass.; 186

aramaïsmes $43-44,66,70,184 ;$ cf. sémitismes

article I6, I42-I44, I46-I47, I48, I49 asyndète $5,130,132$ atticisme $46-47,52,58,64,70,123$, I62-163, $180-181$

attraction du relatif $4 \mathrm{I}$

Canon de Muratori 31-35

Clément de Rome 7, 33-34, 48, I86

comparatif 89-94

construction ad sensum ${ }_{13}^{8}$

datif de la figure étymologique 86 , I 50-1 53

- instrumental 86-88

- avec oúal I8o

- de relation 9-II, 82, I 23

- sociatif 94-100

deux voies 30-3I

diaspora $38,42-44,48-49,177,179$

dualité 130

ellipse I49, 175

emprunts $2,5,6,36-37,44,45,53$, $56,155,163-182,184,185$; cf. importation

épexégèse 23.92

Flavius Josèphe $3^{8}, 66-67,68,69,70$ formules de généralisation 27

futur 4I, 73. 74 génitif absolu 9, 23

- auctoris 147-150

- de comparaison 92-94

- "hébraiqque" 9, I Io-I I 3, I45, I84

- inhaerentiae $1 \mathrm{I} 2$

- partitif 123-I24, I84

- de qualité i Io

grec biblique 8-10, 38-45, 50, 53-54, 64 et pass.; 185,186

- non-littéraire $38,46,58,64$, I04, I 16, I I 7, I 8 , I 44, I6I, I6 4

- post-classique II, 45-47, 58-59 et pass.

hébraïsmes $43,77,150-153,180,184$; cf. sémitismes

hyperbate Ioo

impératif 25,27

importation 36-37, 53, 69, I76; cf. emprunts, interférence

infinitif $25,76-77,83,87$, I I 6-1 22

- absolu en hébreu 15I, I 84

interférence $36-38,55-56$; cf. aramaïsmes, hébraïsmes, importation, latinismes, littéralisme, sémitismes, substitution

interjection $153-155,179-182,184$

Jean $26,64,79,128$

jeûne I $4^{0}-I_{4} 2, I 68-179$

Juifs, langues employées 9-ro, 38-50, 177

langue de groupe $38-45$, I49, I75

- stylisée $4{ }^{I-42}$

latinismes I-8, II-I 2, 36, 45-47, 50-57 et pass.; 122-125, I60-162, I $83-185$

littéralisme 39-40, 53, 55-56, 184; cf. interférence

littérature rabbinique $49,67-68$, I 22, I73, $174-175$ 
Luc $64,72,76,77,79,80,128,152$, 160,164

-, evangile 179

-, Actes 12

Lucien 52

Matthieu 30-31, 50-51, 64, 79, 128, 143, 149, 179

négation 25, 132-134, 184

nom 32, 163, 165-167, 168

nom de nombre 115

Nouveau Testament 9, 24-26, 39,

53-54, 58 et pass.; 186

optatif 9, 10, 62-66, 184

ordre des mots Io, $16,23,146,185$

Palestine 9-10, 32, 42, 170, I76

parataxe 9, 10, 23, II6-122, 184

parfait $57-60,184$

participe 9, 23, 25, 70-74, 78-80, II6I 22, I 47-1 50, I5I-I 53,182

particules $16,18,56$

passif 124, 125

Pasteur d'Hermas, auteur(s) 7, Io, I9-31, 33, 185-186

-, -, connaissance des langues 6-14. 47-51, 55-56, 185

-, date $7,19-20,31-35,186$

- , éditions $3-5,15-18$

-, lieu d'origine 6-7, 12-14, 24, 31-32, 50,183

- et le Nouveau Testament 7, 9-10, I21, 182, I86

-, rétroversion 2-5, 7-8, 12

-, sources 8, 9, 10, 12-13, 20, 28-31, $47-48,98$

-, style I8-19, 23-3I, 33-34, 47, I20I $21,142,152,153,178-179,182$

-, tradition manuscrite $2-5,7,15-18$, 60,63 et pass.; 121

- , versions $I, 3-4,5,15-16,67,76$ et pass.

-, vocabulaire $9,19,23,27,29-30$, $31,155-182,184,185$

-. vulgarismes" 15-18 patronymique 145

Paul 32, 64, 128

Pentateuque 40, 41, 152, 153

périphrases 64, 66-74, 137, 151, 183, 184

pluriel 126-129, 184, 185

plurilinguisme 9-10, 38, 43, 55-56

plus-que-parfait $58,60-62,178,184$

Polybe 69

prépositions 16, 82-109, 183-184

présent historique $25,4 \mathrm{I}, 80, \mathrm{I} 55$, I 85

- des verbes signifiant aller $4 \mathrm{I}$

pronoms I6, 4I, 87-88, III, 124, I37138, 142, 144

proposition infinitive $9,87,124-125$

questions rhétoriques 27

Qumran 12, 68, 145, 147, 150, 173

redoublement distributif $9,113-116$, 184

Rome 6-7, 12-13, 24, 31-32, 48-51, $52-53,69,177,179,183$

sémitismes 2, 8-1 2, 36, 38-45, 51-56

Septante Io, 38-45, 53-54, 58 et pass.; 186

Simonidès, Konstantinos I-5, 8

statio $6,141,168-179,184$

-, dossier patristique 169, I71, 172, I74, 175

style narratif $57-59,68-70$, I53-I55, 178,184

subjonctif $\quad 63-65$

substitution $36-37,45,68$, I76; cf. interférence

suffixes $25,37,144$

superlatif $25,92-94,185$

Synoptiques $42,54,66,68,69,70,79$

verbes signifiant accompagner 95

- - aller 4I, I05

- - combattre 95,98

- - dire 25, 4I, 69, 77-81, 183

- - mélanger 95

- de sentiment 83,87

vocabulaire $43,55-56$ 
Het woord ó $\mu \alpha \lambda$ ó $\varsigma$ kan in de Herder van Hermas niet als een 'significant term of repentance' beschouwd worden. In verband daarmee dient ook het vermoeden van Michaels, dat Hermas' gebruik van ó $\mu$ גós op contact met de Ebionieten wijst, afgewezen te worden.

Cf I $\mathrm{R}$ Michaels, The 'Level Ground' in the Shepherd of Hermas, Zeitschnft fur die neutestamentliche Wissenschaft 59, I 968 , blz $245-250$

\section{II}

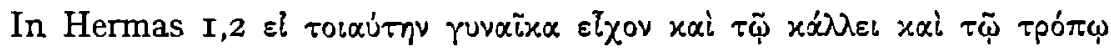
duidt $\tau \tilde{\omega} \tau \rho \delta \pi \omega$ niet het uiterlijk waarneembaar gedrag, maar het karakter van Rhode aan. Het is een toevoeging aan het oorspronkelijke verhaal, en dient om het amoureuze element daarin te temperen en Hermas' handelwijze te verontschuldigen.

\section{III}

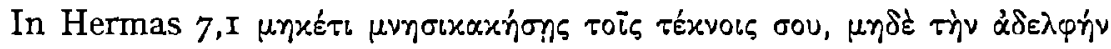

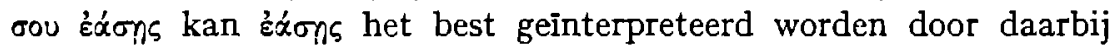
$\mu \nu \eta \sigma i x \alpha x \tilde{\eta} \sigma \alpha \iota$ aan te vullen.

\section{IV}

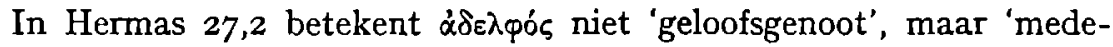
mens'.

Tegen L Pernveden, The Conccpt of the Church in the Shepherd of Hermas, Lund I966, blz. 240 n I 
In Hermas 32,I bevestigt de lezing xai van de Codex Athous de uit de antieke vertalingen gereconstrueerde lezing xúpte.

\section{VI}

Het zoeken naar aramaismen in de evangeliën is nutteloze moeite indien men zich niet zorgvuldig vergewist van de frequentie van de verschijnselen in kwestie in het Grieks van de vroege keizertijd.

\section{VII}

De 'fouten' tegen het Grieks in de Openbaring van Johannes bewijzen niet dat de auteur werd beinvloed door een hem meer vertrouwde Semietische taal of dat de Griekse tekst de vertaling is van een Semietisch origineel.

Tegen G. Mussies, The Morphology of Koine Greek as Used in the Apocalypse of St John, Leıden 1971, blz. 31 I-312.

\section{VIII}

In het Proto-evangelie van Jacobus II,3 kan de lezing van de Papyrus

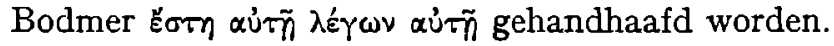

\section{IX}

$M \varepsilon \tau \dot{\alpha}+$ genitivus wordt niet eerst vanaf de vierde eeuw na Christus, maar reeds vanaf de derde eeuw voor Christus instrumentaal gebruikt.

Tegen J. Humbert, La disparitron du datif en grec, Parıs I930, blz. I99.

\section{$\mathrm{X}$}

In Vergilius Aen. 4,256 is het voorkomen van volabat in de toepassing van een vergelijking, één regel na het voorkomen van volat in de vergelijking zelf, een reden te meer om de passage $4,25^{6-25^{8}}$ als een alternatieve versie te beschouwen. 
Het gebruik, in woordenboeken op beperkte corpora van teksten, om volledigheid in het opgeven van plaatsen met een teken aan te geven, verdient overgenomen te worden in grammatica's op dergelijke corpora.

\section{XII}

Bij het schoolonderzoek voor de vakken Grieks en Latijn dient het begrip van de gelezen autcurs mede getoetst te worden met behulp van teksten die niet te voren behandeld zijn.

\section{XIII}

De suggestie van K. L. Poll, dat de verwezenlijking van de Contourennota het Nederlandse onderwijs naar nieuwe middeleeuwen zal voeren, geeft blijk van onderschatting van het onderwijs in de middeleeuwen.

Cf. NRC - Handelsblad 30 apnl 1976, Cultureel Supplement blz. 6 
Stellingen behorende bij Antonius Hilhorst,

Semitismes et Latinismes dans le pasteur d'Hermas

Nijmegen, 1976. 

